University of Louisville

ThinkIR: The University of Louisville's Institutional Repository

Electronic Theses and Dissertations

$5-2012$

\title{
Sulfur oxygenation of ruthenium-dithiolate nitrile hydratase mimic enhances ligand lability and hydrolysis activity.
}

Cesar Antonio Masitas Castillo

University of Louisville

Follow this and additional works at: https://ir.library.louisville.edu/etd

\section{Recommended Citation}

Castillo, Cesar Antonio Masitas, "Sulfur oxygenation of ruthenium-dithiolate nitrile hydratase mimic enhances ligand lability and hydrolysis activity." (2012). Electronic Theses and Dissertations. Paper 223. https://doi.org/10.18297/etd/223

This Doctoral Dissertation is brought to you for free and open access by ThinkIR: The University of Louisville's Institutional Repository. It has been accepted for inclusion in Electronic Theses and Dissertations by an authorized administrator of ThinkIR: The University of Louisville's Institutional Repository. This title appears here courtesy of the author, who has retained all other copyrights. For more information, please contact thinkir@louisville.edu. 


\title{
SULFUR OXYGENATION OF RUTHENIUM-DITHIOLATE NITRILE HYDRATASE MIMIC ENHANCES LIGAND LABILITY AND HYDROLYSIS ACTIVITY
}

\author{
By
}

César Antonio Masitas Castillo

B.S., Universidad Peruana Cayetano Heredia, 2000

M.S., University of Louisville, 2010

\author{
A Thesis \\ Submitted to the Faculty of the \\ College of Arts and Sciences of the University of Louisville \\ In Partial Fulfillment of the Requirements \\ For the Degree of
}

Doctor in Philosophy

Department of Chemistry University of Louisville Louisville, Kentucky

May 2012 
SULFUR OXYGENATION OF RUTHENIUM-DITHIOLATE NITRILE HYDRATASE MIMIC ENHANCES LIGAND LABILITY AND HYDROLYSIS ACTIVITY

By

Mr. César Antonio Masitas Castillo

B.S., Universidad Peruana Cayetano Heredia, 2000

M.S., University of Louisville, 2010

A Thesis Approved on

March 27, 2012

By the following Thesis Committee:

Thesis Director. Dr. Craig A. Grapperhaus

Dr. Robert M. Buchanan

Dr. Mark E. Nobie

Dr. Christopher T. Burns

Dr. Moises A Carreon 


\section{DEDICATION}

This dissertation is dedicated to my parents

Mrs. Beatriz Castillo Rojas

and

Mr. Ernesto Masitas Carmelo

to all my brothers

Flavio, my twin Rafael, Gustavo and Alejandro

and to all my friends and family around the world.

Spiritus ubi vult spirat 


\section{ACKNOWLEDGEMENTS}

I would like to thank my advisor, Dr. Craig A. Grapperhaus, for his guidance and patience over the course of my graduate studies, and for the opportunities and time to grow up academically and professionally outside the walls of the chemistry department.

I would like to thank several other faculty members at University of Louisville, my more deep thanks to the committee members Dr. Buchanan, Dr. Noble and Dr. Burns for their assistance with my progress toward my doctorate studies and educational guidance. Special thanks to Dr. Carreon for his evaluation of my dissertation.

I want to express my gratitude and many thanks to the personal of the Chemistry Department at University of Louisville for their helpful in my daily research work. To Dr. Mark S. Mashuta manager of crystallography facility for his hard and detailed work and enlighten conversations. I am thankful to Dr. Kozlowski and his student Manoj Kumar for their collaboration and guidance in the analysis of the computational data in this manuscript. Also I want to thank Dr. Neil Stolowich for his time, assistance with NMR spectroscopy and kinetic studies set up discussion. I cannot forget the assistant of Dr. William Richmond for his technical expertise in GC-MS and Tracy Larson in the stockroom for his friendship and help.

Many students help me through my doctoral studies in the chemistry department and for this reason they deserve special thanks. Inside the Grapperhaus' Group, Martin G.

O’Toole, Jinlan Cui, Kagna Ouch, Larry Wood, Rajat and Davinder Kumar, It was a 
pleasure share experiences with this group in these last 5 years. I want to express my specials thanks to Maria, Jessica Koehler, Marek Wlodarczyk and Samuel Asem for their truly and deeply friendship and assistance during my time in Louisville.

I would like to thanks my parents, towards their effort and dedication they allow me to move forward in my professional career. Special thanks to all my brothers for their time and productive conversation in these past years. 


\begin{abstract}
SULFUR OXYGENATION OF RUTHENIUM-DITHIOLATE NITRILE HYDRATASE

MIMIC ENHANCES LIGAND LABILITY AND HYDROLYSIS ACTIVITY

Mr. César Antonio Masitas Castillo, M.S., University of Louisville.
\end{abstract}

March 27, 2012

Nitrile hydratase (NHase) is a metalloenzyme that contains a non-heme iron (III) or non-corin cobalt (III) in a non-redox active role. NHase is used as biocatalyst in the industry for the hydrolysis of nitriles to amides. The trivalent metal ions are sixcoordinate with the ligand sphere made up of three cysteines, two amide nitrogens, and a water/hydroxide or nitrile substrate. Interestingly, the $\mathrm{N}_{2} \mathrm{~S}_{3}$ active site contains two posttranslationally modified cysteine residues, resulting in an unusual thiolato (RS-), sulfenate $(\mathrm{RS}(\mathrm{O})-)$, sulfinate $\left(\mathrm{RS}(\mathrm{O})_{2}{ }^{-}\right)$donor set. Synthetic work to mimic the unusual donor set by direct oxygenation of a metallodithiolate precursor with dioxygen has been reported by our research group. In this research we have investigated the $\mathrm{N}_{2} \mathrm{~S}_{3}$ ligand 4,7bis-methylmercaptopropyl-1-thia-4,7-diazacyclononane $\quad(\text { bmmp-TASN })^{2-}$ to systematically probe the metal's influence on sulfur reactivity. As result of this research the ruthenium complex (bmmp-TASN)RuPPh $(1)$ reacts with dioxygen to yield (bmmp$\left.\mathrm{O}_{2}-\mathrm{TASN}\right) \mathrm{RuPPh}_{3}$ (2), (bmmp-O $\left.-\mathrm{TASN}\right) \mathrm{RuPPh}_{3}$ (3) and (bmmp-O $\left.-\mathrm{TASN}\right) \mathrm{RuPPh}_{3}$ (4). By careful control and optimization of the reaction conditions, high yields and functional synthetic models are obtained. Catalytic activity is reported in 1,2 and 3 with $85 \pm 11$ turnover in 18 hours for the formation of benzamide for compound 3. 


\section{TABLE OF CONTENTS}

PAGE

ACKNOWLEDGEMENTS .......................................................................................

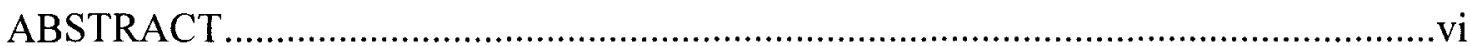

LIST OF FIGURES …………………................................................................

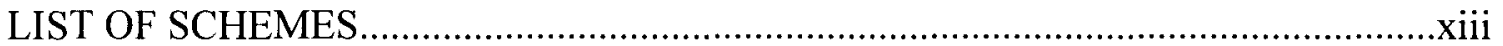

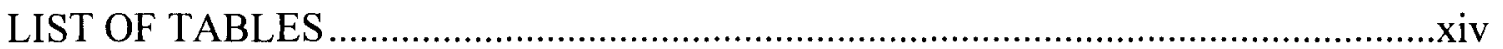

\section{CHAPTER}

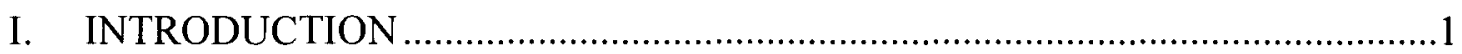

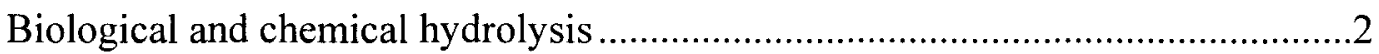

Nitrile hydratase in biological systems ..........................................................

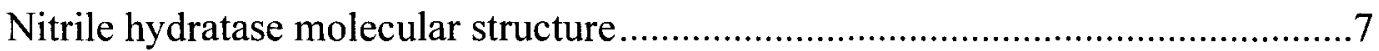

Mechanism insight based on structural information in the enzyme …...................

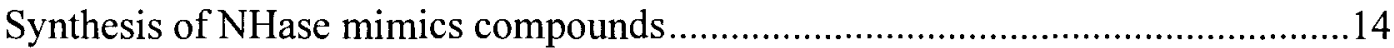

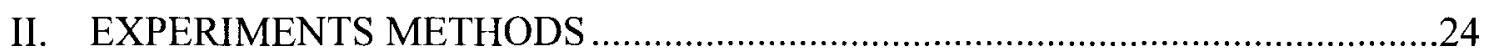

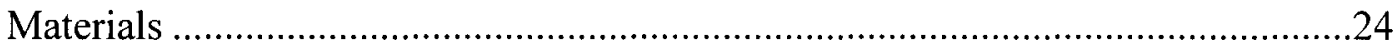

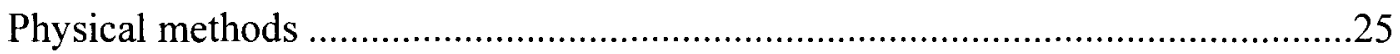

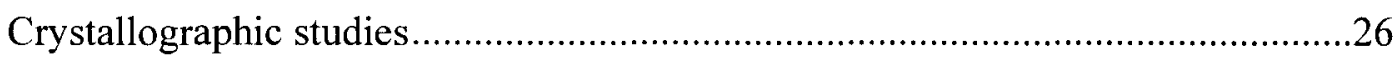

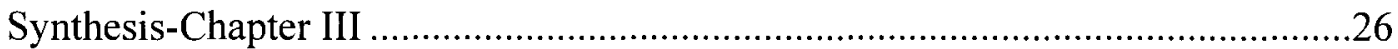




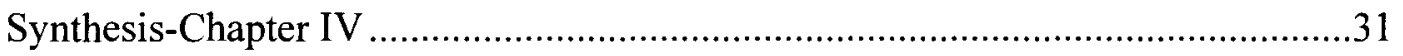

Computational methodology ...............................................................................

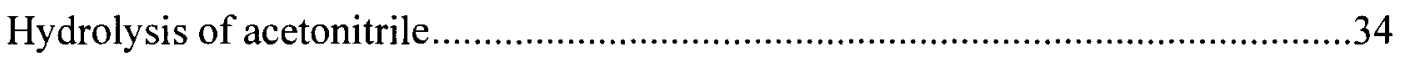

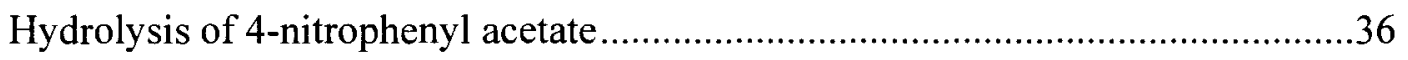

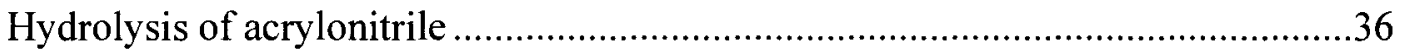

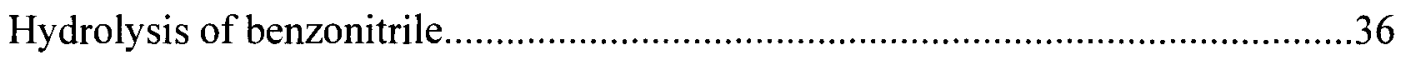

III. CONTROLLED SULFUR-OXYGENATION OF RUTHENIUM (II)

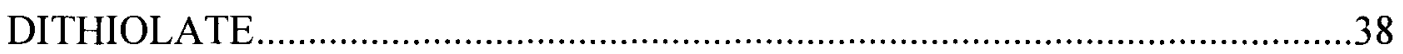

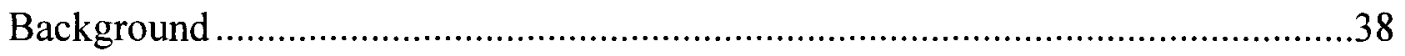

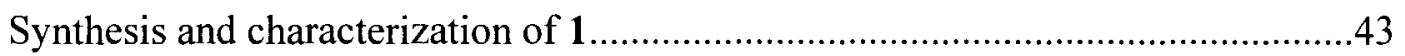

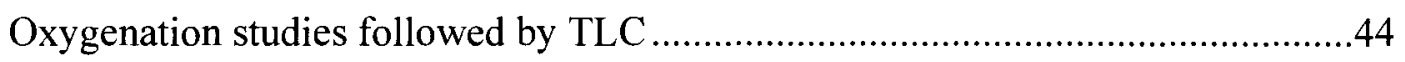

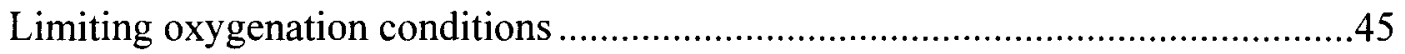

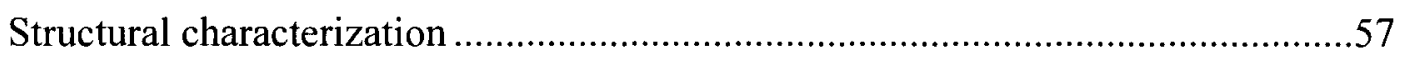

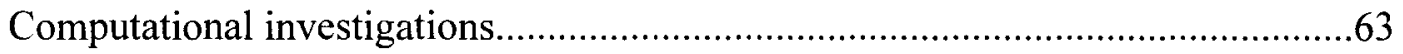

Sulfur oxygenation effect on the complex (bmmp-TASN)RuPPh 3

probed by multi-edge X-ray absorption spectroscopy ……….............................67

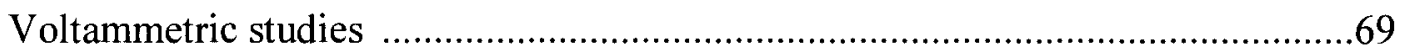

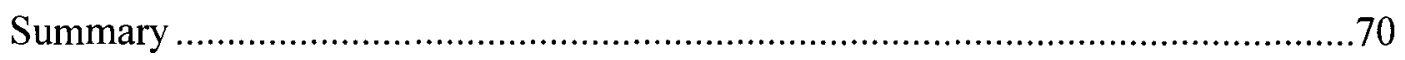

IV. REACTIVITY BEHAVIOR OF (bmmp-TASN)RuPPh

Substrate exchange in bmmp-TASN models preliminary results............................74

Phosphine exchange in the metal complexes (bmmp-TASN)RuPPh

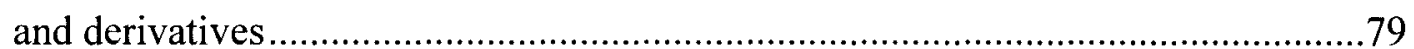

X-ray structure analyses of (bmmp- $\left.\mathrm{O}_{2}-\mathrm{TASN}\right) \mathrm{RuPMePh}_{2}(\mathbf{8})$, 
(bmmp- $\left.\mathrm{O}_{3}-\mathrm{TASN}\right) \mathrm{RuPMePh}_{2}(9)$, their analogues precursors and

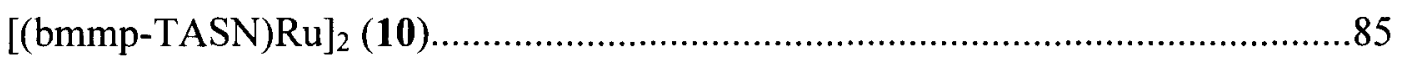

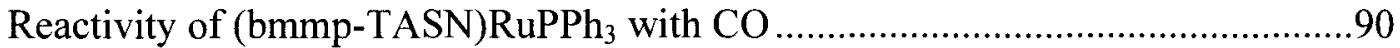

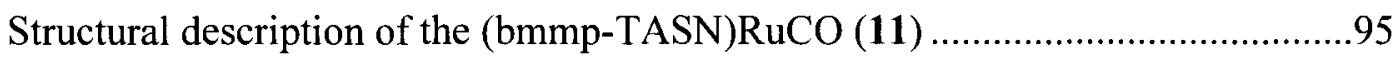

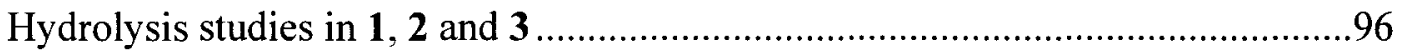

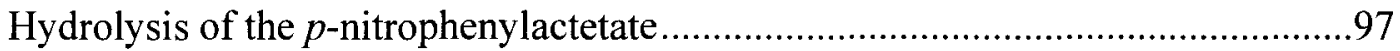

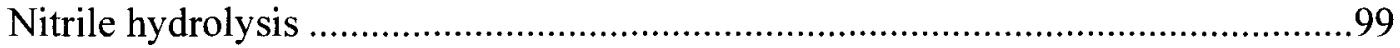

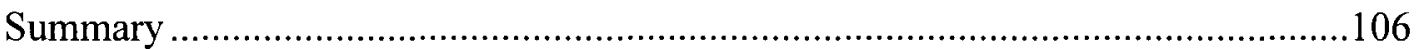

$\mathrm{V}$ CONCLUSIONS AND FUTURE DIRECTIONS ................................................108

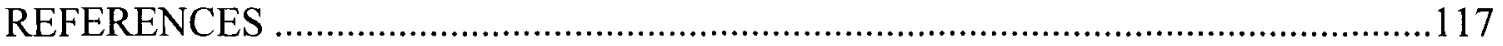

APPENDIX

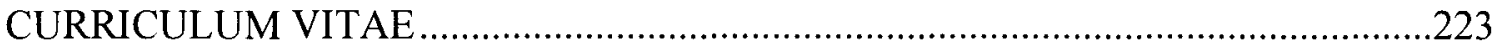




\section{LIST OF FIGURES}

FIGURE PAGE

1. Nitrile hydratase hydrolysis reaction ........................................................

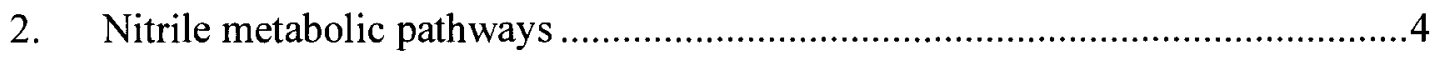

3. Post-translational NHase active site..............................................................

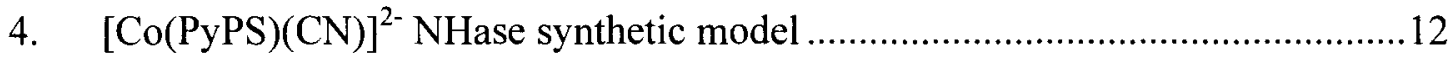

5. NHase models that modulate charge and affect the affinity of the apical

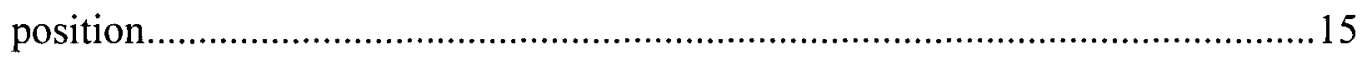

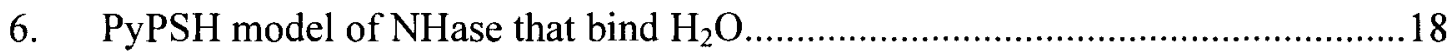

7. Thiolate sulfur oxygenation control nitric oxide photolability ...........................19

8. Synthesis scheme for the complex $\left[\mathrm{CoIII}\left(\mathrm{L}-\mathrm{N}_{2} \mathrm{SOSO}\right)(\mathrm{tBuNC})_{2}\right]^{-}$and

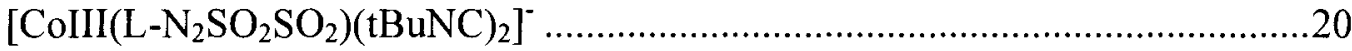

9. Comparison of NHase active site and bmmp-TASN model................................21

10. Spin state of iron influences Fe-SR reactivity with $\mathrm{O}_{2} \ldots \ldots \ldots \ldots \ldots \ldots \ldots \ldots \ldots \ldots \ldots \ldots \ldots . . .23$

11. Active site of NO-inactivated Fe-containing NHase …........................................38

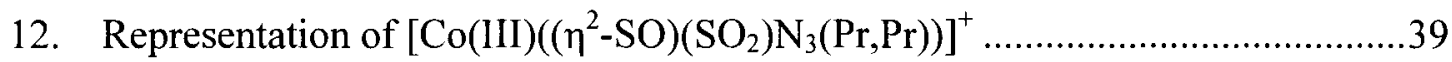

13. Synthesis and characterization of nickel sulfur oxygen derivatives ....................41

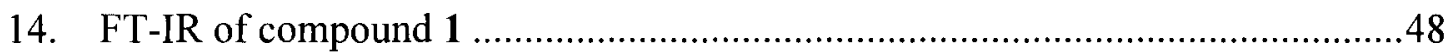

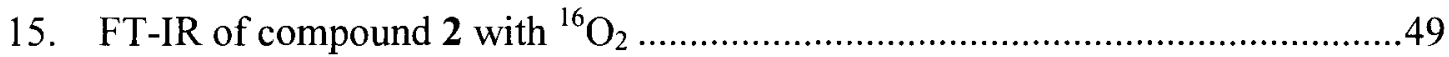

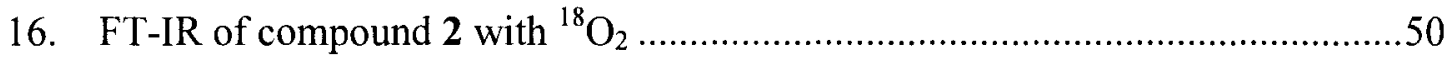

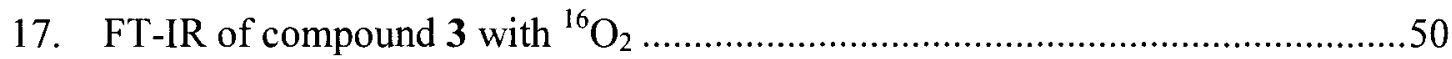


18. FT-IR difference spectra highlighting the ${ }^{18} \mathrm{O}_{2}$-sensitive sulfinato stretching frequencies

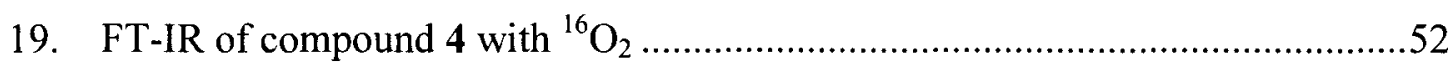

19. Calculated isotopic distribution for compound 2 using ${ }^{16} \mathrm{O}_{2} \ldots \ldots \ldots \ldots \ldots \ldots \ldots \ldots \ldots \ldots . . .52$

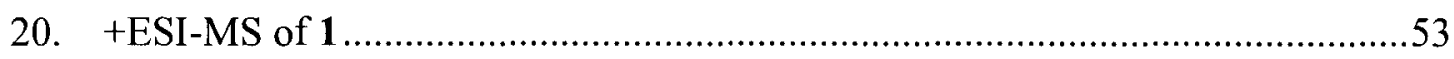

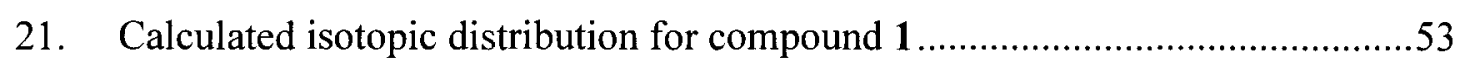

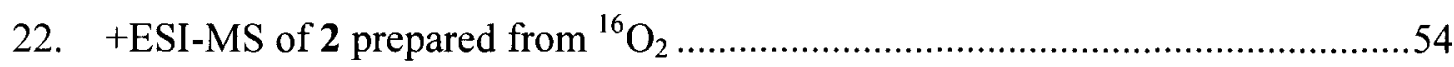

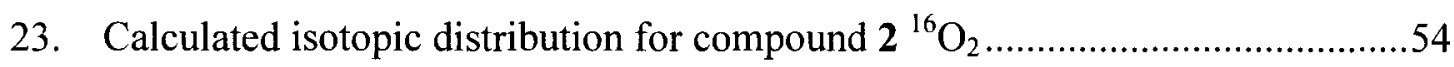

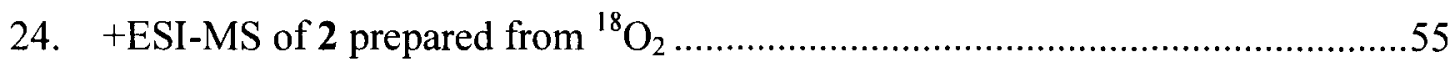

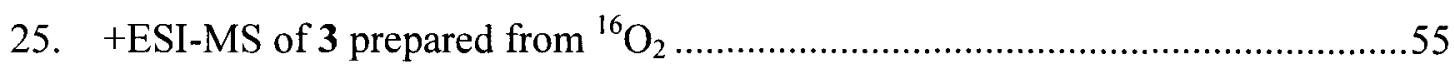

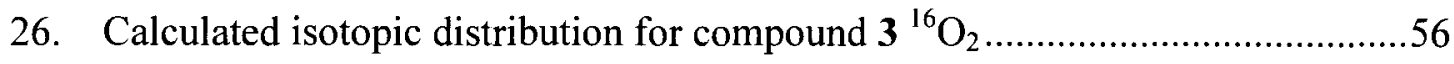

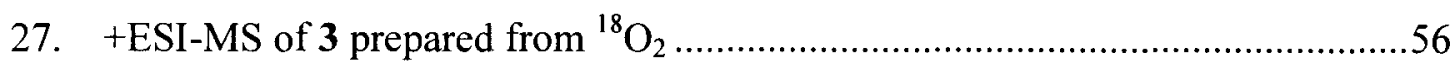

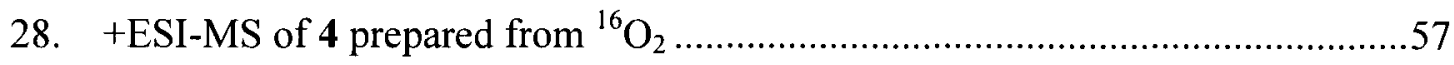

29. Calculated isotopic distribution for compound 4...........................................57

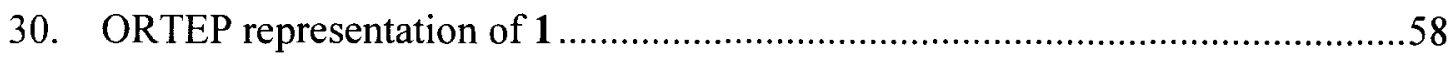

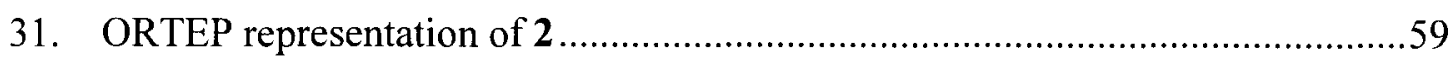

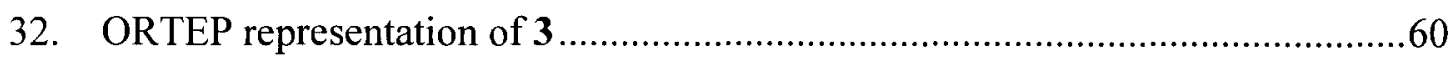

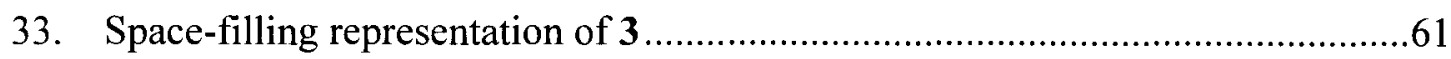

34. Plot of optimized (BP86/LANDZ+6-31G(d)) Ru-L bond distance versus

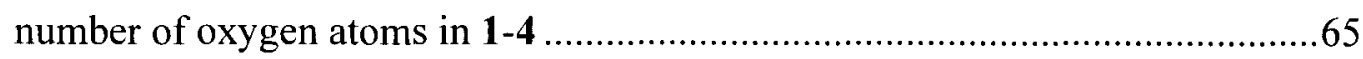

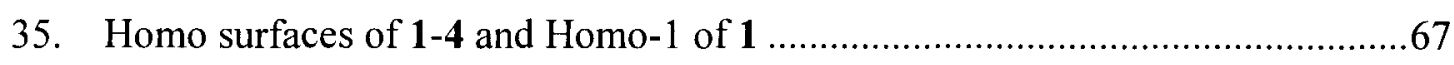

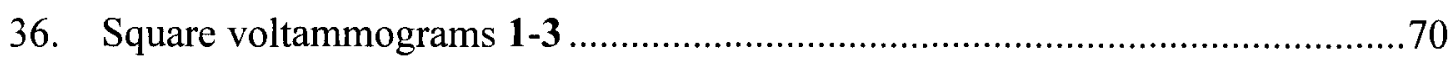

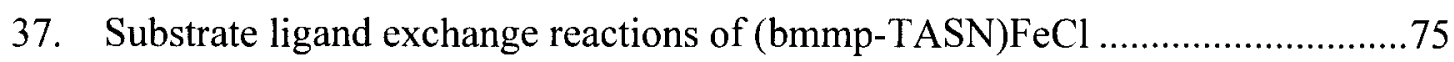


38. [(bmmp-TASN)Fe]Tf is the first NHase model complex that coordinates water and nitrile

39. Sulfur-oxygenation increases the lability of $\mathrm{PPh}_{3}$ allowing a series of

S-oxygenates to be prepared

40. Triphenylphosphine exchange using methylenbis[diphenylphosphine] .82

41. ${ }^{31} \mathrm{P}$ NMR of free triphenylphosphine and compounds 1,2 and 3 at $50{ }^{\circ} \mathrm{C} \ldots \ldots . . .84$

42. ${ }^{31} \mathrm{P}$ NMR of free triphenylphosphine $\mathrm{PMePh}_{2}$ and compounds 7,8 and 9 at $50{ }^{\circ} \mathrm{C}$ 85

43. ORTEP representation of (bmmp- $\left.\mathrm{O}_{2}-\mathrm{TASN}\right) \mathrm{RuPMePh}_{2}(\mathbf{8})$ .87

44. ORTEP representation of (bmmp- $\left.\mathrm{O}_{3}-\mathrm{TASN}\right) \mathrm{RuPMePh}_{2}(9)$ .88

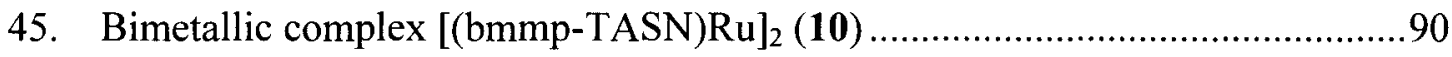

46. Synthesis of (bmmp-TASN)RuCO and ligand frame rearrangement ..................91

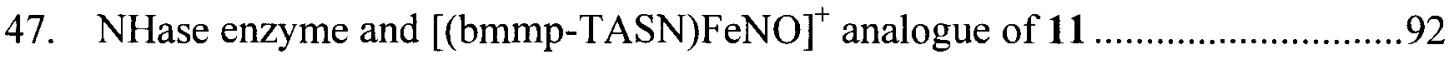

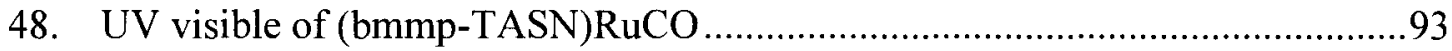

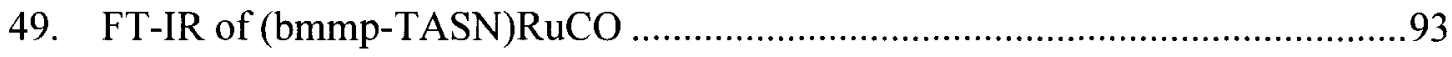

50. ORTEP representation of (bmmp-TASN)RuCO (11) ……………………........96

51. Hydrolysis of $p$-nitrophenylacetate in the presence of $\mathbf{3}$ is confirmed by the changes in the UV-Visible spectrum .............................................................

52. ${ }^{1} \mathrm{H}$ NMR of $\mathrm{CDCl}_{3}$ extract after acetonitrile hydrolysis trial with $3 \ldots \ldots \ldots \ldots \ldots \ldots 101$

53. ${ }^{1}$ H NMR hydrolysis of acetonitrile to acetamide by compound $3 \ldots \ldots \ldots \ldots \ldots \ldots \ldots \ldots . . .102$

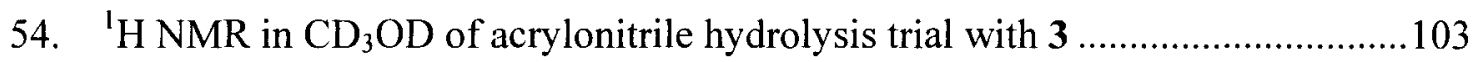

55. FT-IR of benzamide isolated from hydrolysis of benzonitrile in the presence of 3 104 


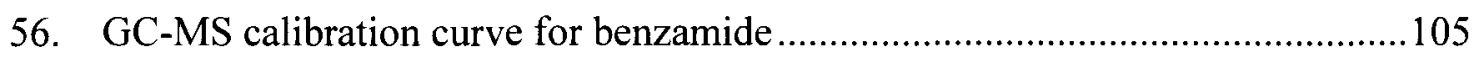

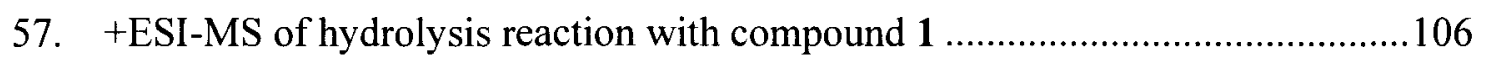

A1. FT-IR of $\mathrm{O}_{2}+($ bmmp-TASN)RuPPh 3 after 96 hours as $\mathrm{KBr}$ pellets.................129

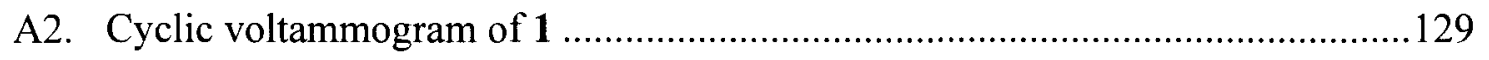

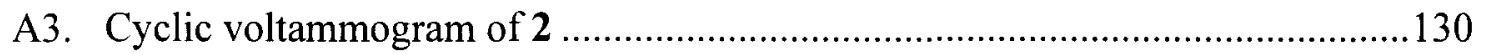

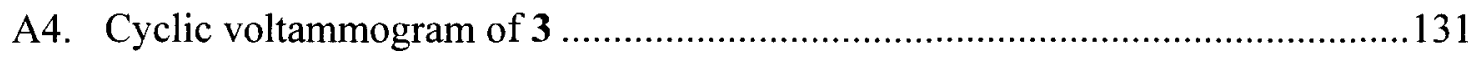

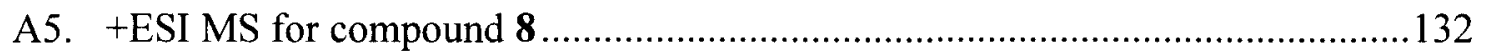

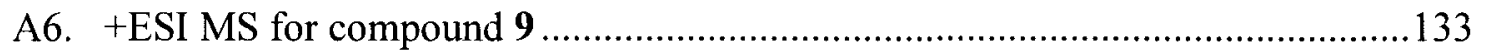

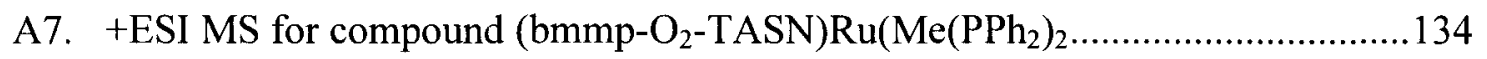

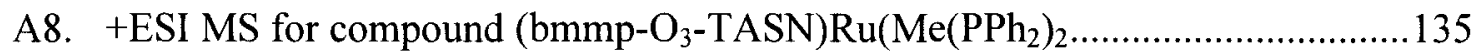




\section{LIST OF SCHEMES}

SCHEME

PAGE

1. Inner mechanism proposed for the PtNHase, active site of NHase enzyme with function nucleotide assignment.............................................................. 11

2. NHase proposed mechanism, nitrile bind the metal center and sulfenate acts as

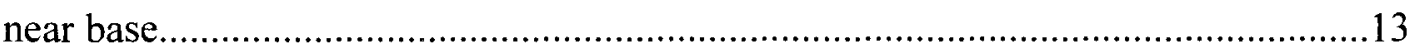

3. Co-nitrile and Co-amide structures isolated in a NHase model by Kovacs...............17

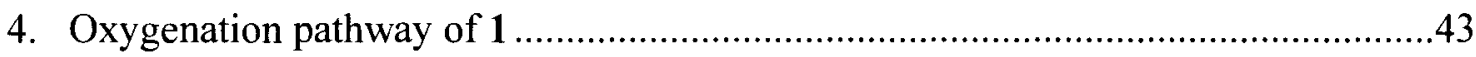

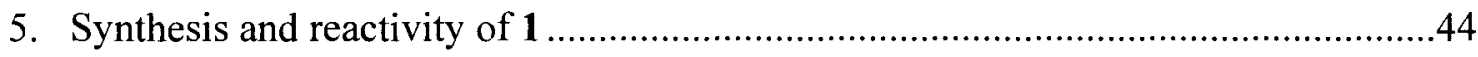

6. Dissociative mechanism for the formation of compound 11 ..............................94

7. Associative or interchange mechanism for the formation of compound 11 ............94 


\section{LIST OF TABLES}

TABLE

PAGE

1. Selected experimental and computational (BP86/LANL2DZ+6-31G(d)) bond distances $(\AA)$ and angles (deg) for 1-4 .63

2. ${ }^{31} \mathrm{P}$ NMR assignments for Figures 37 with the use of $\mathrm{PPh}_{3}$ and for Figure 38 with the use of $\mathrm{PMePh}_{2}$ . .85

3. Selected bond lengths determined by X-ray of compound $\mathbf{8}$ . .87

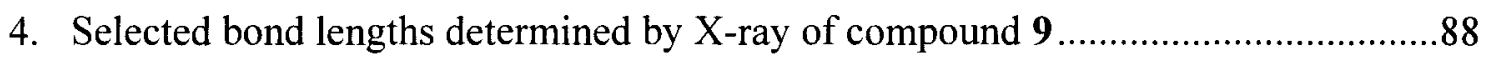

5. Selected bond lengths determined by X-ray of compound $11 \ldots \ldots \ldots \ldots \ldots \ldots \ldots \ldots \ldots \ldots \ldots \ldots . . . .96$

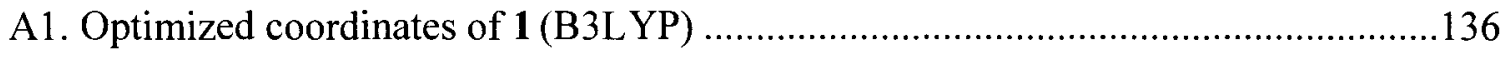

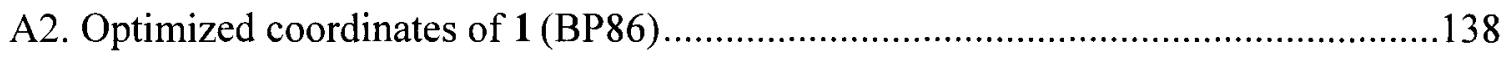

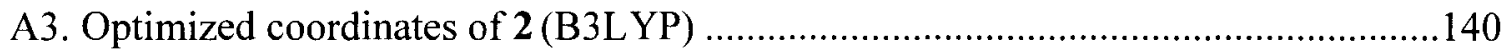

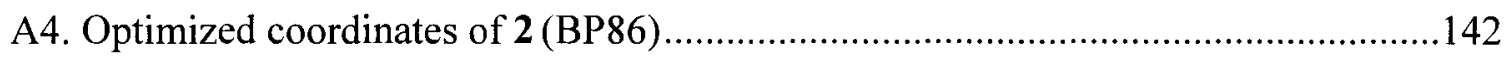

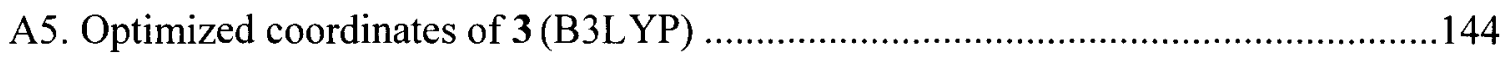

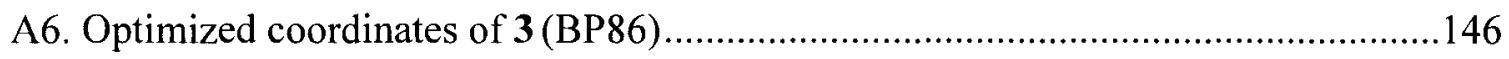

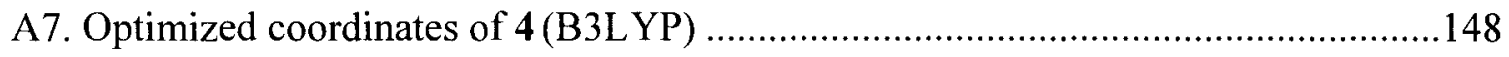

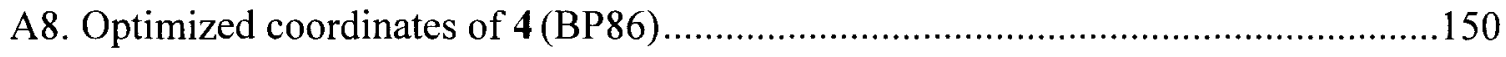

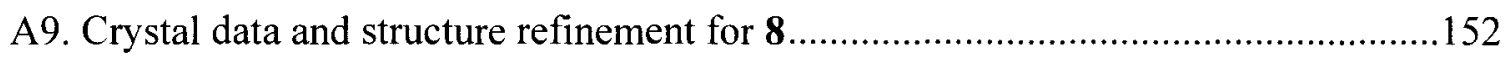

A10. Atomic coordinates $\left(\times 10^{4}\right)$ and equivalent isotropic displacement parameters $\left(\AA^{2} \times 10^{3}\right)$ for 8

A11. Bond lengths $[\AA]$ and angles $\left[{ }^{\circ}\right]$ for 8 155 
A13. Hydrogen coordinates $\left(\times 10^{4}\right)$ and isotropic displacement parameters $\left(\AA^{2} \times 10^{3}\right)$ for 8

A14. Torsion angles $\left[{ }^{\circ}\right]$ for 8

A15. Crystal data and structure refinement for 9

180

A16. Atomic coordinates $\left(\times 10^{4}\right)$ and equivalent isotropic displacement parameters $\left(\AA^{2} \times 10^{3}\right)$ for 9 181

A17. Bond lengths $[\AA]$ and angles $\left[{ }^{\circ}\right]$ for 9 .183

A18. Anisotropic displacement parameters $\left(\AA^{2} \times 10^{3}\right)$ for 9

A19. Hydrogen coordinates $\left(\times 10^{4}\right)$ and isotropic displacement parameters

$$
\left(\AA^{2} \times 10^{3}\right) \text { for } 9
$$

A20. Torsion angles $\left[{ }^{\circ}\right]$ for 9

A21. Crystal data and structure refinement for $\mathbf{1 0}$ .203

A22. Atomic coordinates $\left(\times 10^{4}\right)$ and equivalent isotropic displacement parameters $\left(\AA^{2} \times 10^{3}\right)$ for $\mathbf{1 0}$ .204

A23. Bond lengths $[\AA]$ and angles $\left[{ }^{\circ}\right]$ for $\mathbf{1 0}$ .205

A24. Anisotropic displacement parameters $\left(\AA^{2} \times 10^{3}\right)$ for $\mathbf{1 0}$ 209

A25. Hydrogen coordinates $\left(\times 10^{4}\right)$ and isotropic displacement parameters $\left(\AA^{2} \times 10^{3}\right)$ for $\mathbf{1 0}$ 210

A26. Torsion angles $\left[{ }^{\circ}\right]$ for $\mathbf{1 0}$ .211

A27. Crystal data and structure refinement for $\mathbf{1 1}$ .213

A28. Atomic coordinates $\left(\times 10^{4}\right)$ and equivalent isotropic displacement parameters $\left(\AA^{2} \times 10^{3}\right)$ for 11 


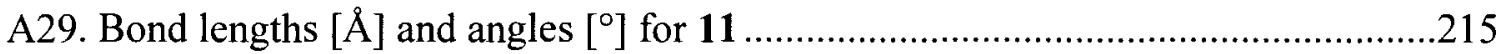

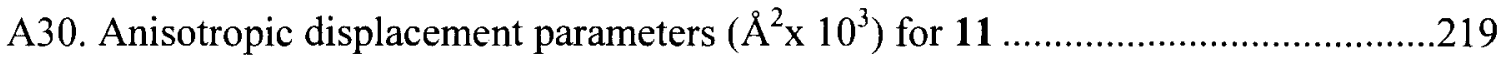

A31. Hydrogen coordinates $\left(\times 10^{4}\right)$ and isotropic displacement parameters

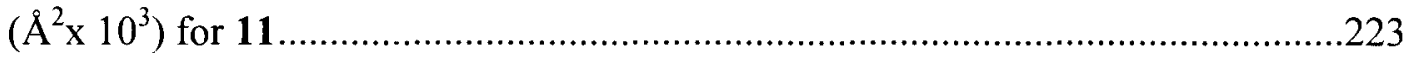

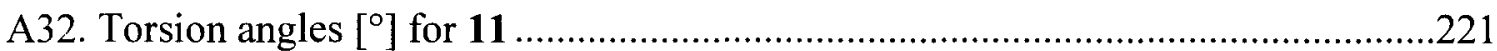




\section{CHAPTER I}

\section{INTRODUCTION}

Nitrile hydratase (NHase) is a crucial enzyme of the nitrile metabolism in several microorganisms that catalyzes the hydration of nitriles to their corresponding higher value amides at ambient pressure, temperature and at physiological $\mathrm{pH}$, Figure $1 .^{1-5}$ Microbial NHases have great potential as biocatalysts for organic chemical processing because these enzymes can convert nitriles to amides in a chemo-, regio-, and/or enantioselective manner. ${ }^{4}$ One of the most attractive features of nitrile metabolizing enzymes is their ability to selectively hydrolyze one cyano group of a dinitrile to its corresponding amide, something that is not possible using conventional chemical methods. ${ }^{6-10}$ However, NHase enzymes are under continuous enzymological investigation to magnify its potential for the synthesis of different amides. Several of the disadvantages of NHases involve their limited substrate acceptability, low enantioselectivity and thermostability. As a consequence of these disadvantages, it is required to study and improve the design of better catalysts for the hydrolysis of a wide range of organic nitriles at the industrial and environmental level. ${ }^{11}$

$$
\mathrm{RC} \equiv \mathrm{N}+\mathrm{H}_{2} \mathrm{O} \stackrel{\text { NHase }}{\longrightarrow} \stackrel{\stackrel{\mathrm{O}}{\|}}{\stackrel{\mathrm{C}}{\mathrm{R}^{-}} \mathrm{NH}_{2}}
$$

Figure 1. Nitrile hydratase hydrolysis reaction. 


\section{Biological and chemical hydrolysis}

Nitrile hydratase has captured substantial interest as a catalyst for commercial bioprocesses in industry. The bioprocess includes a large-scale production of acrylamide ${ }^{12}$ nicotinamide ${ }^{13}$ and 5-cyanovaleramide. ${ }^{13}$ The bio-hydrolysis process has a gain over traditional chemical nitrile hydrolysis routes. The advantages of the bio-process are the synthesis of a metallo-enzyme catalyst through active expression of the NHase. Other advantage is the use of a water matrix using physiological buffer of neutral to slightly basic $\mathrm{pH}$. The most remarkable property is the low or mild temperature $<30^{\circ} \mathrm{C}$ operation of the catalyst that allow the hydrolysis of acrylonitrile. The hydrolysis process use a narrow range of nitrile substrate that can be applied in the chemo-, stereo-, and region- selective amide synthesis. The process requires a simple organic-phase extraction after a complete and high yield conversion of substrate and not by product formation.

In contrast, the chemical hydrolysis process is performed under acid or base conditions in a water media at $200-300{ }^{\circ} \mathrm{C}$. The chemical process requires high pressure but it can be applied to a broad-range of nitriles without chemo-stereo or regionselectivity specificity. The one cost efficient step in the hydrolysis process is the recycling of the metallic copper or copper chromite catalyst, that is only possible in a heterogeneous reaction matrix using a transition metal catalysis. The extraction and purification of the product is performed through organic-phase extraction and multi-step purification of product with the objective to remove trace of sub product such as organic acid and by product like carbon monoxide and $\mathrm{HCN}$ with a low yield of amide. ${ }^{11}$

Biologically speaking, nitriles are starting materials and intermediates in the metabolism of several microorganisms, plants and animals. Hydrolysis of nitrile to 
carboxylic acid and amine is an important reaction in organic synthesis and in nature. At industrial level, there are two approaches to face the hydrolysis of nitriles; chemical hydrolysis and enzymatic hydrolysis by nitrile hydratase enzyme. The enzyme was discovered in the bacterium Arthrobacter sp. J-1 and studied by Asano et al. in the Department of Agricultural Chemistry at University of Kyoto Japan. ${ }^{14}$ Further studies in the year 2000 by Kato et al identified the Arthrobacter sp. J-1 as Rhodoccoccus rhodochrous $\mathrm{J} 1 .^{15}$ The early work of Asano et al in acrylonitrile conversion to acrylamide had a great impact in academia and industry as an efficient process for the hydrolysis of nitriles. As a result of this work, the industrial and big scale process of hydrolysis conversion moved from a chemical synthesis process to a biochemical enzymatic process. In the nitrile metabolism pathway, the nitrile compounds follows two different route to produce carboxylic acids. ${ }^{16}$ In the first pathway, nitriles undergo a direct transformation to corresponding carboxylic acids and ammonia by the activity of the nitrilase enzyme. In the second pathway, the nitriles are first hydrolyzed by NHase to amide, and later to carboxylic acid and ammonia by the activity of the amidase enzyme, both pathways are illustrated in the Figure 2 . 

1) Nitrilases
$\mathrm{R}-\mathrm{C} \equiv \mathrm{N}$

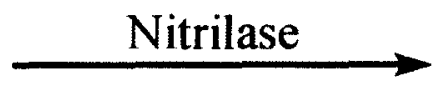
$\mathrm{R}^{\prime \prime} \mathrm{C}_{\mathrm{OH}}^{\mathrm{O}}+$
$\mathrm{NH}_{3}$

2) Nitrile Hydratases

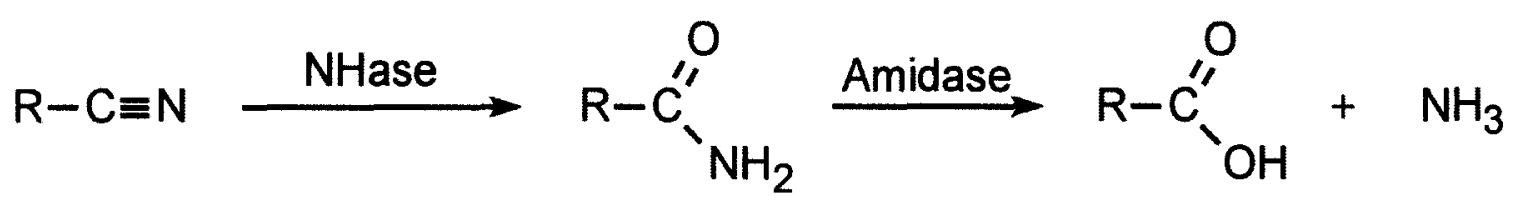

Figure 2. Nitrile metabolic pathways.

\section{Nitrile hydratase in biological systems}

Typically, a nitrile metabolizing microorganism has either a nitrilase or NHase and amidase system for the hydrolysis of nitriles. Nevertheless, some microbes such as $R$. rhodochrous J1, R. rhodochrous LL 100-21, R. rhodochrous PA-34 and N. globerula NHB-2 have both nitrilase and NHase-amidase hydrolysis systems. ${ }^{11}$ A big number of microorganism, belonging to various species of following diverse genera of Proteobacteria such as Acidovorax, Agrobacterium, Bacillus, Bradyrhizobium, Burkholderia, Comanonas, Klebsiella, Mezorhizobium, Moraxella, Pantoea, Pseudomonas, Rhizobium, Rhodopseudomonas, Serratia, Actinobacteria such as Amycolaptosis, Arthrobacter, Brevibacterium, Corynebacterium, Microbacterium, Micrococcus, Nocardia, Pseudonocardia, Rhodococcus have been described to display NHase activity, and their role in the synthesis of amides or bioremediation of nitriles have been very well documented, showing the maximum hydrolysis activity. ${ }^{11}$ 
Evolutionary development studies show that the NHases are entirely bacterial enzymes and have an enormous presence in a large number of the genera of phyla Proteobacteria, Actionobacteria, Cyanobacteria and Firmicutes. Phylogenetic systematic studies provides the molecular evidence of NHase gene in genera such as Azorhizobium, Methyalocella, Acidiphilium, Rhodomicrobium, Roseovarius, Roseobacter, Rhodobacterales, Thalassiobium, Octadecabacter, Dinoroseobacter, Methylobacterium, Legionella, Variovorax, Acinetocacter, Bordetella, Silicibacter, Phaeobacter, Ruegeria, Oceanibulbus, Sinorhizobium, Hoeflea, Sulfitobacter, Oceanicola and Labrenzia of phylum - Proteobacteria; Mycobacterium, Rhodobacteraceae, Streptomyces, Geodermatophillus and Rubrobacter of phylum - Actinobacteria; and Acaryochloris and Synechococcus of phylum - Cyanobacteria, nevertheless NHase enzymatic activity is still not stated in them. Remarkably, phylogenetic studies of NHases confirm the absence of molecular proof of NHase gene in yeasts and molds. There is no evidence of NHase activity in plants; nevertheless, phylogenetic studies indicate the molecular proof of the existence of NHase gene in Ricinus communis.

The hydrolysis activity of the nitrile hydratase has been attributed to the asymmetric configuration of its active site, Figure 3 . As mention before the first industrial scale organic biotransformation was the NHase-mediated hydrolysis of acrylonitrile, which also signifies the first biotechnology application in the petroleum industry. ${ }^{17}$ Annual NHase catalyzed acrylamide production has reached 95 Kiloton (25\% of worldwide output) and 3500 tons of nicotinamide are produced annually with biocatalysts. ${ }^{18}$ At least 10 amides have been produced on batch scales or larger. ${ }^{11,19-26}$ All of these make attractive the study and improvement of this catalyst. 


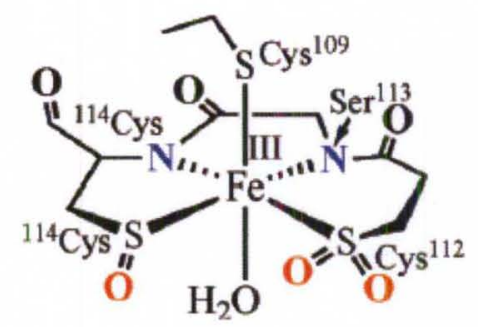

Figure 3. Post-translational NHase active site.

While the NHase biocatalysis has been pioneering the production, its volumes of production fail to meet commercial demands. Also, the biocatalysts process is subject to enzyme inactivation by the substrate or product during turnover. In addition, substantial waste is generated as compared to known chemical methods. Nevertheless NHase continues to attract attention on the industrial scale. Additional uses of the NHase include bioremediation of toxic nitriles ${ }^{11,27,28}$ such as those in pharmaceutical waste-water, ${ }^{29}$ shale oil, ${ }^{30}$ and herbicide ${ }^{31}$ and contaminated soil. ${ }^{32}$

The related Co-containing thiocyanate hydrolase (SCNase) shares a similar active site NHase. ${ }^{33,34}$ Biologically, SCNase catalyzes thiocyanate degradation to carbonyl sulfides, ammonia and hydroxide $\left(\mathrm{SCN}^{-}+2 \mathrm{H}_{2} \mathrm{O} \rightarrow \mathrm{COS}+\mathrm{NH}_{3}+\mathrm{OH}^{-}\right) .^{33}$ Thiocyanate is a natural product of cyanide detoxification by the ubiquitous enzyme thiosulfate transferase (rhodanase). ${ }^{35} \mathrm{SCNase}$ is found in $\mathrm{CN}^{-}$containing waste water sludge from coal gasification factories. $^{34}$

Nitrile hydratase is present in gram-positive bacteria, which have a strong cell wall. One of the key steps to increase the recovery of the NHase is the fracturing of this cellular wall. Ultra sonication, French press and bead-beater process have been used as 
protocols to break the cell wall. These protocols can be used at the same time with hydrolytic enzymes to improve the recovery of NHase. The final purification is carry out in the cell fill extract using ammonium sulfate extraction and column chromatography. The purified NHase extract have molecular mass from 54kDa to 530KDa. The NHase are composed of two different subunits $\alpha$ and $\beta$, each of these sub units have a molecular weight between $22 \mathrm{KDa}$ to $29 \mathrm{KDa} .{ }^{11}$

\section{Nitrile hydratase molecular structure}

Nitrile hydratase contains a non-heme iron (III) or non-corrin cobalt (III) both of which are non-redox active. In addition to these two metals, a recent publication by Okamoto and Eltis have identified one cobalt, two copper and one zinc per functional NHase enzyme; however the configuration of these metals in the holoenzyme have not yet been determined. ${ }^{36}$ The optimal conditions for catalysis of the NHase enzyme are physiological $\mathrm{pH}$ that can varied between 6.5 to 8.5 and temperature between 20 to 35 ${ }^{\circ} \mathrm{C}$. In a few NHases maxima activity at $60{ }^{\circ} \mathrm{C}$ has been reported. The effect of $\mathrm{pH}$ on the enzyme is observed at low $\mathrm{pH}$ where the enzyme suffers of denaturalization and subunit dissociation. Another effect of the low $\mathrm{pH}$ is the ionization of some relevant residues. ${ }^{37}$

The NHase families have several amino acid sequences in the $\alpha$ - and $\beta$-subunit. However, the structural studies on the NHases enzymes indicate that the region of $\alpha$ subunit $\mathrm{VC}(\mathrm{T} / \mathrm{S}) \mathrm{LCSC}(\mathrm{Y} / \mathrm{T})$ is highly conserved. The cobalt-type NHase have threonine (T) and tyrosine (Y) as third and eighth amino acid residues of metal binding first coordination sphere, which are replaced by serine (S) and threonine (T), respectively, in iron-containing NHase. ${ }^{38}$ The catalytic center of the NHase is buried in the interior of the protein, therefore the substrate and the product have to immerse and ascend from the 
hydrolysis pocket. The distance between the metal center in Co-type NHase and the protein surface is $15 \AA^{39}$ The transport of the substrate and product is accomplished through an interface channel between the subunits $\alpha$ and $\beta .^{40}$ It has been postulated that the specificity of the NHase is due to the stereospecificity and substrate affinity profile of the channel. ${ }^{40}$ This statement is in concordance with the limited substrate acceptance and low enantioselectivity of the NHase.

In the active site of the NHase enzyme the trivalent metal ion is six-coordinate in a low-spin coordination environment with the ligand sphere consisting of three cysteines, two amide nitrogens, $\left(\mathrm{N}_{2} \mathrm{~S}_{3}\right.$ coordination sphere) and a water/hydroxide or $\mathrm{NO}$ in the sixth coordination site. ${ }^{1,41,42}$ Some Fe-type NHases show photoreactivity and bind nitric oxide (NO), whereas Co-type NHase not. ${ }^{43-45}$

Interestingly, the $\mathrm{N}_{2} \mathrm{~S}_{3}$ active site contains two post-translationally modified cysteine residues. A sulfenate/sulfenic acid ( $\mathrm{RSO} / \mathrm{RSOH})$ and a sulfinate $\left(\mathrm{RSO}_{2}{ }^{-}\right)$donor are found in the positions cis to the suggested substrate binding site, while the trans position contain an unmodified thiolate ( $\mathrm{RS}^{-}$). NHase cultured under anaerobic conditions lacks S-oxygenation and is catalytically inactive until exposed to $\mathrm{O}_{2}$, which initiates Soxygenation and instills activity. ${ }^{46}$ This asymmetric S-oxygenated environment is also observed in thiocyanate hydrolase ${ }^{47}$ (SCNase), a related enzyme with different substrate preferences that could result from changes in charge distribution and/or substrate accessibility.

\section{Mechanism insight based on structural information in the enzyme}

The crystal structures of Fe- and Co-NHases have been reported ${ }^{48,49}$ at resolutions of $2.65 \AA$ in the Fe active form, and in the NO-bound inactive state, at $1.7 \AA$. Using time- 
resolved X-ray crystallography with Fe-NHase and t-butylisonitrile as a surrogate substrate, Odaka and co-workers observed analogues of coordinated nitrile substrates and amide product complexes strongly suggesting a nitrile-bound pathway. ${ }^{50}$ An alternate mechanism proposed by Holtz based on through kinetic investigations prefers a conserved serine residue to the sulfenate donor as the proximal base. ${ }^{51,52}$

Very recent NHase mutants reported by Odaka confirm serine's importance, but call its role as base into question as it is not required for the hydrolysis. ${ }^{53}$ Further, Odaka prepared inactive mutants with "over-oxygenation" of sulfenate to the less basic sulfinate. Deactivation of NHase by oxidants has been noted elsewhere, ${ }^{54}$ but a novel Fe-NHase recently characterized by high-resolution X-ray crystallography displays bis-sulfinate coordination, no activity has been described. ${ }^{55}$ Fewer studies are reported for SCNase, but over-oxygenation also inactivates that enzyme, which is presumed to follow a similar mechanism as NHase. ${ }^{56}$

From the point of view of the catalytic properties, Fe- and Co-NHase are very similar although Fe preferentially hydrates small aliphatic nitriles, ${ }^{57}$ whereas Co exhibits a high affinity for aromatic nitriles ${ }^{58,59}$. Recent studies indicate that post-translational modification of cysteine-112 and 114 to sulfinate and sulfenate derivatives is essential for catalytic activity and the photo-dissociation of NO. The probable functions of the Soxygenated cysteines include modulation of the Lewis acidity of the metal center, ${ }^{60}$ promotion of NO binding/photo-regulation, ${ }^{45,50,61-65}$ utilization as a proximal base to activate $\mathrm{H}_{2} \mathrm{O},{ }^{50,64,65}$ or modification of substrate/product binding affinity. ${ }^{66}$

Although the catalytic mechanism of hydrolysis remains unclear, there are few mechanism proposed. Initially, three mechanism of hydrolysis where suggested: ${ }^{48}(\mathrm{I})$ an 
inner mechanism, (II) an outer-sphere mechanism and (III) a second outer-sphere mechanism. Over the last 10 years, there has been continuing interest in unraveling the NHase mechanism from biochemical, bioinorganic, and computational perspectives. Recently, based on analyses of the protein structure of Pseudonocardia thermophila JCM $3095 P t$ NHase, an inner mechanism has been proposed.$^{52}$ This mechanism involves a catalytic chord formed between Trp72, Tyr68, and RSer112, Scheme 1. In this mechanism, Tyr68 is deprotonated at $\mathrm{pH} 7.6$ and forms a hydrogen bond with Trp 72 . This interaction stabilizes the charge associated with a deprotonated tyrosine residue and Tyr68 functions as the active site general base. Displacement of the metal-bound water molecule by a nitrile activates the $\mathrm{CN}$ bond toward nucleophilic attack and places the water molecule in the proper orientation to facilitate proton transfer from water molecule to the nitrile $\mathrm{N}$ atom. The second transfer occurs between the active site water molecule and Try $68 .{ }^{52}$ Once proton transfer occurs, the resulting imidate can tautomerize to form an amide with a subsequent proton transfer from RSer116, which functions to shuttle protons from Tyr68. Finally, the amide product is displaced by a water molecule providing the regenerated catalyst. No matter the rationality of the last approach, it lacks a dynamic approach and spectroscopic analysis, for this reason it is necessary to more accurately study this enzyme and its properties from the perspective of bioinorganic modeling. 


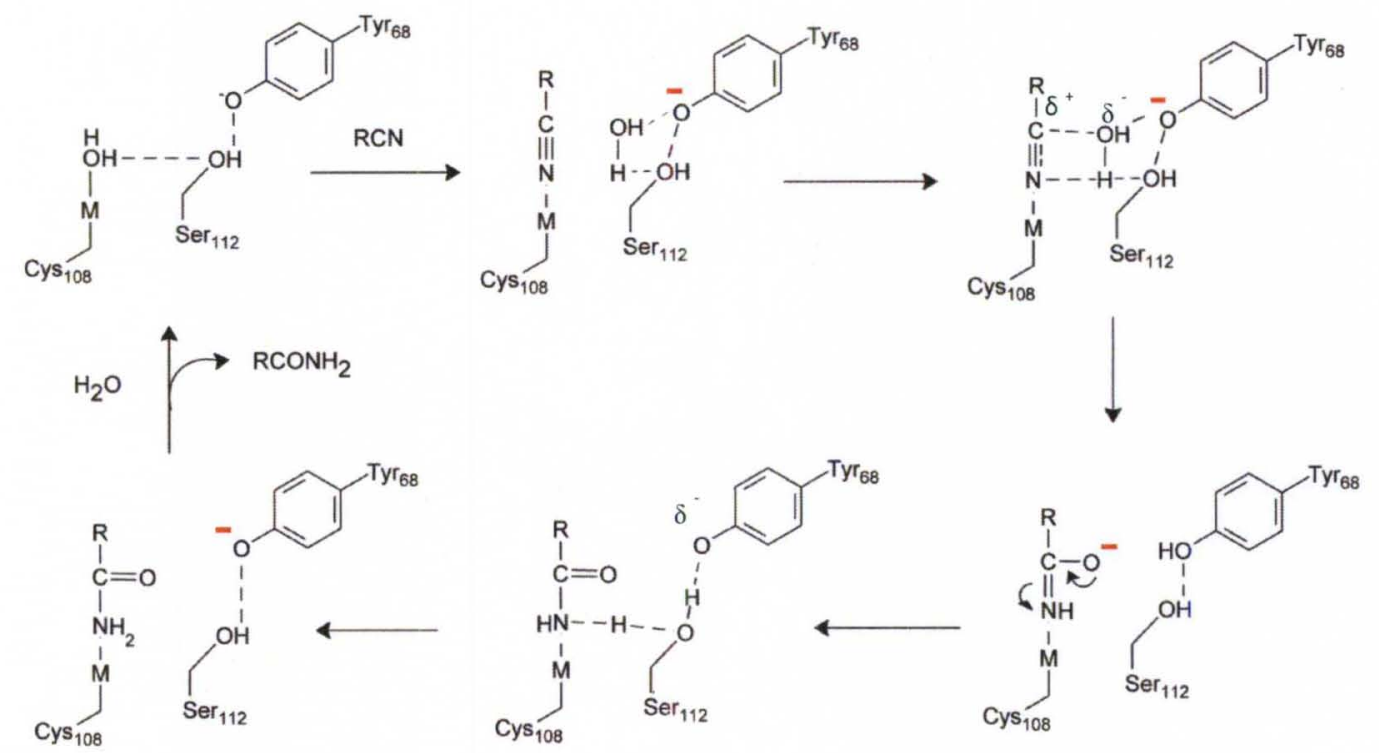

Scheme 1. Inner mechanism proposed for the PtNHase, active site of NHase enzyme with function nucleotide assignment.

The design of nitrile hydratase synthetic models using metals like platinum (II), ${ }^{67}$ ruthenium (II), ${ }^{68-71}$ cobalt (III) ${ }^{72}$ and nickel $(0)^{73,74}$ support that displacement of hydroxide by nitrile and undergoes hydrolysis of the metal bound nitrile by a water molecule. This generates a metal- bound iminol that rearranges to release the amide product. Of all these catalysts, the $\mathrm{Co}$ (III) model studied by Mascharak et al. is the only one that pursued the biomimetic design of the nitrile hydratase with the use of the compound $[\mathrm{Co}(\mathrm{PyPS})(\mathrm{CN})]^{2-}$, Figure 4 , in $5 \mathrm{ml}$ of Tris buffer $(10 \mathrm{mM}, \mathrm{pH} 9.5)$ and $1.5 \mathrm{~mL}$ of acetonitrile at $50{ }^{\circ} \mathrm{C}$ with the generation of $0.56 \mathrm{mmol}$ (18 turnovers after 4 hours). 


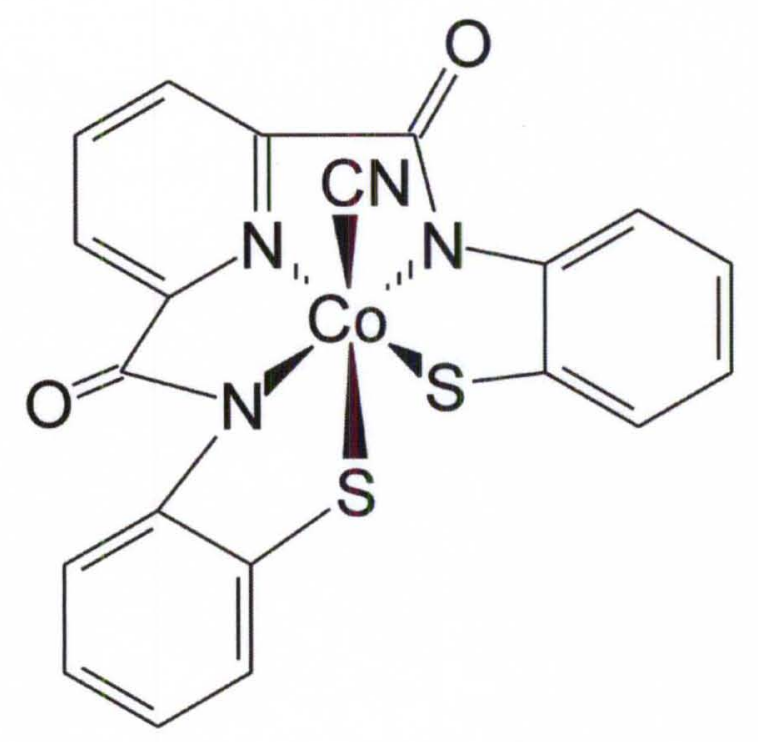

Figure 4. $[\mathrm{Co}(\mathrm{PyPS})(\mathrm{CN})]^{2-}$ NHase synthetic model.

In the outher-sphere hydrolysis mechanism (II), the coordinated hydroxide perform a nucleophilic attack into the nitrile and subsequently the iminolate species generated rearrange to amide keeping the coordination through the oxygen. In the third mechanism or second-outer sphere, the hydroxide coordinated to the metal center extract a proton of a nearby water molecule. The hydroxide performs a nucleophilic attack in the nitrile near to the active site.

Recent studies based on DFT calculations proposed two new mechanisms called first shell and second shell mechanism. ${ }^{64,75}$ The second shell mechanism involves the formation of a metal-bound hydroxide (nucleophile) that attacks the electron deficient carbon of the nitrile. Subsequent rearrangement of the metal-bound iminol intermediate yields an oxygen-bound amide that exchange with water to reset the process. In the first shell mechanism, Scheme 2, the attack of a metal-coordinated nitrile (A) by water activated through interaction with the sulfenate yields a metal-coordinated imidate 
intermediate and a metal-coordinated sulfenic acid (B). Deprotonation of the acid by the imidate provides the iminol complex (C), which tautomerizes to the amide product (D)

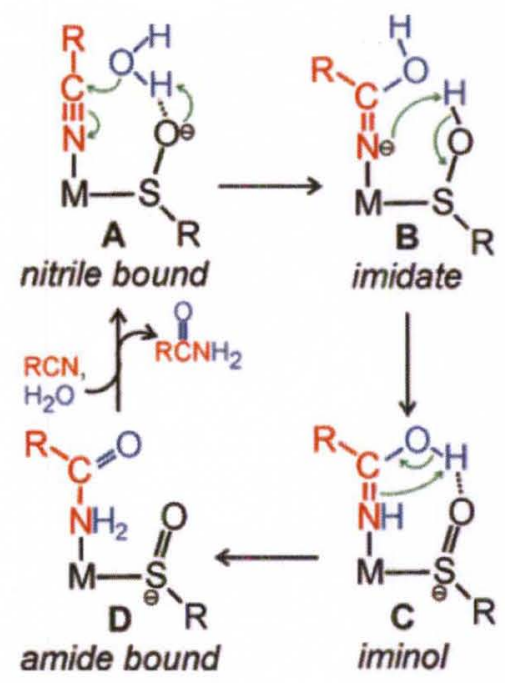

Scheme 2. NHase proposed mechanism, nitrile bind the metal center and sulfenate acts as near base.

The approach adopted by inorganic groups involves the study of the NHase enzyme based on the design of small molecular complexes. These models reproduce structural, electronic, and functional properties of the enzyme's active site. The systematic modification of the structural frame in these models provides insight regarding complex properties, such as ligand affinity, Lewis acidity, oxidation/reduction properties and spectroscopic characteristics. However, model studies based on the Fe-NHase enzyme are complicated by several factors. First, there are inherent synthetic difficulties associated with iron-thiolate chemistry ${ }^{76,77}$ including undesired metal and ligand-centered reactivity as well as internal redox processes. These challenges commonly results in isolation of iron-oxo clusters and/or ligand disulfide. Nevertheless, iron-thiolates can 
react with oxygen in a spin-state dependent process to yield various sulfur-oxygenated derivatives. ${ }^{78}$ As such, the spin-state plays an important role in synthetic design.

\section{Syntheses of NHase mimics compounds}

The syntheses and studies of NHase model complexes with $\mathrm{N}_{2} \mathrm{~S}_{2}, \mathrm{~N}_{3} \mathrm{~S}_{2}$, and $\mathrm{N}_{2} \mathrm{~S}_{3}$ donor frameworks have been fruitful to address structural changes and correlate them with the NHases properties. In this research area over the last 10 years the work of the Artaud, Grapperhaus, Kovacs and Mascharak groups are most notable. Nevertheless, the NHase mechanism remains elusive and studies of $\mathrm{O}_{2}$ reactivity that reproduce the posttranslational modifications are limited. While it is well known that $\mathrm{O}_{2}$ reacts with some metal-thiolates to yield sulfinates $\left(\mathrm{SO}_{2} \mathrm{R}\right)^{79}$, additional products including sulfonates $\left(\mathrm{SO}_{3} \mathrm{R}\right)$ or intractable products such as disulfides or oxoclusters are obtained. Additionally, O-atom transfer reagents remain the best candidate to prepare sulfenates $(\mathrm{S}(\mathrm{O}) \mathrm{R})$, however this approach to generate sulfur oxygen derivatives is restricted by the reactivity of the compounds produced and isolation problems. Sulfenate synthesis is limited by the potential for sulfenate disproportionation to sulfinate and thiolate. As such, metal-coordinated sulfenates have been reported in few cases and usually with the use of O-atom reagents. For example tert-butyl $N$-sulfonyloxaziridine ${ }^{80}$ was used to isolate mono iron-sufenate/thiolate models, while $\mathrm{H}_{2} \mathrm{O}_{2}$ was employed for a mixed sulfenate/sulfinate ${ }^{79}$ nickel complex, a vanadium(V)- $\eta^{2}, \eta^{2}$ side-on disulfenate complex, ${ }^{81}$ and cobalt $\eta^{2-}$ sulfenate, ${ }^{82}$ and finally $\mathrm{H}_{2} \mathrm{O}_{2} \cdot$ urea $^{83,84}$ was used for a cobalt disulfenate.

Julie Kovacs' group at the University of Washington focused on imine nitrogen donors (neutral, strong sigma donors) and amide nitrogen donors with thiolates in her NHase models, such as the complex $\left[\mathrm{Fe}^{\mathrm{III}}\left(\left(\text { tame- } \mathrm{N}_{2} \mathrm{~S}\right) \mathrm{S}_{2} \mathrm{Me}_{2}\right)\right]^{2-}$ (Figure 5 compound C). 
The anionic bis- carboxamide, tris-thiolate $\mathrm{N}_{2} \mathrm{~S}_{3}$ coordination sphere is potentially similar to the unmodified form of NHase. Studies of complexes such as $\left[\mathrm{Fe}{ }^{\mathrm{III}}\left(\left(\operatorname{tame}-\mathrm{N}_{3}\right) \mathrm{S}_{2} \mathrm{Me}_{2}\right)\right]^{+}$ (A), $\left[\mathrm{Fe}^{\mathrm{III}}\left(\mathrm{Et}_{-} \mathrm{N}_{2} \mathrm{~S}_{2} \mathrm{Me}_{2}\right)(\mathrm{py})\right]^{-}(\mathbf{B})$, and $\left[\mathrm{Fe}^{\mathrm{III}}\left(\left(\text { tame- } \mathrm{N}_{2} \mathrm{~S}\right) \mathrm{S}_{2} \mathrm{Me}_{2}\right)\right]^{2-}(\mathbf{C})$ reveal how anionic charge build up (from cationic $\mathbf{A}$ to anionic $\mathbf{B}$ and dianionic $\mathbf{C}$ ) and $\mathrm{Fe}$ spin-state influence apical ligand affinity, Figure 5.

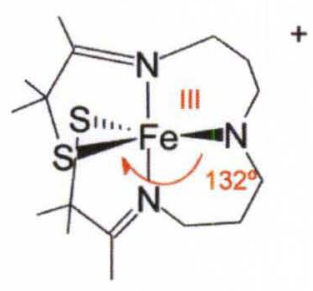

$\operatorname{Pr}, \operatorname{Pr}$

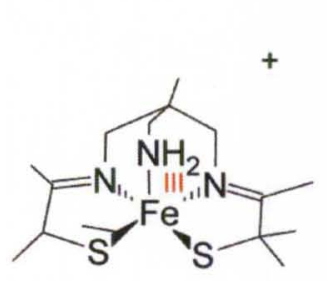

A

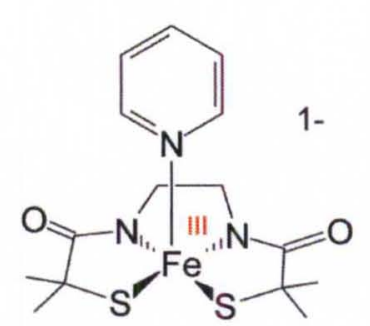

B

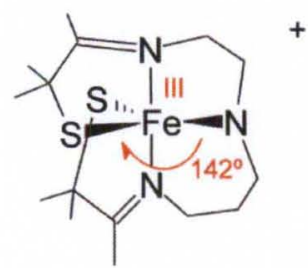

Et, $\mathrm{Pr}$

Figure 5. NHase models that modulate charge and affect the affinity of the apical position.

The strong $\mathrm{N}_{2} \mathrm{~S}_{2}$ basal plane ligand field and anionic charge of equatorial carboxamides in the thiolate-ligated complexes results in a weakened apical ligand interaction even with strong donors. The substitution of apical nitrogen by a thiolate pulls the $\mathrm{Fe}^{3+}$ from the square planar plane $\mathrm{N}_{2} \mathrm{~S}_{2}$. This structural rearrangement is associated with the better affinity of the iron for the thiolate. The spin state $S=3 / 2$ and its half $d_{z}^{2}$ occupied orbital are considered the cause of the low affinity for apical substrates. This low affinity for substrates diverges from affinity of the sulfur oxygenated NHase active site. The active site has a low-spin configuration, an empty apical site orbital and at the same time higher affinity for apical ligands such NO. The thiolates compounds in Figure 
5 have a $S=3 / 2$ spin state. They require the pairing of electrons to accomplish a $S=1 / 2$ configuration and in this way coordinate a substrate in the apical position. This configuration will generate a barrier for ligand binding formation in these penta coordinated complexes. These results strongly suggest the need of a post-translational sulfur oxygenation of the square plane NHase cysteinates to coordinate substrates. This oxygenation is essential in order to weaken the equatorial ligand field and increase the Lewis acidity of the $d_{z}{ }^{2}$ orbital pointing toward the vacant apical site. In this way the affinity for substrates is increases and the reactivity of $\mathrm{H}_{2} \mathrm{O}$ or nitiles for the hydrolysis mechanism is possible. The series of complexes synthesized by the Kovacs group support the nitrile bound mechanism, The penta-coordinate complexes $\mathrm{A}, \mathrm{B}$, and $\mathrm{C}$ bind $\mathrm{NO}$, $\mathrm{SCN}^{-}$and $\mathrm{N}_{3}^{-}$at room temperature and $\left.\left[\mathrm{Fe}^{\mathrm{III}}(\operatorname{Pr}, \mathrm{Pr}) \mathrm{S}_{2}{ }^{\mathrm{Me} 2}\right)\right]^{+}(\mathrm{Pr}, \mathrm{Pr})$ and $\left.\left[\mathrm{Fe}^{\mathrm{III}}(\mathrm{Et}, \mathrm{Pr}) \mathrm{S}_{2}{ }^{\mathrm{Me} 2}\right)\right]^{+}(\mathrm{Et}, \mathrm{Pr})$ bind nitriles at low temperature. ${ }^{85}$ Water binding affinity could not be evaluated as the complexes are readily decomposed.

In a recent study, the Kovacs' group supports the proposed role of sulfenate as a proximal base in the nitrile bound mechanism. They trapped and structurally characterized a Co- iminol species similar to the representation in the Scheme 3 and a Co-amidate by using alkoxide and amine donors in place of the difficult to synthesize sulfenate. ${ }^{65}$ In addition, they have also reported one of the only three structurally characterized sulfenate/sufinate complexes. ${ }^{82}$ Other highlights include Co(III) complexes with relatively fast exchange kinetics due to trans-thiolates and collaborative XAS studies to elucidate the protonation state of the sulfenate donor in NHase. ${ }^{86}$ 


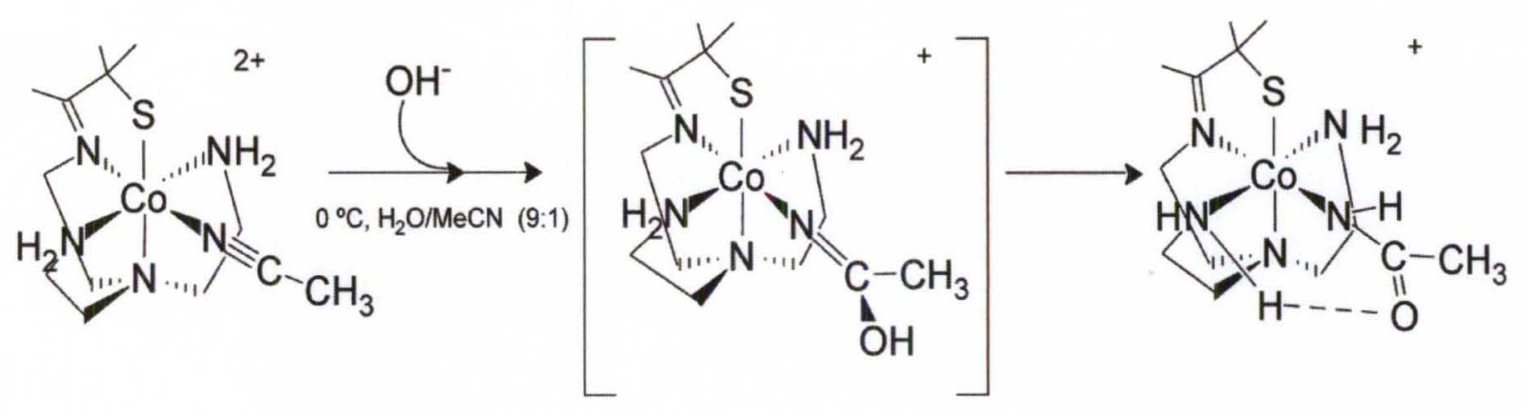

Scheme 3: Co-nitrile and Co-amide structures isolated in a NHase model by Kovacs.

Pradip Mascarak's group at University of California Santa Cruz has focused on the simultaneous coordination of deprotonated amides and thiolates using ligands $\mathrm{N}_{3} \mathrm{~S}_{2}$ coordination system such as $\mathrm{PyPSH}_{2}$ in Figure 6. The Fe complexes are low-spin and the +3 oxidation state is stabilized by the strong $\sigma$-donating ability and anionic charge. The electronic absorption spectra of these complexes are similar to those of Fe-NHase suggesting the green color of the enzyme arises from a thiolate-to metal charge-transfer band. These Fe(III) complexes are stable in aqueous solution and do not undergo hydrolytic decomposition. The Co(III) derivatives are capable of hydrolyzing nitriles to amides with modest catalytic activity $\left(\sim 17\right.$ turnovers, $\left.\mathrm{pH} 9,50{ }^{\circ} \mathrm{C}\right)$ in aqueous buffer. ${ }^{72}$ The cobalt derivative of PyPSH readily binds water lowering its $\mathrm{pKa}$ to 8.3. The monosulfinate derivative of this complex has a lower pKa for the bound water of 7.2. These findings led to the hypothesis that the modified thiolates in NHase modulates the pKa of the bound water in order to facilitate the hydrolysis reaction under physiological conditions. ${ }^{87}$ The proper disposition of donor groups, especially the trans thiolate-S donor, allows for rapid lability of the sixth site in these $\mathrm{Co}(\mathrm{III})$ complexes. In addition to water this model PyPsX can bind $\mathrm{X}=\mathrm{CH}_{3} \mathrm{OH}$, pyridine, and $\mathrm{CN}^{-}$, but show no interaction with nitriles in solution The work provides support for both the inner-sphere and outer- 
sphere mechanisms of hydrolysis involving bound water. More recently, Mascharak has neatly shown that S-oxygenation facilitates photolability in Fe-NO complexes. ${ }^{88}$

The crystal structure of the Fe NHase inactive form at $1.7 \AA$ has an NO as ligand<smiles>O=C(Nc1ccccc1S)c1cccc(C(=O)Nc2ccccc2S)n1</smiles><smiles>O=C(O[Pb]12Sc3ccccc3C(=O)N1c1cccc(n1)C2=O)c1ccccc1</smiles>

Figure 6. PyPSH model of NHase that bind $\mathrm{H}_{2} \mathrm{O}$.

substrate. The NO inactivates the catalytically activity of the enzyme. However upon exposure to light the NO is released and the enzyme regains activity. This experiment suggests that the catalytically activity of the NHase is photo-regulated by NO. Masharack et al. synthesized a NHase analogue with the NO motif. The complex $\left[\left(\mathrm{Cl}_{2} \mathrm{Ph}-\right.\right.$ PepS)Fe(NO)(DMAP)] in Figure 7 has a NO reactivity that shows lability in coordinating solvents such as $\mathrm{MeCN}, \mathrm{THF}$ or DMF at room temperature and at $40^{\circ} \mathrm{C}$. In non-coordinating solvents the $\mathrm{NO}$ complex is stable at room temperature and do not show photolability. The synthesis of sulfur oxygenated derivatives, characterized by FT-IR and EPR spectroscopy suggests the NO release upon photolysis of $\left[\left(\mathrm{Cl}_{2} \mathrm{Ph}-\right.\right.$ $\left.\left.\mathrm{PepSO}_{4}\right) \mathrm{Fe}(\mathrm{NO})(\mathrm{DMAP})\right]^{\text {. Th Th }}$ research implies an important role for the sulfur oxygenation in controlling NO photorelease from Fe-NHase. ${ }^{88}$ 


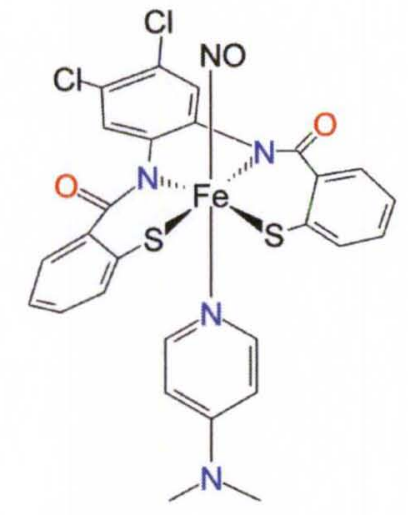

$\left[\left(\mathrm{Cl}_{2} \mathrm{Ph}-\mathrm{PepS}\right) \mathrm{Fe}(\mathrm{NO})(\mathrm{DMAP})\right]^{-}$

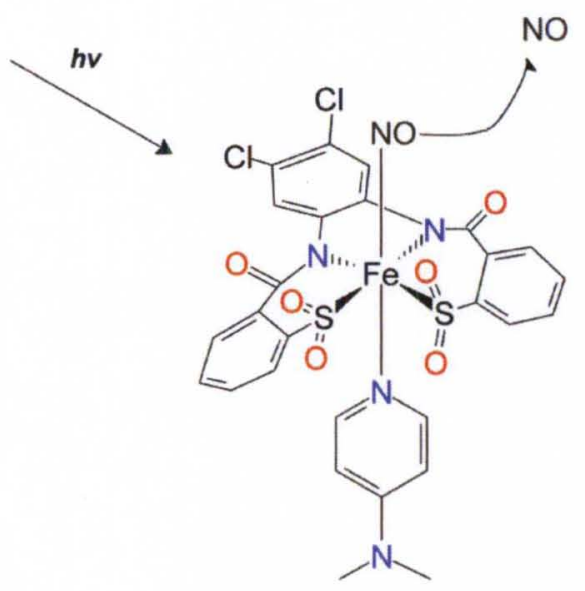

$\left.\left[\left(\mathrm{Cl}_{2} \mathrm{Ph}-\mathrm{PepSO}\right)_{4}\right) \mathrm{Fe}(\mathrm{NO})(\mathrm{DMAP})\right]^{-}$

Figure 7. Thiolate sulfur oxygenation controls nitric oxide photolability.

Research work performed by Isabell Artaud and Jean Claude Chottard at the Université René Descartes (Paris, France) has also focused on deprotonated carboxamides and thiolates. The model complex $\left[\mathrm{Co}^{\mathrm{III}}\left(\mathrm{L}-\mathrm{N}_{2} \mathrm{SOSO}\right)(t \mathrm{BuNC})_{2}\right]^{-}$has a tetradentate diamide-dithiolate in the equatorial plane and non-labile tert-butylisonitriles in the axial positions as show in Figure 8. Although the metal center is coordinatively saturated, the complex catalytically hydrolyzes nitriles (50 turnovers, $4^{\circ} \mathrm{C}, \mathrm{pH} 4.8$ ). When isotopically labled $\mathrm{H}_{2}{ }^{18} \mathrm{O}$ was introduced into the solution, ${ }^{89}$ the labeled oxygen atoms were incorporated into the sulfenate moieties, suggesting the S-oxygenate is the oxygen source for the hydration of nitriles. This study reinforce the recent result of Masafumi Odaka of the Tokio University of Agriculture and Technology that address the sulfenate important role on the catalytic activity of the NHase enzyme. ${ }^{56}$ 


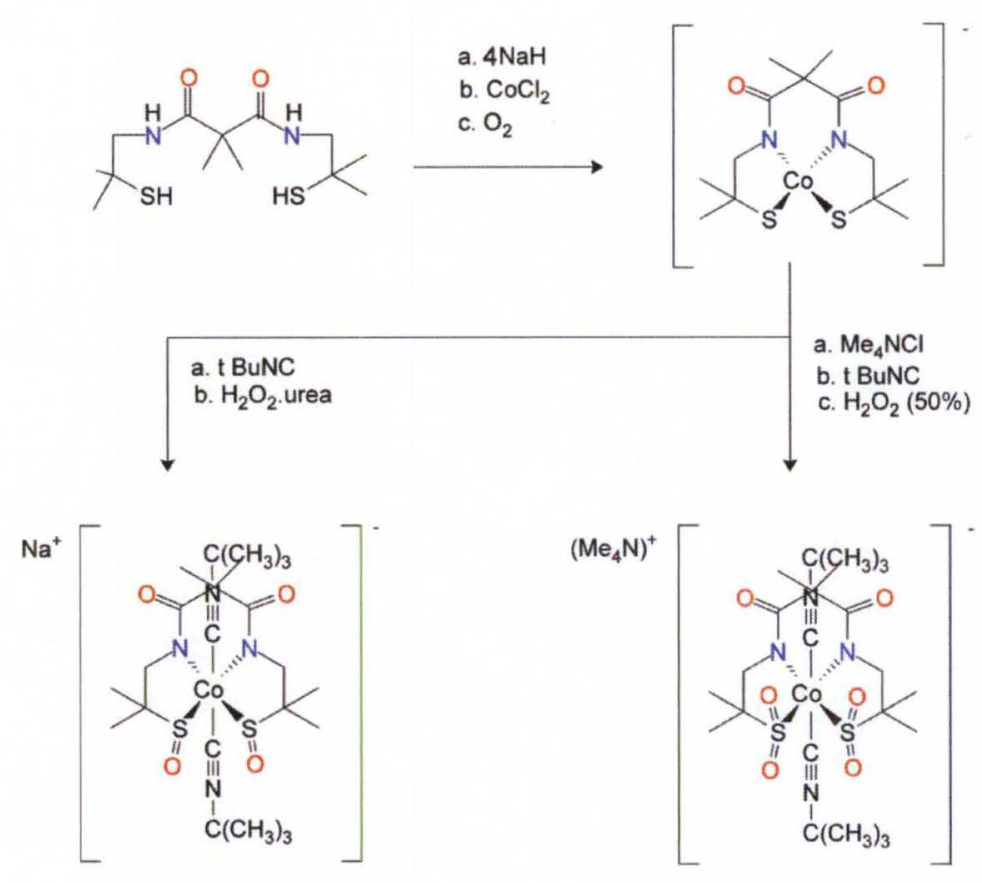

Figure 8. Synthesis scheme for the complex $\left[\mathrm{CoIII}\left(\mathrm{L}-\mathrm{N}_{2} \mathrm{SOSO}\right)(\mathrm{tBuNC})_{2}\right]^{-}$and $\left[\mathrm{CoIII}\left(\mathrm{L}-\mathrm{N}_{2} \mathrm{SO}_{2} \mathrm{SO}_{2}\right)(\mathrm{tBuNC})_{2}\right]^{-}$.

After all these years of NHase research only three NHase models with hydrolytic activity have been reported. Maschrack's proposed $\mathrm{H}_{2} \mathrm{O}$-bound mechanism with 17 turnovers, ${ }^{60,72}$ Sherear suggests a nitrile-coordinated intermediate for his Cometallopeptide mimics $\mathrm{N}_{2} \mathrm{~S}_{3}$, which include a spectroscopically detected sulfenate/sulfinate donor set using electrospray ionization-mass spectrometry (ESI-MS), gel-permeation chromatography (GPC), Co K-edge X-ray absorption spectroscopy, vibrational, and optical spectroscopies..$^{90}$ Its mixed S-oxygenation allows up to 58 turnovers at $\mathrm{pH}=8.0$, but like NHase is inactive in the mono- or bis-sulfinate form. Finally, a ligand-centered pathway is proposed by Chottard for his coordinatively saturated bis-sulfenate Co-complex with axial isonitrile donors, which functions best (50 turnovers) under a $\mathrm{pH}$ of $4.8 .^{91}$ The bis-sulfinate derivative is inactive. Similar complexes are reported by Yano, although hydrolytic was not noted. The related bis-sulfinate of 
Artuad is active (14 turnover, $\mathrm{pH}=7$ ), but only after substitution of isonitriles with $\mathrm{CN}^{-}$ and $\mathrm{H}_{2} \mathrm{O} .^{92}$

Although several NHase mechanisms including water-bound and outer-sphere pathways have been proposed using a bioinorganic and biochemistry approach, the most supported mechanism involves a nitrile-bound pathway. Attack of a metal-coordinated nitrile (I) by water activates with the sulfenate yields a metal-coordinated imidate intermediate and a metal-coordinated sulfenic acid (II). Deprotonation of the acid by the imidate provides the iminol complex (III) with structural rearrangements ends in an amide product (IV), Scheme 2.

The Grapperhaus' group at University of Louisville has focused on complexes with the ligand L (4,7-bis-(2'-methyl-2'-mercaptopropyl)-1-thia-4,7-diazacyclononane, bmmp-TASN) that have a $\mathrm{N}_{2} \mathrm{~S}_{3}$ environment, Figure 9. The bmmp-TASN ligand has a facial $\mathrm{N}_{2} \mathrm{~S}$ donor with unreactive sulfur donor and reactive cis sulfur site in the active center of the enzyme in addition of the exchangeable $6^{\text {th }}$ coordination position, as observed in the Figure 9.
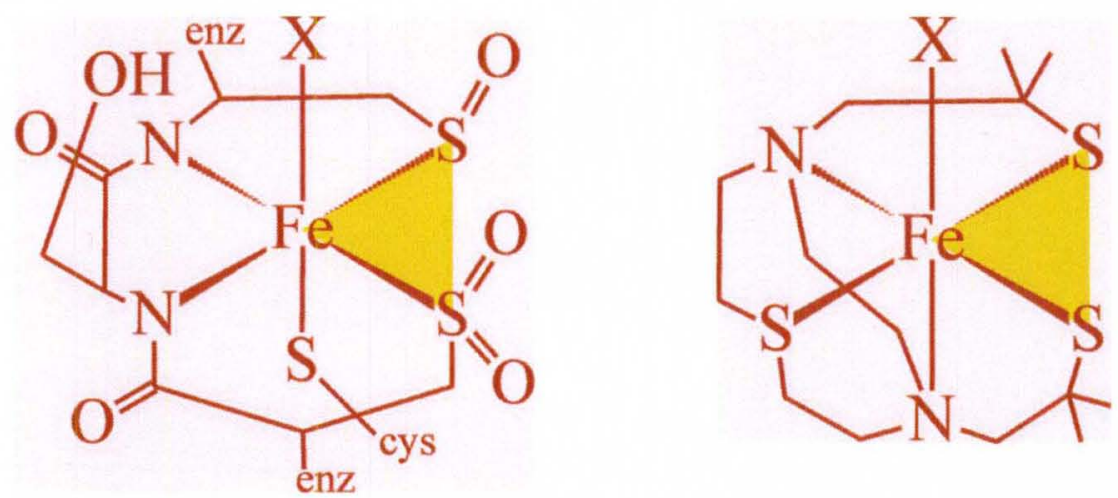

Figure 9. Comparison of NHase active site and bmmp-TASN model. 
The objective of this model is to systematically probe the metal's influence on sulfur reactivity and give insight about the role of the sulfur oxygenated thiolates. Previously, the synthesis of $\mathrm{LFeCl} \mathbf{1}$ ' was reported from $\mathrm{H}_{2} \mathrm{~L}, \mathrm{NEt}_{3}$, and $\left[\mathrm{NBu}_{4}\right]\left[\mathrm{FeCl}_{4}\right]$ in ethanol. $^{93}$ The oxygen sensitivity of 1' and a related iron(III) dithiolate complex LFeCN 2' was reported. Oxygen exposure of the high-spin complex 1' results in disulfide formation and decomplexation of the metal with subsequent iron-oxo cluster formation. ${ }^{94}$ Oxygen exposure of the low-spin complex 2' yields the disulfonate complex (bmmp- $\mathrm{O}_{6}$ TASN)FeCN 4', Figure 10. The corresponding nickel dithiolate, LNiCN 5', yields the related disulfonate derivative, (bmmp- $\mathrm{O}_{6}$-TASN)NiCN 6' upon addition of $\mathrm{H}_{2} \mathrm{O}_{2}$. Further evaluation of 1' and 2' using density functional theory calculations indicate that the lowspin complex 2' contains Fe-S $\mathrm{S}_{\text {thiolate }}$ bonds with calculated covalencies of $75 \%$ and $86 \%$, while the high-spin complex 1' contains Fe-S $\mathrm{S}_{\text {thiolate }}$ bonds with calculated covalencies of $11 \%$ and $40 \%$. These results suggest that spin-state and Fe-S bond covalency play major roles in determining the reaction pathway associated with oxygen exposure. ${ }^{78}$ Based on the sulfur-oxygenation low-spin 2' results, we synthesized the low spin Ru derivative $\mathrm{LRuPPh}_{3} 1$ to evaluate its oxygen sensitivity and resulting sulfur oxygen derivatives. 


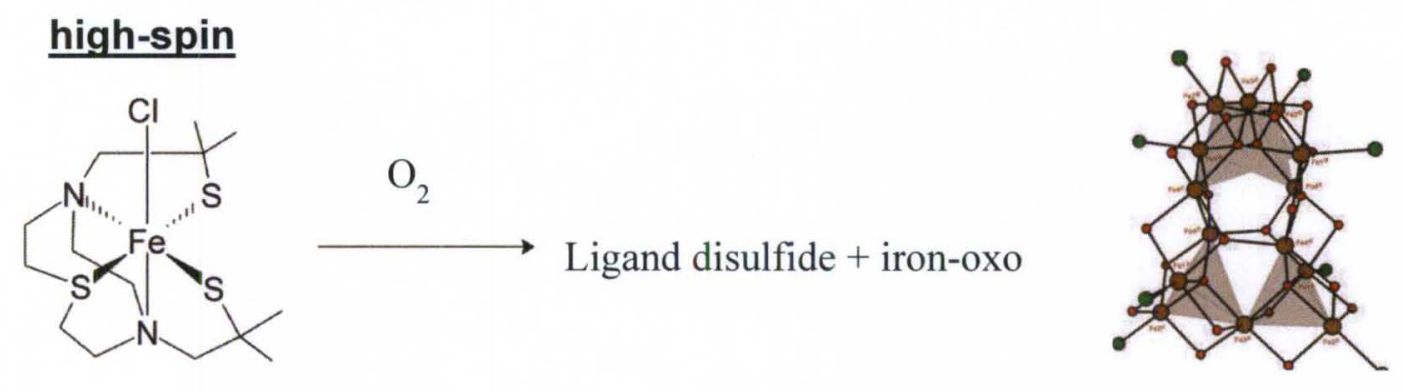

\section{low-spin}
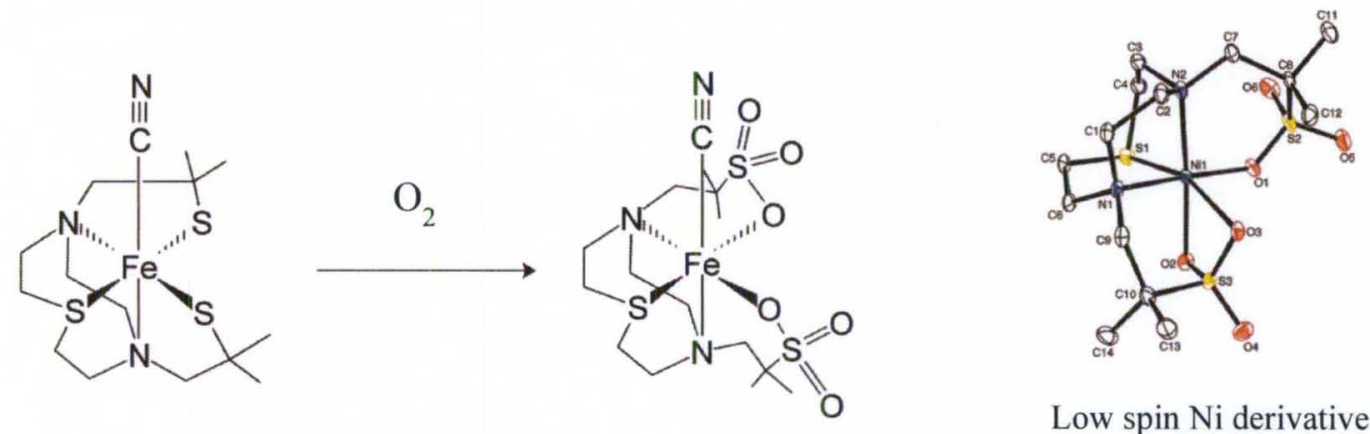

Low spin Ni derivative (bmmp- $\left.\mathrm{O}_{6}-\mathrm{TASN}\right) \mathrm{Ni}$

Figure 10. Spin state of iron influences $\mathrm{Fe}-\mathrm{SR}$ reactivity with $\mathrm{O}_{2}$. 


\section{CHAPTER II}

\section{EXPERIMENTAL METHODS}

\section{Materials}

All chemicals were obtained from commercially available sources and were used as received unless otherwise noted. All glassware was flame-dried under vacuum three times prior to use and refilled with argon. All solvents were dried and freshly distilled using conventional techniques under a nitrogen atmosphere before degassing using freeze-pump-thaw techniques or purging with argon for 15 minutes. Standard Schlenk techniques under an argon atmosphere or an argon-filled glovebox were utilized for the manipulation of all compounds in this study. Oxygen addition was accomplished with the use of a $23 \mathrm{ml}$ gas transfer bulb (fabricated by Wilmad-LabGlass) connected to a Schlenk line.

1-Thia-4,7-diazacyclononane (TASN) was prepared according to the method of Hoffmann et al. ${ }^{95}$ The ligand $\mathrm{H}_{2}(\mathrm{bmmp-TASN})$ was prepared as previously reported and stored as oil under argon in the glovebox. ${ }^{93,96} \mathrm{RuCl}_{2}\left(\mathrm{PPh}_{3}\right)_{3}$ was prepared according to literature methods. ${ }^{97}$ Oxygen $(99.8 \%$ ) was obtained from Welder's Supply, Louisville, KY. Reactions were carried out under argon atmosphere at room temperature, unless otherwise specified. 


\section{Physical methods}

Elemental analyses were obtained from Midwest Microlab (Indianapolis, IN). Infrared spectra were recorded as $\mathrm{KBr}$ pellets using a Thermo Nicolet Avatar 360 spectrometer at $4 \mathrm{~cm}^{-1}$ resolution. The infrared spectra was analyzed in the OMNIC $6.0 \mathrm{a}$ Thermo Nicolet Corporation Copyright 1992-2001 and in the KnowItAll ${ }^{\circledR}$ Informatic

System 8.2 Copyright 2001-2010. ${ }^{1} \mathrm{H},{ }^{13} \mathrm{C}$ and ${ }^{31} \mathrm{P}$ NMR spectra were obtained on a Varian Inova $500 \mathrm{MHz}$ spectrometer and the analysis of the data was performed in MestReNova Version 7.0.2-8636 (C) 2011 Mestrelab Research S.R.L. NMR measurements were carried out under argon atmosphere in J-Young NMR tubes at $25^{\circ} \mathrm{C}$, unless otherwise specified. Electrospray Ionization mass spectrometry (+ESI-MS) was performed by the Laboratory for Biological Mass Spectrometry at Texas A\&M University.

Cyclic voltammetry (CV) was performed using a EG\&G Princeton Applied Research potentiostat/galvanostat model 273 potentiostat with a three electrode cell (glassy carbon working electrode, platinum wire counter electrode, and Ag pseudo reference electrode) at room temperature in an argon filled glovebox. All potentials were scaled to a ferrocene standard used as internal reference. The cyclic voltammetry and square wave were set up in the Model 270/250 research electrochemistry software 4.40 copyright 1996 EG\&G instruments INC. The analysis and plot of electrochemistry data was performed in SigmaPlot for Windows Version 11.0 Copyright $₫ 2008$.

Electronic absorption spectra were recorded with an Agilent 8453 diode array spectrometer with an air free $1 \mathrm{~cm}$ path length quartz cell or an air free $0.5 \mathrm{~cm}$ path length quartz cell with a condenser for temperature dependence experiments in the hydrolysis 
assays with ester substrates. Analysis and process of data were performed in UV-Visible ChemStation copyright (C) Agilent Technologies 95-00.

\section{Crystallographic studies}

Crystals were mounted on a glass fiber for collection of x-ray data on a Bruker SMART APEX CCD diffractometer. The SMART ${ }^{98}$ software package (v 5.632) was used to acquire thirty-second frame $\omega$-scan exposures of data at $100 \mathrm{~K}$ to a $2 \theta \max =56.22^{\circ}$ using monochromated MoKa radiation (0.71073 $\AA$ ) from a sealed tube and a monocapillary. Frame data were processed using $\operatorname{SAINT}^{99}$ (v 6.45) to determine final unit

cell parameters. The structure was solved by Patterson methods using SHELXS-90 ${ }^{100}$ and refined by least squares methods on $\mathrm{F}^{2}$ using SHELXL-97 $7^{101}$ incorporated into the SHELXTL $^{102}$ (v 6.14) suite of programs. All non-hydrogen atoms were refined with anisotropic atomic displacement parameters. Hydrogen atoms were placed in their geometrically generated positions and refined as a riding model. Crystallographic parameters for 1, 2 and $\mathbf{3}$ are published. ${ }^{129,141}$ The 8, 9, 10 and $\mathbf{1 1}$ crystal structure are presented in this thesis, crystallography parameter are included in the appendix. The crystallographic data was collected and processed by Dr. Mark Mashuta.

\section{Synthesis-Chapter III}

(4,7-bis-(2'-methyl-2'-mercaptopropyl)-1-thia-4,7-

diazacyclononane)(triphenylphosphine) ruthenium (II) (bmmp-TASN)RuPPh (1)

A suspension of $650 \mathrm{mg}(2.51 \mathrm{mmol})$ of $\mathrm{H}_{2}$ (bmmp-TASN) in dry-degassed $20 \mathrm{~mL}$ of THF was cooled to $-78^{\circ} \mathrm{C}$ in a liquid nitrogen - acetone bath and $1.20 \mathrm{~mL}(12.7 \mathrm{mmol})$ of $n$-butyl lithium (2M in hexane) was added using a glass syringe (Precaution; $n$-butyl 
lithium reacts violently with water). The solution was allowed to slowly warm to room temperature and then transferred via cannula to a Schlenk flask containing $1.50 \mathrm{~g}(5.72$ mmol) of $\mathrm{RuCl}_{2}\left(\mathrm{PPh}_{3}\right)_{3}$. The resulting solution was stirred for 12 hours and then refluxed for 15 minutes. An orange solid is observed on the walls of Schlenk flask 30 minutes after the beginning of the reaction. The resulting orange solid was isolated by filtration due to its low solubility in THF and washed with diethyl ether under air free conditions. The orange solid was then washed with $100 \mathrm{ml}$ of $\mathrm{H}_{2} \mathrm{O}$ and 3 portions of $20 \mathrm{ml}$ of diethyl ether. This final washing step does not required air - free methods. The complex is stable in the solid state, but readily reacts with dioxygen in solution. Yield: $344 \mathrm{mg}(0.503$ mmol, $20 \%$ ). Electronic absorption (acetonitrile $\left(22^{\circ} \mathrm{C}\right)$ ): $\lambda \max (\varepsilon) 438(883)$. IR ( $\mathrm{KBr}$ pellet), $\mathrm{cm}^{-1}$ : $2902(\mathrm{br}), 1483(\mathrm{~m}), 1457(\mathrm{~m}), 1431(\mathrm{~m}), 1133(\mathrm{~m}), 1085(\mathrm{~m}), 1066(\mathrm{~m})$, $1030(\mathrm{~m}), 1014(\mathrm{~m}), 751(\mathrm{~s}), 701(\mathrm{~m}), 696(\mathrm{~m}) 592(\mathrm{~m}), 516(\mathrm{~m}) . \mathrm{E}_{1 / 2}=-851 \mathrm{mV},(+) \mathrm{ESI}-$ MS, $m / z$ calcd. For $\mathrm{C}_{32} \mathrm{H}_{43} \mathrm{~N}_{2} \mathrm{PRuS}_{3},[\mathrm{M}+] 684.14$ found, 684.11. Anal Calcd for $\mathrm{C}_{32} \mathrm{H}_{43} \mathrm{~N}_{2} \mathrm{PRuS}{ }_{3}: \mathrm{C}: 56.20 \%, \mathrm{H}: 6.34 \%, \mathrm{~N}: 4.10 \%$. found C: $55.13 \%, \mathrm{H}: 6.52 \%, \mathrm{~N}: 3.92 \%$.

${ }^{31} \mathrm{P}$ NMR $43.37 \mathrm{ppm}$ in $\mathrm{CD}_{3} \mathrm{OD}$ referenced to $\mathrm{PPh}_{3}-6.23 \mathrm{ppm}$ at $25^{\circ} \mathrm{C}$. X-ray quality crystals of 1 were obtained by slow evaporation of a methanolic solution under air-free conditions.

\section{$($ bmmp-O - TASN)RuPPh 3 (2)}

A suspension of $1(128 \mathrm{mg}, 0.18 \mathrm{mmol})$ was stirred in $50 \mathrm{~mL}$ of dry, degassed $\mathrm{MeOH}$ in a $250 \mathrm{~mL}$ Schlenk flask with a connected gas sample bulb with a volume of 23 $\mathrm{mL}$ charged with $\mathrm{O}_{2}(0.94 \mathrm{mmol})$ at a pressure of 1 atm. The flask containing 1 was evacuated just to the point of solvent boiling at which time the valve to the gas sample bulb was opened introducing the $\mathrm{O}_{2}$. The solution was stirred for $5 \mathrm{~min}$ after which the 
solution was filtered to remove any trace solids followed by evacuation of the solvent. The crude product was dissolved in degassed methanol. Slow evaporation of the solvent under air-free conditions in the glovebox yielded pure $\mathbf{2}$ as orange crystals. Yield: $93 \mathrm{mg}$ $(0.13 \mathrm{mmol}, 72 \%)$. Electronic absorption (acetonitrile $\left.\left(22^{\circ} \mathrm{C}\right)\right): \lambda \max (\varepsilon) 338(1200), 385$ (764). IR (KBr pellet), cm-1: 3058 (m), 2981 (br), 2961 (br), 2918 (br), 1948 (br), 1635(m), $1580(\mathrm{~s}), 1489$ (s), 1477 (s), 1458 (s), 1432 (s), 1139 (s), 1020 (s), 916 (s), 870 (s), $751(\mathrm{~s}), 703$ (s), 696 (s). $\mathrm{E}_{1 / 2}=-393 \mathrm{mV},(+)$ ESI-MS, $\mathrm{m} / \mathrm{z}$ calcd for $\mathrm{C}_{32} \mathrm{H}_{43} \mathrm{~N}_{2} \mathrm{PRuS}_{3} \mathrm{O}_{2},[\mathrm{M}]^{+} 716.1268$ found, 715.6971 Anal. Calcd for $\mathrm{C}_{32} \mathrm{H}_{43} \mathrm{~N}_{2} \mathrm{PRuS}_{3} \mathrm{O}_{2}$ : C: $53.69 \%, \mathrm{H}: 6.05 \%$, N: $3.91 \%$ found C: $52.90 \%, \mathrm{H}: 6.17 \%, \mathrm{~N}: 3.99 \% .{ }^{31} \mathrm{P}$ NMR 34.34 ppm in $\mathrm{CD}_{3} \mathrm{OD}$ referenced to $\mathrm{PPh}_{3}-6.23 \mathrm{ppm}$ at $25^{\circ} \mathrm{C}$.

(bmmp-O ${ }_{3}$-TASN)RuPPh 3 (3)

To a suspension of $204 \mathrm{mg}(0.298 \mathrm{mmol})$ of 1 in $75 \mathrm{~mL}$ dry, degassed MeOH was added $23 \mathrm{ml}(0.94) \mathrm{mmol}$ of $\mathrm{O}_{2}$. The addition of $\mathrm{O}_{2}$ was performer under atmospheric pressure. The solution was stirred for 12 hours after which the solvent was removed under vacuum. The yellow crude product was dissolved in degassed water and filtrated. The yellow solution was concentrated and dissolved in methanol. Slow evaporation of the solvent under air-free conditions yielded pure 3 as yellow plates. Yield: $179 \mathrm{mg}(0.245$ mmol, 82\%) Electronic absorption (acetonitrile $\left(22^{\circ} \mathrm{C}\right)$ ): $\lambda \max (\varepsilon) 336(1486) . \mathrm{IR}(\mathrm{KBr}$ pellet), $\mathrm{cm}^{-1}: 2925(\mathrm{br}), 1482(\mathrm{~m}), 1461(\mathrm{~m}), 1432(\mathrm{~m}), 1402(\mathrm{~m}), 1137$ (s), $1086(\mathrm{~s}), 1020$ (s), $923(\mathrm{~m}), 914(\mathrm{~m}), 750(\mathrm{~s}), 695(\mathrm{~s}), 595(\mathrm{~s}) 526(\mathrm{~m}), 515(\mathrm{~m}) . \mathrm{E}_{1 / 2}=-263 \mathrm{mV},(+) \mathrm{ESI}-$ MS, $m / z$ calcd for $\mathrm{C}_{32} \mathrm{H}_{43} \mathrm{~N}_{2} \mathrm{PRuS}_{3} \mathrm{O}_{3}$, [M+] 732.1217 found, 731.1147 Anal Calcd for $\mathrm{C}_{32} \mathrm{H}_{43} \mathrm{~N}_{2} \mathrm{PRuS}_{3} \mathrm{O}_{3}:$ C: $52.51 \%, \mathrm{H}: 5.92 \%, \mathrm{~N}: 3.83 \%$ found $\mathrm{C}: 51.36 \%, \mathrm{H}: 6.06 \%, \mathrm{~N}$ : 3.89\%. ${ }^{31} \mathrm{P}$ NMR $33.68 \mathrm{ppm}$ in $\mathrm{CD}_{3} \mathrm{OD}$ referenced to $\mathrm{PPh}_{3}-6.23 \mathrm{ppm}$ at $25^{\circ} \mathrm{C}$. 


\section{Alternative protocol for synthesis of compound 3 staring from compound 2}

To a suspension of $200 \mathrm{mg}(0.279 \mathrm{mmol})$ of 2 in $75 \mathrm{~mL}$ dry, degassed MeOH was added $23 \mathrm{ml}(0.94 \mathrm{mmol})$ of $\mathrm{O}_{2}$. The addition of $\mathrm{O}_{2}$ was performer under atmospheric pressure. The solution was stirred for 12 hours after which the solvent was removed under vacuum. The yellow crude product was dissolved in degassed water and filtrated to obtain purified compound 3. The yellow solution was concentrated and dissolved in methanol. Slow evaporation of the solvent under air-free conditions yielded pure $\mathbf{3}$ as yellow plates, the product was characterized by electrochemistry with an $E_{1 / 2}=-263 \mathrm{mV}$ $[\mathrm{M}+\mathrm{H}]+733.12$ found, 733.13 . Yield: $120 \mathrm{mg}(0.164 \mathrm{mmol})$.

\section{$($ bmmp-O4-TASN)RuPPh 3 (4)}

A suspension of $50 \mathrm{mg}(0.066 \mathrm{mmol})$ of 1 in $50 \mathrm{~mL}$ of dry, degassed MeOH was gently purged with $\mathrm{O}_{2}$ for $2 \mathrm{~h}$. The system was closed under an $\mathrm{O}_{2}$ atmosphere and stirred for $120 \mathrm{~h}$ after which the solvent was removed under vacuum. The crude product was dissolved in water and filtered to remove insoluble products. Evaporation of the filtrate yields 4. Yield: $15 \mathrm{mg}(0.020 \mathrm{mmol}, 30 \%)$. Electronic absorption IR (KBr pellet), $\mathrm{cm}^{-1}$ : 2970 (br), 2918 (br), 1948 (br), 1668 (s), 1580 (s), 1462 (s), 1437 (s), $1136(\mathrm{~s}), 1120$ (s), 1029 (s), 1015 (s), 921 (s), 874 (s), 754 (s), 721 (s), 697 (s). (+)ESI-MS, $m / z$ calcd for $\mathrm{C}_{32} \mathrm{H}_{43} \mathrm{~N}_{2} \mathrm{PRuS}_{3} \mathrm{O}_{4},[\mathrm{M}]^{+} 748.12$ found, 748.12. Three attempts to obtain elemental analyses consistent with the ESI-MS were unsuccessful. This is ascribed to the partial degradation of $\mathbf{4}$ in water during isolation. The "best" analysis assumed Anal. Calcd For $\mathrm{C}_{32} \mathrm{H}_{43} \mathrm{~N}_{2} \mathrm{PRuS}_{3} \mathrm{O}_{4} \cdot 0.5 \mathrm{PPh} 3: \mathrm{C}: 56.02 \%, \mathrm{H}: 5.79 \%, \mathrm{~N}: 3.19 \%$ found $\mathrm{C}: 52.41 \%, \mathrm{H}$ : $6.10 \%, \mathrm{~N}: 3.09 \%$. 
(bmmp-TASN)RuPPh + excess $\mathrm{O}_{2}$

A suspension of $296 \mathrm{mg}(0.433 \mathrm{mmol})$ of 1 in dry degassed $150 \mathrm{ml}$ of MeOH was purged with $\mathrm{O}_{2}$ for 15 minutes and then stirred under an $\mathrm{O}_{2}$ atmosphere saturation for 96 hours. The solution was solvent removed under vacuum yielding a brown, intractable solid. IR (KBr pellet) $\mathrm{cm}^{-1}: 2920$ (br), 1440 (m), 1431 (m), 1267 (s), 1123 (s), 1091 (s), 1017 (s), 924 (s), 869 (s), 799 (s), 747 (s), 699 (s), 593 (s) 523 (m).

\section{Isotopic Labeling Studies (bmmp- ${ }^{18} \mathrm{O}_{2}$-TASN)RuPPh ${ }_{3}(5)$}

The ${ }^{18} \mathrm{O}$ isotopically labeled derivative of 2 was obtained via direct oxygenation of 1 with ${ }^{18} \mathrm{O}_{2}$. A suspension of $1(128 \mathrm{mg}, 0.187 \mathrm{mmol})$ was stirred in $50 \mathrm{~mL}$ of dry, degassed $\mathrm{MeOH}$ in a $250 \mathrm{~mL}$ Schlenk flask with a connected gas sample bulb with a volume of $23 \mathrm{~mL}$ charged with $\mathrm{O}_{2}(0.94 \mathrm{mmol})$ at a pressure of 1 atm. The flask containing 1 was evacuated just to the point of solvent boiling at which time the valve to the gas sample bulb was opened introducing the $\mathrm{O}_{2}$. The solution was stirred for 5 min after which the solution was filtered to remove any trace solids followed by evacuation of the solvent. The crude product was dissolved in degassed methanol. Slow evaporation of the solvent under air-free conditions in the glovebox yielded pure $\mathbf{5}$ as orange crystals. Yield: $93 \mathrm{mg}(0.13 \mathrm{mmol}, 72 \%) .(+)$ ESI-MS, $m / z$ calcd for $\mathrm{C}_{32} \mathrm{H}_{43} \mathrm{~N}_{2} \mathrm{PRuS}_{3}{ }^{18} \mathrm{O}_{2},[\mathrm{M}]^{+}$ 719.93 found, 720.13. IR (KBr pellet) $\mathrm{cm}^{-1}: 2962$ (br) 2921 (br), 1435 (s), 1104 (s), 1082 (s), $751(\mathrm{~s}), 696(\mathrm{~s}), 530(\mathrm{~s})$.

\section{$\left(\right.$ bmmp- $\left.{ }^{18} \mathrm{O}_{3}-\mathrm{TASN}\right) \mathrm{RuPPh}_{3}(6)$}

The ${ }^{18} \mathrm{O}$ isotopically labeled derivative of $\mathbf{3}$ was obtained via direct oxygenation of 1 with ${ }^{18} \mathrm{O}_{2}$. To a suspension of $1(20.0 \mathrm{mg}, 0.03 \mathrm{mmol})$ in dry degassed $\mathrm{MeOH}(10$ $\mathrm{mL}$ ) was added $0.14 \mathrm{mmol}$ of ${ }^{18} \mathrm{O}_{2}$. The reaction was stirred for 12 hours at room 
temperature after which the solvent was removed under vacuum. The crude product was dissolved in methanol. Slow evaporation of the solvent under air-free conditions yielded isotopically labeled 6. Yield: $18 \mathrm{mg}(0.03 \mathrm{mmol}, 82 \%)$. IR (KBr pellet), $\mathrm{cm}^{-1}: 2925(\mathrm{br})$, $1683(\mathrm{~m}), 1462(\mathrm{~m}), 1437(\mathrm{~m}), 1402(\mathrm{~m}), 1119(\mathrm{~s}), 1092(\mathrm{~s}), 1020(\mathrm{~s}), 998(\mathrm{~s}), 980(\mathrm{~m}) 900$ (s), 877 (m), 751 (s), 696 (s), 589 (s) 526 (m), 517 (m). +ESI-MS, m/z calcd for $\mathrm{C}_{32} \mathrm{H}_{43} \mathrm{~N}_{2} \mathrm{PRuS}_{3}{ }^{18} \mathrm{O}_{3},[\mathrm{M}+] 731.93$ found, 737.12. IR (KBr pellet) 2925(br), $1683(\mathrm{~m})$, $1462(\mathrm{~m}), 1437(\mathrm{~m}), 1402(\mathrm{~m}), 1119(\mathrm{~s}), 1092(\mathrm{~s}), 1020(\mathrm{~s}), 998(\mathrm{~s}), 980(\mathrm{~m}) 900(\mathrm{~s}), 877$ (m), $751(\mathrm{~s}), 696(\mathrm{~s}), 589(\mathrm{~s}) 526(\mathrm{~m}), 517(\mathrm{~m})$.

\section{Synthesis-Chapter IV}

(bmmp-TASN)Ru(PMePh $)$ (7)

A solution of 1 (21.5 mg, $0.0314 \mathrm{mmol})$ was stirred in $22 \mathrm{~mL}$ of dry, degassed chlorobenzene in a $250 \mathrm{~mL}$ Schlenk flask. $29 \mu 1(31.20 \mathrm{mg} 0.16 \mathrm{mmol})$ of methyldiphenylphosphine was transfer to the solution in the glovebox using a Eppendorf Research $^{\mathbb{B}}$ adjustable volume pipette of $1000 \mu \mathrm{l}$. The flask containing 1 and methyldiphenylphosphine was heated at $100{ }^{\circ} \mathrm{C}$ for 36 hours using and oil bath. The solution was concentrated under air free conditions at room temperature using the Schlenk line. The yellow solid was transfer to the glovebox inside the Schlenk flask. Yield $3.5 \mathrm{mg}(0.0056 \mathrm{~mol}, 20 \%){ }^{31} \mathrm{P}$ NMR $34.64 \mathrm{ppm}$ in $\mathrm{CD}_{3} \mathrm{OD}$ referenced to $\mathrm{PPh}_{3}$ $6.23 \mathrm{ppm}$ at $25^{\circ} \mathrm{C}$.

\section{$($ bmmp-O 2 -TASN)Ru(PMePh $)(8)$}

A solution of 2 (11 mg, $0.015 \mathrm{mmol})$ was stirred in $5 \mathrm{~mL}$ of dry, degassed $\mathrm{MeOH}$ in a $50 \mathrm{~mL}$ Schlenk flask. $20.2 \mathrm{mg}(0.10 \mathrm{mmol})$ of methyldiphenylphosphine was added 
to the solution inside the glovebox. The flask containing the solution was heated at $62{ }^{\circ} \mathrm{C}$ for 36 hours. The solvent was evacuated under air free conditions at room temperature and the crude was dissolved in degassed $\mathrm{MeOH}$ under air-free condition for crystallization using solvent evaporation technique in a test tube inside the glovebox. After two days the solution was filtered using a cotton pipette setup and crystals were collected from the walls of the tube at the fifth day from the setup of the crystallization. Yield: $9.0 \mathrm{mg}(0.013 \mathrm{mmol}, 92 \%)$. (+)ESI-MS, $\mathrm{m} / z$ calcd for $\mathrm{C}_{27} \mathrm{H}_{41} \mathrm{~N}_{2} \mathrm{O}_{2} \mathrm{PRuS}_{3}$, $[\mathrm{M}+\mathrm{Na}]^{+} 676.1009$ found, $677.1144{ }^{31} \mathrm{P}$ NMR $25.49 \mathrm{ppm}$ in $\mathrm{CD}_{3} \mathrm{OD}$ referenced to $\mathrm{PPh}_{3}$ $6.23 \mathrm{ppm}$ at $25^{\circ} \mathrm{C}$. X-ray quality crystals of 8 were obtained by slow evaporation of a methanolic solution under air-free conditions.

\section{(bmmp-O 3 -TASN)Ru(PMePh $)$ (9)}

A solution of 3 (12 $\mathrm{mg}, 0.016 \mathrm{mmol})$ was stirred in $5 \mathrm{~mL}$ of dry, degassed $\mathrm{MeOH}$ in a $50 \mathrm{~mL}$ Schlenk flask. $15.0 \mathrm{mg}(0.075 \mathrm{mmol})$ of methyldiphenylphosphine was added to the solution inside the glovebox. The flask containing the solution was heated at $62{ }^{\circ} \mathrm{C}$ for 36 hours. The solvent was evacuated under air free conditions at room temperature and the crude was dissolved in degassed $\mathrm{MeOH}$ under air-free condition for crystallization using solvent evaporation technique. After two days the solution was filtered using a cotton pipette setup and crystals were collected from the walls of the tube at the fifth day from the setup of the crystallization. Yield: $10 \mathrm{mg}(0.015 \mathrm{mmol}, 94 \%)$. (+)ESI-MS, $m / z$ calcd for $\mathrm{C}_{27} \mathrm{H}_{41} \mathrm{~N}_{2} \mathrm{O}_{3} \mathrm{PRuS}_{3},[\mathrm{M}+\mathrm{Na}]^{+} 693.0958$ found, $693.1192{ }^{31} \mathrm{P}$ NMR $22.54 \mathrm{ppm}$ in $\mathrm{CD}_{3} \mathrm{OD}$ referenced to $\mathrm{PPh}_{3}-6.23 \mathrm{ppm}$ at $25^{\circ} \mathrm{C}$. X-ray quality crystals of 9 were obtained by slow evaporation of a methanolic solution under air-free conditions. 


\section{$\left[(\text { bmmp-TASN)Ru }]_{2}(10)\right.$}

A suspension of $650 \mathrm{mg}(2.51 \mathrm{mmol})$ of $\mathrm{H}_{2}(\mathrm{bmmp}$-TASN $)$ in dry-degassed $20 \mathrm{~mL}$ of THF was cooled to $-78{ }^{\circ} \mathrm{C}$ in an acetone bath and $1.20 \mathrm{~mL}(12.7 \mathrm{mmol})$ of $n$-butyl lithium (2M in hexane) was added using a glass syringe (Precaution; $n$-butyl lithium reacts violently with water). The solution was allowed to slowly warm to room temperature and transfer via cannula to a Schlenk flask containing $1.50 \mathrm{~g}(5.72 \mathrm{mmol})$ of $\mathrm{RuCl}_{2}\left(\mathrm{PPh}_{3}\right)_{3}$. The resulting solution was stirred for 12 hours and then refluxed for 15 minutes. Orange solid is observer in the wall of Schlenk flask 30 minutes after the beginning of the reaction. The resulting orange solid was isolated by filtration due to its low solubility in THF and washed with diethyl ether under air free conditions. The orange solid was then washed with $100 \mathrm{ml}$ of $\mathrm{H}_{2} \mathrm{O}$ and 3 portions of $20 \mathrm{ml}$ of diethyl ether. The solid was dissolved in methanol for crystallization by ether diffusion. After one month, the crystals of the bimetallic compound were recovered from the walls of the air free reaction tube. Three attempts to reproduce the bimetallic product were unsuccessful. X-ray quality crystals of $\mathbf{1 0}$ were obtained by solvent diffusion methanolether under air-free conditions. In each attempt 1 was isolated as a metal powder containing product.

\section{(bmmp-TASN)RuCO (11)}

A suspension of $1(50 \mathrm{mg}, 0.073 \mathrm{mmol})$ was stirred in $25 \mathrm{~mL}$ of dry, degassed $\mathrm{MeOH}$ in a $50 \mathrm{~mL}$ Schlenk flask. The flask was connected to a $\mathrm{CO}$ gas line, the sample was bubble by 20 minutes and the solution was stirred for 20 hours at $70{ }^{\circ} \mathrm{C}$ followed by evacuation of the solvent. The yellow crude product was dissolved in methanol. Slow evaporation of the solvent under air-free conditions yielded pure $\mathbf{9}$ as yellow crystals. 
Yield: $25 \mathrm{mg}(0.055 \mathrm{mmol}, 75 \%)$. Electronic absorption (methanol $\left.\left(22{ }^{\circ} \mathrm{C}\right)\right): \lambda \max (\varepsilon)$ 331 (956). IR (KBr pellet), $\mathrm{cm}^{-1}: 2887$ (br), 1889(s), 1358(s), 1084(s), 1049(s), 880 (s), (+)ESI-MS, $m / z$ calcd. For $\mathrm{C}_{15} \mathrm{H}_{28} \mathrm{ON}_{2} \mathrm{RuS}_{3}\left[\mathrm{M}^{+}\right] 450$ found, 453 and Anal Calcd for $\mathrm{C}_{32} \mathrm{H}_{43} \mathrm{~N}_{2} \mathrm{PRuS}$ : C: $40.07 \%, \mathrm{H}: 6.28 \%, \mathrm{~N}: 6.23 \%$. found $\mathrm{C}: 39.79 \%, \mathrm{H}: 6.13 \%, \mathrm{~N}: 5.98 \%$. X-ray quality crystals of $\mathbf{1 1}$ were obtained by slow evaporation of a methanolic solution under air-free conditions in the glovebox.

\section{Computational methodology}

Geometry optimization and subsequent molecular orbital (MO) analysis of complexes 1-4 were performed using the Gaussian 03 suite of programs. Density functional theory (DFT) calculations employed the $\mathrm{BP}^{66,86}$ and $\mathrm{B} 3 \mathrm{LYP}^{64,75,103,104}$ functionals based on its fruitful estimation of geometries for numerous transition metal complexes. For these calculations, the LANL2DZ basis set was used for the Ru atom while the 6-31G(d) basis set was applied for all other atoms. Input coordinates for compounds 1-4 were taken from the crystallographic coordinates of 3 with the addition or abstraction of oxygen atoms as needed to complete the family of derivatives. Optimized coordinates are listed in Tables A1-A4 of the Appendix. Similar results were obtained with the BP86 and B3LYP functionals. The former provided a slightly better correlation with experimental bond distances and angles and was used for analyses of the frontier orbitals. Molecular orbitals were visualized from cube files using ChemBio3D Ultra 12.0 and the VMD software package. ${ }^{105}$

\section{Hydrolysis of acetonitrile}

Attempts to hydrolyze acetonitrile with $\mathbf{3}$ were performed following the protocol

described by Chottard et al. ${ }^{89}$ A mixture of $3(5 \mathrm{mg}, 0.007 \mathrm{mmol})$ and 1,4- 
dimethoxybenzene $(7 \mathrm{mg}, 0.050 \mathrm{mmol})$ in $\mathrm{CH}_{3} \mathrm{CN}(0.5 \mathrm{~mL})$ and a $\mathrm{HOAc} / \mathrm{NaOAc}$ buffer ( $1 \mathrm{M}, \mathrm{pH}=4.8,0.5 \mathrm{~mL}$ ) was stirred at room temperature for 5 days under an argon atmosphere. The solvent was removed under reduced pressure to yield a yellow residue that was extracted with chloroform. The organic extract was dried under reduced pressure and the solid residue dissolved in $\mathrm{CD}_{3} \mathrm{OD}$. Analysis by ${ }^{1} \mathrm{H}$ and ${ }^{13} \mathrm{C}$ NMR failed to yield the expected resonances for acetamide in a trail at $4{ }^{\circ} \mathrm{C}$. Attempts at $4{ }^{\circ} \mathrm{C}$ with $\mathbf{1}$ and 2 also show no evidence of nitrile hydrolysis.

Solutions of 1, 2 and 3 in 1:1:1 mixture of acetonitrile, methanol, and PIPES buffer $(\mathrm{pH}=7.0)$ were tested for 5 days at $25^{\circ} \mathrm{C}$. The mixture was composed of $1(5 \mathrm{mg}$, $7 \mu \mathrm{mol})$ in $\mathrm{CH}_{3} \mathrm{CN}(0.5 \mathrm{ml})$, methanol $(0.5 \mathrm{ml})$ and PIPES $(1 \mathrm{M}, \mathrm{pH}=7.0,0.5 \mathrm{ml})$. The identity of the acetamide was confirmed by ${ }^{1} \mathrm{H}$ NMR and ${ }^{13} \mathrm{C}$ NMR and It was quantified using 1,4-dimethoxybenzene $(7 \mathrm{mg}, 0.050 \mathrm{mmol})$ as an internal standard.

Acetonitrile experiment using compounds 1, 2 and 3 were performed in a NMR Jyoung tube in a mixture of $500 \mu \mathrm{l}$ of $\mathrm{MeOH}$ deuterated and $200 \mu \mathrm{l} \mathrm{D} 2 \mathrm{O}$. For compound $\mathbf{3}$, It was used $2.2 \mathrm{mg}(3.0 \mu \mathrm{mol})$ of compound and $2.2 \mathrm{mg}(6.7 \mu \mathrm{mol})$ of $\left(\mathrm{C}_{4} \mathrm{H}_{9}\right)_{4} \mathrm{~N}^{+} \mathrm{BF}_{4}{ }^{-}$as reference. It was used $5 \mu \mathrm{L}(94.6 \mu \mathrm{mol})$ of acetonitrile as substrate. The mixture $\mathrm{MeoH}$ and $\mathrm{D}_{2} \mathrm{O}$ was used to help to solubilize the compound 3 . The reference $\left(\mathrm{C}_{4} \mathrm{H}_{9}\right)_{4} \mathrm{~N}^{+} \mathrm{BF}_{4}^{-}$ was integrated in the region $0.94 \mathrm{ppm}$ to 1.01 for the equivalent of 3 protons. The experiment was performed for 11 day in an oil bath. The sample was only removed from the bath for ${ }^{1} \mathrm{H}$ NMR determinations of 20 minutes. The ${ }^{1} \mathrm{H}$ NMR was performed with a relax delay $1.0 \mathrm{sec}, 256$ repetitions with a total time of 15 minutes, at $25^{\circ} \mathrm{C}$ in a 400 $\mathrm{MHz}$ instrument. Similar experiments were performed in compound $\mathbf{1}$ and $\mathbf{2}$. 


\section{Hydrolysis of 4-nitrophenyl acetate}

A mixture of 1 ( $5 \mathrm{mg}, 7 \mu \mathrm{mol})$ and 4-nitrophenyl acetate $(16 \mathrm{mg}, 0.088 \mathrm{mmol})$ in $\mathrm{MeOH} / \mathrm{H}_{2} \mathrm{O}(8.2 \mathrm{~mL} / 8.2 \mathrm{~mL})$ and citrate buffer $(1 \mathrm{M}, \mathrm{pH}=4.8,1.8 \mathrm{~mL})$ was stirred at room temperature or $4{ }^{\circ} \mathrm{C}$ for 5 days. The reaction was monitored by UV-visible spectroscopy, and results were compared to a control sample with no added metal complex. The same method was applied to solutions containing 2 and 3. Hydrolysis of $\mathrm{CH}_{3} \mathrm{CN}$ : A mixture of 1 (5 mg, $\left.7 \mu \mathrm{mol}\right)$ and 1,4-dimethoxybenzene ( $7 \mathrm{mg}, 0.050 \mathrm{mmol}$ ) in $\mathrm{CH}_{3} \mathrm{CN}(0.5 \mathrm{~mL})$ and an HOAc/NaOAc $(1 \mathrm{M}, \mathrm{pH}=4.8,0.5 \mathrm{~mL})$ or PIPES $(1 \mathrm{M}, \mathrm{pH}=$ 7.0, $0.5 \mathrm{~mL}$ ) buffer was stirred at room temperature and at $4{ }^{\circ} \mathrm{C}$ for 5 days. After chloroform extraction, the organic soluble residue was dissolved in $\mathrm{CD}_{3} \mathrm{OD}$ for ${ }^{1} \mathrm{H}$ and ${ }^{13} \mathrm{C}$ NMR analysis. The same procedure was applied to $\mathbf{2}$ and $\mathbf{3}$.

\section{Hydrolysis of acrylonitrile}

$200 \mu \mathrm{l}(11.11 \mathrm{mmol})$ of water deuterated was added to a solution of acrylonitrile $5 \mu \mathrm{l},(0.08 \mathrm{mmol}), 2.2 \mathrm{mg}\left(3.01 \times 10^{-3} \mathrm{mmol}\right)$ of $3,3.7 \mathrm{mg}(0.01 \mathrm{mmol})$ of $\left(\mathrm{C}_{4} \mathrm{H}_{9}\right)_{4} \mathrm{~N}^{+} \mathrm{BF}_{4}^{-}$ and $500 \mu \mathrm{l}$ of $\mathrm{CD}_{3} \mathrm{OD}$ using a Eppendorf Research ${ }^{(\mathbb{B})}$, adjustable-volume pipette of 1000 $\mu \mathrm{l}$ in a NMR J-young tube in the glovebox. The solution was heated to $70{ }^{\circ} \mathrm{C}$ for 20 days. The ${ }^{1} \mathrm{H}$ NMR resonances $\left(\mathrm{D}_{2} \mathrm{O}\right)$ of the solution before heating were at $\delta 6.29(\mathrm{dd}, J=18.0$ $\left.\mathrm{Hz}, 2 \mathrm{H}, \mathrm{H}_{2}=\mathrm{CHCN}\right)$ and $5.82\left(\mathrm{q}, J=12.0 \mathrm{~Hz}, 1 \mathrm{H}, \mathrm{H}_{2} \mathrm{C}=\mathrm{C} H \mathrm{CN}\right)$. During heating, these resonances decreased and the resonances for acrylamide appeared: $\delta 6.24(\mathrm{t}, J=1.5 \mathrm{~Hz}$, $\left.1 \mathrm{H}, \mathrm{H}_{2} \mathrm{C}=\mathrm{CHCONH}_{2}\right)$ and $5.83\left(\mathrm{t}, J=3.0 \mathrm{~Hz}, 2 \mathrm{H}, \mathrm{H}_{2} \mathrm{C}=\mathrm{CHCONH}_{2}\right)$.

\section{Hydrolysis of benzonitrile}

$1500 \mu \mathrm{l}(83.30 \mathrm{mmol})$ of water deuterated was added to a solution of benzonitrile

$500 \mu \mathrm{l},(4.85 \mathrm{mmol})$ and $500 \mu \mathrm{l}$ of stock solution of $5.4 \times 10^{-4} \mathrm{M}\left(2.70 \times 10^{-7} \mathrm{mmol}\right)$ of 
complex 1-3 using a micropipette into an air free reaction tube in the glovebox. The solution turned yellow in the aquous phase. The benzonitrile formed a clear layer on the top of the solution due to its poor solubility in the aquous layer. The sample was heated at $124^{\circ} \mathrm{C}$ in an oil bath for 18 hours in an oil bath. A white precipitate formed when the solution is cooled down to room temperature after the trial is completed. The solvent was removed under reduced pressure to yield a yellow residue that was extracted with ether. The organic extract was passed through a silica gel column. Analysis by ${ }^{1} \mathrm{H}$ and ${ }^{13} \mathrm{C}$ NMR yield the expected resonances for benzamide. These results were complemented and verified with FT-IR analysis. Yield: $85 \pm 11$ turnovers based in mols of catalyst. IR ( $\mathrm{KBr}$ pellet), $\mathrm{cm}^{-1}: 3390$ (s) 2930 (br) 1670 (s) 1580 (s) 1470 (br) 1415 (br) 1120 (s). Additional hydrolysis studies with complexes $\mathbf{1}$ and $\mathbf{2}$ were conducted according to the protocol listed above. The reaction yields $65 \pm 1$ turnovers for compound 1 and $86 \pm 9$ turnovers for compound 2 . The analysis of the blank following the same conditions with exception of the catalyst gave $7 \pm 2$ turnovers. 


\section{CHAPTER III}

\section{CONTROLLED SULFUR-OXYGENATION OF A RUTHENIUM(II)- DITHIOLATE}

\section{Background}

The hydrolytic metalloenzymes nitrile hydratase (NHase) and thiocyanate hydrolase (SCNase) share an infrequent post-translational modification. ${ }^{42,45-47,56,106}$ Metal-coordinated thiolates in these enzymes react with dioxygen to yield sulfuroxygenated derivatives as a requirement for catalytic activity. The active site of NHase contains a low-spin $\mathrm{Fe}(\mathrm{III})$ or $\mathrm{Co}(\mathrm{III})$ in an $\mathrm{N}_{2} \mathrm{~S}_{3}$ donor set composed of two carboxamido nitrogen donors and three cysteine derived sulfurs, Figure 11.

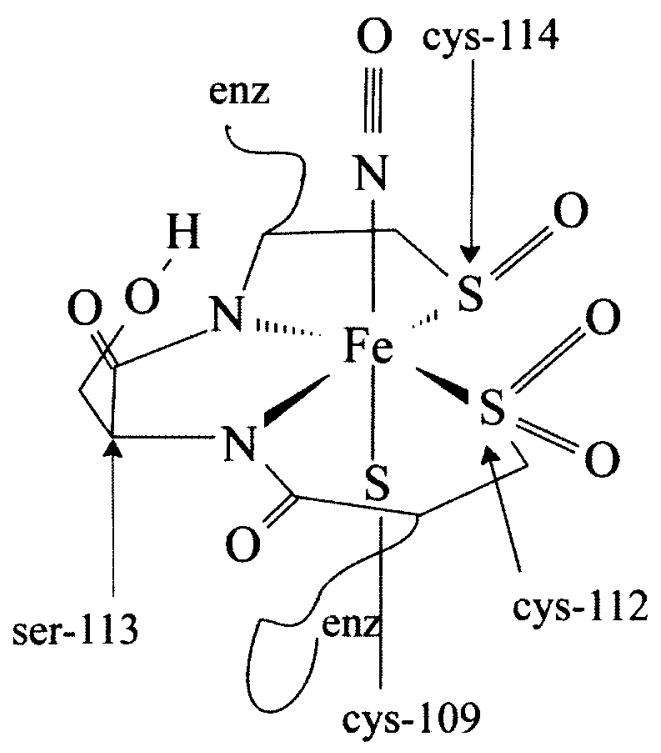

Figure 11. Active site of NO-inactivated Fe-containing NHase. 
The active sites of nitrile hydratase (NHase) ${ }^{106,107}$ and thiocyanate hydrolase $(\mathrm{SCNase})^{47}$ share common asymmetric thiolate ( $\left.\mathrm{RS}^{-}\right)$, sulfenate $\left(\mathrm{RSO}^{-}\right) /$sulfinate $\left(\mathrm{RSO}_{2}{ }^{-}\right)$ donor set that results from the post-translational modification of two cysteines via sulfur oxygenation of metal-coordinated cysteine under aerobic conditions. The synthesis and studies of small-molecule provide numerous examples of metal sulfinates prepared upon $\mathrm{O}_{2}$ oxidation, but metal sulfenates are rare because they tend to oxidize further to sulfinates or sulfonates $\left(\mathrm{RSO}_{3}{ }^{-}\right)$or disproportionate into disulfides. Consequently, only three mixed sulfenate/sulfinate complexes have been structurally reported. ${ }^{79,82,108}$ Of these, the only one isolated from aerobic oxidation is a sulfenic acid (RSOH)/sulfinate derivative of $\left[\mathrm{Ru}(\mathrm{DPPBT})_{3}\right]^{-}(\mathrm{DPPBT}=2$ diphenylphosphinobenzenethiolate $)$ for which no yield is reported. ${ }^{108}$ A more biologically relevant $\left(\mathrm{N}_{3} \mathrm{~S}_{2}\right) \mathrm{Co}$, Figure 12, example reported by Kovacs et al. is readily isolated by $\mathrm{H}_{2} \mathrm{O}_{2}$ oxidation of the sulfinate precursor due to $\eta^{2}$ coordination of the sulfenate, which prevents further reactivity but does not mimic coordination of the active sites. ${ }^{82}$

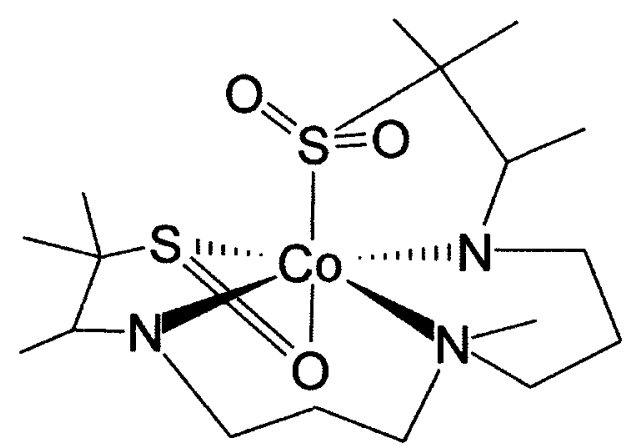

Figure 12. Representation of $\left[\mathrm{Co}(\mathrm{III})\left(\left(\eta^{2}-\mathrm{SO}\right)\left(\mathrm{SO}_{2}\right) \mathrm{N}_{3}(\mathrm{Pr}, \mathrm{Pr})\right)\right]^{+}$. 
In the apo- and native SCNase enzyme, the structure of the side chains as well as the main chain in the first coordination sphere are well preserved with those in Co and Fe types NHase. ${ }^{47}$ Like NHase two of the three cysteine thiolates of the SCNase have been post-translationally oxygenated resulting in the thiolate $\left(\mathrm{RS}^{-}\right)$, sulfenate ( $\mathrm{RSO}^{-}$), sulfinate $\left(\mathrm{RSO}_{2}{ }^{\circ}\right)$ donor combination. ${ }^{42,47,106}$ A number of small molecule active site mimics have been prepared to help delineate these proposed roles and the sulfur-oxygenation pathway. $3,78,80,82,87-89,93,96,109-112$

The oxygen reactivity of thiolates has been explored in the last 30 years due to its implications in degradation of enzymatic and catalytic sites, disulfide formation, demetallation and oxo-cluster formation. In this area, it has been reported oxygen uptake by metal-sulfur complexes. This modification results in a positive shift in the redox potential and structural rearrangement such as the metal bond distances in the complexes. Initial studies for sulfenate complex synthesis were performed using $\mathrm{Co}$ (III) complex by Deutsch et al. ${ }^{113}$ These studies of thiolate reactivity were following up with $\mathrm{H}_{2} \mathrm{O}_{2}$ kinetic studies for the oxidation of the thiolate to sulfenate and the subsequent oxidation to sulfinate. ${ }^{114}$ In addition, it has been performed studies of the reactivity of sulfur bonded $\operatorname{Co(III)}$ complexes. ${ }^{115}$

Studies by Schrauzer, Maroney and Darensbourg were focus in the synthesis and characterization of nickel dithiolate complexes. The Darensbourg group focused in the complex [1,5-bis(2-mercaptoethyl)-1,5-diazacyclooctanato]nickel(II) dithiolate complex and its sterically hindered derivatives. A family of oxygen derivatives where generated upon reaction with ${ }^{3} \Sigma \mathrm{O}_{2},{ }^{1} \Delta \mathrm{O}_{2}$, or $\mathrm{H}_{2} \mathrm{O}_{2}$ with a yield that range from $18 \%$ to $85 \%$ as we can observe in the Figure 13. ${ }^{16,117}$ 

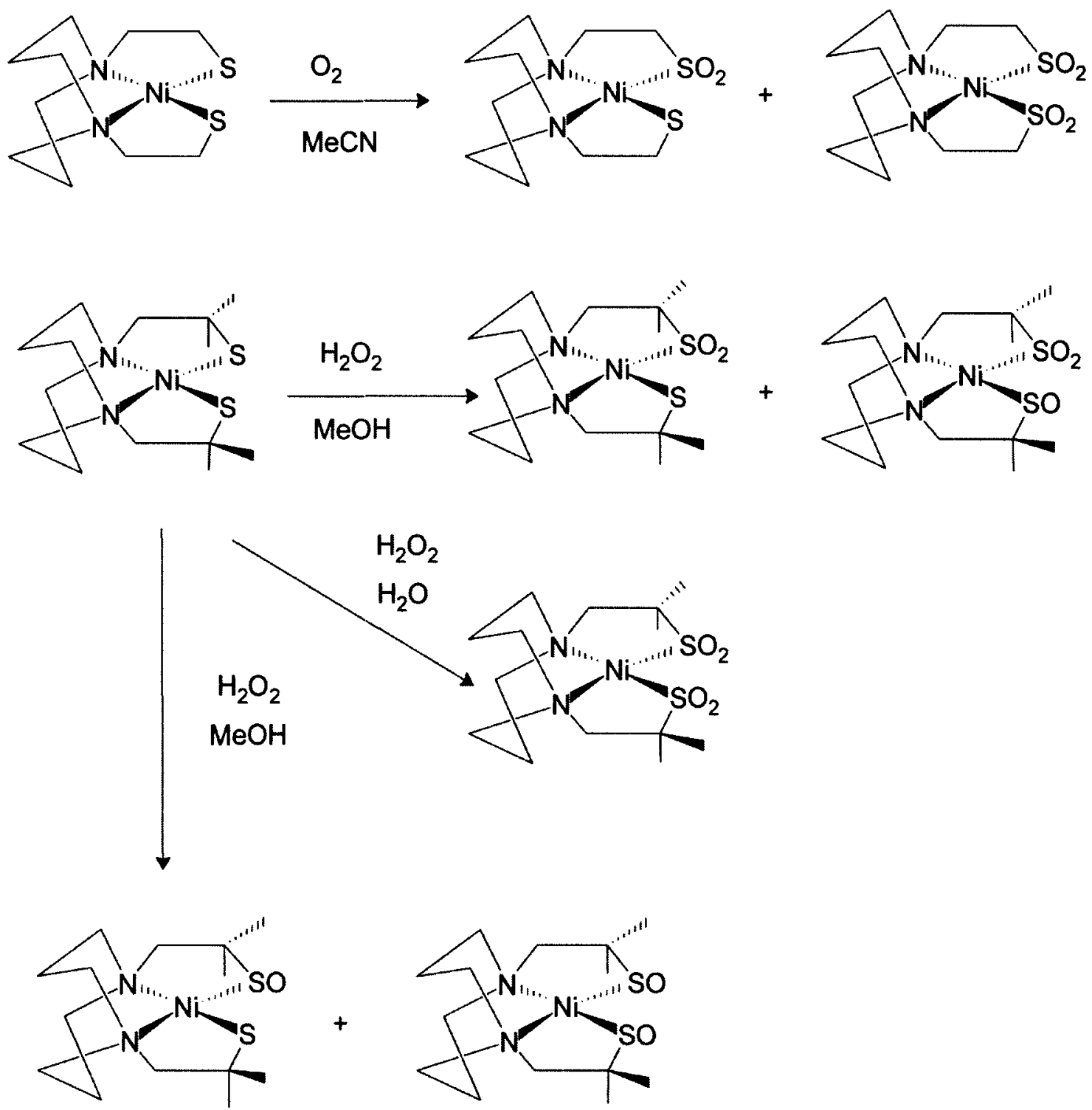

Figure 13. Synthesis and characterization of nickel sulfur oxygen derivatives.

The typical approach to prepare sulfur-oxygenated mimics of NHase or SCNase involves the reaction of metal-thiolate precursors with $\mathrm{O}$-atom transfer agents such as $\mathrm{H}_{2} \mathrm{O}_{2}, \quad N$-sulfonyloxaziridine, $\quad(1 S)-(+)-(10$-camphorsulfonyl)oxaziridine, or urea hydrogen peroxide. ${ }^{80,82,88,89,112,118}$ These efforts have been successful for the isolation of 
sulfenate, ${ }^{80,89,112,118}$ sulfinate, ${ }^{88,89}$ and a mixed sulfenate/sulfinate complex with $\eta^{2}$ sulfenate coordination. ${ }^{82} \mathrm{~A}$ major advantage of $\mathrm{O}$-atom transfer agents is the ability to quantitatively add oxidizing equivalents at controlled rates. Less well developed are reactions employing dioxygen, which is the proposed reagent in the enzymatic systems. ${ }^{42,46,56}$ While several sulfinate complexes have been reported upon $\mathrm{O}_{2}$ exposure of thiolate precursors, ${ }^{82,116,119-127}$ isolation of sulfenate ${ }^{120,128}$ or mixed sulfenate/sulfinate complexes from $\mathrm{O}_{2}$ are rare due to the reactivity of the sulfenate..$^{79,108,129}$

As noted in chapter 1, the Grapperhaus laboratory has focused on NHase model complexes based on the penta coordinate $\mathrm{N}_{2} \mathrm{~S}_{3}$ chelate 4,7-bis-(2'-methyl-2'mercaptopropyl)-1-thia-4,7-diazacyclononane (bmmp- TASN) ${ }^{78,93,96,109}$ With this ligand, a series of iron complexes was synthesized and their oxygen sensitivity was investigated. ${ }^{93}$ Oxygen exposure of the high-spin complex (bmmp-TASN)FeCl yields ligand disulfide with decomplexation of the metal and subsequent iron-oxo cluster formation. ${ }^{94}$ In contrast, $\mathrm{O}_{2}$ addition to the low-spin complex (bmmp-TASN)FeCN generates the bis-sulfonate $\left(2 \mathrm{RSO}^{3-}\right)$ complex (bmmp- $\left.\mathrm{O}_{6}-\mathrm{TASN}\right) \mathrm{FeCN}{ }^{78}$ While the lowspin complex promoted sulfur oxygenation, the reaction proceeded beyond the oxygenation level (sulfenate/sulfinate) observed in NHase and SCNase. As proof of concept the low-spin ruthenium(II) complex (bmmp-TASN)RuPPh $(\mathbf{1})$ was synthesized and its $\mathrm{O}_{2}$ reactivity under limited $\mathrm{O}_{2}$ conditions explored to yield a family of ruthenium sulfur oxygen derivatives as it is observed in Scheme 4. 

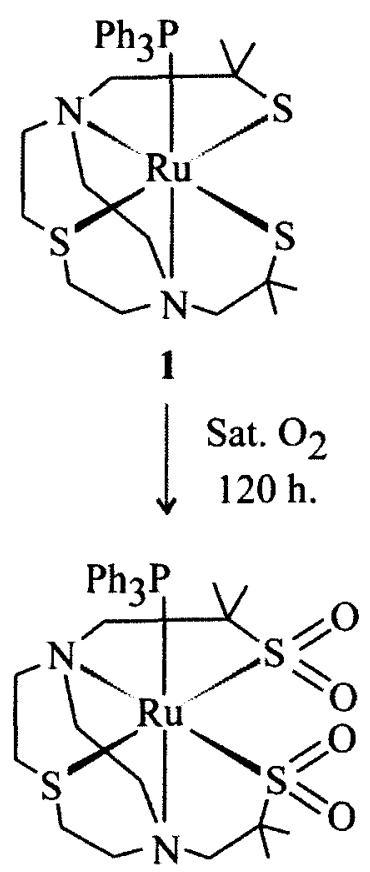

4
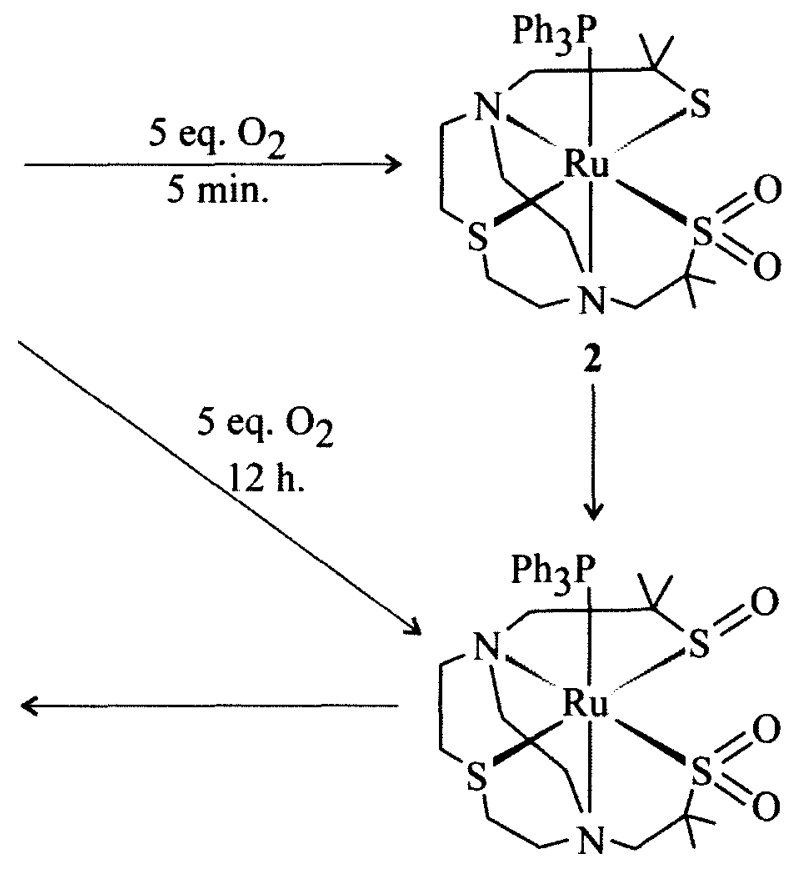

3

Scheme 4. Oxygenation pathway of 1 .

\section{Synthesis and characterization of 1}

Complex 1 is isolated from the metal precursor $\mathrm{RuCl}_{2}\left(\mathrm{PPh}_{3}\right)_{3}$ and $\mathrm{H}_{2}$ (bmmpTASN) upon deprotonation of the ligand with $n$-butyl lithium in tetrahydrofuran as an air- and water-stable orange solid. The complex contains a $\mathrm{Ru}(\mathrm{II})$ ion with a dithiolate pentacoordinate ligand in an octahedral configuration. The reaction is performed overnight but the formation of the product begins after 20 minutes. The protocol of isolation involves the air free filtration of the product and washing the product with ether to remove unreactive material. Further work up involves water washing under aerobic condition to remove phosphine oxide. In an $\mathrm{O}_{2}$-saturated methanol solution, 1 reacts over 96 hours to yield an intractable brown product with an FT-IR spectrum (Figure A1 in the Supporting Information) reminiscent of our previously reported iron disulfonate derivative. $^{78}$ 
Initial studies of sulfur oxygenation in compound $\mathbf{1}$ with oxygen transfer reagent such as $\mathrm{H}_{2} \mathrm{O}_{2}$ and thiourea peroxide generate a mixture of sulfur oxygen derivatives. The sulfur oxygenated derivatives are not isolable by column chromatography or thin layer chromatography (TLC) preparative. All the products of oxygenation are air sensitive in solution. Repeated attempts to isolate analytically pure samples from this product mixture were unsuccessful. The formation of a family of sulfur oxygen derivatives can be avoided by limiting the quantity of $\mathrm{O}_{2}$ and the reaction time as it is observed in Scheme 5 .
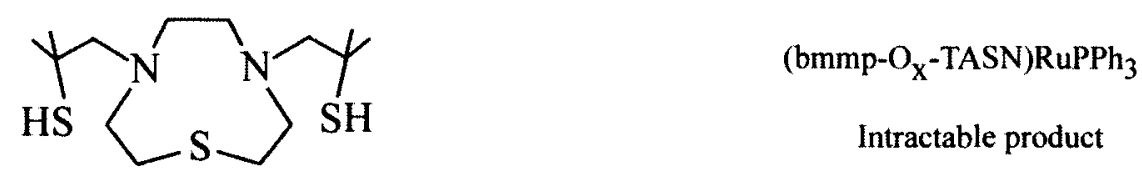

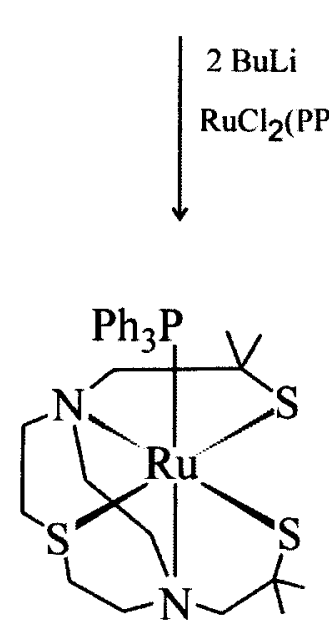

1

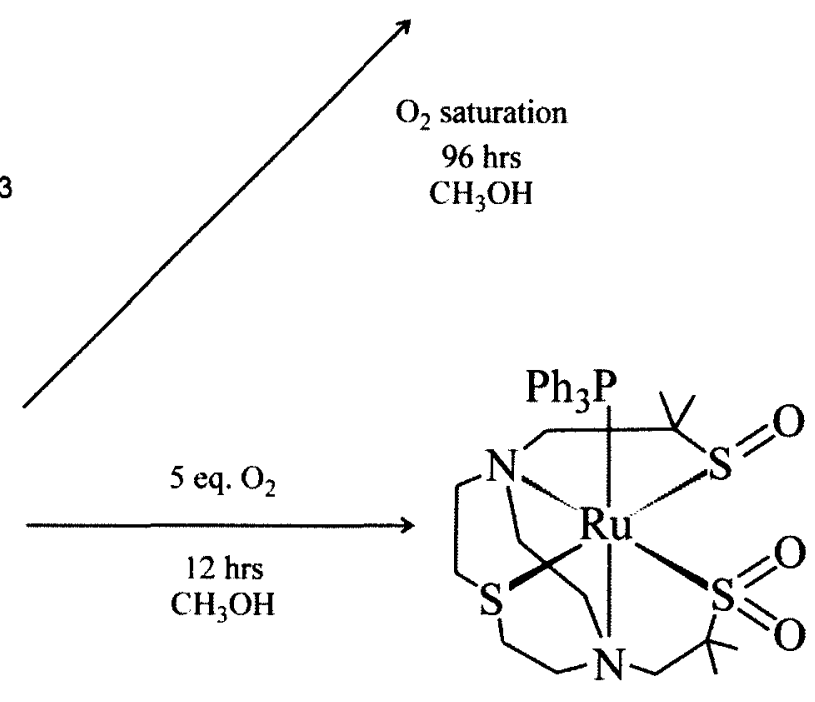

3

Scheme 5. Synthesis and reactivity of 1.

\section{Oxygenation studies followed by TLC}

Oxygenation studies of the complex 1 were monitored by TLC. These studies were achieved with the isolation of complexes and subsequent TLC studies under air free conditions in the glovebox. Methanol solution saturated with potassium chloride allows 
the separation between the 1-4 complexes. The initial reactivity studies were performed with FT-IR and TLC in parallel to determinate the time frame of the reaction. The formation of the intense bands at 1139 and $1020 \mathrm{~cm}^{-1}$ attributed to the asymmetric and symmetric $\mathrm{S}=\mathrm{O}$ stretches of the sulfinate donor after 5 minutes of the reaction set up the first reaction time of this study. Monitoring the reaction by silica gel thin layer chromatography (TLC) revealed additional products. As outlined in Scheme 4, the oxygenation of 1 proceeds in a series of steps with distinct time frames. Immediately following $\mathrm{O}_{2}$ exposure, the thiolate/sulfinate complex (bmmp- $\left.\mathrm{O}_{2}-\mathrm{TASN}\right) \mathrm{RuPPh}_{3} 2$ is observed, $R_{f}=0.56$, along with the thiolate precursor $1, R_{f}=0.68$. After 5 minutes, 2 is the sole product and only traces of $\mathbf{1}$ are detected. Reaction times longer than 5 minutes yield a mixture of 2 and $3\left(R_{f}=0.45\right)$ yielding pure 3 after 15 minutes. From these results it can be inferred that 2 is a precursor to 3 . This is confirmed by the direct conversion of isolated 2 to 3 upon $\mathrm{O}_{2}$ addition. Longer reaction times, 120 hours, under limiting $\mathrm{O}_{2}$ conditions yield the disulfinate complex (bmmp- $\left.\mathrm{O}_{4}-\mathrm{TASN}\right) \mathrm{RuPPh}_{3}(\mathbf{4}), \mathrm{R}_{\mathrm{f}}=$ 0.2. Complex 4 can also be generated from isolated 3 or from 1 with excess $\mathrm{O}_{2}$. In the presence of water, $\mathbf{4}$ degrades to "intractable product" initially obtained in open-air oxygenation studies. ${ }^{129}$

\section{Limiting oxygen conditions}

In the $\mathrm{O}_{2}$ limited reactions, 5 equivalents of $\mathrm{O}_{2}$ were added to a solution of 1 under an argon atmosphere. The solution was stirred for 5 minutes until the solution change from orange to claret and no solid is present in the solution. After that, the solution was filtered using a fritted filter flask under air-free conditions to remove any trace of unreactive staring material followed by evacuation of the solvent. The claret 
crude product was dissolved in degassed methanol and purified using crystallization by solvent evaporation, which yielded yellow-orange crystals of the mono sulfinate derivative 2 in $72 \%$ yield after 72 hours. Further exposition of compound 1 to $\mathrm{O}_{2}$ gives mixture products of compound $\mathbf{2}$ and $\mathbf{3}$. The formation of a sulfinate motif in compound $\mathbf{1}$ changes its solubility properties. Compound $\mathbf{2}$ is partially soluble in water and as a consequence of this solubility compound 2 react with $\mathrm{O}_{2}$ in aqueous solution and require air-free conditions for its manipulation. The mechanism of oxygenation of the thiolate species is unknown. Mechanistic studies are been performed by Davinder Kumar, a current student in the Grapperhaus Group.

In the $\mathrm{O}_{2}$ limited reactions, 5 equivalents of $\mathrm{O}_{2}$ were added to a solution of 1 under an argon atmosphere. After 12 hours, the solution changed color from orange to claret and finally yellow. The solvent was removed under vacuum, and the yellow solid residue was dissolved in degassed methanol, which yielded crystals of the sulfenate/sulfinate derivative 3 in $82 \%$ yield upon slow evaporation under air-free conditions. The formation of sulfenate motif in the compound $\mathbf{3}$ increases its solubility in water, property that is used as protocol of purification. Additional air or $\mathrm{O}_{2}$ exposure results in complex degradation. While limiting the quantity of $\mathrm{O}$-atom-transfer reagents is a common tactic in attempts to obtain partially sulfur-oxygenated derivatives of metal thiolates. ${ }^{79}$ Intentionally limiting the $\mathrm{O}_{2}$ supply for their controlled oxygenation has not been exploited.

The importance of limiting $\mathrm{O}_{2} /$ metal thiolate interactions to achieve partial oxygenation was suggested by the results with $\left[\mathrm{Ru}(\mathrm{DPPBT})_{3}\right]^{-}$. When suspensions of $\left[\mathrm{Ru}(\mathrm{DPPBT})_{3}\right]^{-}$as the poorly soluble $\mathrm{HNEt}_{3}{ }^{+}$salt were exposed to air, the mixed sulfenic 
acid/sulfinate product was obtained. ${ }^{108}$ However, homogeneous solutions of the complex as the $\mathrm{PPN}^{+}$(Bis(triphenylphosphine)iminium) salt reproducibly yield the disulfinate derivative. ${ }^{130}$ As an additional example, the product distribution of singlet oxygen addition to an $\left(\mathrm{N}_{2} \mathrm{~S}_{2}\right) \mathrm{Ni}$ complex shifts toward the sulfenate/sulfinate derivative as the complex concentration increases and the relative $\mathrm{O}_{2}$ concentration decreases. ${ }^{131}$

To extend the family of oxygen derivatives, compound 1 was exposed to a gentle purge with $\mathrm{O}_{2}$ for 2 hours and stirred for 120 hours. The product of the synthesis is air and moisture sensitive. During the reaction the compound change of color form orange to claret and to yellow in 72 hours. The yellow residue is compound $\mathbf{4}$, and turns green when exposed to air and water.

FT-IR and isotopic labeling studies employing ${ }^{16} \mathrm{O}_{2}$ and ${ }^{18} \mathrm{O}_{2}$ confirm $\mathrm{O}_{2}$ as the $\mathrm{O}-$ atom source in the conversion of $\mathbf{1}$ to $\mathbf{2}$ and $\mathbf{3}$. In the Figure 14, the FT-IR of compound $\mathbf{1}$ displays a finger print of the compound between 500 and 1500 wavenumbers $\left(\mathrm{cm}^{-1}\right), \mathrm{C}-\mathrm{H}$ alkyl stretches between 2800 and 3000 wavenumbers $\left(\mathrm{cm}^{-1}\right)$ and $\mathrm{C}-\mathrm{H}$ aromatic stretches above 3000 wavenumbers $\left(\mathrm{cm}^{-1}\right)$. 


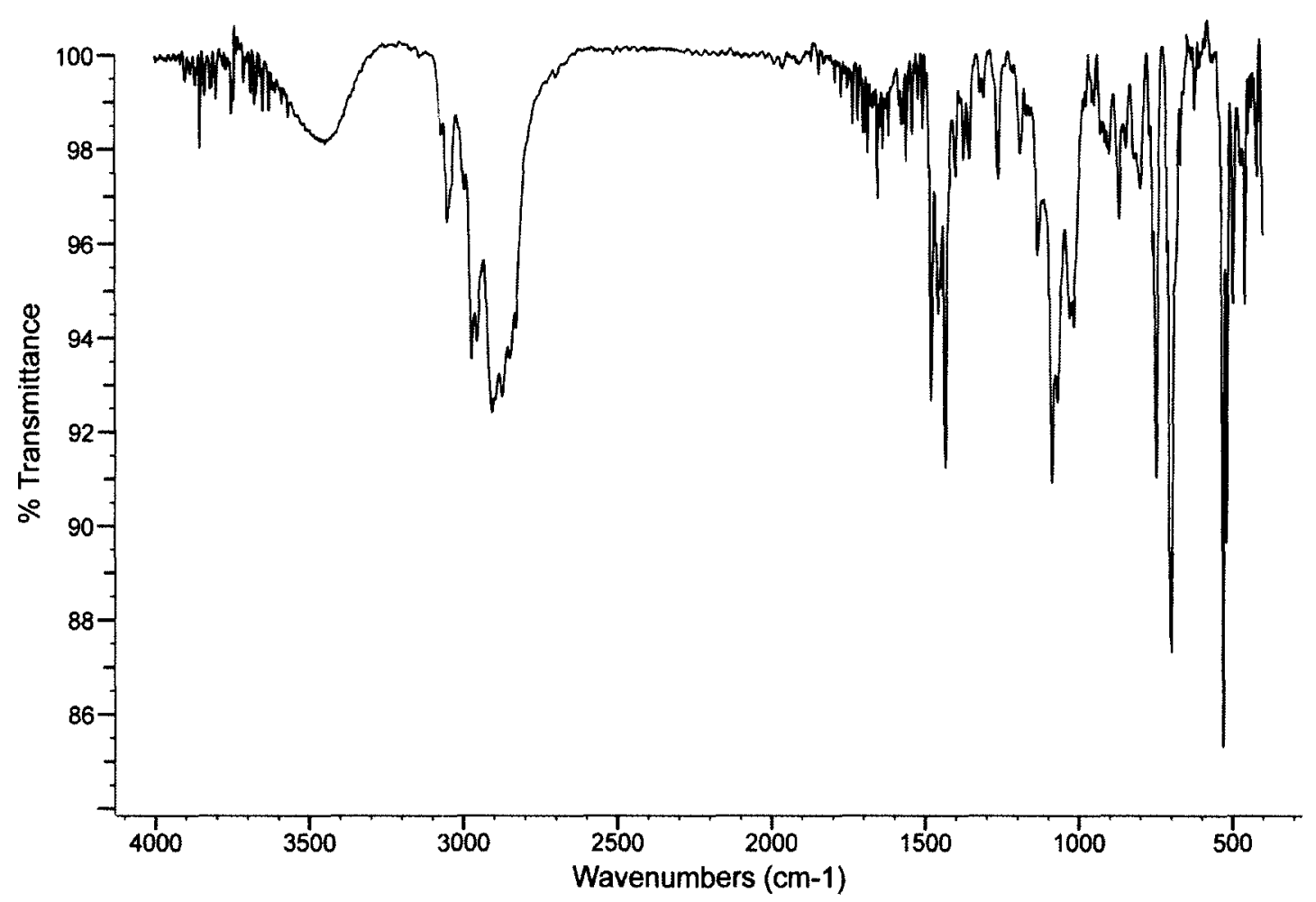

Figure 14. FT-IR of compound 1.

In FT-IR of compound 2 displays sulfinate stretches as strong bands at 1139 and $1020 \mathrm{~cm}^{-1}$ attributed to asymmetric and symmetric $\mathrm{S}=\mathrm{O}$ stretches, Figure 15. Synthesis of 2 employing ${ }^{18} \mathrm{O}_{2}$ as oxygen source shifts these bands to 1104 and $990 \mathrm{~cm}^{-1}$, Figure 16 . These shift of 35 and $30 \mathrm{~cm}^{-1}$ to 1104 and $990 \mathrm{~cm}^{-1}$, respectively, for samples of 2 prepared with ${ }^{18} \mathrm{O}_{2}$ are based on a simple harmonic oscillator approximation. The isotopic shifts are consistent with those observed for ${ }^{34} \mathrm{~S}$-labeled NHase ${ }^{132}$ and other ${ }^{18} \mathrm{O}$-labeled metal sulfinates. ${ }^{16,133}$ 


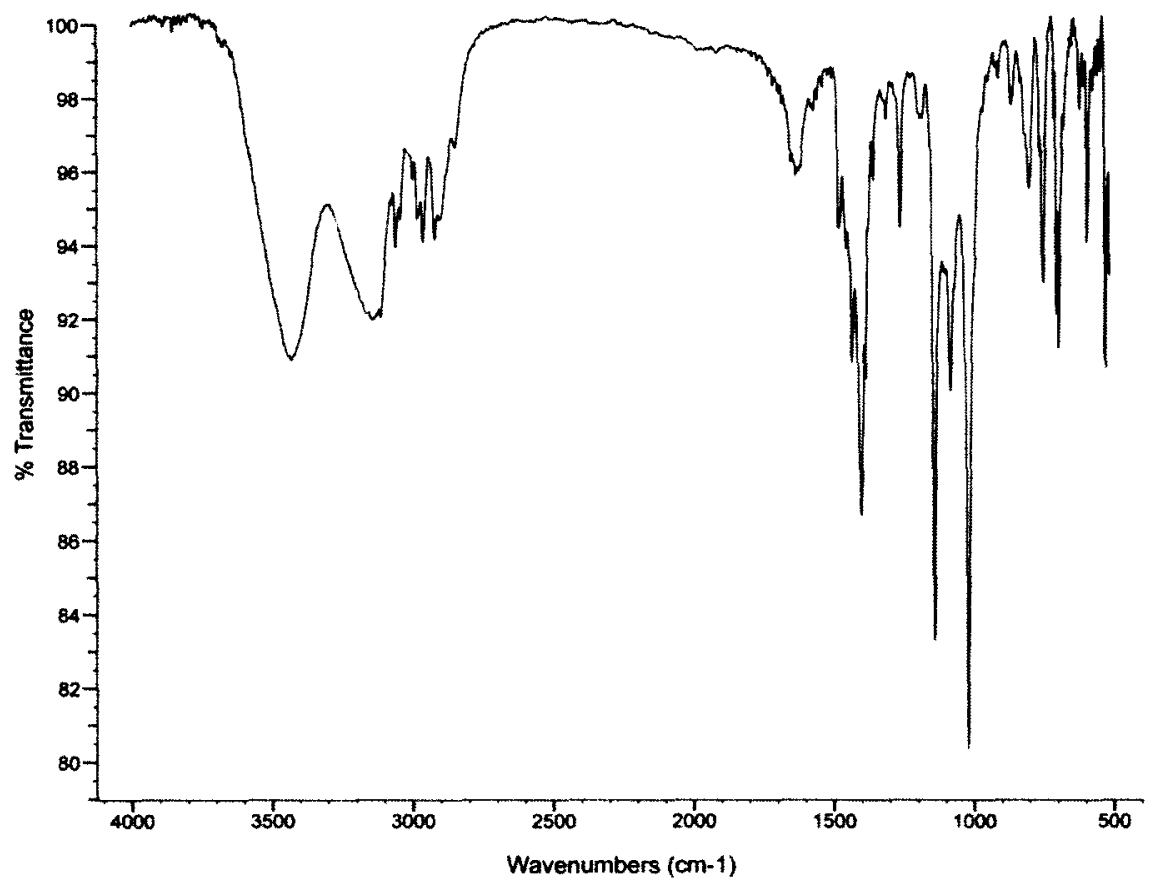

Figure 15. FT-IR of compound 2 with ${ }^{16} \mathrm{O}_{2}$.

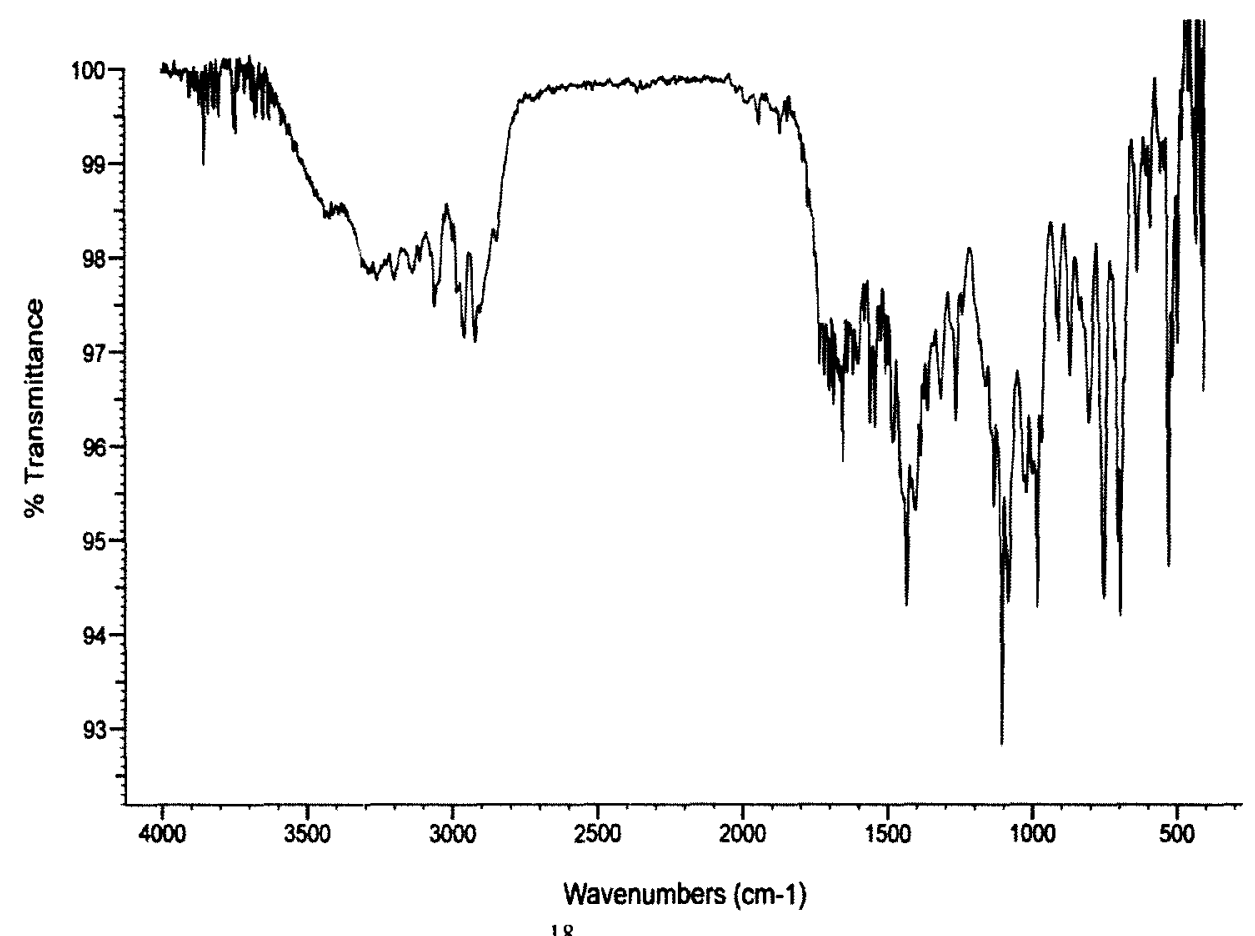

Figure 16. FT-IR of compound 2 with ${ }^{18} \mathrm{O}_{2}$

In the Figure 17, the FT-IR of compound 3 displays a finger print of the compound between 500 and 1500 wavenumbers $\left(\mathrm{cm}^{-1}\right), \mathrm{C}-\mathrm{H}$ alkyl stretches between 
2800 and 3000 wavenumbers $\left(\mathrm{cm}^{-1}\right)$ and $\mathrm{C}-\mathrm{H}$ aromatic stretches above 3000 wavenumbers $\left(\mathrm{cm}^{-1}\right)$.

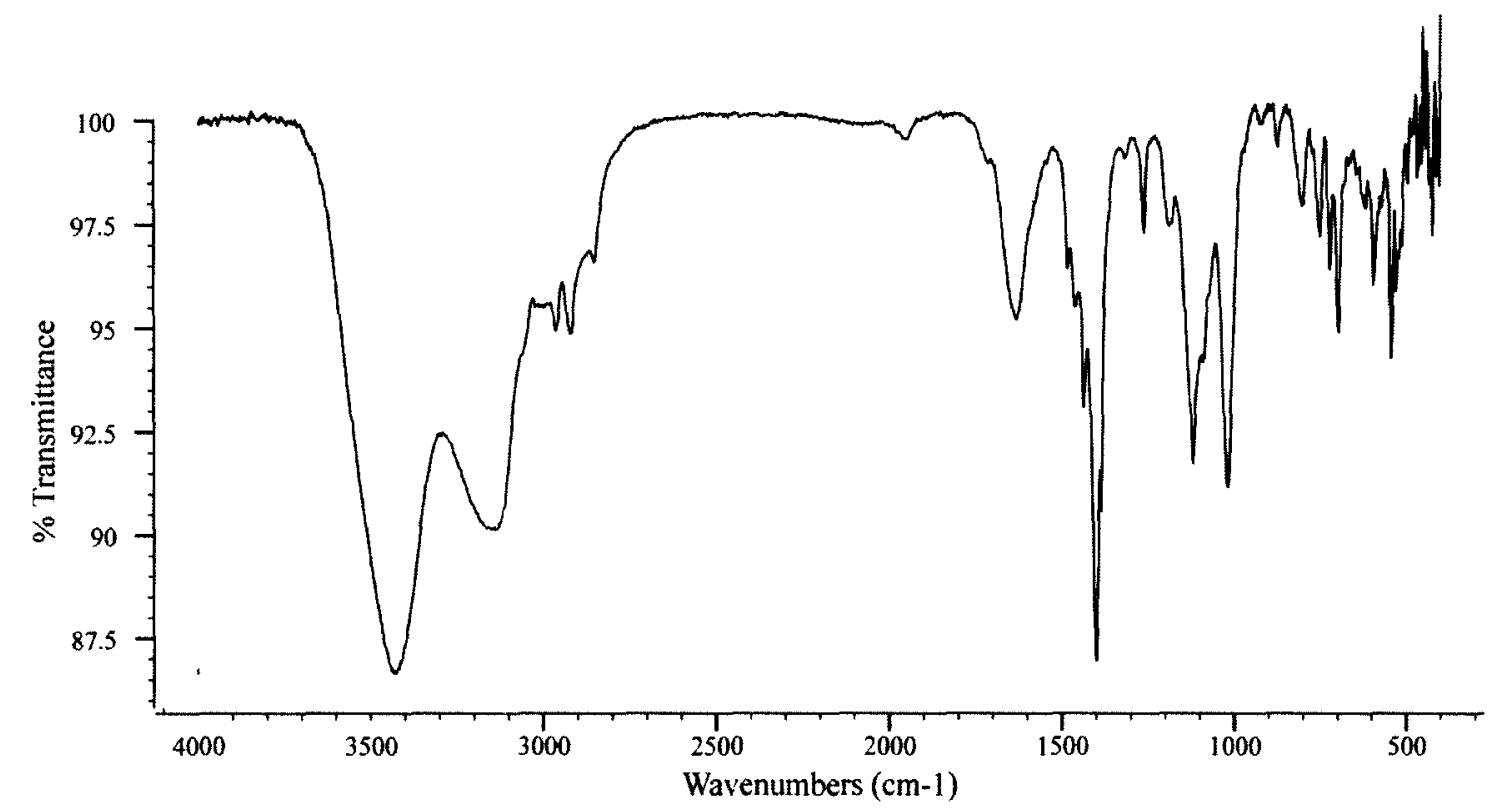

Figure 17. FT-IR of compound 3 with ${ }^{16} \mathrm{O}_{2}$

The difference IR spectrum of 1 and 3 prepared with ${ }^{16} \mathrm{O}_{2}$ in Figure 18a displays intense bands at 1140 and $1020 \mathrm{~cm}^{-1}$ attributed to the asymmetric and symmetric $\mathrm{S}=\mathrm{O}$ stretches of the sulfinate donor. These bands shift by 45 and $38 \mathrm{~cm}^{-1}$ to 1095 and 982 $\mathrm{cm}^{-1}$, respectively, for samples of 3 prepared with ${ }^{18} \mathrm{O}_{2}$ in Figure $18 \mathrm{~b}$. The isotopic shifts are larger than those observed for ${ }^{34} \mathrm{~S}$-labeled NHase ${ }^{132}$ but consistent with a simple harmonic oscillator approximation and other ${ }^{18} \mathrm{O}$-labeled metal sulfinates. ${ }^{16,133}$ 


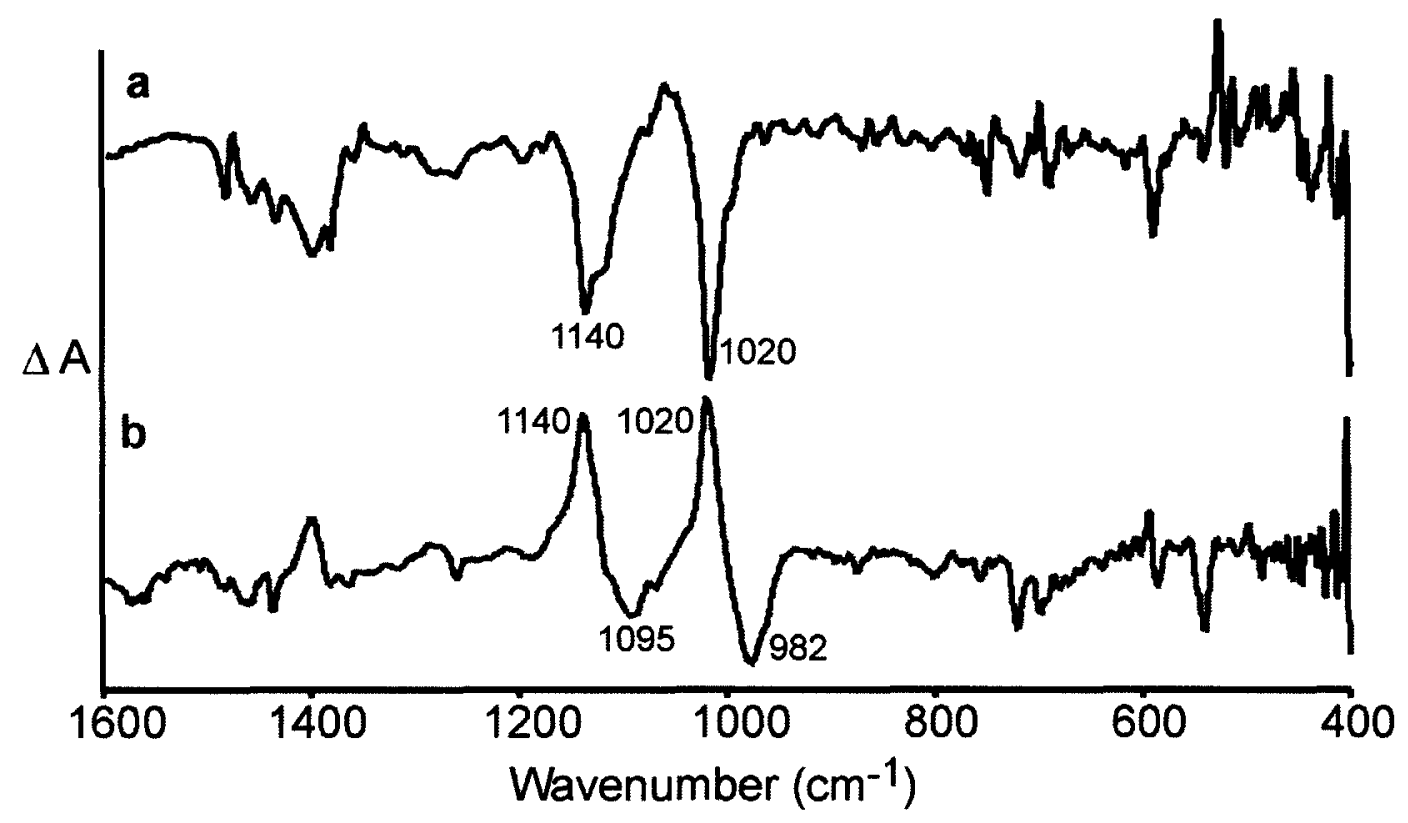

Figure 18. FT-IR difference spectra highlighting the ${ }^{18} \mathrm{O}_{2}$-sensitive sulfinate stretching frequencies of (a) 1 and 3 prepared under ${ }^{16} \mathrm{O}_{2}$ (black line) and (b) 3 prepared under ${ }^{16} \mathrm{O}_{2}$ and ${ }^{18} \mathrm{O}_{2}$ (red line).

The FT-IR in the Figure 18 shows in the spectrum $\mathbf{A}$, the two stretches for the symmetric and asymmetric frequencies of the SO of the sulfinate when the compound 3 is synthesized form ${ }^{16} \mathrm{O}_{2}$. This spectrum is the result of the subtraction of spectrum (compound 3 - compound 1), to emphasize the sulfinate stretches.

In the FT-IR characterization of compound 4, Figure 19, reveals four strong bands at $1136,1120,1029$ and $1015 \mathrm{~cm}^{-1}$ attributed to the asymmetric and symmetric $\mathrm{S}=\mathrm{O}$ stretches associated with two sulfinate stretches. The energies of sulfinate stretches are similar to those reported by Darensbourg in their complete series of nickel sulfur oxygenates. ${ }^{79}$ Oxygen labeling studies were no performed in compound 4. 


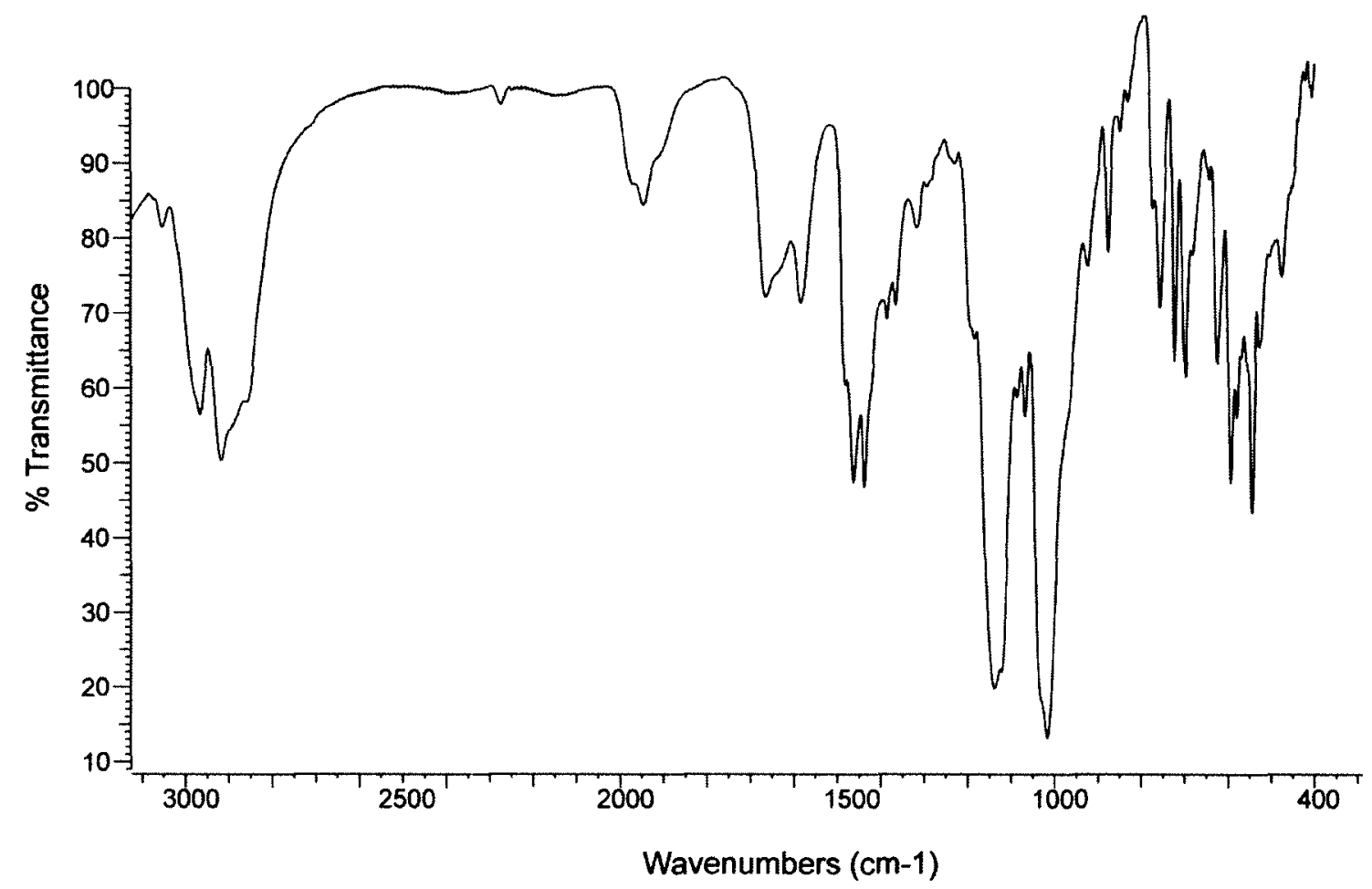

Figure 19. FT-IR of compound 4 with ${ }^{16} \mathrm{O}_{2}$.

The identification and S-oxygenation of the complexes 1-4 was confirmed by mass spectroscopy. +ESI-MS was recorded for each complex prepared from natural abundance $\mathrm{O}_{2}$ and $99.99 \%{ }^{18} \mathrm{O}_{2}$, Figures 20-29. The complex 1 displays a parent peak at $\mathrm{m} / \mathrm{z} 684.1363$, Figure 20, consistent with a theoretical value of 684.1369 for [1] $]^{+}$, Figure 21. The isotopical distribution shows and accuracy of 0.0006 


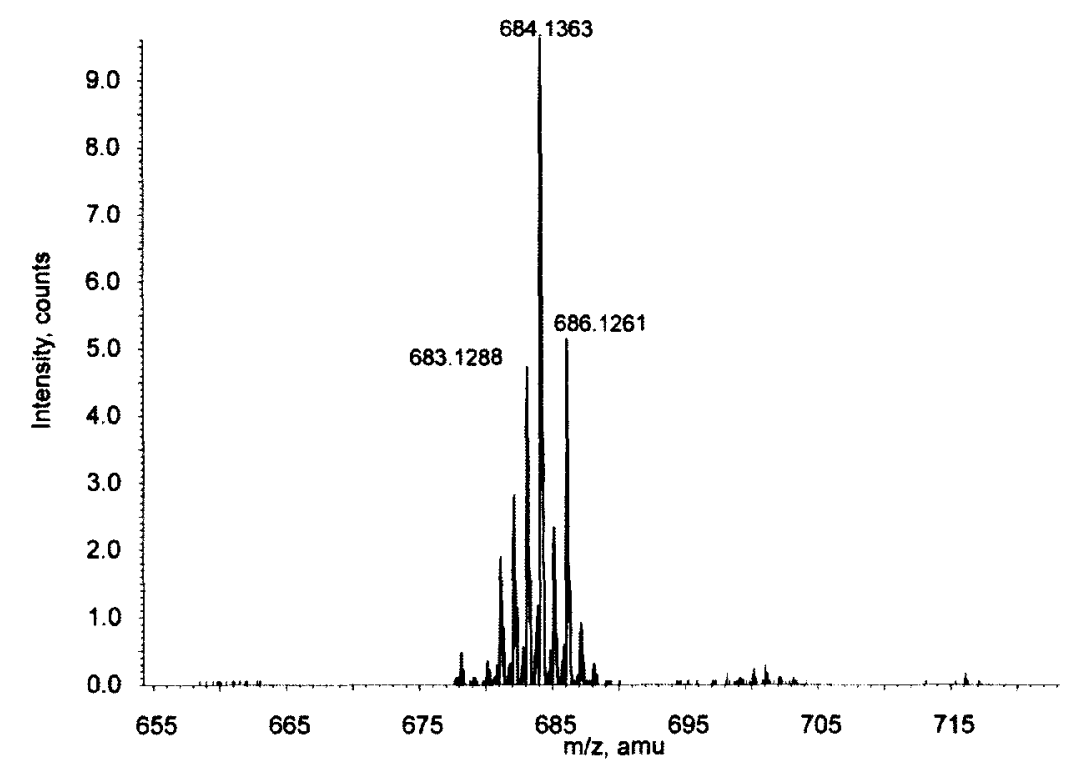

Figure 20. +ESI-MS of 1.

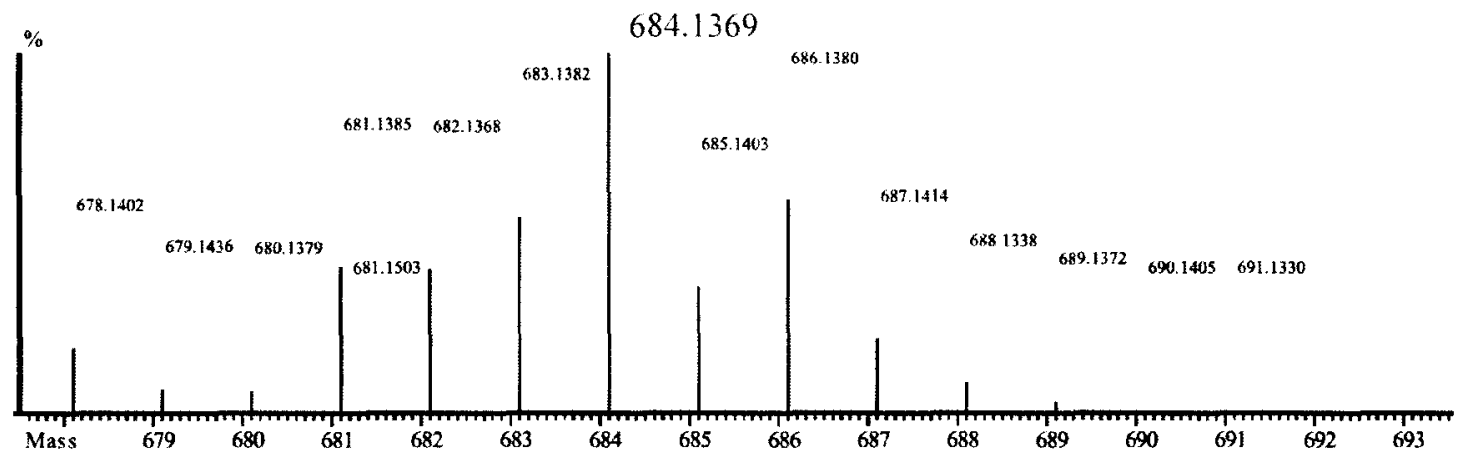

Figure 21. Calculated isotopic distribution for compound 1.

Complex 2 displays a parent peak at $\mathrm{m} / \mathrm{z}=715.6971$ consistent with a theoretical value of 716.1268 for $[2]^{+}$, Figure 22. The isotopical distribution shows and accuracy of 0.4297 , Figure 23 . While + ESI-MS typically yields $[\mathrm{M}+\mathrm{H}]^{+}$or $[\mathrm{M}+\mathrm{Na}]^{+}$ions, the direct oxidation of neutral species at the probe tip to yield $[\mathrm{M}]^{++}$is known for easily oxidized 
samples such as 1-4. ${ }^{134}$ Samples of 2 prepared with ${ }^{18} \mathrm{O}_{2}$ show the expected shift to $\mathrm{m} / \mathrm{z}=$ 720.1338 confirming $\mathrm{O}_{2}$ as the source of both $\mathrm{O}$-atoms in the product, Figure 24 .

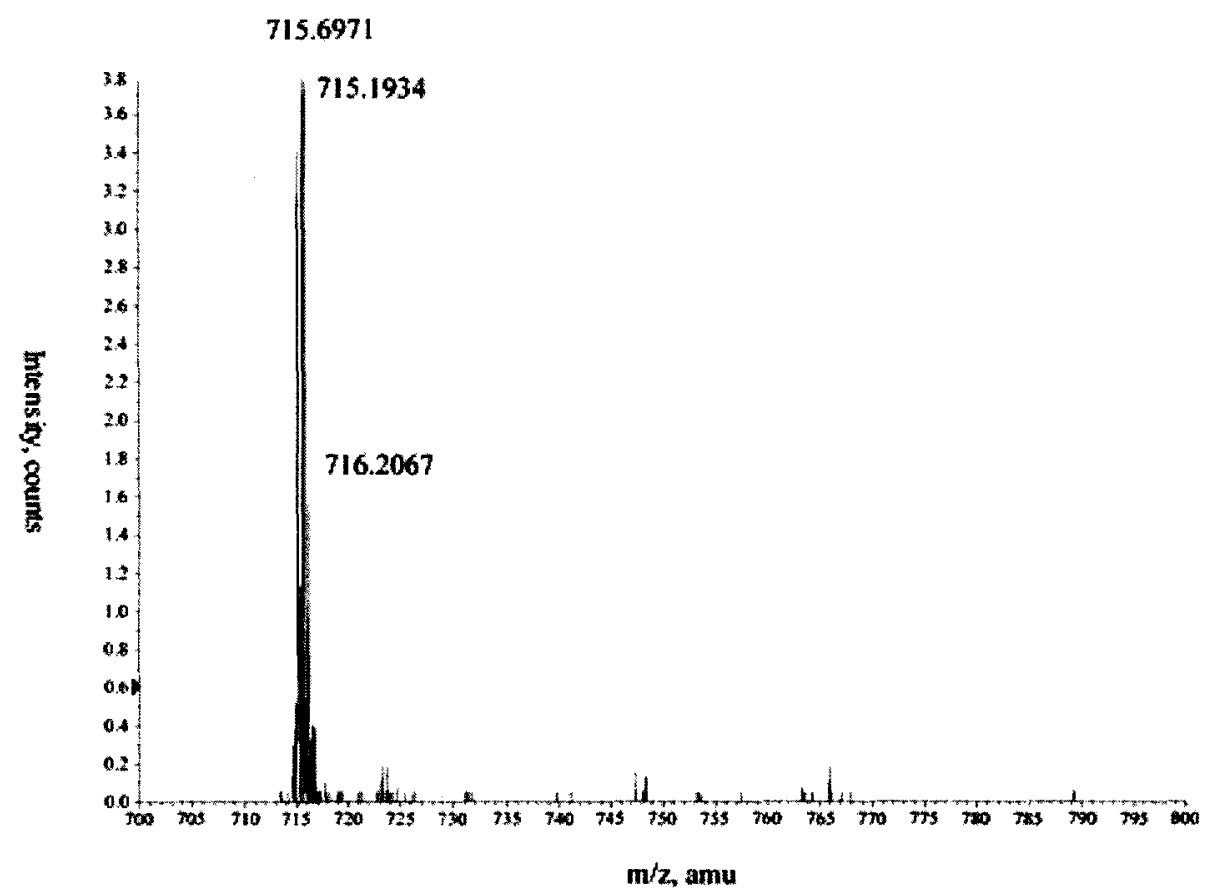

Figure 22. +ESI-MS of 2 prepared from ${ }^{16} \mathrm{O}_{2}$.

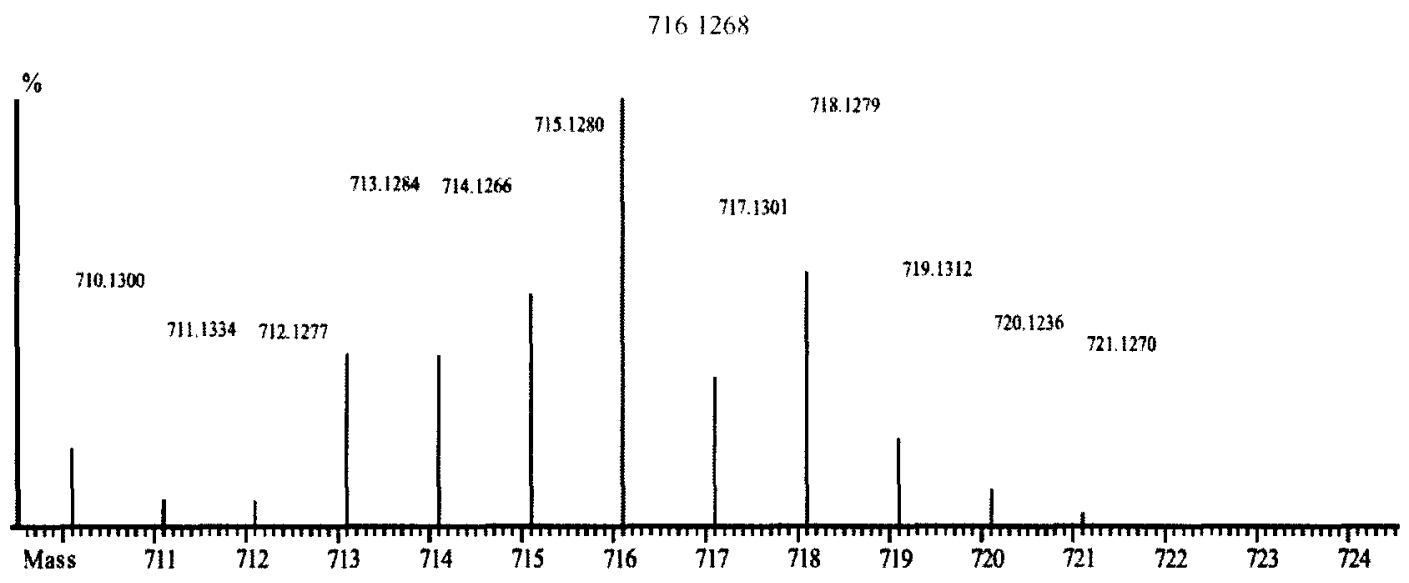

Figure 23. Calculated isotopic distribution for compound 2 using ${ }^{16} \mathrm{O}_{2}$. 


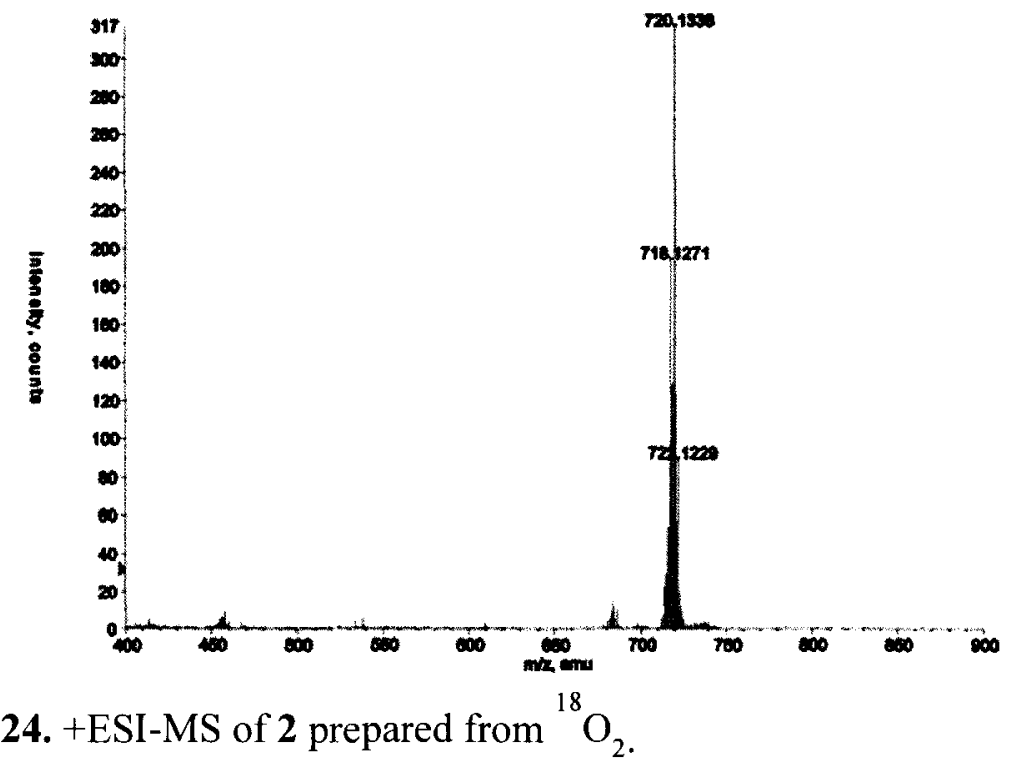

The mixed sulfenate/sulfinate complex 3 displays a peak at $\mathrm{m} / \mathrm{z}=731.1147$, Figure 25, the parent peak of the isotopic distribution is diphase by $1.007 \mathrm{~m} / \mathrm{z}$ of the experimental value, Figure 26. The experimental value shifts by 6.0116 to 737.1263 when the sample is prepared with ${ }^{18} \mathrm{O}_{2}$, Figure $27 .{ }^{129}$ This results indicate that the thiolates of the complex uptake the oxygen from $\mathrm{O}_{2}$ and no from the methanol.

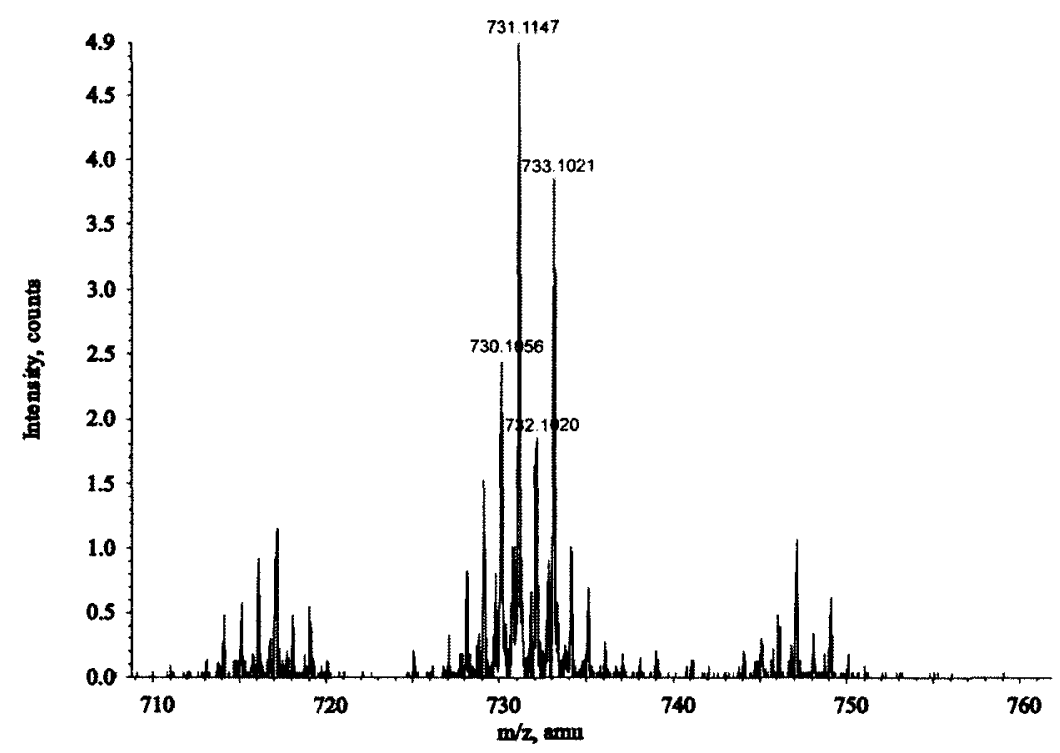

Figure 25. +ESI-MS of 3 prepared from ${ }^{16} \mathrm{O}_{2}$. 


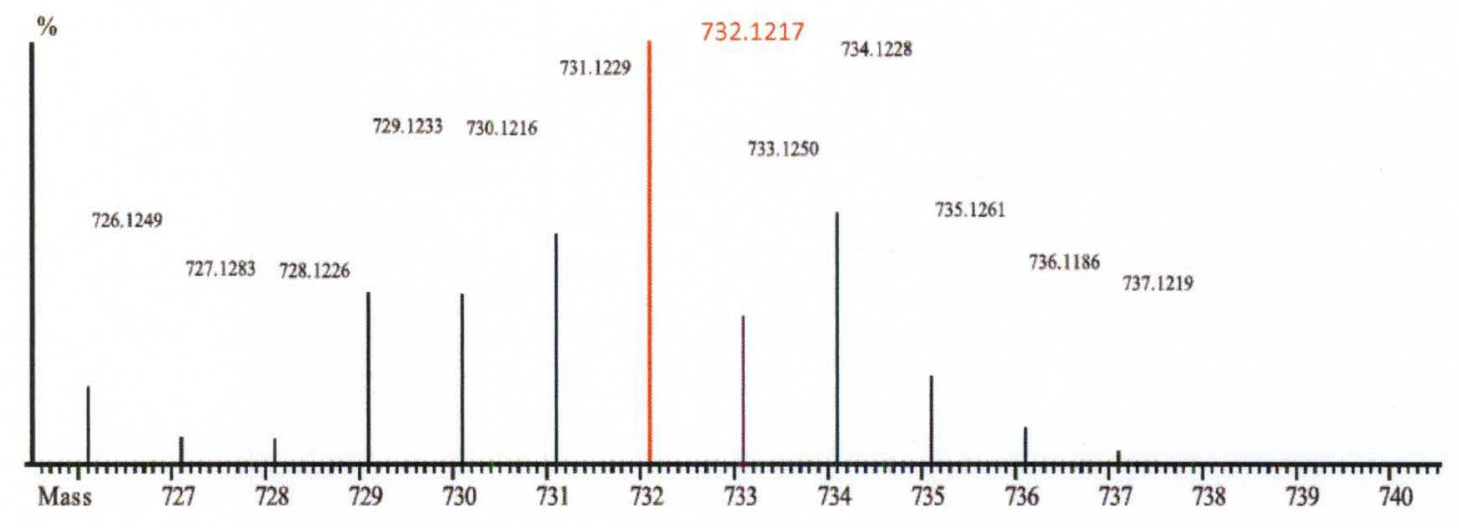

Figure 26. Calculated isotopic distribution for compound $3{ }^{16} \mathrm{O}_{2}$.

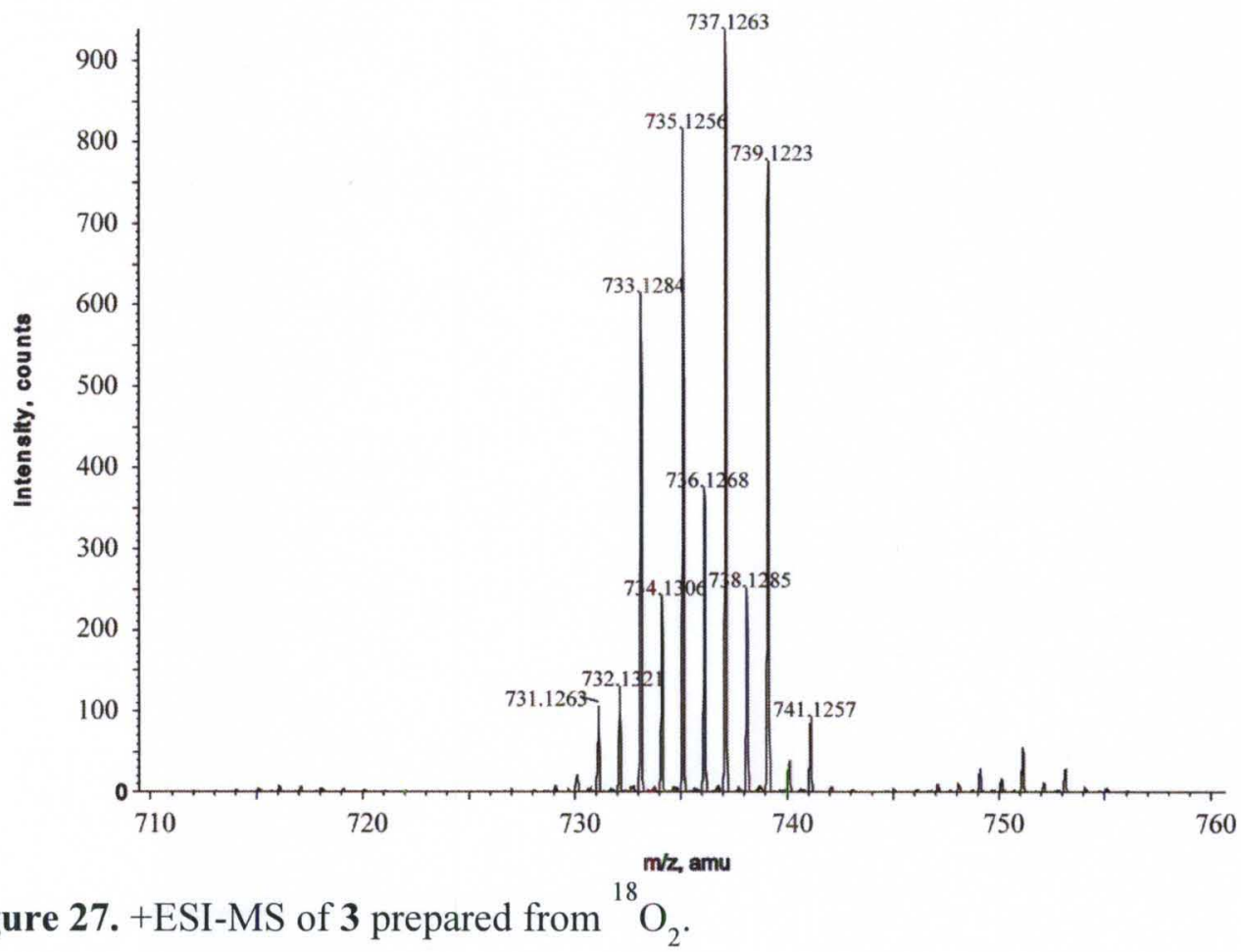

The +ESI-MS of the disulfinate complex $\mathbf{4}$ has only small intensity at the theoretical value of $m / z=748.1185$, Figure 28. A larger peak at $m / z=485.6471$ associated with $\mathrm{PPh}_{3}$ dissociation is observed along with free $\mathrm{PPh}_{3}$ at $m / z=262.0911$. 
The isotopical distribution parent peak calculated for compound $\mathbf{4}$ is diphase by 0.0019 $m / z$, Figure 29 .
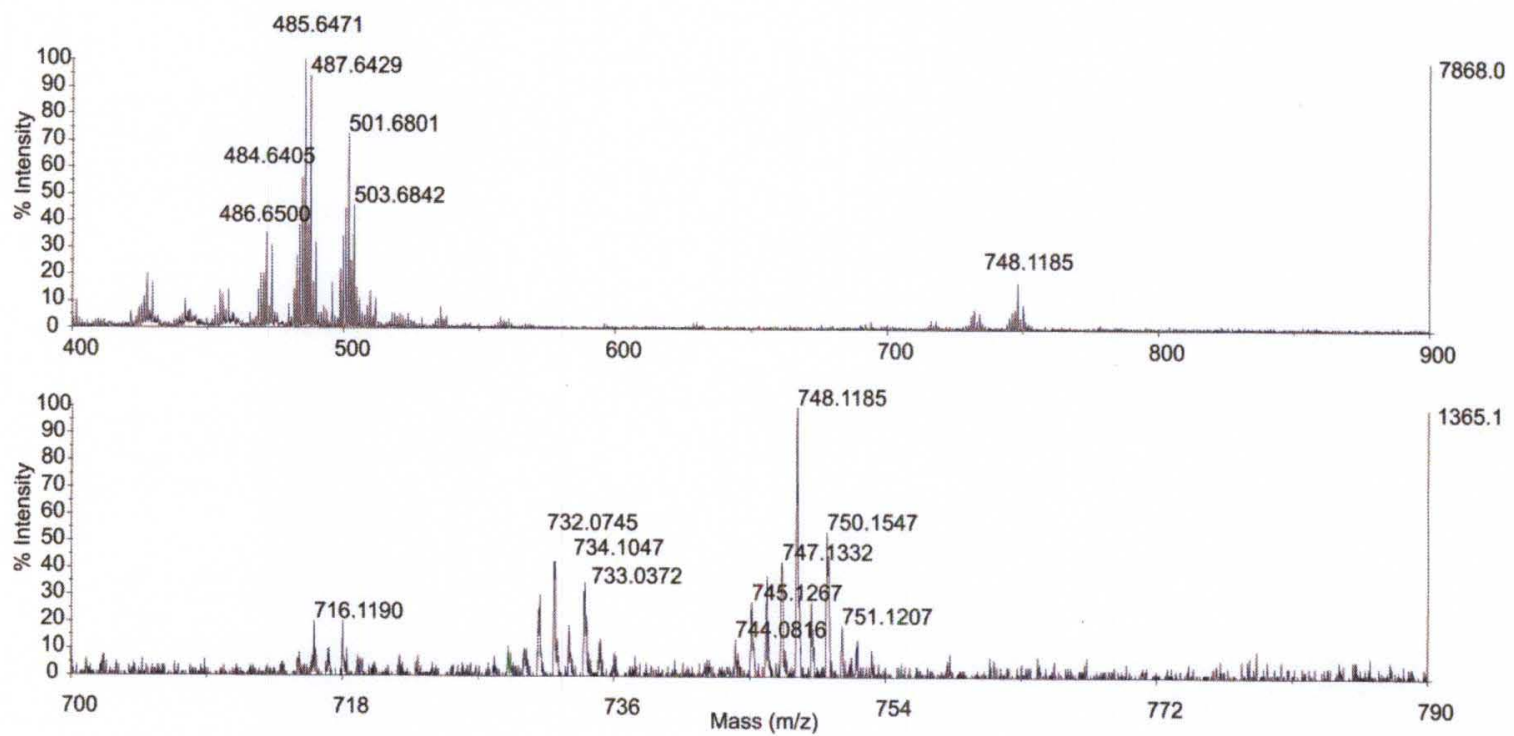

Figure 28. +ESI-MS of $\mathbf{4}$ prepared from ${ }^{16} \mathrm{O}_{2}$.

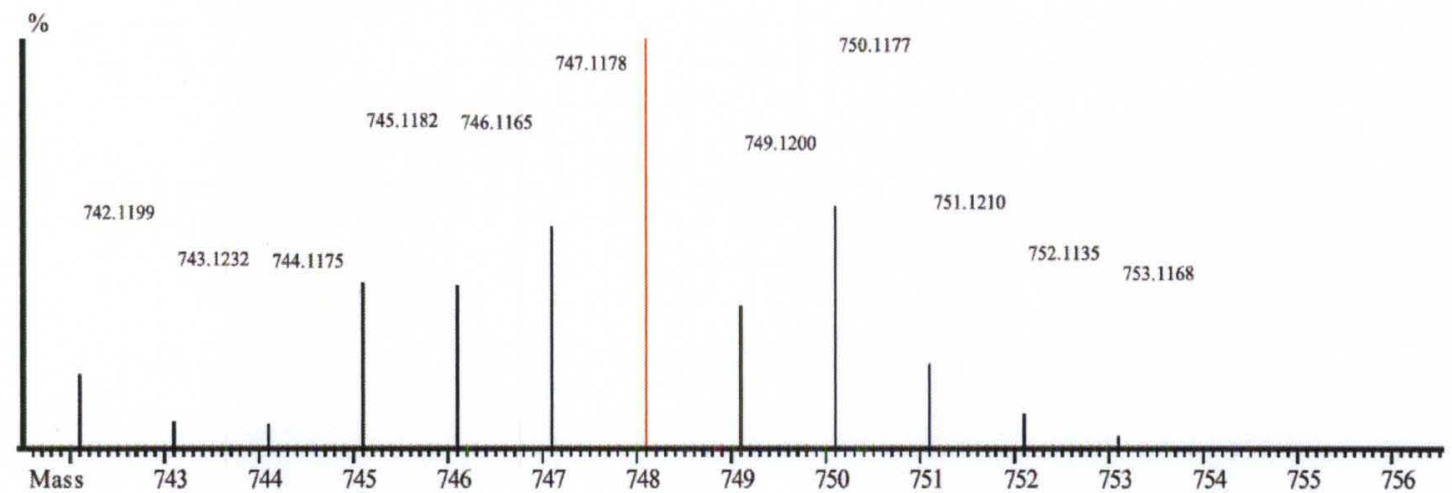

Figure 29. Calculated isotopic distribution for compound 4.

\section{Structural characterization.}

Orange block shaped crystals of $\mathbf{1}$ in the monoclinic space group $\mathrm{P} 2 / n$ were obtained upon slow evaporation of methanol solutions of the complex under air free 
conditions. The final unit cell parameters: $\mathrm{a}=11.085(4) \AA . \mathrm{b}=16.885(6) \AA \mathrm{c}=16.110(6)$ $\AA, \beta=95.848(6)^{\circ}, \mathrm{V}=2999.7(19) \AA^{3}, D_{\text {calc }}=1.514 \mathrm{Mg} / \mathrm{m} 3, Z=4$. Complex 1 contains a six-coordinate ruthenium(II) ion in an $\mathrm{N}_{2} \mathrm{~S}_{3} \mathrm{P}$ donor set with one thioether (S1), one sulfinate (S2), and one thiolate (S3) sulfur donor, Figure 30. The TASN ligand backbone (N1, N2, S1) occupies one face of a distorted octahedron. The three sulfur donors are arranged in a meridional fashion as in other six-coordinate bmmp-TASN based complexes. ${ }^{78,93,96}$ The sulfinate sulfur S2 of 1 sits trans to the amine N2. ${ }^{129}$ The thiolate sulfur $\mathrm{S} 3$ of 1 occupies the position trans to the thioether $\mathrm{S} 1$.

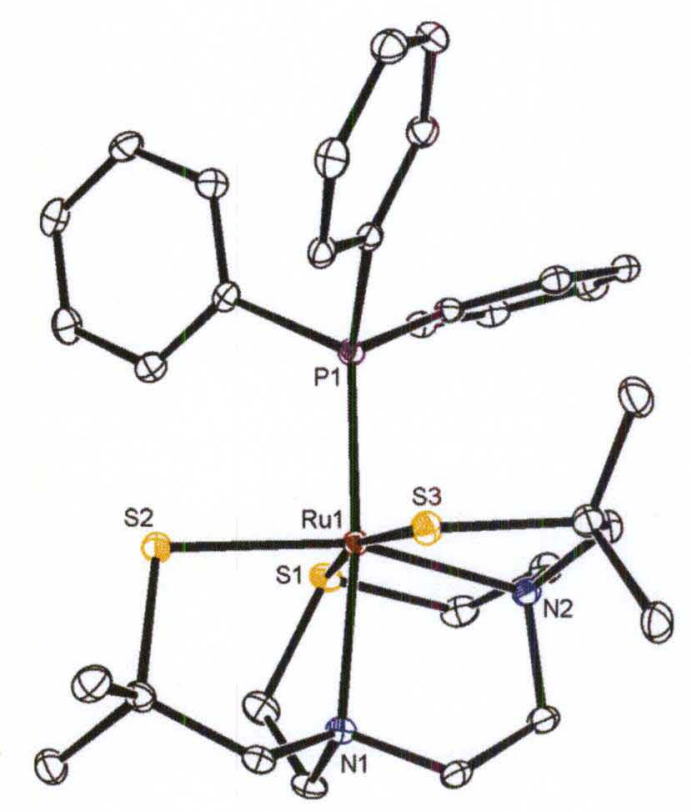

Figure 30. ORTEP representation of $\mathbf{1}$ showing $40 \%$ probability ellipsoids. $\mathrm{H}$ atoms and methanol solvates have been omitted to clearly illustrate the oxygenation of S2.

Orange block shaped crystals of 2 in the monoclinic space group $\mathrm{P} 2{ }_{1} / n$ were obtained upon slow evaporation of methanol solutions of the complex under air free conditions. Complex 2 contains a six-coordinate ruthenium(II) ion in an $\mathrm{N}_{2} \mathrm{~S}_{3} \mathrm{P}$ donor set with one thioether (S1), one sulfinate (S2), and one thiolate (S3) sulfur donor, Figure 31. 
The nine-membered heterocyclic TASN ring (N1, N2, S1) occupies one face of a distorted octahedron. The three sulfur donors are arranged in the same distribution as in other six-coordinate bmmp-TASN based complexes. ${ }^{78,93,96}$ The sulfinate sulfur S2 of 2 sits trans to the amine N2 in the same position as in $1{ }^{129}$ The thiolate sulfur S3 of 2 occupies the position trans to the thioether S1.

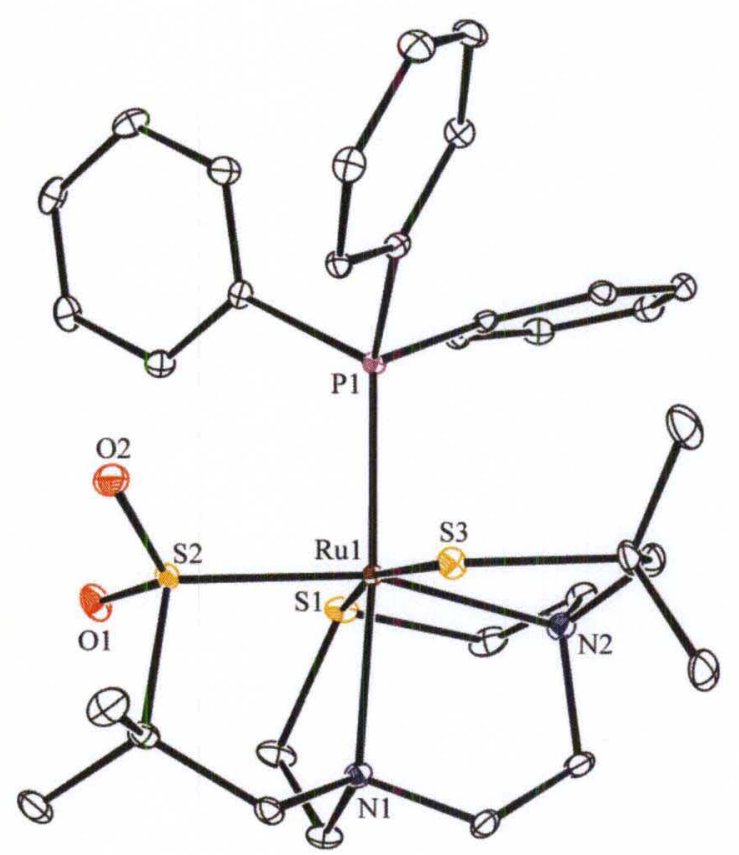

Figure 31. ORTEP representation of 2 showing $40 \%$ probability ellipsoids. H atoms and methanol solvates have been omitted to clearly illustrate the oxygenation of S2.

A large yellow plate crystal of $\mathbf{3}$ in the monoclinic space group P-1 were obtained upon slow evaporation of methanol solutions of the complex under air free conditions. The complex 3 in Figure 32 contains a six-coordinate ruthenium(II) ion in an $\mathrm{N}_{2} \mathrm{~S}_{3} \mathrm{P}$ donor set and reproduce the same structural arrangement that $\mathbf{1}$ and $\mathbf{2}$. The two $\mathrm{O}$ atoms $\mathrm{O} 1$ and $\mathrm{O} 2$ of the sulfinate donor (S2) are directed roughly along the S1-Ru-S3 bond axis with torsion angles of $-12.63(12)^{\circ}$ and $+35.55(13)^{\circ}$ for O1-S2-Ru1-S1 and O2-S2-Ru1- 
$\mathrm{S} 3$, respectively. The sulfenate oxygen $(\mathrm{O} 3)$ is oriented toward N1 along the P1-Ru-N1 axis with an O3-S3-Ru1-N1 torsion angle of $-16.47(14)^{\circ}$. As shown in Figure 33, the triphenylphosphine donor restricts access to the remaining potential oxygenation site on $\mathrm{S} 3$, which may retard the rate of further oxygenation under limited $\mathrm{O}_{2}$.

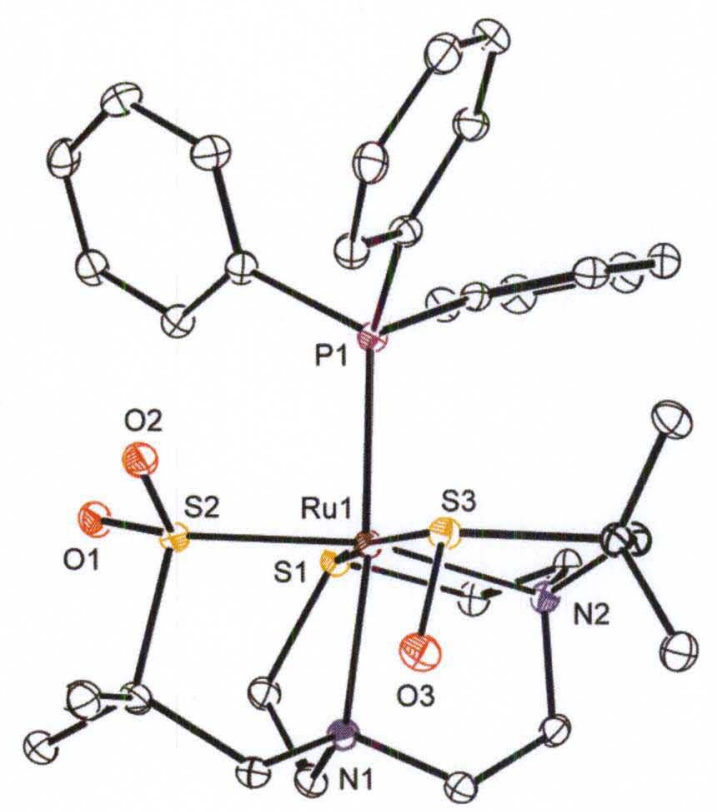

Figure 32. ORTEP representation of 3 showing $40 \%$ probability ellipsoids. H atoms and methanol solvates have been omitted to clearly illustrate the asymmetric oxygenation of S2 and S3. 


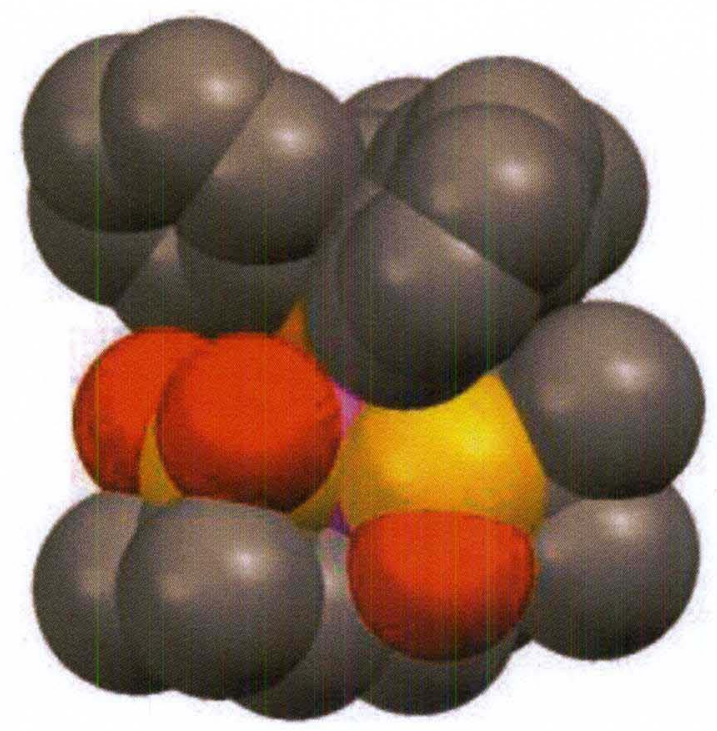

Figure 33. Space-filling representation of 3 illustrating the steric crowding imposed by the phenyl substituents around the sulfenato sulfur, S3.

X-ray crystallographic analyses of $\mathbf{1 , 2}$ and $\mathbf{3}$ reveal similar $\left(\mathrm{N}_{2} \mathrm{~S}_{3}\right) \mathrm{RuPPh}_{3}$ donor environments, with a $\mathrm{NS}_{3}$ square planar equatorial environment and an amine and phosphine donor in an axial positions. The S2 sulfur is oxidized in a sulfinate in $\mathbf{2}$ and $\mathbf{3}$ and $\mathrm{S} 3$ is the sulfur oxidized to sulfenate in 3. As shown in the ORTEP representations of 1-3 in Figures 30-32, respectively all the complexes display a facially coordinated TASN ring (N1, N2, and S1), two pendant sulfur donors (S2 and S3), and a triphenylphosphine (P1) trans to the nitrogen (N1).

The sulfur-oxygenation of a metal coordinated thiolate eliminates a four electron $\mathrm{d} \pi-\mathrm{p} \pi$ antibonding interaction between the metal and the sulfur. ${ }^{66,80,133,135,136}$ Additionally, oxidation of the sulfur decreases its ionic radius. The net result is a decreased Ru-S bond distance. ${ }^{133}$ The Ru-S bond distances to the oxygenated sulfur donors S2 and S3 are

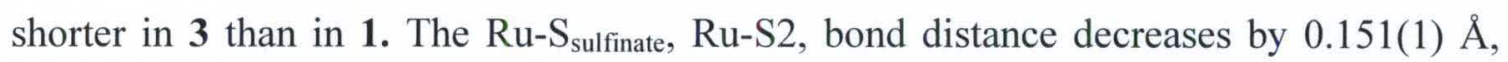


while the Ru-S $\mathrm{S}_{\text {sulfenate }}$ bond distance, Ru-S3, shortens by only $0.026(1) \AA$. Consistent with this explanation, 3 displays significantly longer bond distances to its $\pi$-accepting ligands than 1. The Ru-P1 bond distance to the triphenylphosphine increases by $0.088(1) \AA$, and the Ru- $\mathrm{S}_{\text {thioether }}, \mathrm{Ru}-\mathrm{S} 1$, bond distance similarly increases by $0.072(1) \AA$. This is similar to a recent theoretical prediction by Mascharak et al. of a $0.023 \AA$ increase in the Fe-NO bond distance upon sulfur oxygenation of a dithiolate iron nitrosyl. ${ }^{112}$ The average S-O distance for the sulfinate, $\mathrm{S} 2$, of $1.48 \AA$ falls in the usual range $(1.42-1.48 \AA){ }^{82,133,137}$ The sulfenate S-O bond is more polarized, resulting in a longer S-O distance of 1.556(3) $\AA$, which also lies in the typical range (1.50-1.60 $\AA)^{81,82,113,133}$ Additional comparison of crystallographic data of the family of compounds $\mathbf{1 - 3}$ reveals significant distinctions as a function of sulfur oxygenation. The Ru-S sulfinate distances of 2.2473(6) in 2 is $0.158(1)$ shorter than the corresponding Ru-S thiolate distance in 1. Similarly, the Ru-S thiolate distances of 2.3943(6) in 2 is $0.045(1)$ longer than corresponding Ru-S sulfenate bond length in 3 .

Further, the loss of $\pi$-donating ability induced by S-oxygenation increases metalligand bond distances with traditional $\pi$-acceptors. The Ru-P bond distance consistently increases from $2.2911(10)$ to $2.3519(6)$ to $2.3790(9) \AA$ for $\mathbf{1}, \mathbf{2}$, and 3, respectively. The same trend is noted in the Ru-S thioether bond distances, as we can observe in Table 1. 


\begin{tabular}{lccccccc}
\hline & \multicolumn{5}{c}{1} & \multicolumn{3}{c}{3} & 4 \\
\hline & Experimental & Theoretical & Experimental & Theoretical & Experimental & Theoretical & Theoretical \\
\hline Ru1-S1 & $2.2900(10)$ & 2.33949 & $2.3102(6)$ & 2.36409 & $2.3622(9)$ & 2.43248 & 2.40525 \\
Ru1-S2 & $2.4057(9)$ & 2.44426 & $2.2473(6)$ & 2.32732 & $2.2548(9)$ & 2.33608 & 2.34758 \\
Ru1-S3 & $2.3754(10)$ & 2.4401 & $2.3943(6)$ & 2.43876 & $2.3493(9)$ & 2.39406 & 2.38256 \\
Ru1-P1 & $2.2911(10)$ & 2.32479 & $2.3519(6)$ & 2.3811 & $2.3790(9)$ & 2.41051 & 2.44965 \\
Ru1-N1 & $2.198(2)$ & 2.25183 & $2.1927(19)$ & 2.26026 & $2.178(3)$ & 2.23921 & 2.25745 \\
Ru1-N2 & $2.178(2)$ & 2.22881 & $2.200(2)$ & 2.26699 & $2.192(3)$ & 2.2614 & 2.28146 \\
S2-O1 & & & $1.4906(17)$ & 1.53291 & $1.489(3)$ & 1.5317 & 1.53436 \\
S2-O2 & & & $1.4658(18)$ & 1.5133 & $1.471(3)$ & 1.5148 & 1.51018 \\
S3-O3 & & & & & $1.556(3)$ & 1.5755 & 1.53122 \\
S3-O4 & & & & & & & 1.50548 \\
P1-Ru-S2 & $95.44(2)$ & 91.20 & $93.99(2)$ & 93.43 & $90.72(3)$ & 92.50 & 93.91 \\
P1-Ru-S3 & $92.42(3)$ & 93.34 & $93.16(2)$ & 93.29 & $91.59(3)$ & 91.10 & 94.06 \\
S2-Ru-S3 & $94.93(3)$ & 95.61 & $97.09(2)$ & 97.81 & $94.13(3)$ & 96.42 & 98.52 \\
\hline
\end{tabular}

Table 1. Selected experimental and computational (BP86/LANL2DZ+6-31G(d)) bond distances $(\AA)$ and angles (deg) for 1-4.

\section{Computational investigations}

The geometry optimization and subsequent molecular orbital analysis of complexes 1-4 were performed using the BP86 functional with the LANL2DZ basis set for $\mathrm{Ru}$ and the $6-31 \mathrm{G}(\mathrm{d})$ basis set for all other atoms. The calculated metal-ligand bond distances accurately reproduce the experimental values for complexes $\mathbf{1 - 3}$ within 0.02 to $0.08 \AA$, Table 1. Although no experimental data is available for 4 , the optimized bond distances are consistent with the trends observed for 1-3, Figure 34. Calculated bond angles reproduce experimental values within $4^{\circ}$. Similar results were obtained when the B3L YP functional was analyzed. 
The optimized metal ligand distances reflect changes in the electronic configuration as a function of sulfur oxygenation and its metal bond distances. As shown in Figure 34, the effects of sulfur oxygenation in the series 1-4 are revealed by the Ru-S2, $\mathrm{Ru}-\mathrm{S} 3$, and Ru-P bond distances. As expected, large changes in Ru-S bond distances occur upon oxygenation of the sulfur donor. A decrease of $0.12 \AA$ in the Ru-S2 bond distance occurs when S2 is oxygenated from a thiolate in $\mathbf{1}$ to a sulfinate in $\mathbf{2}$. The Ru-S2 bond distance then only slightly increases in 3 and 4 . The largest decrease in the Ru-S3 bond distance, $0.04 \AA$, is observed when the thiolate of $\mathbf{2}$ is oxidized to a sulfenate in $\mathbf{3}$. Only small changes are observed between the S3-thiolates of 1 and 2 and the S3oxygenates of 3 and 4 . While changes in the Ru-S bond distances reflect bonding changes at that specific atom, the $\mathrm{Ru}-\mathrm{P}$ bond distance consistently increases with each subsequent O-atom added to the sulfur donors. As shown in Figure 34, there is a direct correlation between the Ru-P bond distance and the level of sulfur oxygenation. The data fits a straight line according to the equation $\mathrm{y}=2.331+(0.040) \times\left(\mathrm{R}^{2}=0.98\right)$, where $\mathrm{y}$ is the $\mathrm{Ru}-$ $\mathrm{P}$ bond distance in $\AA$ and $\mathrm{x}$ is the number of O-atoms added to sulfur. For the bissulfinate complex 4 , the theoretical bond length increases to $2.450 \AA$. The elongation of the Ru-P bond as a function of sulfur oxygenation supports the suggestion that post translational modifications in NHase or SCNase regulates substrate/product binding. 


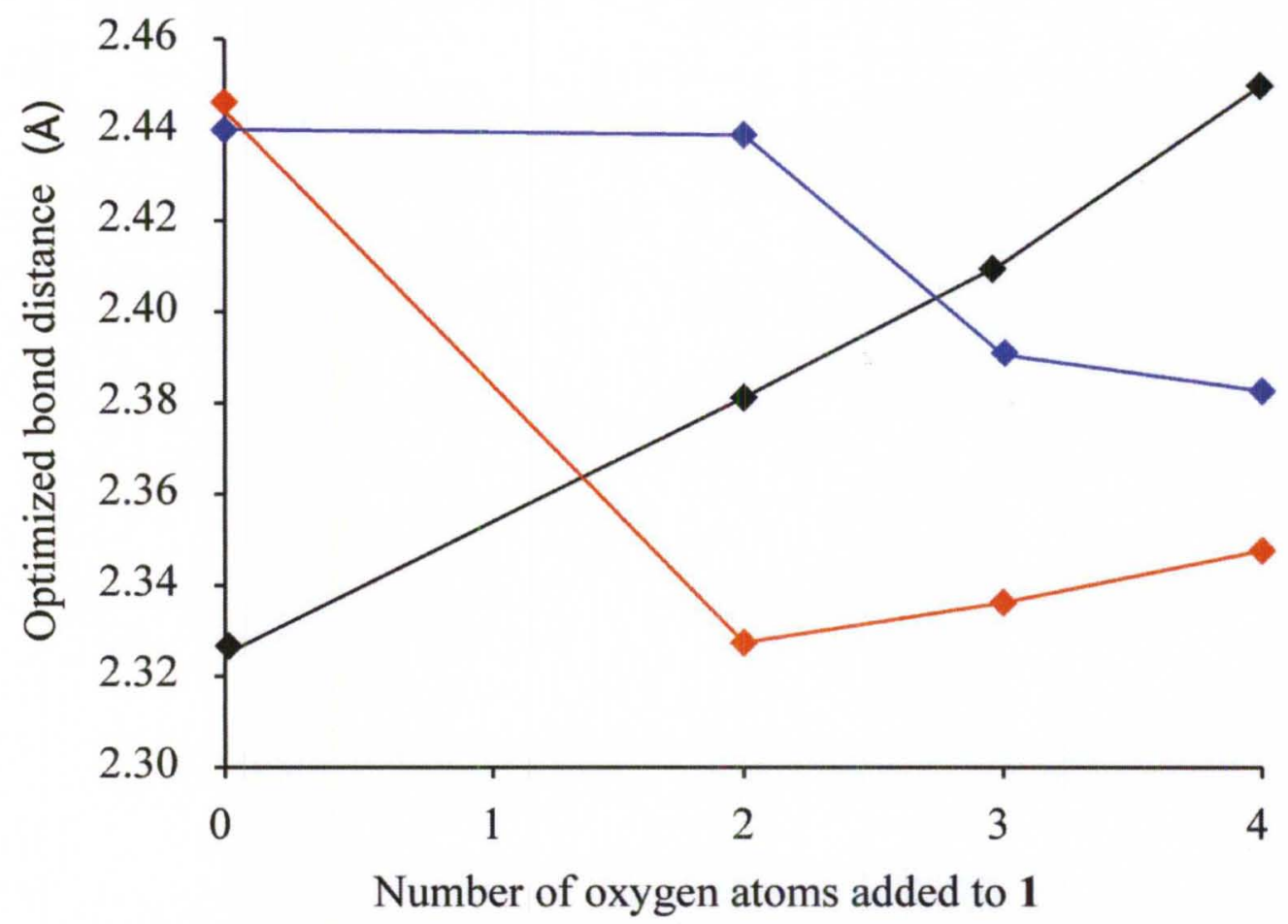

Figure 34. Plot of optimized (BP86/LANDZ+6-31G(d)) Ru-Lbond distance versus number of oxygen atoms in 1-4. Ru-P (black), Ru-S2 (red), Ru-S3 (blue).

Analysis of the frontier orbitals of 1-4 shows a direct connection between sulfur oxygenation and changes in the Ru-P bonding. In 1, the occupied frontier molecular orbitals are dominated by metal-sulfur interactions as observed in other metalthiolates. ${ }^{66,78,136,138-140}$ The highest occupied molecular orbital (HOMO) and HOMO-1, Figure 35 , show $\pi^{*}$ interactions between filled " $\mathrm{t}_{2 \mathrm{~g}}$ " orbitals on the metal and p-type lone pairs on the sulfur on S2 and S3, respectively. Additionally, the HOMO-1 includes a significant $\sigma$-bonding interaction between metal-centered orbital and the phosphine ligand that strengthens the Ru-P bond. This $\sigma$-bonding interaction can be considered as an "outlet" for the 4 electron $\mathrm{d} \pi$-p $\pi$ repulsion described for " $\mathrm{t}_{2 \mathrm{~g}}$-rich" metal-thiolates. A similar $\sigma$-donation is observed in the HOMO between the metal and the thioether sulfur S1, with an additional polarization of the metal orbital toward the phosphine donor. In 
(2), one of the thiolates, S2, has been oxygenated and no longer participates in $\pi$ bonding/antibonding with $\mathrm{Ru}$. The HOMO of $\mathbf{2}$ is localized on the remaining thiolate, $\mathrm{S} 3$, and $\mathrm{Ru}$. The HOMO of $\mathbf{2}$ is similar to the HOMO-1 of $\mathbf{1}$ with a $\pi^{*}$ interaction between Ru and $\mathrm{S}$ and a strong $\sigma$-bonding interaction between $\mathrm{Ru}$ and $\mathrm{P}$. Complexes $\mathbf{3}$ and 4 lack thiolate donors and do not show the same type of interactions as $\mathbf{1}$ and $\mathbf{2}$. Rather, the HOMOs of $\mathbf{3}$ and $\mathbf{4}$ are dominated by S-O $\pi^{*}$ interactions that are $\sigma$-bonding with respect to the metal sulfur-oxygenate bond. As in the HOMO of $\mathbf{1}$, the metal centered orbital is slightly polarized toward the phosphine ligand. Overall, Ru-S interactions reinforce the $\mathrm{Ru}-\mathrm{P}$ bond through the HOMO and HOMO-1 of $\mathbf{1}$ and the HOMO of $\mathbf{2}$. The Ru-P bond is only weakly affected by Ru-S interactions in the HOMOs of $\mathbf{3}$ and $\mathbf{4}$. 

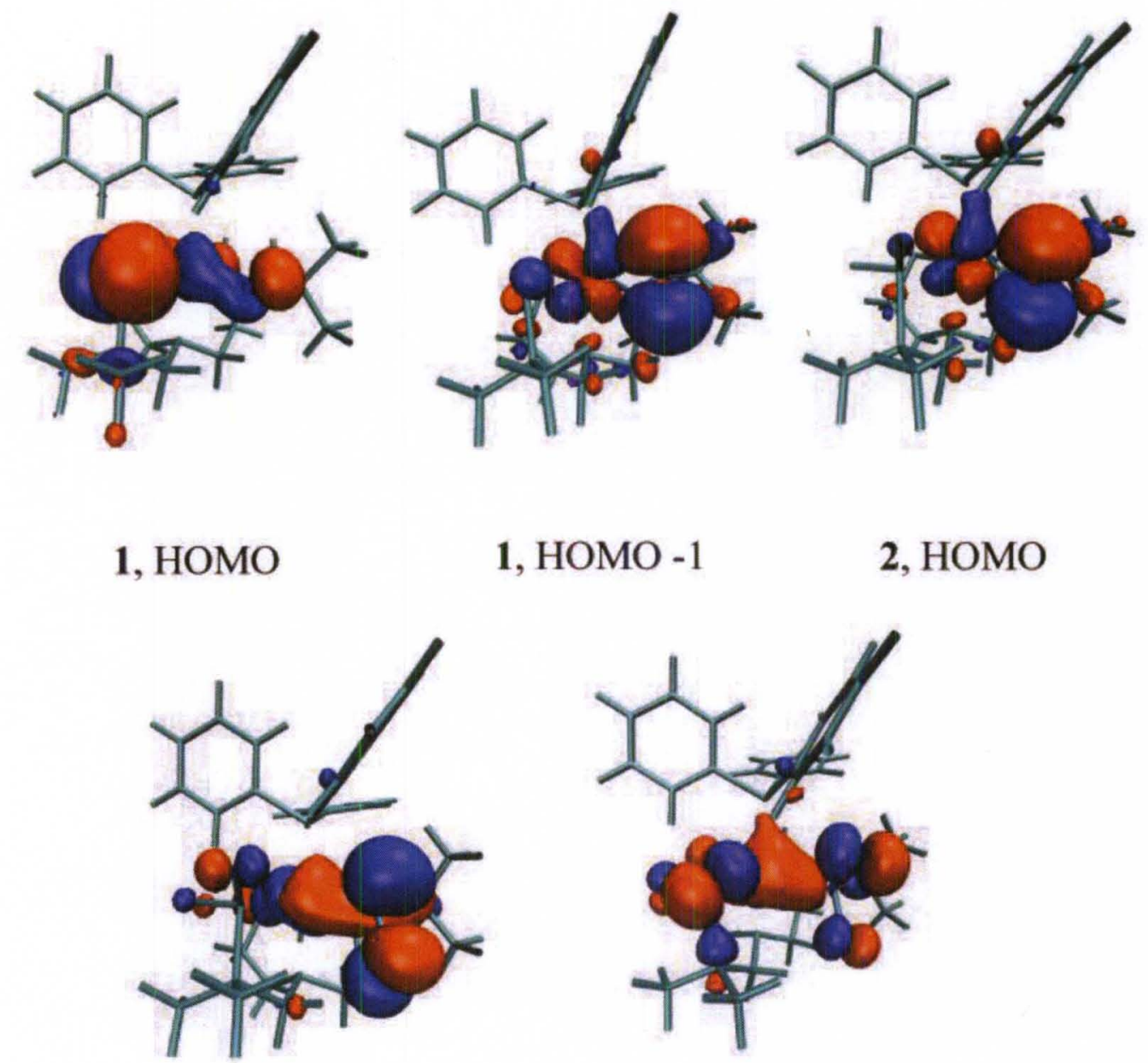

1, HOMO -1

2, HOMO

\section{3, HOMO}

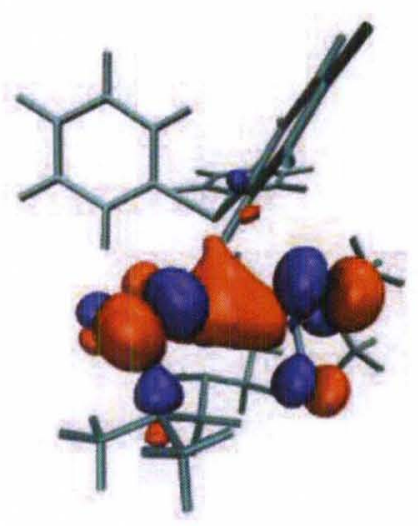

Figure 35. Homo surfaces of 1-4 and Homo-1 of $\mathbf{1}$.

Sulfur oxygenation effect on the complex (bmmp-TASN)RuPPh $\mathrm{h}_{3}$ probed by multiedge X-ray absorption spectroscopy

In collaboration with Jason Shearer at University of Nevada Reno, it was investigated the effect of the sulfur oxygenation in compound 1 . These studies used Ru L-edge, and ligand (N-, S- and P-) K edge X-ray absorption spectroscopies to investigate the effect of the sulfur oxygenation in the electronic structure of compound 1-3. In this 
study is included N K-edge spectroscopy, technique that is used for first time to obtain Nmetal covalency parameters. The most relevant result was that the complexes becomes progressively more ionic, and hence the Ru(II) center becomes a harder Lewis-acid.

In the X-ray absorption studies performed in the National Synchroton Light Source, Brookhaven National Laboratory, it was demonstrated that the bonding in the complex 1-3 is dominated by $\mathrm{S}-\mathrm{Ru}$ interactions. The $\mathrm{Ru}-\mathrm{P}$ and $\mathrm{Ru}-\mathrm{N}$ interaction contribute in a lesser degree in the bonding interactions. Upon oxygenation of compound 1 the thiolates-ruthenium bond distance decrease in $\mathrm{S} 2$ and $\mathrm{S} 3$ for compound 2 and 3 . As this occur the covalency of the compound decrease and the charge of the ruthenium center increase. The ruthenium increase charge goes from -0.073 in RuS to +0.0831 in $\mathrm{RuSO}_{2}$ to +0.263 in $\mathrm{RuSO}_{2} / \mathrm{SO}$ and as consequence the ruthenium center become more Lewis-acid. One of the consequences of the harder ruthenium is the week interaction with the soft phosphine and increase interaction with harder amines (relative to phosphine), both effects were observed in X-ray and DFT calculations.

From a mechanistic perspective, the increase in the hard Lewis acidity nature of the metal ion would aid to the catalysis. In a nitrile metal bond mechanistic model, the increased hardness of the metal would better polarize the nitrile $\mathrm{CN}$ bond aiding in attack by free water. In a water bind mechanism, the coordination of the water is favored over the coordination of a soft nitrile. Furthermore this last mechanism is favored for the increased charge in the metal as consequence of the sulfur oxygenation. The increase in charge will lower the $p K a$ of the water generating the hydroxide for a nitrile attack. 


\section{Voltammetric studies.}

Electrochemical studies of $\mathbf{1 - 3}$ were conducted in acetonitrile solution with $0.1 \mathrm{M}$ tetrabutylammonium hexafluorophosphate (TBAHFP) as supporting electrolyte under air-free conditions in the glovebox. All potentials are referenced to the ferrocenium/ferrocene $(\mathrm{Fc}+/ \mathrm{Fc})$ couple. The square wave voltammograms, Figure 36, clearly outline the changes in the $\mathrm{Ru}(\mathrm{III} / \mathrm{II})$ reduction potential as a function of sulfur oxygenation. The $\mathrm{Ru}(\mathrm{II})$-dithiolato precursor 1 displays a $\mathrm{Ru}(\mathrm{III} / \mathrm{II})$ redox couple with $E_{1 / 2}=-851 \mathrm{mV}$. Oxygenation of 1 to 2 stabilizes the lower oxidation because of electron withdrawing effects of the two $\mathrm{O}$ atoms. The $\mathrm{E}_{1 / 2}$ of 2 is shifted by $+458 \mathrm{mV}$ with respect to 1 resulting in a $\mathrm{Ru}(\mathrm{III} / \mathrm{II})$ potential of $-393 \mathrm{mV}$. The shift is similar to that observed upon oxygenation of other Ru thiolates. ${ }^{130}$ The $E_{1 / 2}$ of $3,-263 \mathrm{mV}$, reflects an additional shift of $+130 \mathrm{mV}$ as compared to 2 as another O-atom is added. The cathodic shift for a single sulfenate O-atom is less than half of the shift induced by the two O-atoms of the sulfinate as previously noted by Darensbourg et al. ${ }^{79}$ The reversibility of each redox event was established by cyclic voltammetry, Supporting Information, Figures A2-A4. Each complex displays a quasi-reversible $\mathrm{Ru}(\mathrm{III} / \mathrm{II})$ couple with $\Delta E$ values of 109,73 , and $79 \mathrm{mV}$ for 1,2 , and 3 at a scan rate of $200 \mathrm{mV} / \mathrm{s}$. 


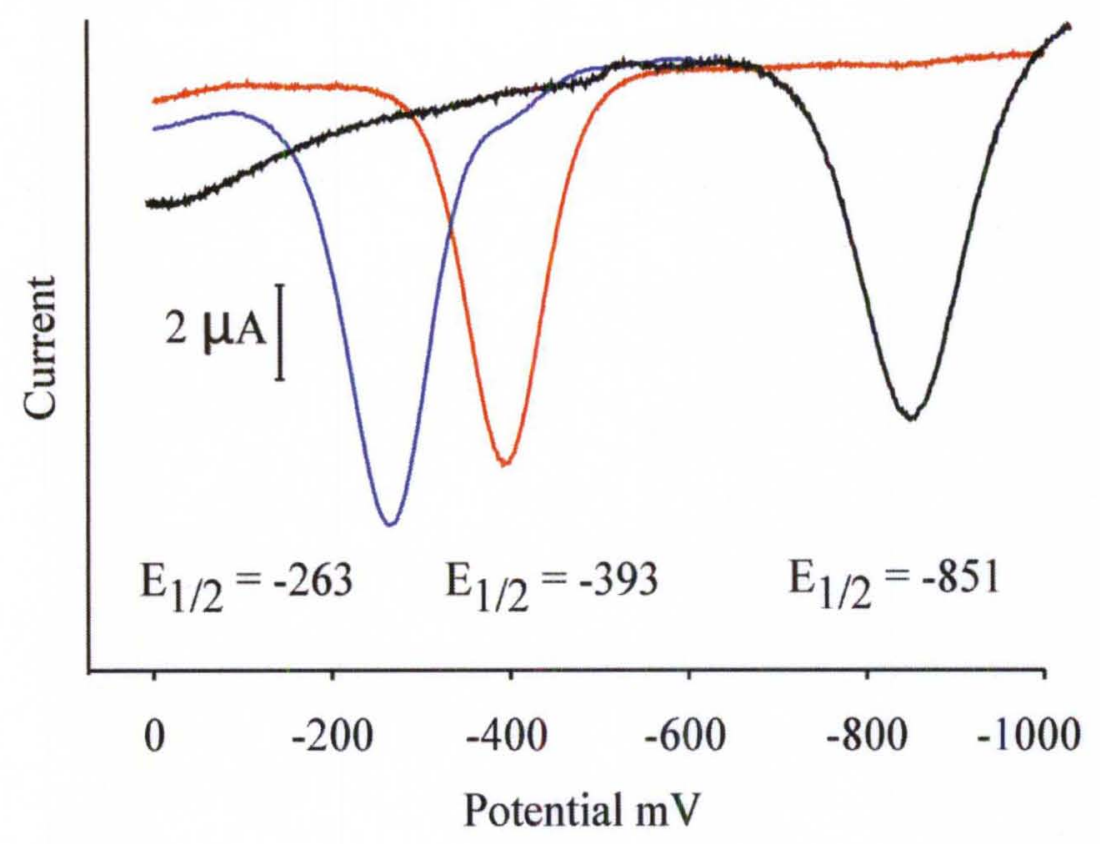

Figure 36. Square voltammograms 1-3.

\section{Summary.}

We reported the synthesis of (bmmp-TASN)RuPPh limiting dioxygen conditions to (bmmp- $\left.\mathrm{O}_{2}-\mathrm{TASN}\right) \mathrm{RuPPh}_{3} \quad$ (2), (bmmp- $\mathrm{O}_{3}$ TASN)RuPPh 3 (3), and (bmmp-O 4 -TASN)RuPPh $(4) .{ }^{129,141}$ The sulfenate/sulfinate $\left(\mathrm{RSO}^{-}\right) /\left(\mathrm{RSO}^{2-}\right)$ donor set of $\mathbf{3}$ mimics the unusual post-translational modification of the NHase and SCNase active sites. The asymmetric sulfur-oxygenate 3 represents one of only three sulfenate/sulfinate derivatives prepared by direct reaction with dioxygen. ${ }^{79,108}$ High yields of $\mathbf{3}$ were obtained by limiting the quantity ( 5 equivalents) and duration (12 hours) of dioxygen exposure.

This research work offers insight into the controlled sulfur oxygenation of metal thiolates and the resulting changes in the electronic structure. The previous hypothesis of the Grapperhaus group that " $\mathrm{t}_{2 \mathrm{~g}}$-rich" low-spin complexes favor sulfur oxygenation is 
supported by the reactivity of $\mathbf{1}$ with $\mathrm{O}_{2}$. Further, partial sulfur oxygenation is achievable using limited $\mathrm{O}_{2}$ conditions, as demonstrated by $\mathbf{3}$ and other reported sulfenate/sulfinate complexes. In 1 , the steric bulk of $\mathrm{PPh}_{3}$ slows oxygenation beyond 3 but does not prevent it, as demonstrated under excess $\mathrm{O}_{2}$ conditions. These results suggest that asymmetric oxygenation of nitrile hydratase and thiocyanate hydrolase may also be facilitated by limited $\mathrm{O}_{2}$ at the active site without the necessity for single $\mathrm{O}$-atom-transfer reagents. Structurally sulfur oxygenation shortens the M-S bond while lengthening the metalligand bonds to $\pi$ acceptors. In combination with the previously documented labilizing effect of the trans-thiolate, ${ }^{142,143}$ sulfur oxygenation may promote ligand exchange. As demonstrated by Mascharak, sulfur oxygenation facilitates photodissociation of NO. ${ }^{112}$ It is also expected to enhance coordination of $\pi$ donors, such as $\mathrm{HO}^{-}$, and may help to discriminate substrate coordination.

Finally, we report systematic studies under limited $\mathrm{O}_{2}$ conditions. By fixing the quantity of $\mathrm{O}_{2}$ and the duration of the reaction, conditions were optimized to reproducibly obtain the monosulfinate (bmmp- $\left.\mathrm{O}_{2}-\mathrm{TASN}\right)-\mathrm{RuPPh}_{3} \quad \mathbf{2}$, the asymmetric sulfenate/sulfinate (bmmp- $\left.\mathrm{O}_{2}-\mathrm{TASN}\right)-\mathrm{RuPPh}_{3} \quad \mathbf{3}$ and bissulfinate (bmmp-O $\mathrm{O}_{4}$ TASN)RuPPh 4 derivatives without the need for $\mathrm{O}$-atom transfer reagents or purification by column chromatography. This approach has yielded a unique "family" of complexes, 1-4, that differ only in the degree of S-oxygenation, Scheme 4. Although the number of reported S-oxygenates is growing, families containing three or more oxygenated derivatives are scant. ${ }^{144}$ Based on a systematic synthesis design, the pioneering Ni- and Pd-dithiolate systems developed by Darensbourg provide a benchmark for completeness, because they offer no prospects to probe the effects of S-oxygenation at a variable ligand 
site. Challenge that it is being addressed in this research, analysis of the structural parameters of 1- 4 reveals a systematic increase in the Ru-P bond distance as a function of S-oxygenation. This trend is reproduced computationally by density functional theory calculations providing insight into the potential of S-oxygenation to regulate substrate/product binding at the NHase active site and to formulate the hypothesis that this sulfur oxygenation allows exchange substrate in our model, this hypothesis will be tested in the next chapter. 


\section{CHAPTER IV}

\section{REACTIVITY BEHAVIOR OF (bmmp-TASN)RuPPh}

The stabilization, coordination and activation of small molecules such as NO, $\mathrm{RCN}, \mathrm{RC}(\mathrm{O}) \mathrm{NH}_{2}, \mathrm{H}_{2} \mathrm{O}$ by transition metal with sulfur-nitrogen coordination complexes such as the (bmmp-TASN)Ru is of critical interest for understanding the molecular mechanisms of NHase and SCNase. It is proposed that these molecules interact with a low spin $\mathrm{Fe}$ (III) or $\mathrm{Co}$ (III) in the first coordination sphere of the NHase. The NHase of Rhodococcus erythropolis $\mathrm{N} 771$ exhibit catalytic activity converting tert-butylisonitrile $(t \mathrm{BuNC})$ to tert-butylamine; its nitrile substrate was structurally characterized by time-

resolved X-ray crystallography. ${ }^{50}$ These studies address a nitrile mechanism where the substrate was coordinated to the iron and then attacked by a solvent molecule activated by $\alpha \mathrm{Cys}^{114}-\mathrm{SO}(\mathrm{H})$.

In a recent published, studies achieved by the Kovac's group supported the proposed role of the sulfenate as a proximal base. Kovac et al trapped and structurally characterized a Co-iminol and Co-amidate, which is tautomer of the imidate intermediate by using alkoxide and amine donor in place of the sulfenate. ${ }^{65}$ However, there are no available catalytic models that reproduce the activity of the enzyme and at the same time the functionality of the post-translational modification remains unexplained. 
At the moment there is not a synthetic compound that model, the basic structural and functional properties of the NHase metal center. To be more specific, the basic features are the metal thiolate-nitrogen coordination, systematic oxygenation of the thiolate, substrate-product exchange in the active site and catalytic activity of the model. In the search for such models, we found that the (bmmp-TASN)RuX is an unequal example in the research community that satisfies the coordination and structural requirement for a catalytic model of the NHase.

\section{Substrate exchange in bmmp-TASN models preliminary results}

The Grapperhaus group has been performing studies in the NHase (bmmpTASN)Fe(III) model in the area of exchange substrate reactivity, using $\mathrm{NaOH}, \mathrm{NO}$, $\mathrm{Et}_{4} \mathrm{NCN}$ and TIOTf. In the latter, the trifluoromethanesulfonate (OTf) was used as a weakly coordinated ion to test solvent coordination solvent and binding reactivity. These

studies cover the first NHase model complex [(bmmp-TASN)Fe]OTf reported to coordinate nitrile and water, both substrates of the enzyme, and achieved broad substrate binding studies. ${ }^{109}$ 

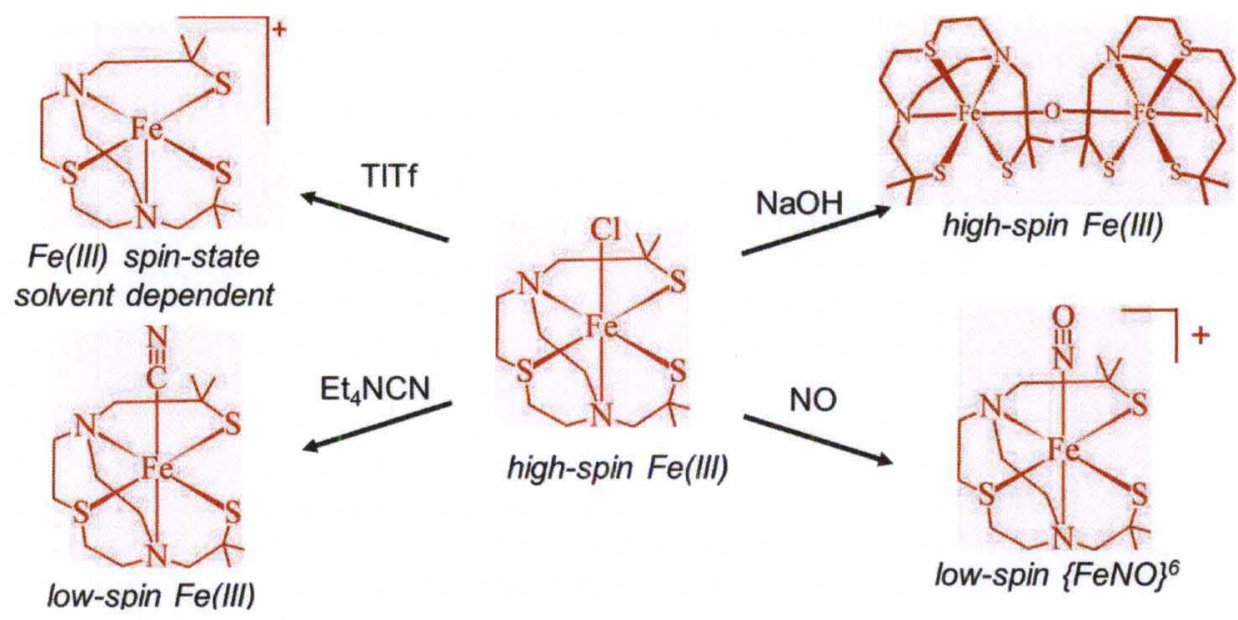

Figure 37. Substrate ligand exchange reactions of (bmmp-TASN)FeCl yield derivative complexes with spin-states that are dependent on the variable ligand allowing comparisons of spin-state effects for closely related complexes. Metathesis of chloride with thallium triflate yields $[(\mathrm{bmmp}-\mathrm{TASN}) \mathrm{Fe}]^{+}$with an open-coordinate sight available for solvent (substrate) binding.

The Grapperhaus groups preliminary studies utilized a penta-cordinated, square pyramidal $\left(\mathrm{N}_{2} \mathrm{~S}_{3}\right) \mathrm{Fe}$ core to bind coordination solvents in the $6^{\text {th }}$ axial position. Solvents that coordinate include $\mathrm{H}_{2} \mathrm{O}, \mathrm{CH}_{3} \mathrm{CN}$, DMF, $\mathrm{MeOH}$, DMSO and pyridine. The triflate derivative was synthesized using a suspension of $\mathrm{LFeCl}$ and dropwise cannula addition of thalium triflate in acetonitrile under air free conditions. Several trials to synthesize the (bmmp-TASN)FeOTf with traditional $\mathrm{Ag}^{+}$salts failed due to $\mathrm{Ag}^{+}$thiolate reactivity. In a series of competition evaluation of binding preference of $\mathrm{H}_{2} \mathrm{O}>$ amide $>$ nitrile, it was determined that the Fe(III) model supports a water bound mechanism. However, no evidence of hydrolysis was observed in this iron model. 


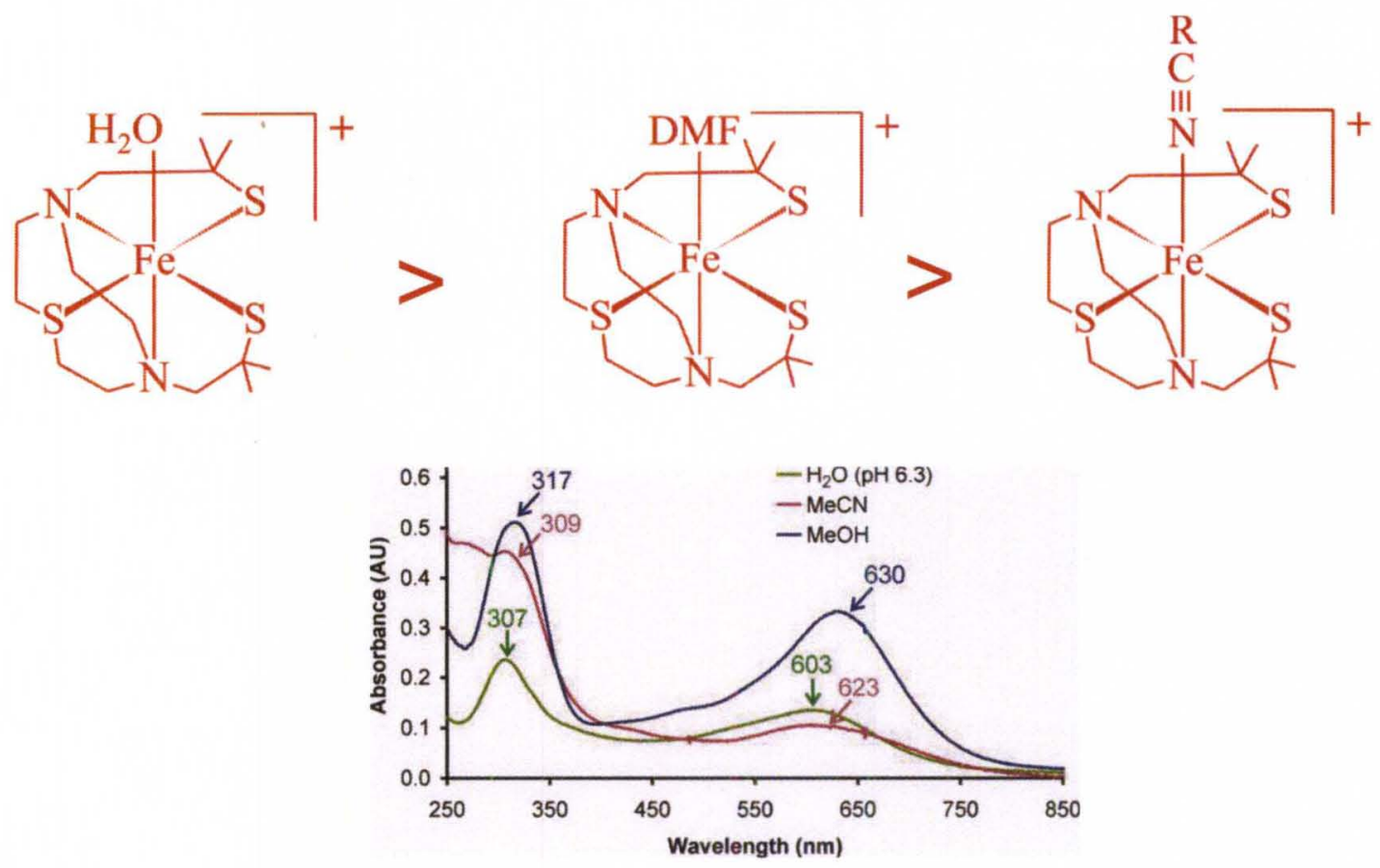

Figure 38. [(bmmp-TASN)Fe]Tf is the first NHase model complex that coordinates water and nitrile. Nitrile coordination is only observed at low temperature. DMF readily displaces nitriles and $\mathrm{H}_{2} \mathrm{O}$ displaces both nitriles and DMF.

The aqua derivative $\left[(\mathrm{bmmp}-\mathrm{TASN}) \mathrm{Fe}-\mathrm{OH}_{2}\right]^{+}$reveals reversible deprotonation events that include the formation of a $\mu$-hydroxo $\left[\left[(\text { bmmp-TASN)Fe }]_{2} \mathrm{OH}\right] \mathrm{OTf}, \mu\right.$-oxo $[(\text { bmmp-TASN }) \mathrm{Fe}]_{2} \mathrm{O}$ complexes. These events take place with biologically relevant $\mathrm{pKa}$ values $(\mathrm{pKa} 1=5.4(1), \mathrm{pKa} 2=6.52(1))$. The first deprotonation yields an unsupported $\mu$ $\mathrm{OH}$ bridge di-iron complex that was structurally characterized by X-ray crystallography. Subsequent deprotonation yields the $\mu$-oxo bridge complex $[(\mathrm{bmmp}-\mathrm{TASN}) \mathrm{Fe}]_{2} \mathrm{O}$ in a reversible process. The [(bmmp-TASN)Fe $] \mathrm{X}\left(\mathrm{X}=\mathrm{H}_{2} \mathrm{O}, \mu-\mathrm{OH}\right.$ and $\mu$-O) complexes lack of hydrolytic activity at a variety of $\mathrm{pH}$ values covering the two protonation states indicating insufficient nucleophilicity of the bridging $\mathrm{HO}^{-}$and strong substrate coordination. 
The Grapperhaus group performed EPR experiments in collaboration with Prof. Brian Bennett from the National Biomedical EPR center, Medical College of Wisconsin to study substrate exchange properties in the penta-coordinated model [(bmmpTASN)Fe $]^{+}$OTf. The spin-state of [(bmmp-TASN)Fe-Solvent]OTf depends on the substrate (coordination solvent) with low-spin Fe(III) complexes observed in acetonitrile $\left(\mathrm{g}_{1}=2.27, \mathrm{~g}_{2}=2.18\right.$, and $\left.\mathrm{g}_{3}=1.98\right)$ or benzonitrile $\left(\mathrm{g}_{1}=2.28, \mathrm{~g}_{2}=2.18, \mathrm{~g}_{3}=2.00\right)$. The nitrile derivatives are in equilibrium with a low-spin 5-coordinate species, [(bmmpTASN $) \mathrm{Fe}]^{+}\left(\mathrm{g}_{1}=2.04, \mathrm{~g}_{2}=2.02\right.$ and $\left.\mathrm{g}_{3}=2.01\right)$; this equilibrium is supported by spectroscopic characterization at $40^{\circ} \mathrm{C}$ in acetonitrile, solutions of $[\mathrm{LFe}]^{+}$are green with absorbance bands at 315,427 , and $603 \mathrm{~nm}$; similar to the spectrum observed in dichloromethane and consistent with a five coordinate complex. Nevertheless, upon cooling to room temperature and below, the acetonitrile solution turns blue color as the peak at $427 \mathrm{~nm}$ loses intensity and the low energy band shifts toward $596 \mathrm{~nm}$. Using other donor solvents such as $\mathrm{H}_{2} \mathrm{O}$, DMF, DMSO, $\mathrm{MeOH}$ or pyridine, 6-coordinated high spin $\mathrm{Fe}(\mathrm{III})$ complexes with the substrate $c$ is to the thiolates show significant strain in the rhombic zero-field splitting term E/D. These results helped to narrow our study of the (bmmp-TASN)FeX NHase system to focus on (bmmp-TASN)RuPPh ${ }_{3}$ which will maintain a low-spin state and would facilitate substrate exchange property required for a catalytic turnover and avoid metal oxo bridged species due to the high Lewis acid character of iron compare with ruthenium.

On the road to synthesize a functional model of NHase capable of exchanging substrates and displaying has hydrolytic activity, we decided to explore the sulfur oxygenation of the model (bmmp-TASN)RuPPh 3 . The bmmp-TASN ligand has been a 
focus of the Grapperhaus group with the objective to reproduce the unusual and synthetic challenging post-translational modification of the nitrile hydratase enzyme. The structural and spectroscopic characterizations of the family derivatives from the thiolate complex (bmmp-TASN)RuPPh ${ }_{3}$ has been described in the previous chapter. As noted previously, low spin $\mathrm{Fe}$ (III) complexes favor S-oxygenation and not disulfide formation or oxo clusters, inspiring investigations with (bmmp-TASN)RuPPh${ }_{3}$.

Analysis of the structural parameters using X-ray crystallography and Density Functional Theory calculations in the family of S-oxygenates reveals a systematic increase in the Ru-P bond distances as a function of S-oxygenation. This trend, which is in opposition to some expectations of tighter Ru-P binding resulting from decreased Sdonation, is supported experimentally by multi-edge X-ray absorption spectroscopy in collaboration with Prof. Jason Shearer of the Chemistry Department at University of Nevada, Reno. The XAS studies include N K-edge spectroscopy, which to our knowledge, has not been previously utilized to extract covalency parameters in metal complexes. The results demonstrate dramatic reduction in metal-ligand covalency with each sequential oxygenation with significant hardening of Ru. This leads to enhanced lability of the soft $\mathrm{PPh}_{3}$ donor.

The sequence of S-oxygenates $\mathrm{L}^{\mathrm{n}} \mathrm{RuPPh}_{3}(\mathrm{n}=1-4)$ synthesized in the Grapperhaus' Lab, make available an unparalleled set of functional complexes to study the outcome of S-oxygenation on catalytic activity, substrate binds, structural rearrangement of the ligand due to substrate binding, coordination solvent preference, and ligand exchange. Even though the number of reported S-oxygenated is growing, families of three or more oxygenated derivatives are scare and just few successful studies 
have reproduced the sulfenate/sulfinate first metal coordination sphere. The groundbreaking Ni- and Pd-dithiolate systems developed by Darensbourg group helped establish this field, such as (N,N'-bis(2-mercaptoethyl)-1,5-diazacyclooctanato)Ni(II) (Ni(bme-daco)), (N,N'-2-mercaptoethyl-2-sulfinatoethyl-1,5-diazacyclootanato)Ni(II) (Ni(mse-daco)), and [N,N'-bis(2-sulfinatoethyl)-1,5-diazacyclooctanato]Ni(II) (Ni(bspdaco)), ${ }^{116,135}$ However these models offer no opportunity for substrate coordination or ligand exchange reactions. Our system masters this limitation with a variable donor site. As we will described in our results below, ligand exchange of $\mathrm{PPh}_{3}$ for other donors provides functional hydrolytic mimic and establish unprecedented synthetic routes to Soxygenates derivatives. Phosphine exchange reactions are known for select Ru-thiolates reported by Sellmann. ${ }^{145,146}$ In this study we found that the model (bmmp-TASN)RuPPh and its family of derivatives can exchange its triphenylphosphine by other phosphines, $\mathrm{CO}$ and the models (1-3) are hydrolytically actives. We proposed to exploit the enhanced lability of our S-oxygenates to initiate similar ligand exchanges to obtain hydrolytically more active complexes and understand the functional effects of posttranslational oxygen modification in thiolates.

\section{Phosphine exchange in the metal complexes (bmmp-TASN)RuPPh ${ }_{3}$ and derivatives}

In this section we will present ${ }^{31} \mathrm{P}$ NMR spectra and +ESI-MS results that support the triphenylphosphine exchange in compound 1,2 and 3 . These results are consequence of preliminary results presented in the last chapter. The preliminary results that support this study include the Ru-P bond distances elongation calculated from X-ray analysis and DFT calculations. Also, the increase in the bond covalencies and metal hardening observed in the XAS studies suggest phosphine lability. Finally, experimentally it was 
observed that under air free conditions compound $\mathbf{3}$ change colors from orange to green upon heating, with a return to orange 3 upon cooling.

Results of our initial synthetic trails at $50{ }^{\circ} \mathrm{C}$ are summarized in Figure 39 and +ESI-MS spectrums in A5 and A6. Phosphine exchange readily occurs for compound 2 and 3 using 5 equivalents of $\mathrm{PMePh}_{2}$ at $50{ }^{\circ} \mathrm{C}$ in $\mathrm{MeOH}$. These results confirms our hypothesis that triphenylphosphine can be exchanged in sulfur-oxygenated compounds. In these experiments, the sulfinate/thiolate compound $\mathbf{2}$ and the sulfinate/sulfenate compound 3 exchange triphenylphosphine for methyl diphenylphosphine as confirmed by + ESI-MS, Figure 39.

The identification of phosphine exchange in the complexes (bmmp- $\mathrm{O}_{2-}$ TASN)RuPMePPh 2 (8) and (bmmp-O ${ }_{3}-\mathrm{TASN} \mathrm{RuPMePPh}_{2}$ (9) were confirmed by mass spectroscopy. +ESI-MS was recorded for each complex after the phosphine exchange reaction. Compound 8 displays a parent peak at $m / z$ 677.1140, Figure A5, consistent with a theoretical value of $677.1009 \mathrm{~m} / z$ for $[8+\mathrm{Na}]^{+}$. The isotopical distribution shows and accuracy of $0.0131 \mathrm{~m} / \mathrm{z}$. Compound 9 displays a parent peak at $m / z$ 693.1192, Figure A6, consistent with a theoretical value of $693.0958 \mathrm{~m} / \mathrm{z}$ for $[9+\mathrm{Na}]^{+}$. The isotopical distribution shows and accuracy of $0.0234 \mathrm{~m} / z$. 


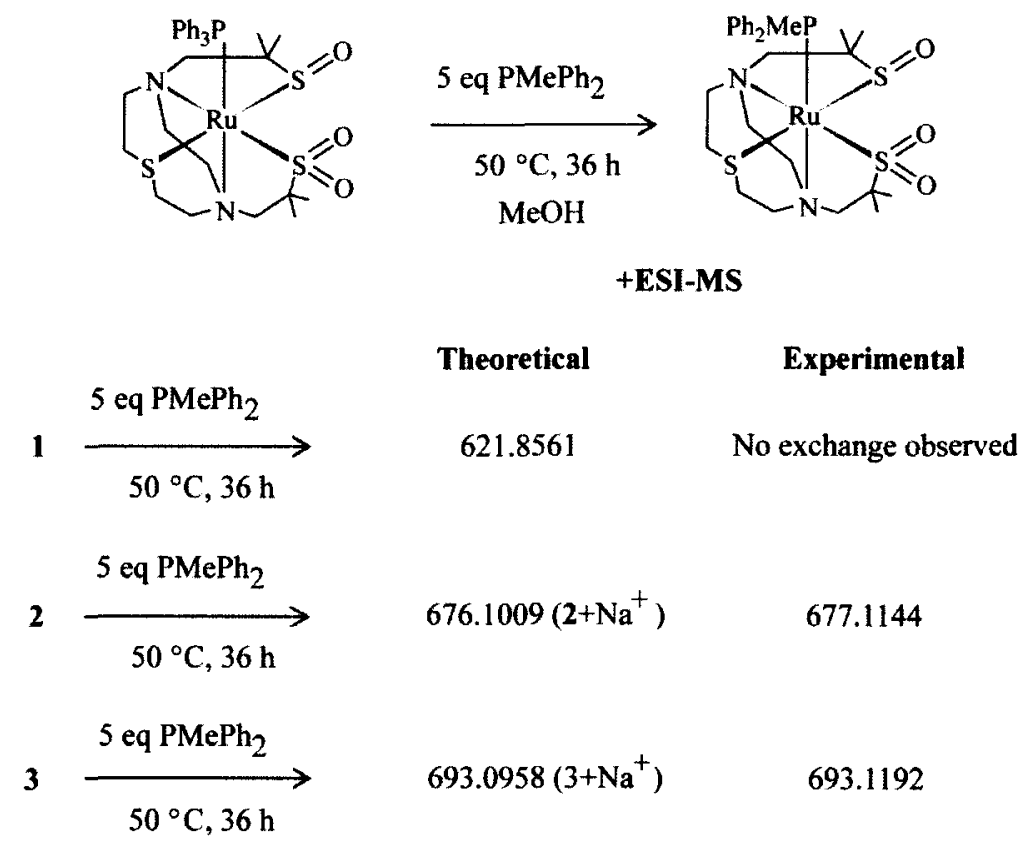

Figure 39. Sulfur-oxygenation increases the lability of $\mathrm{PPh}_{3}$ allowing a series of $\mathrm{S}$ oxygenates to be prepared.

Further investigation in phosphine exchange required the assessment of other phosphines. For this reason, we used the bulky phosphine, methylenebis[ diphenylphosphine]. The +ESI-MS results shows that phosphine exchange was again successful for $\mathbf{2}$ and $\mathbf{3}$, but not for $\mathbf{1}$, Figure 40 and +ESI-MS spectrums in A7 and A8.

The identification of phosphine exchange in the complexes (bmmp- $\mathrm{O}_{2}$ $\mathrm{TASN}) \mathrm{Ru}\left(\mathrm{MePPh}_{2}\right)_{2}$ and $\left(\mathrm{bmmp}-\mathrm{O}_{3}-\mathrm{TASN}\right) \mathrm{Ru}\left(\mathrm{MePPh}_{2}\right)_{2}$ were confirmed by mass spectroscopy. (+)ESI-MS was recorded for each complex after the phosphine exchange reaction. Compound (bmmp- $\left.\mathrm{O}_{2}-\mathrm{TASN}\right) \mathrm{Ru}\left(\mathrm{MePPh}_{2}\right)_{2}$ displays a parent peak at $\mathrm{m} / \mathrm{z}$ 839.1629 , Figure A7, consistent with a theoretical value of $838.1553 \mathrm{~m} / \mathrm{z}$ for [(bmmp- $\mathrm{O}_{2}$ TASN)Ru(MePPh$\left.)_{2}\right]^{+}$. The isotopical distribution shows and accuracy of $1.0076 \mathrm{~m} / \mathrm{z}$. Compound (bmmp- $\left.\mathrm{O}_{3}-\mathrm{TASN}\right) \mathrm{Ru}\left(\mathrm{MePPh}_{2}\right)_{2}$ displays a parent peak at $\mathrm{m} / \mathrm{z}$ 855.1582, 
Figure A8, consistent with a theoretical value of $854.1502 \mathrm{~m} / \mathrm{z}$ for $\left[\left(\mathrm{bmmp}-\mathrm{O}_{3}\right.\right.$ TASN)Ru(MePPh $\left.)_{2}\right]^{+}$. The isotopical distribution shows and accuracy of $1.0008 \mathrm{~m} / z$.
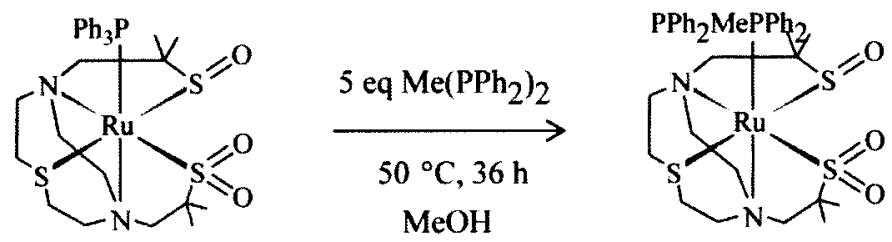

+ESI-MS

Theoretical

Experimental
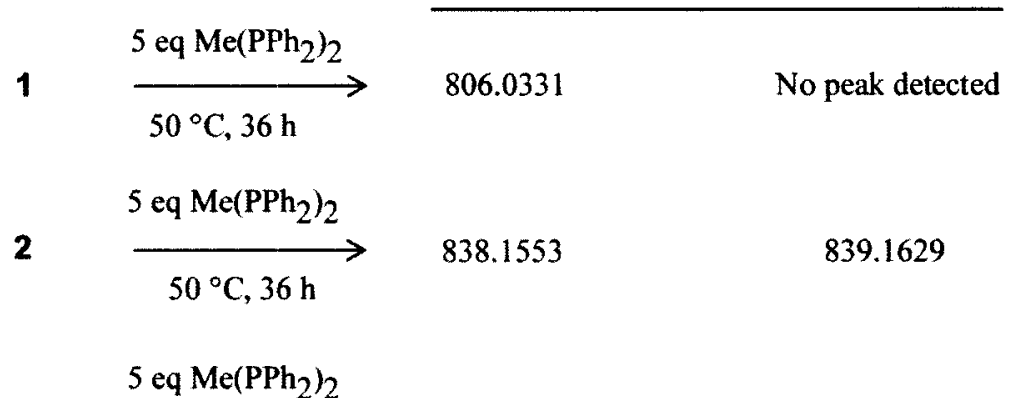

838.1553

839.1629

3

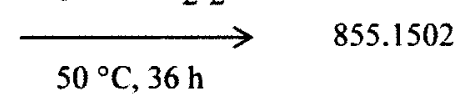

855.1582

Figure 40. Triphenylphosphine exchange using methylenebis[diphenylphosphine]

To achieve the triphenylphosphine exchange in $\mathbf{1}$ extensive heating was applied. Compound 1 has the smallest Ru-P bond distance in the sulfur oxygenated series. Complex 1 was heated to $100{ }^{\circ} \mathrm{C}$ in the presence of 5 equivalents of methyldiphenylphosphine in chlorobenzene. The mixture was heated at $100{ }^{\circ} \mathrm{C}$ for 36 hours under an argon atmosphere. After this period of time the chlorobenzene was removed under a vacuum at room temperature. The yellow-amber solid was transferred to a glovebox. 
Phosphine exchange experiments were performed using ${ }^{31} \mathrm{P}$ NMR in deuterated methanol. The ${ }^{31} \mathrm{P}$ NMR spectrum shows free triphenylphosphine at $-6.23 \mathrm{ppm}$, excess methyldiphenylphosphine at $-27.83 \mathrm{ppm}$ and the exchange product (bmmpTASN)RuPMePh $34.64 \mathrm{ppm}$. Similar experiments over a temperature range of $50{ }^{\circ} \mathrm{C}$ and $100{ }^{\circ} \mathrm{C}$ indicates that the phosphine exchange is temperature dependent. The dissociation of the ligand at moderate temperatures allows the exchange of phosphine, while the product are stable with respect to phosphine or solvent exchange at $25^{\circ} \mathrm{C}$. This is confirmed by electrochemical studies at room temperature. Both cyclic voltammetry and square wave voltammetry reveals a single oxidation-reduction event in compounds $\mathbf{1}$, 2 and 3. As we can observe in the $\mathrm{CV}$ of the (bmmp-TASN) $\mathrm{RuPPh}_{3}$ and derivatives, Figures A2-A4.

The ${ }^{31} \mathrm{P}$ NMR spectrum for $\mathbf{1}, \mathbf{2}$ and $\mathbf{3}$ and free triphenylphosphine is presented in Figure 41 . The free triphenylphosphine displays a peak at $-6.23 \mathrm{ppm}$. The ${ }^{31} \mathrm{P}$ NMR experiment was performed in an air free J-young NMR tube using degassed deuterated methanol at $50{ }^{\circ} \mathrm{C}$ after 1024 repetitions to observe the peaks in the NMR due to the low solubility of the complex in methanol. The results of ${ }^{31} \mathrm{P}$ NMR experiments show a tendency with, a ruthenium dithiolate (bmmp-TASN) $\mathrm{RuPPh}_{3}$ at $43.37 \mathrm{ppm}$, a ruthenium thiolate/sulfinate (bmmp- $\mathrm{O}_{2}-\mathrm{TASN} \mathrm{RuPPh}_{3}$ at $34.343 \mathrm{ppm}$ and a ruthenium sulfinate/sulfenate (bmmp- $\left.\mathrm{O}_{2}-\mathrm{TASN}\right) \mathrm{RuPPh}_{3}$ at $33.68 \mathrm{ppm}$. 


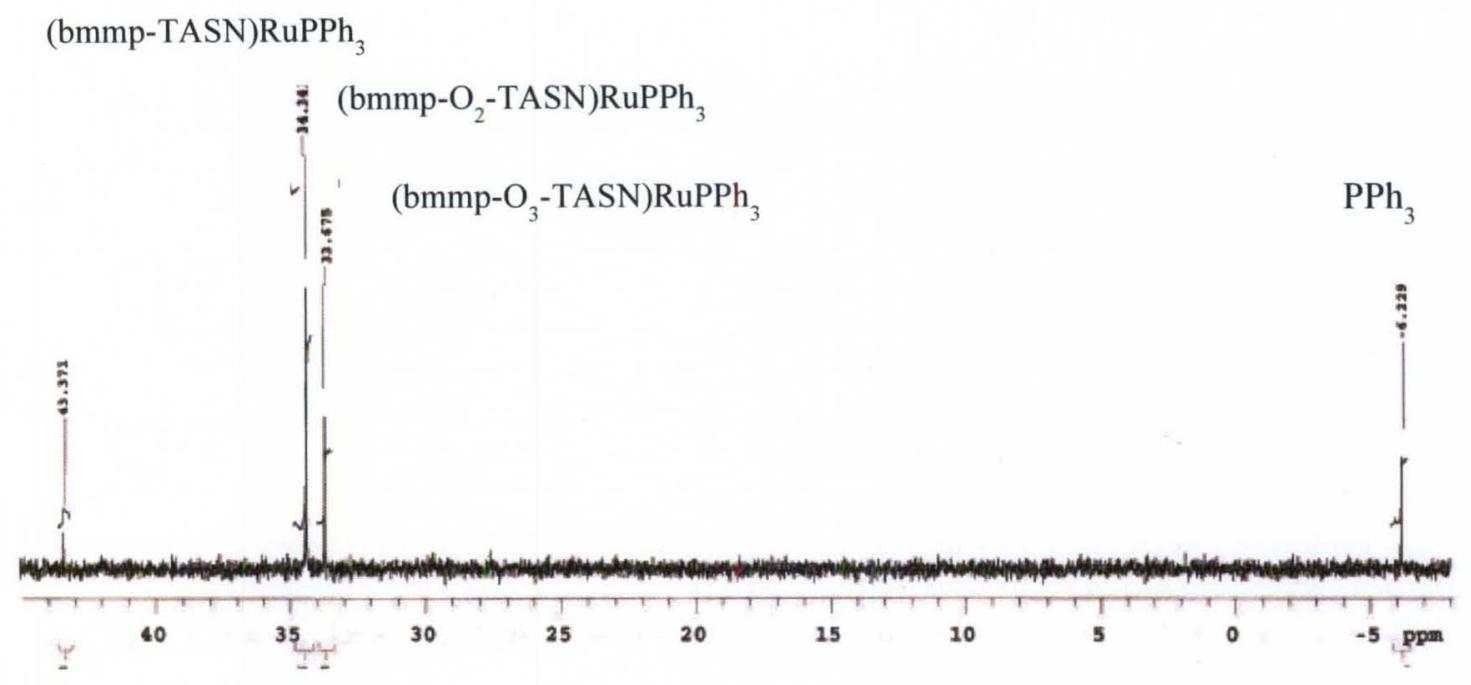

Figure 41. ${ }^{31} \mathrm{P}$ NMR of free triphenylphosphine and compounds $\mathbf{1 , 2}$ and 3 at $50{ }^{\circ} \mathrm{C}$.

To a mixture of $\mathbf{1 , 2}$ and $\mathbf{3}$ in chlorobenze were added $\mathrm{PMePh}_{2}$. The solution was stirred for 36 hours at $100{ }^{\circ} \mathrm{C}$ and the solvent was removed via vacuum under air free conditions. The residue was dissolved in deuterated methanol in a J-young tube and the ${ }^{31} \mathrm{P}$ NMR spectrum was collected. The results show excess $\mathrm{PMePh}_{2}$ at $-27.83 \mathrm{ppm}$, the ruthenium dithiolate exchange product (bmmp-TASN)RuPMePh 2 at $34.64 \mathrm{ppm}$, the ruthenium thiolate/sulfinate exchange product (bmmp- $\left.\mathrm{O}_{2}-\mathrm{TASN}\right) \mathrm{RuPMePh}$ at 25.54 $\mathrm{ppm}$, and the ruthenium sulfinate/sulfenate exchange product (bmmp- $\mathrm{O}_{2}$ TASN)RuPMePh 2 at $22.54 \mathrm{ppm}$. The free $\mathrm{PPh}_{3}$ in this experiment is observed at -6.23 ppm. For each complex the ${ }^{31} \mathrm{P}$ resonance is shifted by $-0.488 \mathrm{ppm}$ with respect to the $\mathrm{PPh}_{3}$ precursor, Table 2 . 


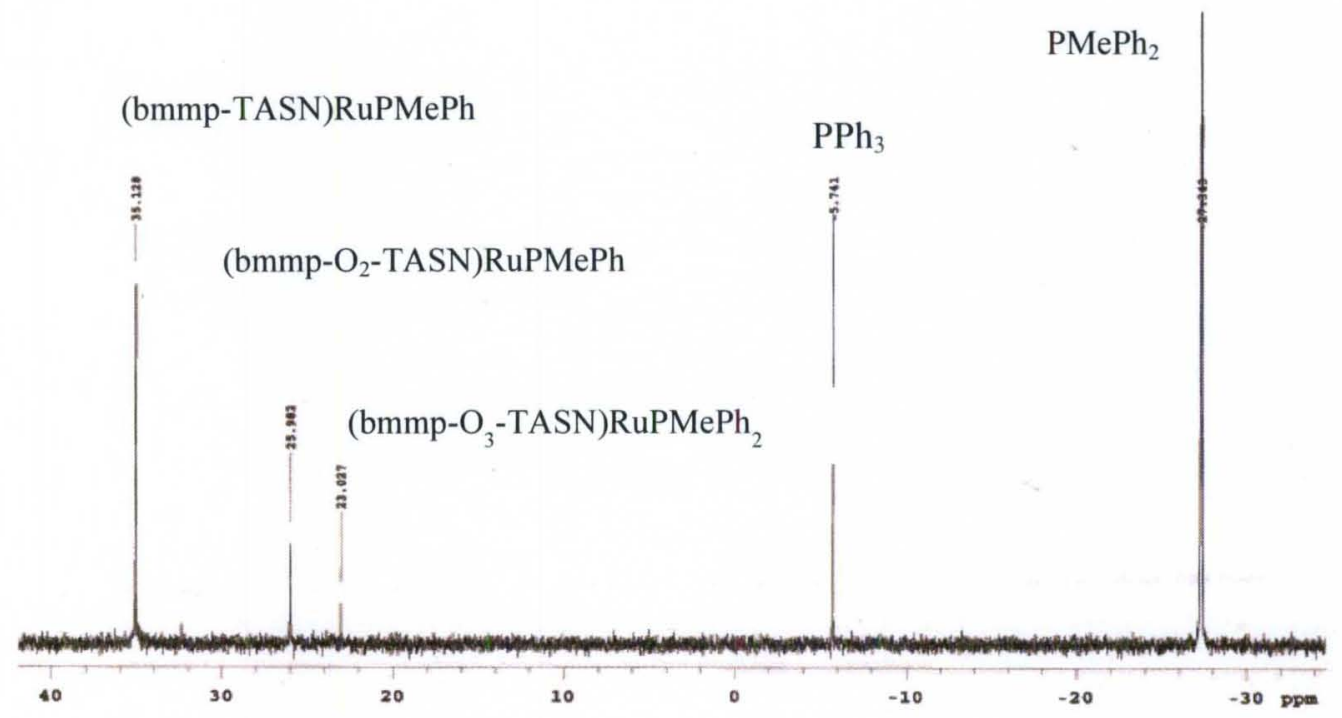

Figure 42. ${ }^{31} \mathrm{P}$ NMR of free triphenylphosphine $\mathrm{PMePh}_{2}$ and compounds 7, 8 and 9 at $50{ }^{\circ} \mathrm{C}$.

$\mathrm{PPh}_{3}$

Free $\mathrm{PPh}_{3} \quad-6.229$

Free $\mathrm{PMePh}_{2}$

Thiolate

$\mathrm{SO} 2$

$\mathrm{SO} 3$
33.675
$\mathrm{PMePh}_{2}$

22.539

Table 2. ${ }^{31} \mathrm{P}$ NMR assignments for Figures 37 with the use of $\mathrm{PPh}_{3}$ and for Figure 38 with the use of $\mathrm{PMePh}_{2}{ }^{31} \mathrm{P} \mathrm{NMR}$ values were internally referenced to free $\mathrm{PPh}_{3}$.

X-ray structure analyses of (bmmp-O $-\mathrm{TASN}_{2} \mathrm{RuPMePh}_{2} \quad(8), \quad\left(\mathrm{bmmp}-\mathrm{O}_{3}-\right.$ TASN)RuPMePh $(9)$, their analogues precursors and $[(\mathrm{bmmp}-\mathrm{TASN}) \mathrm{Ru}]_{2}(10)$

The phosphine exchange studies resulted in the syntheses of new ruthenium complexes with the [(bmmp-TASN)Ru] framework. Of these, two new compounds were structurally characterized by X-ray diffraction studies; (bmmp- $\left.\mathrm{O}_{2}-\mathrm{TASN}\right) \mathrm{RuPMePh} \mathrm{Ph}_{2} \mathbf{8}$ 
and (bmmp- $\left.\mathrm{O}_{3}-\mathrm{TASN}\right) \mathrm{RuPMePh}$ 9. X-ray quality crystals of 7 were not obtained. The molecular structures of $\mathbf{8}$ and $\mathbf{9}$ were elucidated by X-ray structure determination. Complex 8 crystalize in a triclinic space group P-1; with $\mathrm{a}=9.7483(3) \AA$, $\mathrm{b}=14.4088(5)$ $\AA, \mathrm{c}=24.8048(7) \AA ; \alpha=75.203(3)^{\circ}, \beta=80.610(3)^{\circ}, \gamma=81.507(3)^{\circ} ; Z=2 ; R 1 / w R 2=$ 0.0532/0.1279. Complex 9 crystalize in a triclinic space group P-1; a = 8.7767(3) $\AA, \mathrm{b}=$ $15.8615(5) \AA, c=21.4533(7) \AA ; \alpha=89.823(3)^{\circ}, \beta=79.435(3)^{\circ}, \gamma=76.449(3)^{\circ} ; Z=4$; $R 1 / w R 2=0.0289 / 0.0584$. Compound 8 and $\mathbf{9}$ exhibit pseudooctahedral six-coordinated $\mathrm{Ru}(\mathrm{II})$ centers.

The molecular structure of $\mathbf{8}$ with selected distances is shown in Figure 43 and Table 3. The ruthenium center is in a pseudo octahedral environment. As in the precursor complex 2, compound $\mathbf{8}$ displays a facially coordinated TASN ring (N1, N2, and S1), two pendant sulfur donors (S2 and S3), and a single phosphorus donor (P1). The two oxygen atoms, $\mathrm{O} 1$ and $\mathrm{O} 2$, of the sulfinate donor $(\mathrm{S} 2)$ are directed roughly along the S1-Ru-S3 bond axis with torsion angles of $-11.74(13)^{\circ}$ and $+40.42(12)^{\circ}$ for O1-S2-Ru1-S1 and O2S2-Ru1-S3, respectively. 
Table 3. Selected bond lengths determined by X-ray of compound $\mathbf{8}$.

$\begin{array}{lll}\mathrm{Ru} 1-\mathrm{N} 1 & 2.188(3) & \AA \\ \mathrm{Ru} 1-\mathrm{N} 2 & 2.190(3) & \AA \\ \mathrm{Ru} 1-\mathrm{P} 1 & 2.3045(8) & \AA \\ \mathrm{Ru} 1-\mathrm{S} 1 & 2.3163(8) & \AA \\ \mathrm{Ru} 1-\mathrm{S} 2 & 2.2384(8) & \AA \\ \mathrm{Ru} 1-\mathrm{S} 3 & 2.3940(8) & \AA \\ \mathrm{S} 2-\mathrm{O} 1 & 1.478(3) & \AA \\ \mathrm{S} 2-\mathrm{O} 2 & 1.485(3) & \AA\end{array}$

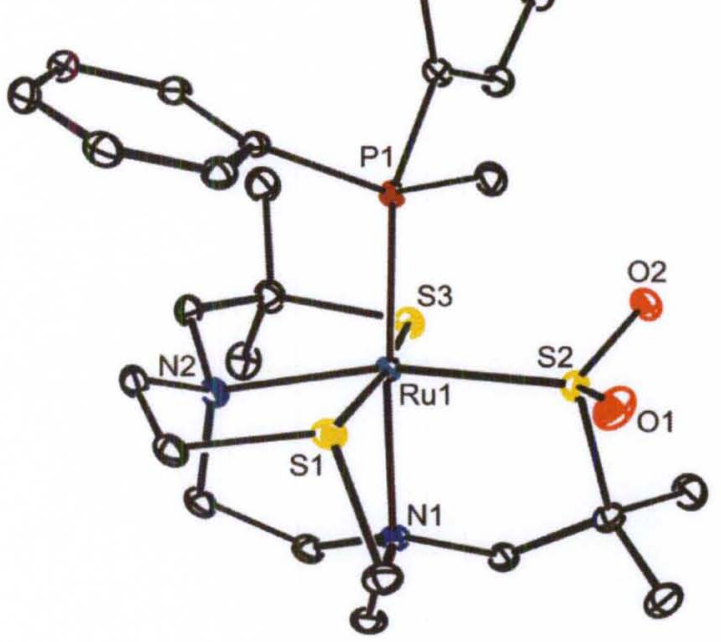

Figure 43. ORTEP representation of (bmmp- $\left.\mathrm{O}_{2}-\mathrm{TASN}\right) \mathrm{RuPMePh}(\mathbf{8})$.

The yellow plate crystals of $\mathbf{9}$, Figure 44 , are similar to $\mathbf{1}, \mathbf{2}, \mathbf{3}$ and $\mathbf{8}$ with a facially coordinated TASN ring (N1, N2, and S1), two pendant sulfur donors (S2 and S3), and a single phosphine (P1). The two oxygen atoms $\mathrm{O} 1$ and $\mathrm{O} 2$ of the sulfinate donor (S2) are directed roughly along the S1-Ru-S3 bond axis with torsion angles of $-12.31(6)^{\circ}$ and $+35.82(6)^{\circ}$ for $\mathrm{O} 1-\mathrm{S} 2-\mathrm{Ru} 1-\mathrm{S} 1$ and $\mathrm{O} 2-\mathrm{S} 2-\mathrm{Ru} 1-\mathrm{S} 3$, respectively. The sulfenate oxygen (O3) is oriented toward N1 along the P1-Ru-N1 axis with an O3-S3-Ru1-N1 torsion angle of $-16.46(8)^{\circ}$. These result clearly show that the formation of S3-O3 bond in compound 9 release a structural stress in the penta heteroatom member ring Ru1-N2C9-C10-S3. The loss of a $\pi$ coordination orbital between a d-Ru and a p-S allows a decrease in the torsion angle O2-S2-Ru1-S3 from $+40.42(12)^{\circ}$ in $\mathbf{8}$ to $+35.82(6)^{\circ}$ in $\mathbf{9}$. The loss of $\pi$-donating ability induced by the S-oxygenation increases the metal-ligand bond distances with traditional $\pi$-acceptors. The Ru-P1 bond distance consistently increases from $2.3045(8)$ to $2.3276(4) \AA$ for $\mathbf{8}$, and 9, respectively. The same trend is 
noted in the Ru-S1 thioether bond distances from $2.3163(8)$ to $2.3508(4) \AA$ for $\mathbf{8}$, and $\mathbf{9}$, respectively.

Table 4. Selected bond lengths determined by x-ray of compound 9 .

$\begin{array}{lll}\text { Ru1-N1 } & 2.1746(13) & \AA \\ \text { Ru1-N2 } & 2.1852(14) & \AA \\ \text { Ru1-P1 } & 2.3276(4) & \AA \\ \text { Ru1-S1 } & 2.3508(4) & \AA \\ \text { Ru1-S2 } & 2.2562(4) & \AA \\ \text { Ru1-S3 } & 2.3532(4) & \AA \\ \text { S2-O1 } & 1.4862(12) & \AA \\ \text { S2-O2 } & 1.4767(12) & \AA \\ \text { S3-O3 } & 1.5197(14) & \AA\end{array}$

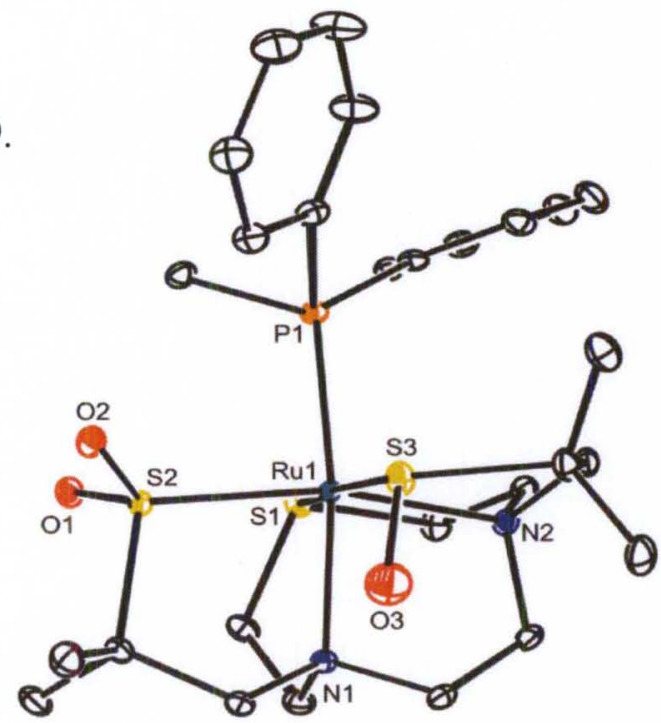

Figure 44. ORTEP representation of (bmmp-O $\left.{ }_{3}-\mathrm{TASN}\right) \mathrm{RuPMePh}_{2}(\mathbf{9})$.

The structural comparison between the two series of phosphine sulfur oxygenated show that the methyldiphenylphosphine is a better base than triphenylphosphine due to the increase in electronic donation from the methyl group into the $\sigma$ orbital between the Ru-P. This $\sigma$ donation effect can be observed through the reduction in the bond distance between Ru1-P1. For compound $\mathbf{2}$ and $\mathbf{8}$, these bond distance are 2.3519(6) $\AA$ and $2.3045(8) \AA$ respectively, this make a $0.0474 \AA$ of difference; for compound 3 and 9 the bond distance are $2.3790(9) \AA$ and $2.3276(4) \AA$ respectively, this make a $0.0514 \AA$ of difference. This approximately $0.05 \AA$ units bond distance effect can be explained as result of small cone angle between the $\mathrm{PPh}_{3}$ with a cone angle of $145^{\circ}$ and $\mathrm{PMePh}_{2}$ with a cone angle of $136^{\circ}$ this reduction of volume allow a better accessibility into the axial pocket of the metal center. Further investigation has to be performed to clearly determine if this bond reduction is caused by an electronic or steric effect or a combination of both. 
The ORTEP representations of $\mathbf{3}$ and $\mathbf{9}$ are shown in Figure 32 and 44 respectively. Selected distances are listed in Table 1 and 4. The comparison of the two structures indicates a zero net effect in the bond distance of the ligand frame due to exchange of the triphenylphosphine for a better nucleophile such as methyl diphenylphosphine. This result suggests that a Ru1-P1 bond distance dependent of the steric effect or cone angle of the substrate. The average Ru-S $2.390 \AA$ and Ru-N $2.192 \AA$ distances are statistically the same as those in Ru1-N2 2.192(3) $\AA$ and 2.1852(14) $\AA$ for 3 and 9 respectively. For the Ru1-N1 bond trans to the phosphine those values are 2.178(3) $\AA$ and $2.1746(13) \AA$ for 3 and 9 respectively. In Ru1-S2 the bond distances are 2.2548(9) $\AA$ and 2.2562(4) $\AA$ for 3 and 9 respectively, and for Ru1-S3 2.3493(9) $\AA$ and 2.3532(4) $\AA$ are 3 and 9 respectively. The direct effect on the ligand frame due to the exchange of the triphenylphosphine is observed in Ru-S1 (thioether) with 2.3622(9) $\AA$ and 2.3508(4) $\AA$ for 3 and 9.

In addition to the phosphine exchange compounds $\mathbf{8}$ and $\mathbf{9}$, the phosphine free bimetallic complex $[(\mathrm{bmmp}-\mathrm{TASN}) \mathrm{Ru}]_{2}(\mathbf{1 0})$ was structurally characterized, Figure 45. Complex $\mathbf{1 0}$ was obtained upon slow crystallization of compound $\mathbf{1}$ through solvent diffusion of methanol-ether under air-free condition for a period of one month. Repeated attempts to reproduce $\mathbf{1 0}$ were unsuccessful. 


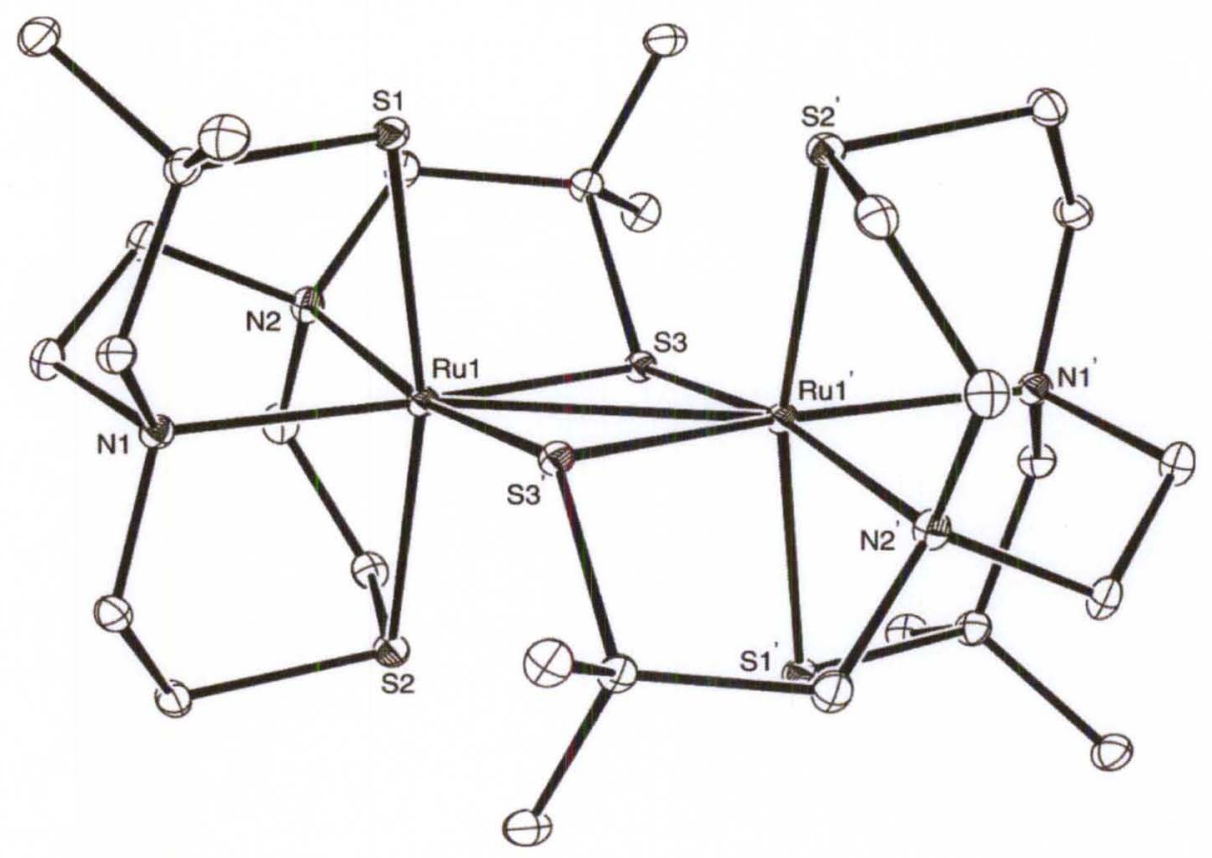

Figure 45. Bimetallic complex $[(\mathrm{bmmp}-\mathrm{TASN}) \mathrm{Ru}]_{2}(\mathbf{1 0})$.

\section{Reactivity of (bmmp-TASN)RuPPh ${ }_{3}(11)$ with CO}

The exchange properties of the compounds $\mathbf{1}, \mathbf{2}$ and $\mathbf{3}$ were confirmed by the replacing of the triphenylphosphine with other phosphines. It was of interest to the Grapperhaus group to evaluate strategic routes to remove the phosphine donors all together to promote substrate binding. If obtained transition metal complexes with sulfur and nitrogen coordination spheres similar to the NHase or SCNase could be realized. As noted previously complexes which model structural and functional features of these active sites are of interest for biochemistry, spectroscopic and technological reasons. The relevant post-translational modification of the cysteines in these enzymes was systematically reproduced in the (bmmp-TASN) $\mathrm{RuPPh}_{3}$ model. In this section efforts to prepare and isolate (bmmp-TASN)RuCO (11) are described. The CO derivative represents an alternative to synthesis of analogue derivatives after the remotion of $\mathrm{CO}$ thermally, photochemically or by chemical oxidation. Once the $\mathrm{CO}$ is removed from the 
metal center, it will be of special interest the synthesis of nitrile, amide, nitric oxide and $\mathrm{H}_{2} \mathrm{O}$ derivatives.

The thiolate complex 1 was treated with $\mathrm{CO}$ for 20 minutes and then stirred for 20 hours at $70{ }^{\circ} \mathrm{C}$ in degassed methanol to yield (bmmp-TASN)RuCO derivative. Interestingly, the bmmp-TASN ligand has rearranged in this complex to generate an $\mathrm{N}_{2} \mathrm{~S}_{2}$ donor plane with a single $\mathrm{S}$ (thioether) donor trans to the variable coordination site alike to the NHase and SCNase active sites, Figure 46 and 47.

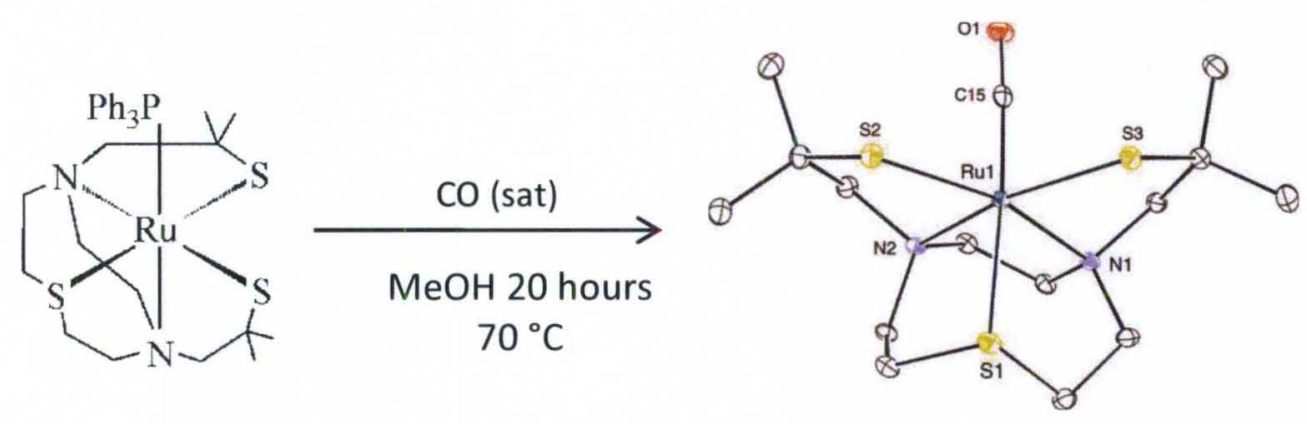

Figure 46. Synthesis of (bmmp-TASN)RuCO and ligand frame rearrangement. 


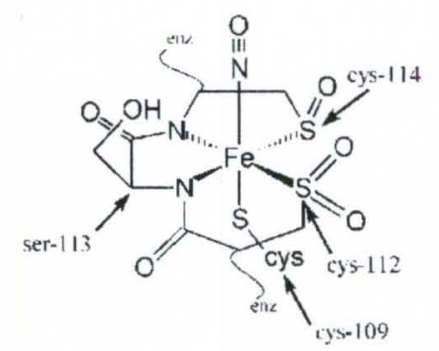

(bmmp-TASN)RuCO has the same $\mathrm{N}_{2} \mathrm{~S}_{3}$ donor arrangement as NHase

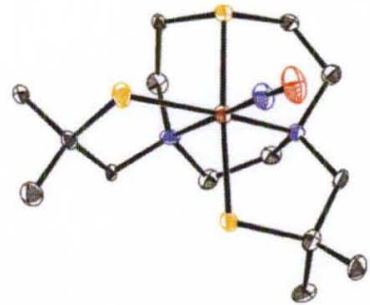

$$
\begin{aligned}
& \text { [(bmmp-TASN)FeNO }]^{+} \text {has } \\
& \text { the typical ligand } \\
& \text { arrangement of our ligand }
\end{aligned}
$$

Figure 47. NHase enzyme and [(bmmp-TASN)FeNO $]^{+}$analogue of 11.

The CO-bound derivative of $\mathbf{1}$ is room temperature stable, making it easy to isolate as a yellow solid. The UV visible spectrum of $\mathbf{1 1}$ shown in Figure 48 is dominated by a band at $331 \mathrm{~nm}$. As the solution is compared with is precursor analogue 1 , the band at $438 \mathrm{~nm}$ is absent. The identity of the product $\mathbf{1 1}$ as a ruthenium-bound $\mathrm{CO}$ species is supported by the appearance in IR of an intense band at $1888 \mathrm{~cm}^{-}{ }^{1}$, Figure 49. A band in this region is characteristic of $\mathrm{Ru}(\mathrm{II})-\mathrm{CO}$ species. ${ }^{147,148}$ The $v_{\mathrm{CO}}$ stretching frequency $1888 \mathrm{~cm}^{-1}$ indicate strong $\pi$-back-bonding, as compared to free $\operatorname{CO} 2155 \mathrm{~cm}^{-1}$. The two thiolates stabilize the $\pi$-interaction between the ruthenium(II) center and $\mathrm{CO}$. 


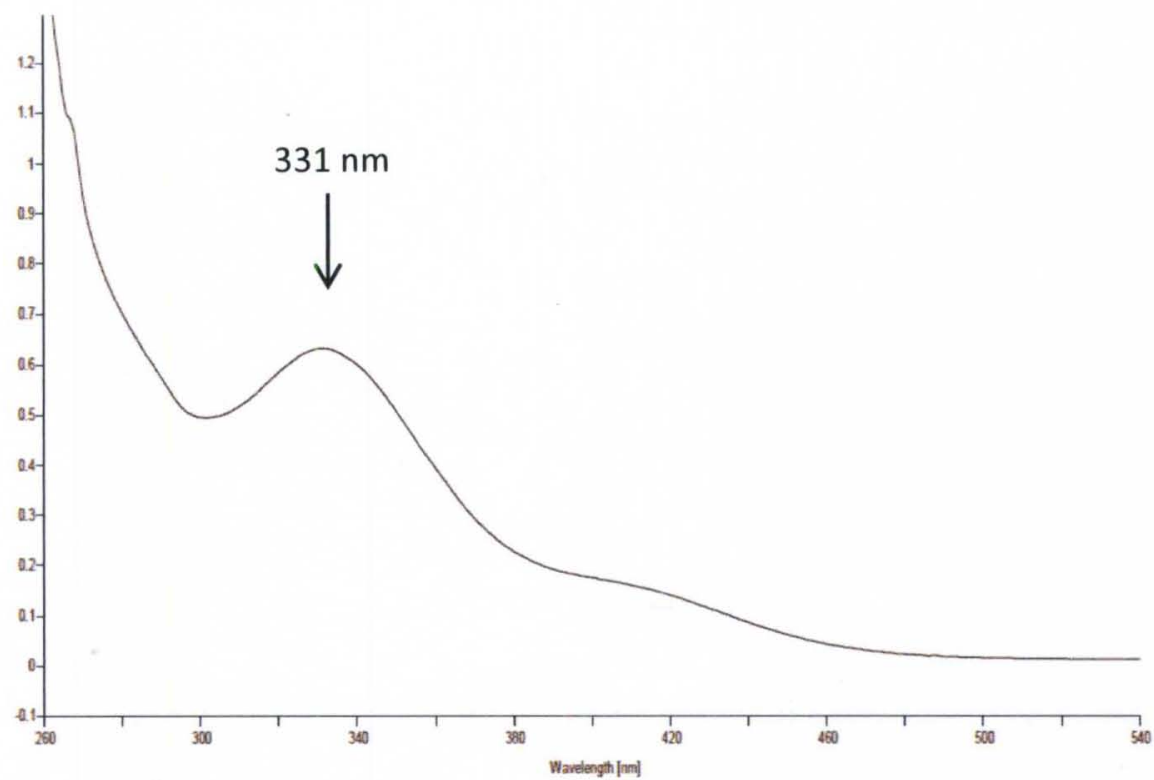

Figure 48. UV visible of (bmmp-TASN)RuCO.

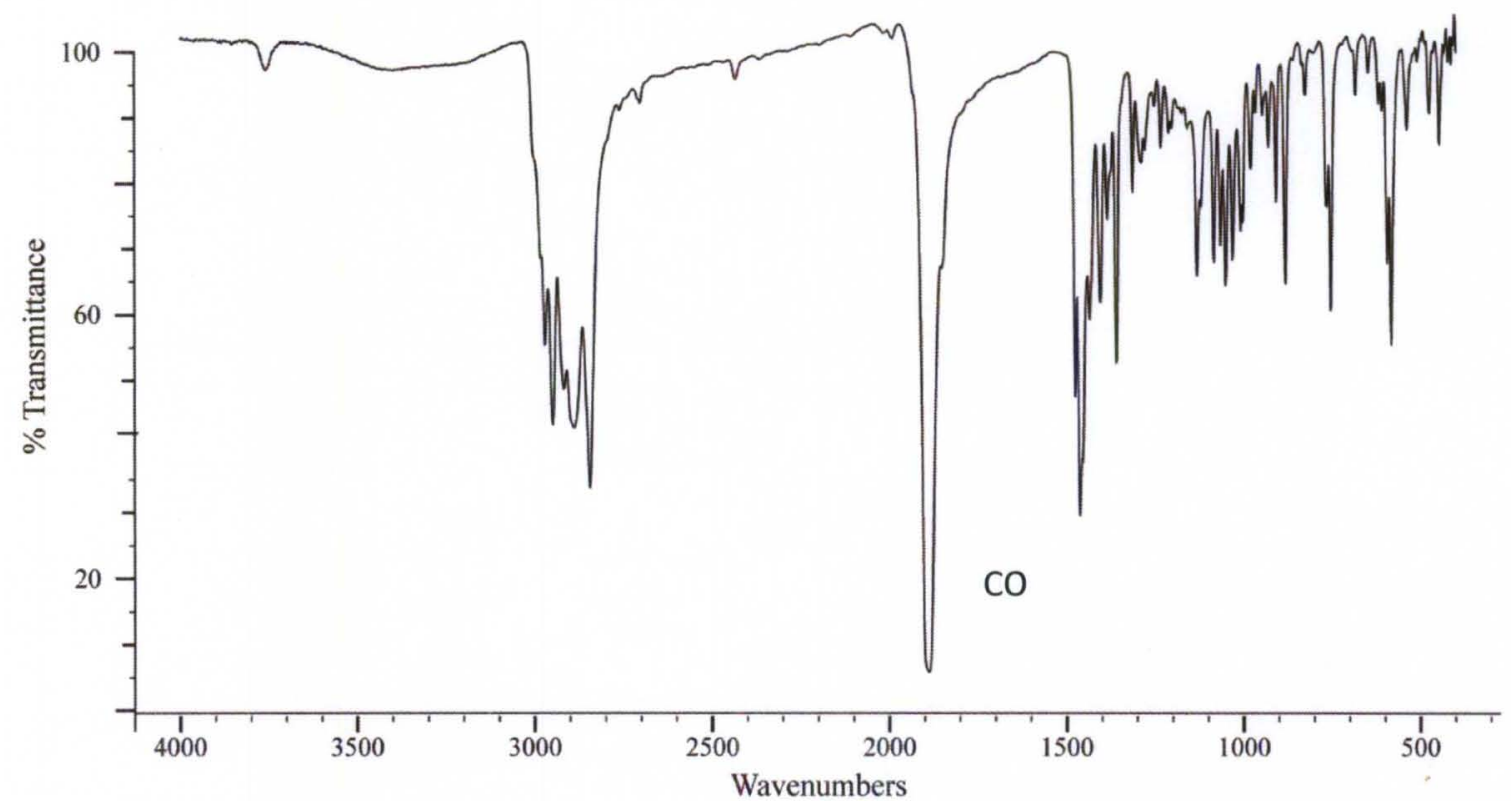

Figure 49. FT-IR of (bmmp-TASN)RuCO.

The displacement reaction and rearrangement of the compound (bmmpTASN)RuCO can be explained for two different routes. The first, Scheme 6 , it is a 
dissociative mechanism in which $\mathrm{PPh}_{3}$ is displaced making a pentacoordinate or methanol coordinate complex. Binding of the $\mathrm{CO}$ causes a structural rearrangement of the ligand frame by electronic effect. The second route, Scheme 7, is an associative or interchange mechanism. The coordination of $\mathrm{CO}$ to the octahedral complex initiates displacement and elimination of the $\mathrm{PPh}_{3}$ with structural rearrangement of the complex.
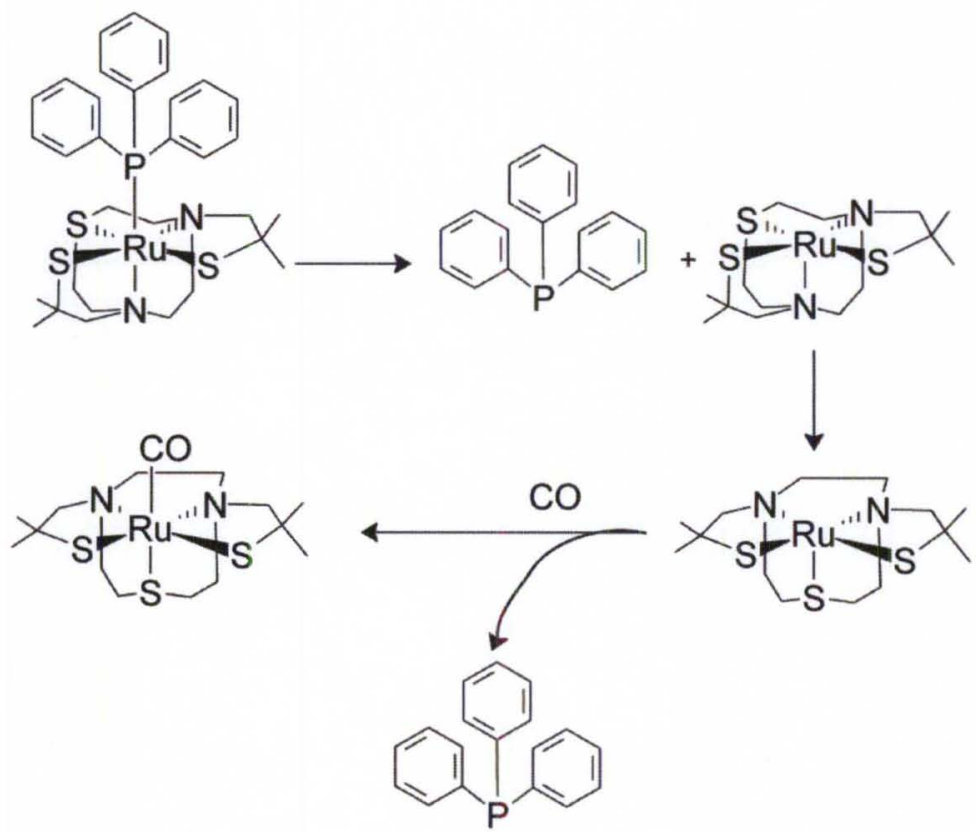

Scheme 6. Dissociative mechanism for the formation of compound 11.
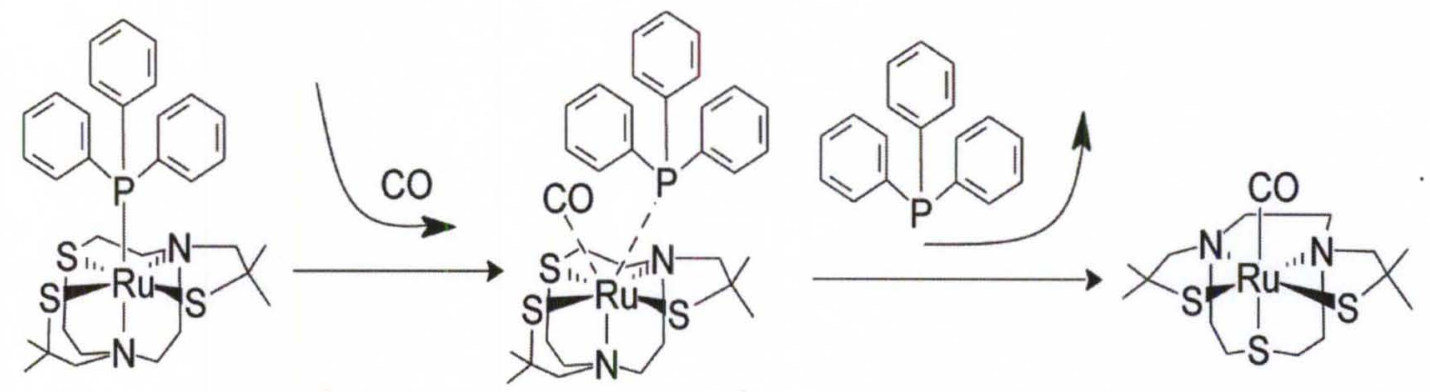

Scheme 7. Associative or interchange mechanism for the formation of compound $\mathbf{1 1 .}$ 
It is expected that the differences in the overall ligand field strength will weaken $\mathrm{CO}$ coordination upon sulfur oxygenation. We expect to observe the weakening of the CO through X-ray crystallography and the IR stretches shift around $1900-1940 \mathrm{~cm}^{-1}$. The complexes will be investigated by Davinder Kumar.

\section{Structural description of the (bmmp-TASN)RuCO (11)}

Yellow plate crystals of $\mathbf{1 1}$ in the space group P 1 21/n 1 were obtained upon slow evaporation of methanol solutions of the complex under air free conditions. The final unit cell parameters: $\mathrm{a}=9.2404(2) \AA . \mathrm{b}=20.7973(4) \AA \mathrm{c}=10.6722(3) \AA, \alpha=90^{\circ}, \beta=$ $94.916(2)^{\circ}, \gamma=90^{\circ} \mathrm{V}=2043.40(8) \AA^{3}, D_{\text {calc }}=1.553 \mathrm{Mg} / \mathrm{m}^{3}, \mathrm{Z}=4$. Complex 11 contains a six-coordinate ruthenium(II) ion in an $\mathrm{N}_{2} \mathrm{~S}_{3} \mathrm{C}$ donor set with one thioether (S1), two thiolate (S2 and S3), sulfur donor, Figure 50. The TASN ligand backbone (N1, N2, S1) occupies one face of a distorted octahedron. The three sulfur donors occupy other face of the octahedron. The thiolate sulfur S2 and S3 are trans to the nitrogen N1 and N2 respectively. The thioether $\mathrm{S} 1$ is trans to the $\mathrm{C} 15$ of the $\mathrm{CO}$.

The $v_{\mathrm{CO}}$ stretch of $\mathbf{1 1}$ suggests that there is significant $\pi$ back-donation to the $\mathrm{CO}$ ligand from the $\mathrm{Ru}$, reflecting the strong electron donating properties of the thiolate ligands and the effect of the thioether trans to the CO. This $\pi$ back donation is reflected in the small reduction in the bond distance between the metal center and atoms of the equatorial plane $\mathrm{N}_{2} \mathrm{~S}_{2}$. As we observe in the Figure 50 compound $\mathbf{1 1}$ has a distorted octahedral configuration with the two thiolate S2 and S3 ligands in cis configuration and the thioether trans to the carbon monoxide ligand, This arrangement of atoms is altered from the analogue precursor compound $\mathbf{1}$. 


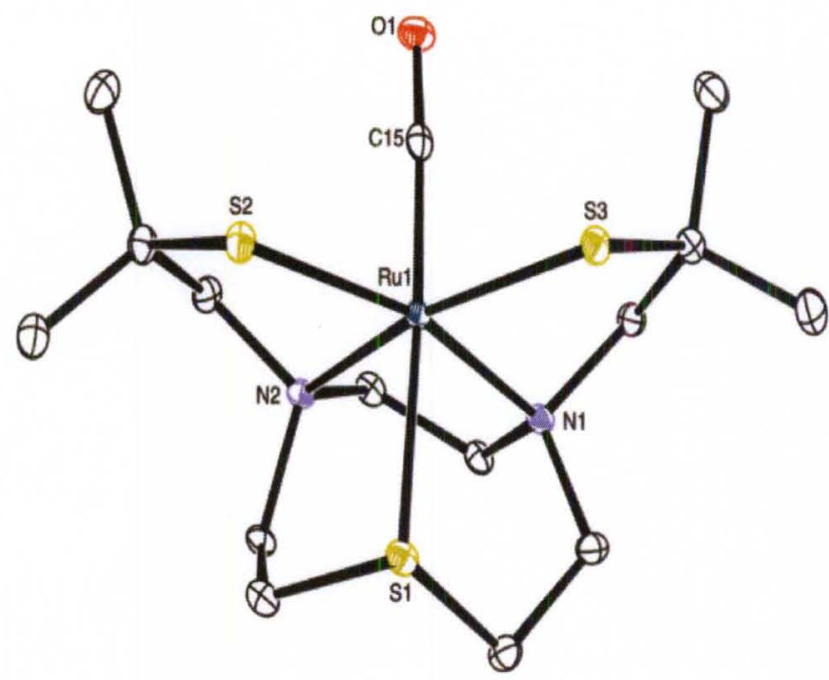

Table 5. Selected bond lengths determined by x-ray of compound 11 .

C15-O1 1.158(3) $\AA$ Ru1-C15 1.822(2) $\AA$ Ru1-N1 2.1354(19) $\AA$ Ru1-N2 2.1215(19) $\AA$ Ru1-S1 2.4379(6) $\AA$ Ru1-S2 2.3854(6) $\AA$ Ru1-S3 2.3854(6) $\AA$

Figure 50. ORTEP representation of (bmmp-TASN)RuCO (11).

The bond distances presented in Table 5 indicate an almost symmetric bond distance in the square planar $\mathrm{N}_{2} \mathrm{~S}_{2}$. The ligand rearrangement that is observed in the complex (bmmp-TASN)RuCO is proposed in the reactivity cycle upon nitrile coordination. The previous reported coordination of nitrile, water, and amides of [(bmmp-TASN)Fe]OTf suggest similar binding may occur for the (1-4) upon $\mathrm{PPh}_{3}$ dissociation. This supposition is supported by the catalytic activity and +ESI-MS of the product of the catalysis as it will described in the next section.

\section{Hydrolysis studies of 1,2 and 3.}

Hydrolysis activity was investigated through the evaluation of a series of substrate candidates to test the activity of the family of S-oxygenated $\mathbf{1 , 2}$ and $\mathbf{3}$. The substrates investigated include $p$-nitrophenylactetate, which was followed by UV-visible, acetonitrile, acrylonitrile and benzonitrile with the exception of the ester all reaction were followed by ${ }^{1} \mathrm{H}$ NMR, FT- IR and GC-MS. The results support that the phosphine lability 
at elevated temperatures is required for hydrolysis activity. At this time, it remains unclear if the coordinated substrate is water or nitrile.

\section{Hydrolysis of the p-nitrophenylacetate}

We began our hydrolysis studies with the $p$-nitrophenylactetate. This ester is excellent candidates for hydrolysis test since it is relatively easy hydrolyze. The concept being that if the ruthenium complexes cannot hydrolyze the ester, it will be less probable to hydrolyze a nitrile. The use of this ester provides us with a versatile strategy to screen our complex in a short time frame and with the use of a minimal amount of complex in each trial. This hydrolysis trial has the advantage that use tractable compound in UVVisible, the $p$-nitrophenylactetate and its product of hydrolysis the $p$-nitrophenol. The absorption bands for both structures are well resolved and significantly separated, the $p$ nitrophenyl acetate compound has a band at $276 \mathrm{~nm}, p$-nitrophenolate at $317 \mathrm{~nm}$ and $p$ nitrophenol at $405 \mathrm{~nm}$.

The initial studies involve the test of hydrolysis activity at low temperature; a mixture of $1(5 \mathrm{mg}, 7 \mu \mathrm{mol})$ and 4-nitrophenyl acetate $(16 \mathrm{mg}, 0.088 \mathrm{mmol})$ in $\mathrm{MeOH} / \mathrm{H}_{2} \mathrm{O}(8.2 \mathrm{~mL} / 8.2 \mathrm{~mL})$ and citrate buffer $(1 \mathrm{M}, \mathrm{pH}=4.8,1.8 \mathrm{~mL})$ was stirred at $4{ }^{\circ} \mathrm{C}$ and at room temperature for 5 days. The reaction was monitored by UV-visible spectroscopy, and results were compared to a control sample with no added metal complex. The same methodology was applied to solutions containing $\mathbf{2}$ and $\mathbf{3}$. From these studies with the $p$-nitrophenylactetate, we concluded that the hydrolysis reaction at $4{ }^{\circ} \mathrm{C}$ and at room temperature show not reactivity for the three compounds, indicating the necessity to raise the temperature to release the triphenylphosphine and expose the metal center to the substrate. 
The next hydrolysis reactions were performed at $50^{\circ} \mathrm{C}$, following the same basic procedure but with pipes buffer at $\mathrm{pH}=6.75$. The substrate was added to the solution, and the UV-visible absorption spectra were recorded at regular intervals. Hydrolysis of $p$ nitrophenylacetate in the presence of $\mathbf{3}$ is confirmed by the changes in the UV-visible spectrum. Spectra were recorded every 3000 seconds for 10 hours. These results are shown in the figure below.
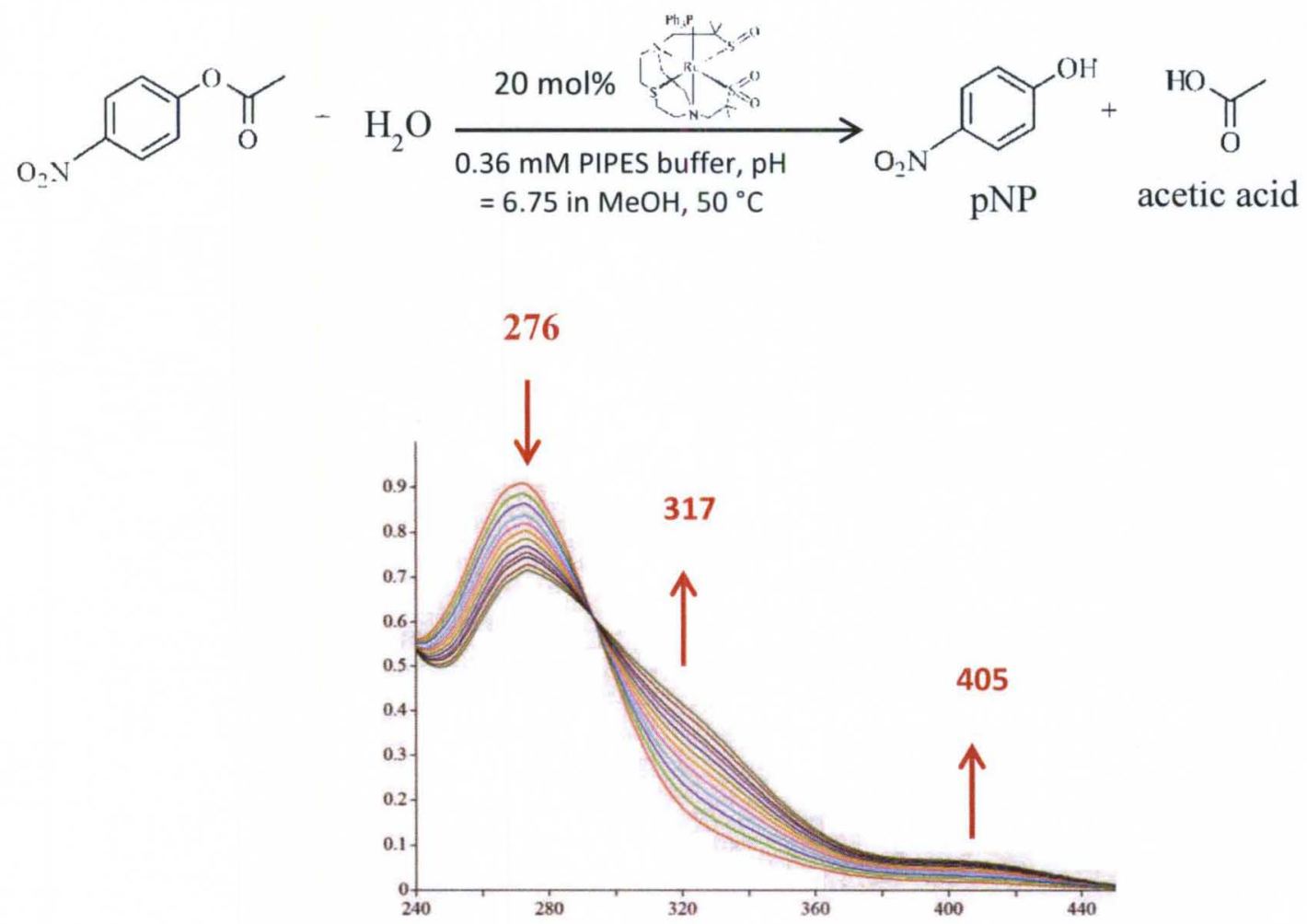

Figure 51. Hydrolysis of p-nitrophenylacetate in the presence of $\mathbf{3}$ is confirmed by the changes in the UV-visible spectrum. Spectra were recorded every $3000 \mathrm{~s}$. Control experiments in the absence of $\mathbf{3}$ do not show hydrolysis.

As it can be observed from the figure 51 , the $p$-nitrophenylacetate at $276 \mathrm{~nm}$ is converted to $p$-nitrophenolate at $317 \mathrm{~nm}$ and p-nitrophenol at $405 \mathrm{~nm}$. The presence of an isosbestic point at $292 \mathrm{~nm}$ suggests that the substrate is being hydrolyzed to the product 
without intermediate as it is not observed any other additional band. These experiments show that compound $\mathbf{3}$ can hydrolyze at least 2 equivalents of substrate over a period of 10 hours. Control experiments in the absence of $\mathbf{3}$ do not show hydrolysis. Condition improvements like $\mathrm{pH}$, temperature or increasing of substrate concentration have not been tested.

This preliminary screening was designed to prove the hydrolytic catalytic activity of compound 3. The kinetic data indicate low turnover and for this reason we decided to not to pursue further studies. However the result obtained in this experiment show that compound $\mathbf{3}$ is a promising candidate for further hydrolysis studies.

\section{Nitrile hydrolysis}

The polarized S-O bond of the sulfenate has been suggested as a nucleophile for nitrile hydrolysis. ${ }^{80}$ Previously, Chottard et al. reported the slow, catalytic (18 turnovers after $17 \mathrm{~h}$ ) hydrolysis of acetonitrile in a coordinativly saturated, exchange-inert cobalt(III) sulfenate ${ }^{89}$ Attempts to hydrolyze acetonitrile with $\mathbf{3}$ following the same protocol yielded no quantifiable acetamide at $4^{\circ} \mathrm{C}$. This may be attributed to steric influences of the $\mathrm{PPh}_{3}$ ligand or the reduced Lewis acidity of ruthenium(II) in 3 as compared to cobalt(III) in the Chottard system or mainly due to the coordination of the $\mathrm{PPh}_{3}$ to the metal.

The initial trials of hydrolysis of $\mathrm{CH}_{3} \mathrm{CN}$ involve the use of a mixture of 1 ( $5 \mathrm{mg}$, $7 \mu \mathrm{mol})$ and 1,4-dimethoxybenzene (internal reference) $(7 \mathrm{mg}, 0.050 \mathrm{mmol})$ in $\mathrm{CH}_{3} \mathrm{CN}$ $(0.5 \mathrm{~mL})$ and an $\mathrm{HOAc} / \mathrm{NaOAc}(1 \mathrm{M}, \mathrm{pH}=4.8,0.5 \mathrm{~mL})$ or PIPES $(1 \mathrm{M}, \mathrm{pH}=7.0,0.5$ $\mathrm{mL}$ ) buffer, solution that was stirred at $4{ }^{\circ} \mathrm{C}$ for 5 days. After chloroform extraction, the

organic soluble residue was dissolved in $\mathrm{CD}_{3} \mathrm{OD}$ for ${ }^{1} \mathrm{H}$ and ${ }^{13} \mathrm{C}$ NMR analysis. The same 
procedure was applied to $\mathbf{2}$ and $\mathbf{3}$. As mentioned above acetamide was not detected in ${ }^{1} \mathrm{H}$ or ${ }^{13} \mathrm{C}$ NMR.

The compounds 1-3 were investigated in solution to probe their potential hydrolytic activity. Metal complex that promoted hydrolysis requires dissociation of the phosphine to provide an open coordination site for substrate binding or direct participation of the S-oxygenate moieties in the hydrolysis. Structural studies (vide infra) reveal an increased Ru-P bond distance as a function of S-oxygenation suggesting the phosphine may become labile in solution generating a transient five-coordinate intermediate.

Solutions of, $\mathbf{2}$ and $\mathbf{3}$ in 1:1:1 mixture of acetonitrile, methanol, and PIPES buffer $(\mathrm{pH}=7.0)$ yield small quantities of acetamide after stirring under inert conditions for 5 days at $25{ }^{\circ} \mathrm{C}$. The identity of the acetamide was confirmed by ${ }^{1} \mathrm{H}$ and ${ }^{13} \mathrm{C}$ NMR, Figure 52, following evaporation of solvent and extraction of soluble products into $\mathrm{CDCl}_{3}$. Attempts to quantify acetamide using 1,4-dimethoxybenzene as an internal standard yielded inconsistent results. ${ }^{141}$ In all cases, the acetamide integration was significantly greater than the standard, but proportional to free $\mathrm{PPh}_{3}$. No acetamide is observed for 1 under identical conditions. In contrast, the acetonitrile hydrolysis activity of $\mathbf{2}$ and $\mathbf{3}$ is consistent with partial $\mathrm{PPh}_{3}$ dissociation in solution. It should be noted that exchange inert $\mathrm{Co}(\mathrm{III})$ sulfenate complexes have been reported to hydrolyze nitriles via a ligandcentered mechanism in buffers at $\mathrm{pH}=4.8 .{ }^{89}$ However, under these conditions no hydrolysis was observed for 1-3. Further studies are underway to exchange the coordinated phosphine for more labile ligands. Complex 4 degraded to an intractable orange product upon the addition of water precluding catalytic attempts. 


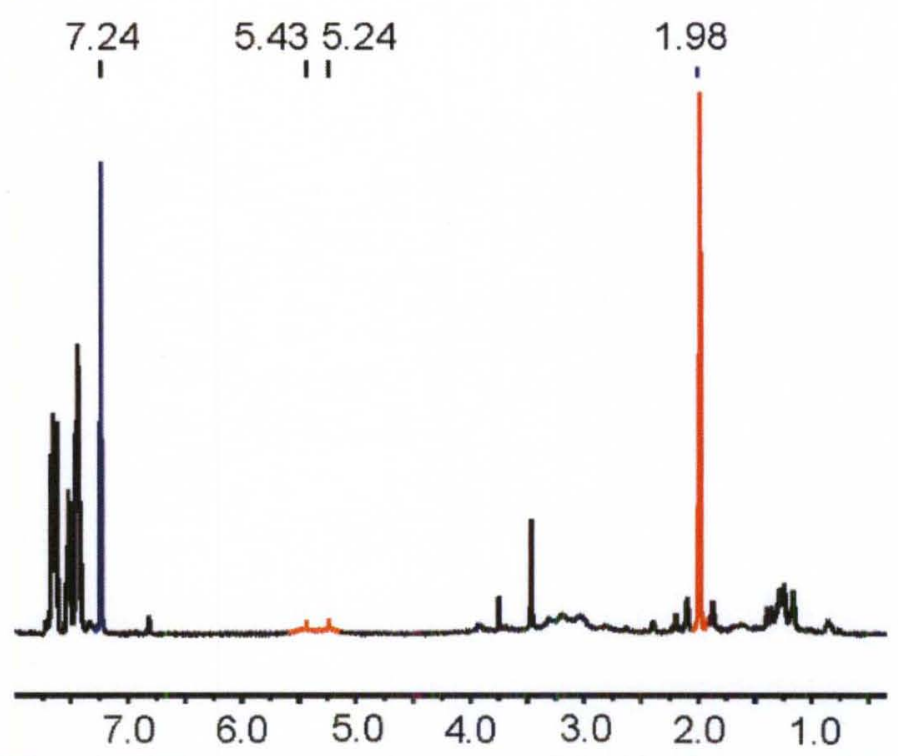

Figure 52. ${ }^{1} \mathrm{H}$ NMR of $\mathrm{CDCl}_{3}$ extract after acetonitrile hydrolysis trial with 3 reveals peaks at 1.98 and $5.34 \mathrm{ppm}$ associated with acetamide at $25^{\circ} \mathrm{C}$.

Further catalytic studies involve the use of higher temperature to evaluate hydrolysis activity; as result of these studies we detect the formation of acetamide in compound 3 at $75{ }^{\circ} \mathrm{C}$ as we observe in the Figure 53. The temperature in this experiment is above the $50{ }^{\circ} \mathrm{C}$ required to exchange the triphenylphosphine. This result strongly suggests the necessity to expose the metal center to generate any catalytic activity.

The experiment was performed in a NMR J-young tube in a mixture of $500 \mu \mathrm{l}$ of $\mathrm{MeOH}$ deuterated and $200 \mu 1 \mathrm{D}_{2} \mathrm{O}$. It was used $2.2 \mathrm{mg}(3.0 \mu \mathrm{mol})$ of compound 3 and 2.2 mg $(6.7 \mu \mathrm{mol})$ of $\left(\mathrm{C}_{4} \mathrm{H}_{9}\right)_{4} \mathrm{~N}^{+} \mathrm{BF}_{4}{ }^{-}$as reference. The substrate was acetonitrile $5 \mu \mathrm{L}(94.6$ $\mu$ mol). The mixture $\mathrm{MeOH}$ and $\mathrm{D}_{2} \mathrm{O}$ was used to help to solubilize the compound 3. The reference $\left(\mathrm{C}_{4} \mathrm{H}_{9}\right)_{4} \mathrm{~N}^{+} \mathrm{BF}_{4}{ }^{-}$was integrated in the region $0.94 \mathrm{ppm}$ to 1.01 for the equivalent of 3 protons. The experiment was performed for 11 day in an oil bath. The sample was only removed from the bath for ${ }^{1} \mathrm{H}$ NMR determinations of 20 minutes. The ${ }^{1} \mathrm{H}$ NMR was performed with a relaxation delay of 1.0 seconds, 256 repetitions with a total time of 15 minutes, at $25^{\circ} \mathrm{C}$ in a $400 \mathrm{MHz}$ instrument. 
Unfortunately, besides being a positive test of nitrile active complex the yield obtained indicates 2 turnovers after 11 days for the hydrolysis reaction and for this reason it was required to evaluate other nitriles that are easier to hydrolyze.

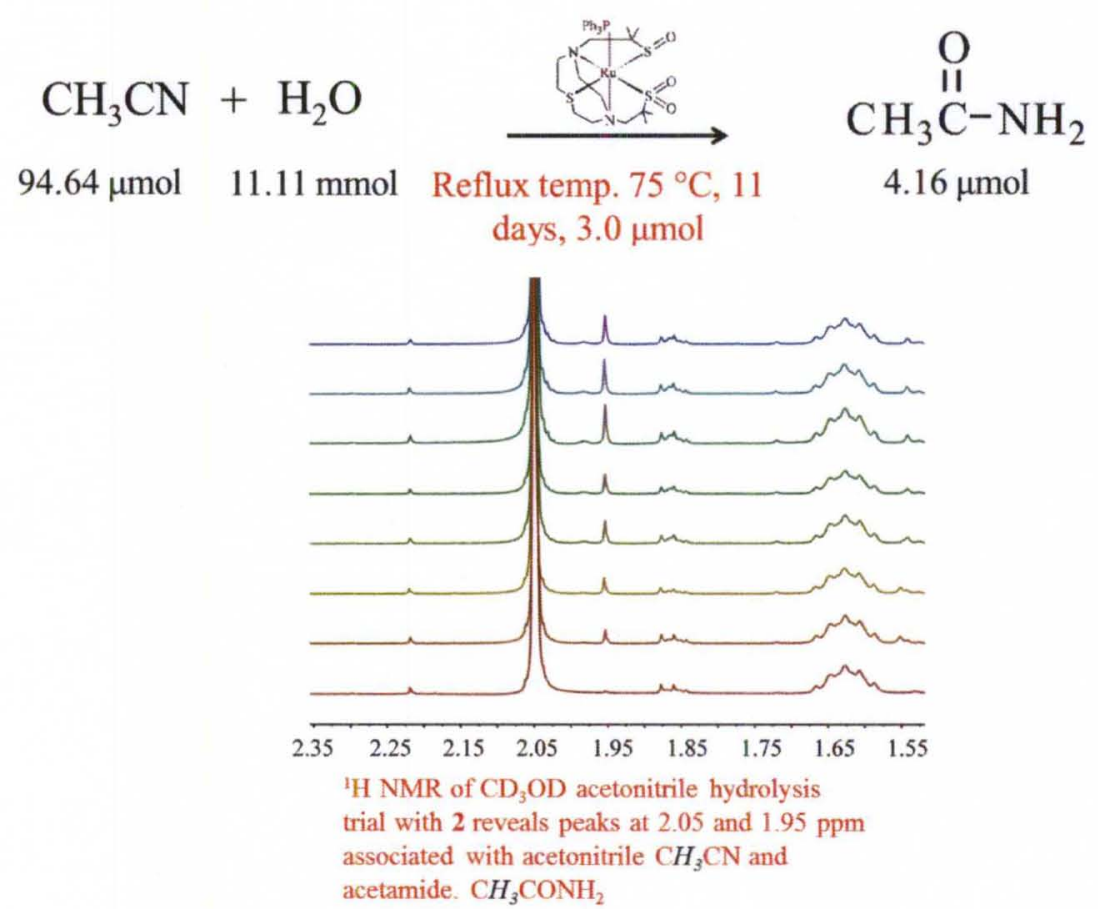

Figure 53. Hydrolysis of acetonitrile to acetamide by compound 3 .

The two other substrate candidates that we decided to analyze were acrylonitrile and benzonitrile; both compounds are easy to hydrolyze for NHases models. Shearer et alin a recent publication reported 58 turnovers at $25{ }^{\circ} \mathrm{C}$ in 18 hours for the formation of acrylamide. The acrylonitrile unfortunately present an inconvenient, it is thermal unstable and polymerize around $50{ }^{\circ} \mathrm{C}$, as result of the polymerization during the hydrolysis studies at $75^{\circ} \mathrm{C}$, it was observed a decrease in the staring material faster that acrylamide formation. The hydrolysis of acrylonitrile to acrylamide is observed for 3 (2.2 mg, 3 $\mu \mathrm{mol}$ ) in a 5:2 mixture of $\mathrm{MeOH}$ :Water (Total volume $0.7 \mathrm{~mL}$ ). Using as reference 3.7 $\mathrm{mg}, 11.2 \mu \mathrm{mol}\left(\mathrm{C}_{4} \mathrm{H}_{9}\right)_{4} \mathrm{~N}^{+} \mathrm{BF}_{4}$ at reflux temperature $\left(75^{\circ} \mathrm{C}\right)$ and $50: 1$ substrate $10 \mu \mathrm{l}, 150$ 
$\mu$ mol of acrylonitrile to catalyst. No acrylamide was detected in control reactions. After 2850 minutes the hydrolysis stops with 25 turnovers as it can be observed in Figure 54 .

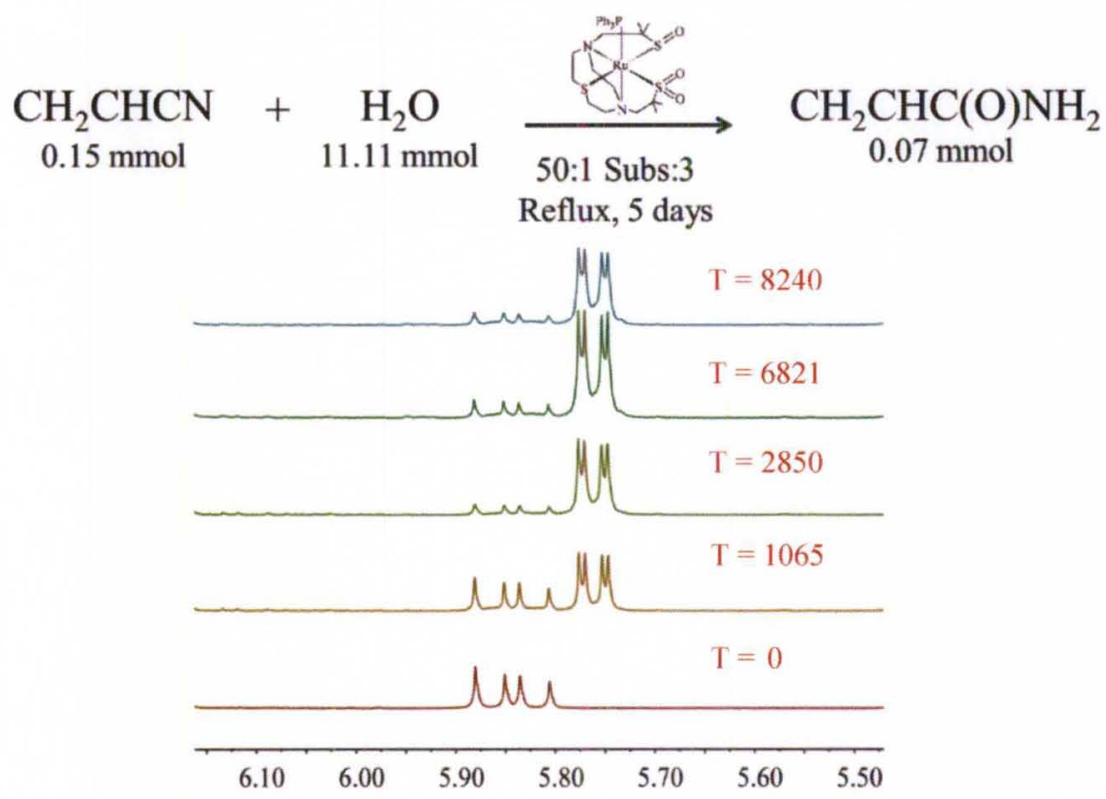

Figure 54. ${ }^{1} \mathrm{H}$ NMR in $\mathrm{CD}_{3} \mathrm{OD}$ of acrylonitrile hydrolysis trial with $\mathbf{3}$ reveals groups peaks at $5.84 \mathrm{q}$ and $5.76 \mathrm{dd}$ ppm associated with $\mathrm{CH}_{2} \mathrm{CHCN}$ and $\mathrm{CH}_{2} \mathrm{CHC}(\mathrm{O}) \mathrm{NH}_{2}$.

The other candidate, benzonitrile is easy to hydrolyze in a matrix of water and ruthenium complex at $100{ }^{\circ} \mathrm{C}$ and $124{ }^{\circ} \mathrm{C}$, The results of these studies indicate an hydrolyze activity in the three compounds $\mathbf{1 - 3}$ with the highest activity in compound $\mathbf{3}$. In the Figure 55, it is presented the FT-IR of the product isolated after the reaction at $100{ }^{\circ} \mathrm{C}$ for compound 3 with $9 \pm 1$ turnovers. The FT-IR matches the spectrum of a pure benzamide. Compound 2 shows $10 \pm 1$ turnovers and compound 1 show 6 turnovers. The protocol involve in this reaction required the use $1200 \mu \mathrm{l}$ benzonitrile and $2000 \mu \mathrm{l}$ of $\mathrm{D}_{2} \mathrm{O}$ using a reaction flask at $100{ }^{\circ} \mathrm{C}$ for 5 days. 


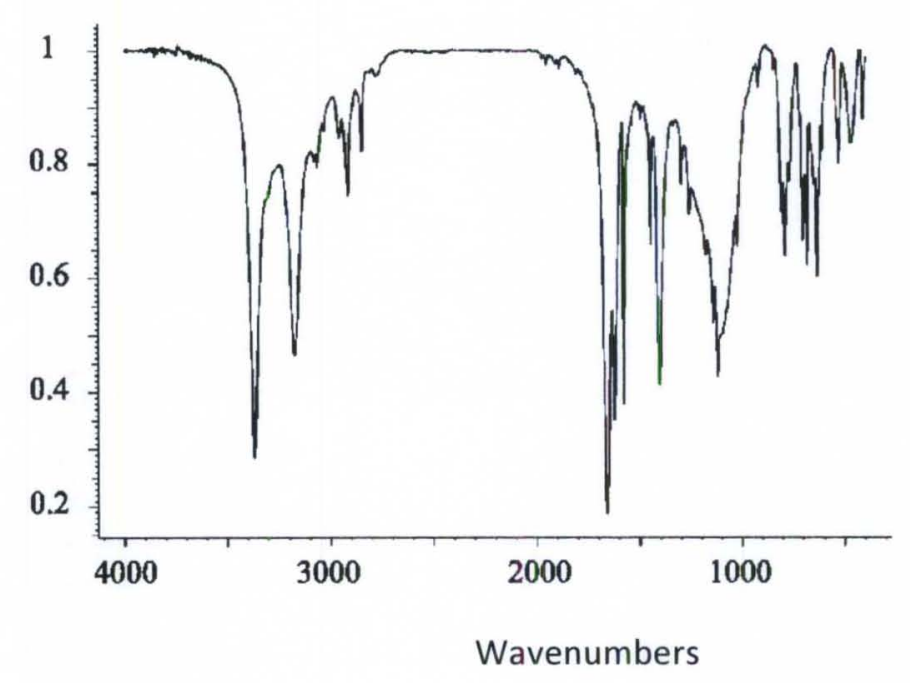

Figure 55. FT-IR of benzamide isolated from hydrolysis of benzonitrile in the presence of 3 .

Additional trails performed at $124{ }^{\circ} \mathrm{C}$ indicate $65 \pm 1$ turnovers for compound $\mathbf{1}$, $86 \pm 9$ for compound $\mathbf{2}$ and $85 \pm 11$ for compound 3 . It was observed that the ruthenium complex $\mathbf{1}$ has the low solubility in polar solvents and at the same time the low reactivity with 65 turnovers by 18 hours at $124{ }^{\circ} \mathrm{C}$. The likely reason for this reactivity is the competition between the $\mathrm{PPh}_{3}$ substrate and the benzonitrile or water. In presence of air the activity of $\mathbf{1}$ drop to $29 \pm 2$ turnovers. This result can be explained based on the sulfur oxygenation of the complex and water sensitivity of complex 4 . The number of moles of catalyst used in these trails was $2.7 \times 10^{-7}$, the number mmoles of benzonitrile substrate was 4.9 and the number of mmoles of $\mathrm{D}_{2} \mathrm{O}$ 82.9. The quantification was performed by GC-MS. The calibration curve is presented in the Figure 56. 


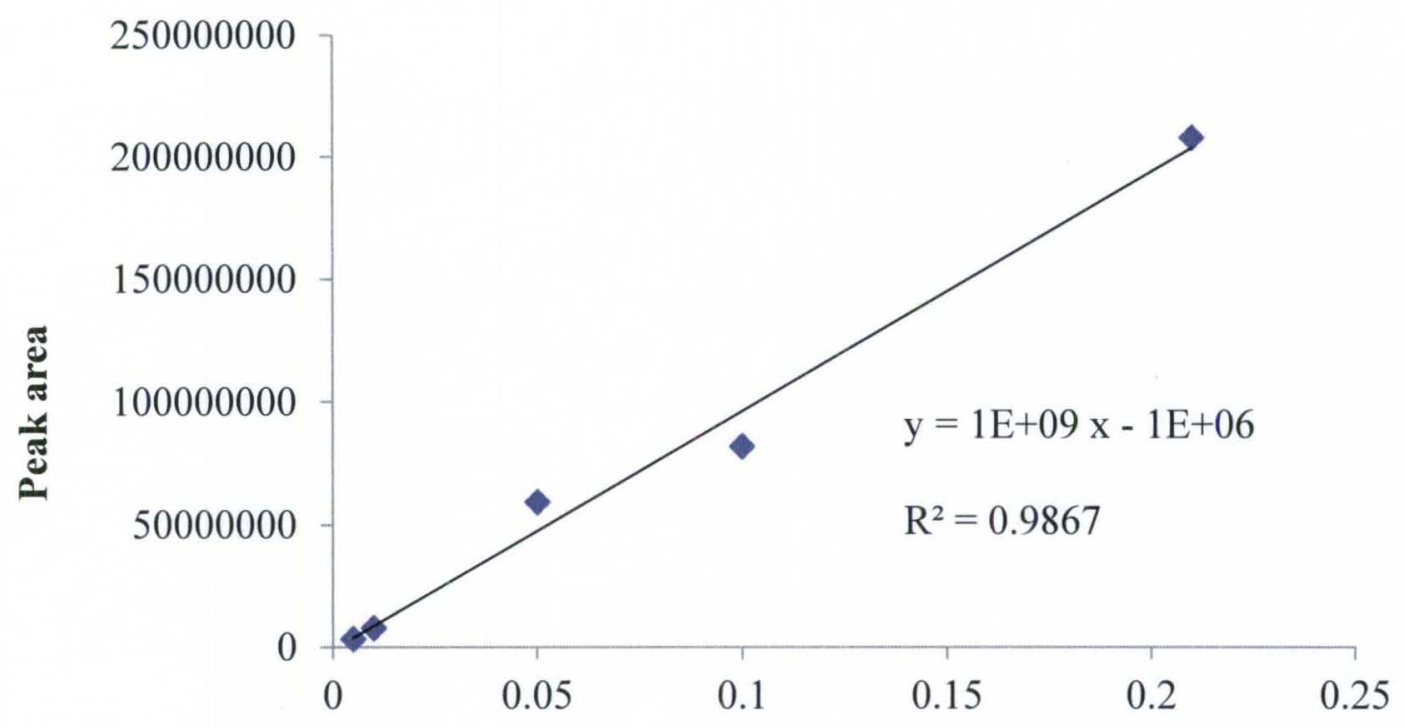

Conc. $(\%)$ of benzamide in ether

Figure 56. GC-MS calibration curve for benzamide.

Preliminary results of +ESI-MS in the product of the benzonitrile hydrolysis reaction with compound $\mathbf{1}$ indicate a nitrile bound mechanism. The mass spectral analysis of an isolated green residue product of the hydrolysis of benzonitrile with complex 1 displays a $\mathrm{m} / \mathrm{z}$ peaks at 574.15 and 590.16 consistent with (bmmp-TASN)RuNCPh and (bmmp-TASN)RuNH ${ }_{2} \mathrm{C}(\mathrm{O}) \mathrm{Ph}$, respectively, suggesting discreet substrate and product derivatives Figure 57, further research is underway to isolated and fully characterized these new compounds. 


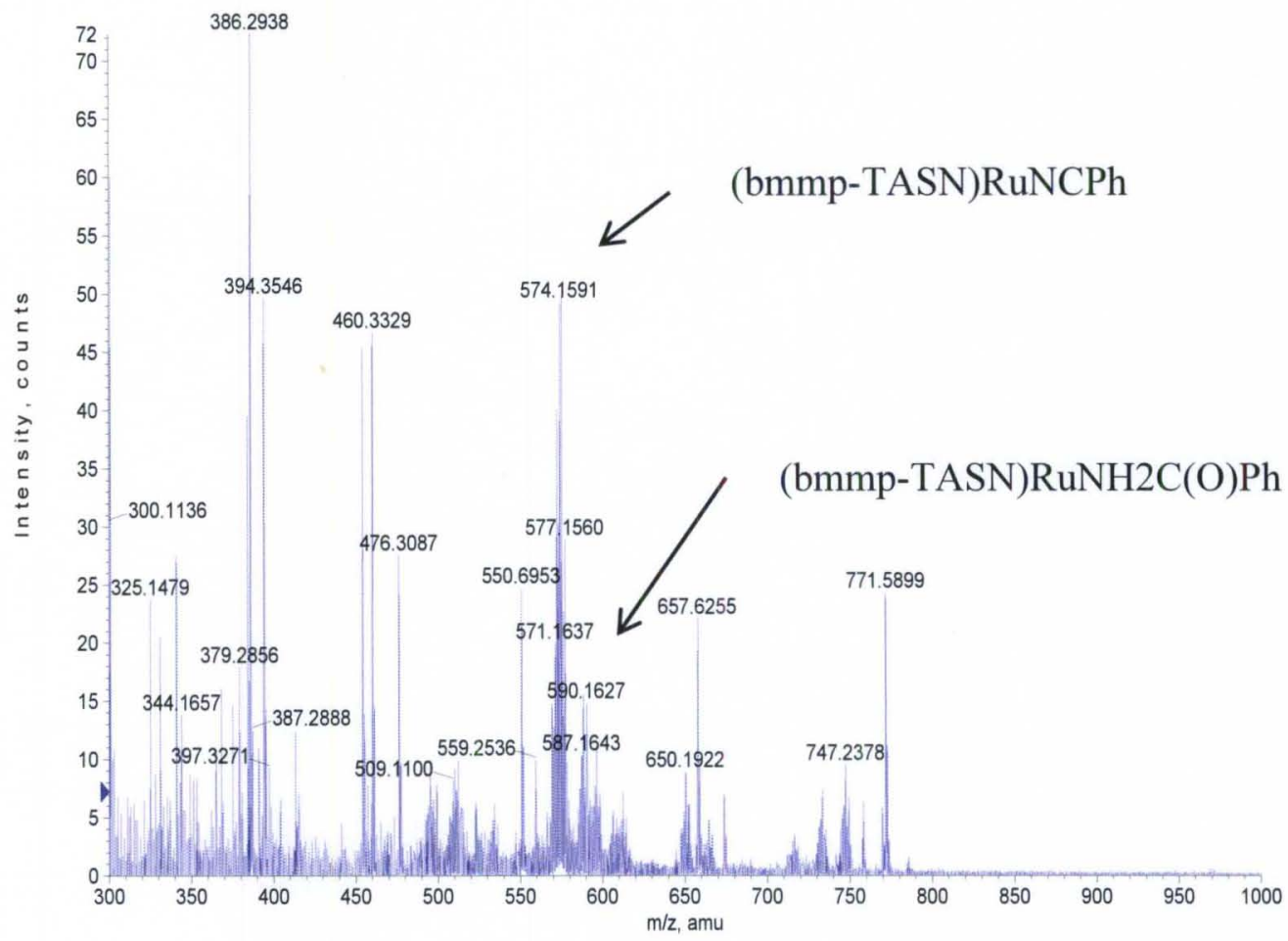

Figure 57. +ESI-MS of benzonitrile hydrolysis reaction with compound 1.

\section{Summary}

Studies with (bmmp-TASN) RuPPh 3 and S-oxygenates 2-3 reveal significant benzonitrile hydrolysis at moderate temperatures $124{ }^{\circ} \mathrm{C}$. Benzonitrile is an ideal substrate due to its product the benzamide is easily isolated by sublimation from the crude reaction mixture and because after the hydrolysis reaction the benzamide precipitate from the reaction matrix. The sulfenate/sulfinate complex $\mathbf{3}$ shows up to $85 \pm$ 11 turnovers in 18 hours. Control reactions without Ru catalyst yield $7 \pm 2$ Turnovers. Turnovers numbers have not yet been optimized, but some hydrolysis is also observed for (1-2) We have also examined hydrolysis of acrylonitrile, which offer the advantage of in situ reaction monitoring by ${ }^{1} \mathrm{H}$ NMR. As shown in Figure 54, the relative intensity of the acrylamide vinyl protons increases with time, when compound $\mathbf{3}$ promoted hydrolysis. It 
is expect the hydrolysis proceeds via a nitrile- or water-bound complex following thermally induced $\mathrm{PPh}_{3}$ dissociation. As mentioned in this chapter the thermally dissociation of $\mathrm{PPh}_{3}$ for phosphine exchange has an inverse correlation with the oxygenation of thiolates, at $100{ }^{\circ} \mathrm{C}$, for the thiolate ligand and $50^{\circ} \mathrm{C}$ for sulfenate/sulfinate derivate for phosphine exchange. 


\section{CHAPTER V}

\section{CONCLUSIONS AND FUTURE DIRECTIONS}

The research in the area of nitrile hydratase has been fruitful in the last 20 years. From the beginning, this area of study has been oriented in both industrial and academic direction. It was remarkable that nitrile hydratase was the first enzyme used in a biotechnological process; the transform of acrylonitrile to acrylamide. It was this process that demonstrated the technological advantage of the nitrile hydratase enzyme to produce acrylamide at industrial level. This process cannot be accomplished by chemical means due to the highest temperatures of 200 to $300{ }^{\circ} \mathrm{C}$ and higher pressures required in the chemical hydrolysis. Since under these conditions acrylonitrile will polymerize and decompose.

It was the NHase, with its low temperature range of hydrolysis activity between 25 to $35^{\circ} \mathrm{C}$, that allowed the industrial synthesis of acrylamides. This technological breakthrough and the unusual active center of the NHase drove the research in academia. At the academic level, the NHase represent an ideally case of study to understand metal thiolate reactivity and post-translational modifications.

The main targets of study in the area of metal thiolate reactivity and NHase are the understanding of the metal thiolate reactivity with oxygen, the reproduction of the 
synthetically challenge sulfenate/sulfinate active site of the NHase enzyme, the study of the NHase mechanism and the designing of a more efficient NHase synthetic model.

As a bioinorganic synthetic chemistry group, we took the challenge to study the NHase system and respond some of the unsolved question in the area. This thesis was oriented to synthesize the best functional synthetic NHase model in the research community. The conclusion and future direction of this research are presented in the next paragraphs.

\section{Synthesis of (bmmp-TASN)RuPPh 3 and sulfur oxygen derivatives}

In this research we reported the synthesis of (bmmp-TASN) $\mathrm{RuPPh}_{3}(\mathbf{1})$ and its derivatives compounds under limiting dioxygen conditions. The family of oxygen derivatives include (bmmp-O $\left.\mathrm{O}_{2}-\mathrm{TASN}\right) \mathrm{RuPPh}_{3}(\mathbf{2}), \quad\left(\mathrm{bmmp}-\mathrm{O}_{3}-\mathrm{TASN}\right) \mathrm{RuPPh}_{3}(\mathbf{3})$, and (bmmp-O $\left.\mathrm{O}_{4}-\mathrm{TASN}\right) \mathrm{RuPPh}_{3}(4){ }^{129,141}$ The sulfenate/sulfinate $\left(\mathrm{RSO}^{-}\right) /\left(\mathrm{RSO}^{2-}\right)$ donor set of 3 mimics the unusual post-translational modification of the NHase and SCNase active sites. The asymmetric sulfur-oxygenate $\mathbf{3}$ represents one of only three sulfenate/sulfinate derivatives prepared by direct reaction with dioxygen. ${ }^{79,108}$ High yields of $\mathbf{3}$ were obtained by limiting the quantity ( 5 equivalents) and duration (12 hours) of dioxygen exposure. The sulfur oxygen derivate are oxygen sensible and their synthesis required a new synthetic approach.

\section{$\mathrm{O}_{2}$ delivery an innovative approach for the synthesis of sulfur oxygen derivatives}

The typical approach to prepare sulfur-oxygenated mimics of NHase or SCNase involves the reaction of metal-thiolate precursors with O-atom transfer agents such as $\mathrm{H}_{2} \mathrm{O}_{2}, \quad \mathrm{~N}$-sulfonyloxaziridine, $\quad(1 S)-(+)-(10$-camphorsulfonyl)oxaziridine, $\quad$ or $\quad$ urea hydrogen peroxide. ${ }^{80,82,88,89,112,118}$ These efforts have been successful for the isolation of 
sulfenate, ${ }^{80,89,112,118}$ sulfinate, ${ }^{88,89}$ and a mixed sulfenate/sulfinate complex with $\eta^{2}$ sulfenate coordination. ${ }^{82}$ A major advantage of O-atom transfer agents is the ability to quantitatively add oxidizing equivalents at controlled rates. However the application of this approach in our system was not successful. A new synthetic strategy was applied for the synthesis of the sulfur oxygenated reactive products. Less well developed are reactions employing dioxygen, which is the proposed reagent in the enzymatic systems. ${ }^{42,46,56}$ While several sulfinate complexes have been reported upon $\mathrm{O}_{2}$ exposure of thiolate precursors, ${ }^{82,116,119-127}$ isolation of sulfenate ${ }^{120,128}$ or mixed sulfenate/sulfinate complexes from $\mathrm{O}_{2}$ are rare due to the reactivity of the sulfenate. ${ }^{79,108,129,142,143}$ Our strategy for the synthesis of a family of sulfur oxygen derivative involves the quantitative delivery of oxygen using an oxygen transfer bulb. The optimizations of the conditions for a sequential oxygenation were obtained with the use of FT-IR and TLC.

As part of the sulfur oxygenation study, we report this innovative approach to synthesize sulfur oxygen derivatives. A family of sulfur oxygenated derivatives has been prepared through the controlled oxygenation of 1 under limiting $\mathrm{O}_{2}$ conditions. The key synthetic features of our approach include restricted quantities of $\mathrm{O}_{2}$ and short reaction times. Prior studies were largely conducted open to air or in oxygen saturated solutions under an $\mathrm{O}_{2}$ atmosphere for hours or days. By fixing the quantity of $\mathrm{O}_{2}$ and the duration of the reaction, conditions were optimized to reproducibly obtain high yields of $\mathbf{2}, \mathbf{3}$, or 4 without the need for $\mathrm{O}$-atom transfer reagents or purification by column chromatography. The S-oxygenation of 1 proceeds in a series of stepwise additions. Rapid addition of $\mathrm{O}_{2}$ to 1 yields 2 . This step is promoted by the $t_{2 g}{ }^{6}$ electron configuration of $R u(I I)$. As reported previously, the $\pi$ and $\pi^{*}$ interactions between thiolate sulfur donors and $\mathrm{t}_{2 \mathrm{~g}}$-rich 
metal ions increase the covalency of the metal-sulfur bond and promote sulfur oxygenation. The second oxygenation step, $\mathbf{2}$ to $\mathbf{3}$, proceeds significantly more slowly than the first. This is attributable, at least in part, to steric interactions between the remaining thiolate and $\mathrm{PPh}_{3}$. Further oxygenation of 3 to 4 is further hindered by the $\mathrm{PPh}_{3}$ and occurs only on longer time scales. Each S-oxygenate, 2-4, is stable in acetonitrile and methanol solutions in the absence of $\mathrm{O}_{2}$. Complex 4 degrades in the presence of water. This approach has yielded a unique "family" of complexes, 1-4, that differ only in the degree of S-oxygenation. Although the number of reported S-oxygenates is growing, families containing three or more oxygenated derivatives are scant. ${ }^{144}$

\section{Relevance of the oxygenation study of metal-thiolate complexes}

With the synthesis of the family of sulfur oxygen derivatives, this thesis offers insight into the controlled sulfur oxygenation of metal thiolates and the resulting changes in the electronic structure. The relevance of this study is not only subscribed to the NHase or SCNase systems but also to post-translational modification of cysteines during redox signaling and oxidative stress. The cysteine residues, thiolates, suffer oxidative modification that will leads to functional alterations in the proteins. This modification has a broad biological impact in health and diseases from molecular to the cellular and organismal level. In our study we observe and characterize the effect of the oxidation with $\mathrm{O}_{2}$ of two thiolates in a NHase model system using for a first time a family of sulfur oxygen derivatives to understand the effect of this oxidation. Until now the oxidative modification in cysteine has been reviewed from a biochemistry approach with reactive oxygen species, in cellular redox systems and in apoptosis ${ }^{149}$ but not in a synthetic catalytic center. One of the results of this oxidative modification in our NHase synthetic 
model is the improvement in catalytic activity in a $25 \%$ for the NHase analogue with respect to the thiolate derivative. It can be speculated that the activation of the NHase is due to the effect of sulfur oxygenation and the elongation of the Metal-NO bond distance and subsequent exposure of the metal to the substrate.

\section{Low spin state configuration leads to metal-thiolate oxygenation complex}

Through our studies of the ruthenium dithiolate oxygen reactivity we confirm the previous hypothesis of the Grapperhaus group that " $\mathrm{t}_{2 \mathrm{~g}}$-rich" low-spin complexes favor sulfur oxygenation. This hypothesis is supported by the reactivity of $\mathbf{1}$ a ruthenium(II) low spin complex with $\mathrm{O}_{2}$. Further, partial sulfur oxygenation is achievable using limited $\mathrm{O}_{2}$ conditions, as demonstrated by 23 and 4. In 1, the steric bulk of $\mathrm{PPh}_{3}$ slows oxygenation beyond 4 but does not prevent it, as demonstrated under excess $\mathrm{O}_{2}$ conditions. These results suggest that asymmetric oxygenation of nitrile hydratase and thiocyanate hydrolase may also be facilitated by limited $\mathrm{O}_{2}$ at the active site without the necessity for single O-atom-transfer reagents. Structurally sulfur oxygenation shortens the M-S bond while lengthening the metal-ligand bonds to $\pi$ acceptors. In combination with the previously documented labilizing effect of the trans-thiolate, ${ }^{142,143}$ sulfur oxygenation may promote ligand exchange. It is also expected to enhance coordination of $\pi$ donors, such as $\mathrm{HO}^{-}$, and may help to discriminate substrate coordination.

\section{Study of a pentacoordinate system that allow substrate exchange}

Based on a systematic synthesis design, the pioneering $\mathrm{Ni}$ - and $\mathrm{Pd}$-dithiolate systems developed by Darensbourg provide a benchmark for completeness, because they offer no prospects to probe the effects of S-oxygenation at a variable ligand site. To face this challenge we used a pentacoordinate ligand designed for the Grapperhaus group to 
allow us exchange the six coordinate substrate. The analysis of the structural parameters of 1-4 reveals a systematic increase in the $\mathrm{Ru}-\mathrm{P}$ bond distance as a function of $\mathrm{S}$ oxygenation. This trend is reproduced computationally by density functional theory calculations providing insight into the potential of S-oxygenation to regulate substrate/product binding at the NHase active site and exchange substrates.

The ligand bmmp-TASN has been employed to model the donors at active sites of NHase and SCNase. As in the enzymes, ligand coordination positions two reactive thiolate donors on the same octahedral face as a sixth, variable ligand. The $\mathrm{t}_{2 \mathrm{~g}}{ }^{6}$ electron configuration of $\mathrm{Ru}(\mathrm{II})$ in $\mathbf{1 - 3}$ reproduces key electronic features of the low-spin $\mathrm{t}_{2 \mathrm{~g}}{ }^{5}$ and $\mathrm{t}_{2 \mathrm{~g}}{ }^{6}$ configurations of $\mathrm{Fe}(\mathrm{III})$ and $\mathrm{Co}(\mathrm{III})$ at the enzyme active sites. Further, as noted in the current thesis, the $\mathrm{Ru}-\mathrm{S} \pi^{*}$ interactions stabilizes $\mathrm{Ru}-\mathrm{P} \sigma$-bonding. Upon oxygenation, the $\mathrm{Ru}-\mathrm{S} \pi^{*}$ interaction is relieved, weakening the $\mathrm{Ru}-\mathrm{P}$ bond as evidenced by the direct correlation between the oxygenation level and Ru-P bond distance. These results allow us postulate our complexes 1-3 as ideal candidates to analyze $\mathrm{H}_{2} \mathrm{O}$, nitriles and $\mathrm{NO}$ derivatives. These substrates are postulated as part of the NHase mechanism.

\section{Sulfur oxygenation $X$-ray absorption studies in a ruthenium NHase mimic}

NHases require oxygenated cysteinate residues to be catalytically activity. The functional reasons for these post-translational modifications are on debate. In this thesis, It was investigated the influence of sequential thiolate oxygenation on a series of $\mathrm{Ru}(\mathrm{II})$ complex 1, 2 and 3. It had previously been observed by X-ray and by computational calculations that upon sequential oxygenation the $\mathrm{Ru}-\mathrm{P}$ bond-length increased.

Through the use of X-ray absorption spectroscopy it has been confirmed that the bonding in these complexes is largely dominated by $\mathrm{S}-\mathrm{Ru}$ interactions, with the $\mathrm{Ru}-\mathrm{P}$ 
and $\mathrm{Ru}-\mathrm{N}$ interactions contributing to a lesser degree. Upon oxygenation the bonding interactions between the oxygenated thiolates and the Ru-center decreases. As this occurs the covalency of the complex decreases overall and the charge on the Ru-center increases as a result; the atomic charge on $\mathrm{Ru}$ increases from -0.073 in $\mathbf{1}$ to +0.0831 in $\mathbf{2}$ to +0.263 in 3. In terms of hard/soft acid/base chemistry the Ru-center is becoming a harder Lewisacid. Thus, bonding between the progressively harder $\mathrm{Ru}$-center and the soft $\mathrm{PPh}_{3}$ ligand would be expected to become weaker, which is what is observed.

Compound 3 mimic the asymmetric environment of the catalytic center of the NHase

One of the most remarkable result in this thesis was the synthesis of the sulfenate/sulfinate (bmmp- $\left.\mathrm{O}_{3}-\mathrm{TASN}\right) \mathrm{RuPPh}_{3}$ compound 3. The compound $\mathbf{3}$ provide us with an unusual synthetic model with a mixed S-oxygenation environment observed at the active site of NHase and SCNase. This model complex situates the Grappaerhus' group in an ideal position to investigate the effects of the sulfur oxygenation in a NHase mimic with catalytic activity. To our knowledge, only three mixed sulfenate/sulfinate complexes have been structurally characterized. Of these, only one is isolated from aerobic oxidation, but that complex is coordinative saturated and not provide an exchange substrate position as consequence it is not a useful mimic. This complex is a NHase model complex reported by Kovacs, that is isolated via $\mathrm{H}_{2} \mathrm{O}_{2}$ oxidation of the thiolate sulfinate precursor, but the generated sulfenate ligand is coordinated in a side-on, $\eta^{2}$ fashion that saturates the metal coordination sphere and prevents further reactivity studies as mentioned in chapter one. We state that our (bmmp- $\left.\mathrm{O}_{3}-\mathrm{TASN}\right) \mathrm{RuPPh}_{3}$ complex best mimics the S-oxygenation of NHase and SCNase as: it is isolated directly from aerobic 
oxidation; it maintains $\eta^{1}$-coordination of the sulfenate ligand and specially because it is built on a pentadentate ligand framework that allows a variable coordination site cis to the S-oxygenates. Finally this model is hydrolytically active and it is functional catalyst.

\section{Sulfur oxygen derivatives 2 and 3 facilitate phosphine exchange}

Notably, the increase in bond length in the family of sulfur oxygen derivatives correlates with the phosphine exchange reactivity with compound 2 and 3 at $50{ }^{\circ} \mathrm{C}$ in methanol and for compound 1 at $100{ }^{\circ} \mathrm{C}$ chlorobenzene. It is expected that the same M-L bond distance trend would be observed for Fe(III) and Co(III). In support of this, Mascharak et al. have shown that S-oxygenation of an iron-thiolate facilitates photodissociation of NO. Our series of complexes confirms the observation for that isolated example to show a direct correlation between the number of $\mathrm{O}$-atoms and the M-L bond distance. These results are consistent with the notion that sulfur oxygenation increases substrate/product lability as it is the case of exchange of triphenylphosphine by methyldiphenylphosphine. Synthesis of a thiolate derivative is accomplished with the use of $\mathrm{CO}$ in methanol at $70{ }^{\circ} \mathrm{C}$.

\section{(bmmp-TASN)RuPPh ${ }_{3}$ and sulfur oxygen derivatives are hydrolysis active catalyst}

The hypothesis of hydrolysis activity has been investigated through the evaluation of a series of hydrolysable substrate candidates, such compounds were screening to test its activity, and between them we can mention $p$-nitrophenylactetate with the UV-visible studies, acetonitrile, acrylonitrile and benzonitrile with ${ }^{1} \mathrm{H}$ NMR and FT-IR studies.

Studies with (bmmp-TASN)RuPPh 3 (1) and S-oxygenates (2-3) reveal significant benzonitrile hydrolysis at moderate temperatures $124{ }^{\circ} \mathrm{C}$. Benzonitrile is an ideal substrate due to its product the benzamide is easily isolated by sublimation from the crude reaction mixture and because after the hydrolysis reaction the benzamide 
precipitated from the reaction matrix. The sulfenate/sulfinate complex (3) shows up to 85 \pm 11 turnovers over 18 hours. Control reactions without $\mathrm{Ru}$ catalyst yield $7 \pm 2$ Turnovers. Turnovers numbers have not yet been optimized, but some hydrolysis is also observed for (1-2). Compound 2 generates $86 \pm 9$ turnovers, and compound 1 generates $65 \pm 1$ turnovers.

We have also examined hydrolysis of acrylonitrile, which offer the advantage of in situ reaction monitoring by ${ }^{1} \mathrm{H}$ NMR. The relative intensity of the acrylamide vinyl protons increases with the time, when compound $\mathbf{3}$ promoted hydrolysis. It was reported 25 turnovers after 2850 minutes. It is expect the hydrolysis proceeds via a nitrile- or water-bound complex following thermally induced $\mathrm{PPh}_{3}$ dissociation. As mentioned above the thermally dissociation of $\mathrm{PPh}_{3}$ for phosphine exchange has an inverse correlation with the oxygenation of thiolates, at $100^{\circ} \mathrm{C}$ for the thiolate and at $50^{\circ} \mathrm{C}$ for sulfenate/sulfinate derivate for phosphine exchange. 


\section{REFERENCES}

1. Akimasa Miyanaga; Shinya Fushinobu; Kiyoshi Ito; Hirofumi Shoun; Takayosh Wakagi, Eur. J. of Biochem. 2004, 271, 429-438.

2. Petrillo, K. L.; Wu, S.; Hann, E. C.; Cooling, F. B.; Ben-Bassat, A.; Gavagan, J. E.; DiCosimo, R.; Payne, M. S., Appl. Microbiol. Biotechnol. 2005, 67, 664-670.

3. Kovacs, J. A., Chem. Rev. 2004, 104, 825-848.

4. Banerjee, A.; Sharma, R.; Banerjee, U. C., Appl. Microbiol. Biotechnol. 2002 $60,33-44$

5. Hjort, C. M.; Godtfredsen, S. E.; Emborg, C., J. Chem. Technol. Biotechnol. 1990, 48, 217-226.

6. Wu, Z. L.; Li, Z. Y., Chem. Commun. 2003, 386-387.

7. Dadd, M. R.; Claridge, T. D. W.; Walton, R.; Pettman, A. J.; Knowles, C. J., Enzyme Microb. Technol. 2001, 29, 20-27.

8. Martinkova, L.; Klempier, N.; Prepechalova, I.; Prikrylova, V.; Ovesna, M.; Griengl, H.; Kren, V., Biotechnol Lett. 1998, 20, 909-912.

9. Cowan, D.; Cramp, R.; Pereira, R.; Graham, D.; Almatawah, Q., Extremophiles 1998, 2, 207-216.

10. Tauber, M. M.; Cavaco-Paulo, A.; Robra, K. H.; Gubitz, G. M., Appl. Environ. Microbiol. 2000, 66, 1634-1638. 
11. Prasad, S.; Bhalla, T. C., Biotechnol. Adv. 2010, 28, 725-741.

12. Kobayashi, M.; Nagasawa, T.; Yamada, H., Trends in Biotechnol. 1992, 10, $402-$ 408.

13. Nagasawa, T.; Mathew, C. D.; Mauger, J.; Yamada, H., Appl. Environ. Microbio.l 1988, 54, 1766-1769.

14. Asano, Y.; Tani, Y.; Yamada, H., Agric. Biol. Chem. 1980, 44, 2251-2252.

15. Kato, Y.; Nakamura, K.; Sakiyama, H.; Mayhew, S. G.; Asano, Y., Biochemistry. 2000, 39, 800-809.

16. Bandyopadhyay Ak Fau - Nagasawa, T.; Nagasawa T Fau - Asano, Y.; Asano Y Fau - Fujishiro, K.; Fujishiro K Fau - Tani, Y.; Tani Y Fau - Yamada, H.; Yamada, H., Appl. Environ. Microbiol. 1986.

17. Kobayashi, M.; Shimizu, S., Nat. Biotech. 1998, 16, 733-736.

18. Shaw, N. M.; Robins, K. T.; Kiener, A., Adv. Synth. Catal. 2003, 345, 425-435.

19. Dieter Sellmann, B. H., Annette Rösler, Frank W. Heinemann, Angew. Chem. Int. Ed. 2001, 40, 1505-1507.

20. Bunch, A. W., Antonie. Leeuwenhoek. 1998, 74, 89-97.

21. Hann, E. C.; Eisenberg, A.; Fager, S. K.; Perkins, N. E.; Gallagher, F. G.; Cooper, S. M.; Gavagan, J. E.; Stieglitz, B.; Hennessey, S. M.; DiCosimo, R., Bioorg. Med. Chem. 1999, 7, 2239-2245.

22. Mauger, J.; Nagasawa, T.; Yamada, H., Tetrahedron 1989, 45, 1347-1354.

23. Nagasawa, T.; Shimizu, H.; Yamada, H., Appl. Microbiol. Biotechnol. 1993, $40,189-195$.

24. Nagasawa, T.; Yamada, H., Trends. Biotechnol. 1989, 7, 153-158. 
25. Prasad, S.; Raj, J.; Bhalla, T., Indian. J. Microbiol. 2007, 47, 34-41.

26. Raj, J.; Seth, A.; Prasad, S.; Bhalla, T., Appl. Microbiol. Biotechnol. 2007, 74, 535-539.

27. Kobayashi, M.; Shimizu, S., Nat. Biotechnol. 1998, 16, 733-736.

28. Kohyama, E.; Yoshimura, A.; Aoshima, D.; Yoshida, T.; Kawamoto, H.; Nagasawa, T., Appl. Microbiol. Biotechnol. 2006, 72, 600-606.

29. Li, T.; Liu, J.; Bai, R.; Ohandja, D.-G.; Wong, F.-S., Water Res. 2007, 41, $3465-3473$.

30. Aislabie, J.; Atlas, R. M., Appl. Environ. Microbiol. 1988, 54, 2197-2202.

31. Holtze, M. S.; Sørensen, S. R.; Sørensen, J.; Aamand, J., Environ. Pollut. 2008, $154,155-168$.

32. Baxter, J.; Garton, N.; Cummings, S., Folia. Microbiol. 2006, 51, 591-597.

33. Katayama, Y.; Narahara, Y.; Inoue, Y.; Amano, F.; Kanagawa, T.; Kuraishi, H., J. Biol. Chem. 1992, 267, 9170-9175.

34. Katayama, Y.; Matsushita, Y.; Kaneko, M.; Kondo, M.; Mizuno, T.; Nyunoya, H., J. Bacteriol. 1998, 180, 2583-2589.

35. Westley, J., Fundam. Appl. Toxicol. 1983, 3, 377-382.

36. Okamoto, S.; Eltis, L. D., Mol. Microbiol. 2007, 65, 828-838.

37. Cramp, R. A.; Cowan, D. A., Biochim. Biophys. Acta, Protein Struct. Mol. Enzymol. 1999, $1431,249-260$.

38. Payne, M. S.; Wu, S.; Fallon, R. D.; Tudor, G.; Stieglitz, B.; Turner, I. M.; Nelson, M. J., Biochemistry. 1997, 36, 5447-5454.

39. Taştan Bishop, A. Ö.; Sewell, T., Biochem. Biophys. Res. Commun. 2006, 
$343,319-325$.

40. Fau, K; Nowak, Biophys. J. 2008, 94, 3824-3838.

41. Hourai, S.; Miki, M.; Takashima, Y.; Mitsuda, S.; Yanagi, K., Biochem. Biophys. Res. Commun. 2003, 312, 340-345.

42. Miyanaga, A.; Fushinobu, S.; Ito, K.; Wakagi, T., Biochem. Biophys. Res. Commun. 2001, 288, 1169-1174.

43. Odaka, M.; Fujii, K.; Hoshino, M.; Noguchi, T.; Tsujimura, M.; Nagashima, S.; Yohda, M.; Nagamune, T.; Inoue, Y.; Endo, I., J. Am. Chem. Soc. 1997, 119, 3785-3791.

44. Bonnet, D.; Artaud, I.; Moali, C.; Petre, D.; Mansuy, D., FEBS Lett. 1997, 409, 216-220.

45. Endo, I.; Odaka, M.; Yohda, M., Trends. Biotechnol. 1999, 17, 244-249.

46. Murakami, T.; Nojiri, M.; Nakayama, H.; Dohmae, N.; Takio, K.; Odaka, M.; Endo, I.; Nagamune, T.; Yohda, M., Protein Sci. 2000, 9, 1024-1030.

47. Arakawa, T.; Kawano, Y.; Kataoka, S.; Katayama, Y.; Kamiya, N.; Yohda, M.; Odaka, M., J. Mol. Biol. 2007, 366, 1497-1509.

48. Huang, W. J.; Jia, J.; Cummings, J.; Nelson, M.; Schneider, G.; Lindqvist, Y., Structure 1997, 5, 691-699.

49. Nagashima, S.; Nakasako, M.; Dohmae, N.; Tsujimura, M.; Takio, K.; Odaka, M.; Yohda, M.; Kamiya, N.; Endo, I., Nat. Struct. Biol. 1998, 5, 347-351.

50. Hashimoto, K.; Suzuki, H.; Taniguchi, K.; Noguchi, T.; Yohda, M.; Odaka, M., J. Biol. Chem. 2008, 283, 36617-36623.

51. Rao, S.; Holz, R. C., Biochemistry 2008, 47, 12057-12064. 
52. Mitra, S.; Holz, R. C., J. Biol. Chem. 2007, 282, 7397-7404.

53. Yamanaka, Y.; Hashimoto, K.; Ohtaki, A.; Noguchi, K.; Yohda, M.; Odaka, M., J. Biol. Inorg. Chem. 2010, 15, 655-665.

54. Song, L.; Wang, M.; Yang, X.; Qian, S., Biotechnol. J. 2007, 2, 717-724.

55. Song, L.; Wang, M.; Shi, J.; Xue, Z.; Wang, M.-X.; Qian, S., Biochem. Biophys. Res. Commun. 2007, 362, 319-324.

56. Arakawa, T.; Kawano, Y.; Katayama, Y.; Nakayama, H.; Dohmae, N.; Yohda, M.; Odaka, M., J. Am. Chem. Soc. 2009, 131, 14838-14843.

57. Nagasawa, T.; Nanba, H.; Ryuno, K.; Takeuchi, K.; Yamada, H., Eur. J. Biochem. 1987, 162, 691-698.

58. Nagasawa, T.; Takeuchi, K.; Yamada, H., Eur. J. Biochem. 1991, 196, 581-589.

59. Wieser, M.; Takeuchi, K.; Wada, Y.; Yamada, H.; Nagasawa, T., FEMS Microbiol. Lett. 1998, 169, 17-22.

60. Tyler, L. A.; Noveron, J. C.; Olmstead, M. M.; Mascharak, P. K., Inorg. Chem. 2003, $42,5751-5761$.

61. Noguchi, T.; Honda, J.; Nagamune, T.; Sasabe, H.; Inoue, Y.; Endo, I., FEBS Lett. 1995, 358, 9-12.

62. Noguchi, T.; Hoshino, M.; Tsujimura, M.; Odaka, M.; Inoue, Y.; Endo, I., Biochemistry. 1996, 35, 16777-16781.

63. Tsujimura, M.; Odaka, M.; Nagashima, S.; Yohda, M.; Endo, I., J. Biochem. 1996, $119,407-413$.

64. Hopmann, K. H.; Guo, J. D.; Himo, F., Inorg. Chem. 2007, 46, 4850-4856.

65. Swartz, R. D.; Coggins, M. K.; Kaminsky, W.; Kovacs, J. A., J. Am. Chem. Soc. 
2011, 133, 3954-3963.

66. Dey, A.; Jeffrey, S. P.; Darensbourg, M.; Hodgson, K. O.; Hedman, B.; Solomon, E. I., Inorg. Chem. 2007, 46, 4989-4996.

67. Jensen, C. M.; Trogler, W. C., J. Am. Chem. Soc. 1986, 108, 723-729

68. Garcia-Alvarez, R.; Diez, J.; Crochet, P.; Cadierno, V., Organometallics. 2010, 29, 3955-3965.

69. Cadierno, V.; Díez, J.; Francos, J.; Gimeno, J., Chem. Eur. J. 2010, 16, 9808-9817.

70. García-Álvarez, R.; Díez, J.; Crochet, P.; Cadierno, V., Organometallics. 2011, 30, $5442-5451$.

71. Muranaka, M.; Hyodo, I.; Okumura, W.; Oshiki, T., Catal. Today. 2011, 164, $552-555$.

72. Noveron, J. C.; Olmstead, M. M.; Mascharak, P. K., J. Am. Chem. Soc. 1999, $121,3553-3554$.

73. Crisóstomo, C.; Crestani, M. G.; García, J. J., Inorg. Chim. Acta. 2010, 363, 1092-1096.

74. Crisóstomo, C.; Crestani, M. G.; García, J. J., J. Mol. Catal. A: Chem. 2007, $266,139-148$.

75. Hopmann, K. H.; Himo, F., Eur. J. of Inorg. Chem. 2008, 1406-1412.

76. Deutsch, E.; Root, M. J.; Nosco, D. L., Adv. Inorg Bioinorg. Mech. 1982, 1, 269-389.

77. Ellis, K. J.; Lappin, A. G.; McAuley, A., J. Chem. Soc. 1975, 1930-1934.

78. O'Toole, M. G.; Kreso, M.; Kozlowski, P. M.; Mashuta, M. S.; Grapperhaus, C. A., J. Biol. Inorg. Chem. 2008, 13, 1219-1230.

79. Buonomo, R. M.; Font, I.; Maguire, M. J.; Reibenspies, J. H.; Tuntulani, T.; 
Darensbourg, M. Y., J. Am. Chem. Soc. 1995, 117, 963-973.

80. Lugo-Mas, P.; Dey, A.; Xu, L.; Davin, S. D.; Benedict, J.; Kaminsky, W.; Hodgson, K. O.; Hedman, B.; Solomon, E. I.; Kovacs, J. A., J. Am. Chem. Soc. 2006, 128, 11211-11221.

81. Cornman, C. R.; Stauffer, T. C.; Boyle, P. D., J. Am. Chem. Soc. 1997, 119, 5986-5987.

82. Kung, I.; Schweitzer, D.; Shearer, J.; Taylor, W. D.; Jackson, H. L.; Lovell, S.;

Kovacs, J. A., J. Am. Chem. Soc. 2000, 122, 8299-8300.

83. Heinrich, L.; Li, Y.; Vaissermann, J.; Chottard, J. C., Eur. J. Inorg. Chem. 2001, 1407-1409.

84. Heinrich, L.; Li, Y.; Vaissermann, J.; Chottard, G.; Chottard, J.-C., Angew. Chem Int. Ed. 1999, 38, 3526-3528.

85. Shearer, J.; Jackson, H. L.; Schweitzer, D.; Rittenberg, D. K.; Leavy, T. M.; Kaminsky, W.; Scarrow, R. C.; Kovacs, J. A., J. Am. Chem. Soc. 2002, 124, $11417-11428$

86. Dey, A.; Chow, M.; Taniguchi, K.; Lugo-Mas, P.; Davin, S.; Maeda, M.; Kovacs, J. A.; Odaka, M.; Hodgson, K. O.; Hedman, B.; Solomon, E. I., J. Am. Chem. Soc. 2006, $128,533-541$.

87. Harrop, T. C.; Olmstead, M. M.; Mascharak, P. K., Inorg. Chem. 2005, 44, 9527-9533.

88. Rose, M. J.; Betterley, N. M.; Mascharak, P. K., J. Am. Chem. Soc. 2009, 131, 8340-8341.

89. Heinrich, L.; Mary-Verla, A.; Li, Y.; Vaissermann, J.; Chottard, J. C., Eur. 
J. Inorg. Chem. 2001, 2203-2206.

90. Shearer, J.; Callan, P. E.; Amie, J., Inorg. Chem. 2010, 49, 9064-9077.

91. Laurent Heinrich, A. M.-V., Yun Li, Jacqueline Vaissermann, Jean-Claude Chottard, Eur. J. Inorg. Chem. 2001, 2001, 2203-2206.

92. Rat, M.; Sousa, Rodolphe Alves d.; Tomas, A.; Frapart, Y.; Tuchagues, J.-P.; Artaud, I., Eur. J. Inorg. Chem. 2003, 2003, 759-765.

93. Grapperhaus, C. A.; Li, M.; Patra, A. K.; Poturovic, S.; Kozlowski, P. M.; Zgierski, M. Z.; Mashuta, M. S., Inorg. Chem. 2003, 42, 4382-4388.

94. Grapperhaus, C. A.; O'Toole, M. G.; Mashuta, M. S., Inorg. Chem. Commun. 2006, 9, 1204-1206.

95. Hoffmann, P.; Steinhoff, A.; Mattes, R., Z. Naturforsch., B: Chem.Sci 1987, 42, $867-873$.

96. Grapperhaus, C. A.; Patra, A. K.; Mashuta, M. S., Inorg. Chem. 2002, 41, 1039-1041.

97. Parry, R., Inorg. Synth. 1970, 12, 237-240.

98. SMART (v.5632), Bruker Advance X-ray Solutions, Inc: Madison, WI, 2005.

99. SAINT (V6.45A), Bruker Advanced X-ray Solutions, Inc.: Madison, WI 2003.

100. Sheldrick, G. M., Acta Crystallogr., Sect A: Found. Crystallogr. 1990, A46, 467.

101. Sheldrick, G. M. SHELXL-97. Program for the Refinement of Crystal Structures, University Gottingen Gottingen, Germany, 1997.

102. SHELXTL (v6.14), Program Library for Structure Solution and Molecular Graphics, Bruker Advanced X-ray Solutions, Inc: Madison, WI, 2000.

103. Boone, A. J.; Chang, C. H.; Greene, S. N.; Herz, T.; Richards, N. G. J., Coord. 
Chem. Rev. 2003, 238-239, 291-314.

104. Peplowski, L.; Kubiak, K.; Zelek, S.; Nowak, W., Int. J. Quantum Chem. 2008, 108, 161-179.

105. Humphrey, W.; Dalke, A.; Schulten, K., J. Mol. Graphics 1996, 14, 33-38.

106. Shigehiro, S.; Nakasako, M.; Dohmae, N.; Tsujimura, M.; Tokoi, K.; Odaka, M.; Yohda, M.; Kamiya, N.; Endo, I., Nat. Struct. Biol. 1998, 5, 347-351.

107. Endo, I.; Nakasako, M.; Nagashima, S.; Dohmae, N.; Tsujimura, M.; Takio, K.; Odaka, M.; Yohda, M.; Kamiya, N., J. Inorg. Biochem. 1999, 74, 22-22.

108. Dilworth, J.; Zheng, Y.: Lu, S.; Wu, Q., Transition Met. Chem. 1992, 17, 364-368.

109. O'Toole, M. G.; Bennett, B.; Mashuta, M. S.; Grapperhaus, C. A., Inorg. Chem. 2009, 48, 2300-2308.

110. Lugo-Mas, P.; Taylor, W.; Schweitzer, D.; Theisen, R. M.; Xu, L.; Shearer, J.; Swartz, R. D.; Gleaves, M. C.; DiPasquale, A.; Kaminsky, W.; Kovacs, J. A., Inorg. Chem. 2008, 47, 11228-11236.

111. Harrop, T. C.; Mascharak, P. K., Acc. Chem. Res. 2004, 37, 253-260.

112. Rose, M. J.; Betterley, N. M.; Oliver, A. G.; Mascharak, P. K., Inorg. Chem. 2010, 49, 1854-1864.

113. Adzamli, I. K.; Libson, K.; Lydon, J. D.; Elder, R. C.; Deutsch, E., Inorg. Chem. 1979, 18, 303-311.

114. Herting, D. L.; Sloan, C. P.; Cabral, A. W.; Krueger, J. H. Inorg. Chem, 1978, 17, 1649-54. 
115. Lydon, J. D.; Deutsch, E., Inorg. Chem. 1982, 21, 3180-3185.

116. Farmer, P. J.; Solouki, T.; Mills, D. K.; Soma, T.; Russell, D. H.; Reibenspies, J. H.; Darensbourg, M. Y., J. Am. Chem. Soc. 1992, 114, 4601-4605.

117. Farmer, P. J.; Solouki, T.; Soma, T.; Russell, D. H.; Darensbourg, M. Y., Inorg. Chem. 1993, 32, 4171-4172.

118. Yano, T.; Wasada-Tsutsui, Y.; Arii, H.; Yamaguchi, S.; Funahashi, Y.; Ozawa, T.; Masuda, H., Inorg. Chem. 2007, 46, 10345-10353.

119. Mirza, S. A.; Pressler, M. A.; Kumar, M.; Day, R. O.; Maroney, M. J., Inorg. Chem. 1993, 32, 977-987.

120. Farmer, P. J.; Verpeaux, J.-N.; Amatore, C.; Darensbourg, M. Y.; Musie, G., J. Am. Chem. Soc. 1994, 116, 9355-9356.

121. Noveron, J. C.; Olmstead, M. M.; Mascharak, P. K., J. Am. Chem. Soc. 2001, 123, 3247-3259.

122. Kaasjager, V. E.; Bouwman, E.; Gorter, S.; Reedijk, J.; Grapperhaus, C. A.; Reibenspies, J. H.; Smee, J. J.; Darensbourg, M. Y.; Derecskei-Kovacs, A.; Thomson, L. M., Inorg. Chem. 2002, 41, 1837-1844.

123. Lee, C.-M.; Hsieh, C.-H.; Dutta, A.; Lee, G.-H.; Liaw, W.-F., J. Am. Chem. Soc. 2003, 125, 11492-11493.

124. Galardon, E.; Giorgi, M.; Artaud, I., Chem. Commun. 2004, 286-287.

125. Chen, H.-W.; Lin, C.-W.; Chen, C.-C.; Yang, L.-B.; Chiang, M.-H.; Liaw, W.-F., Inorg. Chem. 2005, 44, 3226-3232.

126. Lee, C.-M.; Chen, C.-H.; Chen, H.-W.; Hsu, J.-L.; Lee, G.-H.; Liaw, W.-F., Inorg. Chem. 2005, 44, 6670-6679. 
127. Chohan, B. S.; Maroney, M. J., Inorg. Chem. 2006, 45, 1906-1908.

128. Grapperhaus, C. A.; Darensbourg, M. Y.; Sumner, L. W.; Russell, D. H., J. Am. Chem. Soc. 1996, 118, 1791-1792.

129. Masitas, C. A.; Mashuta, M. S.; Grapperhaus, C. A., Inorg. Chem. 2010, 49, 5344-5346.

130. Grapperhaus, C. A.; Poturovic, S.; Mashuta, M. S., Inorg. Chem. 2005, 44, 8185-8187.

131. Grapperhaus, C. A.; Maguire, M. J.; Tuntulani, T.; Darensbourg, M. Y., Inorg. Chem. 1997, 36, 1860-1866.

132. Noguchi, T.; Nojiri, M.; Takei, K.-i.; Odaka, M.; Kamiya, N., Biochemistry 2003, $42,11642-11650$.

133. Grapperhaus, C. A.; Darensbourg, M. Y., Acc. Chem. Res. 1998, 31, 451-459.

134. Henderson, W.; McIndoe, J. S., Ionisation Techniques. In Mass Spectrometry of Inorganic, Coordination and Organometallic Compounds, John Wiley \& Sons, Ltd: 2005; pp 47-105.

135. Farmer, P. J.; Reibenspies, J. H.; Lindahl, P. A.; Darensbourg, M. Y., J. Am. Chem. Soc. 1993, $115,4665-4674$.

136. Grapperhaus, C. A.; Mullins, C. S.; Kozlowski, P. M.; Mashuta, M. S., Inorg. Chem. 2004, 43, 2859-2866.

137. Kumar, M.; Colpas, G. J.; Day, R. O.; Maroney, M. J., J. Am. Chem. Soc. 1989, 111, 8323-8325.

138. Bellefeuille, J. A.; Grapperhaus, C. A.; Derecskei-Kovacs, A.; Reibenspies, J. H.; 
Darensbourg, M. Y., Inorg. Chim. Acta 2000, 300, 73-81.

139. Chang, C. H.; Boone, A. J.; Bartlett, R. J.; Richards, N. G. J., Inorg. Chem. 2003, 43, 458-472.

140. Kennepohl, P.; Neese, F.; Schweitzer, D.; Jackson, H. L.; Kovacs, J. A.; Solomon, E. I., Inorg. Chem. 2005, 44, 1826-1836.

141. Masitas, C. A.; Kumar, M.; Mashuta, M. S.; Kozlowski, P. M.; Grapperhaus, C. A., Inorg. Chem. 2010, 49, 10875-10881.

142. Brines, L. M.; Kovacs, J. A., Eur. J. Inorg. Chem. 2007, 29-38.

143. Brines, L. M.; Shearer, J.; Fender, J. K.; Schweitzer, D.; Shoner, S. C.; Barnhart, D.; Kaminsky, W.; Lovell, S.; Kovacs, J. A., Inorg. Chem. 2007, 46, 9267-9277.

144. Tuntulani, T.; Musie, G.; Reibenspies, J. H.; Darensbourg, M. Y., Inorg. Chem. 1995, 34, 6279-6286.

145. Sellmann, D.; Ruf, R.; Knoch, F.; Moll, M., Inorg. Chem. 1995, 34, 4745-4755.

146. Sellmann, D.; Engl, K.; Heinemann, F. W.; Sieler, J., Eur. J. Inorg. Chem. 2000, 1079-1089.

147. Prabhakaran, R.; Huang, R.; Karvembu, R.; Jayabalakrishnan, C.; Natarajan, K., Inorg. Chim. Acta 2007, 360, 691-694.

148. Gunasekaran, N.; Remya, N.; Radhakrishnan, S.; Karvembu, R., J. Coord. Chem. 2011, 64, 491-501.

149. Circu, M. L.; Aw, T. Y., Free Radical Biol. Med. 2010, 48, 749-762. 


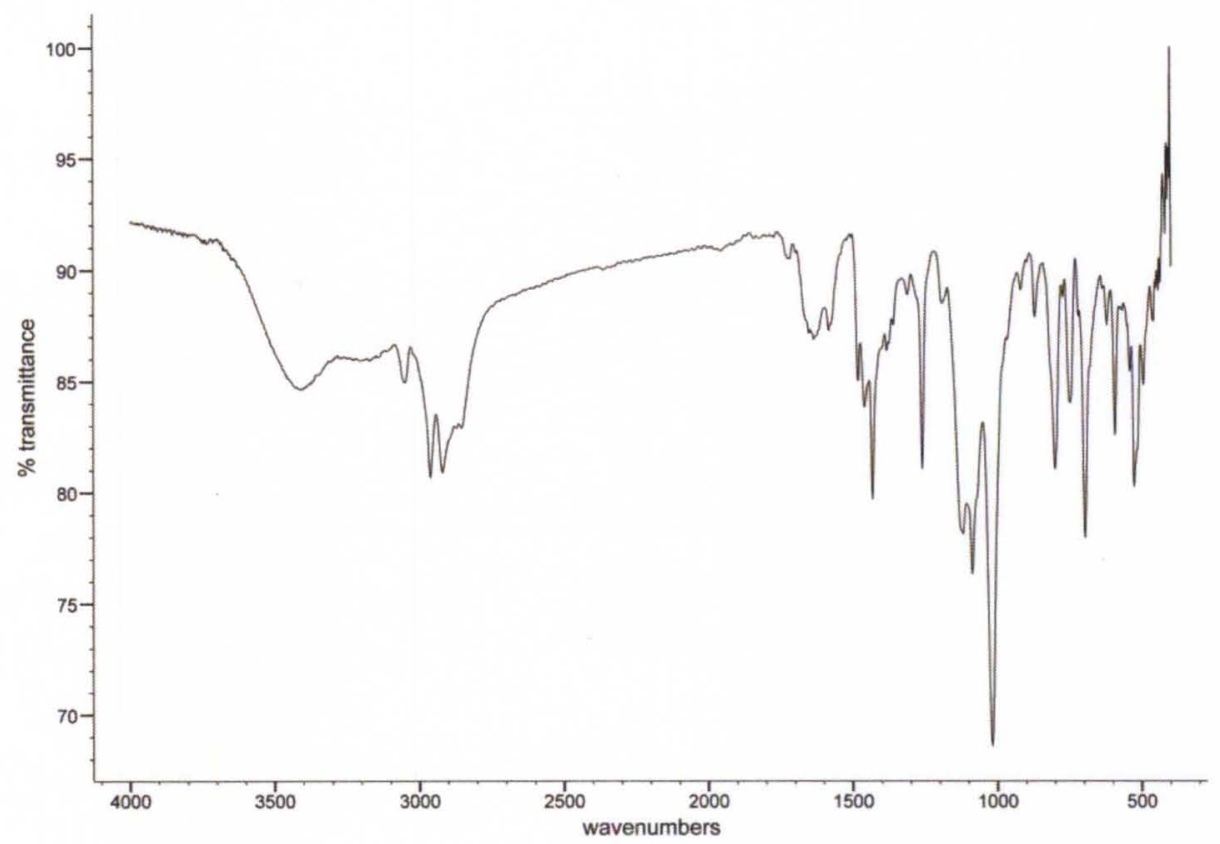

Figure A1. FT-IR of $\mathrm{O}_{2}+($ bmmp-TASN)RuPPh 3 after 96 hours as $\mathrm{KBr}$ pellets.

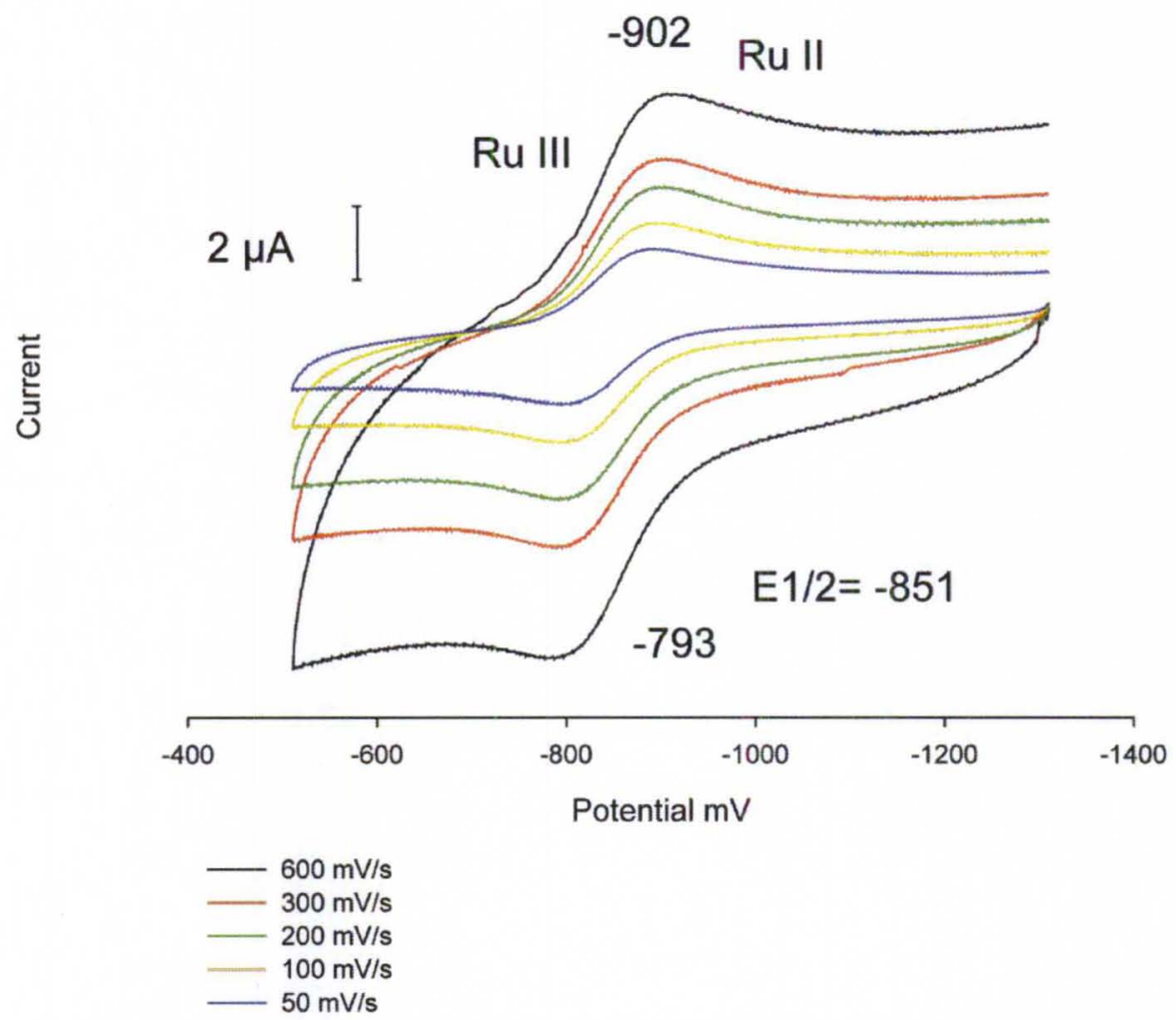

Figure A2. Cyclic voltammogram of 1 in acetonitrile with $0.1 \mathrm{M}$ tetrabutylammonium hexafluorophosphate as supporting electrolyte. Potentials referenced to $\mathrm{Fc} / \mathrm{Fc}^{+}$. 


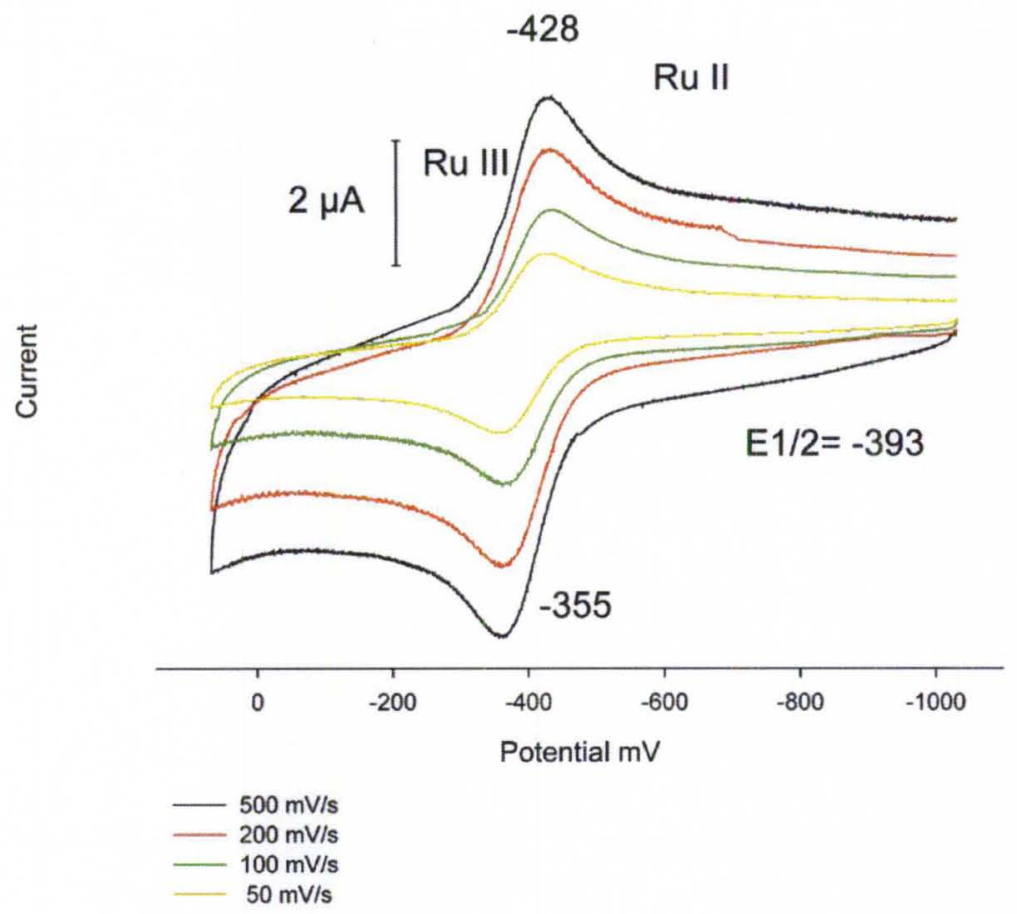

Figure A3. Cyclic voltammogram of $\mathbf{2}$ in acetonitrile with $0.1 \mathrm{M}$ tetrabutylammonium hexafluorophosphate as supporting electrolyte. Potentials referenced to $\mathrm{Fc} / \mathrm{Fc}^{+}$. 


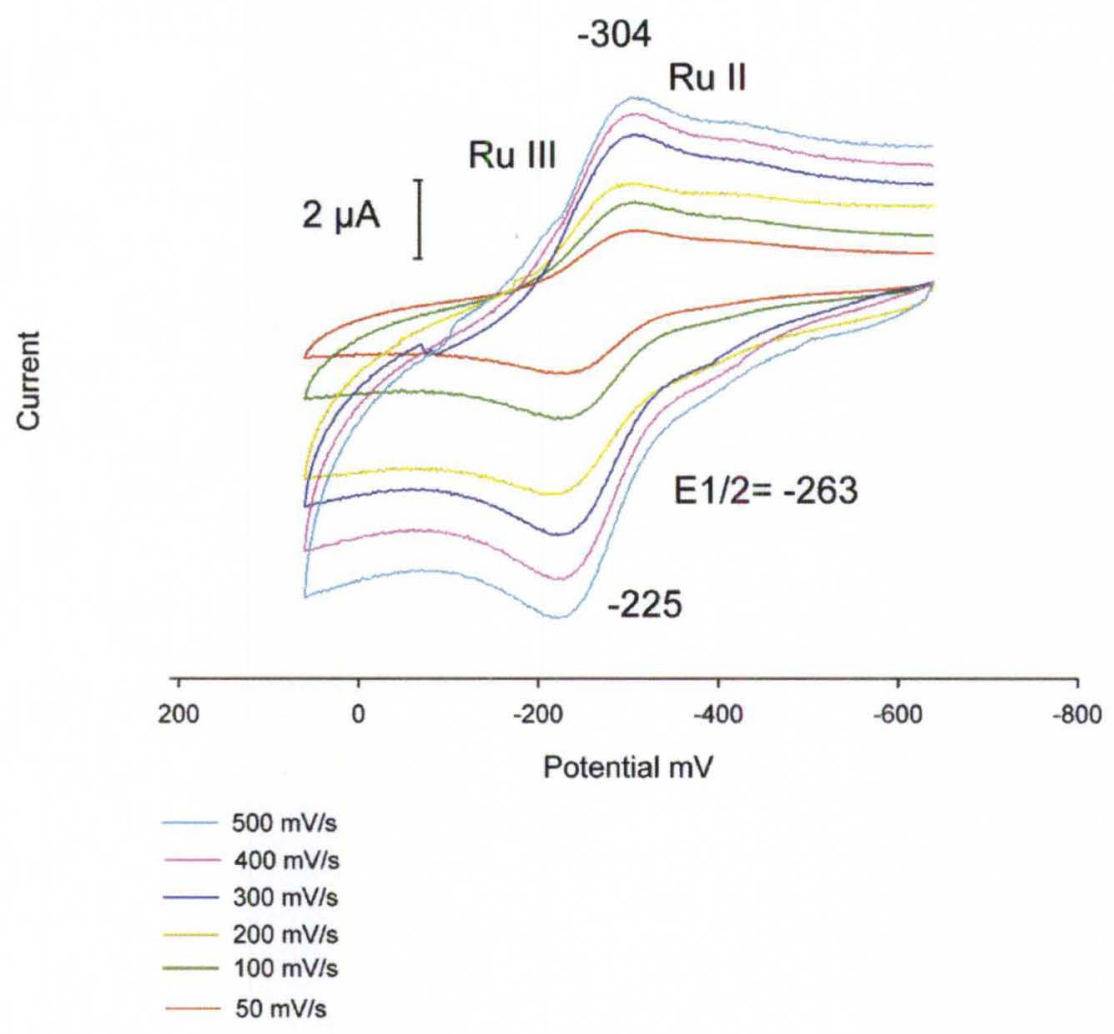

Figure A4. Cyclic voltammogram of 3 in acetonitrile with $0.1 \mathrm{M}$ tetrabutylammonium hexafluorophosphate as supporting electrolyte. Potentials referenced to $\mathrm{Fc} / \mathrm{Fc}^{+}$. 
+TOF MS: 0.017 to $0.183 \mathrm{~min}$ from 07211020 wiff

Max. 1010.9 counts.

$a=3.55581864568315810 e-004, t 0=4.64411847614901490 e+001$
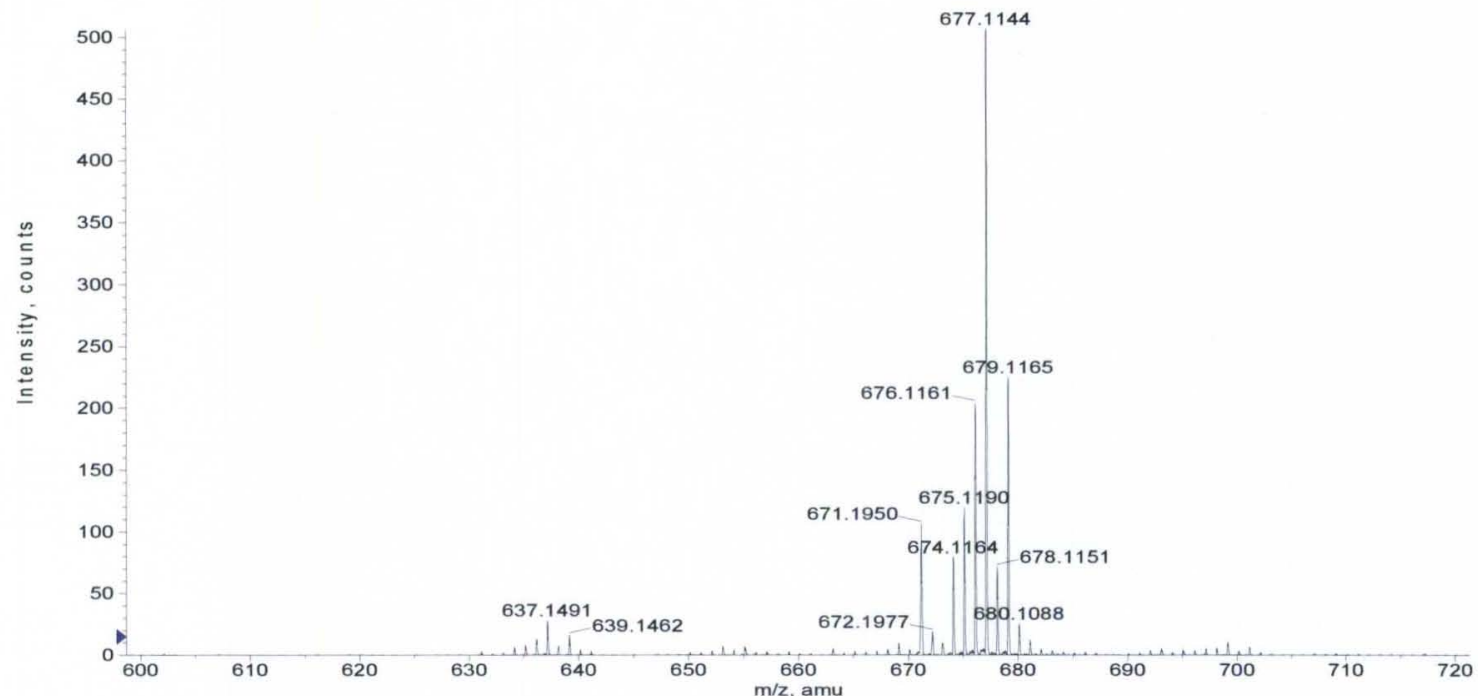

+TOF MS: 0.017 to $0.183 \mathrm{~min}$ from 07211020 wiff

Max. 10109 counts. 作
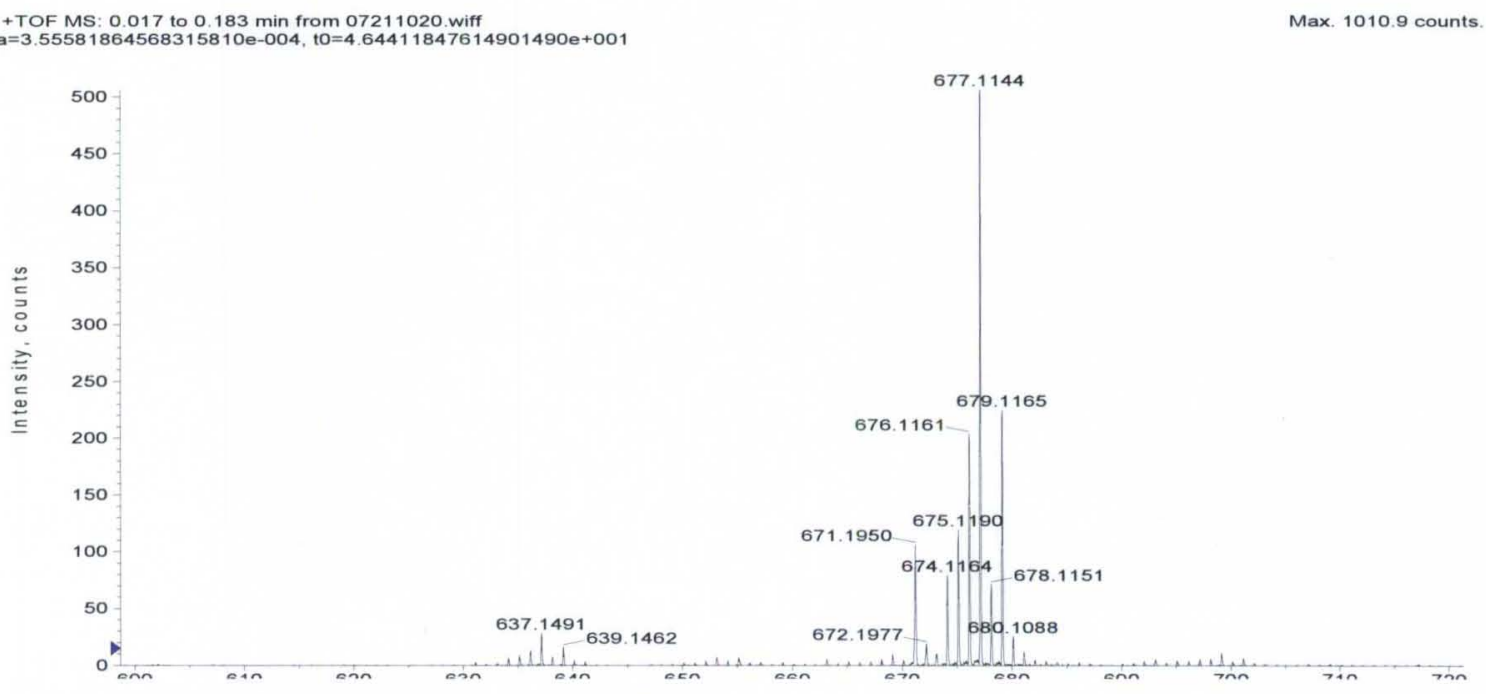

Figure A5. +ESI MS for compound 8. 
+TOF MS: 0.083 to $0.167 \mathrm{~min}$ from 07211021 wifi $a=3.55581864568315810 e-004$, to $=4.64411847614901490 e+001$

Max. 469.3 counts

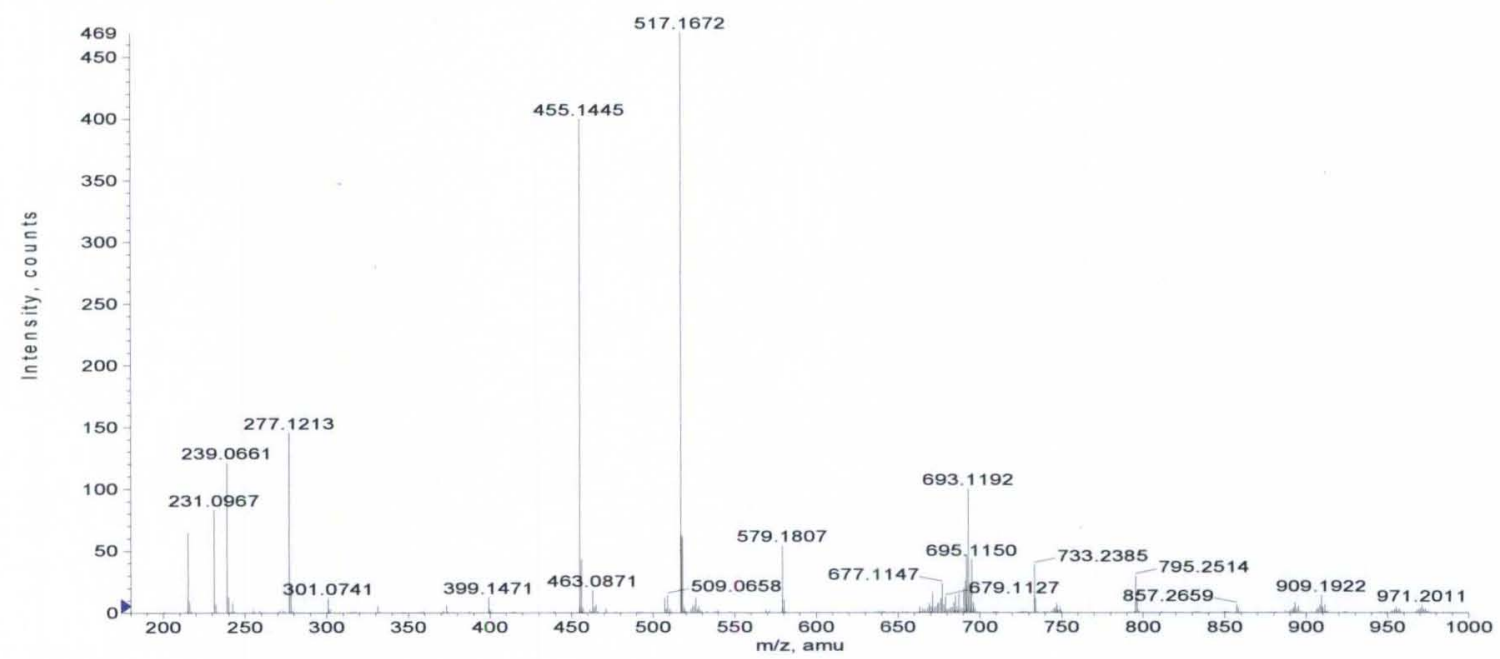

+TOF MS: 0.083 to $0.167 \mathrm{~min}$ from 07211021 wiff

$a=3.55581864568315810 e-004, t 0=4.64411847614901490 e+001$

Max. 469.3 counts

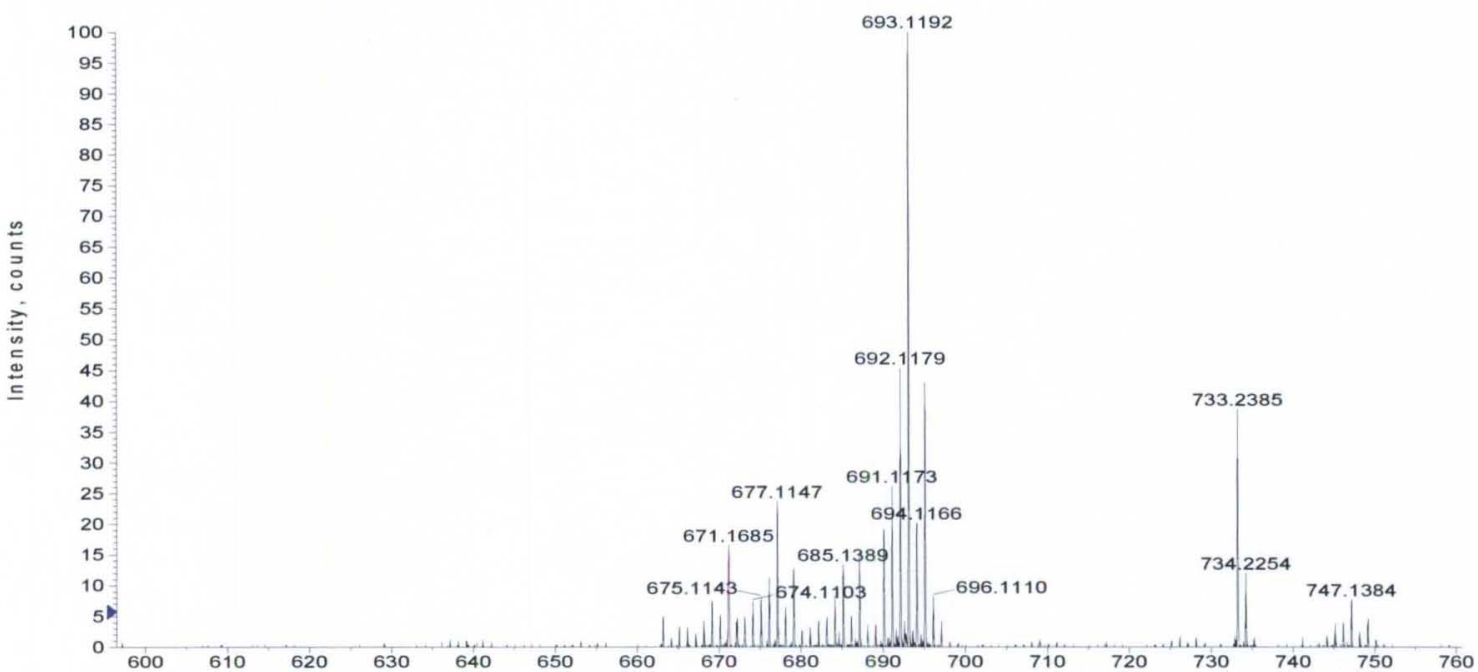

Figure A6. +ESI MS for compound 9 . 


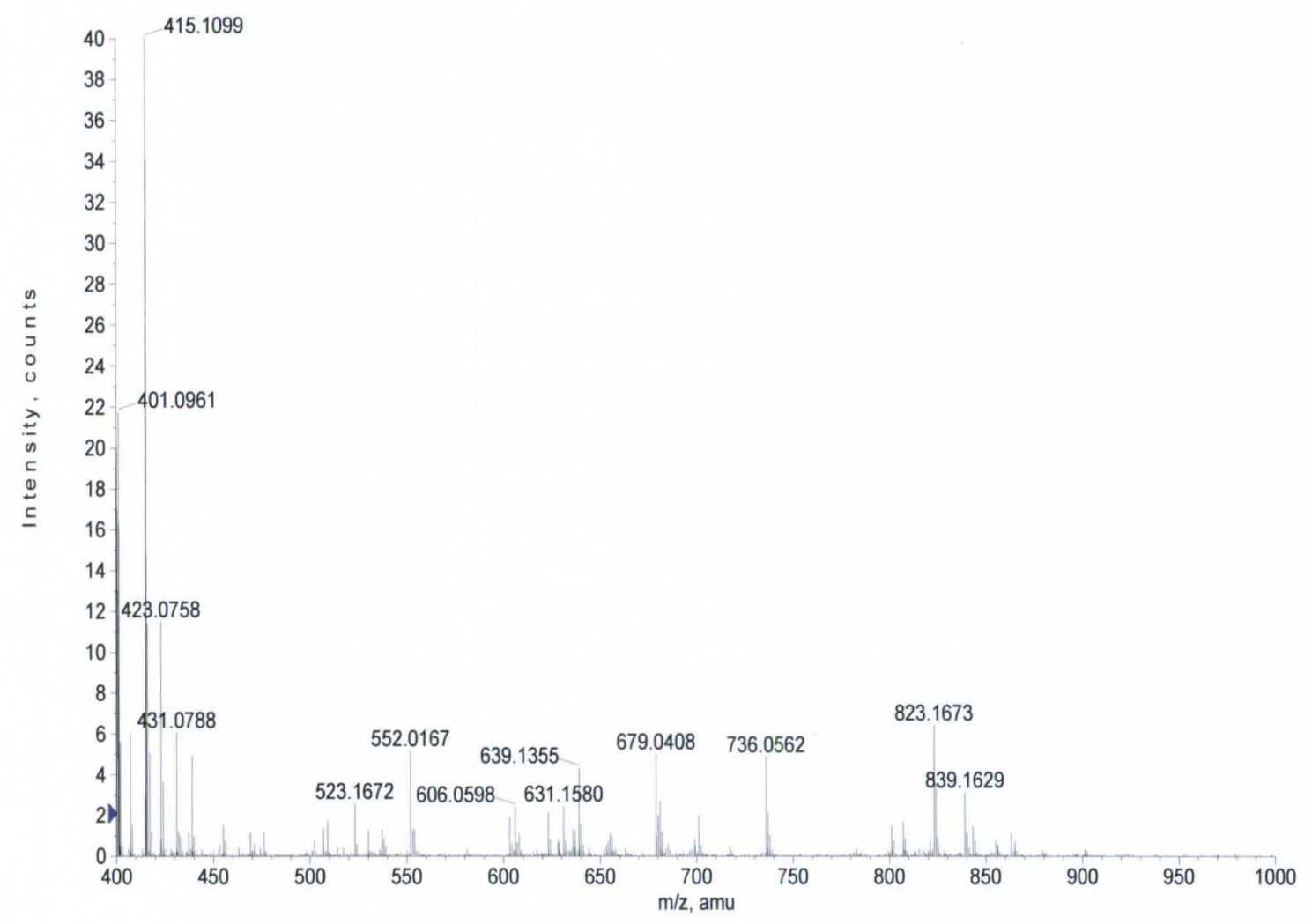

Figure A7. +ESI MS for compound (bmmp- $\left.\mathrm{O}_{2}-\mathrm{TASN}\right) \mathrm{Ru}\left(\mathrm{Me}\left(\mathrm{PPh}_{2}\right)_{2}\right.$. 
+TOF MS: 0.417 to $1.783 \mathrm{~min}$ from 01251032.wiff $a=3.55603402798775250 \mathrm{e}-004, \mathrm{t} 0=4.71856293243654360 \mathrm{e}+001$

Max. 2.9 counts.

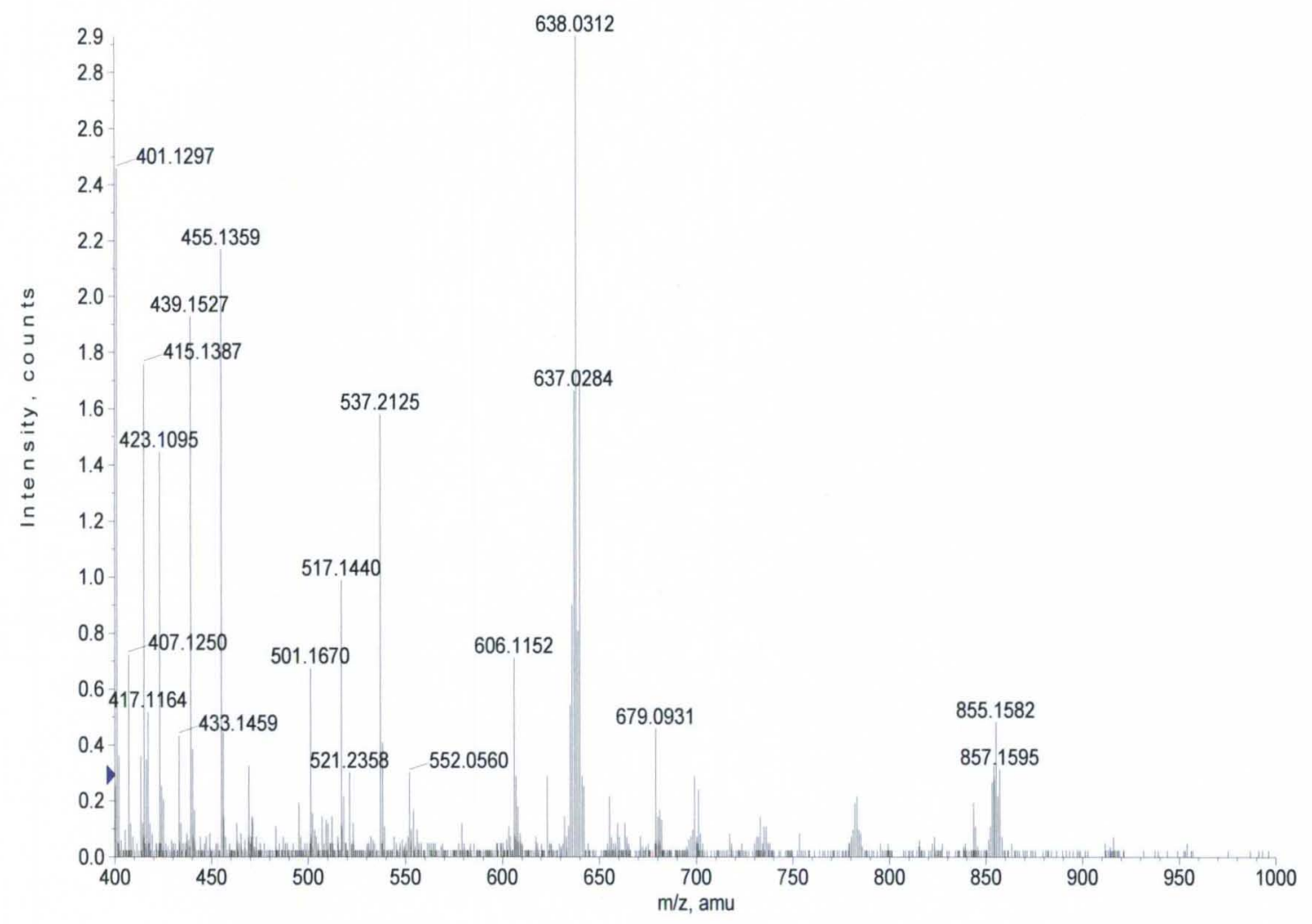

Figure A8. +ESI MS for compound (bmmp- $\left.\mathrm{O}_{3}-\mathrm{TASN}\right) \mathrm{Ru}\left(\mathrm{Me}\left(\mathrm{PPh}_{2}\right)_{2}\right.$. 
TABLE A1 Optimized coordinates of 1 (B3LYP)

\begin{tabular}{|c|c|c|c|}
\hline $\mathrm{C}$ & -2.140479 & -3.835227 & 1.847745 \\
\hline $\mathrm{C}$ & 0.297767 & -3.589939 & 2.173125 \\
\hline $\mathrm{C}$ & -0.917465 & -2.987333 & 1.434361 \\
\hline C & 2.039153 & 0.854411 & 4.097420 \\
\hline $\mathrm{C}$ & 3.301908 & 0.320181 & 4.360255 \\
\hline C & -0.613516 & -3.069885 & -0.084967 \\
\hline C & -3.571911 & -1.364095 & -0.193658 \\
\hline $\mathrm{C}$ & $-3,318107$ & 2.447163 & 2.663376 \\
\hline C & -2.745212 & -2.280445 & -1.087370 \\
\hline C & -3.703127 & 0.811114 & 0.848766 \\
\hline C & 1.492245 & 0.781115 & 2.816152 \\
\hline $\mathrm{C}$ & 4.025498 & -0.275513 & 3.327261 \\
\hline $\mathrm{C}$ & -3.123587 & 2.206678 & 1.150846 \\
\hline $\mathrm{C}$ & 2.211772 & 0.181743 & 1.769189 \\
\hline $\mathrm{C}$ & 3.487606 & -0.337598 & 2.039182 \\
\hline C & -3.848526 & 3.343469 & 0.402970 \\
\hline $\mathrm{C}$ & -0.662705 & -2.109464 & -2.335331 \\
\hline C & -3.636171 & 0.592262 & -1.650032 \\
\hline $\mathrm{C}$ & 2.845440 & -2.209687 & -0.493640 \\
\hline $\mathrm{C}$ & 3.486717 & -3.120576 & -1.338171 \\
\hline $\mathrm{C}$ & 2.461357 & -0.937758 & -0.951386 \\
\hline $\mathrm{C}$ & -1.086656 & -0.949513 & -3.247982 \\
\hline $\mathrm{C}$ & -2.620699 & 1.403362 & -2.453635 \\
\hline $\mathrm{C}$ & 3.751160 & -2.784448 & -2.666199 \\
\hline $\mathrm{C}$ & 2.218108 & 1.851854 & -0.526729 \\
\hline C & 3.614874 & 2.014075 & -0.466673 \\
\hline C & 2.738502 & -0.615193 & -2.293366 \\
\hline $\mathrm{C}$ & 1.449893 & 2.908704 & -1.028257 \\
\hline C & 3.372635 & -1.526169 & -3.140168 \\
\hline C & 4.220992 & 3.189502 & -0.903505 \\
\hline C & 2.057779 & 4.091449 & -1.463902 \\
\hline C & 3.441851 & 4.235417 & -1.407232 \\
\hline $\mathrm{H}$ & -2.299349 & -3.745959 & 2.926897 \\
\hline $\mathrm{H}$ & -1.971161 & -4.898038 & 1.618775 \\
\hline $\mathrm{H}$ & 0.098290 & -3.629604 & 3.248540 \\
\hline $\mathrm{H}$ & -3.071905 & -3.536418 & 1.361729 \\
\hline $\mathrm{H}$ & 0.509947 & -4.614920 & 1.830342 \\
\hline $\mathrm{H}$ & 1.467904 & 1.322992 & 4.894432 \\
\hline $\mathrm{H}$ & 3.720573 & 0.369953 & 5.362337 \\
\hline
\end{tabular}




\begin{tabular}{|c|c|c|c|}
\hline $\mathrm{H}$ & 1.190665 & -2.976085 & 2.033020 \\
\hline $\mathrm{H}$ & -3.407762 & -1.628208 & 0.848603 \\
\hline $\mathrm{H}$ & -4.383546 & 2.409366 & 2.938862 \\
\hline $\mathrm{H}$ & -0.862292 & -4.081357 & -0.454653 \\
\hline $\mathrm{H}$ & -2.779504 & 1.696753 & 3.249035 \\
\hline $\mathrm{H}$ & -2.978713 & -3.325520 & -0.850365 \\
\hline $\mathrm{H}$ & -3.474561 & 0.174661 & 1.704052 \\
\hline$H$ & -4.642103 & -1.489406 & -0.429563 \\
\hline $\mathrm{H}$ & -4.801168 & 0.872349 & 0.747660 \\
\hline $\mathrm{H}$ & 0.456738 & -2.926496 & -0.228050 \\
\hline $\mathrm{H}$ & -2.933225 & 3.433839 & 2.940733 \\
\hline $\mathrm{H}$ & 0.508410 & 1.195875 & 2.621763 \\
\hline $\mathrm{H}$ & 5.013173 & -0.688708 & 3.516842 \\
\hline $\mathrm{H}$ & -3.032985 & -2.141289 & -2.132114 \\
\hline $\mathrm{H}$ & -4.911631 & 3.379880 & 0.684406 \\
\hline $\mathrm{H}$ & 2.655485 & -2.494394 & 0.535271 \\
\hline $\mathrm{H}$ & -0.924170 & -3.057785 & -2.836868 \\
\hline $\mathrm{H}$ & -3.965862 & -0.254257 & -2.259305 \\
\hline $\mathrm{H}$ & 3.781997 & -4.092570 & -0.951170 \\
\hline $\mathrm{H}$ & -4.527764 & 1.209741 & -1.484632 \\
\hline $\mathrm{H}$ & 4.076384 & -0.791703 & 1.249360 \\
\hline $\mathrm{H}$ & -3.398617 & 4.306405 & 0.665084 \\
\hline $\mathrm{H}$ & 0.420301 & -2.088856 & -2.214687 \\
\hline $\mathrm{H}$ & -3.790870 & 3.248932 & -0.684601 \\
\hline $\mathrm{H}$ & -2.114866 & -1.045630 & -3.607629 \\
\hline $\mathrm{H}$ & -2.462406 & 2.389073 & -2.010081 \\
\hline $\mathrm{H}$ & 4.238430 & 1.218833 & -0.071383 \\
\hline $\mathrm{H}$ & -0.437947 & -0.944019 & -4.128850 \\
\hline $\mathrm{H}$ & 4.251518 & -3.491304 & -3.322654 \\
\hline $\mathrm{H}$ & -2.967276 & 1.531934 & -3.485397 \\
\hline $\mathrm{H}$ & 0.370286 & 2.828873 & -1.048295 \\
\hline $\mathrm{H}$ & 2.468219 & 0.363730 & -2.678027 \\
\hline H & 5.301866 & 3.290938 & -0.846169 \\
\hline $\mathrm{H}$ & 3.580824 & -1.245683 & -4.169781 \\
\hline $\mathrm{H}$ & 1.437986 & 4.901281 & -1.840247 \\
\hline $\mathrm{H}$ & 3.913369 & 5.155006 & -1.744648 \\
\hline $\mathrm{N}$ & -1.264039 & -2.054535 & -0.972994 \\
\hline$N$ & -3.155823 & 0.058210 & -0.330122 \\
\hline $\mathrm{P}$ & 1.447078 & 0.250397 & 0.076453 \\
\hline$S$ & -1.121851 & -1.204769 & 1.993296 \\
\hline$S$ & -1.291696 & 2.262745 & 0.765757 \\
\hline$S$ & -0.914853 & 0.716503 & -2.420997 \\
\hline $\mathrm{Ru}$ & -0.909045 & 0.006284 & -0.139162 \\
\hline
\end{tabular}


TABLE A2 Optimized coordinates of 1 (BP86)

\begin{tabular}{|c|c|c|c|}
\hline $\mathrm{C}$ & -2.131546 & -3.825739 & 1.859745 \\
\hline C & 0.309585 & -3.548301 & 2.217010 \\
\hline C & -0.905619 & -2.975099 & 1.447133 \\
\hline $\mathrm{C}$ & 1.990536 & 0.844279 & 4.120143 \\
\hline $\mathrm{C}$ & 3.261510 & 0.308356 & 4.385356 \\
\hline $\mathrm{C}$ & -0.579889 & -3.067921 & -0.068478 \\
\hline $\mathrm{C}$ & -3.546896 & -1.389451 & -0.206361 \\
\hline $\mathrm{C}$ & -3.325452 & 2.437085 & 2.649352 \\
\hline $\mathrm{C}$ & -2.711305 & -2.294095 & -1.105908 \\
\hline $\mathrm{C}$ & -3.706260 & 0.794377 & 0.827868 \\
\hline C & 1.442710 & 0.773219 & 2.830520 \\
\hline $\mathrm{C}$ & 3.993437 & -0.285406 & 3.346251 \\
\hline $\mathrm{C}$ & -3.131202 & 2.191428 & 1.133488 \\
\hline $\mathrm{C}$ & 2.171548 & 0.174794 & 1.778637 \\
\hline $\mathrm{C}$ & 3.455294 & -0.345529 & 2.048944 \\
\hline $\mathrm{C}$ & -3.838679 & 3.334896 & 0.372582 \\
\hline $\mathrm{C}$ & -0.608018 & -2.117080 & -2.335872 \\
\hline $\mathrm{C}$ & -3.618647 & 0.569514 & -1.683730 \\
\hline C & 2.842165 & -2.203098 & -0.501847 \\
\hline $\mathrm{C}$ & 3.493876 & -3.107861 & -1.358328 \\
\hline $\mathrm{C}$ & 2.447561 & -0.922092 & -0.952486 \\
\hline $\mathrm{C}$ & -1.034720 & -0.960764 & -3.253247 \\
\hline C & -2.597980 & 1.392660 & -2.471701 \\
\hline $\mathrm{C}$ & 3.757891 & -2.757005 & -2.691434 \\
\hline $\mathrm{C}$ & 2.178995 & 1.862695 & -0.512140 \\
\hline C & 3.582600 & 2.023532 & -0.435675 \\
\hline $\mathrm{C}$ & 2.725832 & -0.584094 & -2.299331 \\
\hline $\mathrm{C}$ & 1.409630 & 2.926892 & -1.017881 \\
\hline $\mathrm{C}$ & 3.369853 & -1.489393 & -3.157720 \\
\hline $\mathrm{C}$ & 4.196185 & 3.208867 & -0.861974 \\
\hline $\mathrm{C}$ & 2.025036 & 4.119049 & -1.441598 \\
\hline $\mathrm{C}$ & 3.416991 & 4.263596 & -1.369297 \\
\hline $\mathrm{H}$ & -2.320349 & -3.700518 & 2.939824 \\
\hline $\mathrm{H}$ & -1.940753 & -4.902089 & 1.670682 \\
\hline $\mathrm{H}$ & 0.091607 & -3.570727 & 3.298641 \\
\hline $\mathrm{H}$ & -3.061708 & -3.554919 & 1.334987 \\
\hline $\mathrm{H}$ & 0.544624 & -4.584647 & 1.894622 \\
\hline $\mathrm{H}$ & 1.412725 & 1.313907 & 4.924677 \\
\hline $\mathrm{H}$ & 3.681700 & 0.356470 & 5.397016 \\
\hline $\mathrm{H}$ & 1.201331 & -2.915580 & 2.078293 \\
\hline
\end{tabular}




\begin{tabular}{|c|c|c|c|}
\hline $\mathrm{H}$ & -3.356250 & -1.645600 & 0.847201 \\
\hline $\mathrm{H}$ & -4.400029 & 2.409622 & 2.926911 \\
\hline $\mathrm{H}$ & -0.826135 & -4.090189 & -0.440943 \\
\hline H & -2.787800 & 1.676413 & 3.239814 \\
\hline $\mathrm{H}$ & -2.936428 & -3.354498 & -0.881418 \\
\hline $\mathrm{H}$ & -3.478242 & 0.149986 & 1.692588 \\
\hline $\mathrm{H}$ & -4.628114 & -1.526286 & -0.430022 \\
\hline $\mathrm{H}$ & -4.813420 & 0.849766 & 0.712386 \\
\hline $\mathrm{H}$ & 0.501512 & -2.918275 & -0.203120 \\
\hline $\mathrm{H}$ & -2.928310 & 3.429042 & 2.926730 \\
\hline $\mathrm{H}$ & 0.451039 & 1.194032 & 2.625971 \\
\hline $\mathrm{H}$ & 4.990505 & -0.699787 & 3.538061 \\
\hline $\mathrm{H}$ & -2.987926 & -2.141395 & -2.162621 \\
\hline $\mathrm{H}$ & -4.908303 & 3.396225 & 0.660689 \\
\hline $\mathrm{H}$ & 2.653218 & -2.496748 & 0.535683 \\
\hline $\mathrm{H}$ & -0.864139 & -3.081009 & -2.831792 \\
\hline $\mathbf{H}$ & -3.930668 & -0.293741 & -2.297472 \\
\hline $\mathrm{H}$ & 3.799781 & -4.088770 & -0.975568 \\
\hline $\mathrm{H}$ & -4.530069 & 1.176699 & -1.520615 \\
\hline $\mathrm{H}$ & 4.049513 & -0.799957 & 1.249221 \\
\hline $\mathrm{H}$ & -3.364544 & 4.299904 & 0.622139 \\
\hline $\mathrm{H}$ & 0.483534 & -2.087663 & -2.199304 \\
\hline H & -3.787570 & 3.222805 & -0.723076 \\
\hline $\mathrm{H}$ & -2.069288 & -1.058210 & -3.625431 \\
\hline $\mathrm{H}$ & -2.449590 & 2.386475 & -2.016517 \\
\hline H & 4.204628 & 1.216502 & -0.034295 \\
\hline $\mathrm{H}$ & -0.370165 & -0.935685 & -4.133319 \\
\hline $\mathrm{H}$ & 4.267120 & -3.461188 & -3.359296 \\
\hline H & -2.921523 & 1.520886 & -3.520765 \\
\hline $\mathrm{H}$ & 0.318668 & 2.840050 & -1.045371 \\
\hline $\mathrm{H}$ & 2.446061 & 0.406442 & -2.675879 \\
\hline $\mathrm{H}$ & 5.285702 & 3.311450 & -0.792882 \\
\hline $\mathrm{H}$ & 3.579810 & -1.196388 & -4.193452 \\
\hline $\mathrm{H}$ & 1.404308 & 4.938738 & -1.822375 \\
\hline $\mathrm{H}$ & 3.895630 & 5.193424 & -1.698787 \\
\hline$N$ & -1.225920 & -2.051609 & -0.970427 \\
\hline$N$ & -3.139562 & 0.041920 & -0.349744 \\
\hline $\mathrm{P}$ & 1.402704 & 0.247933 & 0.078039 \\
\hline$S$ & -1.127448 & -1.173649 & 1.974557 \\
\hline$S$ & -1.285265 & 2.230443 & 0.757387 \\
\hline $\mathrm{S}$ & -0.877260 & 0.706931 & -2.383202 \\
\hline Ru & -0.896692 & -0.003854 & -0.154391 \\
\hline
\end{tabular}


TABLE A3 Optimized coordinates of 2 (B3LYP)

\begin{tabular}{|c|c|c|c|}
\hline $\mathrm{C}$ & 3.433335 & 0.119184 & \\
\hline C & 4.118403 & -0.470413 & 3.255793 \\
\hline C & 2.192551 & 0.716138 & 4.089062 \\
\hline C & 0.560282 & -3.509690 & $2.285438^{\prime}$ \\
\hline $\mathrm{C}$ & 1.624010 & 0.708546 & 2.814965 \\
\hline C & -1.852695 & -3.963407 & 1.972080 \\
\hline $\mathrm{C}$ & 2.301800 & 0.107929 & 1.740505 \\
\hline C & -0.698644 & -3.040912 & 1.523451 \\
\hline C & 2.929479 & -2.163155 & -0.655126 \\
\hline $\mathrm{C}$ & -0.384233 & -3.167038 & 0.009275 \\
\hline $\mathrm{C}$ & 3.642416 & 2.079455 & -0.321356 \\
\hline $\mathrm{C}$ & 3.566062 & -3.017958 & -1.559955 \\
\hline C & 4.253632 & 3.246561 & -0.771743 \\
\hline $\mathrm{C}$ & -3.378324 & 2.071369 & 2.622911 \\
\hline C & 2.503247 & -0.880418 & -1.040720 \\
\hline $\mathrm{C}$ & 2.261376 & 1.872599 & -0.498927 \\
\hline C & 3.783479 & -2.613699 & -2.877226 \\
\hline $\mathrm{C}$ & 3.495840 & 4.234987 & -1.407213 \\
\hline $\mathrm{C}$ & -3.446766 & -1.713843 & -0.203388 \\
\hline $\mathrm{C}$ & -3.297364 & 1.882669 & 1.101288 \\
\hline $\mathrm{C}$ & -3.784889 & 0.459572 & 0.771295 \\
\hline $\mathrm{C}$ & -2.542919 & -2.585328 & -1.061045 \\
\hline C & 2.738083 & -0.486817 & -2.371765 \\
\hline $\mathrm{C}$ & -0.459692 & -2.296436 & -2.276499 \\
\hline C & 1.510590 & 2.869004 & -1.131952 \\
\hline $\mathrm{C}$ & 3.365419 & -1.342647 & -3.278783 \\
\hline $\mathrm{C}$ & 2.127811 & 4.042784 & -1.580315 \\
\hline $\mathrm{C}$ & -4.103599 & 2.983895 & 0.400832 \\
\hline C & -0.945652 & -1.199234 & -3.234184 \\
\hline C & -3.648581 & 0.179699 & -1.729165 \\
\hline $\mathrm{C}$ & -2.692976 & 1.027922 & -2.569232 \\
\hline $\mathbf{H}$ & 3.867602 & 0.120510 & 5.314570 \\
\hline $\mathrm{H}$ & 5.092696 & -0.925710 & 3.415618 \\
\hline $\mathrm{H}$ & 1.655509 & 1.190368 & 4.906102 \\
\hline $\mathrm{H}$ & 0.365145 & -3.516243 & 3.362292 \\
\hline $\mathrm{H}$ & 0.850769 & -4.529700 & 1.988811 \\
\hline $\mathrm{H}$ & 1.402861 & -2.835071 & 2.113811 \\
\hline $\mathrm{H}$ & -2.031680 & -3.834380 & 3.044031 \\
\hline H & -1.593073 & -5.018191 & 1.798873 \\
\hline $\mathrm{H}$ & 4.127939 & -0.910372 & 1.162326 \\
\hline $\mathrm{H}$ & 0.668396 & 1.194624 & 2.650310 \\
\hline
\end{tabular}




\begin{tabular}{|c|c|c|c|}
\hline $\mathrm{H}$ & 2.775917 & -2.503381 & 0.362730 \\
\hline $\mathrm{H}$ & 4.246905 & 1.329540 & 0.177657 \\
\hline $\mathrm{H}$ & -2.799040 & -3.768266 & 1.462143 \\
\hline $\mathrm{H}$ & 3.894375 & -3.999589 & -1.227878 \\
\hline $\mathrm{H}$ & 5.321152 & 3.386566 & -0.621186 \\
\hline $\mathrm{H}$ & -2.744639 & 1.351955 & 3.148677 \\
\hline $\mathrm{H}$ & 0.674112 & -2.962951 & -0.135581 \\
\hline $\mathrm{H}$ & -0.562573 & -4.208179 & -0.315434 \\
\hline $\mathrm{H}$ & -3.049234 & 3.074395 & 2.903585 \\
\hline $\mathrm{H}$ & -4.415196 & 1.935296 & 2.960636 \\
\hline $\mathrm{H}$ & -3.276283 & -1.934981 & 0.847314 \\
\hline $\mathrm{H}$ & -3.573259 & -0.165802 & 1.638139 \\
\hline $\mathrm{H}$ & -2.699275 & -3.639548 & -0.800364 \\
\hline $\mathrm{H}$ & 4.279518 & -3.277013 & -3.580649 \\
\hline $\mathrm{H}$ & 0.615459 & -2.196600 & -2.138729 \\
\hline $\mathrm{H}$ & 3.971346 & 5.148916 & -1.754465 \\
\hline $\mathrm{H}$ & -4.501853 & -1.922798 & -0.444618 \\
\hline $\mathrm{H}$ & -4.875513 & 0.469941 & 0.613335 \\
\hline $\mathrm{H}$ & -0.643499 & -3.277413 & -2.748688 \\
\hline $\mathrm{H}$ & 2.437256 & 0.503282 & -2.699911 \\
\hline $\mathrm{H}$ & -2.823061 & -2.491639 & -2.112374 \\
\hline $\mathrm{H}$ & -3.683508 & 3.968868 & 0.617925 \\
\hline H & 0.438384 & 2.771464 & -1.243904 \\
\hline $\mathrm{H}$ & 3.538531 & -1.008116 & -4.298577 \\
\hline $\mathrm{H}$ & -5.138935 & 2.960593 & 0.767681 \\
\hline $\mathrm{H}$ & 1.521199 & 4.809281 & -2.055232 \\
\hline $\mathrm{H}$ & -4.120839 & 2.877989 & -0.686254 \\
\hline $\mathrm{H}$ & -3.914407 & -0.716655 & -2.296415 \\
\hline $\mathrm{H}$ & -0.274251 & -1.172903 & -4.097334 \\
\hline $\mathrm{H}$ & -4.581367 & 0.739199 & -1.589725 \\
\hline $\mathrm{H}$ & -1.952346 & -1.387576 & -3.617272 \\
\hline $\mathrm{H}$ & -2.621640 & 2.050110 & -2.191433 \\
\hline $\mathrm{H}$ & -3.024577 & 1.044244 & -3.613366 \\
\hline$N$ & -1.090712 & -2.239775 & -0.928220 \\
\hline$N$ & -3.141055 & -0.265841 & -0.379529 \\
\hline $\mathrm{O}$ & -0.744857 & 2.620358 & 1.788985 \\
\hline $\mathrm{O}$ & -1.525621 & 3.005022 & -0.586576 \\
\hline $\mathrm{P}$ & 1.510638 & 0.254964 & 0.065782 \\
\hline$S$ & -1.047587 & -1.259517 & 2.017778 \\
\hline$S$ & -1.437260 & 2.049339 & 0.588805 \\
\hline$S$ & -0.931176 & 0.504778 & -2.474021 \\
\hline $\mathrm{Ru}$ & -0.896727 & -0.109503 & -0.145997 \\
\hline
\end{tabular}


TABLE A4 Optimized coordinates of 2 (BP86)

\begin{tabular}{|c|c|c|c|}
\hline $\mathrm{C}$ & $3.40488 \mathrm{I}$ & -0.131487 & 4.326814 \\
\hline $\mathrm{C}$ & 4.092042 & -0.664092 & 3.225934 \\
\hline $\mathrm{C}$ & 2.159925 & 0.488065 & 4.131933 \\
\hline $\mathrm{C}$ & 0.619685 & -3.521032 & 2.196841 \\
\hline $\mathrm{C}$ & 3.530994 & -0.586148 & 1.938865 \\
\hline $\mathrm{C}$ & 1.586491 & 0.556016 & 2.852983 \\
\hline $\mathrm{C}$ & -1.794343 & -4.028478 & 1.872561 \\
\hline $\mathrm{C}$ & 2.266203 & 0.010904 & 1.740045 \\
\hline $\mathrm{C}$ & -0.654609 & -3.075587 & 1.439953 \\
\hline $\mathrm{C}$ & 2.928568 & -2.106410 & -0.796474 \\
\hline $\mathrm{C}$ & -0.338201 & -3.160018 & -0.078854 \\
\hline $\mathrm{C}$ & 3.591946 & 2.128063 & -0.131256 \\
\hline $\mathrm{C}$ & 3.573658 & -2.897623 & -1.763789 \\
\hline $\mathrm{C}$ & 4.214799 & 3.317347 & -0.532239 \\
\hline $\mathrm{C}$ & -3.386278 & 1.994191 & 2.674275 \\
\hline $\mathrm{C}$ & 2.476469 & -0.801354 & -1.101837 \\
\hline $\mathrm{C}$ & 2.219296 & 1.907890 & -0.392844 \\
\hline C & 3.771859 & -2.406395 & -3.063186 \\
\hline $\mathrm{C}$ & 3.477172 & 4.310739 & -1.199648 \\
\hline $\mathrm{C}$ & -3.417473 & -1.743785 & -0.244610 \\
\hline $\mathrm{C}$ & -3.314090 & 1.826534 & 1.147671 \\
\hline $\mathrm{C}$ & -3.775214 & 0.401642 & 0.793440 \\
\hline C & -2.508627 & -2.574330 & -1.139495 \\
\hline $\mathrm{C}$ & 2.693850 & -0.318581 & -2.415199 \\
\hline $\mathrm{C}$ & -0.420949 & -2.222629 & -2.347787 \\
\hline $\mathrm{C}$ & 1.486387 & 2.908291 & -1.057291 \\
\hline $\mathrm{C}$ & 3.328066 & -1.111977 & -3.384096 \\
\hline $\mathrm{C}$ & 2.115136 & 4.103043 & -1.454327 \\
\hline $\mathrm{C}$ & -4.11907 & 2.936409 & 0.455157 \\
\hline $\mathrm{C}$ & -0.921439 & -1.097963 & -3.266629 \\
\hline C & -3.644220 & 0.195125 & -1.728279 \\
\hline $\mathrm{C}$ & -2.697878 & 1.093216 & -2.527248 \\
\hline $\mathrm{H}$ & 3.842851 & -0.190220 & 5.330137 \\
\hline$H$ & 5.073556 & -1.134079 & 3.359511 \\
\hline $\mathrm{H}$ & 1.622214 & 0.921982 & 4.982578 \\
\hline $\mathrm{H}$ & 0.423485 & -3.549772 & 3.282383 \\
\hline $\mathrm{H}$ & 0.943005 & -4.535769 & 1882350 \\
\hline $\mathrm{H}$ & 1.446804 & -2.810353 & 2.033760 \\
\hline $\mathrm{H}$ & -1.988592 & -3.909895 & 2.952450 \\
\hline $\mathrm{H}$ & -1.507169 & -5.084839 & 1.694164 \\
\hline $\mathrm{H}$ & 4.100991 & -0.980933 & 1.092207 \\
\hline
\end{tabular}




\begin{tabular}{|c|c|c|c|}
\hline $\mathrm{H}$ & 0.627356 & 1.065184 & 2.708360 \\
\hline $\mathrm{H}$ & 2.787904 & -2.511956 & 0.210173 \\
\hline $\mathrm{H}$ & 4.178507 & 1.370774 & 0.398474 \\
\hline H & -2.747745 & -3.849427 & 1.348775 \\
\hline $\mathrm{H}$ & 3.924549 & -3.900518 & -1.493926 \\
\hline $\mathrm{H}$ & 5.278592 & 3.470462 & -0.315763 \\
\hline $\mathrm{H}$ & -2.741801 & 1.262036 & 3.188512 \\
\hline $\mathrm{H}$ & 0.728518 & -2.941568 & -0.223588 \\
\hline $\mathrm{H}$ & -0.514013 & -4.201994 & -0.436157 \\
\hline $\mathrm{H}$ & -3.053021 & 3.001846 & 2.969312 \\
\hline $\mathrm{H}$ & -4.428729 & 1.851512 & 3.021810 \\
\hline $\mathrm{H}$ & -3.216545 & -1.986645 & 0.809847 \\
\hline $\mathrm{H}$ & -3.529524 & -0.248348 & 1.648485 \\
\hline $\mathbf{H}$ & -2.646397 & -3.650353 & -0.91687 \\
\hline $\mathrm{H}$ & 4.275190 & -3.022358 & -3.816872 \\
\hline $\mathrm{H}$ & 0.663189 & -2.115807 & -2.20210 \\
\hline $\mathrm{H}$ & 3.963256 & 5.243750 & -1.50814 \\
\hline $\mathrm{H}$ & -4.483704 & -1.958626 & -0.47550 \\
\hline $\mathrm{H}$ & -4.877706 & 0.385682 & 0.644749 \\
\hline $\mathrm{H}$ & -0.599700 & -3.201829 & -2.84814 \\
\hline $\mathrm{H}$ & 2.371673 & 0.695518 & $-2.67578 t$ \\
\hline $\mathrm{H}$ & -2.789884 & -2.441658 & -2.19757 \\
\hline $\mathrm{H}$ & -3.692538 & 3.926760 & 0.68072 \\
\hline $\mathrm{H}$ & 0.411122 & 2.793865 & -1.21974 \\
\hline $\mathrm{H}$ & 3.488763 & -0.709321 & -4.39107 \\
\hline $\mathrm{H}$ & -5.163305 & 2.913375 & 0.824860 \\
\hline $\mathrm{H}$ & 1.521353 & 4.876649 & -1.954473 \\
\hline $\mathrm{H}$ & -4.138362 & 2.838508 & -0.642152 \\
\hline $\mathrm{H}$ & -3.885002 & -0.699381 & -2.328819 \\
\hline $\mathrm{H}$ & -0.246172 & -1.023384 & -4.135665 \\
\hline $\mathbf{H}$ & -4.600900 & 0.727667 & -1.566979 \\
\hline $\mathrm{H}$ & -1.938217 & -1.278662 & -3.656089 \\
\hline $\mathrm{H}$ & -2.632829 & 2.107517 & -2.097415 \\
\hline $\mathrm{H}$ & -3.015095 & 1.150658 & -3.584157 \\
\hline$N$ & -1.055682 & -2.206275 & -0.990612 \\
\hline $\mathrm{N}$ & -3.123660 & -0.283119 & -0.385301 \\
\hline $\mathrm{O}$ & -0.734063 & 2.546652 & 1.872135 \\
\hline $\mathrm{O}$ & -1.524052 & 3.027828 & -0.509212 \\
\hline $\mathrm{P}$ & 1.460526 & 0.253280 & 0.073405 \\
\hline$S$ & -1.046200 & -1.301819 & 1.965758 \\
\hline $\mathrm{S}$ & -1.426159 & 2.013917 & 0.636305 \\
\hline $\mathrm{S}$ & -0.919835 & 0.580177 & -2.415530 \\
\hline $\mathrm{Ru}$ & -0.882241 & -0.106574 & -0.153694 \\
\hline
\end{tabular}


TABLE A5 Optimized coordinates of 3 (B3LYP)

\begin{tabular}{|c|c|c|c|}
\hline $\mathrm{C}$ & 3.268532 & -0.410516 & 4.400847 \\
\hline C & 3.979679 & -0.930304 & 3.318757 \\
\hline $\mathrm{C}$ & 2.074586 & 0.275145 & 4.173747 \\
\hline $\mathrm{C}$ & 0.560272 & -3.600510 & 1.896396 \\
\hline $\mathrm{C}$ & 3.493105 & -0.773853 & 2.018642 \\
\hline $\mathrm{C}$ & 1.576041 & 0.424596 & 2.878626 \\
\hline C & -1.859196 & -4.036962 & 1.508318 \\
\hline C & 2.277968 & -0.108633 & 1.782973 \\
\hline $\mathrm{C}$ & -0.686883 & -3.120990 & 1.131642 \\
\hline C & 2.979710 & -2.108153 & -0.848635 \\
\hline C & -0.365801 & -3.129115 & -0.379512 \\
\hline C & 3.738122 & 2.047923 & 0.046947 \\
\hline C & 3.645248 & -2.856469 & -1.824159 \\
\hline $\mathrm{C}$ & 4.393384 & 3.243216 & -0.235848 \\
\hline $\mathrm{C}$ & -3.505760 & 1.981448 & 2.548684 \\
\hline C & 2.588290 & -0.779528 & -1.089940 \\
\hline $\mathrm{C}$ & 2.367092 & 1.893892 & -0.233376 \\
\hline C & 3.924998 & -2.297594 & -3.071220 \\
\hline $\mathrm{C}$ & 3.690525 & 4.311480 & -0.801657 \\
\hline C & -3.419611 & -1.580311 & -0.549635 \\
\hline $\mathrm{C}$ & -3.300670 & 1.906277 & 1.027882 \\
\hline C & -3.799046 & 0.532138 & 0.547460 \\
\hline C & -2.496141 & -2.410005 & -1.431283 \\
\hline C & 2.886778 & -0.229922 & -2.351257 \\
\hline C & -0.365859 & -2.042967 & -2.561173 \\
\hline $\mathrm{C}$ & 1.670488 & 2.968360 & -0.795451 \\
\hline $\mathrm{C}$ & 3.540899 & -0.979889 & -3.330124 \\
\hline $\mathrm{C}$ & 2.332608 & 4.169981 & -1.074367 \\
\hline C & -4.002351 & 3.092331 & 0.353360 \\
\hline $\mathrm{C}$ & -0.797213 & -0.858292 & -3.434244 \\
\hline C & -3.481462 & 0.429214 & -1.946979 \\
\hline $\mathrm{C}$ & -2.476033 & 1.357824 & -2.623524 \\
\hline $\mathrm{H}$ & 3.648071 & -0.530953 & 5.412310 \\
\hline H & 4.920607 & -1.450629 & 3.479471 \\
\hline $\mathrm{H}$ & 1.519657 & 0.698205 & 5.006623 \\
\hline H & 0.331852 & -3.716483 & 2.960472 \\
\hline $\mathrm{H}$ & 0.904388 & -4.574228 & 1.517602 \\
\hline $\mathrm{H}$ & 1.386660 & -2.887934 & 1.818770 \\
\hline $\mathrm{H}$ & -2.096323 & -3.938675 & 2.569622 \\
\hline H & -1.578729 & -5.079616 & 1.305296 \\
\hline $\mathrm{H}$ & 4.080921 & -1.161275 & 1.194099 \\
\hline H & 0.658981 & 0.984753 & 2.718452 \\
\hline
\end{tabular}




\begin{tabular}{|c|c|c|c|}
\hline $\mathrm{H}$ & 2.776861 & -2.569701 & 0.110783 \\
\hline $\mathrm{H}$ & 4.299637 & 1.234878 & 0.495125 \\
\hline $\mathrm{H}$ & -2.779451 & -3.823400 & 0.961755 \\
\hline $\mathrm{H}$ & 3.946697 & -3.876997 & -1.602173 \\
\hline $\mathrm{H}$ & 5.451715 & 3.342605 & -0.008187 \\
\hline $\mathrm{H}$ & -2.992515 & 1.162803 & 3.058161 \\
\hline H & 0.701180 & -2.961060 & -0.513153 \\
\hline $\mathrm{H}$ & -0.584181 & -4.131353 & -0.790121 \\
\hline $\mathrm{H}$ & -3.127243 & 2.928078 & 2.941665 \\
\hline $\mathrm{H}$ & -4.577928 & 1.911143 & 2.778946 \\
\hline $\mathrm{H}$ & -3.329159 & -1.869480 & 0.499279 \\
\hline $\mathrm{H}$ & -3.668956 & -0.164695 & 1.376758 \\
\hline $\mathrm{H}$ & -2.676718 & -3.474775 & -1.241110 \\
\hline $\mathrm{H}$ & 4.443150 & -2.878366 & -3.829495 \\
\hline $\mathrm{H}$ & 0.705383 & -1.972626 & -2.378781 \\
\hline $\mathrm{H}$ & 4.200243 & 5.247206 & -1.017260 \\
\hline $\mathrm{H}$ & -4.463351 & -1.743171 & -0.865293 \\
\hline $\mathrm{H}$ & -4.868773 & 0.593229 & 0.291858 \\
\hline $\mathrm{H}$ & -0.546655 & -2.976136 & -3.122158 \\
\hline $\mathrm{H}$ & 2.617155 & 0.799218 & -2.565919 \\
\hline $\mathrm{H}$ & -2.735811 & -2.251856 & -2.485524 \\
\hline $\mathrm{H}$ & -3.571763 & 4.040910 & 0.682970 \\
\hline $\mathrm{H}$ & 0.604376 & 2.906734 & -0.973486 \\
\hline $\mathrm{H}$ & 3.762347 & -0.525962 & -4.292705 \\
\hline $\mathrm{H}$ & -5.064892 & 3.080053 & 0.631562 \\
\hline $\mathrm{H}$ & 1.767923 & 4.997455 & -1.495537 \\
\hline $\mathrm{H}$ & -3.934695 & 3.071680 & -0.736772 \\
\hline H & -3.686034 & -0.414091 & -2.612152 \\
\hline $\mathrm{H}$ & -0.107903 & -0.788256 & -4.280901 \\
\hline $\mathrm{H}$ & -4.431118 & 0.966845 & -1.835097 \\
\hline $\mathrm{H}$ & -1.801101 & -0.981657 & -3.849026 \\
\hline $\mathrm{H}$ & -2.397205 & 2.317604 & -2.107124 \\
\hline $\mathrm{H}$ & -2.764204 & 1.524125 & -3.667518 \\
\hline$N$ & -1.039420 & -2.100233 & -1.231948 \\
\hline $\mathrm{N}$ & -3.077840 & -0.125473 & -0.604671 \\
\hline $\mathrm{O}$ & -2.506666 & -1.433441 & 2.372882 \\
\hline $\mathrm{O}$ & -0.772301 & 2.424603 & 1.993653 \\
\hline $\mathrm{O}$ & -1.344439 & 3.096246 & -0.382911 \\
\hline $\mathrm{P}$ & 1.567289 & 0.237716 & 0.102378 \\
\hline$S$ & -1.047491 & -1.336220 & 1.821748 \\
\hline$S$ & -1.398504 & 2.024499 & 0.690793 \\
\hline$S$ & -0.739341 & 0.768194 & -2.533919 \\
\hline $\mathrm{Ru}$ & -0.869835 & -0.075719 & -0.228630 \\
\hline
\end{tabular}


TABLE A6 Optimized coordinates of 3 (BP86)

\begin{tabular}{|c|c|c|c|}
\hline $\mathrm{C}$ & 3.254140 & -0.754292 & 4.357940 \\
\hline C & 3.967111 & -1.185998 & 3.228576 \\
\hline C & 2.051109 & -0.050822 & 4.190579 \\
\hline $\mathrm{C}$ & 0.607514 & -3.675172 & 1.707019 \\
\hline $\mathrm{C}$ & 3.471211 & -0.927933 & 1.939026 \\
\hline C & 1.542152 & 0.199206 & 2.906366 \\
\hline C & -1.818448 & -4.122435 & 1.298425 \\
\hline $\mathrm{C}$ & 2.244210 & -0.248837 & 1.763405 \\
\hline C & -0.647584 & -3.189061 & 0.956162 \\
\hline C & 2.972169 & -2.022784 & -1.016868 \\
\hline C & -0.330541 & -3.107534 & -0.551791 \\
\hline C & 3.638332 & 2.107277 & 0.349926 \\
\hline C & 3.653644 & -2.684040 & -2.053865 \\
\hline $\mathrm{C}$ & 4.300133 & 3.321199 & 0.120434 \\
\hline C & -3.488150 & 1.861856 & 2.654548 \\
\hline $\mathrm{C}$ & 2.559782 & -0.675993 & -1.153270 \\
\hline $\mathrm{C}$ & 2.306616 & 1.918142 & -0.086666 \\
\hline $\mathrm{C}$ & 3.926256 & -2.019415 & -3.259448 \\
\hline $\mathrm{C}$ & 3.644285 & 4.368980 & -0.547945 \\
\hline C & -3.406363 & -1.570650 & -0.616400 \\
\hline $\mathrm{C}$ & -3.300039 & 1.843138 & 1.128186 \\
\hline $\mathrm{C}$ & -3.786331 & 0.487826 & 0.593431 \\
\hline $\mathrm{C}$ & -2.484284 & -2.348364 & -1.549989 \\
\hline C & 2.851786 & -0.019132 & -2.372553 \\
\hline C & -0.356005 & -1.898161 & -2.676510 \\
\hline C & 1.653818 & 2.974775 & -0.749359 \\
\hline $\mathrm{C}$ & 3.519951 & -0.683468 & -3.413428 \\
\hline C & 2.321289 & 4.192244 & -0.974732 \\
\hline $\mathrm{C}$ & -3.987832 & 3.062164 & 0.495451 \\
\hline C & -0.809998 & -0.671909 & -3.479102 \\
\hline C & -3.475849 & 0.519359 & -1.913703 \\
\hline $\mathrm{C}$ & -2.476053 & 1.503455 & -2.519152 \\
\hline $\mathrm{H}$ & 3.643015 & -0.953727 & 5.363381 \\
\hline H & 4.920864 & -1.714182 & 3.344864 \\
\hline $\mathrm{H}$ & 1.497170 & 0.310462 & 5.064376 \\
\hline $\mathrm{H}$ & 0.378445 & -3.831613 & 2.775260 \\
\hline $\mathrm{H}$ & 0.971773 & -4.638183 & 1.294189 \\
\hline $\mathrm{H}$ & 1.428571 & -2.939444 & 1.654411 \\
\hline $\mathrm{H}$ & -2.061802 & -4.058941 & 2.370797 \\
\hline H & -1.532231 & -5.166171 & 1.061879 \\
\hline $\mathrm{H}$ & 4.063661 & -1.238829 & 1.073127 \\
\hline $\mathrm{H}$ & 0.622603 & 0.785353 & 2.783312 \\
\hline
\end{tabular}




\begin{tabular}{|c|c|c|c|}
\hline $\mathrm{H}$ & 2.775919 & -2.565873 & -0.087299 \\
\hline H & 4.162342 & 1.307573 & 0.882638 \\
\hline H & -2.746682 & -3.893601 & 0.751917 \\
\hline H & 3.974707 & -3.722641 & -1.911558 \\
\hline $\mathrm{H}$ & 5.330549 & 3.449423 & 0.472347 \\
\hline H & -2.974799 & 1.009762 & 3.127935 \\
\hline $\mathrm{H}$ & 0.743508 & -2.910538 & -0.682022 \\
\hline $\mathrm{H}$ & -0.541097 & -4.093204 & -1.030562 \\
\hline $\mathrm{H}$ & -3.086394 & 2.794153 & 3.083073 \\
\hline $\mathrm{H}$ & -4.566911 & 1.798177 & 2.899896 \\
\hline $\mathrm{H}$ & -3.301483 & -1.912233 & 0.429580 \\
\hline $\mathrm{H}$ & -3.632548 & -0.255229 & 1.395664 \\
\hline $\mathrm{H}$ & -2.652879 & -3.433622 & -1.412756 \\
\hline $\mathrm{H}$ & 4.457867 & -2.534390 & -4.067566 \\
\hline H & 0.725306 & -1.822191 & -2.490730 \\
\hline $\mathrm{H}$ & 4.160870 & 5.320100 & -0.722701 \\
\hline $\mathrm{H}$ & -4.462819 & -1.719623 & -0.931259 \\
\hline $\mathrm{H}$ & -4.869135 & 0.542107 & 0.344703 \\
\hline H & -0.531025 & -2.813721 & -3.286198 \\
\hline $\mathrm{H}$ & 2.565296 & 1.030305 & -2.500975 \\
\hline $\mathrm{H}$ & -2.731785 & -2.136973 & -2.604227 \\
\hline $\mathrm{H}$ & -3.530710 & 4.000492 & 0.847801 \\
\hline H & 0.601278 & 2.885572 & -1.026107 \\
\hline $\mathrm{H}$ & 3.737134 & -0.146002 & -4.343995 \\
\hline $\mathrm{H}$ & -5.056418 & 3.062970 & 0.787883 \\
\hline H & 1.787596 & 5.008226 & -1.475316 \\
\hline $\mathrm{H}$ & -3.935951 & 3.071904 & -0.605389 \\
\hline $\mathrm{H}$ & -3.661272 & -0.296697 & -2.634003 \\
\hline $\mathrm{H}$ & -0.133286 & -0.545093 & -4.341105 \\
\hline H & -4.446675 & 1.032805 & -1.770945 \\
\hline $\mathrm{H}$ & -1.833317 & -0.772217 & -3.878848 \\
\hline $\mathrm{H}$ & -2.385121 & 2.425042 & -1.915934 \\
\hline H & -2.763927 & 1.757883 & -3.555349 \\
\hline $\mathrm{N}$ & -1.023804 & -2.030633 & -1.340639 \\
\hline $\mathrm{N}$ & -3.063844 & -0.107927 & -0.597687 \\
\hline $\mathrm{O}$ & -2.490030 & -1.565204 & 2.299841 \\
\hline $\mathrm{O}$ & -0.738851 & 2.276211 & 2.140732 \\
\hline $\mathrm{O}$ & -1.309418 & 3.130314 & -0.201611 \\
\hline$P$ & 1.511951 & 0.227630 & 0.116538 \\
\hline$S$ & -1.025852 & -1.413132 & 1.738364 \\
\hline$S$ & -1.363318 & 1.968714 & 0.795327 \\
\hline$S$ & -0.733558 & 0.896568 & -2.463978 \\
\hline Ru & -0.854866 & -0.066105 & -0.233391 \\
\hline
\end{tabular}


TABLE A7 Optimized coordinates of 4 (B3LYP)

\begin{tabular}{|c|c|c|c|}
\hline $\mathrm{C}$ & -3.660133 & -0.283464 & 4.260329 \\
\hline $\mathrm{C}$ & -4.273253 & 0.423390 & 3.225840 \\
\hline $\mathrm{C}$ & -2.434854 & -0.911090 & 4.033612 \\
\hline C & -0.171444 & 3.804330 & 1.886042 \\
\hline $\mathrm{C}$ & -3.662001 & 0.502292 & 1.972802 \\
\hline C & -1.811947 & -0.825823 & 2.788674 \\
\hline $\mathrm{C}$ & 2.255132 & 3.959018 & 1.392219 \\
\hline $\mathrm{C}$ & -2.420994 & -0.113629 & 1.743679 \\
\hline C & 0.977189 & 3.160368 & 1.094569 \\
\hline C & -2.837952 & 2.330425 & -0.506321 \\
\hline $\mathrm{C}$ & 0.583539 & 3.135569 & -0.397882 \\
\hline C & -3.861111 & -1.808804 & -0.536604 \\
\hline $\mathrm{C}$ & -3.450190 & 3.270570 & -1.340195 \\
\hline C & -4.542587 & -2.937465 & -0.982327 \\
\hline C & 3.468541 & -2.115533 & 2.497320 \\
\hline $\mathrm{C}$ & -2.538664 & 1.036301 & -0.964996 \\
\hline C & -2.452868 & -1.760336 & -0.550838 \\
\hline C & -3.768979 & 2.940851 & -2.658021 \\
\hline $\mathrm{C}$ & -3.831497 & -4.050069 & -1.443423 \\
\hline $\mathrm{C}$ & 3.531607 & 1.385995 & -0.668554 \\
\hline $\mathrm{C}$ & 3.251527 & -2.064843 & 0.977337 \\
\hline C & 3.817843 & -0.731225 & 0.455453 \\
\hline $\mathrm{C}$ & 2.623724 & 2.261870 & -1.521108 \\
\hline C & -2.873730 & 0.718657 & -2.294863 \\
\hline C & 0.436633 & 2.031509 & -2.559658 \\
\hline C & -1.751161 & -2.881178 & -1.004276 \\
\hline $\mathrm{C}$ & -3.477670 & 1.659625 & -3.131409 \\
\hline $\mathrm{C}$ & -2.440333 & -4.017559 & -1.446055 \\
\hline C & 3.885289 & -3.298231 & 0.320346 \\
\hline $\mathrm{C}$ & 0.764835 & 0.818364 & -3.437598 \\
\hline C & 3.429658 & -0.634244 & -2.028893 \\
\hline $\mathrm{C}$ & 2.348769 & -1.486666 & -2.686687 \\
\hline $\mathrm{H}$ & -4.136820 & -0.346843 & 5.235357 \\
\hline H & -5.231917 & 0.910728 & 3.385658 \\
\hline $\mathrm{H}$ & -1.947730 & -1.465237 & 4.831103 \\
\hline $\mathrm{H}$ & 0.115950 & 3.942528 & 2.931138 \\
\hline $\mathrm{H}$ & -0.413918 & 4.788849 & 1.461862 \\
\hline $\mathrm{H}$ & -1.068403 & 3.183254 & 1.886060 \\
\hline $\mathrm{H}$ & 2.512091 & 3.876520 & 2.450372 \\
\hline H & 2.079492 & 5.018305 & 1.159089 \\
\hline $\mathrm{H}$ & -4.171670 & 1.039653 & 1.180888 \\
\hline
\end{tabular}




\begin{tabular}{|c|c|c|c|}
\hline $\mathrm{H}$ & -0.856818 & -1.315400 & 2.634773 \\
\hline $\mathrm{H}$ & -2.608186 & 2.606689 & 0.516636 \\
\hline $\mathrm{H}$ & -4.434102 & -0.963835 & -0.169781 \\
\hline $\mathrm{H}$ & 3.131402 & 3.635790 & 0.828428 \\
\hline $\mathrm{H}$ & -3.681539 & 4.259264 & -0.952475 \\
\hline $\mathrm{H}$ & -5.629376 & -2.951232 & -0.961901 \\
\hline $\mathrm{H}$ & 2.987154 & -1.271996 & 2.997546 \\
\hline $\mathrm{H}$ & -0.496216 & 3.012925 & -0.469546 \\
\hline $\mathrm{H}$ & 0.828882 & 4.113507 & -0.847833 \\
\hline $\mathrm{H}$ & 3.062615 & -3.041120 & 2.911736 \\
\hline $\mathrm{H}$ & 4.544686 & -2.076887 & 2.715923 \\
\hline $\mathrm{H}$ & 3.513627 & 1.707366 & 0.370369 \\
\hline $\mathrm{H}$ & 3.772100 & -0.020515 & 1.277722 \\
\hline $\mathrm{H}$ & 2.883092 & 3.314895 & -1.358653 \\
\hline $\mathrm{H}$ & -4.247615 & 3.670193 & -3.305854 \\
\hline$H$ & -0.628768 & 2.027407 & -2.339000 \\
\hline $\mathrm{H}$ & -4.362710 & -4.934430 & -1.786310 \\
\hline $\mathrm{H}$ & 4.566679 & 1.475006 & -1.036346 \\
\hline $\mathrm{H}$ & 4.869739 & -0.866725 & 0.159626 \\
\hline $\mathrm{H}$ & 0.654790 & 2.949638 & -3.131696 \\
\hline $\mathrm{H}$ & -2.677866 & -0.278376 & -2.676701 \\
\hline $\mathrm{H}$ & 2.807717 & 2.071010 & -2.580149 \\
\hline $\mathrm{H}$ & 3.412594 & -4.217082 & 0.675486 \\
\hline $\mathrm{H}$ & -0.668983 & -2.907451 & -0.986199 \\
\hline H & -3.732893 & 1.383745 & -4.151502 \\
\hline $\mathrm{H}$ & 4.950190 & -3.333035 & 0.586754 \\
\hline $\mathrm{H}$ & -1.871038 & -4.880068 & -1.782412 \\
\hline $\mathrm{H}$ & 3.802939 & -3.294906 & -0.768989 \\
\hline $\mathrm{H}$ & 3.684094 & 0.190326 & -2.700176 \\
\hline $\mathrm{H}$ & 0.036468 & 0.776621 & -4.252858 \\
\hline $\mathrm{H}$ & 4.339239 & -1.238552 & -1.931214 \\
\hline $\mathrm{H}$ & 1.754667 & 0.881799 & -3.897310 \\
\hline $\mathrm{H}$ & 2.232142 & -2.450973 & -2.187118 \\
\hline $\mathrm{H}$ & 2.585390 & -1.643106 & -3.744963 \\
\hline$N$ & 1.159595 & 2.052280 & -1.256775 \\
\hline$N$ & 3.095044 & -0.044149 & -0.678994 \\
\hline $\mathrm{O}$ & 2.597950 & 1.321324 & 2.286476 \\
\hline $\mathrm{O}$ & 0.728852 & -2.542235 & 1.952100 \\
\hline $\mathrm{O}$ & 1.246882 & -3.140401 & -0.450267 \\
\hline $\mathrm{P}$ & -1.612881 & -0.206919 & 0.075847 \\
\hline$S$ & 1.178089 & 1.323421 & 1.763617 \\
\hline $\mathrm{S}$ & 1.345473 & -2.110175 & 0.660197 \\
\hline$S$ & 0.656955 & -0.795445 & -2.518224 \\
\hline $\mathrm{Ru}$ & 0.883088 & 0.025326 & -0.230239 \\
\hline $\mathrm{O}$ & 0.142306 & 1.210764 & 2.829836 \\
\hline
\end{tabular}


TABLE A8 Optimized coordinates of 4 (BP86)

\begin{tabular}{|c|c|c|c|}
\hline C & -3.630911 & -0.250566 & 4.276451 \\
\hline C & -4.225556 & 0.483275 & 3.239422 \\
\hline $\mathrm{C}$ & -2.413031 & -0.910212 & 4.047799 \\
\hline C & -0.206015 & 3.785660 & 1.905374 \\
\hline $\mathrm{C}$ & -3.603816 & 0.558731 & 1.981545 \\
\hline $\mathrm{C}$ & -1.779060 & -0.829461 & 2.799574 \\
\hline $\mathrm{C}$ & 2.224980 & 3.975837 & 1.384583 \\
\hline C & -2.370265 & -0.089050 & 1.752972 \\
\hline C & 0.946098 & 3.174516 & 1.089843 \\
\hline C & -2.819080 & 2.319551 & -0.552648 \\
\hline $\mathrm{C}$ & 0.541548 & 3.141766 & -0.398519 \\
\hline $\mathrm{C}$ & -3.828694 & -1.805185 & -0.454654 \\
\hline C & -3.453282 & 3.234367 & -1.411966 \\
\hline C & -4.537153 & -2.932650 & -0.887945 \\
\hline $\mathrm{C}$ & 3.503253 & -2.116541 & 2.470121 \\
\hline $\mathrm{C}$ & -2.510832 & 1.007217 & -0.979350 \\
\hline C & -2.413727 & -1.771042 & -0.510587 \\
\hline $\mathrm{C}$ & -3.783501 & 2.861695 & -2.724155 \\
\hline $\mathrm{C}$ & -3.846265 & -4.055519 & -1.377437 \\
\hline C & 3.506910 & 1.418829 & -0.684259 \\
\hline $\mathrm{C}$ & 3.285668 & -2.046969 & 0.948995 \\
\hline $\mathrm{C}$ & 3.821835 & -0.699140 & 0.438768 \\
\hline C & 2.586043 & 2.279921 & -1.539931 \\
\hline $\mathrm{C}$ & -2.860476 & 0.645219 & -2.303150 \\
\hline C & 0.384008 & 2.025094 & -2.560874 \\
\hline $\mathrm{C}$ & -1.730143 & -2.901195 & -0.991688 \\
\hline C & -3.484052 & 1.561847 & -3.165248 \\
\hline $\mathrm{C}$ & -2.446866 & -4.035215 & -1.420006 \\
\hline C & 3.910571 & -3.272507 & 0.265160 \\
\hline $\mathrm{C}$ & 0.718346 & 0.809859 & -3.435186 \\
\hline $\mathrm{C}$ & 3.408082 & -0.608767 & -2.054341 \\
\hline $\mathrm{C}$ & 2.327252 & -1.484029 & -2.684626 \\
\hline $\mathrm{H}$ & -4.117734 & -0.311275 & 5.256852 \\
\hline $\mathrm{H}$ & -5.181316 & 0.995994 & 3.399892 \\
\hline $\mathrm{H}$ & -1.939668 & -1.488353 & 4.849137 \\
\hline $\mathrm{H}$ & 0.097355 & 3.922783 & 2.955788 \\
\hline $\mathrm{H}$ & -0.483746 & 4.775920 & I. 492478 \\
\hline H & -1.093876 & 3.134433 & 1.916504 \\
\hline $\mathrm{H}$ & 2.512161 & 3.857555 & 2.441226 \\
\hline $\mathrm{H}$ & 2.034262 & 5.050161 & 1.191433 \\
\hline $\mathrm{H}$ & -4.102232 & 1.116410 & 1.182941 \\
\hline
\end{tabular}




\begin{tabular}{|c|c|c|c|}
\hline $\mathrm{H}$ & -0.827629 & -1.346823 & 2.637436 \\
\hline $\mathrm{H}$ & -2.578004 & 2.629826 & 0.468738 \\
\hline$H$ & -4.384664 & -0.948256 & -0.061255 \\
\hline $\mathrm{H}$ & 3.096904 & 3.678035 & 0.782034 \\
\hline $\mathrm{H}$ & -3.693230 & 4.239703 & -1.047071 \\
\hline $\mathrm{H}$ & -5.632095 & -2.936503 & -0.834523 \\
\hline $\mathrm{H}$ & 3.024045 & -1.266653 & 2.982010 \\
\hline $\mathrm{H}$ & -0.548569 & 3.012571 & -0.468134 \\
\hline $\mathrm{H}$ & 0.784854 & 4.125004 & -0.864345 \\
\hline $\mathrm{H}$ & 3.084908 & -3.050408 & 2.877928 \\
\hline $\mathrm{H}$ & 4.588218 & -2.089810 & 2.692964 \\
\hline $\mathrm{H}$ & 3.479834 & 1.739096 & 0.368308 \\
\hline $\mathrm{H}$ & 3.750045 & 0.014606 & 1.273174 \\
\hline $\mathrm{H}$ & 2.833238 & 3.348062 & -1.388173 \\
\hline $\mathrm{H}$ & -4.279055 & 3.573677 & -3.393548 \\
\hline $\mathrm{H}$ & -0.688576 & 2.009442 & -2.324087 \\
\hline $\mathrm{H}$ & -4.399878 & -4.940898 & -1.711330 \\
\hline $\mathrm{H}$ & 4.551445 & 1.514674 & -1.053360 \\
\hline $\mathrm{H}$ & 4.886487 & -0.803434 & 0.136669 \\
\hline $\mathrm{H}$ & 0.592481 & 2.955250 & -3.136253 \\
\hline $\mathrm{H}$ & -2.658821 & -0.371296 & -2.657662 \\
\hline $\mathrm{H}$ & 2.760240 & 2.078826 & -2.609706 \\
\hline $\mathrm{H}$ & 3.435704 & -4.203942 & 0.612307 \\
\hline $\mathrm{H}$ & -0.636454 & -2.935400 & -0.996342 \\
\hline $\mathrm{H}$ & -3.749920 & 1.251179 & -4.182449 \\
\hline $\mathrm{H}$ & 4.987035 & -3.314325 & 0.522628 \\
\hline H & -1.891170 & -4.909268 & -1.778604 \\
\hline $\mathrm{H}$ & 3.818336 & -3.251686 & -0.832513 \\
\hline $\mathrm{H}$ & 3.635655 & 0.229097 & -2.736036 \\
\hline $\mathrm{H}$ & -0.021202 & 0.747784 & -4.251574 \\
\hline $\mathrm{H}$ & 4.340928 & -1.196848 & -1.963149 \\
\hline $\mathrm{H}$ & 1.715648 & 0.872618 & -3.903061 \\
\hline $\mathrm{H}$ & 2.219852 & -2.449256 & -2.159298 \\
\hline $\mathrm{H}$ & 2.540593 & -1.654412 & -3.755373 \\
\hline$N$ & 1.120861 & 2.049953 & -1.256424 \\
\hline$N$ & 3.077187 & -0.020539 & -0.693357 \\
\hline O & 2.615267 & 1.329982 & 2.268853 \\
\hline $\mathrm{O}$ & 0.750259 & -2.529308 & 1.965865 \\
\hline $\mathrm{O}$ & 1.249524 & -3.151747 & -0.460009 \\
\hline$P$ & -1.550286 & -0.203890 & 0.082277 \\
\hline$S$ & 1.172968 & 1.309077 & 1.755084 \\
\hline $\mathrm{S}$ & 1.347729 & -2.095435 & 0.648510 \\
\hline $\mathrm{S}$ & 0.626943 & -0.796742 & -2.476989 \\
\hline $\mathrm{Ru}$ & 0.868239 & 0.027919 & -0.230450 \\
\hline $\mathrm{O}$ & 0.148649 & 1.188294 & 2.851738 \\
\hline
\end{tabular}


Table A9. Crystal data and structure refinement for 8 .

\author{
Identification code \\ Empirical formula \\ Formula weight \\ Temperature \\ Wavelength \\ Crystal system \\ Space group \\ Unit cell dimensions \\ Volume \\ Z \\ Density (calculated) \\ Absorption coefficient \\ $\mathrm{F}(000)$ \\ Crystal size \\ Theta range for data collection \\ Index ranges \\ Reflections collected \\ Independent reflections \\ Completeness to theta $=26.32^{\circ}$ \\ Absorption correction \\ Max. and min. transmission \\ Refinement method \\ Data / restraints / parameters \\ Goodness-of-fit on $F^{2}$ \\ Final $R$ indices [ $1>2$ sigma(I)] \\ $\mathrm{R}$ indices (all data) \\ Largest diff. peak and hole
}

cag 175lt

C62.75 H88.50 N5 O4 P2.50 Ru2 S6

1448.81

$100(2) \mathrm{K}$

$0.71073 \AA$

Triclinic

P-1

$\mathrm{a}=9.7483(3) \AA$

$\mathrm{b}=14.4088(5) \AA$

$\mathrm{c}=24.8048(7) \AA$

$3302.85(19) \AA^{3}$

2

$1.457 \mathrm{Mg} / \mathrm{m}^{3}$

$0.757 \mathrm{~mm}^{-1}$

1507

$0.25 \times 0.22 \times 0.18 \mathrm{~mm}^{3}$

3.25 to $29.10^{\circ}$.

$-13<=\mathrm{h}<=12,-19<=\mathrm{k}<=18,-31<=\mathrm{l}<=32$

66164

16098 [R(int) $=0.0279]$

$99.8 \%$

Semi-empirical from equivalents

1.00000 and 0.96524

Full-matrix least-squares on $\mathrm{F}^{2}$

$16098 / 0 / 709$

1.085

$\mathrm{R} 1=0.0418, w \mathrm{R} 2=0.1244$

$\mathrm{R} 1=0.0532, w \mathrm{R} 2=0.1279$

4.052 and $-0.944 \mathrm{e} . \AA^{-3}$ 
Table A10. Atomic coordinates ( $\left.\times 10^{4}\right)$ and equivalent isotropic displacement parameters $\left(\AA^{2} \times 10^{3}\right)$ for 8. U(eq) is defined as one third of the trace of the orthogonalized $U^{\mathrm{ij}}$ tensor.

\begin{tabular}{|c|c|c|c|c|}
\hline & $\mathrm{x}$ & $\mathrm{y}$ & $\mathrm{z}$ & $\mathrm{U}(\mathrm{eq})$ \\
\hline $\mathrm{Ru}(1)$ & $6456(1)$ & $4133(1)$ & $3143(1)$ & $11(1)$ \\
\hline $\mathrm{S}(1)$ & $4581(1)$ & $4437(1)$ & $2634(1)$ & $16(1)$ \\
\hline $\mathrm{S}(2)$ & $8028(1)$ & $3597(1)$ & $2495(1)$ & $15(1)$ \\
\hline $\mathrm{S}(3)$ & $8153(1)$ & $4095(1)$ & $3748(1)$ & $15(1)$ \\
\hline$P(1)$ & $5841(1)$ & 2592(1) & $3519(1)$ & $11(1)$ \\
\hline$O(1)$ & $7439(3)$ & $3402(2)$ & $2028(1)$ & $26(1)$ \\
\hline$O(2)$ & $9125(3)$ & $2819(2)$ & $2708(1)$ & $23(1)$ \\
\hline $\mathrm{N}(1)$ & $6962(3)$ & $5572(2)$ & $2672(1)$ & $14(1)$ \\
\hline $\mathrm{N}(2)$ & $5145(3)$ & $5004(2)$ & $3679(1)$ & $15(1)$ \\
\hline$C(1)$ & $6783(3)$ & $6170(2)$ & $3097(1)$ & $16(1)$ \\
\hline$C(2)$ & $5395(3)$ & $6051(2)$ & $3464(2)$ & $17(1)$ \\
\hline$C(3)$ & $3635(3)$ & $4941(2)$ & $3662(2)$ & $17(1)$ \\
\hline $\mathrm{C}(4)$ & $3287(3)$ & $5112(2)$ & $3066(2)$ & $18(1)$ \\
\hline$C(5)$ & $5211(4)$ & $5391(2)$ & 2052(2) & $20(1)$ \\
\hline$C(6)$ & $6007(4)$ & $6064(2)$ & $2235(2)$ & $18(1)$ \\
\hline$C(7)$ & $8477(3)$ & $5502(2)$ & $2435(2)$ & $17(1)$ \\
\hline $\mathrm{C}(8)$ & $9011(3)$ & $4686(2)$ & $2132(2)$ & $18(1)$ \\
\hline $\mathrm{C}(9)$ & $5471(3)$ & $4616(2)$ & $4266(1)$ & $17(1)$ \\
\hline$C(10)$ & $7026(4)$ & $4438(2)$ & $4360(2)$ & $18(1)$ \\
\hline$C(11)$ & $10568(4)$ & $4413(3)$ & $2204(2)$ & $25(1)$ \\
\hline$C(12)$ & $8865(4)$ & $4967(3)$ & $1509(2)$ & $26(1)$ \\
\hline$C(13)$ & $7556(4)$ & $5305(3)$ & $4486(2)$ & $23(1)$ \\
\hline$C(14)$ & $7154(4)$ & $3585(3)$ & $4875(2)$ & $24(1)$ \\
\hline$C(15)$ & $4045(3)$ & $2528(2)$ & $3881(1)$ & $14(1)$ \\
\hline$C(16)$ & $3729(3)$ & $2480(2)$ & $4458(1)$ & $17(1)$ \\
\hline$C(17)$ & $2356(4)$ & $2567(2)$ & $4711(2)$ & $21(1)$ \\
\hline$C(18)$ & $1267(4)$ & $2709(3)$ & $4388(2)$ & $23(1)$ \\
\hline$C(19)$ & $1557(4)$ & $2748(2)$ & $3818(2)$ & $22(1)$ \\
\hline$C(20)$ & $2925(3)$ & $2652(2)$ & $3568(2)$ & $17(1)$ \\
\hline $\mathrm{C}(21)$ & $6772(3)$ & $1600(2)$ & 3999(1) & $14(1)$ \\
\hline$C(22)$ & $6158(4)$ & $748(2)$ & $4246(2)$ & $19(1)$ \\
\hline$C(23)$ & $6887(4)$ & $-48(2)$ & $4560(2)$ & $23(1)$ \\
\hline$C(24)$ & $8251(4)$ & $-2(3)$ & $4640(2)$ & $22(1)$ \\
\hline$C(25)$ & $8869(4)$ & $832(3)$ & $4402(2)$ & $21(1)$ \\
\hline$C(26)$ & $8145(3)$ & $1628(2)$ & $4077(2)$ & $17(1)$ \\
\hline $\mathrm{C}(27)$ & $5799(3)$ & $1949(2)$ & $2975(1)$ & $17(1)$ \\
\hline $\mathrm{Ru}(2)$ & $2196(1)$ & $9662(1)$ & $2156(1)$ & $11(1)$ \\
\hline $\mathrm{S}(4)$ & $2730(1)$ & $10603(1)$ & $2706(1)$ & $14(1)$ \\
\hline $\mathrm{S}(5)$ & $2410(1)$ & $8264(1)$ & $2802(1)$ & $14(1)$ \\
\hline$S(6)$ & $1323(1)$ & $9004(1)$ & $1496(1)$ & $16(1)$ \\
\hline $\mathrm{P}(2)$ & $4556(1)$ & $9410(1)$ & $1838(1)$ & $13(1)$ \\
\hline$O(3)$ & $3075(3)$ & $8294(2)$ & $3293(1)$ & $22(1)$ \\
\hline$O(4)$ & 2976(3) & $7394(2)$ & $2589(1)$ & $22(1)$ \\
\hline $\mathrm{N}(3)$ & $62(3)$ & $9856(2)$ & $2583(1)$ & $14(1)$ \\
\hline $\mathrm{N}(4)$ & $1439(3)$ & $11037(2)$ & $1616(1)$ & $14(1)$ \\
\hline$C(28)$ & $-799(3)$ & $10425(2)$ & $2140(2)$ & $17(1)$ \\
\hline $\mathrm{C}(29)$ & $-91(3)$ & $11293(2)$ & $1796(2)$ & $17(1)$ \\
\hline $\mathrm{C}(30)$ & $2210(3)$ & $11824(2)$ & $1669(1)$ & $16(1)$ \\
\hline$C(31)$ & $2254(3)$ & $11825(2)$ & $2282(2)$ & $17(1)$ \\
\hline$C(32)$ & $1251(3)$ & $10449(3)$ & $3258(1)$ & $18(1)$ \\
\hline$C(33)$ & $-102(3)$ & $10406(2)$ & $3038(2)$ & $17(1)$ \\
\hline
\end{tabular}




\begin{tabular}{|c|c|c|c|c|}
\hline$C(34)$ & $-449(3)$ & $8875(2)$ & 2794(2) & $18(1)$ \\
\hline$C(35)$ & $533(3)$ & $8071(2)$ & $3120(2)$ & $19(1)$ \\
\hline$C(36)$ & $1228(3)$ & $10073(2)$ & $893(1)$ & $17(1)$ \\
\hline$C(37)$ & $1715(3)$ & $10956(2)$ & $1019(1)$ & $17(1)$ \\
\hline$C(38)$ & $211(4)$ & $7119(3)$ & $3035(2)$ & $28(1)$ \\
\hline$C(39)$ & $335(4)$ & $8017(3)$ & $3753(2)$ & $26(1)$ \\
\hline$C(40)$ & $-253(4)$ & $10277(3)$ & $726(2)$ & $23(1)$ \\
\hline $\mathrm{C}(41)$ & $2243(4)$ & $9833(3)$ & $391(2)$ & $23(1)$ \\
\hline $\mathrm{C}(42)$ & $5372(3)$ & $10496(2)$ & $1434(1)$ & $14(1)$ \\
\hline $\mathrm{C}(43)$ & $5775(3)$ & $11115(2)$ & $1709(2)$ & $18(1)$ \\
\hline$C(44)$ & $6305(4)$ & $11978(3)$ & $1412(2)$ & $23(1)$ \\
\hline$C(45)$ & $6406(4)$ & $12218(3)$ & $830(2)$ & $26(1)$ \\
\hline$C(46)$ & 5994(4) & $11612(3)$ & $551(2)$ & $22(1)$ \\
\hline $\mathrm{C}(47)$ & $5501(3)$ & $10756(2)$ & $847(2)$ & $18(1)$ \\
\hline $\mathrm{C}(48)$ & $5415(3)$ & $8564(2)$ & $1407(2)$ & $18(1)$ \\
\hline$C(49)$ & $6878(4)$ & $8513(3)$ & $1266(2)$ & $24(1)$ \\
\hline$C(50)$ & $7578(4)$ & $7902(3)$ & $939(2)$ & $28(1)$ \\
\hline$C(51)$ & $6856(4)$ & $7342(3)$ & $732(2)$ & $27(1)$ \\
\hline$C(52)$ & $5425(4)$ & $7372(3)$ & $874(2)$ & $24(1)$ \\
\hline$C(53)$ & $4702(4)$ & $7972(2)$ & $1217(2)$ & $20(1)$ \\
\hline$C(54)$ & $5596(3)$ & $9008(3)$ & $2425(2)$ & $19(1)$ \\
\hline $\mathrm{N}(200)$ & $6416(4)$ & 7939(3) & 3928(2) & $44(1)$ \\
\hline$C(200)$ & 3994(5) & $8816(3)$ & $4229(2)$ & $36(1)$ \\
\hline$C(201)$ & $5339(5)$ & $8321(3)$ & $4056(2)$ & $32(1)$ \\
\hline $\mathrm{P}(3)$ & $6377(2)$ & $5709(2)$ & $9577(1)$ & $32(1)$ \\
\hline$C(500)$ & $6160(9)$ & $5883(7)$ & $8853(4)$ & $36(2)$ \\
\hline$C(300)$ & $7602(3)$ & $4600(2)$ & $9683(2)$ & $30(1)$ \\
\hline$C(305)$ & $8496(4)$ & $4441(3)$ & $10087(2)$ & $30(1)$ \\
\hline$C(304)$ & 9434(4) & $3611(3)$ & $10174(2)$ & $30(1)$ \\
\hline$C(303)$ & $9478(4)$ & 2939(3) & $9856(2)$ & $30(1)$ \\
\hline$C(302)$ & $8584(4)$ & 3098(3) & 9452(2) & $30(1)$ \\
\hline$C(301)$ & $7646(4)$ & 3929(3) & $9366(2)$ & $30(1)$ \\
\hline$C(400)$ & $5001(4)$ & 4997(3) & 9999(2) & $29(1)$ \\
\hline$C(404)$ & $3615(5)$ & $5141(4)$ & $9681(2)$ & $29(1)$ \\
\hline$C(403)$ & $2419(6)$ & $4729(5)$ & $9975(3)$ & $29(1)$ \\
\hline$C(402)$ & $3560(5)$ & $4400(4)$ & $10796(2)$ & $29(1)$ \\
\hline$C(401)$ & $4718(4)$ & 4793(3) & $10520(2)$ & $29(1)$ \\
\hline
\end{tabular}


Table A11. Bond lengths $[\AA]$ and angles $\left[{ }^{\circ}\right]$ for 8 .

\begin{tabular}{|c|c|}
\hline $\mathrm{Ru}(1)-\mathrm{N}(1)$ & $2.188(3)$ \\
\hline $\mathrm{Ru}(1)-\mathrm{N}(2)$ & $2.190(3)$ \\
\hline $\mathrm{Ru}(1)-\mathrm{S}(2)$ & $2.2384(8)$ \\
\hline $\mathrm{Ru}(1)-\mathrm{P}(1)$ & $2.3045(8)$ \\
\hline $\mathrm{Ru}(1)-\mathrm{S}(1)$ & $2.3163(8)$ \\
\hline $\mathrm{Ru}(1)-\mathrm{S}(3)$ & $2.3940(8)$ \\
\hline$S(1)-C(5)$ & $1.816(4)$ \\
\hline$S(1)-C(4)$ & $1.848(3)$ \\
\hline $\mathrm{S}(2)-\mathrm{O}(1)$ & $1.478(3)$ \\
\hline $\mathrm{S}(2)-\mathrm{O}(2)$ & $1.485(3)$ \\
\hline$S(2)-C(8)$ & $1.905(3)$ \\
\hline$S(3)-C(10)$ & $1.853(4)$ \\
\hline$P(1)-C(27)$ & $1.831(3)$ \\
\hline$P(1)-C(15)$ & $1.836(3)$ \\
\hline$P(1)-C(21)$ & $1.840(3)$ \\
\hline $\mathrm{N}(1)-\mathrm{C}(7)$ & $1.497(4)$ \\
\hline $\mathrm{N}(1)-\mathrm{C}(1)$ & $1.498(4)$ \\
\hline $\mathrm{N}(1)-\mathrm{C}(6)$ & $1.511(4)$ \\
\hline $\mathrm{N}(2)-\mathrm{C}(9)$ & $1.490(4)$ \\
\hline $\mathrm{N}(2)-\mathrm{C}(3)$ & $1.496(4)$ \\
\hline $\mathrm{N}(2)-\mathrm{C}(2)$ & $1.509(4)$ \\
\hline$C(1)-C(2)$ & $1.509(5)$ \\
\hline $\mathrm{C}(1)-\mathrm{H}(1 \mathrm{~A})$ & 0.9700 \\
\hline $\mathrm{C}(1)-\mathrm{H}(1 \mathrm{~B})$ & 0.9700 \\
\hline $\mathrm{C}(2)-\mathrm{H}(2 \mathrm{~A})$ & 0.9700 \\
\hline $\mathrm{C}(2)-\mathrm{H}(2 \mathrm{~B})$ & 0.9700 \\
\hline$C(3)-C(4)$ & $1.522(5)$ \\
\hline $\mathrm{C}(3)-\mathrm{H}(3 \mathrm{~A})$ & 0.9700 \\
\hline $\mathrm{C}(3)-\mathrm{H}(3 \mathrm{~B})$ & 0.9700 \\
\hline $\mathrm{C}(4)-\mathrm{H}(4 \mathrm{~A})$ & 0.9700 \\
\hline $\mathrm{C}(4)-\mathrm{H}(4 \mathrm{~B})$ & 0.9700 \\
\hline$C(5)-C(6)$ & $1.521(5)$ \\
\hline $\mathrm{C}(5)-\mathrm{H}(5 \mathrm{~A})$ & 0.9700 \\
\hline $\mathrm{C}(5)-\mathrm{H}(5 \mathrm{~B})$ & 0.9700 \\
\hline $\mathrm{C}(6)-\mathrm{H}(6 \mathrm{~A})$ & 0.9700 \\
\hline $\mathrm{C}(6)-\mathrm{H}(6 \mathrm{~B})$ & 0.9700 \\
\hline$C(7)-C(8)$ & $1.537(5)$ \\
\hline $\mathrm{C}(7)-\mathrm{H}(7 \mathrm{~A})$ & 0.9700 \\
\hline $\mathrm{C}(7)-\mathrm{H}(7 \mathrm{~B})$ & 0.9700 \\
\hline$C(8)-C(12)$ & $1.518(5)$ \\
\hline$C(8)-C(11)$ & $1.539(5)$ \\
\hline $\mathrm{C}(9)-\mathrm{C}(10)$ & $1.545(5)$ \\
\hline$C(9)-H(9 A)$ & 0.9700 \\
\hline $\mathrm{C}(9)-\mathrm{H}(9 \mathrm{~B})$ & 0.9700 \\
\hline$C(10)-C(14)$ & $1.538(5)$ \\
\hline$C(10)-C(13)$ & $1.538(5)$ \\
\hline $\mathrm{C}(11)-\mathrm{H}(11 \mathrm{~A})$ & 0.9600 \\
\hline $\mathrm{C}(11)-\mathrm{H}(11 \mathrm{~B})$ & 0.9600 \\
\hline $\mathrm{C}(11)-\mathrm{H}(11 \mathrm{C})$ & 0.9600 \\
\hline $\mathrm{C}(12)-\mathrm{H}(12 \mathrm{~A})$ & 0.9600 \\
\hline $\mathrm{C}(12)-\mathrm{H}(12 \mathrm{~B})$ & 0.9600 \\
\hline $\mathrm{C}(12)-\mathrm{H}(12 \mathrm{C})$ & 0.9600 \\
\hline $\mathrm{C}(13)-\mathrm{H}(13 \mathrm{~A})$ & 0.9600 \\
\hline $\mathrm{C}(13)-\mathrm{H}(13 \mathrm{~B})$ & 0.9600 \\
\hline
\end{tabular}




\begin{tabular}{|c|c|}
\hline$C(13)-H(13 C)$ & 0.9600 \\
\hline $\mathrm{C}(14)-\mathrm{H}(14 \mathrm{~A})$ & 0.9600 \\
\hline$C(14)-H(14 B)$ & 0.9600 \\
\hline$C(14)-H(14 C)$ & 0.9600 \\
\hline$C(15)-C(16)$ & $1.400(5)$ \\
\hline$C(15)-C(20)$ & $1.405(5)$ \\
\hline$C(16)-C(17)$ & $1.385(5)$ \\
\hline $\mathrm{C}(16)-\mathrm{H}(16)$ & 0.9300 \\
\hline$C(17)-C(18)$ & $1.393(5)$ \\
\hline $\mathrm{C}(17)-\mathrm{H}(17)$ & 0.9300 \\
\hline$C(18)-C(19)$ & $1.384(6)$ \\
\hline $\mathrm{C}(18)-\mathrm{H}(18)$ & 0.9300 \\
\hline$C(19)-C(20)$ & $1.379(5)$ \\
\hline $\mathrm{C}(19)-\mathrm{H}(19)$ & 0.9300 \\
\hline $\mathrm{C}(20)-\mathrm{H}(20)$ & 0.9300 \\
\hline$C(21)-C(26)$ & $1.390(5)$ \\
\hline$C(21)-C(22)$ & $1.397(5)$ \\
\hline$C(22)-C(23)$ & $1.382(5)$ \\
\hline $\mathrm{C}(22)-\mathrm{H}(22)$ & 0.9300 \\
\hline$C(23)-C(24)$ & $1.390(5)$ \\
\hline $\mathrm{C}(23)-\mathrm{H}(23)$ & 0.9300 \\
\hline$C(24)-C(25)$ & $1.374(5)$ \\
\hline $\mathrm{C}(24)-\mathrm{H}(24)$ & 0.9300 \\
\hline$C(25)-C(26)$ & $1.393(5)$ \\
\hline $\mathrm{C}(25)-\mathrm{H}(25)$ & 0.9300 \\
\hline $\mathrm{C}(26)-\mathrm{H}(26)$ & 0.9300 \\
\hline$C(27)-H(27 A)$ & 0.9600 \\
\hline $\mathrm{C}(27)-\mathrm{H}(27 \mathrm{~B})$ & 0.9600 \\
\hline $\mathrm{C}(27)-\mathrm{H}(27 \mathrm{C})$ & 0.9600 \\
\hline $\mathrm{Ru}(2)-\mathrm{N}(3)$ & $2.188(3)$ \\
\hline $\mathrm{Ru}(2)-\mathrm{N}(4)$ & $2.190(3)$ \\
\hline $\mathrm{Ru}(2)-\mathrm{S}(5)$ & $2.2365(8)$ \\
\hline $\operatorname{Ru}(2)-S(4)$ & $2.3136(8)$ \\
\hline$R u(2)-P(2)$ & $2.3145(8)$ \\
\hline $\mathrm{Ru}(2)-\mathrm{S}(6)$ & $2.4062(8)$ \\
\hline$S(4)-C(32)$ & $1.816(3)$ \\
\hline$S(4)-C(31)$ & $1.842(3)$ \\
\hline$S(5)-O(3)$ & $1.482(3)$ \\
\hline$S(5)-O(4)$ & $1.483(2)$ \\
\hline$S(5)-C(35)$ & $1.901(3)$ \\
\hline$S(6)-C(36)$ & $1.856(3)$ \\
\hline$P(2)-C(54)$ & $1.834(3)$ \\
\hline$P(2)-C(42)$ & $1.842(3)$ \\
\hline$P(2)-C(48)$ & $1.843(3)$ \\
\hline $\mathrm{N}(3)-\mathrm{C}(28)$ & $1.491(4)$ \\
\hline$N(3)-C(34)$ & $1.504(4)$ \\
\hline$N(3)-C(33)$ & $1.513(4)$ \\
\hline$N(4)-C(30)$ & $1.492(4)$ \\
\hline$N(4)-C(37)$ & $1.494(4)$ \\
\hline $\mathrm{N}(4)-\mathrm{C}(29)$ & $1.505(4)$ \\
\hline$C(28)-C(29)$ & $1.512(5)$ \\
\hline $\mathrm{C}(28)-\mathrm{H}(28 \mathrm{~A})$ & 0.9700 \\
\hline $\mathrm{C}(28)-\mathrm{H}(28 \mathrm{~B})$ & 0.9700 \\
\hline$C(29)-H(29 A)$ & 0.9700 \\
\hline $\mathrm{C}(29)-\mathrm{H}(29 \mathrm{~B})$ & 0.9700 \\
\hline $\mathrm{C}(30)-\mathrm{C}(31)$ & $1.528(5)$ \\
\hline
\end{tabular}




\begin{tabular}{|c|c|}
\hline $\mathrm{C}(30)-\mathrm{H}(30 \mathrm{~A})$ & 0.9700 \\
\hline $\mathrm{C}(30)-\mathrm{H}(30 \mathrm{~B})$ & 0.9700 \\
\hline $\mathrm{C}(31)-\mathrm{H}(31 \mathrm{~A})$ & 0.9700 \\
\hline $\mathrm{C}(31)-\mathrm{H}(31 \mathrm{~B})$ & 0.9700 \\
\hline $\mathrm{C}(32)-\mathrm{C}(33)$ & $1.523(4)$ \\
\hline $\mathrm{C}(32)-\mathrm{H}(32 \mathrm{~A})$ & 0.9700 \\
\hline $\mathrm{C}(32)-\mathrm{H}(32 \mathrm{~B})$ & 0.9700 \\
\hline $\mathrm{C}(33)-\mathrm{H}(33 \mathrm{~A})$ & 0.9700 \\
\hline $\mathrm{C}(33)-\mathrm{H}(33 \mathrm{~B})$ & 0.9700 \\
\hline$C(34)-C(35)$ & $1.537(5)$ \\
\hline $\mathrm{C}(34)-\mathrm{H}(34 \mathrm{~A})$ & 0.9700 \\
\hline $\mathrm{C}(34)-\mathrm{H}(34 \mathrm{~B})$ & 0.9700 \\
\hline$C(35)-C(38)$ & $1.524(5)$ \\
\hline$C(35)-C(39)$ & $1.532(5)$ \\
\hline$C(36)-C(40)$ & $1.533(5)$ \\
\hline$C(36)-C(37)$ & $1.540(5)$ \\
\hline$C(36)-C(41)$ & $1.538(5)$ \\
\hline $\mathrm{C}(37)-\mathrm{H}(37 \mathrm{~A})$ & 0.9700 \\
\hline $\mathrm{C}(37)-\mathrm{H}(37 \mathrm{~B})$ & 0.9700 \\
\hline $\mathrm{C}(38)-\mathrm{H}(38 \mathrm{~A})$ & 0.9600 \\
\hline $\mathrm{C}(38)-\mathrm{H}(38 \mathrm{~B})$ & 0.9600 \\
\hline $\mathrm{C}(38)-\mathrm{H}(38 \mathrm{C})$ & 0.9600 \\
\hline$C(39)-H(39 A)$ & 0.9600 \\
\hline $\mathrm{C}(39)-\mathrm{H}(39 \mathrm{~B})$ & 0.9600 \\
\hline $\mathrm{C}(39)-\mathrm{H}(39 \mathrm{C})$ & 0.9600 \\
\hline $\mathrm{C}(40)-\mathrm{H}(40 \mathrm{~A})$ & 0.9600 \\
\hline $\mathrm{C}(40)-\mathrm{H}(40 \mathrm{~B})$ & 0.9600 \\
\hline $\mathrm{C}(40)-\mathrm{H}(40 \mathrm{C})$ & 0.9600 \\
\hline $\mathrm{C}(41)-\mathrm{H}(41 \mathrm{~A})$ & 0.9600 \\
\hline $\mathrm{C}(41)-\mathrm{H}(41 \mathrm{~B})$ & 0.9600 \\
\hline $\mathrm{C}(41)-\mathrm{H}(41 \mathrm{C})$ & 0.9600 \\
\hline$C(42)-C(43)$ & $1.385(5)$ \\
\hline$C(42)-C(47)$ & $1.396(5)$ \\
\hline$C(43)-C(44)$ & $1.398(5)$ \\
\hline $\mathrm{C}(43)-\mathrm{H}(43)$ & 0.9300 \\
\hline$C(44)-C(45)$ & $1.385(6)$ \\
\hline $\mathrm{C}(44)-\mathrm{H}(44)$ & 0.9300 \\
\hline$C(45)-C(46)$ & $1.379(5)$ \\
\hline $\mathrm{C}(45)-\mathrm{H}(45)$ & 0.9300 \\
\hline$C(46)-C(47)$ & $1.375(5)$ \\
\hline $\mathrm{C}(46)-\mathrm{H}(46)$ & 0.9300 \\
\hline $\mathrm{C}(47)-\mathrm{H}(47)$ & 0.9300 \\
\hline$C(48)-C(53)$ & $1.387(5)$ \\
\hline$C(48)-C(49)$ & $1.407(5)$ \\
\hline$C(49)-C(50)$ & $1.381(5)$ \\
\hline $\mathrm{C}(49)-\mathrm{H}(49)$ & 0.9300 \\
\hline $\mathrm{C}(50)-\mathrm{C}(51)$ & $1.382(6)$ \\
\hline $\mathrm{C}(50)-\mathrm{H}(50)$ & 0.9300 \\
\hline $\mathrm{C}(51)-\mathrm{C}(52)$ & $1.379(5)$ \\
\hline $\mathrm{C}(51)-\mathrm{H}(51)$ & 0.9300 \\
\hline$C(52)-C(53)$ & $1.402(5)$ \\
\hline $\mathrm{C}(52)-\mathrm{H}(52)$ & 0.9300 \\
\hline $\mathrm{C}(53)-\mathrm{H}(53)$ & 0.9300 \\
\hline $\mathrm{C}(54)-\mathrm{H}(54 \mathrm{~A})$ & 0.9600 \\
\hline $\mathrm{C}(54)-\mathrm{H}(54 \mathrm{~B})$ & 0.9600 \\
\hline $\mathrm{C}(54)-\mathrm{H}(54 \mathrm{C})$ & 0.9600 \\
\hline
\end{tabular}




\begin{tabular}{|c|c|}
\hline$N(200)-C(201)$ & $1.148(6)$ \\
\hline$C(200)-C(201)$ & $1.452(6)$ \\
\hline $\mathrm{C}(200)-\mathrm{H}(20 \mathrm{~A})$ & 0.9600 \\
\hline $\mathrm{C}(200)-\mathrm{H}(20 \mathrm{~B})$ & 0.9600 \\
\hline $\mathrm{C}(200)-\mathrm{H}(20 \mathrm{C})$ & 0.9600 \\
\hline $\mathrm{P}(3)-\mathrm{C}(402) \# 1$ & $0.968(7)$ \\
\hline$P(3)-C(401) \# 1$ & $1.457(6)$ \\
\hline$P(3)-C(403) \# 1$ & $1.690(6)$ \\
\hline$P(3)-C(400)$ & $1.830(5)$ \\
\hline$P(3)-C(300)$ & $1.836(4)$ \\
\hline$P(3)-C(500)$ & $1.793(10)$ \\
\hline$P(3)-C(404) \# 1$ & $1.932(5)$ \\
\hline$C(500)-C(402) \# 1$ & $0.929(10)$ \\
\hline$C(500)-C(401) \# 1$ & $1.772(10)$ \\
\hline $\mathrm{C}(500)-\mathrm{H}(50 \mathrm{~A})$ & 0.9600 \\
\hline $\mathrm{C}(500)-\mathrm{H}(50 \mathrm{~B})$ & 0.9600 \\
\hline $\mathrm{C}(500)-\mathrm{H}(50 \mathrm{C})$ & 0.9600 \\
\hline$C(300)-C(305)$ & $1.3873(16)$ \\
\hline$C(300)-C(301)$ & $1.3873(16)$ \\
\hline $\mathrm{C}(300)-\mathrm{C}(403) \# 1$ & $1.436(8)$ \\
\hline$C(300)-C(404) \# 1$ & $1.895(7)$ \\
\hline$C(300)-C(402) \# 1$ & $1.963(7)$ \\
\hline$C(305)-C(403) \# 1$ & $1.373(8)$ \\
\hline$C(305)-C(304)$ & $1.3871(16)$ \\
\hline$C(304)-C(303)$ & $1.3873(15)$ \\
\hline $\mathrm{C}(304)-\mathrm{H}(304)$ & 0.9300 \\
\hline$C(303)-C(302)$ & $1.3873(15)$ \\
\hline $\mathrm{C}(303)-\mathrm{H}(303)$ & 0.9300 \\
\hline$C(302)-C(301)$ & $1.3873(16)$ \\
\hline $\mathrm{C}(302)-\mathrm{H}(302)$ & 0.9300 \\
\hline $\mathrm{C}(301)-\mathrm{H}(301)$ & 0.9300 \\
\hline$C(400)-C(401)$ & $1.2439(14)$ \\
\hline$C(400)-C(401) \# 1$ & $1.238(8)$ \\
\hline $\mathrm{C}(400)-\mathrm{C}(404) \# 1$ & $1.637(8)$ \\
\hline$C(400)-C(404)$ & $1.6332(19)$ \\
\hline$C(400)-P(3) \# 1$ & $1.828(5)$ \\
\hline$C(404)-C(403)$ & $1.3963(16)$ \\
\hline$C(404)-C(401) \# 1$ & $1.629(10)$ \\
\hline$C(404)-C(300) \# 1$ & $1.895(8)$ \\
\hline $\mathrm{C}(404)-\mathrm{P}(3) \# 1$ & $1.932(6)$ \\
\hline$C(403)-C(305) \# 1$ & $1.373(14)$ \\
\hline$C(403)-C(300) \# 1$ & $1.436(8)$ \\
\hline$C(403)-P(3) \# 1$ & $1.691(6)$ \\
\hline $\mathrm{C}(402)-\mathrm{P}(3) \# 1$ & $0.968(6)$ \\
\hline $\mathrm{C}(402)-\mathrm{C}(500) \# 1$ & $0.930(10)$ \\
\hline$C(402)-C(401)$ & $1.3432(15)$ \\
\hline$C(402)-C(300) \# 1$ & $1.963(7)$ \\
\hline $\mathrm{C}(401)-\mathrm{P}(3) \# 1$ & $1.457(5)$ \\
\hline $\mathrm{C}(401)-\mathrm{C}(404) \# 1$ & $1.629(7)$ \\
\hline $\mathrm{C}(401)-\mathrm{C}(500) \# 1$ & $1.773(10)$ \\
\hline$N(1)-R u(1)-N(2)$ & $80.43(10)$ \\
\hline$N(1)-R u(1)-S(2)$ & $85.23(7)$ \\
\hline $\mathrm{N}(2)-\mathrm{Ru}(1)-\mathrm{S}(2)$ & $165.37(7)$ \\
\hline$N(1)-R u(1)-P(1)$ & $171.67(8)$ \\
\hline $\mathrm{N}(2)-\mathrm{Ru}(1)-\mathrm{P}(1)$ & $104.35(7)$ \\
\hline $\mathrm{S}(2)-\mathrm{Ru}(1)-\mathrm{P}(1)$ & $90.24(3)$ \\
\hline
\end{tabular}




\begin{tabular}{|c|c|}
\hline$N(1)-R u(1)-S(1)$ & $85.41(7)$ \\
\hline$N(2)-R u(1)-S(1)$ & $85.50(7)$ \\
\hline$S(2)-R u(1)-S(1)$ & $96.29(3)$ \\
\hline$P(1)-R u(1)-S(1)$ & $88.15(3)$ \\
\hline$N(1)-R u(1)-S(3)$ & $89.29(7)$ \\
\hline $\mathrm{N}(2)-\mathrm{Ru}(1)-\mathrm{S}(3)$ & $83.06(7)$ \\
\hline$S(2)-R u(1)-S(3)$ & $93.94(3)$ \\
\hline$P(1)-R u(1)-S(3)$ & $98.00(3)$ \\
\hline$S(1)-R u(1)-S(3)$ & $168.05(3)$ \\
\hline$C(5)-S(1)-C(4)$ & $102.56(16)$ \\
\hline$C(5)-S(1)-R u(1)$ & $99.15(11)$ \\
\hline$C(4)-S(1)-R u(1)$ & $100.48(11)$ \\
\hline$O(1)-S(2)-O(2)$ & $111.87(16)$ \\
\hline $\mathrm{O}(1)-\mathrm{S}(2)-\mathrm{C}(8)$ & $102.64(15)$ \\
\hline $\mathrm{O}(2)-\mathrm{S}(2)-\mathrm{C}(8)$ & $104.87(15)$ \\
\hline$O(1)-S(2)-R u(1)$ & $115.19(11)$ \\
\hline $\mathrm{O}(2)-\mathrm{S}(2)-\mathrm{Ru}(1)$ & $116.55(11)$ \\
\hline$C(8)-S(2)-R u(1)$ & $103.68(11)$ \\
\hline $\mathrm{C}(10)-\mathrm{S}(3)-\mathrm{Ru}(1)$ & $101.31(11)$ \\
\hline$C(27)-P(1)-C(15)$ & $100.53(15)$ \\
\hline $\mathrm{C}(27)-\mathrm{P}(1)-\mathrm{C}(21)$ & $97.33(15)$ \\
\hline$C(15)-P(1)-C(21)$ & $100.14(15)$ \\
\hline$C(27)-P(1)-R u(1)$ & $112.13(11)$ \\
\hline$C(15)-P(1)-R u(1)$ & $115.01(10)$ \\
\hline$C(21)-P(1)-R u(1)$ & $127.42(11)$ \\
\hline$C(7)-N(1)-C(1)$ & $107.1(2)$ \\
\hline$C(7)-N(1)-C(6)$ & $113.3(3)$ \\
\hline $\mathrm{C}(1)-\mathrm{N}(1)-\mathrm{C}(6)$ & $107.5(2)$ \\
\hline $\mathrm{C}(7)-\mathrm{N}(1)-\mathrm{Ru}(1)$ & $108.52(18)$ \\
\hline $\mathrm{C}(1)-\mathrm{N}(1)-\mathrm{Ru}(1)$ & $105.84(19)$ \\
\hline $\mathrm{C}(6)-\mathrm{N}(1)-\mathrm{Ru}(1)$ & $114.13(19)$ \\
\hline$C(9)-N(2)-C(3)$ & $109.4(3)$ \\
\hline $\mathrm{C}(9)-\mathrm{N}(2)-\mathrm{C}(2)$ & $111.5(3)$ \\
\hline $\mathrm{C}(3)-\mathrm{N}(2)-\mathrm{C}(2)$ & $107.6(2)$ \\
\hline $\mathrm{C}(9)-\mathrm{N}(2)-\mathrm{Ru}(1)$ & $108.46(19)$ \\
\hline $\mathrm{C}(3)-\mathrm{N}(2)-\mathrm{Ru}(1)$ & $109.70(19)$ \\
\hline $\mathrm{C}(2)-\mathrm{N}(2)-\mathrm{Ru}(1)$ & $110.2(2)$ \\
\hline $\mathrm{N}(1)-\mathrm{C}(1)-\mathrm{C}(2)$ & $110.1(3)$ \\
\hline $\mathrm{N}(1)-\mathrm{C}(1)-\mathrm{H}(1 \mathrm{~A})$ & 109.6 \\
\hline $\mathrm{C}(2)-\mathrm{C}(1)-\mathrm{H}(1 \mathrm{~A})$ & 109.6 \\
\hline $\mathrm{N}(1)-\mathrm{C}(1)-\mathrm{H}(1 \mathrm{~B})$ & 109.6 \\
\hline $\mathrm{C}(2)-\mathrm{C}(1)-\mathrm{H}(1 \mathrm{~B})$ & 109.6 \\
\hline$H(1 A)-C(1)-H(1 B)$ & 108.2 \\
\hline $\mathrm{N}(2)-\mathrm{C}(2)-\mathrm{C}(1)$ & $112.0(3)$ \\
\hline $\mathrm{N}(2)-\mathrm{C}(2)-\mathrm{H}(2 \mathrm{~A})$ & 109.2 \\
\hline $\mathrm{C}(1)-\mathrm{C}(2)-\mathrm{H}(2 \mathrm{~A})$ & 109.2 \\
\hline $\mathrm{N}(2)-\mathrm{C}(2)-\mathrm{H}(2 \mathrm{~B})$ & 109.2 \\
\hline $\mathrm{C}(1)-\mathrm{C}(2)-\mathrm{H}(2 \mathrm{~B})$ & 109.2 \\
\hline $\mathrm{H}(2 \mathrm{~A})-\mathrm{C}(2)-\mathrm{H}(2 \mathrm{~B})$ & 107.9 \\
\hline $\mathrm{N}(2)-\mathrm{C}(3)-\mathrm{C}(4)$ & $112.6(3)$ \\
\hline $\mathrm{N}(2)-\mathrm{C}(3)-\mathrm{H}(3 \mathrm{~A})$ & 109.1 \\
\hline $\mathrm{C}(4)-\mathrm{C}(3)-\mathrm{H}(3 \mathrm{~A})$ & 109.1 \\
\hline $\mathrm{N}(2)-\mathrm{C}(3)-\mathrm{H}(3 \mathrm{~B})$ & 109.1 \\
\hline $\mathrm{C}(4)-\mathrm{C}(3)-\mathrm{H}(3 \mathrm{~B})$ & 109.1 \\
\hline $\mathrm{H}(3 \mathrm{~A})-\mathrm{C}(3)-\mathrm{H}(3 \mathrm{~B})$ & 107.8 \\
\hline $\mathrm{C}(3)-\mathrm{C}(4)-\mathrm{S}(1)$ & $112.3(2)$ \\
\hline
\end{tabular}




$\begin{array}{ll}\text { C(3)-C(4)-H(4A) } & 109.1 \\ \text { S(1)-C(4)-H(4A) } & 109.1 \\ \text { C(3)-C(4)-H(4B) } & 109.1 \\ \text { S(1)-C(4)-H(4B) } & 109.1 \\ \text { H(4A)-C(4)-H(4B) } & 107.9 \\ \text { C(6)-C(5)-S(1) } & 112.7(2) \\ \text { C(6)-C(5)-H(5A) } & 109.1 \\ \text { S(1)-C(5)-H(5A) } & 109.1 \\ \text { C(6)-C(5)-H(5B) } & 109.1 \\ \text { S(1)-C(5)-H(5B) } & 109.1 \\ \text { H(5A)-C(5)-H(5B) } & 107.8 \\ \text { N(1)-C(6)-C(5) } & 115.0(3) \\ \text { N(1)-C(6)-H(6A) } & 108.5 \\ \text { C(5)-C(6)-H(6A) } & 108.5 \\ \text { N(1)-C(6)-H(6B) } & 108.5 \\ \text { C(5)-C(6)-H(6B) } & 108.5 \\ \text { H(6A)-C(6)-H(6B) } & 107.5 \\ \text { N(1)-C(7)-C(8) } & 116.6(3) \\ \text { N(1)-C(7)-H(7A) } & 108.2 \\ \text { C(8)-C(7)-H(7A) } & 108.2 \\ \text { N(1)-C(7)-H(7B) } & 108.2 \\ \text { C(8)-C(7)-H(7B) } & 108.2 \\ \text { H(7A)-C(7)-H(7B) } & 107.3 \\ \text { C(12)-C(8)-C(7) } & 113.7(3) \\ \text { C(12)-C(8)-C(11) } & 109.1(3) \\ \text { C(7)-C(8)-C(11) } & 106.6(3) \\ \text { C(12)-C(8)-S(2) } & 109.9(2) \\ \text { C(7)-C(8)-S(2) } & 108.7(2) \\ \text { C(11)-C(8)-S(2) } & 108.7(2) \\ \text { N(2)-C(9)-C(10) } & 117.7(3) \\ \text { N(2)-C(9)-H(9A) } & 107.9 \\ \text { C(10)-C(9)-H(9A) } & 107.9 \\ \text { N(2)-C(9)-H(9B) } & 107.9 \\ \text { C(10)-C(9)-H(9B) } & 107.9 \\ \text { H(9A)-C(9)-H(9B) } & 107.2 \\ \text { C(14)-C(10)-C(13) } & 107.7(3) \\ \text { C(14)-C(10)-C(9) } & 106.7(3) \\ \text { C(13)-C(10)-C(9) } & 113.5(3) \\ \text { C(14)-C(10)-S(3) } & 108.2(2) \\ \text { C(13)-C(10)-S(3) } & 109.6(2) \\ \text { C(9)-C(10)-S(3) } & 11.0(2) \\ \text { C(8)-C(11)-H(11A) } & 109.5 \\ \text { C(8)-C(11)-H(11B) } & 109.5 \\ \text { H(11A)-C(11)-H(11B) } & 109.5 \\ \text { C(8)-C(11)-H(11C) } & 109.5 \\ \text { H(11A)-C(11)-H(11C) } & 109.5 \\ \text { H(11B)-C(11)-H(11C) } & 109.5 \\ \text { C(8)-C(12)-H(12A) } & 109.5 \\ \text { C(8)-C(12)-H(12B) } & 109.5 \\ \text { H(12A)-C(12)-H(12B) } & 109.5 \\ \text { C(8)-C(12)-H(12C) } & 109.5 \\ \text { H(12A)-C(12)-H(12C) } & 109.5 \\ \text { H(12B)-C(12)-H(12C) } & 109.5 \\ \text { C(10)-C(13)-H(13A) } & 109.5 \\ \text { C(10)-C(13)-H(13B) } & 109.5 \\ \text { H(13A)-C(13)-H(13B) } & 109.5 \\ & \\ & \end{array}$




\begin{tabular}{|c|c|}
\hline $\mathrm{C}(10)-\mathrm{C}(13)-\mathrm{H}(13 \mathrm{C})$ & 109.5 \\
\hline $\mathrm{H}(13 \mathrm{~A})-\mathrm{C}(13)-\mathrm{H}(13 \mathrm{C})$ & 109.5 \\
\hline $\mathrm{H}(13 \mathrm{~B})-\mathrm{C}(13)-\mathrm{H}(13 \mathrm{C})$ & 109.5 \\
\hline $\mathrm{C}(10)-\mathrm{C}(14)-\mathrm{H}(14 \mathrm{~A})$ & 109.5 \\
\hline $\mathrm{C}(10)-\mathrm{C}(14)-\mathrm{H}(14 \mathrm{~B})$ & 109.5 \\
\hline $\mathrm{H}(14 \mathrm{~A})-\mathrm{C}(14)-\mathrm{H}(14 \mathrm{~B})$ & 109.5 \\
\hline $\mathrm{C}(10)-\mathrm{C}(14)-\mathrm{H}(14 \mathrm{C})$ & 109.5 \\
\hline $\mathrm{H}(14 \mathrm{~A})-\mathrm{C}(14)-\mathrm{H}(14 \mathrm{C})$ & 109.5 \\
\hline $\mathrm{H}(14 \mathrm{~B})-\mathrm{C}(14)-\mathrm{H}(14 \mathrm{C})$ & 109.5 \\
\hline$C(16)-C(15)-C(20)$ & $117.9(3)$ \\
\hline$C(16)-C(15)-P(1)$ & $121.6(2)$ \\
\hline$C(20)-C(15)-P(1)$ & $119.9(3)$ \\
\hline$C(17)-C(16)-C(15)$ & $121.0(3)$ \\
\hline$C(17)-C(16)-H(16)$ & 119.5 \\
\hline $\mathrm{C}(15)-\mathrm{C}(16)-\mathrm{H}(16)$ & 119.5 \\
\hline$C(16)-C(17)-C(18)$ & $119.8(3)$ \\
\hline $\mathrm{C}(16)-\mathrm{C}(17)-\mathrm{H}(17)$ & 120.1 \\
\hline $\mathrm{C}(18)-\mathrm{C}(17)-\mathrm{H}(17)$ & 120.1 \\
\hline $\mathrm{C}(19)-\mathrm{C}(18)-\mathrm{C}(17)$ & $120.0(3)$ \\
\hline$C(19)-C(18)-H(18)$ & 120.0 \\
\hline $\mathrm{C}(17)-\mathrm{C}(18)-\mathrm{H}(18)$ & 120.0 \\
\hline$C(20)-C(19)-C(18)$ & $120.1(3)$ \\
\hline $\mathrm{C}(20)-\mathrm{C}(19)-\mathrm{H}(19)$ & 120.0 \\
\hline $\mathrm{C}(18)-\mathrm{C}(19)-\mathrm{H}(19)$ & 120.0 \\
\hline$C(19)-C(20)-C(15)$ & $121.1(3)$ \\
\hline $\mathrm{C}(19)-\mathrm{C}(20)-\mathrm{H}(20)$ & 119.5 \\
\hline$C(15)-C(20)-H(20)$ & 119.5 \\
\hline$C(26)-C(21)-C(22)$ & $118.2(3)$ \\
\hline$C(26)-C(21)-P(1)$ & $121.5(2)$ \\
\hline$C(22)-C(21)-P(1)$ & $119.9(2)$ \\
\hline $\mathrm{C}(23)-\mathrm{C}(22)-\mathrm{C}(21)$ & $121.2(3)$ \\
\hline $\mathrm{C}(23)-\mathrm{C}(22)-\mathrm{H}(22)$ & 119.4 \\
\hline $\mathrm{C}(21)-\mathrm{C}(22)-\mathrm{H}(22)$ & 119.4 \\
\hline$C(22)-C(23)-C(24)$ & $119.7(3)$ \\
\hline$C(22)-C(23)-H(23)$ & 120.1 \\
\hline $\mathrm{C}(24)-\mathrm{C}(23)-\mathrm{H}(23)$ & 120.1 \\
\hline$C(25)-C(24)-C(23)$ & $119.8(3)$ \\
\hline $\mathrm{C}(25)-\mathrm{C}(24)-\mathrm{H}(24)$ & 120.1 \\
\hline $\mathrm{C}(23)-\mathrm{C}(24)-\mathrm{H}(24)$ & 120.1 \\
\hline$C(24)-C(25)-C(26)$ & $120.5(3)$ \\
\hline$C(24)-C(25)-H(25)$ & 119.7 \\
\hline$C(26)-C(25)-H(25)$ & 119.7 \\
\hline$C(21)-C(26)-C(25)$ & $120.5(3)$ \\
\hline$C(21)-C(26)-H(26)$ & 119.8 \\
\hline$C(25)-C(26)-H(26)$ & 119.8 \\
\hline$P(1)-C(27)-H(27 A)$ & 109.5 \\
\hline $\mathrm{P}(1)-\mathrm{C}(27)-\mathrm{H}(27 \mathrm{~B})$ & 109.5 \\
\hline $\mathrm{H}(27 \mathrm{~A})-\mathrm{C}(27)-\mathrm{H}(27 \mathrm{~B})$ & 109.5 \\
\hline $\mathrm{P}(1)-\mathrm{C}(27)-\mathrm{H}(27 \mathrm{C})$ & 109.5 \\
\hline $\mathrm{H}(27 \mathrm{~A})-\mathrm{C}(27)-\mathrm{H}(27 \mathrm{C})$ & 109.5 \\
\hline $\mathrm{H}(27 \mathrm{~B})-\mathrm{C}(27)-\mathrm{H}(27 \mathrm{C})$ & 109.5 \\
\hline $\mathrm{N}(3)-\mathrm{Ru}(2)-\mathrm{N}(4)$ & $80.42(10)$ \\
\hline N(3)-Ru(2)-S(5) & $85.43(7)$ \\
\hline $\mathrm{N}(4)-\mathrm{Ru}(2)-\mathrm{S}(5)$ & $165.70(7)$ \\
\hline $\mathrm{N}(3)-\mathrm{Ru}(2)-\mathrm{S}(4)$ & $85.08(7)$ \\
\hline N(4)-Ru(2)-S(4) & $85.15(7)$ \\
\hline
\end{tabular}




\begin{tabular}{|c|c|}
\hline$S(5)-R u(2)-S(4)$ & $95.74(3)$ \\
\hline$N(3)-R u(2)-P(2)$ & $171.41(8)$ \\
\hline $\mathrm{N}(4)-\mathrm{Ru}(2)-\mathrm{P}(2)$ & $105.19(7)$ \\
\hline $\mathrm{S}(5)-\mathrm{Ru}(2)-\mathrm{P}(2)$ & $89.10(3)$ \\
\hline$S(4)-R u(2)-P(2)$ & $88.89(3)$ \\
\hline$N(3)-R u(2)-S(6)$ & $89.29(7)$ \\
\hline$N(4)-R u(2)-S(6)$ & $82.62(7)$ \\
\hline $\mathrm{S}(5)-\mathrm{Ru}(2)-\mathrm{S}(6)$ & $95.22(3)$ \\
\hline$S(4)-R u(2)-S(6)$ & $167.24(3)$ \\
\hline$P(2)-R u(2)-S(6)$ & $97.81(3)$ \\
\hline$C(32)-S(4)-C(31)$ & $102.15(16)$ \\
\hline$C(32)-S(4)-R u(2)$ & $99.65(11)$ \\
\hline$C(31)-S(4)-R u(2)$ & $100.95(11)$ \\
\hline $\mathrm{O}(3)-\mathrm{S}(5)-\mathrm{O}(4)$ & $111.28(15)$ \\
\hline $\mathrm{O}(3)-\mathrm{S}(5)-\mathrm{C}(35)$ & $102.99(15)$ \\
\hline $\mathrm{O}(4)-\mathrm{S}(5)-\mathrm{C}(35)$ & $105.38(15)$ \\
\hline$O(3)-S(5)-\operatorname{Ru}(2)$ & $115.18(11)$ \\
\hline$O(4)-S(5)-R u(2)$ & $116.47(11)$ \\
\hline$C(35)-S(5)-R u(2)$ & $103.72(11)$ \\
\hline$C(36)-S(6)-R u(2)$ & $100.85(11)$ \\
\hline $\mathrm{C}(54)-\mathrm{P}(2)-\mathrm{C}(42)$ & $100.91(15)$ \\
\hline$C(54)-P(2)-C(48)$ & $98.96(16)$ \\
\hline$C(42)-P(2)-C(48)$ & $98.69(15)$ \\
\hline$C(54)-P(2)-R u(2)$ & $111.24(11)$ \\
\hline$C(42)-P(2)-R u(2)$ & $115.57(10)$ \\
\hline $\mathrm{C}(48)-\mathrm{P}(2)-\mathrm{Ru}(2)$ & $127.41(11)$ \\
\hline$C(28)-N(3)-C(34)$ & $107.5(2)$ \\
\hline$C(28)-N(3)-C(33)$ & $107.7(2)$ \\
\hline$C(34)-N(3)-C(33)$ & $112.9(3)$ \\
\hline $\mathrm{C}(28)-\mathrm{N}(3)-\mathrm{Ru}(2)$ & $105.93(19)$ \\
\hline$C(34)-N(3)-R u(2)$ & $107.72(19)$ \\
\hline$C(33)-N(3)-R u(2)$ & $114.65(19)$ \\
\hline$C(30)-N(4)-C(37)$ & $108.3(3)$ \\
\hline$C(30)-N(4)-C(29)$ & $107.8(2)$ \\
\hline$C(37)-N(4)-C(29)$ & $111.9(2)$ \\
\hline$C(30)-N(4)-R u(2)$ & $109.87(19)$ \\
\hline$C(37)-N(4)-R u(2)$ & $108.86(19)$ \\
\hline$C(29)-N(4)-R u(2)$ & $110.0(2)$ \\
\hline$N(3)-C(28)-C(29)$ & $110.1(3)$ \\
\hline $\mathrm{N}(3)-\mathrm{C}(28)-\mathrm{H}(28 \mathrm{~A})$ & 109.6 \\
\hline $\mathrm{C}(29)-\mathrm{C}(28)-\mathrm{H}(28 \mathrm{~A})$ & 109.6 \\
\hline $\mathrm{N}(3)-\mathrm{C}(28)-\mathrm{H}(28 \mathrm{~B})$ & 109.6 \\
\hline $\mathrm{C}(29)-\mathrm{C}(28)-\mathrm{H}(28 \mathrm{~B})$ & 109.6 \\
\hline $\mathrm{H}(28 \mathrm{~A})-\mathrm{C}(28)-\mathrm{H}(28 \mathrm{~B})$ & 108.2 \\
\hline $\mathrm{N}(4)-\mathrm{C}(29)-\mathrm{C}(28)$ & $112.4(3)$ \\
\hline $\mathrm{N}(4)-\mathrm{C}(29)-\mathrm{H}(29 \mathrm{~A})$ & 109.1 \\
\hline $\mathrm{C}(28)-\mathrm{C}(29)-\mathrm{H}(29 \mathrm{~A})$ & 109.1 \\
\hline $\mathrm{N}(4)-\mathrm{C}(29)-\mathrm{H}(29 \mathrm{~B})$ & 109.1 \\
\hline $\mathrm{C}(28)-\mathrm{C}(29)-\mathrm{H}(29 \mathrm{~B})$ & 109.1 \\
\hline $\mathrm{H}(29 \mathrm{~A})-\mathrm{C}(29)-\mathrm{H}(29 \mathrm{~B})$ & 107.9 \\
\hline$N(4)-C(30)-C(31)$ & $112.4(3)$ \\
\hline $\mathrm{N}(4)-\mathrm{C}(30)-\mathrm{H}(30 \mathrm{~A})$ & 109.1 \\
\hline $\mathrm{C}(31)-\mathrm{C}(30)-\mathrm{H}(30 \mathrm{~A})$ & 109.1 \\
\hline $\mathrm{N}(4)-\mathrm{C}(30)-\mathrm{H}(30 \mathrm{~B})$ & 109.1 \\
\hline $\mathrm{C}(31)-\mathrm{C}(30)-\mathrm{H}(30 \mathrm{~B})$ & 109.1 \\
\hline$H(30 A)-C(30)-H(30 B)$ & 107.9 \\
\hline
\end{tabular}




$\begin{array}{ll}\text { C(30)-C(31)-S(4) } & 111.8(2) \\ \text { C(30)-C(31)-H(31A) } & 109.3 \\ \text { S(4)-C(31)-H(31A) } & 109.3 \\ \text { C(30)-C(31)-H(31B) } & 109.3 \\ \text { S(4)-C(31)-H(31B) } & 109.3 \\ \text { H(31A)-C(31)-H(31B) } & 107.9 \\ \text { C(33)-C(32)-S(4) } & 112.6(2) \\ \text { C(33)-C(32)-H(32A) } & 109.1 \\ \text { S(4)-C(32)-H(32A) } & 109.1 \\ \text { C(33)-C(32)-H(32B) } & 109.1 \\ \text { S(4)-C(32)-H(32B) } & 109.1 \\ \mathrm{H}(32 A)-C(32)-H(32 B) & 107.8 \\ \text { N(3)-C(33)-C(32) } & 114.8(3) \\ \text { N(3)-C(33)-H(33A) } & 108.6 \\ \text { C(32)-C(33)-H(33A) } & 108.6 \\ \text { N(3)-C(33)-H(33B) } & 108.6 \\ \text { C(32)-C(33)-H(33B) } & 108.6 \\ \text { H(33A)-C(33)-H(33B) } & 107.5 \\ \text { N(3)-C(34)-C(35) } & 116.6(3) \\ \text { N(3)-C(34)-H(34A) } & 108.1 \\ \text { C(35)-C(34)-H(34A) } & 108.1 \\ \text { N(3)-C(34)-H(34B) } & 108.1 \\ \text { C(35)-C(34)-H(34B) } & 108.1 \\ \text { H(34A)-C(34)-H(34B) } & 107.3 \\ \text { C(38)-C(35)-C(39) } & 108.5(3) \\ \text { C(38)-C(35)-C(34) } & 106.9(3) \\ \text { C(39)-C(35)-C(34) } & 113.5(3) \\ \text { C(38)-C(35)-S(5) } & 108.8(2) \\ \text { C(39)-C(35)-S(5) } & 110.5(2) \\ \text { C(34)-C(35)-S(5) } & 108.5(2) \\ \text { C(40)-C(36)-C(37) } & 112.6(3) \\ \text { C(40)-C(36)-C(41) } & 108.0(3) \\ \text { C(37)-C(36)-C(41) } & 106.5(3) \\ \text { C(40)-C(36)-S(6) } & 109.7(2) \\ \text { C(37)-C(36)-S(6) } & 111.7(2) \\ \text { C(41)-C(36)-S(6) } & 108.1(2) \\ \text { N(4)-C(37)-C(36) } & 116.7(3) \\ \text { N(4)-C(37)-H(37A) } & 108.1 \\ \text { C(36)-C(37)-H(37A) } & 108.1 \\ \text { N(4)-C(37)-H(37B) } & 108.1 \\ \text { C(36)-C(37)-H(37B) } & 108.1 \\ \text { H(37A)-C(37)-H(37B) } & 107.3 \\ \text { C(35)-C(38)-H(38A) } & 109.5 \\ \text { C(35)-C(38)-H(38B) } & 109.5 \\ \text { H(38A)-C(38)-H(38B) } & 109.5 \\ \text { C(35)-C(38)-H(38C) } & 109.5 \\ \text { H(38A)-C(38)-H(38C) } & 109.5 \\ \text { H(38B)-C(38)-H(38C) } & 109.5 \\ \text { C(35)-C(39)-H(39A) } & 109.5 \\ \text { C(35)-C(39)-H(39B) } & 109.5 \\ \text { H(39A)-C(39)-H(39B) } & 109.5 \\ \text { C(35)-C(39)-H(39C) } & 109.5 \\ \text { H(39A)-C(39)-H(39C) } & 109.5 \\ \text { H(39B)-C(39)-H(39C) } & 109.5 \\ \text { C(36)-C(40)-H(40A) } & 109.5 \\ \text { C(36)-C(40)-H(40B) } & 109.5 \\ & \\ & \end{array}$




$\begin{array}{ll}\mathrm{H}(40 \mathrm{~A})-\mathrm{C}(40)-\mathrm{H}(40 \mathrm{~B}) & 109.5 \\ \mathrm{C}(36)-\mathrm{C}(40)-\mathrm{H}(40 \mathrm{C}) & 109.5 \\ \mathrm{H}(40 \mathrm{~A})-\mathrm{C}(40)-\mathrm{H}(40 \mathrm{C}) & 109.5 \\ \mathrm{H}(40 \mathrm{~B})-\mathrm{C}(40)-\mathrm{H}(40 \mathrm{C}) & 109.5 \\ \mathrm{C}(36)-\mathrm{C}(41)-\mathrm{H}(41 \mathrm{~A}) & 109.5 \\ \mathrm{C}(36)-\mathrm{C}(41)-\mathrm{H}(41 \mathrm{~B}) & 109.5 \\ \mathrm{H}(41 \mathrm{~A})-\mathrm{C}(41)-\mathrm{H}(41 \mathrm{~B}) & 109.5 \\ \mathrm{C}(36)-\mathrm{C}(41)-\mathrm{H}(41 \mathrm{C}) & 109.5 \\ \mathrm{H}(41 \mathrm{~A})-\mathrm{C}(41)-\mathrm{H}(41 \mathrm{C}) & 109.5 \\ \mathrm{H}(41 \mathrm{~B})-\mathrm{C}(41)-\mathrm{H}(41 \mathrm{C}) & 109.5 \\ \mathrm{C}(43)-\mathrm{C}(42)-\mathrm{C}(47) & 118.4(3) \\ \mathrm{C}(43)-\mathrm{C}(42)-\mathrm{P}(2) & 120.3(3) \\ \mathrm{C}(47)-\mathrm{C}(42)-\mathrm{P}(2) & 121.1(2) \\ \mathrm{C}(42)-\mathrm{C}(43)-\mathrm{C}(44) & 121.3(3) \\ \mathrm{C}(42)-\mathrm{C}(43)-\mathrm{H}(43) & 119.3 \\ \mathrm{C}(44)-\mathrm{C}(43)-\mathrm{H}(43) & 119.3 \\ \mathrm{C}(45)-\mathrm{C}(44)-\mathrm{C}(43) & 118.8(3) \\ \mathrm{C}(45)-\mathrm{C}(44)-\mathrm{H}(44) & 120.6 \\ \mathrm{C}(43)-\mathrm{C}(44)-\mathrm{H}(44) & 120.6 \\ \mathrm{C}(46)-\mathrm{C}(45)-\mathrm{C}(44) & 120.4(3) \\ \mathrm{C}(46)-\mathrm{C}(45)-\mathrm{H}(45) & 119.8 \\ \mathrm{C}(44)-\mathrm{C}(45)-\mathrm{H}(45) & 119.8 \\ \mathrm{C}(47)-\mathrm{C}(46)-\mathrm{C}(45) & 120.4(3) \\ \mathrm{C}(47)-\mathrm{C}(46)-\mathrm{H}(46) & 119.8 \\ \mathrm{C}(45)-\mathrm{C}(46)-\mathrm{H}(46) & 119.8 \\ \mathrm{C}(46)-\mathrm{C}(47)-\mathrm{C}(42) & 120.7(3) \\ \mathrm{C}(46)-\mathrm{C}(47)-\mathrm{H}(47) & 119.6 \\ \mathrm{C}(42)-\mathrm{C}(47)-\mathrm{H}(47) & 119.6 \\ \mathrm{C}(53)-\mathrm{C}(48)-\mathrm{C}(49) & 118.1(3) \\ \mathrm{C}(53)-\mathrm{C}(48)-\mathrm{P}(2) & 123.7(3) \\ \mathrm{C}(49)-\mathrm{C}(48)-\mathrm{P}(2) & 118.2(3) \\ \mathrm{C}(50)-\mathrm{C}(49)-\mathrm{C}(48) & 120.8(4) \\ \mathrm{C}(50)-\mathrm{C}(49)-\mathrm{H}(49) & 119.6 \\ \mathrm{C}(48)-\mathrm{C}(49)-\mathrm{H}(49) & 119.6 \\ \mathrm{C}(49)-\mathrm{C}(50)-\mathrm{C}(51) & 120.8(4) \\ \mathrm{C}(49)-\mathrm{C}(50)-\mathrm{H}(50) & 119.6 \\ \mathrm{C}(51)-\mathrm{C}(50)-\mathrm{H}(50) & 119.6 \\ \mathrm{C}(52)-\mathrm{C}(51)-\mathrm{C}(50) & 119.0(3) \\ \mathrm{C}(52)-\mathrm{C}(51)-\mathrm{H}(51) & 120.5 \\ \mathrm{C}(50)-\mathrm{C}(51)-\mathrm{H}(51) & 120.5 \\ \mathrm{C}(51)-\mathrm{C}(52)-\mathrm{C}(53) & 120.9(4) \\ \mathrm{C}(51)-\mathrm{C}(52)-\mathrm{H}(52) & 119.6 \\ \mathrm{C}(53)-\mathrm{C}(52)-\mathrm{H}(52) & 119.6 \\ \mathrm{C}(48)-\mathrm{C}(53)-\mathrm{C}(52) & 120.3(3) \\ \mathrm{C}(48)-\mathrm{C}(53)-\mathrm{H}(53) & 119.8 \\ \mathrm{C}(52)-\mathrm{C}(53)-\mathrm{H}(53) & 119.8 \\ \mathrm{P}(2)-\mathrm{C}(54)-\mathrm{H}(54 A) & 109.5 \\ \mathrm{P}(2)-\mathrm{C}(54)-\mathrm{H}(54 \mathrm{~B}) & 109.5 \\ \mathrm{H}(54 \mathrm{~A})-\mathrm{C}(54)-\mathrm{H}(54 \mathrm{~B}) & 109.5 \\ \mathrm{P}(2)-\mathrm{C}(54)-\mathrm{H}(54 \mathrm{C}) & 109.5 \\ \mathrm{H}(54 \mathrm{~A})-\mathrm{C}(54)-\mathrm{H}(54 \mathrm{C}) & 109.5 \\ \mathrm{H}(54 \mathrm{~B})-\mathrm{C}(54)-\mathrm{H}(54 C) & 109.5 \\ \mathrm{C}(201)-\mathrm{C}(200)-\mathrm{H}(20 \mathrm{~A}) & 109.5 \\ \mathrm{C}(201)-\mathrm{C}(200)-\mathrm{H}(20 \mathrm{~B}) & 109.5 \\ \mathrm{H}(20 \mathrm{~A})-\mathrm{C}(200)-\mathrm{H}(20 \mathrm{~B}) & 109.5 \\ \mathrm{C}(201)-\mathrm{C}(200)-\mathrm{H}(20 \mathrm{C}) & 109.5 \\ & \end{array}$




$\begin{array}{lc}\text { H(20A)-C(200)-H(20C) } & 109.5 \\ \text { H(20B)-C(200)-H(20C) } & 109.5 \\ \text { N(200)-C(201)-C(200) } & 178.4(5) \\ \text { C(402)\#1-P(3)-C(401)\#1 } & 63.6(3) \\ \text { C(402)\#1-P(3)-C(403)\#1 } & 125.5(4) \\ \text { C(401)\#1-P(3)-C(403)\#1 } & 127.4(3) \\ \text { C(402)\#1-P(3)-C(400) } & 104.9(4) \\ \text { C(401)\#1-P(3)-C(400) } & 42.4(3) \\ \text { C(403)\#1-P(3)-C(400) } & 96.0(2) \\ \text { C(402)\#1-P(3)-C(300) } & 82.6(3) \\ \text { C(401)\#1-P(3)-C(300) } & 92.2(2) \\ \text { C(403)\#1-P(3)-C(300) } & 47.8(2) \\ \text { C(400)-P(3)-C(300) } & 88.36(19) \\ \text { C(402)\#1-P(3)-C(500) } & 18.7(4) \\ \text { C(401)\#1-P(3)-C(500) } & 65.1(3) \\ \text { C(403)\#1-P(3)-C(500) } & 141.2(4) \\ \text { C(400)-P(3)-C(500) } & 107.3(4) \\ \text { C(300)-P(3)-C(500) } & 101.2(3) \\ \text { C(402)\#1-P(3)-C(404)\#1 } & 133.5(4) \\ \text { C(401)\#1-P(3)-C(404)\#1 } & 89.1(2) \\ \text { C(403)\#1-P(3)-C(404)\#1 } & 44.72(14) \\ \text { C(400)-P(3)-C(404)\#1 } & 51.5(2) \\ \text { C(300)-P(3)-C(404)\#1 } & 60.33(18) \\ \text { C(500)-P(3)-C(404)\#1 } & 148.7(3) \\ \text { C(402)\#1-C(500)-C(401)\#1 } & 48.1(5) \\ \text { C(402)\#1-C(500)-P(3) } & 19.5(5) \\ \text { C(401)\#1-C(500)-P(3) } & 48.2(3) \\ \text { C(402)\#1-C(500)-H(50A) } & 109.5 \\ \text { C(401)\#1-C(500)-H(50A) } & 91.6 \\ \text { P(3)-C(500)-H(50A) } & 90.1 \\ \text { C(402)\#1-C(500)-H(50B) } & 109.5 \\ \text { C(401)\#1-C(500)-H(50B) } & 74.7 \\ \text { P(3)-C(500)-H(50B) } & 120.5 \\ \text { H(50A)-C(500)-H(50B) } & 109.5 \\ \text { C(402)\#1-C(500)-H(50C) } & 109.5 \\ \text { C(401)\#1-C(500)-H(50C) } & 154.7 \\ \text { P(3)-C(500)-H(50C) } & 115.7 \\ \text { H(50A)-C(500)-H(50C) } & 109.5 \\ \text { H(50B)-C(500)-H(50C) } & 109.5 \\ \text { C(305)-C(300)-C(301) } & 120.0 \\ \text { C(305)-C(300)-C(403)\#1 } & 58.2(4) \\ \text { C(301)-C(300)-C(403)\#1 } & 178.1(4) \\ \text { C(305)-C(300)-P(3) } & 118.9(2) \\ \text { C(301)-C(300)-P(3) } & 121.1(2) \\ \text { C(403)\#1-C(300)-P(3) } & 60.8(3) \\ \text { C(305)-C(300)-C(404)\#1 } & 77.2(2) \\ \text { C(301)-C(300)-C(404)\#1 } & 132.6(2) \\ \text { C(403)\#1-C(300)-C(404)\#1 } & 47.1(2) \\ \text { P(3)-C(300)-C(404)\#1 } & 62.36(18) \\ \text { C(305)-C(300)-C(402)\#1 } & 143.9(4) \\ \text { C(301)-C(300)-C(402)\#1 } & 94.1(4) \\ \text { C(403)\#1-C(300)-C(402)\#1 } & 87.8(3) \\ \text { P(3)-C(300)-C(402)\#1 } & 29.3(2) \\ \text { C(404)\#1-C(300)-C(402)\#1 } & 88.5(3) \\ \text { C(403)\#1-C(305)-C(304) } & 177.2(4) \\ \text { C(403)\#1-C(305)-C(300) } & 62.7(4) \\ & \\ & \end{array}$




\begin{tabular}{|c|c|}
\hline$C(304)-C(305)-C(300)$ & 120.0 \\
\hline$C(305)-C(304)-C(303)$ & 120.0 \\
\hline $\mathrm{C}(305)-\mathrm{C}(304)-\mathrm{H}(304)$ & 120.0 \\
\hline $\mathrm{C}(303)-\mathrm{C}(304)-\mathrm{H}(304)$ & 120.0 \\
\hline$C(302)-C(303)-C(304)$ & 120.0 \\
\hline $\mathrm{C}(302)-\mathrm{C}(303)-\mathrm{H}(303)$ & 120.0 \\
\hline $\mathrm{C}(304)-\mathrm{C}(303)-\mathrm{H}(303)$ & 120.0 \\
\hline$C(301)-C(302)-C(303)$ & 120.0 \\
\hline $\mathrm{C}(301)-\mathrm{C}(302)-\mathrm{H}(302)$ & 120.0 \\
\hline $\mathrm{C}(303)-\mathrm{C}(302)-\mathrm{H}(302)$ & 120.0 \\
\hline$C(302)-C(301)-C(300)$ & 120.0 \\
\hline $\mathrm{C}(302)-\mathrm{C}(301)-\mathrm{H}(301)$ & 120.0 \\
\hline $\mathrm{C}(300)-\mathrm{C}(301)-\mathrm{H}(301)$ & 120.0 \\
\hline$C(401)-C(400)-C(401) \# 1$ & $179.6(5)$ \\
\hline$C(401)-C(400)-C(404) \# 1$ & $67.3(3)$ \\
\hline $\mathrm{C}(401) \# 1-\mathrm{C}(400)-\mathrm{C}(404) \# 1$ & $112.6(5)$ \\
\hline$C(401)-C(400)-C(404)$ & 112.5 \\
\hline$C(401) \# 1-C(400)-C(404)$ & $67.5(4)$ \\
\hline$C(404) \# 1-C(400)-C(404)$ & $179.6(3)$ \\
\hline$C(401)-C(400)-P(3)$ & $127.2(2)$ \\
\hline $\mathrm{C}(401) \# 1-\mathrm{C}(400)-\mathrm{P}(3)$ & $52.5(4)$ \\
\hline $\mathrm{C}(404) \# 1-\mathrm{C}(400)-\mathrm{P}(3)$ & $67.5(3)$ \\
\hline$C(404)-C(400)-P(3)$ & $112.50(19)$ \\
\hline $\mathrm{C}(401)-\mathrm{C}(400)-\mathrm{P}(3) \# 1$ & $52.54(18)$ \\
\hline $\mathrm{C}(401) \# 1-\mathrm{C}(400)-\mathrm{P}(3) \# 1$ & $127.7(5)$ \\
\hline $\mathrm{C}(404) \# 1-\mathrm{C}(400)-\mathrm{P}(3) \# 1$ & $112.4(3)$ \\
\hline$C(404)-C(400)-P(3) \# 1$ & $67.60(18)$ \\
\hline$P(3)-C(400)-P(3) \# 1$ & $179.7(3)$ \\
\hline $\mathrm{C}(403)-\mathrm{C}(404)-\mathrm{C}(401) \# 1$ & $156.9(3)$ \\
\hline$C(403)-C(404)-C(400)$ & 119.1 \\
\hline$C(401) \# 1-C(404)-C(400)$ & $44.6(3)$ \\
\hline$C(403)-C(404)-C(300) \# 1$ & $48.9(3)$ \\
\hline $\mathrm{C}(401) \# 1-\mathrm{C}(404)-\mathrm{C}(300) \# 1$ & $132.9(6)$ \\
\hline$C(400)-C(404)-C(300) \# 1$ & $92.6(2)$ \\
\hline $\mathrm{C}(403)-\mathrm{C}(404)-\mathrm{P}(3) \# 1$ & $58.42(16)$ \\
\hline$C(401) \# 1-C(404)-P(3) \# 1$ & $101.5(4)$ \\
\hline$C(400)-C(404)-P(3) \# 1$ & $61.01(16)$ \\
\hline $\mathrm{C}(300) \# 1-\mathrm{C}(404)-\mathrm{P}(3) \# 1$ & $57.31(19)$ \\
\hline $\mathrm{C}(305) \# 1-\mathrm{C}(403)-\mathrm{C}(404)$ & $97.8(7)$ \\
\hline $\mathrm{C}(305) \# 1-\mathrm{C}(403)-\mathrm{C}(300) \# 1$ & $59.2(5)$ \\
\hline$C(404)-C(403)-C(300) \# 1$ & $84.0(3)$ \\
\hline$C(305) \# 1-C(403)-P(3) \# 1$ & $130.5(7)$ \\
\hline C(404)-C(403)-P(3)\#1 & $76.85(19)$ \\
\hline$C(300) \# 1-C(403)-P(3) \# 1$ & $71.4(3)$ \\
\hline$P(3) \# 1-C(402)-C(500) \# 1$ & $141.8(9)$ \\
\hline$P(3) \# 1-C(402)-C(401)$ & $76.3(3)$ \\
\hline $\mathrm{C}(500) \# 1-\mathrm{C}(402)-\mathrm{C}(401)$ & $100.9(7)$ \\
\hline $\mathrm{P}(3) \# 1-\mathrm{C}(402)-\mathrm{C}(300) \# 1$ & $68.1(4)$ \\
\hline $\mathrm{C}(500) \# 1-\mathrm{C}(402)-\mathrm{C}(300) \# 1$ & $149.7(8)$ \\
\hline$C(401)-C(402)-C(300) \# 1$ & $90.5(2)$ \\
\hline$C(400)-C(401)-C(402)$ & 123.7 \\
\hline $\mathrm{C}(400)-\mathrm{C}(401)-\mathrm{P}(3) \# 1$ & $84.8(2)$ \\
\hline$C(402)-C(401)-P(3) \# 1$ & $40.2(2)$ \\
\hline$C(400)-C(401)-C(404) \# 1$ & $67.9(3)$ \\
\hline$C(402)-C(401)-C(404) \# 1$ & $157.4(3)$ \\
\hline$P(3) \# 1-C(401)-C(404) \# 1$ & $137.9(4)$ \\
\hline
\end{tabular}




$\begin{array}{lr}C(400)-C(401)-C(500) \# 1 & 150.6(4) \\ C(402)-C(401)-C(500) \# 1 & 31.0(3) \\ \text { P(3)\#1-C(401)-C(500)\#1 } & 66.6(4) \\ \text { C(404)\#1-C(401)-C(500)\#1 } & 130.2(5)\end{array}$

Symmetry transformations used to generate equivalent atoms:

$\# 1-\mathrm{x}+1,-\mathrm{y}+1,-\mathrm{z}+2$ 
Table A12. Anisotropic displacement parameters $\left(\AA^{2} \times 10^{3}\right)$ for 8 The anisotropic displacement factor exponent takes the form: $-2 \pi^{2}\left[h^{2} a^{* 2} U^{11}+\ldots+2 h k a^{*} b^{*} U^{12}\right]$

\begin{tabular}{|c|c|c|c|c|c|c|}
\hline & $\mathrm{U}^{11}$ & $U^{22}$ & $U^{33}$ & $U^{23}$ & $U^{13}$ & $U^{12}$ \\
\hline $\mathrm{Ru}(1)$ & $10(1)$ & $9(1)$ & $12(1)$ & $-2(1)$ & $-1(1)$ & $0(1)$ \\
\hline$S(1)$ & $15(1)$ & $14(1)$ & $18(1)$ & $-1(1)$ & $-5(1)$ & $-1(1)$ \\
\hline$S(2)$ & $16(1)$ & $14(1)$ & $16(1)$ & $-5(1)$ & $3(1)$ & $-3(1)$ \\
\hline $\mathrm{S}(3)$ & $13(1)$ & $16(1)$ & $17(1)$ & $-5(1)$ & $-3(1)$ & $0(1)$ \\
\hline$P(1)$ & $10(1)$ & $10(1)$ & $13(1)$ & $-2(1)$ & $-1(1)$ & $0(1)$ \\
\hline$O(1)$ & $33(2)$ & $31(1)$ & $20(1)$ & $-11(1)$ & $2(1)$ & $-14(1)$ \\
\hline $\mathrm{O}(2)$ & $19(1)$ & $14(1)$ & $31(2)$ & $-3(1)$ & $6(1)$ & $2(1)$ \\
\hline$N(1)$ & $14(1)$ & $12(1)$ & $14(1)$ & $-1(1)$ & $-1(1)$ & $-1(1)$ \\
\hline$N(2)$ & $12(1)$ & $12(1)$ & $19(2)$ & $-5(1)$ & $0(1)$ & $-1(1)$ \\
\hline$C(1)$ & $17(2)$ & $11(2)$ & $20(2)$ & $-4(1)$ & $-3(1)$ & $-2(1)$ \\
\hline $\mathrm{C}(2)$ & $17(2)$ & $11(2)$ & $24(2)$ & $-6(1)$ & $-3(1)$ & $0(1)$ \\
\hline$C(3)$ & $12(2)$ & $14(2)$ & $23(2)$ & $-6(1)$ & 1(1) & $0(1)$ \\
\hline$C(4)$ & $11(2)$ & $14(2)$ & $28(2)$ & $-3(1)$ & $-4(1)$ & $0(1)$ \\
\hline$C(5)$ & $21(2)$ & $20(2)$ & $17(2)$ & 1(1) & $-5(1)$ & $-3(1)$ \\
\hline$C(6)$ & $19(2)$ & $15(2)$ & $18(2)$ & $2(1)$ & $-4(1)$ & $0(1)$ \\
\hline$C(7)$ & $14(2)$ & $13(2)$ & $21(2)$ & $-1(1)$ & $1(1)$ & $-3(1)$ \\
\hline$C(8)$ & $18(2)$ & $16(2)$ & $18(2)$ & $-3(1)$ & $3(1)$ & $-5(1)$ \\
\hline$C(9)$ & $18(2)$ & $16(2)$ & $16(2)$ & $-7(1)$ & $2(1)$ & $-1(1)$ \\
\hline $\mathrm{C}(10)$ & $20(2)$ & $19(2)$ & $17(2)$ & $-7(1)$ & $-3(1)$ & $0(1)$ \\
\hline$C(11)$ & $16(2)$ & $20(2)$ & $36(2)$ & $-5(2)$ & $4(2)$ & $-4(1)$ \\
\hline$C(12)$ & $31(2)$ & $25(2)$ & $19(2)$ & $-2(2)$ & $5(2)$ & $-7(2)$ \\
\hline$C(13)$ & $22(2)$ & $26(2)$ & $23(2)$ & $-12(2)$ & $-3(1)$ & $-5(1)$ \\
\hline$C(14)$ & $27(2)$ & $24(2)$ & $18(2)$ & $-6(1)$ & $-4(1)$ & $2(1)$ \\
\hline$C(15)$ & $12(2)$ & $10(1)$ & $18(2)$ & $0(1)$ & $0(1)$ & $-2(1)$ \\
\hline$C(16)$ & $16(2)$ & $13(2)$ & $19(2)$ & $0(1)$ & $-1(1)$ & $-1(1)$ \\
\hline $\mathrm{C}(17)$ & $18(2)$ & $17(2)$ & $22(2)$ & $-1(1)$ & $5(1)$ & $0(1)$ \\
\hline $\mathrm{C}(18)$ & $14(2)$ & $19(2)$ & $33(2)$ & $-4(2)$ & $5(1)$ & $-1(1)$ \\
\hline$C(19)$ & $14(2)$ & $17(2)$ & $33(2)$ & $-3(1)$ & $-5(1)$ & $-2(1)$ \\
\hline$C(20)$ & $17(2)$ & $15(2)$ & $20(2)$ & $-4(1)$ & $-2(1)$ & $-4(1)$ \\
\hline$C(21)$ & $15(2)$ & $12(1)$ & $16(2)$ & $-4(1)$ & $-2(1)$ & $0(1)$ \\
\hline$C(22)$ & $17(2)$ & $16(2)$ & $24(2)$ & $0(1)$ & $-4(1)$ & $-4(1)$ \\
\hline$C(23)$ & $28(2)$ & $14(2)$ & $26(2)$ & $2(1)$ & $-6(2)$ & $-5(1)$ \\
\hline $\mathrm{C}(24)$ & $27(2)$ & $19(2)$ & $20(2)$ & $-2(1)$ & $-10(1)$ & $5(1)$ \\
\hline$C(25)$ & $16(2)$ & $23(2)$ & $24(2)$ & $-6(1)$ & $-7(1)$ & $3(1)$ \\
\hline$C(26)$ & $16(2)$ & $16(2)$ & $19(2)$ & $-4(1)$ & $-2(1)$ & $-2(1)$ \\
\hline$C(27)$ & $18(2)$ & $15(2)$ & $19(2)$ & $-7(1)$ & $-1(1)$ & $-2(1)$ \\
\hline $\mathrm{Ru}(2)$ & $10(1)$ & $11(1)$ & $13(1)$ & $-3(1)$ & $-3(1)$ & 1(1) \\
\hline$S(4)$ & $13(1)$ & $15(1)$ & $16(1)$ & $-6(1)$ & $-3(1)$ & $0(1)$ \\
\hline$S(5)$ & $12(1)$ & $13(1)$ & $16(1)$ & $-2(1)$ & $-2(1)$ & $-1(1)$ \\
\hline$S(6)$ & $17(1)$ & $17(1)$ & $18(1)$ & $-6(1)$ & $-6(1)$ & $-1(1)$ \\
\hline$P(2)$ & $12(1)$ & $12(1)$ & $14(1)$ & $-2(1)$ & $-2(1)$ & $1(1)$ \\
\hline $\mathrm{O}(3)$ & $20(1)$ & $28(1)$ & $18(1)$ & $0(1)$ & $-6(1)$ & $-5(1)$ \\
\hline $\mathrm{O}(4)$ & $20(1)$ & $14(1)$ & $27(1)$ & $-3(1)$ & $0(1)$ & $0(1)$ \\
\hline$N(3)$ & $11(1)$ & $16(1)$ & $17(2)$ & $-5(1)$ & $-2(1)$ & $0(1)$ \\
\hline $\mathrm{N}(4)$ & $12(1)$ & $13(1)$ & $18(2)$ & $-4(1)$ & $-4(1)$ & $1(1)$ \\
\hline $\mathrm{C}(28)$ & $12(2)$ & $19(2)$ & $20(2)$ & $-6(1)$ & $-4(1)$ & $2(1)$ \\
\hline$C(29)$ & $12(2)$ & $17(2)$ & $22(2)$ & $-4(1)$ & $-5(1)$ & $4(1)$ \\
\hline $\mathrm{C}(30)$ & $16(2)$ & $13(2)$ & $20(2)$ & $-4(1)$ & $-4(1)$ & $0(1)$ \\
\hline$C(31)$ & $16(2)$ & $13(2)$ & $22(2)$ & $-6(1)$ & $-4(1)$ & $0(1)$ \\
\hline$C(32)$ & $16(2)$ & $23(2)$ & $16(2)$ & $-7(1)$ & $0(1)$ & $-3(1)$ \\
\hline $\mathrm{C}(33)$ & $13(2)$ & $20(2)$ & $18(2)$ & $-7(1)$ & $-1(1)$ & l(1) \\
\hline
\end{tabular}




\begin{tabular}{lllllll}
$\mathrm{C}(34)$ & $13(2)$ & $18(2)$ & $22(2)$ & $-4(1)$ & $-2(1)$ & $-3(1)$ \\
$\mathrm{C}(35)$ & $15(2)$ & $18(2)$ & $23(2)$ & $-2(1)$ & $-2(1)$ & $-4(1)$ \\
$\mathrm{C}(36)$ & $18(2)$ & $19(2)$ & $15(2)$ & $-5(1)$ & $-6(1)$ & $2(1)$ \\
$\mathrm{C}(37)$ & $16(2)$ & $16(2)$ & $18(2)$ & $-2(1)$ & $-5(1)$ & $0(1)$ \\
$\mathrm{C}(38)$ & $20(2)$ & $20(2)$ & $43(2)$ & $-4(2)$ & $-6(2)$ & $-5(1)$ \\
$\mathrm{C}(39)$ & $20(2)$ & $33(2)$ & $21(2)$ & $2(2)$ & $1(1)$ & $-3(2)$ \\
$\mathrm{C}(40)$ & $21(2)$ & $27(2)$ & $20(2)$ & $-4(1)$ & $-9(1)$ & $-1(1)$ \\
$\mathrm{C}(41)$ & $24(2)$ & $27(2)$ & $17(2)$ & $-7(1)$ & $-5(1)$ & $1(1)$ \\
$\mathrm{C}(42)$ & $10(1)$ & $15(2)$ & $16(2)$ & $-3(1)$ & $-1(1)$ & $2(1)$ \\
$\mathrm{C}(43)$ & $15(2)$ & $20(2)$ & $18(2)$ & $-5(1)$ & $-2(1)$ & $0(1)$ \\
$\mathrm{C}(44)$ & $20(2)$ & $20(2)$ & $30(2)$ & $-7(2)$ & $-1(1)$ & $-7(1)$ \\
$\mathrm{C}(45)$ & $26(2)$ & $21(2)$ & $28(2)$ & $-2(2)$ & $1(2)$ & $-8(1)$ \\
$\mathrm{C}(46)$ & $24(2)$ & $22(2)$ & $18(2)$ & $-3(1)$ & $0(1)$ & $-1(1)$ \\
$\mathrm{C}(47)$ & $18(2)$ & $19(2)$ & $17(2)$ & $-6(1)$ & $-3(1)$ & $0(1)$ \\
$\mathrm{C}(48)$ & $19(2)$ & $13(2)$ & $18(2)$ & $-2(1)$ & $0(1)$ & $1(1)$ \\
$\mathrm{C}(49)$ & $19(2)$ & $18(2)$ & $33(2)$ & $-9(2)$ & $6(2)$ & $-2(1)$ \\
$\mathrm{C}(50)$ & $22(2)$ & $21(2)$ & $37(2)$ & $-7(2)$ & $10(2)$ & $1(1)$ \\
$\mathrm{C}(51)$ & $35(2)$ & $16(2)$ & $25(2)$ & $-5(2)$ & $6(2)$ & $4(2)$ \\
$\mathrm{C}(52)$ & $30(2)$ & $20(2)$ & $22(2)$ & $-8(1)$ & $-6(2)$ & $4(1)$ \\
$\mathrm{C}(53)$ & $20(2)$ & $19(2)$ & $18(2)$ & $-4(1)$ & $-4(1)$ & $3(1)$ \\
$\mathrm{C}(54)$ & $14(2)$ & $22(2)$ & $18(2)$ & $1(1)$ & $-2(1)$ & $-1(1)$ \\
$\mathrm{N}(200)$ & $45(2)$ & $44(2)$ & $50(3)$ & $-20(2)$ & $-16(2)$ & $-3(2)$ \\
$\mathrm{C}(200)$ & $41(2)$ & $36(2)$ & $35(2)$ & $-13(2)$ & $-16(2)$ & $-1(2)$ \\
$\mathrm{C}(201)$ & $42(2)$ & $31(2)$ & $31(2)$ & $-9(2)$ & $-17(2)$ & $-6(2)$ \\
$\mathrm{P}(3)$ & $31(1)$ & $22(1)$ & $41(1)$ & $-9(1)$ & $-1(1)$ & $-3(1)$ \\
& & & & & & \\
\hline
\end{tabular}


Table A13. Hydrogen coordinates ( $\times 10^{4}$ ) and isotropic displacement parameters $\left(\AA^{2} \times 10^{3}\right)$ for 8

\begin{tabular}{|c|c|c|c|c|}
\hline & $\mathrm{x}$ & $y$ & $\mathrm{z}$ & $\mathrm{U}(\mathrm{eq})$ \\
\hline$H(1 A)$ & 6825 & 6844 & 2905 & 19 \\
\hline $\mathrm{H}(\mathrm{IB})$ & 7536 & 5970 & 3328 & 19 \\
\hline $\mathrm{H}(2 \mathrm{~A})$ & 5370 & 6335 & 3781 & 20 \\
\hline $\mathrm{H}(2 \mathrm{~B})$ & 4650 & 6396 & 3250 & 20 \\
\hline$H(3 A)$ & 3066 & 5416 & 3843 & 20 \\
\hline$H(3 B)$ & 3399 & 4307 & 3874 & 20 \\
\hline $\mathrm{H}(4 \mathrm{~A})$ & 3255 & 5797 & 2889 & 22 \\
\hline $\mathrm{H}(4 \mathrm{~B})$ & 2368 & 4918 & 3081 & 22 \\
\hline $\mathrm{H}(5 \mathrm{~A})$ & 5820 & 5103 & 1774 & 24 \\
\hline$H(5 B)$ & 4421 & 5764 & 1876 & 24 \\
\hline $\mathrm{H}(6 \mathrm{~A})$ & 6562 & 6416 & 1906 & 22 \\
\hline$H(6 B)$ & 5335 & 6533 & 2385 & 22 \\
\hline $\mathrm{H}(7 \mathrm{~A})$ & 9025 & 5422 & 2740 & 20 \\
\hline $\mathrm{H}(7 \mathrm{~B})$ & 8657 & 6111 & 2173 & 20 \\
\hline$H(9 A)$ & 5073 & 4009 & 4415 & 20 \\
\hline $\mathrm{H}(9 \mathrm{~B})$ & 4993 & 5060 & 4489 & 20 \\
\hline$H(11 \mathrm{~A})$ & 10950 & 3908 & 2016 & 37 \\
\hline $\mathrm{H}(11 \mathrm{~B})$ & 10661 & 4191 & 2597 & 37 \\
\hline $\mathrm{H}(1 \mathrm{lC})$ & 11064 & 4968 & 2045 & 37 \\
\hline$H(12 A)$ & 9434 & 5475 & 1323 & 39 \\
\hline $\mathrm{H}(12 \mathrm{~B})$ & 7905 & 5186 & 1461 & 39 \\
\hline $\mathrm{H}(12 \mathrm{C})$ & 9165 & 4416 & 1351 & 39 \\
\hline$H(13 A)$ & 7047 & 5430 & 4830 & 34 \\
\hline $\mathrm{H}(13 \mathrm{~B})$ & 7417 & 5864 & 4184 & 34 \\
\hline $\mathrm{H}(13 \mathrm{C})$ & 8535 & 5161 & 4523 & 34 \\
\hline $\mathrm{H}(14 \mathrm{~A})$ & 8077 & 3506 & 4980 & 35 \\
\hline $\mathrm{H}(14 \mathrm{~B})$ & 6985 & 3006 & 4785 & 35 \\
\hline $\mathrm{H}(14 \mathrm{C})$ & 6478 & 3711 & 5183 & 35 \\
\hline$H(16)$ & 4453 & 2388 & 4675 & 20 \\
\hline $\mathrm{H}(17)$ & 2161 & 2531 & 5095 & 25 \\
\hline $\mathrm{H}(18)$ & 344 & 2777 & 4555 & 28 \\
\hline $\mathrm{H}(19)$ & 829 & 2839 & 3603 & 26 \\
\hline $\mathrm{H}(20)$ & 3110 & 2670 & 3186 & 21 \\
\hline $\mathrm{H}(22)$ & 5242 & 715 & 4197 & 23 \\
\hline $\mathrm{H}(23)$ & 6465 & -611 & 4718 & 28 \\
\hline $\mathrm{H}(24)$ & 8745 & -533 & 4852 & 27 \\
\hline $\mathrm{H}(25)$ & 9778 & 865 & 4460 & 25 \\
\hline$H(26)$ & 8582 & 2182 & 3910 & 21 \\
\hline $\mathrm{H}(27 \mathrm{~A})$ & 5317 & 1387 & 3136 & 25 \\
\hline $\mathrm{H}(27 \mathrm{~B})$ & 6739 & 1756 & 2825 & 25 \\
\hline $\mathrm{H}(27 \mathrm{C})$ & 5320 & 2366 & 2679 & 25 \\
\hline $\mathrm{H}(28 \mathrm{~A})$ & -1717 & 10636 & 2314 & 20 \\
\hline $\mathrm{H}(28 \mathrm{~B})$ & -919 & 10021 & 1896 & 20 \\
\hline$H(29 A)$ & -553 & 11579 & 1465 & 21 \\
\hline $\mathrm{H}(29 \mathrm{~B})$ & -192 & 11771 & 2017 & 21 \\
\hline $\mathrm{H}(30 \mathrm{~A})$ & 1761 & 12442 & 1481 & 19 \\
\hline $\mathrm{H}(30 \mathrm{~B})$ & 3159 & 11746 & 1482 & 19 \\
\hline $\mathrm{H}(3 \backslash \mathrm{A})$ & 1344 & 12080 & 2440 & 20 \\
\hline$H(31 B)$ & 2930 & 12246 & 2297 & 20 \\
\hline $\mathrm{H}(32 \mathrm{~A})$ & 1454 & 9858 & 3539 & 21 \\
\hline $\mathrm{H}(32 \mathrm{~B})$ & 1126 & 10982 & 3441 & 21 \\
\hline
\end{tabular}




\begin{tabular}{|c|c|c|c|c|}
\hline $\mathrm{H}(33 \mathrm{~A})$ & -756 & 10109 & 3350 & 21 \\
\hline $\mathrm{H}(33 \mathrm{~B})$ & -507 & 11061 & 2891 & 21 \\
\hline $\mathrm{H}(34 \mathrm{~A})$ & -641 & 8669 & 2473 & 21 \\
\hline $\mathrm{H}(34 \mathrm{~B})$ & -1330 & 8941 & 3035 & 21 \\
\hline $\mathrm{H}(37 \mathrm{~A})$ & 2714 & 10947 & 899 & 20 \\
\hline $\mathrm{H}(37 \mathrm{~B})$ & 1263 & 11534 & 790 & 20 \\
\hline $\mathrm{H}(38 \mathrm{~A})$ & 777 & 6594 & 3247 & 42 \\
\hline $\mathrm{H}(38 \mathrm{~B})$ & 414 & 7119 & 2643 & 42 \\
\hline $\mathrm{H}(38 \mathrm{C})$ & -760 & 7045 & 3161 & 42 \\
\hline $\mathrm{H}(39 \mathrm{~A})$ & 460 & 8630 & 3812 & 40 \\
\hline $\mathrm{H}(39 \mathrm{~B})$ & 1011 & 7528 & 3929 & 40 \\
\hline $\mathrm{H}(39 \mathrm{C})$ & -590 & 7859 & 3913 & 40 \\
\hline $\mathrm{H}(40 \mathrm{~A})$ & -273 & 10786 & 390 & 34 \\
\hline$H(40 B)$ & -898 & 10470 & 1024 & 34 \\
\hline $\mathrm{H}(40 \mathrm{C})$ & -514 & 9704 & 659 & 34 \\
\hline $\mathrm{H}(41 \mathrm{~A})$ & 1938 & 9314 & 279 & 34 \\
\hline $\mathrm{H}(4 \mid \mathrm{B})$ & 3165 & 9642 & 498 & 34 \\
\hline $\mathrm{H}(41 \mathrm{C})$ & 2260 & 10393 & 82 & 34 \\
\hline $\mathrm{H}(43)$ & 5692 & 10953 & 2100 & 22 \\
\hline $\mathrm{H}(44)$ & 6585 & 12384 & 1601 & 28 \\
\hline $\mathrm{H}(45)$ & 6753 & 12791 & 626 & 31 \\
\hline $\mathrm{H}(46)$ & 6050 & 11784 & 161 & 27 \\
\hline $\mathrm{H}(47)$ & 5250 & 10344 & 654 & 21 \\
\hline $\mathrm{H}(49)$ & 7379 & 8896 & 1395 & 29 \\
\hline $\mathrm{H}(50)$ & 8547 & 7867 & 857 & 34 \\
\hline $\mathrm{H}(51)$ & 7328 & 6951 & 499 & 33 \\
\hline $\mathrm{H}(52)$ & 4933 & 6989 & 742 & 28 \\
\hline $\mathrm{H}(53)$ & 3740 & 7972 & 1317 & 23 \\
\hline $\mathrm{H}(54 \mathrm{~A})$ & 6571 & 9027 & 2283 & 28 \\
\hline $\mathrm{H}(54 \mathrm{~B})$ & 5434 & 8359 & 2621 & 28 \\
\hline $\mathrm{H}(54 \mathrm{C})$ & 5328 & 9427 & 2678 & 28 \\
\hline $\mathrm{H}(20 \mathrm{~A})$ & 3958 & 8872 & 4609 & 54 \\
\hline $\mathrm{H}(20 \mathrm{~B})$ & 3260 & 8454 & 4209 & 54 \\
\hline $\mathrm{H}(20 \mathrm{C})$ & 3873 & 9448 & 3985 & 54 \\
\hline $\mathrm{H}(50 \mathrm{~A})$ & 5519 & 6450 & 8874 & 54 \\
\hline $\mathrm{H}(50 \mathrm{~B})$ & 5708 & 5433 & 8736 & 54 \\
\hline $\mathrm{H}(50 \mathrm{C})$ & 6964 & 6059 & 8585 & 54 \\
\hline$H(304)$ & 10033 & 3504 & 10444 & 37 \\
\hline $\mathrm{H}(303)$ & 10106 & 2382 & 9914 & 37 \\
\hline$H(302)$ & 8613 & 2647 & 9240 & 37 \\
\hline $\mathrm{H}(301)$ & 7047 & 4035 & 9095 & 37 \\
\hline
\end{tabular}


Table A14. Torsion angles $\left[{ }^{\circ}\right]$ for 8 .

\begin{tabular}{|c|c|}
\hline $\bar{N}(1)-R u(1)-S(1)-C(5)$ & $20.09(14)$ \\
\hline $\mathrm{N}(2)-\mathrm{Ru}(1)-\mathrm{S}(1)-\mathrm{C}(5)$ & $100.83(14)$ \\
\hline$S(2)-R u(1)-S(1)-C(5)$ & $-64.59(12)$ \\
\hline $\mathrm{P}(1)-\mathrm{Ru}(1)-\mathrm{S}(1)-\mathrm{C}(5)$ & $-154.62(12)$ \\
\hline$S(3)-R u(1)-S(1)-C(5)$ & $84.07(19)$ \\
\hline$N(1)-R u(1)-S(1)-C(4)$ & $-84.61(13)$ \\
\hline $\mathrm{N}(2)-\mathrm{Ru}(1)-\mathrm{S}(1)-\mathrm{C}(4)$ & $-3.88(13)$ \\
\hline$S(2)-R u(1)-S(1)-C(4)$ & $-169.29(11)$ \\
\hline $\mathrm{P}(1)-\mathrm{Ru}(1)-\mathrm{S}(1)-\mathrm{C}(4)$ & $100.67(11)$ \\
\hline$S(3)-R u(1)-S(1)-C(4)$ & $-20.63(19)$ \\
\hline $\mathrm{N}(1)-\mathrm{Ru}(1)-\mathrm{S}(2)-\mathrm{O}(1)$ & $-96.58(14)$ \\
\hline $\mathrm{N}(2)-\mathrm{Ru}(1)-\mathrm{S}(2)-\mathrm{O}(1)$ & $-108.0(3)$ \\
\hline $\mathrm{P}(1)-\mathrm{Ru}(1)-\mathrm{S}(2)-\mathrm{O}(1)$ & $76.43(12)$ \\
\hline$S(1)-R u(1)-S(2)-O(1)$ & $-11.74(13)$ \\
\hline $\mathrm{S}(3)-\mathrm{Ru}(1)-\mathrm{S}(2)-\mathrm{O}(1)$ & $174.46(12)$ \\
\hline $\mathrm{N}(1)-\mathrm{Ru}(1)-\mathrm{S}(2)-\mathrm{O}(2)$ & $129.38(14)$ \\
\hline $\mathrm{N}(2)-\mathrm{Ru}(1)-\mathrm{S}(2)-\mathrm{O}(2)$ & $117.9(3)$ \\
\hline $\mathrm{P}(1)-\mathrm{Ru}(1)-\mathrm{S}(2)-\mathrm{O}(2)$ & $-57.62(12)$ \\
\hline $\mathrm{S}(1)-\mathrm{Ru}(1)-\mathrm{S}(2)-\mathrm{O}(2)$ & $-145.78(12)$ \\
\hline$S(3)-R u(1)-S(2)-O(2)$ & $40.42(12)$ \\
\hline $\mathrm{N}(1)-\mathrm{Ru}(1)-\mathrm{S}(2)-\mathrm{C}(8)$ & $14.74(13)$ \\
\hline $\mathrm{N}(2)-\mathrm{Ru}(1)-\mathrm{S}(2)-\mathrm{C}(8)$ & $3.3(3)$ \\
\hline$P(1)-R u(1)-S(2)-C(8)$ & $-172.26(12)$ \\
\hline$S(1)-R u(1)-S(2)-C(8)$ & $99.58(12)$ \\
\hline$S(3)-R u(1)-S(2)-C(8)$ & $-74.22(12)$ \\
\hline $\mathrm{N}(1)-\mathrm{Ru}(1)-\mathrm{S}(3)-\mathrm{C}(10)$ & $98.88(13)$ \\
\hline $\mathrm{N}(2)-\mathrm{Ru}(1)-\mathrm{S}(3)-\mathrm{C}(10)$ & $18.44(13)$ \\
\hline$S(2)-R u(1)-S(3)-C(10)$ & $-175.94(11)$ \\
\hline $\mathrm{P}(1)-\mathrm{Ru}(1)-\mathrm{S}(3)-\mathrm{C}(10)$ & $-85.15(11)$ \\
\hline$S(1)-R u(1)-S(3)-C(10)$ & $35.3(2)$ \\
\hline$N(1)-R u(1)-P(1)-C(27)$ & $17.8(5)$ \\
\hline $\mathrm{N}(2)-\mathrm{Ru}(1)-\mathrm{P}(1)-\mathrm{C}(27)$ & $142.04(14)$ \\
\hline S(2)-Ru(1)-P(1)-C(27) & $-39.12(12)$ \\
\hline$S(1)-R u(1)-P(1)-C(27)$ & $57.17(12)$ \\
\hline$S(3)-R u(1)-P(1)-C(27)$ & $-133.12(12)$ \\
\hline $\mathrm{N}(1)-\mathrm{Ru}(1)-\mathrm{P}(1)-\mathrm{C}(15)$ & $-96.2(5)$ \\
\hline$N(2)-R u(1)-P(1)-C(15)$ & $27.99(14)$ \\
\hline $\mathrm{S}(2)-\mathrm{Ru}(1)-\mathrm{P}(1)-\mathrm{C}(15)$ & $-153.16(12)$ \\
\hline$S(1)-R u(1)-P(1)-C(15)$ & $-56.88(12)$ \\
\hline$S(3)-R u(1)-P(1)-C(15)$ & $112.83(12)$ \\
\hline $\mathrm{N}(1)-\mathrm{Ru}(1)-\mathrm{P}(1)-\mathrm{C}(21)$ & $136.8(5)$ \\
\hline $\mathrm{N}(2)-\mathrm{Ru}(1)-\mathrm{P}(1)-\mathrm{C}(21)$ & $-98.98(15)$ \\
\hline$S(2)-R u(1)-P(1)-C(21)$ & $79.87(14)$ \\
\hline$S(1)-R u(1)-P(1)-C(21)$ & $176.15(14)$ \\
\hline$S(3)-R u(1)-P(1)-C(21)$ & $-14.14(14)$ \\
\hline $\mathrm{N}(2)-\mathrm{Ru}(1)-\mathrm{N}(1)-\mathrm{C}(7)$ & $143.2(2)$ \\
\hline$S(2)-R u(1)-N(1)-C(7)$ & $-33.9(2)$ \\
\hline $\mathrm{P}(1)-\mathrm{Ru}(1)-\mathrm{N}(1)-\mathrm{C}(7)$ & $-91.1(5)$ \\
\hline $\mathrm{S}(1)-\mathrm{Ru}(1)-\mathrm{N}(1)-\mathrm{C}(7)$ & $-130.6(2)$ \\
\hline$S(3)-R u(1)-N(1)-C(7)$ & $60.1(2)$ \\
\hline $\mathrm{N}(2)-\mathrm{Ru}(1)-\mathrm{N}(1)-\mathrm{C}(1)$ & $28.57(19)$ \\
\hline $\mathrm{S}(2)-\mathrm{Ru}(1)-\mathrm{N}(1)-\mathrm{C}(1)$ & $-148.52(18)$ \\
\hline $\mathrm{P}(1)-\mathrm{Ru}(1)-\mathrm{N}(1)-\mathrm{C}(1)$ & $154.2(4)$ \\
\hline$S(1)-R u(1)-N(1)-C(1)$ & $114.77(18)$ \\
\hline
\end{tabular}




\begin{tabular}{|c|c|}
\hline$S(3)-R u(1)-N(1)-C(1)$ & $-54.51(18)$ \\
\hline $\mathrm{N}(2)-\mathrm{Ru}(1)-\mathrm{N}(1)-\mathrm{C}(6)$ & $-89.4(2)$ \\
\hline$S(2)-R u(1)-N(1)-C(6)$ & $93.5(2)$ \\
\hline $\mathrm{P}(1)-\mathrm{Ru}(1)-\mathrm{N}(1)-\mathrm{C}(6)$ & $36.2(6)$ \\
\hline$S(1)-R u(1)-N(1)-C(6)$ & $-3.2(2)$ \\
\hline$S(3)-R u(1)-N(1)-C(6)$ & $-172.5(2)$ \\
\hline $\mathrm{N}(1)-\mathrm{Ru}(1)-\mathrm{N}(2)-\mathrm{C}(9)$ & $-126.8(2)$ \\
\hline $\mathrm{S}(2)-\mathrm{Ru}(1)-\mathrm{N}(2)-\mathrm{C}(9)$ & $-115.3(3)$ \\
\hline $\mathrm{P}(1)-\mathrm{Ru}(1)-\mathrm{N}(2)-\mathrm{C}(9)$ & $60.13(19)$ \\
\hline$S(1)-R u(1)-N(2)-C(9)$ & $147.06(19)$ \\
\hline $\mathrm{S}(3)-\mathrm{Ru}(1)-\mathrm{N}(2)-\mathrm{C}(9)$ & $-36.39(18)$ \\
\hline $\mathrm{N}(1)-\mathrm{Ru}(1)-\mathrm{N}(2)-\mathrm{C}(3)$ & $113.7(2)$ \\
\hline $\mathrm{S}(2)-\mathrm{Ru}(1)-\mathrm{N}(2)-\mathrm{C}(3)$ & $125.3(3)$ \\
\hline$P(1)-R u(1)-N(2)-C(3)$ & $-59.3(2)$ \\
\hline $\mathrm{S}(1)-\mathrm{Ru}(1)-\mathrm{N}(2)-\mathrm{C}(3)$ & $27.64(19)$ \\
\hline $\mathrm{S}(3)-\mathrm{Ru}(1)-\mathrm{N}(2)-\mathrm{C}(3)$ & $-155.8(2)$ \\
\hline$N(1)-R u(1)-N(2)-C(2)$ & $-4.5(2)$ \\
\hline $\mathrm{S}(2)-\mathrm{Ru}(1)-\mathrm{N}(2)-\mathrm{C}(2)$ & $7.0(4)$ \\
\hline $\mathrm{P}(1)-\mathrm{Ru}(1)-\mathrm{N}(2)-\mathrm{C}(2)$ & $-177.54(18)$ \\
\hline $\mathrm{S}(1)-\mathrm{Ru}(1)-\mathrm{N}(2)-\mathrm{C}(2)$ & $-90.6(2)$ \\
\hline $\mathrm{S}(3)-\mathrm{Ru}(1)-\mathrm{N}(2)-\mathrm{C}(2)$ & $85.9(2)$ \\
\hline $\mathrm{C}(7)-\mathrm{N}(1)-\mathrm{C}(1)-\mathrm{C}(2)$ & $-164.5(3)$ \\
\hline $\mathrm{C}(6)-\mathrm{N}(1)-\mathrm{C}(1)-\mathrm{C}(2)$ & $73.4(3)$ \\
\hline $\mathrm{Ru}(1)-\mathrm{N}(1)-\mathrm{C}(1)-\mathrm{C}(2)$ & $-48.9(3)$ \\
\hline $\mathrm{C}(9)-\mathrm{N}(2)-\mathrm{C}(2)-\mathrm{C}(1)$ & $99.5(3)$ \\
\hline $\mathrm{C}(3)-\mathrm{N}(2)-\mathrm{C}(2)-\mathrm{C}(1)$ & $-140.6(3)$ \\
\hline $\mathrm{Ru}(1)-\mathrm{N}(2)-\mathrm{C}(2)-\mathrm{C}(1)$ & $-21.0(3)$ \\
\hline $\mathrm{N}(1)-\mathrm{C}(1)-\mathrm{C}(2)-\mathrm{N}(2)$ & $47.7(4)$ \\
\hline $\mathrm{C}(9)-\mathrm{N}(2)-\mathrm{C}(3)-\mathrm{C}(4)$ & $-167.3(3)$ \\
\hline $\mathrm{C}(2)-\mathrm{N}(2)-\mathrm{C}(3)-\mathrm{C}(4)$ & $71.4(3)$ \\
\hline $\mathrm{Ru}(1)-\mathrm{N}(2)-\mathrm{C}(3)-\mathrm{C}(4)$ & $-48.5(3)$ \\
\hline $\mathrm{N}(2)-\mathrm{C}(3)-\mathrm{C}(4)-\mathrm{S}(1)$ & $45.7(3)$ \\
\hline$C(5)-S(1)-C(4)-C(3)$ & $-121.7(2)$ \\
\hline$R u(1)-S(1)-C(4)-C(3)$ & $-19.8(2)$ \\
\hline$C(4)-S(1)-C(5)-C(6)$ & $65.7(3)$ \\
\hline$R u(1)-S(1)-C(5)-C(6)$ & $-37.3(3)$ \\
\hline$C(7)-N(1)-C(6)-C(5)$ & $104.7(3)$ \\
\hline$C(1)-N(1)-C(6)-C(5)$ & $-137.3(3)$ \\
\hline$R u(1)-N(1)-C(6)-C(5)$ & $-20.2(4)$ \\
\hline $\mathrm{S}(1)-\mathrm{C}(5)-\mathrm{C}(6)-\mathrm{N}(1)$ & $40.1(4)$ \\
\hline$C(1)-N(1)-C(7)-C(8)$ & $161.7(3)$ \\
\hline $\mathrm{C}(6)-\mathrm{N}(1)-\mathrm{C}(7)-\mathrm{C}(8)$ & $-79.9(3)$ \\
\hline $\mathrm{Ru}(1)-\mathrm{N}(1)-\mathrm{C}(7)-\mathrm{C}(8)$ & $47.9(3)$ \\
\hline $\mathrm{N}(1)-\mathrm{C}(7)-\mathrm{C}(8)-\mathrm{C}(12)$ & $88.7(4)$ \\
\hline $\mathrm{N}(1)-\mathrm{C}(7)-\mathrm{C}(8)-\mathrm{C}(11)$ & $-151.0(3)$ \\
\hline $\mathrm{N}(1)-\mathrm{C}(7)-\mathrm{C}(8)-\mathrm{S}(2)$ & $-34.1(3)$ \\
\hline $\mathrm{O}(1)-\mathrm{S}(2)-\mathrm{C}(8)-\mathrm{C}(12)$ & $-0.3(3)$ \\
\hline $\mathrm{O}(2)-\mathrm{S}(2)-\mathrm{C}(8)-\mathrm{C}(12)$ & $116.7(3)$ \\
\hline$R u(1)-S(2)-C(8)-C(12)$ & $-120.5(2)$ \\
\hline $\mathrm{O}(1)-\mathrm{S}(2)-\mathrm{C}(8)-\mathrm{C}(7)$ & $124.8(2)$ \\
\hline$O(2)-S(2)-C(8)-C(7)$ & $-118.2(2)$ \\
\hline$R u(1)-S(2)-C(8)-C(7)$ & $4.5(2)$ \\
\hline $\mathrm{O}(1)-\mathrm{S}(2)-\mathrm{C}(8)-\mathrm{C}(11)$ & $-119.6(3)$ \\
\hline $\mathrm{O}(2)-\mathrm{S}(2)-\mathrm{C}(8)-\mathrm{C}(11)$ & $-2.6(3)$ \\
\hline$R u(1)-S(2)-C(8)-C(11)$ & $120.1(2)$ \\
\hline $\mathrm{C}(3)-\mathrm{N}(2)-\mathrm{C}(9)-\mathrm{C}(10)$ & $169.0(3)$ \\
\hline
\end{tabular}




\begin{tabular}{|c|c|}
\hline $\mathrm{C}(2)-\mathrm{N}(2)-\mathrm{C}(9)-\mathrm{C}(10)$ & $-72.2(3)$ \\
\hline $\mathrm{Ru}(1)-\mathrm{N}(2)-\mathrm{C}(9)-\mathrm{C}(10)$ & $49.3(3)$ \\
\hline $\mathrm{N}(2)-\mathrm{C}(9)-\mathrm{C}(10)-\mathrm{C}(14)$ & $-149.4(3)$ \\
\hline$N(2)-C(9)-C(10)-C(13)$ & $92.2(4)$ \\
\hline$N(2)-C(9)-C(10)-S(3)$ & $-31.7(4)$ \\
\hline $\operatorname{Ru}(1)-S(3)-C(10)-C(14)$ & $116.9(2)$ \\
\hline $\mathrm{Ru}(1)-\mathrm{S}(3)-\mathrm{C}(10)-\mathrm{C}(13)$ & $-126.0(2)$ \\
\hline Ru(1)-S(3)-C(10)-C(9) & $0.1(2)$ \\
\hline $\mathrm{C}(27)-\mathrm{P}(1)-\mathrm{C}(15)-\mathrm{C}(16)$ & $148.2(3)$ \\
\hline $\mathrm{C}(21)-\mathrm{P}(1)-\mathrm{C}(15)-\mathrm{C}(16)$ & $48.7(3)$ \\
\hline $\mathrm{Ru}(1)-\mathrm{P}(1)-\mathrm{C}(15)-\mathrm{C}(16)$ & $-91.2(3)$ \\
\hline$C(27)-P(1)-C(15)-C(20)$ & $-40.0(3)$ \\
\hline$C(21)-P(1)-C(15)-C(20)$ & $-139.5(3)$ \\
\hline $\mathrm{Ru}(1)-\mathrm{P}(1)-\mathrm{C}(15)-\mathrm{C}(20)$ & $80.6(3)$ \\
\hline$C(20)-C(15)-C(16)-C(17)$ & $-1.0(5)$ \\
\hline $\mathrm{P}(1)-\mathrm{C}(15)-\mathrm{C}(16)-\mathrm{C}(17)$ & $170.9(2)$ \\
\hline$C(15)-C(16)-C(17)-C(18)$ & $-0.2(5)$ \\
\hline$C(16)-C(17)-C(18)-C(19)$ & $0.9(5)$ \\
\hline$C(17)-C(18)-C(19)-C(20)$ & $-0.4(5)$ \\
\hline$C(18)-C(19)-C(20)-C(15)$ & $-0.9(5)$ \\
\hline$C(16)-C(15)-C(20)-C(19)$ & $1.6(5)$ \\
\hline$P(1)-C(15)-C(20)-C(19)$ & $-170.5(3)$ \\
\hline $\mathrm{C}(27)-\mathrm{P}(1)-\mathrm{C}(21)-\mathrm{C}(26)$ & $109.5(3)$ \\
\hline$C(15)-P(1)-C(21)-C(26)$ & $-148.3(3)$ \\
\hline$R u(1)-P(1)-C(21)-C(26)$ & $-15.7(3)$ \\
\hline $\mathrm{C}(27)-\mathrm{P}(1)-\mathrm{C}(21)-\mathrm{C}(22)$ & $-63.1(3)$ \\
\hline$C(15)-P(1)-C(21)-C(22)$ & $39.0(3)$ \\
\hline Ru(1)-P(1)-C(21)-C(22) & $171.7(2)$ \\
\hline$C(26)-C(21)-C(22)-C(23)$ & $0.3(5)$ \\
\hline $\mathrm{P}(1)-\mathrm{C}(21)-\mathrm{C}(22)-\mathrm{C}(23)$ & $173.2(3)$ \\
\hline $\mathrm{C}(21)-\mathrm{C}(22)-\mathrm{C}(23)-\mathrm{C}(24)$ & $0.6(6)$ \\
\hline$C(22)-C(23)-C(24)-C(25)$ & $-0.3(6)$ \\
\hline$C(23)-C(24)-C(25)-C(26)$ & $-0.9(6)$ \\
\hline$C(22)-C(21)-C(26)-C(25)$ & $-1.5(5)$ \\
\hline $\mathrm{P}(1)-\mathrm{C}(21)-\mathrm{C}(26)-\mathrm{C}(25)$ & $-174.2(3)$ \\
\hline$C(24)-C(25)-C(26)-C(21)$ & $1.8(5)$ \\
\hline $\mathrm{N}(3)-\mathrm{Ru}(2)-\mathrm{S}(4)-\mathrm{C}(32)$ & $20.25(14)$ \\
\hline $\mathrm{N}(4)-\mathrm{Ru}(2)-\mathrm{S}(4)-\mathrm{C}(32)$ & $101.02(14)$ \\
\hline$S(5)-R u(2)-S(4)-C(32)$ & $-64.65(12)$ \\
\hline$P(2)-R u(2)-S(4)-C(32)$ & $-153.63(12)$ \\
\hline$S(6)-R u(2)-S(4)-C(32)$ & $84.40(18)$ \\
\hline $\mathrm{N}(3)-\mathrm{Ru}(2)-\mathrm{S}(4)-\mathrm{C}(31)$ & $-84.25(13)$ \\
\hline $\mathrm{N}(4)-\mathrm{Ru}(2)-\mathrm{S}(4)-\mathrm{C}(31)$ & $-3.48(13)$ \\
\hline$S(5)-R u(2)-S(4)-C(31)$ & $-169.15(11)$ \\
\hline$P(2)-R u(2)-S(4)-C(31)$ & $101.87(11)$ \\
\hline$S(6)-R u(2)-S(4)-C(31)$ & $-20.11(19)$ \\
\hline $\mathrm{N}(3)-\mathrm{Ru}(2)-\mathrm{S}(5)-\mathrm{O}(3)$ & $-96.84(14)$ \\
\hline $\mathrm{N}(4)-\mathrm{Ru}(2)-\mathrm{S}(5)-\mathrm{O}(3)$ & $-105.1(3)$ \\
\hline $\mathrm{S}(4)-\mathrm{Ru}(2)-\mathrm{S}(5)-\mathrm{O}(3)$ & $-12.26(12)$ \\
\hline $\mathrm{P}(2)-\mathrm{Ru}(2)-\mathrm{S}(5)-\mathrm{O}(3)$ & $76.53(12)$ \\
\hline$S(6)-R u(2)-S(5)-O(3)$ & $174.29(12)$ \\
\hline N(3)-Ru(2)-S(5)-O(4) & $130.14(14)$ \\
\hline $\mathrm{N}(4)-\mathrm{Ru}(2)-\mathrm{S}(5)-\mathrm{O}(4)$ & $121.9(3)$ \\
\hline $\mathrm{S}(4)-\mathrm{Ru}(2)-\mathrm{S}(5)-\mathrm{O}(4)$ & $-145.27(12)$ \\
\hline $\mathrm{P}(2)-\mathrm{Ru}(2)-\mathrm{S}(5)-\mathrm{O}(4)$ & $-56.48(12)$ \\
\hline$S(6)-R u(2)-S(5)-O(4)$ & $41.28(12)$ \\
\hline
\end{tabular}


$\mathrm{N}(3)-\mathrm{Ru}(2)-\mathrm{S}(5)-\mathrm{C}(35)$

$\mathrm{N}(4)-\mathrm{Ru}(2)-\mathrm{S}(5)-\mathrm{C}(35)$

$\mathrm{S}(4)-\mathrm{Ru}(2)-\mathrm{S}(5)-\mathrm{C}(35)$

$\mathrm{P}(2)-\mathrm{Ru}(2)-\mathrm{S}(5)-\mathrm{C}(35)$

$\mathrm{S}(6)-\mathrm{Ru}(2)-\mathrm{S}(5)-\mathrm{C}(35)$

$\mathrm{N}(3)-\mathrm{Ru}(2)-\mathrm{S}(6)-\mathrm{C}(36)$

$\mathrm{N}(4)-\mathrm{Ru}(2)-\mathrm{S}(6)-\mathrm{C}(36)$

$\mathrm{S}(5)-\mathrm{Ru}(2)-\mathrm{S}(6)-\mathrm{C}(36)$

$\mathrm{S}(4)-\mathrm{Ru}(2)-\mathrm{S}(6)-\mathrm{C}(36)$

$\mathrm{P}(2)-\mathrm{Ru}(2)-\mathrm{S}(6)-\mathrm{C}(36)$

$\mathrm{N}(3)-\mathrm{Ru}(2)-\mathrm{P}(2)-\mathrm{C}(54)$

$\mathrm{N}(4)-\mathrm{Ru}(2)-\mathrm{P}(2)-\mathrm{C}(54)$

$\mathrm{S}(5)-\mathrm{Ru}(2)-\mathrm{P}(2)-\mathrm{C}(54)$

$\mathrm{S}(4)-\mathrm{Ru}(2)-\mathrm{P}(2)-\mathrm{C}(54)$

$\mathrm{S}(6)-\mathrm{Ru}(2)-\mathrm{P}(2)-\mathrm{C}(54)$

$\mathrm{N}(3)-\mathrm{Ru}(2)-\mathrm{P}(2)-\mathrm{C}(42)$

$\mathrm{N}(4)-\mathrm{Ru}(2)-\mathrm{P}(2)-\mathrm{C}(42)$

$\mathrm{S}(5)-\mathrm{Ru}(2)-\mathrm{P}(2)-\mathrm{C}(42)$

$\mathrm{S}(4)-\mathrm{Ru}(2)-\mathrm{P}(2)-\mathrm{C}(42)$

$\mathrm{S}(6)-\mathrm{Ru}(2)-\mathrm{P}(2)-\mathrm{C}(42)$

$\mathrm{N}(3)-\mathrm{Ru}(2)-\mathrm{P}(2)-\mathrm{C}(48)$

$\mathrm{N}(4)-\mathrm{Ru}(2)-\mathrm{P}(2)-\mathrm{C}(48)$

$\mathrm{S}(5)-\mathrm{Ru}(2)-\mathrm{P}(2)-\mathrm{C}(48)$

$\mathrm{S}(4)-\mathrm{Ru}(2)-\mathrm{P}(2)-\mathrm{C}(48)$

$\mathrm{S}(6)-\mathrm{Ru}(2)-\mathrm{P}(2)-\mathrm{C}(48)$

$\mathrm{N}(4)-\mathrm{Ru}(2)-\mathrm{N}(3)-\mathrm{C}(28)$

$\mathrm{S}(5)-\mathrm{Ru}(2)-\mathrm{N}(3)-\mathrm{C}(28)$

$\mathrm{S}(4)-\mathrm{Ru}(2)-\mathrm{N}(3)-\mathrm{C}(28)$

$\mathrm{P}(2)-\mathrm{Ru}(2)-\mathrm{N}(3)-\mathrm{C}(28)$

$\mathrm{S}(6)-\mathrm{Ru}(2)-\mathrm{N}(3)-\mathrm{C}(28)$

$\mathrm{N}(4)-\mathrm{Ru}(2)-\mathrm{N}(3)-\mathrm{C}(34)$

S(5)-Ru(2)-N(3)-C(34)

S(4)-Ru(2)-N(3)-C(34)

$\mathrm{P}(2)-\mathrm{Ru}(2)-\mathrm{N}(3)-\mathrm{C}(34)$

$\mathrm{S}(6)-\mathrm{Ru}(2)-\mathrm{N}(3)-\mathrm{C}(34)$

$\mathrm{N}(4)-\mathrm{Ru}(2)-\mathrm{N}(3)-\mathrm{C}(33)$

$\mathrm{S}(5)-\mathrm{Ru}(2)-\mathrm{N}(3)-\mathrm{C}(33)$

$\mathrm{S}(4)-\mathrm{Ru}(2)-\mathrm{N}(3)-\mathrm{C}(33)$

$\mathrm{P}(2)-\mathrm{Ru}(2)-\mathrm{N}(3)-\mathrm{C}(33)$

$\mathrm{S}(6)-\mathrm{Ru}(2)-\mathrm{N}(3)-\mathrm{C}(33)$

$\mathrm{N}(3)-\mathrm{Ru}(2)-\mathrm{N}(4)-\mathrm{C}(30)$

$\mathrm{S}(5)-\mathrm{Ru}(2)-\mathrm{N}(4)-\mathrm{C}(30)$

$\mathrm{S}(4)-\mathrm{Ru}(2)-\mathrm{N}(4)-\mathrm{C}(30)$

$\mathrm{P}(2)-\mathrm{Ru}(2)-\mathrm{N}(4)-\mathrm{C}(30)$

$\mathrm{S}(6)-\mathrm{Ru}(2)-\mathrm{N}(4)-\mathrm{C}(30)$

$\mathrm{N}(3)-\mathrm{Ru}(2)-\mathrm{N}(4)-\mathrm{C}(37)$

$\mathrm{S}(5)-\mathrm{Ru}(2)-\mathrm{N}(4)-\mathrm{C}(37)$

$\mathrm{S}(4)-\mathrm{Ru}(2)-\mathrm{N}(4)-\mathrm{C}(37)$

$\mathrm{P}(2)-\mathrm{Ru}(2)-\mathrm{N}(4)-\mathrm{C}(37)$

$\mathrm{S}(6)-\mathrm{Ru}(2)-\mathrm{N}(4)-\mathrm{C}(37)$

$\mathrm{N}(3)-\mathrm{Ru}(2)-\mathrm{N}(4)-\mathrm{C}(29)$

$\mathrm{S}(5)-\mathrm{Ru}(2)-\mathrm{N}(4)-\mathrm{C}(29)$

$\mathrm{S}(4)-\mathrm{Ru}(2)-\mathrm{N}(4)-\mathrm{C}(29)$

$\mathrm{P}(2)-\mathrm{Ru}(2)-\mathrm{N}(4)-\mathrm{C}(29)$

$\mathrm{S}(6)-\mathrm{Ru}(2)-\mathrm{N}(4)-\mathrm{C}(29)$

C(34)-N(3)-C(28)-C(29)
$14.89(14)$

$6.6(3)$

$99.48(12)$

$-171.73(12)$

$-73.97(12)$

99.33(13)

$18.89(13)$

$-175.33(11)$

$35.60(19)$

$-85.53(11)$

$5.2(5)$

$135.28(14)$

$-45.14(13)$

$50.62(13)$

$-140.29(13)$

$-109.1(5)$

21.01(14)

$-159.42(12)$

$-63.66(12)$

105.44(12)

125.7(5)

$-104.21(16)$

75.37(14)

171.12(14)

$-19.78(14)$

28.91(19)

$-149.02(19)$

$114.80(19)$

$160.4(4)$

$-53.73(18)$

$143.7(2)$

$-34.19(19)$

$-130.4(2)$

$-84.8(5)$

61.10(19)

$-89.7(2)$

92.3(2)

$-3.8(2)$

$41.8(6)$

$-172.4(2)$

$113.5(2)$

121.9(3)

27.7(2)

$-59.8(2)$

$-155.9(2)$

$-128.0(2)$

$-119.6(3)$

146.22(19)

$58.7(2)$

$-37.44(18)$

$-5.0(2)$

3.3(4)

$-90.9(2)$

$-178.39(18)$

$85.5(2)$

$-163.7(3)$ 


\begin{tabular}{|c|c|}
\hline $\mathrm{C}(33)-\mathrm{N}(3)-\mathrm{C}(28)-\mathrm{C}(29)$ & $74.4(3)$ \\
\hline $\mathrm{Ru}(2)-\mathrm{N}(3)-\mathrm{C}(28)-\mathrm{C}(29)$ & $-48.7(3)$ \\
\hline $\mathrm{C}(30)-\mathrm{N}(4)-\mathrm{C}(29)-\mathrm{C}(28)$ & $-140.1(3)$ \\
\hline $\mathrm{C}(37)-\mathrm{N}(4)-\mathrm{C}(29)-\mathrm{C}(28)$ & $100.9(3)$ \\
\hline $\mathrm{Ru}(2)-\mathrm{N}(4)-\mathrm{C}(29)-\mathrm{C}(28)$ & $-20.2(3)$ \\
\hline $\mathrm{N}(3)-\mathrm{C}(28)-\mathrm{C}(29)-\mathrm{N}(4)$ & $47.1(4)$ \\
\hline $\mathrm{C}(37)-\mathrm{N}(4)-\mathrm{C}(30)-\mathrm{C}(31)$ & $-167.8(3)$ \\
\hline$C(29)-N(4)-C(30)-C(31)$ & $70.9(3)$ \\
\hline$R u(2)-N(4)-C(30)-C(31)$ & $-49.0(3)$ \\
\hline$N(4)-C(30)-C(31)-S(4)$ & $46.3(3)$ \\
\hline$C(32)-S(4)-C(31)-C(30)$ & $-122.9(2)$ \\
\hline $\mathrm{Ru}(2)-\mathrm{S}(4)-\mathrm{C}(31)-\mathrm{C}(30)$ & $-20.4(2)$ \\
\hline $\mathrm{C}(31)-\mathrm{S}(4)-\mathrm{C}(32)-\mathrm{C}(33)$ & $66.6(3)$ \\
\hline$R u(2)-S(4)-C(32)-C(33)$ & $-36.9(2)$ \\
\hline $\mathrm{C}(28)-\mathrm{N}(3)-\mathrm{C}(33)-\mathrm{C}(32)$ & $-136.8(3)$ \\
\hline $\mathrm{C}(34)-\mathrm{N}(3)-\mathrm{C}(33)-\mathrm{C}(32)$ & $104.7(3)$ \\
\hline $\mathrm{Ru}(2)-\mathrm{N}(3)-\mathrm{C}(33)-\mathrm{C}(32)$ & $-19.1(4)$ \\
\hline $\mathrm{S}(4)-\mathrm{C}(32)-\mathrm{C}(33)-\mathrm{N}(3)$ & $38.9(4)$ \\
\hline $\mathrm{C}(28)-\mathrm{N}(3)-\mathrm{C}(34)-\mathrm{C}(35)$ & $162.5(3)$ \\
\hline $\mathrm{C}(33)-\mathrm{N}(3)-\mathrm{C}(34)-\mathrm{C}(35)$ & $-78.8(3)$ \\
\hline $\mathrm{Ru}(2)-\mathrm{N}(3)-\mathrm{C}(34)-\mathrm{C}(35)$ & $48.8(3)$ \\
\hline $\mathrm{N}(3)-\mathrm{C}(34)-\mathrm{C}(35)-\mathrm{C}(38)$ & $-152.2(3)$ \\
\hline $\mathrm{N}(3)-\mathrm{C}(34)-\mathrm{C}(35)-\mathrm{C}(39)$ & $88.2(4)$ \\
\hline $\mathrm{N}(3)-\mathrm{C}(34)-\mathrm{C}(35)-\mathrm{S}(5)$ & $-35.1(4)$ \\
\hline $\mathrm{O}(3)-\mathrm{S}(5)-\mathrm{C}(35)-\mathrm{C}(38)$ & $-118.8(3)$ \\
\hline $\mathrm{O}(4)-\mathrm{S}(5)-\mathrm{C}(35)-\mathrm{C}(38)$ & $-2.0(3)$ \\
\hline$R u(2)-S(5)-C(35)-C(38)$ & $120.9(2)$ \\
\hline$O(3)-S(5)-C(35)-C(39)$ & $0.3(3)$ \\
\hline $\mathrm{O}(4)-\mathrm{S}(5)-\mathrm{C}(35)-\mathrm{C}(39)$ & $117.0(3)$ \\
\hline$R u(2)-S(5)-C(35)-C(39)$ & $-120.1(2)$ \\
\hline $\mathrm{O}(3)-\mathrm{S}(5)-\mathrm{C}(35)-\mathrm{C}(34)$ & $125.3(2)$ \\
\hline $\mathrm{O}(4)-\mathrm{S}(5)-\mathrm{C}(35)-\mathrm{C}(34)$ & $-118.0(2)$ \\
\hline $\operatorname{Ru}(2)-S(5)-C(35)-C(34)$ & $4.9(3)$ \\
\hline$R u(2)-S(6)-C(36)-C(40)$ & $-125.5(2)$ \\
\hline$R u(2)-S(6)-C(36)-C(37)$ & $0.1(2)$ \\
\hline$R u(2)-S(6)-C(36)-C(41)$ & $117.0(2)$ \\
\hline$C(30)-N(4)-C(37)-C(36)$ & $169.7(3)$ \\
\hline $\mathrm{C}(29)-\mathrm{N}(4)-\mathrm{C}(37)-\mathrm{C}(36)$ & $-71.6(3)$ \\
\hline $\mathrm{Ru}(2)-\mathrm{N}(4)-\mathrm{C}(37)-\mathrm{C}(36)$ & $50.2(3)$ \\
\hline $\mathrm{C}(40)-\mathrm{C}(36)-\mathrm{C}(37)-\mathrm{N}(4)$ & $92.0(3)$ \\
\hline$C(41)-C(36)-C(37)-N(4)$ & $-149.8(3)$ \\
\hline$S(6)-C(36)-C(37)-N(4)$ & $-31.9(3)$ \\
\hline $\mathrm{C}(54)-\mathrm{P}(2)-\mathrm{C}(42)-\mathrm{C}(43)$ & $-39.6(3)$ \\
\hline $\mathrm{C}(48)-\mathrm{P}(2)-\mathrm{C}(42)-\mathrm{C}(43)$ & $-140.6(3)$ \\
\hline$R u(2)-P(2)-C(42)-C(43)$ & $80.4(3)$ \\
\hline $\mathrm{C}(54)-\mathrm{P}(2)-\mathrm{C}(42)-\mathrm{C}(47)$ & $145.3(3)$ \\
\hline $\mathrm{C}(48)-\mathrm{P}(2)-\mathrm{C}(42)-\mathrm{C}(47)$ & $44.3(3)$ \\
\hline $\mathrm{Ru}(2)-\mathrm{P}(2)-\mathrm{C}(42)-\mathrm{C}(47)$ & $-94.6(3)$ \\
\hline$C(47)-C(42)-C(43)-C(44)$ & $-0.2(5)$ \\
\hline $\mathrm{P}(2)-\mathrm{C}(42)-\mathrm{C}(43)-\mathrm{C}(44)$ & $-175.4(3)$ \\
\hline $\mathrm{C}(42)-\mathrm{C}(43)-\mathrm{C}(44)-\mathrm{C}(45)$ & $0.9(5)$ \\
\hline$C(43)-C(44)-C(45)-C(46)$ & $-0.2(5)$ \\
\hline$C(44)-C(45)-C(46)-C(47)$ & $-1.1(6)$ \\
\hline $\mathrm{C}(45)-\mathrm{C}(46)-\mathrm{C}(47)-\mathrm{C}(42)$ & $1.8(5)$ \\
\hline$C(43)-C(42)-C(47)-C(46)$ & $-1.1(5)$ \\
\hline$P(2)-C(42)-C(47)-C(46)$ & $174.1(3)$ \\
\hline
\end{tabular}




\begin{tabular}{|c|c|}
\hline$C(54)-P(2)-C(48)-C(53)$ & $126.3(3)$ \\
\hline$C(42)-P(2)-C(48)-C(53)$ & $-131.1(3)$ \\
\hline $\mathrm{Ru}(2)-\mathrm{P}(2)-\mathrm{C}(48)-\mathrm{C}(53)$ & $0.7(4)$ \\
\hline$C(54)-P(2)-C(48)-C(49)$ & $-53.4(3)$ \\
\hline $\mathrm{C}(42)-\mathrm{P}(2)-\mathrm{C}(48)-\mathrm{C}(49)$ & $49.2(3)$ \\
\hline $\mathrm{Ru}(2)-\mathrm{P}(2)-\mathrm{C}(48)-\mathrm{C}(49)$ & $-179.0(2)$ \\
\hline $\mathrm{C}(53)-\mathrm{C}(48)-\mathrm{C}(49)-\mathrm{C}(50)$ & $1.1(5)$ \\
\hline$P(2)-C(48)-C(49)-C(50)$ & $-179.2(3)$ \\
\hline$C(48)-C(49)-C(50)-C(51)$ & $1.5(6)$ \\
\hline $\mathrm{C}(49)-\mathrm{C}(50)-\mathrm{C}(51)-\mathrm{C}(52)$ & $-2.6(6)$ \\
\hline $\mathrm{C}(50)-\mathrm{C}(51)-\mathrm{C}(52)-\mathrm{C}(53)$ & $1.1(6)$ \\
\hline $\mathrm{C}(49)-\mathrm{C}(48)-\mathrm{C}(53)-\mathrm{C}(52)$ & $-2.5(5)$ \\
\hline$P(2)-C(48)-C(53)-C(52)$ & $177.7(3)$ \\
\hline$C(51)-C(52)-C(53)-C(48)$ & $1.5(5)$ \\
\hline$C(401) \# 1-P(3)-C(500)-C(402) \# 1$ & $-80.7(14)$ \\
\hline $\mathrm{C}(403) \# 1-\mathrm{P}(3)-\mathrm{C}(500)-\mathrm{C}(402) \# 1$ & $38.8(15)$ \\
\hline $\mathrm{C}(400)-\mathrm{P}(3)-\mathrm{C}(500)-\mathrm{C}(402) \# 1$ & $-85.3(13)$ \\
\hline $\mathrm{C}(300)-\mathrm{P}(3)-\mathrm{C}(500)-\mathrm{C}(402) \# 1$ & $6.5(14)$ \\
\hline $\mathrm{C}(404) \# 1-\mathrm{P}(3)-\mathrm{C}(500)-\mathrm{C}(402) \# 1$ & $-43.3(16)$ \\
\hline$C(402) \# 1-P(3)-C(500)-C(401) \# 1$ & $80.7(14)$ \\
\hline $\mathrm{C}(403) \# 1-\mathrm{P}(3)-\mathrm{C}(500)-\mathrm{C}(401) \# 1$ & $119.5(5)$ \\
\hline $\mathrm{C}(400)-\mathrm{P}(3)-\mathrm{C}(500)-\mathrm{C}(401) \# 1$ & $-4.6(4)$ \\
\hline C(300)-P(3)-C(500)-C(401)\#1 & $87.2(3)$ \\
\hline$C(404) \# 1-P(3)-C(500)-C(401) \# 1$ & $37.4(7)$ \\
\hline C(402)\#1-P(3)-C(300)-C(305) & $154.3(5)$ \\
\hline $\mathrm{C}(401) \# 1-\mathrm{P}(3)-\mathrm{C}(300)-\mathrm{C}(305)$ & $-142.7(3)$ \\
\hline $\mathrm{C}(403) \# 1-\mathrm{P}(3)-\mathrm{C}(300)-\mathrm{C}(305)$ & $-0.9(3)$ \\
\hline C(400)-P(3)-C(300)-C(305) & $-100.4(2)$ \\
\hline $\mathrm{C}(500)-\mathrm{P}(3)-\mathrm{C}(300)-\mathrm{C}(305)$ & $152.2(3)$ \\
\hline $\mathrm{C}(404) \# 1-\mathrm{P}(3)-\mathrm{C}(300)-\mathrm{C}(305)$ & $-54.92(19)$ \\
\hline $\mathrm{C}(402) \# 1-\mathrm{P}(3)-\mathrm{C}(300)-\mathrm{C}(301)$ & $-25.5(4)$ \\
\hline $\mathrm{C}(401) \# 1-\mathrm{P}(3)-\mathrm{C}(300)-\mathrm{C}(301)$ & $37.5(2)$ \\
\hline $\mathrm{C}(403) \# 1-\mathrm{P}(3)-\mathrm{C}(300)-\mathrm{C}(301)$ & $179.3(4)$ \\
\hline$C(400)-P(3)-C(300)-C(301)$ & $79.7(2)$ \\
\hline $\mathrm{C}(500)-\mathrm{P}(3)-\mathrm{C}(300)-\mathrm{C}(301)$ & $-27.6(4)$ \\
\hline $\mathrm{C}(404) \# 1-\mathrm{P}(3)-\mathrm{C}(300)-\mathrm{C}(301)$ & $125.3(2)$ \\
\hline $\mathrm{C}(402) \# 1-\mathrm{P}(3)-\mathrm{C}(300)-\mathrm{C}(403) \# 1$ & $155.2(5)$ \\
\hline $\mathrm{C}(401) \# 1-\mathrm{P}(3)-\mathrm{C}(300)-\mathrm{C}(403) \# 1$ & $-141.8(4)$ \\
\hline $\mathrm{C}(400)-\mathrm{P}(3)-\mathrm{C}(300)-\mathrm{C}(403) \# 1$ & $-99.6(3)$ \\
\hline $\mathrm{C}(500)-\mathrm{P}(3)-\mathrm{C}(300)-\mathrm{C}(403) \# 1$ & $153.1(4)$ \\
\hline $\mathrm{C}(404) \# 1-\mathrm{P}(3)-\mathrm{C}(300)-\mathrm{C}(403) \# 1$ & $-54.0(2)$ \\
\hline $\mathrm{C}(402) \# 1-\mathrm{P}(3)-\mathrm{C}(300)-\mathrm{C}(404) \# 1$ & $-150.7(5)$ \\
\hline $\mathrm{C}(401) \# 1-\mathrm{P}(3)-\mathrm{C}(300)-\mathrm{C}(404) \# 1$ & $-87.8(2)$ \\
\hline $\mathrm{C}(403) \# 1-\mathrm{P}(3)-\mathrm{C}(300)-\mathrm{C}(404) \# 1$ & $54.0(2)$ \\
\hline $\mathrm{C}(400)-\mathrm{P}(3)-\mathrm{C}(300)-\mathrm{C}(404) \# 1$ & $-45.5(3)$ \\
\hline $\mathrm{C}(500)-\mathrm{P}(3)-\mathrm{C}(300)-\mathrm{C}(404) \# 1$ & $-152.8(3)$ \\
\hline $\mathrm{C}(401) \# 1-\mathrm{P}(3)-\mathrm{C}(300)-\mathrm{C}(402) \# 1$ & $63.0(3)$ \\
\hline $\mathrm{C}(403) \# 1-\mathrm{P}(3)-\mathrm{C}(300)-\mathrm{C}(402) \# 1$ & $-155.2(5)$ \\
\hline$C(400)-P(3)-C(300)-C(402) \# 1$ & $105.2(4)$ \\
\hline $\mathrm{C}(500)-\mathrm{P}(3)-\mathrm{C}(300)-\mathrm{C}(402) \# 1$ & $-2.1(5)$ \\
\hline $\mathrm{C}(404) \# 1-\mathrm{P}(3)-\mathrm{C}(300)-\mathrm{C}(402) \# 1$ & $150.7(5)$ \\
\hline$C(301)-C(300)-C(305)-C(403) \# 1$ & $-179.3(4)$ \\
\hline$P(3)-C(300)-C(305)-C(403) \# 1$ & $0.9(3)$ \\
\hline $\mathrm{C}(404) \# 1-\mathrm{C}(300)-\mathrm{C}(305)-\mathrm{C}(403) \# 1$ & $-47.1(2)$ \\
\hline $\mathrm{C}(402) \# 1-\mathrm{C}(300)-\mathrm{C}(305)-\mathrm{C}(403) \# 1$ & $22.0(4)$ \\
\hline $\mathrm{C}(301)-\mathrm{C}(300)-\mathrm{C}(305)-\mathrm{C}(304)$ & 0.0 \\
\hline
\end{tabular}




\begin{tabular}{|c|c|}
\hline$C(403) \# 1-C(300)-C(305)-C(304)$ & $179.3(4)$ \\
\hline$P(3)-C(300)-C(305)-C(304)$ & $-179.83(17)$ \\
\hline $\mathrm{C}(404) \# 1-\mathrm{C}(300)-\mathrm{C}(305)-\mathrm{C}(304)$ & $132.1(2)$ \\
\hline $\mathrm{C}(402) \# 1-\mathrm{C}(300)-\mathrm{C}(305)-\mathrm{C}(304)$ & $-158.8(4)$ \\
\hline $\mathrm{C}(403) \# 1-\mathrm{C}(305)-\mathrm{C}(304)-\mathrm{C}(303)$ & $166(7)$ \\
\hline $\mathrm{C}(300)-\mathrm{C}(305)-\mathrm{C}(304)-\mathrm{C}(303)$ & 0.0 \\
\hline $\mathrm{C}(305)-\mathrm{C}(304)-\mathrm{C}(303)-\mathrm{C}(302)$ & 0.0 \\
\hline $\mathrm{C}(304)-\mathrm{C}(303)-\mathrm{C}(302)-\mathrm{C}(301)$ & 0.0 \\
\hline $\mathrm{C}(303)-\mathrm{C}(302)-\mathrm{C}(301)-\mathrm{C}(300)$ & 0.0 \\
\hline$C(305)-C(300)-C(301)-C(302)$ & 0.0 \\
\hline $\mathrm{C}(403) \# 1-\mathrm{C}(300)-\mathrm{C}(301)-\mathrm{C}(302)$ & $-19(10)$ \\
\hline$P(3)-C(300)-C(301)-C(302)$ & $179.83(17)$ \\
\hline$C(404) \# 1-C(300)-C(301)-C(302)$ & $-100.6(3)$ \\
\hline$C(402) \# 1-C(300)-C(301)-C(302)$ & $167.7(3)$ \\
\hline $\mathrm{C}(402) \# 1-\mathrm{P}(3)-\mathrm{C}(400)-\mathrm{C}(401)$ & $167.3(4)$ \\
\hline $\mathrm{C}(401) \# 1-\mathrm{P}(3)-\mathrm{C}(400)-\mathrm{C}(401)$ & $-179.6(5)$ \\
\hline$C(403) \# 1-P(3)-C(400)-C(401)$ & $38.08(17)$ \\
\hline $\mathrm{C}(300)-\mathrm{P}(3)-\mathrm{C}(400)-\mathrm{C}(401)$ & $85.4(2)$ \\
\hline$C(500)-P(3)-C(400)-C(401)$ & $-173.4(3)$ \\
\hline $\mathrm{C}(404) \# 1-\mathrm{P}(3)-\mathrm{C}(400)-\mathrm{C}(401)$ & $32.96(18)$ \\
\hline $\mathrm{C}(402) \# 1-\mathrm{P}(3)-\mathrm{C}(400)-\mathrm{C}(401) \# 1$ & $-13.1(2)$ \\
\hline $\mathrm{C}(403) \# 1-\mathrm{P}(3)-\mathrm{C}(400)-\mathrm{C}(401) \# 1$ & $-142.3(5)$ \\
\hline $\mathrm{C}(300)-\mathrm{P}(3)-\mathrm{C}(400)-\mathrm{C}(401) \# 1$ & $-95.0(4)$ \\
\hline $\mathrm{C}(500)-\mathrm{P}(3)-\mathrm{C}(400)-\mathrm{C}(401) \# \mathrm{I}$ & $6.2(5)$ \\
\hline $\mathrm{C}(404) \# 1-\mathrm{P}(3)-\mathrm{C}(400)-\mathrm{C}(401) \# 1$ & $-147.4(6)$ \\
\hline $\mathrm{C}(402) \# 1-\mathrm{P}(3)-\mathrm{C}(400)-\mathrm{C}(404) \# 1$ & $134.3(4)$ \\
\hline $\mathrm{C}(401) \# 1-\mathrm{P}(3)-\mathrm{C}(400)-\mathrm{C}(404) \# 1$ & $147.4(6)$ \\
\hline $\mathrm{C}(403) \# 1-\mathrm{P}(3)-\mathrm{C}(400)-\mathrm{C}(404) \# 1$ & $5.11(16)$ \\
\hline $\mathrm{C}(300)-\mathrm{P}(3)-\mathrm{C}(400)-\mathrm{C}(404) \# 1$ & $52.4(3)$ \\
\hline $\mathrm{C}(500)-\mathrm{P}(3)-\mathrm{C}(400)-\mathrm{C}(404) \# 1$ & $153.7(4)$ \\
\hline $\mathrm{C}(402) \# 1-\mathrm{P}(3)-\mathrm{C}(400)-\mathrm{C}(404)$ & $-46.1(3)$ \\
\hline $\mathrm{C}(401) \# 1-\mathrm{P}(3)-\mathrm{C}(400)-\mathrm{C}(404)$ & $-32.9(4)$ \\
\hline$C(403) \# 1-P(3)-C(400)-C(404)$ & $-175.29(18)$ \\
\hline$C(300)-P(3)-C(400)-C(404)$ & $-128.00(16)$ \\
\hline$C(500)-P(3)-C(400)-C(404)$ & $-26.7(3)$ \\
\hline $\mathrm{C}(404) \# 1-\mathrm{P}(3)-\mathrm{C}(400)-\mathrm{C}(404)$ & $179.6(3)$ \\
\hline $\mathrm{C}(402) \# 1-\mathrm{P}(3)-\mathrm{C}(400)-\mathrm{P}(3) \# 1$ & $-152(69)$ \\
\hline $\mathrm{C}(401) \# 1-\mathrm{P}(3)-\mathrm{C}(400)-\mathrm{P}(3) \# 1$ & $-139(69)$ \\
\hline $\mathrm{C}(403) \# 1-\mathrm{P}(3)-\mathrm{C}(400)-\mathrm{P}(3) \# 1$ & $78(69)$ \\
\hline $\mathrm{C}(300)-\mathrm{P}(3)-\mathrm{C}(400)-\mathrm{P}(3) \# 1$ & $126(100)$ \\
\hline $\mathrm{C}(500)-\mathrm{P}(3)-\mathrm{C}(400)-\mathrm{P}(3) \# 1$ & $-133(69)$ \\
\hline C(404)\#1-P(3)-C(400)-P(3)\#1 & 73(69) \\
\hline$C(401)-C(400)-C(404)-C(403)$ & -21.3 \\
\hline $\mathrm{C}(401) \# 1-\mathrm{C}(400)-\mathrm{C}(404)-\mathrm{C}(403)$ & $159.1(5)$ \\
\hline $\mathrm{C}(404) \# 1-\mathrm{C}(400)-\mathrm{C}(404)-\mathrm{C}(403)$ & $-83(71)$ \\
\hline $\mathrm{P}(3)-\mathrm{C}(400)-\mathrm{C}(404)-\mathrm{C}(403)$ & $-173.01(19)$ \\
\hline $\mathrm{P}(3) \# 1-\mathrm{C}(400)-\mathrm{C}(404)-\mathrm{C}(403)$ & $6.64(18)$ \\
\hline C(401)-C(400)-C(404)-C(401)\#1 & $179.5(5)$ \\
\hline$C(404) \# 1-C(400)-C(404)-C(401) \# 1$ & $118(71)$ \\
\hline $\mathrm{P}(3)-\mathrm{C}(400)-\mathrm{C}(404)-\mathrm{C}(401) \# 1$ & $27.8(4)$ \\
\hline $\mathrm{P}(3) \# 1-\mathrm{C}(400)-\mathrm{C}(404)-\mathrm{C}(401) \# 1$ & $-152.5(6)$ \\
\hline$C(401)-C(400)-C(404)-C(300) \# 1$ & $22.0(2)$ \\
\hline $\mathrm{C}(401) \# 1-\mathrm{C}(400)-\mathrm{C}(404)-\mathrm{C}(300) \# 1$ & $-157.6(7)$ \\
\hline $\mathrm{C}(404) \# 1-\mathrm{C}(400)-\mathrm{C}(404)-\mathrm{C}(300) \# 1$ & $-40(71)$ \\
\hline$P(3)-C(400)-C(404)-C(300) \# 1$ & $-129.7(4)$ \\
\hline $\mathrm{P}(3) \# 1-\mathrm{C}(400)-\mathrm{C}(404)-\mathrm{C}(300) \# 1$ & $49.94(19)$ \\
\hline
\end{tabular}




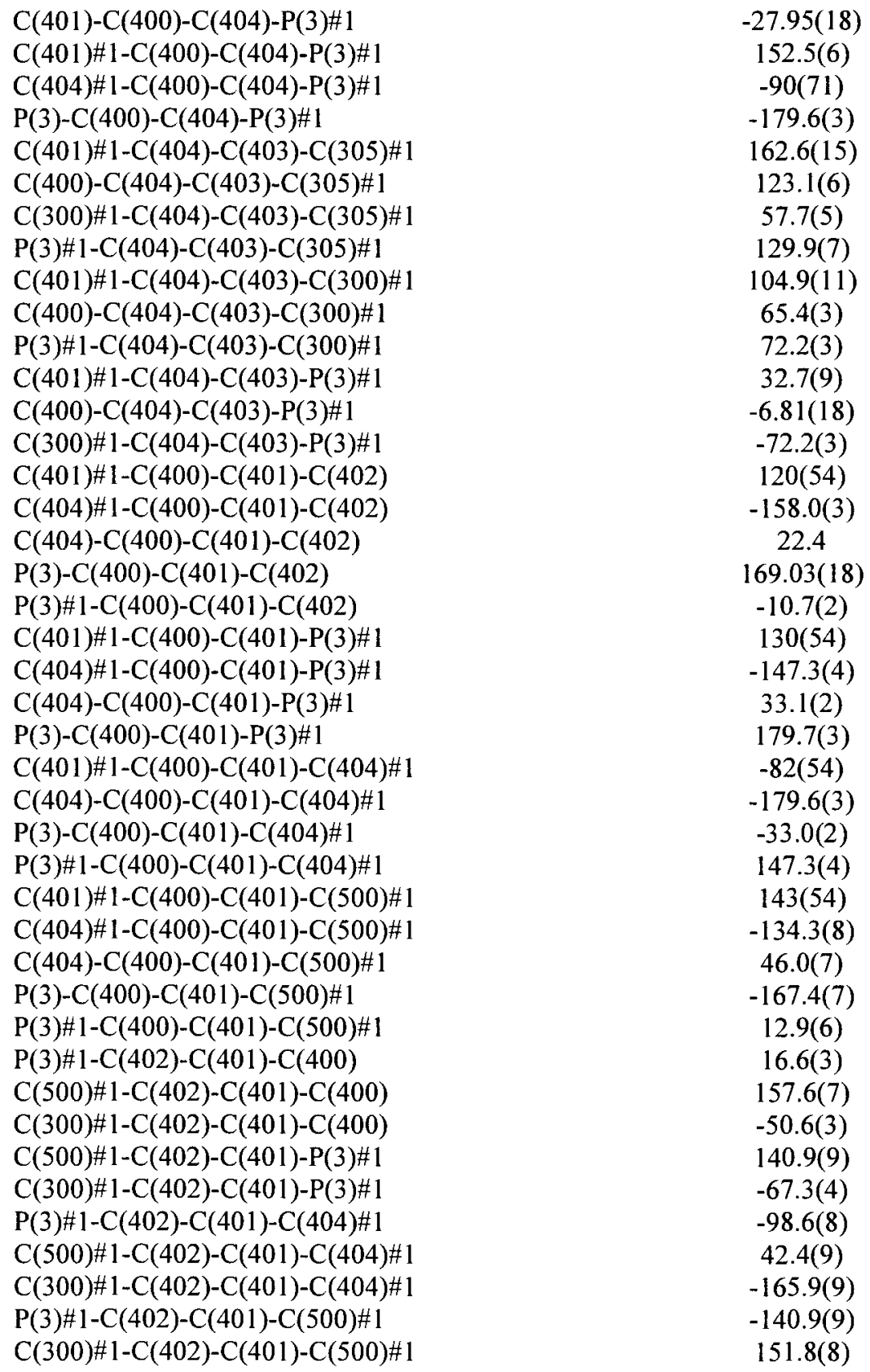

Symmetry transformations used to generate equivalent atoms:

$\# 1-x+1,-y+1,-z+2$ 
Table A15. Crystal data and structure refinement for 9

\author{
Identification code \\ Empirical formula \\ Formula weight \\ Temperature \\ Wavelength \\ Crystal system \\ Space group \\ Unit cell dimensions \\ Volume \\ Z \\ Density (calculated) \\ Absorption coefficient \\ $\mathrm{F}(000)$ \\ Crystal size \\ Theta range for data collection \\ Index ranges \\ Reflections collected \\ Independent reflections \\ Completeness to theta $=26.32^{\circ}$ \\ Absorption correction \\ Max. and min. transmission \\ Refinement method \\ Data / restraints / parameters \\ Goodness-of-fit on $\mathrm{F}^{2}$ \\ Final $R$ indices [ $\mathrm{I}>2$ sigma(I)] \\ $\mathrm{R}$ indices (all data) \\ Largest diff. peak and hole
}

cag174lt

C27 H41 N2 O3 P Ru S3

669.84

$100(2) \mathrm{K}$

$0.71073 \AA$

Triclinic

$\mathrm{P}-1$

$\mathrm{a}=8.7767(3) \AA$

$\mathrm{b}=15.8615(5) \AA$

$\mathrm{c}=21.4533(7) \AA$

$2851.70(16) \AA^{3}$

4

$1.560 \mathrm{Mg} / \mathrm{m}^{3}$

$0.858 \mathrm{~mm}^{-1}$

1392

$0.38 \times 0.20 \times 0.15 \mathrm{~mm}^{3}$

3.21 to $29.09^{\circ}$

$-11<=\mathrm{h}<=11,-21<=\mathrm{k}<=21,-28<=\mathrm{k}<=28$

63219

$14094[\mathrm{R}(\mathrm{int})=0.0252]$

$97.9 \%$

Semi-empirical from equivalents

1.00 and 0.918

Full-matrix least-squares on $\mathrm{F}^{2}$

$14094 / 0 / 677$

1.051

$\mathrm{R} 1=0.0225, \mathrm{wR} 2=0.0571$

$\mathrm{R} 1=0.0289, \mathrm{wR} 2=0.0584$

1.093 and -0.660 e. $\AA^{-3}$ $\alpha=89.823(3)^{\circ}$.

$\beta=79.435(3)^{\circ}$.

$\gamma=76.449(3)^{\circ}$. 
Table A16. Atomic coordinates ( $\left.\times 10^{4}\right)$ and equivalent isotropic displacement parameters $\left(\AA^{2} \times 10^{3}\right)$ for $9 \mathrm{U}(\mathrm{eq})$ is defined as one third of the trace of the orthogonalized $\mathrm{U}^{\mathrm{ij}}$ tensor.

\begin{tabular}{|c|c|c|c|c|}
\hline & $\mathrm{x}$ & $\mathrm{y}$ & $\mathrm{z}$ & $\mathrm{U}(\mathrm{eq})$ \\
\hline $\mathrm{Ru}(1)$ & $5047(1)$ & $1460(1)$ & $1552(1)$ & $8(1)$ \\
\hline $\mathrm{Ru}(2)$ & $3968(1)$ & $2767(1)$ & $5690(1)$ & $8(1)$ \\
\hline$S(1)$ & $6052(1)$ & 192(1) & $897(1)$ & $13(1)$ \\
\hline$S(2)$ & 2601(1) & $1200(1)$ & $1841(1)$ & $11(1)$ \\
\hline$S(3)$ & 4497(1) & $2756(1)$ & $2170(1)$ & $15(1)$ \\
\hline$S(4)$ & 3913(1) & $3830(1)$ & $4926(1)$ & $12(1)$ \\
\hline$S(5)$ & $6650(1)$ & 2381(1) & $5498(1)$ & $11(1)$ \\
\hline$S(6)$ & $3560(1)$ & $1749(1)$ & $6469(1)$ & $12(1)$ \\
\hline $\mathrm{P}(1)$ & $4270(1)$ & $2210(1)$ & $684(1)$ & $11(1)$ \\
\hline $\mathrm{P}(2)$ & $3958(1)$ & $3810(1)$ & $6453(1)$ & $10(1)$ \\
\hline$O(1)$ & $2330(2)$ & $497(1)$ & $1449(1)$ & $17(1)$ \\
\hline $\mathrm{O}(2)$ & $1206(1)$ & $1950(1)$ & $1947(1)$ & $17(1)$ \\
\hline$O(3)$ & $4226(2)$ & $2672(1)$ & $2886(1)$ & $30(1)$ \\
\hline $\mathrm{O}(4)$ & $7396(1)$ & $2979(1)$ & $5079(1)$ & $16(1)$ \\
\hline$O(5)$ & $7492(1)$ & $2138(1)$ & $6032(1)$ & $16(1)$ \\
\hline$O(6)$ & $4012(2)$ & $792(1)$ & $6247(1)$ & $31(1)$ \\
\hline $\mathrm{N}(1)$ & $5638(2)$ & $613(1)$ & $2315(1)$ & $12(1)$ \\
\hline $\mathrm{N}(2)$ & $7552(2)$ & $1493(1)$ & $1466(1)$ & $13(1)$ \\
\hline $\mathrm{N}(3)$ & $4201(2)$ & $1874(1)$ & $4892(1)$ & $12(1)$ \\
\hline $\mathrm{N}(4)$ & $1454(2)$ & $2929(1)$ & $5664(1)$ & $12(1)$ \\
\hline$C(1)$ & 6884(2) & $950(1)$ & $2560(1)$ & $15(1)$ \\
\hline$C(2)$ & $8200(2)$ & 1042(1) & $2018(1)$ & $16(1)$ \\
\hline$C(3)$ & $8543(2)$ & $1028(1)$ & $869(1)$ & $16(1)$ \\
\hline$C(4)$ & $8199(2)$ & $150(1)$ & $758(1)$ & $17(1)$ \\
\hline$C(5)$ & $5887(2)$ & $-603(1)$ & $1500(1)$ & $17(1)$ \\
\hline$C(6)$ & $6299(2)$ & $-333(1)$ & $2116(1)$ & $16(1)$ \\
\hline$C(7)$ & 4214(2) & $748(1)$ & $2844(1)$ & $14(1)$ \\
\hline$C(8)$ & $2632(2)$ & $721(1)$ & $2654(1)$ & $14(1)$ \\
\hline $\mathrm{C}(9)$ & $7689(2)$ & $2417(1)$ & $1451(1)$ & $14(1)$ \\
\hline$C(10)$ & $6503(2)$ & 3054(1) & $1947(1)$ & $16(1)$ \\
\hline$C(11)$ & $1318(2)$ & 1301(1) & $3137(1)$ & $21(1)$ \\
\hline$C(12)$ & $2328(2)$ & $-190(1)$ & $2649(1)$ & $21(1)$ \\
\hline$C(13)$ & $7158(2)$ & $3167(1)$ & $2546(1)$ & $22(1)$ \\
\hline$C(14)$ & $6117(2)$ & $3937(1)$ & $1642(1)$ & $22(1)$ \\
\hline$C(15)$ & $5917(2)$ & $2177(1)$ & 11(1) & $13(1)$ \\
\hline$C(16)$ & $6799(2)$ & 2814(1) & $-67(1)$ & $17(1)$ \\
\hline$C(17)$ & $8154(2)$ & $2713(1)$ & $-533(1)$ & $23(1)$ \\
\hline$C(18)$ & $8660(2)$ & $1977(1)$ & $-936(1)$ & $22(1)$ \\
\hline$C(19)$ & $7794(2)$ & $1346(1)$ & $-871(1)$ & $18(1)$ \\
\hline$C(20)$ & $6443(2)$ & $1443(1)$ & $-405(1)$ & $14(1)$ \\
\hline$C(21)$ & $3079(2)$ & $3339(1)$ & $724(1)$ & $15(1)$ \\
\hline $\mathrm{C}(22)$ & $1861(2)$ & $3605(1)$ & 1249(1) & $18(1)$ \\
\hline$C(23)$ & $797(2)$ & $4413(1)$ & $1268(1)$ & $22(1)$ \\
\hline$C(24)$ & $939(2)$ & $4963(1)$ & $770(1)$ & $27(1)$ \\
\hline$C(25)$ & $2145(3)$ & $4708(1)$ & $250(1)$ & $30(1)$ \\
\hline$C(26)$ & $3205(2)$ & $3900(1)$ & $222(1)$ & $23(1)$ \\
\hline$C(27)$ & 2939(2) & $1720(1)$ & $313(1)$ & $16(1)$ \\
\hline$C(28)$ & $2720(2)$ & $1540(1)$ & $5025(1)$ & $15(1)$ \\
\hline$C(29)$ & $1256(2)$ & $2278(1)$ & $5192(1)$ & $15(1)$ \\
\hline$C(30)$ & $757(2)$ & $3816(1)$ & $5455(1)$ & $14(1)$ \\
\hline$C(31)$ & 1793(2) & $4072(1)$ & $4870(1)$ & $16(1)$ \\
\hline
\end{tabular}




\begin{tabular}{lrrrr}
$C(32)$ & $4799(2)$ & $3136(1)$ & $4220(1)$ & $16(1)$ \\
$C(33)$ & $4293(2)$ & $2282(1)$ & $4252(1)$ & $16(1)$ \\
$C(34)$ & $5577(2)$ & $1118(1)$ & $4908(1)$ & $14(1)$ \\
$C(35)$ & $7139(2)$ & $1333(1)$ & $4994(1)$ & $15(1)$ \\
$C(36)$ & $558(2)$ & $2829(1)$ & $6313(1)$ & $14(1)$ \\
$C(37)$ & $1299(2)$ & $2046(1)$ & $6674(1)$ & $15(1)$ \\
$C(38)$ & $7992(2)$ & $606(1)$ & $5365(1)$ & $20(1)$ \\
$C(39)$ & $8236(2)$ & $1414(1)$ & $4368(1)$ & $22(1)$ \\
$C(40)$ & $850(2)$ & $2316(1)$ & $7381(1)$ & $24(1)$ \\
$C(41)$ & $678(2)$ & $1244(1)$ & $6570(1)$ & $22(1)$ \\
$C(42)$ & $4193(2)$ & $3529(1)$ & $7269(1)$ & $14(1)$ \\
$C(43)$ & $3425(2)$ & $4058(1)$ & $7805(1)$ & $20(1)$ \\
$C(44)$ & $3723(3)$ & $3822(1)$ & $8403(1)$ & $28(1)$ \\
$C(45)$ & $4833(3)$ & $3065(1)$ & $8469(1)$ & $29(1)$ \\
$C(46)$ & $5640(2)$ & $2548(1)$ & $7942(1)$ & $22(1)$ \\
$C(47)$ & $5323(2)$ & $2768(1)$ & $7342(1)$ & $16(1)$ \\
$C(48)$ & $2245(2)$ & $4758(1)$ & $6557(1)$ & $13(1)$ \\
$C(49)$ & $832(2)$ & $4785(1)$ & $6990(1)$ & $15(1)$ \\
$C(50)$ & $-489(2)$ & $5480(1)$ & $7019(1)$ & $18(1)$ \\
$C(51)$ & $-419(2)$ & $6159(1)$ & $6617(1)$ & $18(1)$ \\
$C(52)$ & $967(2)$ & $6142(1)$ & $6180(1)$ & $16(1)$ \\
$C(53)$ & $2288(2)$ & $5446(1)$ & $6150(1)$ & $14(1)$ \\
$C(54)$ & $5632(2)$ & $4332(1)$ & $6248(1)$ & $15(1)$ \\
& & & & \\
\hline
\end{tabular}


Table A17. Bond lengths $[\AA]$ and angles $\left[{ }^{\circ}\right]$ for 9

\begin{tabular}{|c|c|}
\hline $\operatorname{Ru}(1)-N(1)$ & $2.1746(13)$ \\
\hline $\mathrm{Ru}(1)-\mathrm{N}(2)$ & $2.1852(14)$ \\
\hline $\operatorname{Ru}(1)-S(2)$ & $2.2562(4)$ \\
\hline $\operatorname{Ru}(1)-P(1)$ & $2.3276(4)$ \\
\hline $\operatorname{Ru}(1)-S(1)$ & $2.3508(4)$ \\
\hline $\mathrm{Ru}(1)-\mathrm{S}(3)$ & $2.3532(4)$ \\
\hline $\mathrm{Ru}(2)-\mathrm{N}(4)$ & $2.1720(14)$ \\
\hline $\mathrm{Ru}(2)-\mathrm{N}(3)$ & $2.1743(13)$ \\
\hline $\operatorname{Ru}(2)-S(5)$ & $2.2481(4)$ \\
\hline $\mathrm{Ru}(2)-\mathrm{P}(2)$ & $2.3279(4)$ \\
\hline $\operatorname{Ru}(2)-S(4)$ & $2.3455(4)$ \\
\hline $\operatorname{Ru}(2)-S(6)$ & $2.3596(4)$ \\
\hline$S(1)-C(5)$ & $1.8154(17)$ \\
\hline$S(1)-C(4)$ & $1.8378(18)$ \\
\hline $\mathrm{S}(2)-\mathrm{O}(2)$ & $1.4767(12)$ \\
\hline$S(2)-O(1)$ & $1.4862(12)$ \\
\hline$S(2)-C(8)$ & $1.9022(16)$ \\
\hline $\mathrm{S}(3)-\mathrm{O}(3)$ & $1.5197(14)$ \\
\hline$S(3)-C(10)$ & $1.9057(18)$ \\
\hline$S(4)-C(32)$ & $1.8138(17)$ \\
\hline$S(4)-C(31)$ & $1.8359(17)$ \\
\hline $\mathrm{S}(5)-\mathrm{O}(5)$ & $1.4779(12)$ \\
\hline$S(5)-O(4)$ & $1.4856(12)$ \\
\hline$S(5)-C(35)$ & $1.9028(16)$ \\
\hline $\mathrm{S}(6)-\mathrm{O}(6)$ & $1.5303(15)$ \\
\hline$S(6)-C(37)$ & $1.8961(17)$ \\
\hline$P(1)-C(27)$ & $1.8362(17)$ \\
\hline$P(1)-C(15)$ & $1.8377(17)$ \\
\hline$P(1)-C(21)$ & $1.8438(17)$ \\
\hline$P(2)-C(54)$ & $1.8353(17)$ \\
\hline$P(2)-C(48)$ & $1.8419(17)$ \\
\hline$P(2)-C(42)$ & $1.8422(16)$ \\
\hline$N(1)-C(1)$ & $1.498(2)$ \\
\hline $\mathrm{N}(1)-\mathrm{C}(7)$ & $1.500(2)$ \\
\hline $\mathrm{N}(1)-\mathrm{C}(6)$ & $1.509(2)$ \\
\hline $\mathrm{N}(2)-\mathrm{C}(3)$ & $1.495(2)$ \\
\hline$N(2)-C(9)$ & $1.499(2)$ \\
\hline $\mathrm{N}(2)-\mathrm{C}(2)$ & $1.511(2)$ \\
\hline$N(3)-C(34)$ & $1.495(2)$ \\
\hline$N(3)-C(28)$ & $1.496(2)$ \\
\hline$N(3)-C(33)$ & $1.511(2)$ \\
\hline $\mathrm{N}(4)-\mathrm{C}(36)$ & $1.496(2)$ \\
\hline $\mathrm{N}(4)-\mathrm{C}(30)$ & $1.500(2)$ \\
\hline $\mathrm{N}(4)-\mathrm{C}(29)$ & $1.509(2)$ \\
\hline$C(1)-C(2)$ & $1.515(2)$ \\
\hline $\mathrm{C}(1)-\mathrm{H}(1 \mathrm{~A})$ & 0.9700 \\
\hline $\mathrm{C}(1)-\mathrm{H}(1 \mathrm{~B})$ & 0.9700 \\
\hline $\mathrm{C}(2)-\mathrm{H}(2 \mathrm{~A})$ & 0.9700 \\
\hline $\mathrm{C}(2)-\mathrm{H}(2 \mathrm{~B})$ & 0.9700 \\
\hline$C(3)-C(4)$ & $1.522(2)$ \\
\hline $\mathrm{C}(3)-\mathrm{H}(3 \mathrm{~A})$ & 0.9700 \\
\hline $\mathrm{C}(3)-\mathrm{H}(3 \mathrm{~B})$ & 0.9700 \\
\hline $\mathrm{C}(4)-\mathrm{H}(4 \mathrm{~A})$ & 0.9700 \\
\hline $\mathrm{C}(4)-\mathrm{H}(4 \mathrm{~B})$ & 0.9700 \\
\hline
\end{tabular}




\begin{tabular}{|c|c|}
\hline$C(5)-C(6)$ & $1.520(2)$ \\
\hline $\mathrm{C}(5)-\mathrm{H}(5 \mathrm{~A})$ & 0.9700 \\
\hline $\mathrm{C}(5)-\mathrm{H}(5 \mathrm{~B})$ & 0.9700 \\
\hline $\mathrm{C}(6)-\mathrm{H}(6 \mathrm{~A})$ & 0.9700 \\
\hline$C(6)-H(6 B)$ & 0.9700 \\
\hline$C(7)-C(8)$ & $1.528(2)$ \\
\hline $\mathrm{C}(7)-\mathrm{H}(7 \mathrm{~A})$ & 0.9700 \\
\hline $\mathrm{C}(7)-\mathrm{H}(7 \mathrm{~B})$ & 0.9700 \\
\hline $\mathrm{C}(8)-\mathrm{C}(11)$ & $1.524(2)$ \\
\hline $\mathrm{C}(8)-\mathrm{C}(12)$ & $1.530(2)$ \\
\hline $\mathrm{C}(9)-\mathrm{C}(10)$ & $1.531(2)$ \\
\hline$C(9)-H(9 A)$ & 0.9700 \\
\hline $\mathrm{C}(9)-\mathrm{H}(9 \mathrm{~B})$ & 0.9700 \\
\hline $\mathrm{C}(10)-\mathrm{C}(13)$ & $1.529(2)$ \\
\hline$C(10)-C(14)$ & $1.535(2)$ \\
\hline$C(11)-H(11 A)$ & 0.9600 \\
\hline $\mathrm{C}(11)-\mathrm{H}(11 \mathrm{~B})$ & 0.9600 \\
\hline $\mathrm{C}(11)-\mathrm{H}(11 \mathrm{C})$ & 0.9600 \\
\hline $\mathrm{C}(12)-\mathrm{H}(12 \mathrm{~A})$ & 0.9600 \\
\hline $\mathrm{C}(12)-\mathrm{H}(12 \mathrm{~B})$ & 0.9600 \\
\hline $\mathrm{C}(12)-\mathrm{H}(12 \mathrm{C})$ & 0.9600 \\
\hline$C(13)-H(13 A)$ & 0.9600 \\
\hline$C(13)-H(13 B)$ & 0.9600 \\
\hline $\mathrm{C}(13)-\mathrm{H}(13 \mathrm{C})$ & 0.9600 \\
\hline $\mathrm{C}(14)-\mathrm{H}(14 \mathrm{~A})$ & 0.9600 \\
\hline $\mathrm{C}(14)-\mathrm{H}(14 \mathrm{~B})$ & 0.9600 \\
\hline $\mathrm{C}(14)-\mathrm{H}(14 \mathrm{C})$ & 0.9600 \\
\hline$C(15)-C(16)$ & $1.402(2)$ \\
\hline$C(15)-C(20)$ & $1.402(2)$ \\
\hline$C(16)-C(17)$ & $1.384(3)$ \\
\hline $\mathrm{C}(16)-\mathrm{H}(16)$ & 0.9300 \\
\hline$C(17)-C(18)$ & $1.389(3)$ \\
\hline $\mathrm{C}(17)-\mathrm{H}(17)$ & 0.9300 \\
\hline$C(18)-C(19)$ & $1.384(3)$ \\
\hline $\mathrm{C}(18)-\mathrm{H}(18)$ & 0.9300 \\
\hline$C(19)-C(20)$ & $1.383(2)$ \\
\hline $\mathrm{C}(19)-\mathrm{H}(19)$ & 0.9300 \\
\hline $\mathrm{C}(20)-\mathrm{H}(20)$ & 0.9300 \\
\hline $\mathrm{C}(21)-\mathrm{C}(22)$ & $1.392(2)$ \\
\hline$C(21)-C(26)$ & $1.401(2)$ \\
\hline$C(22)-C(23)$ & $1.393(2)$ \\
\hline $\mathrm{C}(22)-\mathrm{H}(22)$ & 0.9300 \\
\hline$C(23)-C(24)$ & $1.385(3)$ \\
\hline $\mathrm{C}(23)-\mathrm{H}(23)$ & 0.9300 \\
\hline$C(24)-C(25)$ & $1.377(3)$ \\
\hline $\mathrm{C}(24)-\mathrm{H}(24)$ & 0.9300 \\
\hline$C(25)-C(26)$ & $1.390(3)$ \\
\hline $\mathrm{C}(25)-\mathrm{H}(25)$ & 0.9300 \\
\hline $\mathrm{C}(26)-\mathrm{H}(26)$ & 0.9300 \\
\hline$C(27)-H(27 A)$ & 0.9600 \\
\hline $\mathrm{C}(27)-\mathrm{H}(27 \mathrm{~B})$ & 0.9600 \\
\hline $\mathrm{C}(27)-\mathrm{H}(27 \mathrm{C})$ & 0.9600 \\
\hline $\mathrm{C}(28)-\mathrm{C}(29)$ & $1.509(2)$ \\
\hline $\mathrm{C}(28)-\mathrm{H}(28 \mathrm{~A})$ & 0.9700 \\
\hline $\mathrm{C}(28)-\mathrm{H}(28 \mathrm{~B})$ & 0.9700 \\
\hline $\mathrm{C}(29)-\mathrm{H}(29 \mathrm{~A})$ & 0.9700 \\
\hline
\end{tabular}




\begin{tabular}{|c|c|}
\hline$C(29)-H(29 B)$ & 0.9700 \\
\hline $\mathrm{C}(30)-\mathrm{C}(31)$ & $1.523(2)$ \\
\hline $\mathrm{C}(30)-\mathrm{H}(30 \mathrm{~A})$ & 0.9700 \\
\hline $\mathrm{C}(30)-\mathrm{H}(30 \mathrm{~B})$ & 0.9700 \\
\hline $\mathrm{C}(31)-\mathrm{H}(31 \mathrm{~A})$ & 0.9700 \\
\hline $\mathrm{C}(31)-\mathrm{H}(31 \mathrm{~B})$ & 0.9700 \\
\hline $\mathrm{C}(32)-\mathrm{C}(33)$ & $1.519(2)$ \\
\hline $\mathrm{C}(32)-\mathrm{H}(32 \mathrm{~A})$ & 0.9700 \\
\hline $\mathrm{C}(32)-\mathrm{H}(32 \mathrm{~B})$ & 0.9700 \\
\hline $\mathrm{C}(33)-\mathrm{H}(33 \mathrm{~A})$ & 0.9700 \\
\hline$C(33)-H(33 B)$ & 0.9700 \\
\hline $\mathrm{C}(34)-\mathrm{C}(35)$ & $1.531(2)$ \\
\hline $\mathrm{C}(34)-\mathrm{H}(34 \mathrm{~A})$ & 0.9700 \\
\hline $\mathrm{C}(34)-\mathrm{H}(34 \mathrm{~B})$ & 0.9700 \\
\hline$C(35)-C(39)$ & $1.525(2)$ \\
\hline$C(35)-C(38)$ & $1.528(2)$ \\
\hline$C(36)-C(37)$ & $1.538(2)$ \\
\hline $\mathrm{C}(36)-\mathrm{H}(36 \mathrm{~A})$ & 0.9700 \\
\hline $\mathrm{C}(36)-\mathrm{H}(36 \mathrm{~B})$ & 0.9700 \\
\hline$C(37)-C(41)$ & $1.527(2)$ \\
\hline$C(37)-C(40)$ & $1.532(3)$ \\
\hline $\mathrm{C}(38)-\mathrm{H}(38 \mathrm{~A})$ & 0.9600 \\
\hline $\mathrm{C}(38)-\mathrm{H}(38 \mathrm{~B})$ & 0.9600 \\
\hline $\mathrm{C}(38)-\mathrm{H}(38 \mathrm{C})$ & 0.9600 \\
\hline $\mathrm{C}(39)-\mathrm{H}(39 \mathrm{~A})$ & 0.9600 \\
\hline $\mathrm{C}(39)-\mathrm{H}(39 \mathrm{~B})$ & 0.9600 \\
\hline $\mathrm{C}(39)-\mathrm{H}(39 \mathrm{C})$ & 0.9600 \\
\hline $\mathrm{C}(40)-\mathrm{H}(40 \mathrm{~A})$ & 0.9600 \\
\hline $\mathrm{C}(40)-\mathrm{H}(40 \mathrm{~B})$ & 0.9600 \\
\hline $\mathrm{C}(40)-\mathrm{H}(40 \mathrm{C})$ & 0.9600 \\
\hline $\mathrm{C}(41)-\mathrm{H}(41 \mathrm{~A})$ & 0.9600 \\
\hline $\mathrm{C}(41)-\mathrm{H}(41 \mathrm{~B})$ & 0.9600 \\
\hline $\mathrm{C}(41)-\mathrm{H}(41 \mathrm{C})$ & 0.9600 \\
\hline$C(42)-C(43)$ & $1.392(2)$ \\
\hline $\mathrm{C}(42)-\mathrm{C}(47)$ & $1.400(2)$ \\
\hline$C(43)-C(44)$ & $1.388(2)$ \\
\hline $\mathrm{C}(43)-\mathrm{H}(43)$ & 0.9300 \\
\hline$C(44)-C(45)$ & $1.383(3)$ \\
\hline $\mathrm{C}(44)-\mathrm{H}(44)$ & 0.9300 \\
\hline$C(45)-C(46)$ & $1.377(3)$ \\
\hline $\mathrm{C}(45)-\mathrm{H}(45)$ & 0.9300 \\
\hline$C(46)-C(47)$ & $1.391(2)$ \\
\hline $\mathrm{C}(46)-\mathrm{H}(46)$ & 0.9300 \\
\hline $\mathrm{C}(47)-\mathrm{H}(47)$ & 0.9300 \\
\hline $\mathrm{C}(48)-\mathrm{C}(49)$ & $1.399(2)$ \\
\hline $\mathrm{C}(48)-\mathrm{C}(53)$ & $1.400(2)$ \\
\hline $\mathrm{C}(49)-\mathrm{C}(50)$ & $1.392(2)$ \\
\hline $\mathrm{C}(49)-\mathrm{H}(49)$ & 0.9300 \\
\hline$C(50)-C(51)$ & $1.384(2)$ \\
\hline $\mathrm{C}(50)-\mathrm{H}(50)$ & 0.9300 \\
\hline$C(51)-C(52)$ & $1.388(3)$ \\
\hline $\mathrm{C}(51)-\mathrm{H}(51)$ & 0.9300 \\
\hline$C(52)-C(53)$ & $1.393(2)$ \\
\hline $\mathrm{C}(52)-\mathrm{H}(52)$ & 0.9300 \\
\hline $\mathrm{C}(53)-\mathrm{H}(53)$ & 0.9300 \\
\hline $\mathrm{C}(54)-\mathrm{H}(54 \mathrm{~A})$ & 0.9600 \\
\hline
\end{tabular}




\begin{tabular}{|c|c|}
\hline $\mathrm{C}(54)-\mathrm{H}(54 \mathrm{~B})$ & 0.9600 \\
\hline $\mathrm{C}(54)-\mathrm{H}(54 \mathrm{C})$ & 0.9600 \\
\hline$N(1)-R u(1)-N(2)$ & $81.18(5)$ \\
\hline$N(1)-R u(1)-S(2)$ & $84.24(4)$ \\
\hline$N(2)-R u(1)-S(2)$ & $165.43(4)$ \\
\hline $\mathrm{N}(1)-\mathrm{Ru}(1)-\mathrm{P}(1)$ & $171.82(4)$ \\
\hline$N(2)-R u(1)-P(1)$ & $103.90(4)$ \\
\hline$S(2)-R u(1)-P(1)$ & $90.574(15)$ \\
\hline$N(1)-R u(1)-S(1)$ & $84.81(4)$ \\
\hline$N(2)-R u(1)-S(1)$ & $84.73(4)$ \\
\hline$S(2)-R u(1)-S(1)$ & $93.868(15)$ \\
\hline$P(1)-R u(1)-S(1)$ & $89.248(15)$ \\
\hline $\mathrm{N}(1)-\mathrm{Ru}(1)-\mathrm{S}(3)$ & $95.06(4)$ \\
\hline$N(2)-R u(1)-S(3)$ & $85.50(4)$ \\
\hline$S(2)-R u(1)-S(3)$ & $95.939(15)$ \\
\hline$P(1)-R u(1)-S(3)$ & $91.759(15)$ \\
\hline S(1)-Ru(1)-S(3) & $170.130(16)$ \\
\hline$N(4)-R u(2)-N(3)$ & $81.28(5)$ \\
\hline $\mathrm{N}(4)-\mathrm{Ru}(2)-\mathrm{S}(5)$ & $165.44(4)$ \\
\hline$N(3)-R u(2)-S(5)$ & $84.19(4)$ \\
\hline$N(4)-R u(2)-P(2)$ & $103.33(4)$ \\
\hline$N(3)-R u(2)-P(2)$ & $172.24(4)$ \\
\hline $\mathrm{S}(5)-\mathrm{Ru}(2)-\mathrm{P}(2)$ & $91.022(15)$ \\
\hline$N(4)-R u(2)-S(4)$ & $85.31(4)$ \\
\hline$N(3)-R u(2)-S(4)$ & $84.89(4)$ \\
\hline$S(5)-R u(2)-S(4)$ & $92.599(15)$ \\
\hline$P(2)-\operatorname{Ru}(2)-S(4)$ & $89.247(15)$ \\
\hline$N(4)-R u(2)-S(6)$ & $85.22(4)$ \\
\hline$N(3)-R u(2)-S(6)$ & $94.79(4)$ \\
\hline$S(5)-R u(2)-S(6)$ & $96.861(15)$ \\
\hline$P(2)-R u(2)-S(6)$ & $91.830(15)$ \\
\hline$S(4)-R u(2)-S(6)$ & $170.457(15)$ \\
\hline$C(5)-S(1)-C(4)$ & $101.62(8)$ \\
\hline $\mathrm{C}(5)-\mathrm{S}(1)-\mathrm{Ru}(1)$ & $98.65(6)$ \\
\hline $\mathrm{C}(4)-\mathrm{S}(1)-\mathrm{Ru}(1)$ & $100.45(6)$ \\
\hline $\mathrm{O}(2)-\mathrm{S}(2)-\mathrm{O}(1)$ & $111.32(7)$ \\
\hline $\mathrm{O}(2)-\mathrm{S}(2)-\mathrm{C}(8)$ & $104.44(7)$ \\
\hline $\mathrm{O}(1)-\mathrm{S}(2)-\mathrm{C}(8)$ & $103.75(7)$ \\
\hline $\mathrm{O}(2)-\mathrm{S}(2)-\mathrm{Ru}(1)$ & $118.15(5)$ \\
\hline $\mathrm{O}(1)-\mathrm{S}(2)-\mathrm{Ru}(1)$ & $113.47(5)$ \\
\hline $\mathrm{C}(8)-\mathrm{S}(2)-\mathrm{Ru}(1)$ & $103.87(5)$ \\
\hline $\mathrm{O}(3)-\mathrm{S}(3)-\mathrm{C}(10)$ & $105.58(8)$ \\
\hline $\mathrm{O}(3)-\mathrm{S}(3)-\mathrm{Ru}(1)$ & $116.47(6)$ \\
\hline$C(10)-S(3)-R u(1)$ & $99.61(5)$ \\
\hline $\mathrm{C}(32)-\mathrm{S}(4)-\mathrm{C}(31)$ & $101.03(8)$ \\
\hline $\mathrm{C}(32)-\mathrm{S}(4)-\mathrm{Ru}(2)$ & $99.25(6)$ \\
\hline $\mathrm{C}(31)-\mathrm{S}(4)-\mathrm{Ru}(2)$ & $100.07(5)$ \\
\hline $\mathrm{O}(5)-\mathrm{S}(5)-\mathrm{O}(4)$ & $110.96(7)$ \\
\hline $\mathrm{O}(5)-\mathrm{S}(5)-\mathrm{C}(35)$ & $103.79(7)$ \\
\hline $\mathrm{O}(4)-\mathrm{S}(5)-\mathrm{C}(35)$ & $103.95(7)$ \\
\hline $\mathrm{O}(5)-\mathrm{S}(5)-\mathrm{Ru}(2)$ & $119.21(5)$ \\
\hline $\mathrm{O}(4)-\mathrm{S}(5)-\mathrm{Ru}(2)$ & $113.35(5)$ \\
\hline $\mathrm{C}(35)-\mathrm{S}(5)-\mathrm{Ru}(2)$ & $103.55(5)$ \\
\hline $\mathrm{O}(6)-\mathrm{S}(6)-\mathrm{C}(37)$ & $105.17(8)$ \\
\hline $\mathrm{O}(6)-\mathrm{S}(6)-\mathrm{Ru}(2)$ & $116.99(6)$ \\
\hline $\mathrm{C}(37)-\mathrm{S}(6)-\mathrm{Ru}(2)$ & $99.78(5)$ \\
\hline
\end{tabular}




\begin{tabular}{|c|c|}
\hline$C(27)-P(1)-C(15)$ & $101.04(8)$ \\
\hline$C(27)-P(1)-C(21)$ & $96.99(8)$ \\
\hline$C(15)-P(1)-C(21)$ & $103.98(8)$ \\
\hline$C(27)-P(1)-R u(1)$ & $112.72(6)$ \\
\hline $\mathrm{C}(15)-\mathrm{P}(1)-\mathrm{Ru}(1)$ & $113.92(5)$ \\
\hline$C(21)-P(1)-R u(1)$ & $124.65(5)$ \\
\hline $\mathrm{C}(54)-\mathrm{P}(2)-\mathrm{C}(48)$ & $101.18(8)$ \\
\hline $\mathrm{C}(54)-\mathrm{P}(2)-\mathrm{C}(42)$ & $98.32(8)$ \\
\hline $\mathrm{C}(48)-\mathrm{P}(2)-\mathrm{C}(42)$ & $104.05(7)$ \\
\hline$C(54)-P(2)-R u(2)$ & $112.68(6)$ \\
\hline $\mathrm{C}(48)-\mathrm{P}(2)-\mathrm{Ru}(2)$ & $115.39(5)$ \\
\hline$C(42)-P(2)-R u(2)$ & $122.07(5)$ \\
\hline $\mathrm{C}(1)-\mathrm{N}(1)-\mathrm{C}(7)$ & $106.76(12)$ \\
\hline$C(1)-N(1)-C(6)$ & $109.03(13)$ \\
\hline$C(7)-N(1)-C(6)$ & $112.07(13)$ \\
\hline $\mathrm{C}(1)-\mathrm{N}(1)-\mathrm{Ru}(1)$ & $104.39(9)$ \\
\hline $\mathrm{C}(7)-\mathrm{N}(1)-\mathrm{Ru}(1)$ & $109.16(9)$ \\
\hline$C(6)-N(1)-R u(1)$ & $114.86(10)$ \\
\hline $\mathrm{C}(3)-\mathrm{N}(2)-\mathrm{C}(9)$ & $108.06(12)$ \\
\hline $\mathrm{C}(3)-\mathrm{N}(2)-\mathrm{C}(2)$ & $107.61(13)$ \\
\hline$C(9)-N(2)-C(2)$ & $111.05(12)$ \\
\hline $\mathrm{C}(3)-\mathrm{N}(2)-\mathrm{Ru}(1)$ & $111.44(10)$ \\
\hline $\mathrm{C}(9)-\mathrm{N}(2)-\mathrm{Ru}(1)$ & $109.33(10)$ \\
\hline $\mathrm{C}(2)-\mathrm{N}(2)-\mathrm{Ru}(1)$ & $109.35(10)$ \\
\hline $\mathrm{C}(34)-\mathrm{N}(3)-\mathrm{C}(28)$ & $107.02(12)$ \\
\hline $\mathrm{C}(34)-\mathrm{N}(3)-\mathrm{C}(33)$ & $112.42(13)$ \\
\hline $\mathrm{C}(28)-\mathrm{N}(3)-\mathrm{C}(33)$ & $108.34(12)$ \\
\hline$C(34)-N(3)-R u(2)$ & $109.02(9)$ \\
\hline $\mathrm{C}(28)-\mathrm{N}(3)-\mathrm{Ru}(2)$ & $104.66(10)$ \\
\hline$C(33)-N(3)-R u(2)$ & $114.81(10)$ \\
\hline $\mathrm{C}(36)-\mathrm{N}(4)-\mathrm{C}(30)$ & $107.80(13)$ \\
\hline $\mathrm{C}(36)-\mathrm{N}(4)-\mathrm{C}(29)$ & $111.16(12)$ \\
\hline $\mathrm{C}(30)-\mathrm{N}(4)-\mathrm{C}(29)$ & $107.47(12)$ \\
\hline$C(36)-N(4)-R u(2)$ & $109.76(9)$ \\
\hline $\mathrm{C}(30)-\mathrm{N}(4)-\mathrm{Ru}(2)$ & $110.81(10)$ \\
\hline$C(29)-N(4)-R u(2)$ & $109.80(10)$ \\
\hline $\mathrm{N}(1)-\mathrm{C}(1)-\mathrm{C}(2)$ & $110.35(13)$ \\
\hline $\mathrm{N}(1)-\mathrm{C}(1)-\mathrm{H}(1 \mathrm{~A})$ & 109.6 \\
\hline $\mathrm{C}(2)-\mathrm{C}(1)-\mathrm{H}(1 \mathrm{~A})$ & 109.6 \\
\hline $\mathrm{N}(1)-\mathrm{C}(1)-\mathrm{H}(1 \mathrm{~B})$ & 109.6 \\
\hline $\mathrm{C}(2)-\mathrm{C}(1)-\mathrm{H}(1 \mathrm{~B})$ & 109.6 \\
\hline $\mathrm{H}(1 \mathrm{~A})-\mathrm{C}(1)-\mathrm{H}(1 \mathrm{~B})$ & 108.1 \\
\hline $\mathrm{N}(2)-\mathrm{C}(2)-\mathrm{C}(1)$ & $112.23(13)$ \\
\hline $\mathrm{N}(2)-\mathrm{C}(2)-\mathrm{H}(2 \mathrm{~A})$ & 109.2 \\
\hline $\mathrm{C}(1)-\mathrm{C}(2)-\mathrm{H}(2 \mathrm{~A})$ & 109.2 \\
\hline $\mathrm{N}(2)-\mathrm{C}(2)-\mathrm{H}(2 \mathrm{~B})$ & 109.2 \\
\hline $\mathrm{C}(1)-\mathrm{C}(2)-\mathrm{H}(2 \mathrm{~B})$ & 109.2 \\
\hline $\mathrm{H}(2 \mathrm{~A})-\mathrm{C}(2)-\mathrm{H}(2 \mathrm{~B})$ & 107.9 \\
\hline $\mathrm{N}(2)-\mathrm{C}(3)-\mathrm{C}(4)$ & $112.65(14)$ \\
\hline $\mathrm{N}(2)-\mathrm{C}(3)-\mathrm{H}(3 \mathrm{~A})$ & 109.1 \\
\hline $\mathrm{C}(4)-\mathrm{C}(3)-\mathrm{H}(3 \mathrm{~A})$ & 109.1 \\
\hline $\mathrm{N}(2)-\mathrm{C}(3)-\mathrm{H}(3 \mathrm{~B})$ & 109.1 \\
\hline $\mathrm{C}(4)-\mathrm{C}(3)-\mathrm{H}(3 \mathrm{~B})$ & 109.1 \\
\hline $\mathrm{H}(3 \mathrm{~A})-\mathrm{C}(3)-\mathrm{H}(3 \mathrm{~B})$ & 107.8 \\
\hline$C(3)-C(4)-S(1)$ & $112.69(12)$ \\
\hline $\mathrm{C}(3)-\mathrm{C}(4)-\mathrm{H}(4 \mathrm{~A})$ & 109.1 \\
\hline
\end{tabular}




\begin{tabular}{|c|c|}
\hline$S(1)-C(4)-H(4 A)$ & 109.1 \\
\hline $\mathrm{C}(3)-\mathrm{C}(4)-\mathrm{H}(4 \mathrm{~B})$ & 109.1 \\
\hline $\mathrm{S}(1)-\mathrm{C}(4)-\mathrm{H}(4 \mathrm{~B})$ & 109.1 \\
\hline $\mathrm{H}(4 \mathrm{~A})-\mathrm{C}(4)-\mathrm{H}(4 \mathrm{~B})$ & 107.8 \\
\hline$C(6)-C(5)-S(1)$ & $112.20(11)$ \\
\hline $\mathrm{C}(6)-\mathrm{C}(5)-\mathrm{H}(5 \mathrm{~A})$ & 109.2 \\
\hline $\mathrm{S}(1)-\mathrm{C}(5)-\mathrm{H}(5 \mathrm{~A})$ & 109.2 \\
\hline $\mathrm{C}(6)-\mathrm{C}(5)-\mathrm{H}(5 \mathrm{~B})$ & 109.2 \\
\hline $\mathrm{S}(1)-\mathrm{C}(5)-\mathrm{H}(5 \mathrm{~B})$ & 109.2 \\
\hline $\mathrm{H}(5 \mathrm{~A})-\mathrm{C}(5)-\mathrm{H}(5 \mathrm{~B})$ & 107.9 \\
\hline$N(1)-C(6)-C(5)$ & $114.79(13)$ \\
\hline $\mathrm{N}(1)-\mathrm{C}(6)-\mathrm{H}(6 \mathrm{~A})$ & 108.6 \\
\hline$C(5)-C(6)-H(6 A)$ & 108.6 \\
\hline $\mathrm{N}(1)-\mathrm{C}(6)-\mathrm{H}(6 \mathrm{~B})$ & 108.6 \\
\hline $\mathrm{C}(5)-\mathrm{C}(6)-\mathrm{H}(6 \mathrm{~B})$ & 108.6 \\
\hline $\mathrm{H}(6 \mathrm{~A})-\mathrm{C}(6)-\mathrm{H}(6 \mathrm{~B})$ & 107.5 \\
\hline$N(1)-C(7)-C(8)$ & $116.12(13)$ \\
\hline $\mathrm{N}(1)-\mathrm{C}(7)-\mathrm{H}(7 \mathrm{~A})$ & 108.3 \\
\hline $\mathrm{C}(8)-\mathrm{C}(7)-\mathrm{H}(7 \mathrm{~A})$ & 108.3 \\
\hline $\mathrm{N}(1)-\mathrm{C}(7)-\mathrm{H}(7 \mathrm{~B})$ & 108.3 \\
\hline $\mathrm{C}(8)-\mathrm{C}(7)-\mathrm{H}(7 \mathrm{~B})$ & 108.3 \\
\hline $\mathrm{H}(7 \mathrm{~A})-\mathrm{C}(7)-\mathrm{H}(7 \mathrm{~B})$ & 107.4 \\
\hline $\mathrm{C}(11)-\mathrm{C}(8)-\mathrm{C}(7)$ & $106.72(14)$ \\
\hline$C(11)-C(8)-C(12)$ & $108.87(14)$ \\
\hline $\mathrm{C}(7)-\mathrm{C}(8)-\mathrm{C}(12)$ & $113.59(14)$ \\
\hline $\mathrm{C}(11)-\mathrm{C}(8)-\mathrm{S}(2)$ & $108.64(12)$ \\
\hline$C(7)-C(8)-S(2)$ & $108.38(11)$ \\
\hline$C(12)-C(8)-S(2)$ & $110.47(11)$ \\
\hline $\mathrm{N}(2)-\mathrm{C}(9)-\mathrm{C}(10)$ & $116.58(13)$ \\
\hline $\mathrm{N}(2)-\mathrm{C}(9)-\mathrm{H}(9 \mathrm{~A})$ & 108.1 \\
\hline$C(10)-C(9)-H(9 A)$ & 108.1 \\
\hline $\mathrm{N}(2)-\mathrm{C}(9)-\mathrm{H}(9 \mathrm{~B})$ & 108.1 \\
\hline $\mathrm{C}(10)-\mathrm{C}(9)-\mathrm{H}(9 \mathrm{~B})$ & 108.1 \\
\hline $\mathrm{H}(9 \mathrm{~A})-\mathrm{C}(9)-\mathrm{H}(9 \mathrm{~B})$ & 107.3 \\
\hline$C(13)-C(10)-C(9)$ & $113.00(15)$ \\
\hline $\mathrm{C}(13)-\mathrm{C}(10)-\mathrm{C}(14)$ & $108.73(14)$ \\
\hline$C(9)-C(10)-C(14)$ & $107.60(14)$ \\
\hline$C(13)-C(10)-S(3)$ & $109.77(12)$ \\
\hline $\mathrm{C}(9)-\mathrm{C}(10)-\mathrm{S}(3)$ & $112.77(11)$ \\
\hline$C(14)-C(10)-S(3)$ & $104.53(12)$ \\
\hline$C(8)-C(11)-H(11 A)$ & 109.5 \\
\hline $\mathrm{C}(8)-\mathrm{C}(11)-\mathrm{H}(11 \mathrm{~B})$ & 109.5 \\
\hline $\mathrm{H}(11 \mathrm{~A})-\mathrm{C}(11)-\mathrm{H}(11 \mathrm{~B})$ & 109.5 \\
\hline $\mathrm{C}(8)-\mathrm{C}(11)-\mathrm{H}(11 \mathrm{C})$ & 109.5 \\
\hline $\mathrm{H}(11 \mathrm{~A})-\mathrm{C}(11)-\mathrm{H}(11 \mathrm{C})$ & 109.5 \\
\hline $\mathrm{H}(11 \mathrm{~B})-\mathrm{C}(11)-\mathrm{H}(11 \mathrm{C})$ & 109.5 \\
\hline $\mathrm{C}(8)-\mathrm{C}(12)-\mathrm{H}(12 \mathrm{~A})$ & 109.5 \\
\hline $\mathrm{C}(8)-\mathrm{C}(12)-\mathrm{H}(12 \mathrm{~B})$ & 109.5 \\
\hline $\mathrm{H}(12 \mathrm{~A})-\mathrm{C}(12)-\mathrm{H}(12 \mathrm{~B})$ & 109.5 \\
\hline $\mathrm{C}(8)-\mathrm{C}(12)-\mathrm{H}(12 \mathrm{C})$ & 109.5 \\
\hline $\mathrm{H}(12 \mathrm{~A})-\mathrm{C}(12)-\mathrm{H}(12 \mathrm{C})$ & 109.5 \\
\hline $\mathrm{H}(12 \mathrm{~B})-\mathrm{C}(12)-\mathrm{H}(12 \mathrm{C})$ & 109.5 \\
\hline $\mathrm{C}(10)-\mathrm{C}(13)-\mathrm{H}(13 \mathrm{~A})$ & 109.5 \\
\hline $\mathrm{C}(10)-\mathrm{C}(13)-\mathrm{H}(13 \mathrm{~B})$ & 109.5 \\
\hline $\mathrm{H}(13 \mathrm{~A})-\mathrm{C}(13)-\mathrm{H}(13 \mathrm{~B})$ & 109.5 \\
\hline $\mathrm{C}(10)-\mathrm{C}(13)-\mathrm{H}(13 \mathrm{C})$ & 109.5 \\
\hline
\end{tabular}




\begin{tabular}{|c|c|}
\hline $\mathrm{H}(13 \mathrm{~A})-\mathrm{C}(13)-\mathrm{H}(13 \mathrm{C})$ & 109.5 \\
\hline$H(13 B)-C(13)-H(13 C)$ & 109.5 \\
\hline $\mathrm{C}(10)-\mathrm{C}(14)-\mathrm{H}(14 \mathrm{~A})$ & 109.5 \\
\hline$C(10)-C(14)-H(14 B)$ & 109.5 \\
\hline $\mathrm{H}(14 \mathrm{~A})-\mathrm{C}(14)-\mathrm{H}(14 \mathrm{~B})$ & 109.5 \\
\hline$C(10)-C(14)-H(14 C)$ & 109.5 \\
\hline $\mathrm{H}(14 \mathrm{~A})-\mathrm{C}(14)-\mathrm{H}(14 \mathrm{C})$ & 109.5 \\
\hline $\mathrm{H}(14 \mathrm{~B})-\mathrm{C}(14)-\mathrm{H}(14 \mathrm{C})$ & 109.5 \\
\hline$C(16)-C(15)-C(20)$ & $117.74(16)$ \\
\hline$C(16)-C(15)-P(1)$ & $122.53(13)$ \\
\hline$C(20)-C(15)-P(1)$ & $119.32(13)$ \\
\hline$C(17)-C(16)-C(15)$ & $120.89(16)$ \\
\hline $\mathrm{C}(17)-\mathrm{C}(16)-\mathrm{H}(16)$ & 119.6 \\
\hline $\mathrm{C}(15)-\mathrm{C}(16)-\mathrm{H}(16)$ & 119.6 \\
\hline$C(16)-C(17)-C(18)$ & $120.36(17)$ \\
\hline $\mathrm{C}(16)-\mathrm{C}(17)-\mathrm{H}(17)$ & 119.8 \\
\hline $\mathrm{C}(18)-\mathrm{C}(17)-\mathrm{H}(17)$ & 119.8 \\
\hline$C(19)-C(18)-C(17)$ & $119.58(17)$ \\
\hline $\mathrm{C}(19)-\mathrm{C}(18)-\mathrm{H}(18)$ & 120.2 \\
\hline $\mathrm{C}(17)-\mathrm{C}(18)-\mathrm{H}(18)$ & 120.2 \\
\hline$C(20)-C(19)-C(18)$ & $120.25(16)$ \\
\hline $\mathrm{C}(20)-\mathrm{C}(19)-\mathrm{H}(19)$ & 119.9 \\
\hline $\mathrm{C}(18)-\mathrm{C}(19)-\mathrm{H}(19)$ & 119.9 \\
\hline$C(19)-C(20)-C(15)$ & $121.17(16)$ \\
\hline $\mathrm{C}(19)-\mathrm{C}(20)-\mathrm{H}(20)$ & 119.4 \\
\hline $\mathrm{C}(15)-\mathrm{C}(20)-\mathrm{H}(20)$ & 119.4 \\
\hline $\mathrm{C}(22)-\mathrm{C}(21)-\mathrm{C}(26)$ & $118.57(16)$ \\
\hline $\mathrm{C}(22)-\mathrm{C}(21)-\mathrm{P}(1)$ & $117.66(13)$ \\
\hline$C(26)-C(21)-P(1)$ & $123.24(13)$ \\
\hline$C(21)-C(22)-C(23)$ & $120.24(16)$ \\
\hline $\mathrm{C}(21)-\mathrm{C}(22)-\mathrm{H}(22)$ & 119.9 \\
\hline $\mathrm{C}(23)-\mathrm{C}(22)-\mathrm{H}(22)$ & 119.9 \\
\hline$C(24)-C(23)-C(22)$ & $120.63(18)$ \\
\hline $\mathrm{C}(24)-\mathrm{C}(23)-\mathrm{H}(23)$ & 119.7 \\
\hline $\mathrm{C}(22)-\mathrm{C}(23)-\mathrm{H}(23)$ & 119.7 \\
\hline $\mathrm{C}(25)-\mathrm{C}(24)-\mathrm{C}(23)$ & $119.59(17)$ \\
\hline $\mathrm{C}(25)-\mathrm{C}(24)-\mathrm{H}(24)$ & 120.2 \\
\hline $\mathrm{C}(23)-\mathrm{C}(24)-\mathrm{H}(24)$ & 120.2 \\
\hline$C(24)-C(25)-C(26)$ & $120.35(18)$ \\
\hline $\mathrm{C}(24)-\mathrm{C}(25)-\mathrm{H}(25)$ & 119.8 \\
\hline $\mathrm{C}(26)-\mathrm{C}(25)-\mathrm{H}(25)$ & 119.8 \\
\hline$C(25)-C(26)-C(21)$ & $120.61(18)$ \\
\hline$C(25)-C(26)-H(26)$ & 119.7 \\
\hline $\mathrm{C}(21)-\mathrm{C}(26)-\mathrm{H}(26)$ & 119.7 \\
\hline $\mathrm{P}(1)-\mathrm{C}(27)-\mathrm{H}(27 \mathrm{~A})$ & 109.5 \\
\hline $\mathrm{P}(1)-\mathrm{C}(27)-\mathrm{H}(27 \mathrm{~B})$ & 109.5 \\
\hline$H(27 A)-C(27)-H(27 B)$ & 109.5 \\
\hline $\mathrm{P}(1)-\mathrm{C}(27)-\mathrm{H}(27 \mathrm{C})$ & 109.5 \\
\hline $\mathrm{H}(27 \mathrm{~A})-\mathrm{C}(27)-\mathrm{H}(27 \mathrm{C})$ & 109.5 \\
\hline $\mathrm{H}(27 \mathrm{~B})-\mathrm{C}(27)-\mathrm{H}(27 \mathrm{C})$ & 109.5 \\
\hline$N(3)-C(28)-C(29)$ & $110.86(13)$ \\
\hline $\mathrm{N}(3)-\mathrm{C}(28)-\mathrm{H}(28 \mathrm{~A})$ & 109.5 \\
\hline $\mathrm{C}(29)-\mathrm{C}(28)-\mathrm{H}(28 \mathrm{~A})$ & 109.5 \\
\hline $\mathrm{N}(3)-\mathrm{C}(28)-\mathrm{H}(28 \mathrm{~B})$ & 109.5 \\
\hline $\mathrm{C}(29)-\mathrm{C}(28)-\mathrm{H}(28 \mathrm{~B})$ & 109.5 \\
\hline $\mathrm{H}(28 \mathrm{~A})-\mathrm{C}(28)-\mathrm{H}(28 \mathrm{~B})$ & 108.1 \\
\hline
\end{tabular}




\begin{tabular}{|c|c|}
\hline $\mathrm{C}(28)-\mathrm{C}(29)-\mathrm{N}(4)$ & $112.23(13)$ \\
\hline $\mathrm{C}(28)-\mathrm{C}(29)-\mathrm{H}(29 \mathrm{~A})$ & 109.2 \\
\hline $\mathrm{N}(4)-\mathrm{C}(29)-\mathrm{H}(29 \mathrm{~A})$ & 109.2 \\
\hline $\mathrm{C}(28)-\mathrm{C}(29)-\mathrm{H}(29 \mathrm{~B})$ & 109.2 \\
\hline $\mathrm{N}(4)-\mathrm{C}(29)-\mathrm{H}(29 \mathrm{~B})$ & 109.2 \\
\hline $\mathrm{H}(29 \mathrm{~A})-\mathrm{C}(29)-\mathrm{H}(29 \mathrm{~B})$ & 107.9 \\
\hline$N(4)-C(30)-C(31)$ & $112.58(14)$ \\
\hline $\mathrm{N}(4)-\mathrm{C}(30)-\mathrm{H}(30 \mathrm{~A})$ & 109.1 \\
\hline $\mathrm{C}(31)-\mathrm{C}(30)-\mathrm{H}(30 \mathrm{~A})$ & 109.1 \\
\hline $\mathrm{N}(4)-\mathrm{C}(30)-\mathrm{H}(30 \mathrm{~B})$ & 109.1 \\
\hline $\mathrm{C}(31)-\mathrm{C}(30)-\mathrm{H}(30 \mathrm{~B})$ & 109.1 \\
\hline $\mathrm{H}(30 \mathrm{~A})-\mathrm{C}(30)-\mathrm{H}(30 \mathrm{~B})$ & 107.8 \\
\hline$C(30)-C(31)-S(4)$ & $112.56(11)$ \\
\hline $\mathrm{C}(30)-\mathrm{C}(31)-\mathrm{H}(31 \mathrm{~A})$ & 109.1 \\
\hline $\mathrm{S}(4)-\mathrm{C}(31)-\mathrm{H}(31 \mathrm{~A})$ & 109.1 \\
\hline $\mathrm{C}(30)-\mathrm{C}(31)-\mathrm{H}(31 \mathrm{~B})$ & 109.1 \\
\hline $\mathrm{S}(4)-\mathrm{C}(31)-\mathrm{H}(31 \mathrm{~B})$ & 109.1 \\
\hline $\mathrm{H}(31 \mathrm{~A})-\mathrm{C}(31)-\mathrm{H}(31 \mathrm{~B})$ & 107.8 \\
\hline $\mathrm{C}(33)-\mathrm{C}(32)-\mathrm{S}(4)$ & $112.37(12)$ \\
\hline$C(33)-C(32)-H(32 A)$ & 109.1 \\
\hline $\mathrm{S}(4)-\mathrm{C}(32)-\mathrm{H}(32 \mathrm{~A})$ & 109.1 \\
\hline $\mathrm{C}(33)-\mathrm{C}(32)-\mathrm{H}(32 \mathrm{~B})$ & 109.1 \\
\hline $\mathrm{S}(4)-\mathrm{C}(32)-\mathrm{H}(32 \mathrm{~B})$ & 109.1 \\
\hline $\mathrm{H}(32 \mathrm{~A})-\mathrm{C}(32)-\mathrm{H}(32 \mathrm{~B})$ & 107.9 \\
\hline $\mathrm{N}(3)-\mathrm{C}(33)-\mathrm{C}(32)$ & $115.29(13)$ \\
\hline $\mathrm{N}(3)-\mathrm{C}(33)-\mathrm{H}(33 \mathrm{~A})$ & 108.5 \\
\hline $\mathrm{C}(32)-\mathrm{C}(33)-\mathrm{H}(33 \mathrm{~A})$ & 108.5 \\
\hline $\mathrm{N}(3)-\mathrm{C}(33)-\mathrm{H}(33 \mathrm{~B})$ & 108.5 \\
\hline $\mathrm{C}(32)-\mathrm{C}(33)-\mathrm{H}(33 \mathrm{~B})$ & 108.5 \\
\hline $\mathrm{H}(33 \mathrm{~A})-\mathrm{C}(33)-\mathrm{H}(33 \mathrm{~B})$ & 107.5 \\
\hline $\mathrm{N}(3)-\mathrm{C}(34)-\mathrm{C}(35)$ & $116.24(13)$ \\
\hline $\mathrm{N}(3)-\mathrm{C}(34)-\mathrm{H}(34 \mathrm{~A})$ & 108.2 \\
\hline $\mathrm{C}(35)-\mathrm{C}(34)-\mathrm{H}(34 \mathrm{~A})$ & 108.2 \\
\hline $\mathrm{N}(3)-\mathrm{C}(34)-\mathrm{H}(34 \mathrm{~B})$ & 108.2 \\
\hline $\mathrm{C}(35)-\mathrm{C}(34)-\mathrm{H}(34 \mathrm{~B})$ & 108.2 \\
\hline $\mathrm{H}(34 \mathrm{~A})-\mathrm{C}(34)-\mathrm{H}(34 \mathrm{~B})$ & 107.4 \\
\hline$C(39)-C(35)-C(38)$ & $109.34(15)$ \\
\hline$C(39)-C(35)-C(34)$ & $113.41(14)$ \\
\hline $\mathrm{C}(38)-\mathrm{C}(35)-\mathrm{C}(34)$ & $107.30(14)$ \\
\hline $\mathrm{C}(39)-\mathrm{C}(35)-\mathrm{S}(5)$ & $110.17(11)$ \\
\hline $\mathrm{C}(38)-\mathrm{C}(35)-\mathrm{S}(5)$ & $107.67(11)$ \\
\hline $\mathrm{C}(34)-\mathrm{C}(35)-\mathrm{S}(5)$ & $108.76(11)$ \\
\hline$N(4)-C(36)-C(37)$ & $116.20(13)$ \\
\hline $\mathrm{N}(4)-\mathrm{C}(36)-\mathrm{H}(36 \mathrm{~A})$ & 108.2 \\
\hline $\mathrm{C}(37)-\mathrm{C}(36)-\mathrm{H}(36 \mathrm{~A})$ & 108.2 \\
\hline $\mathrm{N}(4)-\mathrm{C}(36)-\mathrm{H}(36 \mathrm{~B})$ & 108.2 \\
\hline $\mathrm{C}(37)-\mathrm{C}(36)-\mathrm{H}(36 \mathrm{~B})$ & 108.2 \\
\hline $\mathrm{H}(36 \mathrm{~A})-\mathrm{C}(36)-\mathrm{H}(36 \mathrm{~B})$ & 107.4 \\
\hline $\mathrm{C}(41)-\mathrm{C}(37)-\mathrm{C}(40)$ & $108.60(15)$ \\
\hline$C(41)-C(37)-C(36)$ & $112.81(14)$ \\
\hline$C(40)-C(37)-C(36)$ & $106.90(14)$ \\
\hline $\mathrm{C}(41)-\mathrm{C}(37)-\mathrm{S}(6)$ & $109.20(12)$ \\
\hline $\mathrm{C}(40)-\mathrm{C}(37)-\mathrm{S}(6)$ & $106.54(12)$ \\
\hline$C(36)-C(37)-S(6)$ & $112.50(11)$ \\
\hline$C(35)-C(38)-H(38 A)$ & 109.5 \\
\hline $\mathrm{C}(35)-\mathrm{C}(38)-\mathrm{H}(38 \mathrm{~B})$ & 109.5 \\
\hline
\end{tabular}




$\begin{array}{ll}\text { H(38A)-C(38)-H(38B) } & 109.5 \\ \text { C(35)-C(38)-H(38C) } & 109.5 \\ \text { H(38A)-C(38)-H(38C) } & 109.5 \\ \text { H(38B)-C(38)-H(38C) } & 109.5 \\ \text { C(35)-C(39)-H(39A) } & 109.5 \\ \text { C(35)-C(39)-H(39B) } & 109.5 \\ \text { H(39A)-C(39)-H(39B) } & 109.5 \\ \text { C(35)-C(39)-H(39C) } & 109.5 \\ \text { H(39A)-C(39)-H(39C) } & 109.5 \\ \text { H(39B)-C(39)-H(39C) } & 109.5 \\ \text { C(37)-C(40)-H(40A) } & 109.5 \\ \text { C(37)-C(40)-H(40B) } & 109.5 \\ \text { H(40A)-C(40)-H(40B) } & 109.5 \\ \text { C(37)-C(40)-H(40C) } & 109.5 \\ \text { H(40A)-C(40)-H(40C) } & 109.5 \\ \text { H(40B)-C(40)-H(40C) } & 109.5 \\ \text { C(37)-C(41)-H(41A) } & 109.5 \\ \text { C(37)-C(41)-H(41B) } & 109.5 \\ \text { H(41A)-C(41)-H(41B) } & 109.5 \\ \text { C(37)-C(41)-H(41C) } & 109.5 \\ \text { H(41A)-C(41)-H(41C) } & 109.5 \\ \text { H(41B)-C(41)-H(41C) } & 109.5 \\ \text { C(43)-C(42)-C(47) } & 118.55(15) \\ \text { C(43)-C(42)-P(2) } & 124.20(13) \\ \text { C(47)-C(42)-P(2) } & 117.02(13) \\ \text { C(44)-C(43)-C(42) } & 120.86(16) \\ \text { C(44)-C(43)-H(43) } & 119.6 \\ \text { C(42)-C(43)-H(43) } & 119.6 \\ \text { C(45)-C(44)-C(43) } & 120.04(17) \\ \text { C(45)-C(44)-H(44) } & 120.0 \\ \text { C(43)-C(44)-H(44) } & 120.0 \\ \text { C(46)-C(45)-C(44) } & 119.81(17) \\ \text { C(46)-C(45)-H(45) } & 120.1 \\ \text { C(44)-C(45)-H(45) } & 120.1 \\ \text { C(45)-C(46)-C(47) } & 120.66(17) \\ \text { C(45)-C(46)-H(46) } & 119.7 \\ \text { C(47)-C(46)-H(46) } & 119.7 \\ \text { C(46)-C(47)-C(42) } & 120.04(16) \\ \text { C(46)-C(47)-H(47) } & 120.0 \\ \text { C(42)-C(47)-H(47) } & 120.0 \\ \text { C(49)-C(48)-C(53) } & 118.08(15) \\ \text { C(49)-C(48)-P(2) } & 122.46(12) \\ \text { C(53)-C(48)-P(2) } & 119.18(13) \\ \text { C(50)-C(49)-C(48) } & 120.96(16) \\ \text { C(50)-C(49)-H(49) } & 119.5 \\ \text { C(48)-C(49)-H(49) } & 119.5 \\ \text { C(51)-C(50)-C(49) } & 120.15(17) \\ \text { C(51)-C(50)-H(50) } & 119.9 \\ \text { C(49)-C(50)-H(50) } & 119.9 \\ \text { C(50)-C(51)-C(52) } & 119.81(16) \\ \text { C(50)-C(51)-H(51) } & 120.1 \\ \text { C(52)-C(51)-H(51) } & 120.1 \\ \text { C(51)-C(52)-C(53) } & 120.09(16) \\ \text { C(51)-C(52)-H(52) } & 120.0 \\ \text { C(53)-C(52)-H(52) } & 120.0 \\ \text { C(52)-C(53)-C(48) } & 120.90(16) \\ & \end{array}$




$\begin{array}{ll}\text { C(52)-C(53)-H(53) } & 119.6 \\ \text { C(48)-C(53)-H(53) } & 119.6 \\ \text { P(2)-C(54)-H(54A) } & 109.5 \\ \text { P(2)-C(54)-H(54B) } & 109.5 \\ \text { H(54A)-C(54)-H(54B) } & 109.5 \\ \text { P(2)-C(54)-H(54C) } & 109.5 \\ \text { H(54A)-C(54)-H(54C) } & 109.5 \\ \text { H(54B)-C(54)-H(54C) } & 109.5\end{array}$

Symmetry transformations used to generate equivalent atoms: 
Table A18. Anisotropic displacement parameters $\left(\AA^{2} \times 10^{3}\right)$ for. 9 The anisotropic displacement factor exponent takes the form: $-2 \pi^{2}\left[h^{2} a^{* 2} U^{11}+\ldots+2 h k a^{*} b^{*} U^{12}\right]$

\begin{tabular}{|c|c|c|c|c|c|c|}
\hline & $\mathrm{U}^{11}$ & $\mathrm{U}^{22}$ & $\mathrm{U}^{33}$ & $\mathrm{U}^{23}$ & $\mathrm{U}^{13}$ & $\mathrm{U}^{12}$ \\
\hline $\operatorname{Ru}(1)$ & $8(1)$ & $10(1)$ & $8(1)$ & $1(1)$ & $-2(1)$ & $-2(1)$ \\
\hline $\operatorname{Ru}(2)$ & $7(1)$ & $9(1)$ & $8(1)$ & $1(1)$ & $-2(1)$ & $-2(1)$ \\
\hline$S(1)$ & $14(1)$ & $12(1)$ & $13(1)$ & $-1(1)$ & $-1(1)$ & $-2(1)$ \\
\hline$S(2)$ & $10(1)$ & $13(1)$ & $12(1)$ & l(1) & $-2(1)$ & $-4(1)$ \\
\hline$S(3)$ & $14(1)$ & $16(1)$ & $14(1)$ & $-4(1)$ & $0(1)$ & $-6(1)$ \\
\hline $\mathrm{S}(4)$ & $14(1)$ & $13(1)$ & $10(1)$ & $3(1)$ & $-3(1)$ & $-6(1)$ \\
\hline$S(5)$ & $8(1)$ & $12(1)$ & $12(1)$ & $-1(1)$ & $-1(1)$ & $-3(1)$ \\
\hline$S(6)$ & $10(1)$ & $13(1)$ & $14(1)$ & $5(1)$ & $-2(1)$ & $-3(1)$ \\
\hline $\mathrm{P}(1)$ & $13(1)$ & $11(1)$ & $9(1)$ & $0(1)$ & $-3(1)$ & $-2(1)$ \\
\hline $\mathrm{P}(2)$ & $11(1)$ & $10(1)$ & $9(1)$ & $0(1)$ & $-2(1)$ & $-2(1)$ \\
\hline $\mathrm{O}(1)$ & $19(1)$ & $19(1)$ & $16(1)$ & $0(1)$ & $-5(1)$ & $-10(1)$ \\
\hline $\mathrm{O}(2)$ & $9(1)$ & $18(1)$ & $22(1)$ & $3(1)$ & $-2(1)$ & $-1(1)$ \\
\hline$O(3)$ & $32(1)$ & $36(1)$ & $23(1)$ & $-1(1)$ & $-4(1)$ & $-9(1)$ \\
\hline$O(4)$ & $14(1)$ & $17(1)$ & $17(1)$ & $0(1)$ & $1(1)$ & $-6(1)$ \\
\hline $\mathrm{O}(5)$ & $12(1)$ & $19(1)$ & $17(1)$ & $-1(1)$ & $-5(1)$ & $-1(1)$ \\
\hline$O(6)$ & $31(1)$ & $26(1)$ & $36(1)$ & $5(1)$ & $-6(1)$ & $-6(1)$ \\
\hline $\mathrm{N}(1)$ & $11(1)$ & $14(1)$ & $12(1)$ & $3(1)$ & $-3(1)$ & $-3(1)$ \\
\hline $\mathrm{N}(2)$ & $10(1)$ & $17(1)$ & $11(1)$ & $0(1)$ & $-2(1)$ & $-3(1)$ \\
\hline $\mathrm{N}(3)$ & $13(1)$ & $13(1)$ & $11(1)$ & $0(1)$ & $-3(1)$ & $-5(1)$ \\
\hline$N(4)$ & $10(1)$ & $13(1)$ & $14(1)$ & $4(1)$ & $-4(1)$ & $-3(1)$ \\
\hline$C(1)$ & $14(1)$ & $20(1)$ & $13(1)$ & $3(1)$ & $-6(1)$ & $-5(1)$ \\
\hline$C(2)$ & $11(1)$ & $22(1)$ & $16(1)$ & $4(1)$ & $-6(1)$ & $-3(1)$ \\
\hline$C(3)$ & $10(1)$ & $20(1)$ & $16(1)$ & $0(1)$ & I(1) & $-3(1)$ \\
\hline$C(4)$ & 11(1) & 18(1) & $19(1)$ & $-3(1)$ & $2(1)$ & $0(1)$ \\
\hline$C(5)$ & $20(1)$ & $10(1)$ & $18(1)$ & $2(1)$ & $-1(1)$ & $-3(1)$ \\
\hline$C(6)$ & $16(1)$ & $15(1)$ & $17(1)$ & $4(1)$ & $-3(1)$ & $-1(1)$ \\
\hline$C(7)$ & $14(1)$ & $17(1)$ & $10(1)$ & $3(1)$ & $-2(1)$ & $-5(1)$ \\
\hline$C(8)$ & $14(1)$ & $18(1)$ & $11(1)$ & $3(1)$ & $-1(1)$ & $-6(1)$ \\
\hline $\mathrm{C}(9)$ & $13(1)$ & $18(1)$ & $14(1)$ & $2(1)$ & $-2(1)$ & $-8(1)$ \\
\hline$C(10)$ & $17(1)$ & $19(1)$ & $15(1)$ & $-1(1)$ & $-2(1)$ & $-10(1)$ \\
\hline$C(11)$ & $15(1)$ & $32(1)$ & $14(1)$ & $0(1)$ & $2(1)$ & $-6(1)$ \\
\hline$C(12)$ & $26(1)$ & $23(1)$ & $18(1)$ & $7(1)$ & $-4(1)$ & $-14(1)$ \\
\hline$C(13)$ & $24(1)$ & $28(1)$ & $18(1)$ & $-4(1)$ & $-4(1)$ & $-15(1)$ \\
\hline $\mathrm{C}(14)$ & $26(1)$ & $18(1)$ & $23(1)$ & $-2(1)$ & $-1(1)$ & $-10(1)$ \\
\hline$C(15)$ & $16(1)$ & $15(1)$ & $9(1)$ & $3(1)$ & $-4(1)$ & $-3(1)$ \\
\hline$C(16)$ & $24(1)$ & $16(1)$ & $13(1)$ & $2(1)$ & $-5(1)$ & $-6(1)$ \\
\hline$C(17)$ & $27(1)$ & $24(1)$ & $21(1)$ & $6(1)$ & $-3(1)$ & $-13(1)$ \\
\hline$C(18)$ & $20(1)$ & $29(1)$ & $15(1)$ & $4(1)$ & $2(1)$ & $-6(1)$ \\
\hline$C(19)$ & $20(1)$ & $20(1)$ & $13(1)$ & $0(1)$ & $-3(1)$ & $0(1)$ \\
\hline$C(20)$ & $17(1)$ & $15(1)$ & $12(1)$ & $2(1)$ & $-5(1)$ & $-5(1)$ \\
\hline$C(21)$ & $18(1)$ & $13(1)$ & $15(1)$ & $0(1)$ & $-7(1)$ & $-2(1)$ \\
\hline $\mathrm{C}(22)$ & $18(1)$ & $18(1)$ & $16(1)$ & I(1) & $-4(1)$ & $-3(1)$ \\
\hline$C(23)$ & $20(1)$ & $20(1)$ & $22(1)$ & $-2(1)$ & $-2(1)$ & $0(1)$ \\
\hline$C(24)$ & $29(1)$ & $16(1)$ & $32(1)$ & 1(1) & $-7(1)$ & $4(1)$ \\
\hline$C(25)$ & $39(1)$ & $21(1)$ & $24(1)$ & $8(1)$ & $-4(1)$ & $3(1)$ \\
\hline$C(26)$ & $30(1)$ & $20(1)$ & $15(1)$ & $2(1)$ & $-1(1)$ & $3(1)$ \\
\hline$C(27)$ & $16(1)$ & $20(1)$ & $13(1)$ & I(1) & $-6(1)$ & $-4(1)$ \\
\hline$C(28)$ & $16(1)$ & $16(1)$ & $16(1)$ & $0(1)$ & $-6(1)$ & $-8(1)$ \\
\hline$C(29)$ & $14(1)$ & $16(1)$ & $18(1)$ & $3(1)$ & $-8(1)$ & $-7(1)$ \\
\hline$C(30)$ & $12(1)$ & $12(1)$ & $18(1)$ & $5(1)$ & $-6(1)$ & $-1(1)$ \\
\hline $\mathrm{C}(31)$ & $16(1)$ & $16(1)$ & $18(1)$ & $7(1)$ & $-9(1)$ & $-5(1)$ \\
\hline
\end{tabular}




\begin{tabular}{|c|c|c|c|c|c|c|}
\hline $\mathrm{C}(32)$ & $20(1)$ & $19(1)$ & $9(1)$ & 1(1) & $0(1)$ & $-7(1)$ \\
\hline $\mathrm{C}(33)$ & $22(1)$ & $19(1)$ & $9(1)$ & $O(1)$ & $-4(1)$ & $-7(1)$ \\
\hline$C(34)$ & $15(1)$ & $12(1)$ & $15(1)$ & $-3(1)$ & $-2(1)$ & $-3(1)$ \\
\hline $\mathrm{C}(35)$ & $12(1)$ & $14(1)$ & $16(1)$ & $-4(1)$ & $0(1)$ & $-2(1)$ \\
\hline$C(36)$ & $10(1)$ & $16(1)$ & $16(1)$ & $6(1)$ & $-2(1)$ & $-3(1)$ \\
\hline $\mathrm{C}(37)$ & $10(1)$ & $18(1)$ & $18(1)$ & $7(1)$ & $-1(1)$ & $-3(1)$ \\
\hline$C(38)$ & $15(1)$ & $14(1)$ & $30(1)$ & $-3(1)$ & $-4(1)$ & $0(1)$ \\
\hline$C(39)$ & $18(1)$ & $22(1)$ & $22(1)$ & $-9(1)$ & $6(1)$ & $-7(1)$ \\
\hline $\mathrm{C}(40)$ & $18(1)$ & $29(1)$ & $18(1)$ & $10(1)$ & $3(1)$ & 1(1) \\
\hline $\mathrm{C}(41)$ & $14(1)$ & $21(1)$ & $33(1)$ & $14(1)$ & $-5(1)$ & $-8(1)$ \\
\hline $\mathrm{C}(42)$ & $18(1)$ & $13(1)$ & $12(1)$ & $1(1)$ & $-5(1)$ & $-5(1)$ \\
\hline $\mathrm{C}(43)$ & $26(1)$ & $16(1)$ & $16(1)$ & $-2(1)$ & $-8(1)$ & $2(1)$ \\
\hline $\mathrm{C}(44)$ & $42(1)$ & $24(1)$ & $13(1)$ & $-4(1)$ & $-6(1)$ & $3(1)$ \\
\hline $\mathrm{C}(45)$ & $48(1)$ & $24(1)$ & $14(1)$ & $2(1)$ & $-14(1)$ & $-1(1)$ \\
\hline $\mathrm{C}(46)$ & $31(1)$ & $16(1)$ & $19(1)$ & $2(1)$ & $-13(1)$ & $0(1)$ \\
\hline $\mathrm{C}(47)$ & $20(1)$ & $15(1)$ & $14(1)$ & $0(1)$ & $-6(1)$ & $-3(1)$ \\
\hline $\mathrm{C}(48)$ & $15(1)$ & $12(1)$ & $11(1)$ & $-2(1)$ & $-4(1)$ & $-2(1)$ \\
\hline$C(49)$ & $18(1)$ & $15(1)$ & $13(1)$ & $1(1)$ & $-3(1)$ & $-3(1)$ \\
\hline $\mathrm{C}(50)$ & $15(1)$ & $22(1)$ & $14(1)$ & $-4(1)$ & $-2(1)$ & $-1(1)$ \\
\hline $\mathrm{C}(51)$ & $19(1)$ & $15(1)$ & $19(1)$ & $-4(1)$ & $-9(1)$ & $3(1)$ \\
\hline $\mathrm{C}(52)$ & 21(1) & $12(1)$ & $18(1)$ & 1(1) & $-9(1)$ & $-3(1)$ \\
\hline $\mathrm{C}(53)$ & $15(1)$ & $14(1)$ & $12(1)$ & $-1(1)$ & $-4(1)$ & $-5(1)$ \\
\hline $\mathrm{C}(54)$ & $14(1)$ & $14(1)$ & $18(1)$ & $1(1)$ & $-4(1)$ & $-5(1)$ \\
\hline
\end{tabular}


Table A19. Hydrogen coordinates ( $\left.\times 10^{4}\right)$ and isotropic displacement parameters $\left(\AA^{2} \times 10^{3}\right)$ for 9.

\begin{tabular}{|c|c|c|c|c|}
\hline & $\mathrm{x}$ & $y$ & $z$ & $\mathrm{U}(\mathrm{eq})$ \\
\hline $\mathrm{H}(1 \mathrm{~A})$ & 7331 & 553 & 2861 & 18 \\
\hline $\mathrm{H}(1 \mathrm{~B})$ & 6401 & 1509 & 2779 & 18 \\
\hline$H(2 A)$ & 8872 & 1371 & 2169 & 19 \\
\hline $\mathrm{H}(2 \mathrm{~B})$ & 8857 & 471 & 1873 & 19 \\
\hline $\mathrm{H}(3 \mathrm{~A})$ & 9663 & 946 & 890 & 19 \\
\hline $\mathrm{H}(3 \mathrm{~B})$ & 8339 & 1386 & 512 & 19 \\
\hline $\mathrm{H}(4 \mathrm{~A})$ & 8699 & -262 & 1038 & 21 \\
\hline $\mathrm{H}(4 \mathrm{~B})$ & 8669 & -58 & 324 & 21 \\
\hline $\mathrm{H}(5 \mathrm{~A})$ & 4805 & -681 & 1584 & 20 \\
\hline $\mathrm{H}(5 \mathrm{~B})$ & 6598 & -1156 & 1343 & 20 \\
\hline $\mathrm{H}(6 \mathrm{~A})$ & 5901 & -681 & 2452 & 20 \\
\hline $\mathrm{H}(6 \mathrm{~B})$ & 7451 & -463 & 2070 & 20 \\
\hline $\mathrm{H}(7 \mathrm{~A})$ & 4087 & 1307 & 3056 & 16 \\
\hline $\mathrm{H}(7 \mathrm{~B})$ & 4436 & 307 & 3151 & 16 \\
\hline $\mathrm{H}(9 \mathrm{~A})$ & 7565 & 2627 & 1034 & 17 \\
\hline $\mathrm{H}(9 \mathrm{~B})$ & 8759 & 2427 & 1503 & 17 \\
\hline $\mathrm{H}(11 \mathrm{~A})$ & 296 & 1259 & 3059 & 31 \\
\hline $\mathrm{H}(11 \mathrm{~B})$ & 1407 & 1892 & 3099 & 31 \\
\hline $\mathrm{H}(11 \mathrm{C})$ & 1425 & 1119 & 3557 & 31 \\
\hline $\mathrm{H}(12 \mathrm{~A})$ & 2211 & -393 & 3073 & 31 \\
\hline $\mathrm{H}(12 \mathrm{~B})$ & 3212 & -577 & 2382 & 31 \\
\hline $\mathrm{H}(12 \mathrm{C})$ & 1370 & -172 & 2488 & 31 \\
\hline $\mathrm{H}(13 \mathrm{~A})$ & 7357 & 2624 & 2752 & 33 \\
\hline $\mathrm{H}(13 \mathrm{~B})$ & 6393 & 3596 & 2830 & 33 \\
\hline $\mathrm{H}(13 \mathrm{C})$ & 8136 & 3352 & 2434 & 33 \\
\hline $\mathrm{H}(14 \mathrm{~A})$ & 7091 & 4103 & 1476 & 33 \\
\hline $\mathrm{H}(14 \mathrm{~B})$ & 5464 & 4365 & 1956 & 33 \\
\hline $\mathrm{H}(14 \mathrm{C})$ & 5555 & 3894 & 1304 & 33 \\
\hline $\mathrm{H}(16)$ & 6470 & 3310 & 199 & 21 \\
\hline $\mathrm{H}(17)$ & 8727 & 3140 & -578 & 27 \\
\hline $\mathrm{H}(18)$ & 9575 & 1908 & -1247 & 26 \\
\hline $\mathrm{H}(19)$ & 8122 & 855 & -1143 & 22 \\
\hline $\mathrm{H}(20)$ & 5873 & 1013 & -365 & 17 \\
\hline $\mathrm{H}(22)$ & 1757 & 3241 & 1587 & 21 \\
\hline $\mathrm{H}(23)$ & -16 & 4584 & 1620 & 26 \\
\hline $\mathrm{H}(24)$ & 224 & 5501 & 786 & 32 \\
\hline $\mathrm{H}(25)$ & 2251 & 5079 & -84 & 36 \\
\hline $\mathrm{H}(26)$ & 4004 & 3730 & -135 & 28 \\
\hline $\mathrm{H}(27 \mathrm{~A})$ & 2844 & 1967 & -91 & 24 \\
\hline $\mathrm{H}(27 \mathrm{~B})$ & 1905 & 1830 & 583 & 24 \\
\hline $\mathrm{H}(27 \mathrm{C})$ & 3372 & 1104 & 254 & 24 \\
\hline $\mathrm{H}(28 \mathrm{~A})$ & 2774 & 1151 & 5374 & 18 \\
\hline $\mathrm{H}(28 \mathrm{~B})$ & 2645 & 1215 & 4654 & 18 \\
\hline $\mathrm{H}(29 \mathrm{~A})$ & 348 & 2045 & 5368 & 18 \\
\hline $\mathrm{H}(29 \mathrm{~B})$ & 1036 & 2569 & 4808 & 18 \\
\hline $\mathrm{H}(30 \mathrm{~A})$ & 626 & 4237 & 5800 & 17 \\
\hline $\mathrm{H}(30 \mathrm{~B})$ & -292 & 3832 & 5364 & 17 \\
\hline $\mathrm{H}(31 \mathrm{~A})$ & 1425 & 4688 & 4813 & 19 \\
\hline $\mathrm{H}(31 \mathrm{~B})$ & 1673 & 3763 & 4499 & 19 \\
\hline $\mathrm{H}(32 \mathrm{~A})$ & 4489 & 3438 & 3852 & 19 \\
\hline
\end{tabular}




\begin{tabular}{|c|c|c|c|c|}
\hline $\mathrm{H}(32 \mathrm{~B})$ & 5952 & 3018 & 4166 & 19 \\
\hline$H(33 A)$ & 3254 & 2377 & 4133 & 19 \\
\hline $\mathrm{H}(33 \mathrm{~B})$ & 5041 & 1872 & 3941 & 19 \\
\hline $\mathrm{H}(34 \mathrm{~A})$ & 5780 & 775 & 4514 & 17 \\
\hline $\mathrm{H}(34 \mathrm{~B})$ & 5264 & 758 & 5251 & 17 \\
\hline $\mathrm{H}(36 \mathrm{~A})$ & -507 & 2786 & 6276 & 17 \\
\hline $\mathrm{H}(36 \mathrm{~B})$ & 447 & 3352 & 6567 & 17 \\
\hline $\mathrm{H}(38 \mathrm{~A})$ & 8251 & 68 & 5122 & 30 \\
\hline $\mathrm{H}(38 \mathrm{~B})$ & 7307 & 558 & 5761 & 30 \\
\hline$H(38 C)$ & 8955 & 736 & 5446 & 30 \\
\hline $\mathrm{H}(39 \mathrm{~A})$ & 8558 & 865 & 4136 & 32 \\
\hline $\mathrm{H}(39 \mathrm{~B})$ & 9162 & 1582 & 4453 & 32 \\
\hline $\mathrm{H}(39 \mathrm{C})$ & 7678 & 1845 & 4122 & 32 \\
\hline $\mathrm{H}(40 \mathrm{~A})$ & -286 & 2529 & 7497 & 36 \\
\hline $\mathrm{H}(40 \mathrm{~B})$ & 1363 & 2765 & 7463 & 36 \\
\hline $\mathrm{H}(40 \mathrm{C})$ & 1191 & 1824 & 7626 & 36 \\
\hline $\mathrm{H}(41 \mathrm{~A})$ & 1125 & 787 & 6825 & 33 \\
\hline $\mathrm{H}(41 \mathrm{~B})$ & 982 & 1056 & 6131 & 33 \\
\hline $\mathrm{H}(41 \mathrm{C})$ & -464 & 1386 & 6690 & 33 \\
\hline $\mathrm{H}(43)$ & 2702 & 4578 & 7762 & 24 \\
\hline $\mathrm{H}(44)$ & 3177 & 4172 & 8758 & 33 \\
\hline $\mathrm{H}(45)$ & 5034 & 2905 & 8869 & 34 \\
\hline $\mathrm{H}(46)$ & 6405 & 2046 & 7986 & 27 \\
\hline $\mathrm{H}(47)$ & 5862 & 2409 & 6990 & 20 \\
\hline $\mathrm{H}(49)$ & 773 & 4331 & 7263 & 19 \\
\hline $\mathrm{H}(50)$ & -1421 & 5488 & 7309 & 21 \\
\hline $\mathrm{H}(51)$ & -1298 & 6626 & 6639 & 21 \\
\hline $\mathrm{H}(52)$ & 1014 & 6595 & 5906 & 20 \\
\hline $\mathrm{H}(53)$ & 3212 & 5439 & 5855 & 16 \\
\hline $\mathrm{H}(54 \mathrm{~A})$ & 5454 & 4835 & 6524 & 23 \\
\hline $\mathrm{H}(54 \mathrm{~B})$ & 5714 & 4503 & 5816 & 23 \\
\hline $\mathrm{H}(54 \mathrm{C})$ & 6604 & 3931 & 6297 & 23 \\
\hline
\end{tabular}


Table A20. Torsion angles $\left[{ }^{\circ}\right]$ for 9.

\begin{tabular}{|c|c|}
\hline $\mathrm{N}(1)-\mathrm{Ru}(1)-\mathrm{S}(1)-\mathrm{C}(5)$ & $20.23(7)$ \\
\hline $\mathrm{N}(2)-\mathrm{Ru}(1)-\mathrm{S}(1)-\mathrm{C}(5)$ & $101.82(7)$ \\
\hline$S(2)-R u(1)-S(1)-C(5)$ & $-63.62(6)$ \\
\hline$P(1)-R u(1)-S(1)-C(5)$ & $-154.15(6)$ \\
\hline$S(3)-R u(1)-S(1)-C(5)$ & $109.91(10)$ \\
\hline $\mathrm{N}(1)-\mathrm{Ru}(1)-\mathrm{S}(1)-\mathrm{C}(4)$ & $-83.38(7)$ \\
\hline $\mathrm{N}(2)-\mathrm{Ru}(1)-\mathrm{S}(1)-\mathrm{C}(4)$ & $-1.79(7)$ \\
\hline $\mathrm{S}(2)-\mathrm{Ru}(1)-\mathrm{S}(1)-\mathrm{C}(4)$ & $-167.23(6)$ \\
\hline$P(1)-R u(1)-S(1)-C(4)$ & $102.24(6)$ \\
\hline$S(3)-R u(1)-S(1)-C(4)$ & $6.30(11)$ \\
\hline $\mathrm{N}(1)-\mathrm{Ru}(1)-\mathrm{S}(2)-\mathrm{O}(2)$ & $130.33(7)$ \\
\hline $\mathrm{N}(2)-\mathrm{Ru}(1)-\mathrm{S}(2)-\mathrm{O}(2)$ & $130.78(16)$ \\
\hline $\mathrm{P}(1)-\mathrm{Ru}(1)-\mathrm{S}(2)-\mathrm{O}(2)$ & $-56.01(6)$ \\
\hline$S(1)-R u(1)-S(2)-O(2)$ & $-145.29(6)$ \\
\hline $\mathrm{S}(3)-\mathrm{Ru}(1)-\mathrm{S}(2)-\mathrm{O}(2)$ & $35.82(6)$ \\
\hline $\mathrm{N}(1)-\mathrm{Ru}(1)-\mathrm{S}(2)-\mathrm{O}(1)$ & $-96.69(6)$ \\
\hline $\mathrm{N}(2)-\mathrm{Ru}(1)-\mathrm{S}(2)-\mathrm{O}(1)$ & $-96.24(16)$ \\
\hline $\mathrm{P}(1)-\mathrm{Ru}(1)-\mathrm{S}(2)-\mathrm{O}(1)$ & $76.98(6)$ \\
\hline$S(1)-R u(1)-S(2)-O(1)$ & $-12.31(6)$ \\
\hline$S(3)-R u(1)-S(2)-O(1)$ & $168.81(5)$ \\
\hline $\mathrm{N}(1)-\mathrm{Ru}(1)-\mathrm{S}(2)-\mathrm{C}(8)$ & $15.28(6)$ \\
\hline$N(2)-R u(1)-S(2)-C(8)$ & $15.73(16)$ \\
\hline$P(1)-R u(1)-S(2)-C(8)$ & $-171.06(5)$ \\
\hline$S(1)-R u(1)-S(2)-C(8)$ & $99.66(5)$ \\
\hline$S(3)-R u(1)-S(2)-C(8)$ & $-79.23(5)$ \\
\hline $\mathrm{N}(1)-\mathrm{Ru}(1)-\mathrm{S}(3)-\mathrm{O}(3)$ & $-16.46(8)$ \\
\hline $\mathrm{N}(2)-\mathrm{Ru}(1)-\mathrm{S}(3)-\mathrm{O}(3)$ & $-97.18(8)$ \\
\hline $\mathrm{S}(2)-\mathrm{Ru}(1)-\mathrm{S}(3)-\mathrm{O}(3)$ & $68.26(7)$ \\
\hline $\mathrm{P}(1)-\mathrm{Ru}(1)-\mathrm{S}(3)-\mathrm{O}(3)$ & $159.02(7)$ \\
\hline$S(1)-R u(1)-S(3)-O(3)$ & $-105.25(11)$ \\
\hline $\mathrm{N}(1)-\mathrm{Ru}(1)-\mathrm{S}(3)-\mathrm{C}(10)$ & $96.41(7)$ \\
\hline $\mathrm{N}(2)-\mathrm{Ru}(1)-\mathrm{S}(3)-\mathrm{C}(10)$ & $15.69(7)$ \\
\hline$S(2)-R u(1)-S(3)-C(10)$ & $-178.87(6)$ \\
\hline$P(1)-R u(1)-S(3)-C(10)$ & $-88.11(6)$ \\
\hline$S(1)-R u(1)-S(3)-C(10)$ & $7.62(11)$ \\
\hline $\mathrm{N}(4)-\mathrm{Ru}(2)-\mathrm{S}(4)-\mathrm{C}(32)$ & $-101.09(7)$ \\
\hline $\mathrm{N}(3)-\mathrm{Ru}(2)-\mathrm{S}(4)-\mathrm{C}(32)$ & $-19.45(7)$ \\
\hline$S(5)-R u(2)-S(4)-C(32)$ & $64.47(6)$ \\
\hline $\mathrm{P}(2)-\mathrm{Ru}(2)-\mathrm{S}(4)-\mathrm{C}(32)$ & $155.46(6)$ \\
\hline$S(6)-R u(2)-S(4)-C(32)$ & $-107.97(10)$ \\
\hline $\mathrm{N}(4)-\mathrm{Ru}(2)-\mathrm{S}(4)-\mathrm{C}(31)$ & $1.95(7)$ \\
\hline $\mathrm{N}(3)-\mathrm{Ru}(2)-\mathrm{S}(4)-\mathrm{C}(31)$ & $83.60(7)$ \\
\hline$S(5)-R u(2)-S(4)-C(31)$ & $167.52(6)$ \\
\hline$P(2)-R u(2)-S(4)-C(31)$ & $-101.49(6)$ \\
\hline$S(6)-R u(2)-S(4)-C(31)$ & $-4.92(11)$ \\
\hline $\mathrm{N}(4)-\mathrm{Ru}(2)-\mathrm{S}(5)-\mathrm{O}(5)$ & $-135.68(15)$ \\
\hline $\mathrm{N}(3)-\mathrm{Ru}(2)-\mathrm{S}(5)-\mathrm{O}(5)$ & $-132.39(7)$ \\
\hline $\mathrm{P}(2)-\mathrm{Ru}(2)-\mathrm{S}(5)-\mathrm{O}(5)$ & $53.72(6)$ \\
\hline $\mathrm{S}(4)-\mathrm{Ru}(2)-\mathrm{S}(5)-\mathrm{O}(5)$ & $143.01(6)$ \\
\hline$S(6)-R u(2)-S(5)-O(5)$ & $-38.25(6)$ \\
\hline $\mathrm{N}(4)-\mathrm{Ru}(2)-\mathrm{S}(5)-\mathrm{O}(4)$ & $90.86(15)$ \\
\hline $\mathrm{N}(3)-\mathrm{Ru}(2)-\mathrm{S}(5)-\mathrm{O}(4)$ & $94.15(6)$ \\
\hline $\mathrm{P}(2)-\mathrm{Ru}(2)-\mathrm{S}(5)-\mathrm{O}(4)$ & $-79.74(5)$ \\
\hline$S(4)-R u(2)-S(5)-O(4)$ & $9.55(5)$ \\
\hline
\end{tabular}




\begin{tabular}{|c|c|}
\hline $\mathrm{S}(6)-\mathrm{Ru}(2)-\mathrm{S}(5)-\mathrm{O}(4)$ & $-171.71(5)$ \\
\hline$N(4)-R u(2)-S(5)-C(35)$ & $-21.11(16)$ \\
\hline $\mathrm{N}(3)-\mathrm{Ru}(2)-\mathrm{S}(5)-\mathrm{C}(35)$ & $-17.83(7)$ \\
\hline$P(2)-R u(2)-S(5)-C(35)$ & $168.29(6)$ \\
\hline $\mathrm{S}(4)-\mathrm{Ru}(2)-\mathrm{S}(5)-\mathrm{C}(35)$ & $-102.42(6)$ \\
\hline$S(6)-R u(2)-S(5)-C(35)$ & $76.32(6)$ \\
\hline$N(4)-R u(2)-S(6)-O(6)$ & $97.13(8)$ \\
\hline $\mathrm{N}(3)-\mathrm{Ru}(2)-\mathrm{S}(6)-\mathrm{O}(6)$ & $16.32(8)$ \\
\hline $\mathrm{S}(5)-\mathrm{Ru}(2)-\mathrm{S}(6)-\mathrm{O}(6)$ & $-68.39(7)$ \\
\hline $\mathrm{P}(2)-\mathrm{Ru}(2)-\mathrm{S}(6)-\mathrm{O}(6)$ & $-159.64(7)$ \\
\hline $\mathrm{S}(4)-\mathrm{Ru}(2)-\mathrm{S}(6)-\mathrm{O}(6)$ & $104.00(11)$ \\
\hline$N(4)-R u(2)-S(6)-C(37)$ & $-15.56(7)$ \\
\hline$N(3)-R u(2)-S(6)-C(37)$ & $-96.37(7)$ \\
\hline$S(5)-R u(2)-S(6)-C(37)$ & $178.92(6)$ \\
\hline$P(2)-R u(2)-S(6)-C(37)$ & $87.67(6)$ \\
\hline$S(4)-R u(2)-S(6)-C(37)$ & $-8.69(11)$ \\
\hline$N(1)-R u(1)-P(1)-C(27)$ & 13.1(3) \\
\hline $\mathrm{N}(2)-\mathrm{Ru}(1)-\mathrm{P}(1)-\mathrm{C}(27)$ & $140.85(7)$ \\
\hline$S(2)-R u(1)-P(1)-C(27)$ & $-37.40(6)$ \\
\hline$S(1)-R u(1)-P(1)-C(27)$ & $56.47(6)$ \\
\hline$S(3)-R u(1)-P(1)-C(27)$ & $-133.36(6)$ \\
\hline $\mathrm{N}(1)-\mathrm{Ru}(1)-\mathrm{P}(1)-\mathrm{C}(15)$ & $-101.2(3)$ \\
\hline$N(2)-R u(1)-P(1)-C(15)$ & $26.46(7)$ \\
\hline$S(2)-R u(1)-P(1)-C(15)$ & $-151.78(6)$ \\
\hline$S(1)-R u(1)-P(1)-C(15)$ & $-57.92(6)$ \\
\hline$S(3)-R u(1)-P(1)-C(15)$ & $112.26(6)$ \\
\hline $\mathrm{N}(1)-\mathrm{Ru}(1)-\mathrm{P}(1)-\mathrm{C}(21)$ & $129.9(3)$ \\
\hline$N(2)-R u(1)-P(1)-C(21)$ & $-102.42(8)$ \\
\hline$S(2)-R u(1)-P(1)-C(21)$ & $79.34(7)$ \\
\hline $\mathrm{S}(1)-\mathrm{Ru}(1)-\mathrm{P}(1)-\mathrm{C}(21)$ & $173.20(7)$ \\
\hline$S(3)-R u(1)-P(1)-C(21)$ & $-16.62(7)$ \\
\hline$N(4)-R u(2)-P(2)-C(54)$ & $-142.21(7)$ \\
\hline$N(3)-R u(2)-P(2)-C(54)$ & $-16.4(3)$ \\
\hline $\mathrm{S}(5)-\mathrm{Ru}(2)-\mathrm{P}(2)-\mathrm{C}(54)$ & $35.37(6)$ \\
\hline $\mathrm{S}(4)-\mathrm{Ru}(2)-\mathrm{P}(2)-\mathrm{C}(54)$ & $-57.21(6)$ \\
\hline $\mathrm{S}(6)-\mathrm{Ru}(2)-\mathrm{P}(2)-\mathrm{C}(54)$ & $132.27(6)$ \\
\hline $\mathrm{N}(4)-\mathrm{Ru}(2)-\mathrm{P}(2)-\mathrm{C}(48)$ & $-26.68(7)$ \\
\hline $\mathrm{N}(3)-\mathrm{Ru}(2)-\mathrm{P}(2)-\mathrm{C}(48)$ & $99.2(3)$ \\
\hline$S(5)-R u(2)-P(2)-C(48)$ & $150.91(6)$ \\
\hline$S(4)-R u(2)-P(2)-C(48)$ & $58.32(6)$ \\
\hline$S(6)-R u(2)-P(2)-C(48)$ & $-112.20(6)$ \\
\hline $\mathrm{N}(4)-\mathrm{Ru}(2)-\mathrm{P}(2)-\mathrm{C}(42)$ & $101.24(8)$ \\
\hline $\mathrm{N}(3)-\mathrm{Ru}(2)-\mathrm{P}(2)-\mathrm{C}(42)$ & $-132.9(3)$ \\
\hline$S(5)-R u(2)-P(2)-C(42)$ & $-81.17(7)$ \\
\hline$S(4)-R u(2)-P(2)-C(42)$ & $-173.76(7)$ \\
\hline$S(6)-R u(2)-P(2)-C(42)$ & $15.73(7)$ \\
\hline $\mathrm{N}(2)-\mathrm{Ru}(1)-\mathrm{N}(1)-\mathrm{C}(1)$ & $31.39(10)$ \\
\hline$S(2)-R u(1)-N(1)-C(1)$ & $-148.72(10)$ \\
\hline$P(1)-R u(1)-N(1)-C(1)$ & $160.4(2)$ \\
\hline$S(1)-R u(1)-N(1)-C(1)$ & $116.85(10)$ \\
\hline $\mathrm{S}(3)-\mathrm{Ru}(1)-\mathrm{N}(1)-\mathrm{C}(1)$ & $-53.24(10)$ \\
\hline $\mathrm{N}(2)-\mathrm{Ru}(1)-\mathrm{N}(1)-\mathrm{C}(7)$ & $145.24(10)$ \\
\hline$S(2)-R u(1)-N(1)-C(7)$ & $-34.87(9)$ \\
\hline $\mathrm{P}(1)-\mathrm{Ru}(1)-\mathrm{N}(1)-\mathrm{C}(7)$ & $-85.8(3)$ \\
\hline $\mathrm{S}(1)-\mathrm{Ru}(1)-\mathrm{N}(1)-\mathrm{C}(7)$ & $-129.30(10)$ \\
\hline$S(3)-R u(1)-N(1)-C(7)$ & $60.61(10)$ \\
\hline
\end{tabular}




\begin{tabular}{|c|c|}
\hline$N(2)-R u(1)-N(1)-C(6)$ & $-87.94(11)$ \\
\hline$S(2)-R u(1)-N(1)-C(6)$ & $91.95(11)$ \\
\hline $\mathrm{P}(1)-\mathrm{Ru}(1)-\mathrm{N}(1)-\mathrm{C}(6)$ & $41.1(3)$ \\
\hline$S(1)-R u(1)-N(1)-C(6)$ & $-2.48(10)$ \\
\hline$S(3)-R u(1)-N(1)-C(6)$ & $-172.57(10)$ \\
\hline $\mathrm{N}(1)-\mathrm{Ru}(1)-\mathrm{N}(2)-\mathrm{C}(3)$ & $110.80(11)$ \\
\hline$S(2)-R u(1)-N(2)-C(3)$ & $110.35(15)$ \\
\hline $\mathrm{P}(1)-\mathrm{Ru}(1)-\mathrm{N}(2)-\mathrm{C}(3)$ & $-62.66(10)$ \\
\hline $\mathrm{S}(1)-\mathrm{Ru}(1)-\mathrm{N}(2)-\mathrm{C}(3)$ & $25.26(10)$ \\
\hline $\mathrm{S}(3)-\mathrm{Ru}(1)-\mathrm{N}(2)-\mathrm{C}(3)$ & $-153.36(10)$ \\
\hline $\mathrm{N}(1)-\mathrm{Ru}(1)-\mathrm{N}(2)-\mathrm{C}(9)$ & $-129.81(10)$ \\
\hline $\mathrm{S}(2)-\mathrm{Ru}(1)-\mathrm{N}(2)-\mathrm{C}(9)$ & $-130.26(13)$ \\
\hline $\mathrm{P}(1)-\mathrm{Ru}(1)-\mathrm{N}(2)-\mathrm{C}(9)$ & $56.73(10)$ \\
\hline $\mathrm{S}(1)-\mathrm{Ru}(1)-\mathrm{N}(2)-\mathrm{C}(9)$ & $144.64(10)$ \\
\hline $\mathrm{S}(3)-\mathrm{Ru}(1)-\mathrm{N}(2)-\mathrm{C}(9)$ & $-33.97(9)$ \\
\hline $\mathrm{N}(1)-\mathrm{Ru}(1)-\mathrm{N}(2)-\mathrm{C}(2)$ & $-8.03(10)$ \\
\hline $\mathrm{S}(2)-\mathrm{Ru}(1)-\mathrm{N}(2)-\mathrm{C}(2)$ & $-8.5(2)$ \\
\hline $\mathrm{P}(1)-\mathrm{Ru}(1)-\mathrm{N}(2)-\mathrm{C}(2)$ & $178.51(9)$ \\
\hline $\mathrm{S}(1)-\mathrm{Ru}(1)-\mathrm{N}(2)-\mathrm{C}(2)$ & $-93.58(10)$ \\
\hline $\mathrm{S}(3)-\mathrm{Ru}(1)-\mathrm{N}(2)-\mathrm{C}(2)$ & $87.81(10)$ \\
\hline $\mathrm{N}(4)-\mathrm{Ru}(2)-\mathrm{N}(3)-\mathrm{C}(34)$ & $-144.38(10)$ \\
\hline $\mathrm{S}(5)-\mathrm{Ru}(2)-\mathrm{N}(3)-\mathrm{C}(34)$ & $36.46(9)$ \\
\hline $\mathrm{P}(2)-\mathrm{Ru}(2)-\mathrm{N}(3)-\mathrm{C}(34)$ & $88.5(3)$ \\
\hline $\mathrm{S}(4)-\mathrm{Ru}(2)-\mathrm{N}(3)-\mathrm{C}(34)$ & $129.60(10)$ \\
\hline$S(6)-R u(2)-N(3)-C(34)$ & $-59.97(10)$ \\
\hline $\mathrm{N}(4)-\mathrm{Ru}(2)-\mathrm{N}(3)-\mathrm{C}(28)$ & $-30.16(10)$ \\
\hline $\mathrm{S}(5)-\mathrm{Ru}(2)-\mathrm{N}(3)-\mathrm{C}(28)$ & $150.68(9)$ \\
\hline $\mathrm{P}(2)-\mathrm{Ru}(2)-\mathrm{N}(3)-\mathrm{C}(28)$ & $-157.2(2)$ \\
\hline$S(4)-R u(2)-N(3)-C(28)$ & $-116.18(9)$ \\
\hline$S(6)-R u(2)-N(3)-C(28)$ & $54.25(9)$ \\
\hline $\mathrm{N}(4)-\mathrm{Ru}(2)-\mathrm{N}(3)-\mathrm{C}(33)$ & $88.48(11)$ \\
\hline$S(5)-R u(2)-N(3)-C(33)$ & $-90.68(11)$ \\
\hline$P(2)-R u(2)-N(3)-C(33)$ & $-38.6(3)$ \\
\hline $\mathrm{S}(4)-\mathrm{Ru}(2)-\mathrm{N}(3)-\mathrm{C}(33)$ & $2.46(11)$ \\
\hline$S(6)-R u(2)-N(3)-C(33)$ & $172.89(11)$ \\
\hline $\mathrm{N}(3)-\mathrm{Ru}(2)-\mathrm{N}(4)-\mathrm{C}(36)$ & $129.85(10)$ \\
\hline $\mathrm{S}(5)-\mathrm{Ru}(2)-\mathrm{N}(4)-\mathrm{C}(36)$ & $133.16(13)$ \\
\hline$P(2)-R u(2)-N(4)-C(36)$ & $-56.50(10)$ \\
\hline $\mathrm{S}(4)-\mathrm{Ru}(2)-\mathrm{N}(4)-\mathrm{C}(36)$ & $-144.61(10)$ \\
\hline$S(6)-R u(2)-N(4)-C(36)$ & $34.25(9)$ \\
\hline $\mathrm{N}(3)-\mathrm{Ru}(2)-\mathrm{N}(4)-\mathrm{C}(30)$ & $-111.20(11)$ \\
\hline $\mathrm{S}(5)-\mathrm{Ru}(2)-\mathrm{N}(4)-\mathrm{C}(30)$ & $-107.90(15)$ \\
\hline$P(2)-R u(2)-N(4)-C(30)$ & $62.44(10)$ \\
\hline$S(4)-R u(2)-N(4)-C(30)$ & $-25.66(10)$ \\
\hline$S(6)-R u(2)-N(4)-C(30)$ & $153.19(10)$ \\
\hline$N(3)-R u(2)-N(4)-C(29)$ & $7.37(10)$ \\
\hline$S(5)-R u(2)-N(4)-C(29)$ & $10.7(2)$ \\
\hline $\mathrm{P}(2)-\mathrm{Ru}(2)-\mathrm{N}(4)-\mathrm{C}(29)$ & $-178.99(9)$ \\
\hline $\mathrm{S}(4)-\mathrm{Ru}(2)-\mathrm{N}(4)-\mathrm{C}(29)$ & $92.91(9)$ \\
\hline$S(6)-R u(2)-N(4)-C(29)$ & $-88.23(9)$ \\
\hline $\mathrm{C}(7)-\mathrm{N}(1)-\mathrm{C}(1)-\mathrm{C}(2)$ & $-166.28(13)$ \\
\hline $\mathrm{C}(6)-\mathrm{N}(1)-\mathrm{C}(1)-\mathrm{C}(2)$ & $72.46(16)$ \\
\hline $\mathrm{Ru}(1)-\mathrm{N}(1)-\mathrm{C}(1)-\mathrm{C}(2)$ & $-50.74(14)$ \\
\hline $\mathrm{C}(3)-\mathrm{N}(2)-\mathrm{C}(2)-\mathrm{C}(1)$ & $-138.76(14)$ \\
\hline $\mathrm{C}(9)-\mathrm{N}(2)-\mathrm{C}(2)-\mathrm{C}(1)$ & $103.16(16)$ \\
\hline $\mathrm{Ru}(1)-\mathrm{N}(2)-\mathrm{C}(2)-\mathrm{C}(1)$ & $-17.57(16)$ \\
\hline
\end{tabular}




\begin{tabular}{|c|c|}
\hline$N(1)-C(1)-C(2)-N(2)$ & $46.96(18)$ \\
\hline $\mathrm{C}(9)-\mathrm{N}(2)-\mathrm{C}(3)-\mathrm{C}(4)$ & $-166.60(13)$ \\
\hline $\mathrm{C}(2)-\mathrm{N}(2)-\mathrm{C}(3)-\mathrm{C}(4)$ & $73.40(17)$ \\
\hline $\mathrm{Ru}(1)-\mathrm{N}(2)-\mathrm{C}(3)-\mathrm{C}(4)$ & $-46.47(16)$ \\
\hline$N(2)-C(3)-C(4)-S(1)$ & $45.15(18)$ \\
\hline $\mathrm{C}(5)-\mathrm{S}(1)-\mathrm{C}(4)-\mathrm{C}(3)$ & $-122.59(13)$ \\
\hline$R u(1)-S(1)-C(4)-C(3)$ & $-21.40(13)$ \\
\hline$C(4)-S(1)-C(5)-C(6)$ & $64.50(14)$ \\
\hline $\operatorname{Ru}(1)-\mathrm{S}(1)-\mathrm{C}(5)-\mathrm{C}(6)$ & $-38.13(13)$ \\
\hline $\mathrm{C}(1)-\mathrm{N}(1)-\mathrm{C}(6)-\mathrm{C}(5)$ & $-138.55(15)$ \\
\hline $\mathrm{C}(7)-\mathrm{N}(1)-\mathrm{C}(6)-\mathrm{C}(5)$ & $103.48(16)$ \\
\hline $\mathrm{Ru}(1)-\mathrm{N}(1)-\mathrm{C}(6)-\mathrm{C}(5)$ & $-21.84(18)$ \\
\hline$S(1)-C(5)-C(6)-N(1)$ & $41.89(18)$ \\
\hline $\mathrm{C}(1)-\mathrm{N}(1)-\mathrm{C}(7)-\mathrm{C}(8)$ & $161.69(13)$ \\
\hline $\mathrm{C}(6)-\mathrm{N}(1)-\mathrm{C}(7)-\mathrm{C}(8)$ & $-79.00(17)$ \\
\hline $\mathrm{Ru}(1)-\mathrm{N}(1)-\mathrm{C}(7)-\mathrm{C}(8)$ & $49.40(15)$ \\
\hline$N(1)-C(7)-C(8)-C(11)$ & $-151.28(14)$ \\
\hline $\mathrm{N}(1)-\mathrm{C}(7)-\mathrm{C}(8)-\mathrm{C}(12)$ & $88.74(17)$ \\
\hline$N(1)-C(7)-C(8)-S(2)$ & $-34.44(17)$ \\
\hline $\mathrm{O}(2)-\mathrm{S}(2)-\mathrm{C}(8)-\mathrm{C}(11)$ & $-4.35(13)$ \\
\hline $\mathrm{O}(1)-\mathrm{S}(2)-\mathrm{C}(8)-\mathrm{C}(11)$ & $-121.06(12)$ \\
\hline$R u(1)-S(2)-C(8)-C(11)$ & $120.07(11)$ \\
\hline $\mathrm{O}(2)-\mathrm{S}(2)-\mathrm{C}(8)-\mathrm{C}(7)$ & $-119.95(11)$ \\
\hline $\mathrm{O}(1)-\mathrm{S}(2)-\mathrm{C}(8)-\mathrm{C}(7)$ & $123.34(11)$ \\
\hline $\mathrm{Ru}(1)-\mathrm{S}(2)-\mathrm{C}(8)-\mathrm{C}(7)$ & $4.48(12)$ \\
\hline $\mathrm{O}(2)-\mathrm{S}(2)-\mathrm{C}(8)-\mathrm{C}(12)$ & $115.01(12)$ \\
\hline $\mathrm{O}(1)-\mathrm{S}(2)-\mathrm{C}(8)-\mathrm{C}(12)$ & $-1.70(14)$ \\
\hline $\mathrm{Ru}(1)-\mathrm{S}(2)-\mathrm{C}(8)-\mathrm{C}(12)$ & $-120.56(11)$ \\
\hline $\mathrm{C}(3)-\mathrm{N}(2)-\mathrm{C}(9)-\mathrm{C}(10)$ & $167.60(14)$ \\
\hline $\mathrm{C}(2)-\mathrm{N}(2)-\mathrm{C}(9)-\mathrm{C}(10)$ & $-74.59(17)$ \\
\hline $\mathrm{Ru}(1)-\mathrm{N}(2)-\mathrm{C}(9)-\mathrm{C}(10)$ & $46.15(16)$ \\
\hline $\mathrm{N}(2)-\mathrm{C}(9)-\mathrm{C}(10)-\mathrm{C}(13)$ & $93.74(17)$ \\
\hline $\mathrm{N}(2)-\mathrm{C}(9)-\mathrm{C}(10)-\mathrm{C}(14)$ & $-146.22(14)$ \\
\hline $\mathrm{N}(2)-\mathrm{C}(9)-\mathrm{C}(10)-\mathrm{S}(3)$ & $-31.48(18)$ \\
\hline $\mathrm{O}(3)-\mathrm{S}(3)-\mathrm{C}(10)-\mathrm{C}(13)$ & $-3.30(14)$ \\
\hline $\operatorname{Ru}(1)-S(3)-C(10)-C(13)$ & $-124.40(12)$ \\
\hline $\mathrm{O}(3)-\mathrm{S}(3)-\mathrm{C}(10)-\mathrm{C}(9)$ & $123.65(12)$ \\
\hline $\mathrm{Ru}(1)-\mathrm{S}(3)-\mathrm{C}(10)-\mathrm{C}(9)$ & $2.55(12)$ \\
\hline $\mathrm{O}(3)-\mathrm{S}(3)-\mathrm{C}(10)-\mathrm{C}(14)$ & $-119.76(12)$ \\
\hline$R u(1)-S(3)-C(10)-C(14)$ & $119.14(10)$ \\
\hline$C(27)-P(1)-C(15)-C(16)$ & $147.15(14)$ \\
\hline$C(21)-P(1)-C(15)-C(16)$ & $46.99(16)$ \\
\hline $\mathrm{Ru}(1)-\mathrm{P}(1)-\mathrm{C}(15)-\mathrm{C}(16)$ & $-91.71(14)$ \\
\hline $\mathrm{C}(27)-\mathrm{P}(1)-\mathrm{C}(15)-\mathrm{C}(20)$ & $-40.35(15)$ \\
\hline $\mathrm{C}(21)-\mathrm{P}(1)-\mathrm{C}(15)-\mathrm{C}(20)$ & $-140.50(13)$ \\
\hline$R u(1)-P(1)-C(15)-C(20)$ & $80.79(13)$ \\
\hline $\mathrm{C}(20)-\mathrm{C}(15)-\mathrm{C}(16)-\mathrm{C}(17)$ & $-0.7(2)$ \\
\hline$P(1)-C(15)-C(16)-C(17)$ & $171.87(14)$ \\
\hline$C(15)-C(16)-C(17)-C(18)$ & $0.2(3)$ \\
\hline$C(16)-C(17)-C(18)-C(19)$ & $0.5(3)$ \\
\hline$C(17)-C(18)-C(19)-C(20)$ & $-0.7(3)$ \\
\hline$C(18)-C(19)-C(20)-C(15)$ & $0.2(3)$ \\
\hline$C(16)-C(15)-C(20)-C(19)$ & $0.6(2)$ \\
\hline$P(1)-C(15)-C(20)-C(19)$ & $-172.29(13)$ \\
\hline $\mathrm{C}(27)-\mathrm{P}(1)-\mathrm{C}(21)-\mathrm{C}(22)$ & $85.85(14)$ \\
\hline$C(15)-P(1)-C(21)-C(22)$ & $-170.89(13)$ \\
\hline
\end{tabular}




\begin{tabular}{|c|c|}
\hline$R u(1)-P(1)-C(21)-C(22)$ & $-38.06(16)$ \\
\hline$C(27)-P(1)-C(21)-C(26)$ & $-85.68(16)$ \\
\hline$C(15)-P(1)-C(21)-C(26)$ & $17.58(17)$ \\
\hline$R u(1)-P(1)-C(21)-C(26)$ & $150.41(13)$ \\
\hline$C(26)-C(21)-C(22)-C(23)$ & $-0.1(3)$ \\
\hline $\mathrm{P}(1)-\mathrm{C}(21)-\mathrm{C}(22)-\mathrm{C}(23)$ & $-172.04(14)$ \\
\hline $\mathrm{C}(21)-\mathrm{C}(22)-\mathrm{C}(23)-\mathrm{C}(24)$ & $-0.2(3)$ \\
\hline$C(22)-C(23)-C(24)-C(25)$ & $-0.1(3)$ \\
\hline $\mathrm{C}(23)-\mathrm{C}(24)-\mathrm{C}(25)-\mathrm{C}(26)$ & $0.7(3)$ \\
\hline$C(24)-C(25)-C(26)-C(21)$ & $-1.1(3)$ \\
\hline $\mathrm{C}(22)-\mathrm{C}(21)-\mathrm{C}(26)-\mathrm{C}(25)$ & $0.7(3)$ \\
\hline $\mathrm{P}(1)-\mathrm{C}(21)-\mathrm{C}(26)-\mathrm{C}(25)$ & $172.20(16)$ \\
\hline $\mathrm{C}(34)-\mathrm{N}(3)-\mathrm{C}(28)-\mathrm{C}(29)$ & $164.86(13)$ \\
\hline $\mathrm{C}(33)-\mathrm{N}(3)-\mathrm{C}(28)-\mathrm{C}(29)$ & $-73.70(16)$ \\
\hline $\mathrm{Ru}(2)-\mathrm{N}(3)-\mathrm{C}(28)-\mathrm{C}(29)$ & $49.24(14)$ \\
\hline $\mathrm{N}(3)-\mathrm{C}(28)-\mathrm{C}(29)-\mathrm{N}(4)$ & $-45.93(18)$ \\
\hline $\mathrm{C}(36)-\mathrm{N}(4)-\mathrm{C}(29)-\mathrm{C}(28)$ & $-104.06(15)$ \\
\hline $\mathrm{C}(30)-\mathrm{N}(4)-\mathrm{C}(29)-\mathrm{C}(28)$ & $138.20(14)$ \\
\hline $\mathrm{Ru}(2)-\mathrm{N}(4)-\mathrm{C}(29)-\mathrm{C}(28)$ & $17.59(16)$ \\
\hline $\mathrm{C}(36)-\mathrm{N}(4)-\mathrm{C}(30)-\mathrm{C}(31)$ & $167.28(13)$ \\
\hline $\mathrm{C}(29)-\mathrm{N}(4)-\mathrm{C}(30)-\mathrm{C}(31)$ & $-72.82(16)$ \\
\hline $\mathrm{Ru}(2)-\mathrm{N}(4)-\mathrm{C}(30)-\mathrm{C}(31)$ & $47.16(15)$ \\
\hline $\mathrm{N}(4)-\mathrm{C}(30)-\mathrm{C}(31)-\mathrm{S}(4)$ & $-45.90(17)$ \\
\hline$C(32)-S(4)-C(31)-C(30)$ & $123.22(12)$ \\
\hline $\mathrm{Ru}(2)-\mathrm{S}(4)-\mathrm{C}(31)-\mathrm{C}(30)$ & $21.63(12)$ \\
\hline $\mathrm{C}(31)-\mathrm{S}(4)-\mathrm{C}(32)-\mathrm{C}(33)$ & $-65.76(14)$ \\
\hline $\mathrm{Ru}(2)-\mathrm{S}(4)-\mathrm{C}(32)-\mathrm{C}(33)$ & $36.49(13)$ \\
\hline $\mathrm{C}(34)-\mathrm{N}(3)-\mathrm{C}(33)-\mathrm{C}(32)$ & $-104.52(16)$ \\
\hline $\mathrm{C}(28)-\mathrm{N}(3)-\mathrm{C}(33)-\mathrm{C}(32)$ & $137.42(15)$ \\
\hline $\mathrm{Ru}(2)-\mathrm{N}(3)-\mathrm{C}(33)-\mathrm{C}(32)$ & $20.87(18)$ \\
\hline $\mathrm{S}(4)-\mathrm{C}(32)-\mathrm{C}(33)-\mathrm{N}(3)$ & $-40.01(19)$ \\
\hline $\mathrm{C}(28)-\mathrm{N}(3)-\mathrm{C}(34)-\mathrm{C}(35)$ & $-161.35(14)$ \\
\hline $\mathrm{C}(33)-\mathrm{N}(3)-\mathrm{C}(34)-\mathrm{C}(35)$ & $79.82(17)$ \\
\hline $\mathrm{Ru}(2)-\mathrm{N}(3)-\mathrm{C}(34)-\mathrm{C}(35)$ & $-48.67(16)$ \\
\hline $\mathrm{N}(3)-\mathrm{C}(34)-\mathrm{C}(35)-\mathrm{C}(39)$ & $-91.32(17)$ \\
\hline$N(3)-C(34)-C(35)-C(38)$ & $147.83(14)$ \\
\hline$N(3)-C(34)-C(35)-S(5)$ & $31.62(17)$ \\
\hline $\mathrm{O}(5)-\mathrm{S}(5)-\mathrm{C}(35)-\mathrm{C}(39)$ & $-110.90(13)$ \\
\hline $\mathrm{O}(4)-\mathrm{S}(5)-\mathrm{C}(35)-\mathrm{C}(39)$ & $5.24(14)$ \\
\hline $\mathrm{Ru}(2)-\mathrm{S}(5)-\mathrm{C}(35)-\mathrm{C}(39)$ & $123.92(11)$ \\
\hline $\mathrm{O}(5)-\mathrm{S}(5)-\mathrm{C}(35)-\mathrm{C}(38)$ & $8.26(13)$ \\
\hline $\mathrm{O}(4)-\mathrm{S}(5)-\mathrm{C}(35)-\mathrm{C}(38)$ & $124.40(12)$ \\
\hline $\operatorname{Ru}(2)-S(5)-C(35)-C(38)$ & $-116.92(11)$ \\
\hline $\mathrm{O}(5)-\mathrm{S}(5)-\mathrm{C}(35)-\mathrm{C}(34)$ & $124.22(12)$ \\
\hline $\mathrm{O}(4)-\mathrm{S}(5)-\mathrm{C}(35)-\mathrm{C}(34)$ & $-119.63(12)$ \\
\hline $\mathrm{Ru}(2)-\mathrm{S}(5)-\mathrm{C}(35)-\mathrm{C}(34)$ & $-0.95(12)$ \\
\hline $\mathrm{C}(30)-\mathrm{N}(4)-\mathrm{C}(36)-\mathrm{C}(37)$ & $-167.53(13)$ \\
\hline$C(29)-N(4)-C(36)-C(37)$ & $74.93(17)$ \\
\hline $\mathrm{Ru}(2)-\mathrm{N}(4)-\mathrm{C}(36)-\mathrm{C}(37)$ & $-46.75(16)$ \\
\hline $\mathrm{N}(4)-\mathrm{C}(36)-\mathrm{C}(37)-\mathrm{C}(41)$ & $-92.18(18)$ \\
\hline$N(4)-C(36)-C(37)-C(40)$ & $148.51(15)$ \\
\hline $\mathrm{N}(4)-\mathrm{C}(36)-\mathrm{C}(37)-\mathrm{S}(6)$ & $31.90(18)$ \\
\hline$O(6)-S(6)-C(37)-C(41)$ & $1.50(14)$ \\
\hline $\operatorname{Ru}(2)-\mathrm{S}(6)-\mathrm{C}(37)-\mathrm{C}(41)$ & $123.09(11)$ \\
\hline $\mathrm{O}(6)-\mathrm{S}(6)-\mathrm{C}(37)-\mathrm{C}(40)$ & $118.61(12)$ \\
\hline $\operatorname{Ru}(2)-S(6)-C(37)-C(40)$ & $-119.79(11)$ \\
\hline
\end{tabular}


$\mathrm{O}(6)-\mathrm{S}(6)-\mathrm{C}(37)-\mathrm{C}(36)$

$\mathrm{Ru}(2)-\mathrm{S}(6)-\mathrm{C}(37)-\mathrm{C}(36)$

$\mathrm{C}(54)-\mathrm{P}(2)-\mathrm{C}(42)-\mathrm{C}(43)$

C(48)-P(2)-C(42)-C(43)

$\mathrm{Ru}(2)-\mathrm{P}(2)-\mathrm{C}(42)-\mathrm{C}(43)$

$\mathrm{C}(54)-\mathrm{P}(2)-\mathrm{C}(42)-\mathrm{C}(47)$

$\mathrm{C}(48)-\mathrm{P}(2)-\mathrm{C}(42)-\mathrm{C}(47)$

$\mathrm{Ru}(2)-\mathrm{P}(2)-\mathrm{C}(42)-\mathrm{C}(47)$

$\mathrm{C}(47)-\mathrm{C}(42)-\mathrm{C}(43)-\mathrm{C}(44)$

$\mathrm{P}(2)-\mathrm{C}(42)-\mathrm{C}(43)-\mathrm{C}(44)$

$\mathrm{C}(42)-\mathrm{C}(43)-\mathrm{C}(44)-\mathrm{C}(45)$

$\mathrm{C}(43)-\mathrm{C}(44)-\mathrm{C}(45)-\mathrm{C}(46)$

$\mathrm{C}(44)-\mathrm{C}(45)-\mathrm{C}(46)-\mathrm{C}(47)$

$\mathrm{C}(45)-\mathrm{C}(46)-\mathrm{C}(47)-\mathrm{C}(42)$

$\mathrm{C}(43)-\mathrm{C}(42)-\mathrm{C}(47)-\mathrm{C}(46)$

$\mathrm{P}(2)-\mathrm{C}(42)-\mathrm{C}(47)-\mathrm{C}(46)$

$\mathrm{C}(54)-\mathrm{P}(2)-\mathrm{C}(48)-\mathrm{C}(49)$

$\mathrm{C}(42)-\mathrm{P}(2)-\mathrm{C}(48)-\mathrm{C}(49)$

$\mathrm{Ru}(2)-\mathrm{P}(2)-\mathrm{C}(48)-\mathrm{C}(49)$

$\mathrm{C}(54)-\mathrm{P}(2)-\mathrm{C}(48)-\mathrm{C}(53)$

$-124.57(12)$

$-2.97(12)$

$91.90(16)$

$-11.91(17)$

$-144.64(13)$

$-82.47(14)$

$173.73(13)$

$41.00(16)$

$-2.5(3)$

$-176.77(15)$

2.1(3)

$0.0(3)$

$-1.6(3)$

$1.1(3)$

$0.9(3)$

175.61(14)

$-148.02(14)$

$-46.39(15)$

$90.05(14)$

$38.25(14)$

$\mathrm{C}(42)-\mathrm{P}(2)-\mathrm{C}(48)-\mathrm{C}(53)$

$139.88(13)$

$\mathrm{Ru}(2)-\mathrm{P}(2)-\mathrm{C}(48)-\mathrm{C}(53)$

$-83.68(13)$

$-0.6(2)$

$\mathrm{P}(2)-\mathrm{C}(48)-\mathrm{C}(49)-\mathrm{C}(50)$

$-174.43(13)$

$0.0(3)$

$0.6(2)$

$-0.5(2)$

$-0.2(2)$

$0.7(2)$

174.74(12)

Symmetry transformations used to generate equivalent atoms: 
Table A21. Crystal data and structure refinement for $\mathbf{1 0}$

\author{
Identification code \\ Empirical formula \\ Formula weight \\ Temperature \\ Wavelength \\ Crystal system \\ Space group \\ Unit cell dimensions \\ Volume \\ Z \\ Density (calculated) \\ Absorption coefficient \\ $\mathrm{F}(000)$ \\ Crystal size \\ Crystal color, habit \\ Theta range for data collection \\ Index ranges \\ Reflections collected \\ Independent reflections \\ Completeness to theta $=28.03^{\circ}$ \\ Absorption correction \\ Max. and min. transmission \\ Refinement method \\ Data / restraints / parameters \\ Goodness-of-fit on $\mathrm{F}^{2}$ \\ Final $R$ indices [ $\mathrm{I}>2 \operatorname{sigma}(\mathrm{I})]$ \\ $\mathrm{R}$ indices (all data) \\ Largest diff. peak and hole
}

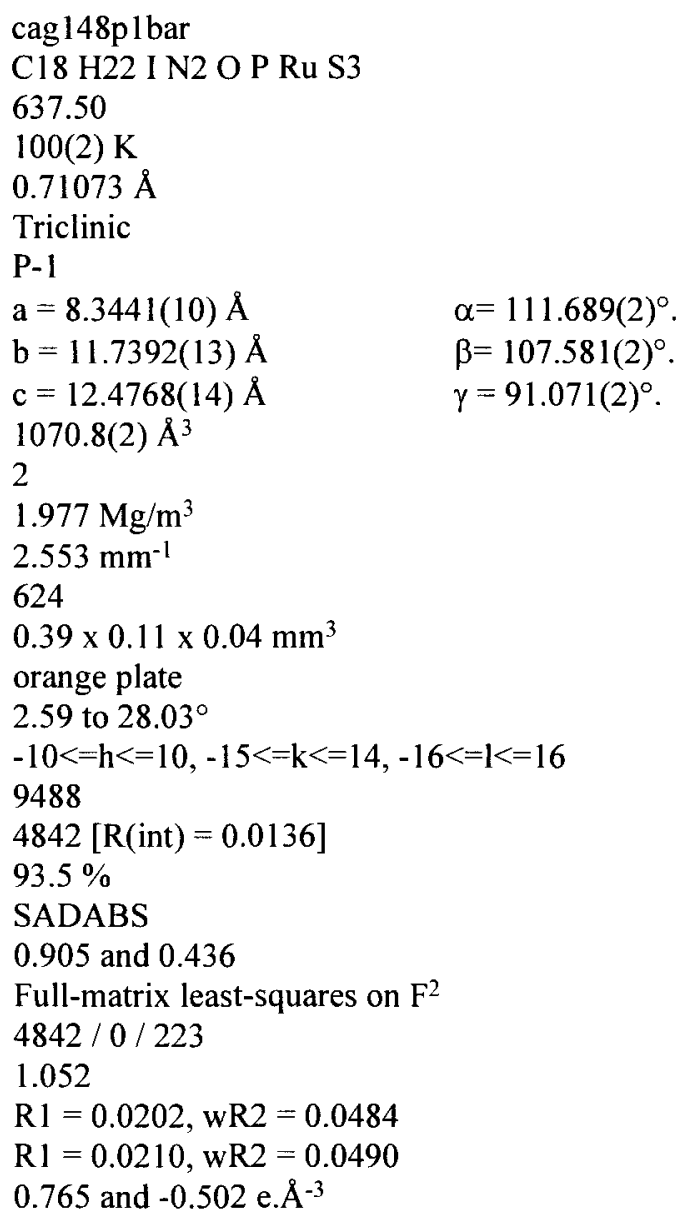


Table A22. Atomic coordinates ( $\left.\times 10^{4}\right)$ and equivalent isotropic displacement parameters $\left(\AA^{2} \times 10^{3}\right)$ for $10 \mathrm{U}(\mathrm{eq})$ is defined as one third of the trace of the orthogonalized $\mathrm{U}^{\mathrm{ij}}$ tensor.

\begin{tabular}{lrrrr}
\hline & $\mathrm{x}$ & $\mathrm{y}$ & $\mathrm{z}$ & $\mathrm{U}(\mathrm{eq})$ \\
\hline $\mathrm{Ru}(1)$ & $5448(1)$ & $244(1)$ & $6262(1)$ & $10(1)$ \\
$\mathrm{S}(1)$ & $8078(1)$ & $-598(1)$ & $6314(1)$ & $14(1)$ \\
$\mathrm{S}(2)$ & $2970(1)$ & $1013(1)$ & $6590(1)$ & $14(1)$ \\
$\mathrm{S}(3)$ & $5500(1)$ & $1633(1)$ & $5357(1)$ & $12(1)$ \\
$\mathrm{N}(1)$ & $5599(2)$ & $-359(2)$ & $7704(2)$ & $13(1)$ \\
$\mathrm{N}(2)$ & $6862(2)$ & $1914(2)$ & $7800(2)$ & $14(1)$ \\
$\mathrm{C}(1)$ & $7958(2)$ & $-1613(2)$ & $7142(2)$ & $15(1)$ \\
$\mathrm{C}(2)$ & $6300(2)$ & $-1567(2)$ & $7452(2)$ & $14(1)$ \\
$\mathrm{C}(5)$ & $3850(2)$ & $-594(2)$ & $7751(2)$ & $15(1)$ \\
$\mathrm{C}(6)$ & $2887(3)$ & $495(2)$ & $7794(2)$ & $16(1)$ \\
$\mathrm{C}(3)$ & $6655(3)$ & $621(2)$ & $8935(2)$ & $16(1)$ \\
$\mathrm{C}(4)$ & $7826(3)$ & $1516(2)$ & $8802(2)$ & $16(1)$ \\
$\mathrm{C}(7)$ & $5730(3)$ & $2833(2)$ & $8293(2)$ & $17(1)$ \\
$\mathrm{C}(8)$ & $3903(3)$ & $2636(2)$ & $7458(2)$ & $16(1)$ \\
$\mathrm{C}(9)$ & $8210(3)$ & $2492(2)$ & $7489(2)$ & $17(1)$ \\
$\mathrm{C}(10)$ & $7664(2)$ & $2535(2)$ & $6206(2)$ & $13(1)$ \\
$\mathrm{C}(11)$ & $7432(3)$ & $3852(2)$ & $6272(2)$ & $18(1)$ \\
$\mathrm{C}(12)$ & $8982(3)$ & $2048(2)$ & $5595(2)$ & $18(1)$ \\
$\mathrm{C}(13)$ & $9545(3)$ & $-1280(2)$ & $8280(2)$ & $18(1)$ \\
$\mathrm{C}(14)$ & $7913(3)$ & $-2964(2)$ & $6301(2)$ & $19(1)$ \\
$\mathrm{O}(1)$ & $6588(2)$ & $2871(1)$ & $1301(2)$ & $25(1)$ \\
$\mathrm{I}$ & $7521(1)$ & $6441(1)$ & $9581(1)$ & $20(1)$ \\
$\mathrm{C}(15)$ & $7706(3)$ & $4024(2)$ & $1975(2)$ & $25(1)$ \\
$\mathrm{C}(16)$ & $7751(3)$ & $4583(2)$ & $3283(2)$ & $32(1)$ \\
& & & & \\
\hline
\end{tabular}


Table A23. Bond lengths $[\AA]$ and angles $\left[^{\circ}\right]$ for 10

\begin{tabular}{|c|c|}
\hline $\mathrm{Ru}(1)-\mathrm{N}(1)$ & $2.1351(16)$ \\
\hline $\mathrm{Ru}(1)-\mathrm{N}(2)$ & $2.1639(16)$ \\
\hline $\mathrm{Ru}(1)-\mathrm{S}(3) \# 1$ & $2.2899(5)$ \\
\hline Ru(1)-S(3) & $2.3068(5)$ \\
\hline $\mathrm{Ru}(1)-\mathrm{S}(2)$ & $2.3589(5)$ \\
\hline $\mathrm{Ru}(1)-\mathrm{S}(1)$ & $2.4170(5)$ \\
\hline $\operatorname{Ru}(1)-\operatorname{Ru}(1) \# 1$ & $2.8349(4)$ \\
\hline$S(1)-C(1)$ & $1.862(2)$ \\
\hline$S(2)-C(8)$ & $1.821(2)$ \\
\hline$S(2)-C(6)$ & $1.834(2)$ \\
\hline$S(3)-C(10)$ & $1.857(2)$ \\
\hline$S(3)-R u(1) \# 1$ & $2.2898(5)$ \\
\hline $\mathrm{N}(1)-\mathrm{C}(5)$ & $1.503(2)$ \\
\hline $\mathrm{N}(1)-\mathrm{C}(2)$ & $1.505(2)$ \\
\hline $\mathrm{N}(1)-\mathrm{C}(3)$ & $1.516(2)$ \\
\hline $\mathrm{N}(2)-\mathrm{C}(4)$ & $1.506(2)$ \\
\hline $\mathrm{N}(2)-\mathrm{C}(9)$ & $1.518(3)$ \\
\hline $\mathrm{N}(2)-\mathrm{C}(7)$ & $1.527(3)$ \\
\hline$C(1)-C(13)$ & $1.535(3)$ \\
\hline$C(1)-C(14)$ & $1.537(3)$ \\
\hline $\mathrm{C}(1)-\mathrm{C}(2)$ & $1.542(3)$ \\
\hline $\mathrm{C}(2)-\mathrm{H}(2 \mathrm{~A})$ & 0.9900 \\
\hline $\mathrm{C}(2)-\mathrm{H}(2 \mathrm{~B})$ & 0.9900 \\
\hline$C(5)-C(6)$ & $1.515(3)$ \\
\hline $\mathrm{C}(5)-\mathrm{H}(5 \mathrm{~A})$ & 0.9900 \\
\hline $\mathrm{C}(5)-\mathrm{H}(5 \mathrm{~B})$ & 0.9900 \\
\hline $\mathrm{C}(6)-\mathrm{H}(6 \mathrm{~A})$ & 0.9900 \\
\hline $\mathrm{C}(6)-\mathrm{H}(6 \mathrm{~B})$ & 0.9900 \\
\hline$C(3)-C(4)$ & $1.508(3)$ \\
\hline $\mathrm{C}(3)-\mathrm{H}(3 \mathrm{~A})$ & 0.9900 \\
\hline$C(3)-H(3 B)$ & 0.9900 \\
\hline $\mathrm{C}(4)-\mathrm{H}(4 \mathrm{~A})$ & 0.9900 \\
\hline $\mathrm{C}(4)-\mathrm{H}(4 \mathrm{~B})$ & 0.9900 \\
\hline$C(7)-C(8)$ & $1.520(3)$ \\
\hline $\mathrm{C}(7)-\mathrm{H}(7 \mathrm{~A})$ & 0.9900 \\
\hline $\mathrm{C}(7)-\mathrm{H}(7 \mathrm{~B})$ & 0.9900 \\
\hline $\mathrm{C}(8)-\mathrm{H}(8 \mathrm{~A})$ & 0.9900 \\
\hline $\mathrm{C}(8)-\mathrm{H}(8 \mathrm{~B})$ & 0.9900 \\
\hline$C(9)-C(10)$ & $1.547(3)$ \\
\hline $\mathrm{C}(9)-\mathrm{H}(9 \mathrm{~A})$ & 0.9900 \\
\hline $\mathrm{C}(9)-\mathrm{H}(9 \mathrm{~B})$ & 0.9900 \\
\hline$C(10)-C(12)$ & $1.525(3)$ \\
\hline$C(10)-C(11)$ & $1.537(3)$ \\
\hline $\mathrm{C}(11)-\mathrm{H}(11 \mathrm{~A})$ & 0.9800 \\
\hline$C(11)-H(11 B)$ & 0.9800 \\
\hline $\mathrm{C}(11)-\mathrm{H}(11 \mathrm{C})$ & 0.9800 \\
\hline $\mathrm{C}(12)-\mathrm{H}(12 \mathrm{~A})$ & 0.9800 \\
\hline $\mathrm{C}(12)-\mathrm{H}(12 \mathrm{~B})$ & 0.9800 \\
\hline$C(12)-H(12 C)$ & 0.9800 \\
\hline $\mathrm{C}(13)-\mathrm{H}(13 \mathrm{~A})$ & 0.9800 \\
\hline $\mathrm{C}(13)-\mathrm{H}(13 \mathrm{~B})$ & 0.9800 \\
\hline $\mathrm{C}(13)-\mathrm{H}(13 \mathrm{C})$ & 0.9800 \\
\hline $\mathrm{C}(14)-\mathrm{H}(14 \mathrm{~A})$ & 0.9800 \\
\hline $\mathrm{C}(14)-\mathrm{H}(14 \mathrm{~B})$ & 0.9800 \\
\hline
\end{tabular}




\begin{tabular}{|c|c|}
\hline $\mathrm{C}(14)-\mathrm{H}(14 \mathrm{C})$ & 0.9800 \\
\hline $\mathrm{O}(1)-\mathrm{C}(15)$ & $1.431(3)$ \\
\hline $\mathrm{O}(1)-\mathrm{H}(1)$ & 0.8400 \\
\hline$C(15)-C(16)$ & $1.505(3)$ \\
\hline $\mathrm{C}(15)-\mathrm{H}(15 \mathrm{~A})$ & 0.9900 \\
\hline $\mathrm{C}(15)-\mathrm{H}(15 \mathrm{~B})$ & 0.9900 \\
\hline$C(16)-H(16 A)$ & 0.9800 \\
\hline$C(16)-H(16 B)$ & 0.9800 \\
\hline $\mathrm{C}(16)-\mathrm{H}(16 \mathrm{C})$ & 0.9800 \\
\hline$N(1)-R u(1)-N(2)$ & $81.21(6)$ \\
\hline $\mathrm{N}(1)-\mathrm{Ru}(1)-\mathrm{S}(3) \# 1$ & $98.67(5)$ \\
\hline $\mathrm{N}(2)-\mathrm{Ru}(1)-\mathrm{S}(3) \# 1$ & $168.08(5)$ \\
\hline N(1)-Ru(1)-S(3) & $157.24(5)$ \\
\hline$N(2)-R u(1)-S(3)$ & $77.46(5)$ \\
\hline$S(3) \# 1-R u(1)-S(3)$ & $103.842(18)$ \\
\hline $\mathrm{N}(1)-\mathrm{Ru}(1)-\mathrm{S}(2)$ & $85.19(4)$ \\
\hline $\mathrm{N}(2)-\mathrm{Ru}(1)-\mathrm{S}(2)$ & $86.77(5)$ \\
\hline$S(3) \# 1-R u(1)-S(2)$ & $105.113(18)$ \\
\hline$S(3)-R u(1)-S(2)$ & $85.762(17)$ \\
\hline$N(1)-R u(1)-S(1)$ & $83.30(4)$ \\
\hline $\mathrm{N}(2)-\mathrm{Ru}(1)-\mathrm{S}(1)$ & $89.19(5)$ \\
\hline$S(3) \# 1-R u(1)-S(1)$ & $78.981(17)$ \\
\hline$S(3)-R u(1)-S(1)$ & $104.111(17)$ \\
\hline $\mathrm{S}(2)-\mathrm{Ru}(1)-\mathrm{S}(1)$ & $168.268(18)$ \\
\hline $\mathrm{N}(1)-\mathrm{Ru}(1)-\mathrm{Ru}(1) \# 1$ & $150.70(4)$ \\
\hline $\mathrm{N}(2)-\mathrm{Ru}(1)-\mathrm{Ru}(1) \# 1$ & $127.86(4)$ \\
\hline$S(3) \# 1-R u(1)-R u(1) \# 1$ & $52.189(13)$ \\
\hline S(3)-Ru(1)-Ru(1)\#1 & $51.653(13)$ \\
\hline$S(2)-R u(1)-R u(1) \# 1$ & $98.653(14)$ \\
\hline$S(1)-R u(1)-R u(1) \# 1$ & $92.522(14)$ \\
\hline$C(1)-S(1)-R u(1)$ & $100.16(6)$ \\
\hline$C(8)-S(2)-C(6)$ & $102.41(10)$ \\
\hline$C(8)-S(2)-R u(1)$ & $97.28(7)$ \\
\hline$C(6)-S(2)-R u(1)$ & $99.64(6)$ \\
\hline$C(10)-S(3)-R u(1) \# 1$ & $131.26(6)$ \\
\hline$C(10)-S(3)-R u(1)$ & $102.94(6)$ \\
\hline $\operatorname{Ru}(1) \# 1-S(3)-\operatorname{Ru}(1)$ & $76.157(18)$ \\
\hline$C(5)-N(1)-C(2)$ & $107.14(14)$ \\
\hline $\mathrm{C}(5)-\mathrm{N}(1)-\mathrm{C}(3)$ & $107.45(15)$ \\
\hline $\mathrm{C}(2)-\mathrm{N}(1)-\mathrm{C}(3)$ & $111.54(15)$ \\
\hline$C(5)-N(1)-R u(1)$ & $109.83(11)$ \\
\hline$C(2)-N(1)-R u(1)$ & $109.61(12)$ \\
\hline $\mathrm{C}(3)-\mathrm{N}(1)-\mathrm{Ru}(1)$ & $111.16(12)$ \\
\hline $\mathrm{C}(4)-\mathrm{N}(2)-\mathrm{C}(9)$ & $105.44(15)$ \\
\hline $\mathrm{C}(4)-\mathrm{N}(2)-\mathrm{C}(7)$ & $107.85(15)$ \\
\hline $\mathrm{C}(9)-\mathrm{N}(2)-\mathrm{C}(7)$ & $112.80(15)$ \\
\hline $\mathrm{C}(4)-\mathrm{N}(2)-\mathrm{Ru}(1)$ & $106.49(11)$ \\
\hline $\mathrm{C}(9)-\mathrm{N}(2)-\mathrm{Ru}(1)$ & $110.37(12)$ \\
\hline$C(7)-N(2)-R u(1)$ & $113.35(12)$ \\
\hline$C(13)-C(1)-C(14)$ & $107.30(16)$ \\
\hline $\mathrm{C}(13)-\mathrm{C}(1)-\mathrm{C}(2)$ & $112.33(17)$ \\
\hline$C(14)-C(1)-C(2)$ & $106.35(16)$ \\
\hline$C(13)-C(1)-S(1)$ & $110.55(14)$ \\
\hline$C(14)-C(1)-S(1)$ & $108.95(14)$ \\
\hline $\mathrm{C}(2)-\mathrm{C}(1)-\mathrm{S}(1)$ & $111.15(13)$ \\
\hline $\mathrm{N}(1)-\mathrm{C}(2)-\mathrm{C}(1)$ & $116.50(15)$ \\
\hline
\end{tabular}




\begin{tabular}{|c|c|}
\hline $\mathrm{N}(1)-\mathrm{C}(2)-\mathrm{H}(2 \mathrm{~A})$ & 108.2 \\
\hline $\mathrm{C}(1)-\mathrm{C}(2)-\mathrm{H}(2 \mathrm{~A})$ & 108.2 \\
\hline $\mathrm{N}(1)-\mathrm{C}(2)-\mathrm{H}(2 \mathrm{~B})$ & 108.2 \\
\hline $\mathrm{C}(1)-\mathrm{C}(2)-\mathrm{H}(2 \mathrm{~B})$ & 108.2 \\
\hline $\mathrm{H}(2 \mathrm{~A})-\mathrm{C}(2)-\mathrm{H}(2 \mathrm{~B})$ & 107.3 \\
\hline $\mathrm{N}(1)-\mathrm{C}(5)-\mathrm{C}(6)$ & $112.44(16)$ \\
\hline $\mathrm{N}(1)-\mathrm{C}(5)-\mathrm{H}(5 \mathrm{~A})$ & 109.1 \\
\hline $\mathrm{C}(6)-\mathrm{C}(5)-\mathrm{H}(5 \mathrm{~A})$ & 109.1 \\
\hline $\mathrm{N}(1)-\mathrm{C}(5)-\mathrm{H}(5 \mathrm{~B})$ & 109.1 \\
\hline $\mathrm{C}(6)-\mathrm{C}(5)-\mathrm{H}(5 \mathrm{~B})$ & 109.1 \\
\hline$H(5 A)-C(5)-H(5 B)$ & 107.8 \\
\hline$C(5)-C(6)-S(2)$ & $110.65(14)$ \\
\hline$C(5)-C(6)-H(6 A)$ & 109.5 \\
\hline$S(2)-C(6)-H(6 A)$ & 109.5 \\
\hline$C(5)-C(6)-H(6 B)$ & 109.5 \\
\hline $\mathrm{S}(2)-\mathrm{C}(6)-\mathrm{H}(6 \mathrm{~B})$ & 109.5 \\
\hline$H(6 A)-C(6)-H(6 B)$ & 108.1 \\
\hline $\mathrm{C}(4)-\mathrm{C}(3)-\mathrm{N}(1)$ & $111.57(16)$ \\
\hline $\mathrm{C}(4)-\mathrm{C}(3)-\mathrm{H}(3 \mathrm{~A})$ & 109.3 \\
\hline $\mathrm{N}(1)-\mathrm{C}(3)-\mathrm{H}(3 \mathrm{~A})$ & 109.3 \\
\hline $\mathrm{C}(4)-\mathrm{C}(3)-\mathrm{H}(3 \mathrm{~B})$ & 109.3 \\
\hline $\mathrm{N}(1)-\mathrm{C}(3)-\mathrm{H}(3 \mathrm{~B})$ & 109.3 \\
\hline $\mathrm{H}(3 \mathrm{~A})-\mathrm{C}(3)-\mathrm{H}(3 \mathrm{~B})$ & 108.0 \\
\hline $\mathrm{N}(2)-\mathrm{C}(4)-\mathrm{C}(3)$ & $109.63(16)$ \\
\hline $\mathrm{N}(2)-\mathrm{C}(4)-\mathrm{H}(4 \mathrm{~A})$ & 109.7 \\
\hline $\mathrm{C}(3)-\mathrm{C}(4)-\mathrm{H}(4 \mathrm{~A})$ & 109.7 \\
\hline $\mathrm{N}(2)-\mathrm{C}(4)-\mathrm{H}(4 \mathrm{~B})$ & 109.7 \\
\hline$C(3)-C(4)-H(4 B)$ & 109.7 \\
\hline $\mathrm{H}(4 \mathrm{~A})-\mathrm{C}(4)-\mathrm{H}(4 \mathrm{~B})$ & 108.2 \\
\hline $\mathrm{C}(8)-\mathrm{C}(7)-\mathrm{N}(2)$ & $116.33(16)$ \\
\hline $\mathrm{C}(8)-\mathrm{C}(7)-\mathrm{H}(7 \mathrm{~A})$ & 108.2 \\
\hline $\mathrm{N}(2)-\mathrm{C}(7)-\mathrm{H}(7 \mathrm{~A})$ & 108.2 \\
\hline $\mathrm{C}(8)-\mathrm{C}(7)-\mathrm{H}(7 \mathrm{~B})$ & 108.2 \\
\hline $\mathrm{N}(2)-\mathrm{C}(7)-\mathrm{H}(7 \mathrm{~B})$ & 108.2 \\
\hline $\mathrm{H}(7 \mathrm{~A})-\mathrm{C}(7)-\mathrm{H}(7 \mathrm{~B})$ & 107.4 \\
\hline$C(7)-C(8)-S(2)$ & $114.06(14)$ \\
\hline $\mathrm{C}(7)-\mathrm{C}(8)-\mathrm{H}(8 \mathrm{~A})$ & 108.7 \\
\hline$S(2)-C(8)-H(8 A)$ & 108.7 \\
\hline $\mathrm{C}(7)-\mathrm{C}(8)-\mathrm{H}(8 \mathrm{~B})$ & 108.7 \\
\hline $\mathrm{S}(2)-\mathrm{C}(8)-\mathrm{H}(8 \mathrm{~B})$ & 108.7 \\
\hline $\mathrm{H}(8 \mathrm{~A})-\mathrm{C}(8)-\mathrm{H}(8 \mathrm{~B})$ & 107.6 \\
\hline $\mathrm{N}(2)-\mathrm{C}(9)-\mathrm{C}(10)$ & $116.45(16)$ \\
\hline $\mathrm{N}(2)-\mathrm{C}(9)-\mathrm{H}(9 \mathrm{~A})$ & 108.2 \\
\hline $\mathrm{C}(10)-\mathrm{C}(9)-\mathrm{H}(9 \mathrm{~A})$ & 108.2 \\
\hline $\mathrm{N}(2)-\mathrm{C}(9)-\mathrm{H}(9 \mathrm{~B})$ & 108.2 \\
\hline $\mathrm{C}(10)-\mathrm{C}(9)-\mathrm{H}(9 \mathrm{~B})$ & 108.2 \\
\hline $\mathrm{H}(9 \mathrm{~A})-\mathrm{C}(9)-\mathrm{H}(9 \mathrm{~B})$ & 107.3 \\
\hline$C(12)-C(10)-C(11)$ & $110.25(16)$ \\
\hline $\mathrm{C}(12)-\mathrm{C}(10)-\mathrm{C}(9)$ & $109.54(16)$ \\
\hline$C(11)-C(10)-C(9)$ & $111.66(16)$ \\
\hline$C(12)-C(10)-S(3)$ & $114.11(13)$ \\
\hline$C(11)-C(10)-S(3)$ & $104.35(13)$ \\
\hline $\mathrm{C}(9)-\mathrm{C}(10)-\mathrm{S}(3)$ & $106.83(13)$ \\
\hline$C(10)-C(11)-H(11 A)$ & 109.5 \\
\hline $\mathrm{C}(10)-\mathrm{C}(11)-\mathrm{H}(11 \mathrm{~B})$ & 109.5 \\
\hline $\mathrm{H}(11 \mathrm{~A})-\mathrm{C}(11)-\mathrm{H}(11 \mathrm{~B})$ & 109.5 \\
\hline
\end{tabular}




\begin{tabular}{|c|c|}
\hline $\mathrm{C}(10)-\mathrm{C}(11)-\mathrm{H}(11 \mathrm{C})$ & 109.5 \\
\hline $\mathrm{H}(11 \mathrm{~A})-\mathrm{C}(11)-\mathrm{H}(11 \mathrm{C})$ & 109.5 \\
\hline $\mathrm{H}(11 \mathrm{~B})-\mathrm{C}(11)-\mathrm{H}(11 \mathrm{C})$ & 109.5 \\
\hline $\mathrm{C}(10)-\mathrm{C}(12)-\mathrm{H}(12 \mathrm{~A})$ & 109.5 \\
\hline$C(10)-C(12)-H(12 B)$ & 109.5 \\
\hline $\mathrm{H}(12 \mathrm{~A})-\mathrm{C}(12)-\mathrm{H}(12 \mathrm{~B})$ & 109.5 \\
\hline $\mathrm{C}(10)-\mathrm{C}(12)-\mathrm{H}(12 \mathrm{C})$ & 109.5 \\
\hline $\mathrm{H}(12 \mathrm{~A})-\mathrm{C}(12)-\mathrm{H}(12 \mathrm{C})$ & 109.5 \\
\hline $\mathrm{H}(12 \mathrm{~B})-\mathrm{C}(12)-\mathrm{H}(12 \mathrm{C})$ & 109.5 \\
\hline $\mathrm{C}(1)-\mathrm{C}(13)-\mathrm{H}(13 \mathrm{~A})$ & 109.5 \\
\hline$C(1)-C(13)-H(13 B)$ & 109.5 \\
\hline $\mathrm{H}(13 \mathrm{~A})-\mathrm{C}(13)-\mathrm{H}(13 \mathrm{~B})$ & 109.5 \\
\hline $\mathrm{C}(1)-\mathrm{C}(13)-\mathrm{H}(13 \mathrm{C})$ & 109.5 \\
\hline $\mathrm{H}(13 \mathrm{~A})-\mathrm{C}(13)-\mathrm{H}(13 \mathrm{C})$ & 109.5 \\
\hline $\mathrm{H}(13 \mathrm{~B})-\mathrm{C}(13)-\mathrm{H}(13 \mathrm{C})$ & 109.5 \\
\hline $\mathrm{C}(1)-\mathrm{C}(14)-\mathrm{H}(14 \mathrm{~A})$ & 109.5 \\
\hline $\mathrm{C}(1)-\mathrm{C}(14)-\mathrm{H}(14 \mathrm{~B})$ & 109.5 \\
\hline $\mathrm{H}(14 \mathrm{~A})-\mathrm{C}(14)-\mathrm{H}(14 \mathrm{~B})$ & 109.5 \\
\hline $\mathrm{C}(1)-\mathrm{C}(14)-\mathrm{H}(14 \mathrm{C})$ & 109.5 \\
\hline $\mathrm{H}(14 \mathrm{~A})-\mathrm{C}(14)-\mathrm{H}(14 \mathrm{C})$ & 109.5 \\
\hline $\mathrm{H}(14 \mathrm{~B})-\mathrm{C}(14)-\mathrm{H}(14 \mathrm{C})$ & 109.5 \\
\hline $\mathrm{C}(15)-\mathrm{O}(1)-\mathrm{H}(1)$ & 109.5 \\
\hline $\mathrm{O}(1)-\mathrm{C}(15)-\mathrm{C}(16)$ & $111.57(19)$ \\
\hline $\mathrm{O}(1)-\mathrm{C}(15)-\mathrm{H}(15 \mathrm{~A})$ & 109.3 \\
\hline $\mathrm{C}(16)-\mathrm{C}(15)-\mathrm{H}(15 \mathrm{~A})$ & 109.3 \\
\hline $\mathrm{O}(1)-\mathrm{C}(15)-\mathrm{H}(15 \mathrm{~B})$ & 109.3 \\
\hline $\mathrm{C}(16)-\mathrm{C}(15)-\mathrm{H}(15 \mathrm{~B})$ & 109.3 \\
\hline $\mathrm{H}(15 \mathrm{~A})-\mathrm{C}(15)-\mathrm{H}(15 \mathrm{~B})$ & 108.0 \\
\hline$C(15)-C(16)-H(16 A)$ & 109.5 \\
\hline $\mathrm{C}(15)-\mathrm{C}(16)-\mathrm{H}(16 \mathrm{~B})$ & 109.5 \\
\hline$H(16 A)-C(16)-H(16 B)$ & 109.5 \\
\hline$C(15)-C(16)-H(16 C)$ & 109.5 \\
\hline $\mathrm{H}(16 \mathrm{~A})-\mathrm{C}(16)-\mathrm{H}(16 \mathrm{C})$ & 109.5 \\
\hline$H(16 B)-C(16)-H(16 C)$ & 109.5 \\
\hline
\end{tabular}

Symmetry transformations used to generate equivalent atoms:

$\# 1-x+1,-y,-z+1$ 
Table A24. Anisotropic displacement parameters $\left(\AA^{2} \times 10^{3}\right)$ for 10 The anisotropic displacement factor exponent takes the form: $-2 \pi^{2}\left[h^{2} a^{* 2} U^{11}+\ldots+2 h k a^{*} b^{*} U^{12}\right]$

\begin{tabular}{lcccccc}
\hline & $\mathrm{U}^{11}$ & $\mathrm{U}^{22}$ & $\mathrm{U}^{33}$ & $\mathrm{U}^{23}$ & $\mathrm{U}^{13}$ & $\mathrm{U}^{12}$ \\
\hline $\mathrm{Ru}(1)$ & $10(1)$ & $10(1)$ & $10(1)$ & $4(1)$ & $3(1)$ & $1(1)$ \\
$\mathrm{S}(1)$ & $12(1)$ & $15(1)$ & $15(1)$ & $8(1)$ & $4(1)$ & $3(1)$ \\
$\mathrm{S}(2)$ & $14(1)$ & $14(1)$ & $15(1)$ & $7(1)$ & $6(1)$ & $4(1)$ \\
$\mathrm{S}(3)$ & $12(1)$ & $11(1)$ & $12(1)$ & $5(1)$ & $3(1)$ & $2(1)$ \\
$\mathrm{N}(1)$ & $13(1)$ & $12(1)$ & $13(1)$ & $5(1)$ & $3(1)$ & $2(1)$ \\
$\mathrm{N}(2)$ & $16(1)$ & $11(1)$ & $13(1)$ & $5(1)$ & $4(1)$ & $1(1)$ \\
$\mathrm{C}(1)$ & $15(1)$ & $15(1)$ & $17(1)$ & $9(1)$ & $4(1)$ & $4(1)$ \\
$\mathrm{C}(2)$ & $15(1)$ & $12(1)$ & $17(1)$ & $8(1)$ & $5(1)$ & $3(1)$ \\
$\mathrm{C}(5)$ & $15(1)$ & $16(1)$ & $17(1)$ & $8(1)$ & $6(1)$ & $1(1)$ \\
$\mathrm{C}(6)$ & $17(1)$ & $18(1)$ & $18(1)$ & $10(1)$ & $8(1)$ & $5(1)$ \\
$\mathrm{C}(3)$ & $19(1)$ & $14(1)$ & $12(1)$ & $5(1)$ & $2(1)$ & $1(1)$ \\
$\mathrm{C}(4)$ & $17(1)$ & $16(1)$ & $11(1)$ & $6(1)$ & $0(1)$ & $0(1)$ \\
$\mathrm{C}(7)$ & $22(1)$ & $12(1)$ & $15(1)$ & $4(1)$ & $7(1)$ & $3(1)$ \\
$\mathrm{C}(8)$ & $20(1)$ & $14(1)$ & $16(1)$ & $6(1)$ & $9(1)$ & $5(1)$ \\
$\mathrm{C}(9)$ & $17(1)$ & $16(1)$ & $16(1)$ & $8(1)$ & $2(1)$ & $-3(1)$ \\
$\mathrm{C}(10)$ & $14(1)$ & $12(1)$ & $14(1)$ & $5(1)$ & $3(1)$ & $0(1)$ \\
$\mathrm{C}(11)$ & $20(1)$ & $12(1)$ & $20(1)$ & $7(1)$ & $4(1)$ & $0(1)$ \\
$\mathrm{C}(12)$ & $13(1)$ & $20(1)$ & $20(1)$ & $7(1)$ & $5(1)$ & $2(1)$ \\
$\mathrm{C}(13)$ & $15(1)$ & $22(1)$ & $20(1)$ & $12(1)$ & $3(1)$ & $3(1)$ \\
$\mathrm{C}(14)$ & $20(1)$ & $15(1)$ & $23(1)$ & $8(1)$ & $8(1)$ & $6(1)$ \\
$\mathrm{O}(1)$ & $28(1)$ & $19(1)$ & $25(1)$ & $6(1)$ & $6(1)$ & $3(1)$ \\
$\mathrm{I}$ & $23(1)$ & $17(1)$ & $18(1)$ & $6(1)$ & $6(1)$ & $0(1)$ \\
$\mathrm{C}(15)$ & $29(1)$ & $19(1)$ & $25(1)$ & $7(1)$ & $9(1)$ & $1(1)$ \\
$\mathrm{C}(16)$ & $34(1)$ & $28(1)$ & $27(1)$ & $4(1)$ & $10(1)$ & $3(1)$ \\
& & & & & & \\
\hline
\end{tabular}


Table A25. Hydrogen coordinates ( $\left.\times 10^{4}\right)$ and isotropic displacement parameters $\left(\AA^{2} \times 10^{3}\right)$ for 10

\begin{tabular}{|c|c|c|c|c|}
\hline & $x$ & $y$ & $\mathrm{z}$ & $\mathrm{U}(\mathrm{eq})$ \\
\hline $\mathrm{H}(2 \mathrm{~A})$ & 5420 & -2223 & 6762 & 17 \\
\hline$H(2 B)$ & 6500 & -1772 & 8179 & 17 \\
\hline $\mathrm{H}(5 \mathrm{~A})$ & 3946 & -769 & 8484 & 18 \\
\hline $\mathrm{H}(5 \mathrm{~B})$ & 3199 & -1336 & 7021 & 18 \\
\hline $\mathrm{H}(6 \mathrm{~A})$ & 3390 & 1187 & 8603 & 19 \\
\hline $\mathrm{H}(6 \mathrm{~B})$ & 1688 & 250 & 7684 & 19 \\
\hline$H(3 A)$ & 5889 & 1084 & 9354 & 19 \\
\hline $\mathrm{H}(3 \mathrm{~B})$ & 7337 & 214 & 9449 & 19 \\
\hline $\mathrm{H}(4 \mathrm{~A})$ & 8289 & 2250 & 9582 & 19 \\
\hline $\mathrm{H}(4 \mathrm{~B})$ & 8789 & 1113 & 8602 & 19 \\
\hline$H(7 A)$ & 5713 & 2802 & 9072 & 20 \\
\hline $\mathrm{H}(7 \mathrm{~B})$ & 6260 & 3678 & 8483 & 20 \\
\hline $\mathrm{H}(8 \mathrm{~A})$ & 3863 & 3034 & 6878 & 19 \\
\hline $\mathrm{H}(8 \mathrm{~B})$ & 3203 & 3054 & 7961 & 19 \\
\hline $\mathrm{H}(9 \mathrm{~A})$ & 9186 & 2027 & 7568 & 20 \\
\hline $\mathrm{H}(9 \mathrm{~B})$ & 8615 & 3351 & 8107 & 20 \\
\hline $\mathrm{H}(11 \mathrm{~A})$ & 8522 & 4399 & 6738 & 27 \\
\hline $\mathrm{H}(1 \mathrm{IB})$ & 6597 & 4159 & 6679 & 27 \\
\hline $\mathrm{H}(11 \mathrm{C})$ & 7030 & 3837 & 5443 & 27 \\
\hline $\mathrm{H}(12 \mathrm{~A})$ & 8684 & 2139 & 4811 & 28 \\
\hline $\mathrm{H}(12 \mathrm{~B})$ & 9003 & 1171 & 5454 & 28 \\
\hline $\mathrm{H}(12 \mathrm{C})$ & 10105 & 2522 & 6126 & 28 \\
\hline $\mathrm{H}(13 \mathrm{~A})$ & 9431 & -1789 & 8730 & 28 \\
\hline$H(13 B)$ & 9665 & -401 & 8807 & 28 \\
\hline $\mathrm{H}(13 \mathrm{C})$ & 10553 & -1435 & 8029 & 28 \\
\hline $\mathrm{H}(14 \mathrm{~A})$ & 8960 & -3046 & 6094 & 29 \\
\hline $\mathrm{H}(14 \mathrm{~B})$ & 6932 & -3200 & 5551 & 29 \\
\hline $\mathrm{H}(14 \mathrm{C})$ & 7818 & -3508 & 6723 & 29 \\
\hline $\mathrm{H}(1)$ & 5583 & 3008 & 1069 & 38 \\
\hline $\mathrm{H}(15 \mathrm{~A})$ & 7323 & 4610 & 1581 & 30 \\
\hline $\mathrm{H}(15 \mathrm{~B})$ & 8866 & 3888 & 1956 & 30 \\
\hline $\mathrm{H}(16 \mathrm{~A})$ & 6605 & 4727 & 3304 & 48 \\
\hline $\mathrm{H}(16 \mathrm{~B})$ & 8521 & 5371 & 3716 & 48 \\
\hline $\mathrm{H}(16 \mathrm{C})$ & 8155 & 4013 & 3680 & 48 \\
\hline
\end{tabular}


Table A26. Torsion angles $\left[{ }^{\circ}\right]$ for $\mathbf{1 0}$

\begin{tabular}{|c|c|}
\hline $\mathrm{N}(1)-\mathrm{Ru}(1)-\mathrm{S}(1)-\mathrm{C}(1)$ & $18.43(8)$ \\
\hline$N(2)-R u(1)-S(1)-C(1)$ & $99.67(8)$ \\
\hline$S(3) \# 1-R u(1)-S(1)-C(1)$ & $-81.80(7)$ \\
\hline$S(3)-R u(1)-S(1)-C(1)$ & $176.53(7)$ \\
\hline$S(2)-R u(1)-S(1)-C(1)$ & $29.84(11)$ \\
\hline $\operatorname{Ru}(1) \# 1-\operatorname{Ru}(1)-\mathrm{S}(1)-\mathrm{C}(1)$ & $-132.47(7)$ \\
\hline$N(1)-R u(1)-S(2)-C(8)$ & $101.78(8)$ \\
\hline $\mathrm{N}(2)-\mathrm{Ru}(1)-\mathrm{S}(2)-\mathrm{C}(8)$ & $20.35(8)$ \\
\hline $\mathrm{S}(3) \# 1-\mathrm{Ru}(1)-\mathrm{S}(2)-\mathrm{C}(8)$ & $-160.51(7)$ \\
\hline$S(3)-R u(1)-S(2)-C(8)$ & $-57.31(7)$ \\
\hline $\mathrm{S}(1)-\mathrm{Ru}(1)-\mathrm{S}(2)-\mathrm{C}(8)$ & $90.41(11)$ \\
\hline $\operatorname{Ru}(1) \# 1-\operatorname{Ru}(1)-\mathrm{S}(2)-\mathrm{C}(8)$ & $-107.47(7)$ \\
\hline$N(1)-R u(1)-S(2)-C(6)$ & $-2.19(8)$ \\
\hline$N(2)-R u(1)-S(2)-C(6)$ & $-83.63(8)$ \\
\hline$S(3) \# 1-R u(1)-S(2)-C(6)$ & $95.51(7)$ \\
\hline$S(3)-R u(1)-S(2)-C(6)$ & $-161.28(7)$ \\
\hline$S(1)-R u(1)-S(2)-C(6)$ & $-13.56(12)$ \\
\hline $\mathrm{Ru}(1) \# 1-\mathrm{Ru}(1)-\mathrm{S}(2)-\mathrm{C}(6)$ & $148.55(7)$ \\
\hline$N(1)-R u(1)-S(3)-C(10)$ & $58.84(13)$ \\
\hline N(2)-Ru(1)-S(3)-C(10) & $38.02(8)$ \\
\hline$S(3) \# 1-R u(1)-S(3)-C(10)$ & $-129.81(7)$ \\
\hline$S(2)-R u(1)-S(3)-C(10)$ & $125.65(7)$ \\
\hline$S(1)-R u(1)-S(3)-C(10)$ & $-47.92(7)$ \\
\hline $\mathrm{Ru}(1) \# 1-\mathrm{Ru}(1)-\mathrm{S}(3)-\mathrm{C}(10)$ & $-129.81(7)$ \\
\hline$N(1)-R u(1)-S(3)-R u(1) \# 1$ & $-171.35(11)$ \\
\hline$N(2)-R u(1)-S(3)-R u(1) \# 1$ & $167.83(5)$ \\
\hline $\mathrm{S}(3) \# 1-\mathrm{Ru}(1)-\mathrm{S}(3)-\mathrm{Ru}(1) \# 1$ & 0.0 \\
\hline$S(2)-\operatorname{Ru}(1)-S(3)-R u(1) \# 1$ & $-104.536(17)$ \\
\hline$S(1)-R u(1)-S(3)-R u(1) \# 1$ & $81.893(17)$ \\
\hline $\mathrm{N}(2)-\mathrm{Ru}(1)-\mathrm{N}(1)-\mathrm{C}(5)$ & $115.06(12)$ \\
\hline $\mathrm{S}(3) \# 1-\mathrm{Ru}(1)-\mathrm{N}(1)-\mathrm{C}(5)$ & $-76.99(11)$ \\
\hline$S(3)-R u(1)-N(1)-C(5)$ & $94.51(15)$ \\
\hline$S(2)-R u(1)-N(1)-C(5)$ & $27.59(11)$ \\
\hline $\mathrm{S}(1)-\mathrm{Ru}(1)-\mathrm{N}(1)-\mathrm{C}(5)$ & $-154.72(12)$ \\
\hline $\mathrm{Ru}(1) \# 1-\mathrm{Ru}(1)-\mathrm{N}(1)-\mathrm{C}(5)$ & $-71.54(15)$ \\
\hline $\mathrm{N}(2)-\mathrm{Ru}(1)-\mathrm{N}(1)-\mathrm{C}(2)$ & $-127.49(12)$ \\
\hline $\mathrm{S}(3) \# 1-\mathrm{Ru}(1)-\mathrm{N}(1)-\mathrm{C}(2)$ & $40.46(12)$ \\
\hline$S(3)-R u(1)-N(1)-C(2)$ & $-148.04(10)$ \\
\hline$S(2)-R u(1)-N(1)-C(2)$ & $145.04(11)$ \\
\hline $\mathrm{S}(1)-\mathrm{Ru}(1)-\mathrm{N}(1)-\mathrm{C}(2)$ & $-37.27(11)$ \\
\hline $\mathrm{Ru}(1) \# 1-\mathrm{Ru}(1)-\mathrm{N}(1)-\mathrm{C}(2)$ & $45.91(17)$ \\
\hline $\mathrm{N}(2)-\mathrm{Ru}(1)-\mathrm{N}(1)-\mathrm{C}(3)$ & $-3.72(12)$ \\
\hline $\mathrm{S}(3) \# 1-\mathrm{Ru}(1)-\mathrm{N}(1)-\mathrm{C}(3)$ & $164.23(11)$ \\
\hline$S(3)-R u(1)-N(1)-C(3)$ & $-24.3(2)$ \\
\hline $\mathrm{S}(2)-\mathrm{Ru}(1)-\mathrm{N}(1)-\mathrm{C}(3)$ & $-91.19(12)$ \\
\hline $\mathrm{S}(1)-\mathrm{Ru}(1)-\mathrm{N}(1)-\mathrm{C}(3)$ & $86.50(12)$ \\
\hline $\mathrm{Ru}(1) \# 1-\mathrm{Ru}(1)-\mathrm{N}(1)-\mathrm{C}(3)$ & $169.68(9)$ \\
\hline$N(1)-R u(1)-N(2)-C(4)$ & $27.35(12)$ \\
\hline $\mathrm{S}(3) \# 1-\mathrm{Ru}(1)-\mathrm{N}(2)-\mathrm{C}(4)$ & $-63.0(3)$ \\
\hline$S(3)-R u(1)-N(2)-C(4)$ & $-160.65(12)$ \\
\hline $\mathrm{S}(2)-\mathrm{Ru}(1)-\mathrm{N}(2)-\mathrm{C}(4)$ & $112.98(12)$ \\
\hline $\mathrm{S}(1)-\mathrm{Ru}(1)-\mathrm{N}(2)-\mathrm{C}(4)$ & $-56.00(11)$ \\
\hline $\mathrm{Ru}(1) \# 1-\mathrm{Ru}(1)-\mathrm{N}(2)-\mathrm{C}(4)$ & $-148.57(10)$ \\
\hline $\mathrm{N}(1)-\mathrm{Ru}(1)-\mathrm{N}(2)-\mathrm{C}(9)$ & $141.31(13)$ \\
\hline
\end{tabular}


$\mathrm{S}(3) \# 1-\mathrm{Ru}(1)-\mathrm{N}(2)-\mathrm{C}(9)$

$51.0(3)$

$\mathrm{S}(3)-\mathrm{Ru}(1)-\mathrm{N}(2)-\mathrm{C}(9)$

$\mathrm{S}(2)-\mathrm{Ru}(1)-\mathrm{N}(2)-\mathrm{C}(9)$

$\mathrm{S}(1)-\mathrm{Ru}(1)-\mathrm{N}(2)-\mathrm{C}(9)$

$\mathrm{Ru}(1) \# 1-\mathrm{Ru}(1)-\mathrm{N}(2)-\mathrm{C}(9)$

$-46.69(11)$

$-133.06(12)$

$57.96(12)$

$-34.61(14)$

$\mathrm{N}(1)-\mathrm{Ru}(1)-\mathrm{N}(2)-\mathrm{C}(7)$

$-91.07(13)$

$\mathrm{S}(3) \# 1-\mathrm{Ru}(1)-\mathrm{N}(2)-\mathrm{C}(7)$

$178.58(16)$

$\mathrm{S}(3)-\mathrm{Ru}(1)-\mathrm{N}(2)-\mathrm{C}(7)$

$80.93(12)$

$\mathrm{S}(2)-\mathrm{Ru}(1)-\mathrm{N}(2)-\mathrm{C}(7)$

$\mathrm{S}(1)-\mathrm{Ru}(1)-\mathrm{N}(2)-\mathrm{C}(7)$

$-5.44(12)$

$\mathrm{Ru}(1) \# 1-\mathrm{Ru}(1)-\mathrm{N}(2)-\mathrm{C}(7)$

$-174.42(12)$

$\operatorname{Ru}(1)-S(1)-C(1)-C(13)$

93.01(13)

$\mathrm{Ru}(1)-\mathrm{S}(1)-\mathrm{C}(1)-\mathrm{C}(14)$

$-124.37(13)$

$\mathrm{Ru}(1)-\mathrm{S}(1)-\mathrm{C}(1)-\mathrm{C}(2)$

$117.95(13)$

$\mathrm{C}(5)-\mathrm{N}(1)-\mathrm{C}(2)-\mathrm{C}(1)$

$1.08(14)$

$169.79(16)$

$\mathrm{C}(3)-\mathrm{N}(1)-\mathrm{C}(2)-\mathrm{C}(1)$

$-72.9(2)$

$\mathrm{Ru}(1)-\mathrm{N}(1)-\mathrm{C}(2)-\mathrm{C}(1)$

$50.67(19)$

$\mathrm{C}(13)-\mathrm{C}(1)-\mathrm{C}(2)-\mathrm{N}(1)$

92.3(2)

$\mathrm{C}(14)-\mathrm{C}(1)-\mathrm{C}(2)-\mathrm{N}(1)$

$-150.63(16)$

$\mathrm{S}(1)-\mathrm{C}(1)-\mathrm{C}(2)-\mathrm{N}(1)$

$-32.2(2)$

$\mathrm{C}(2)-\mathrm{N}(1)-\mathrm{C}(5)-\mathrm{C}(6)$

$\mathrm{C}(3)-\mathrm{N}(1)-\mathrm{C}(5)-\mathrm{C}(6)$

$-170.67(16)$

69.3(2)

$\mathrm{Ru}(1)-\mathrm{N}(1)-\mathrm{C}(5)-\mathrm{C}(6)$

$-51.70(18)$

$\mathrm{N}(1)-\mathrm{C}(5)-\mathrm{C}(6)-\mathrm{S}(2)$

$49.7(2)$

$\mathrm{C}(8)-\mathrm{S}(2)-\mathrm{C}(6)-\mathrm{C}(5)$

$-122.89(15)$

$\mathrm{Ru}(1)-\mathrm{S}(2)-\mathrm{C}(6)-\mathrm{C}(5)$

$-23.16(15)$

$\mathrm{C}(5)-\mathrm{N}(1)-\mathrm{C}(3)-\mathrm{C}(4)$

$-141.45(16)$

$\mathrm{C}(2)-\mathrm{N}(1)-\mathrm{C}(3)-\mathrm{C}(4)$

$101.41(18)$

$-21.25(19)$

$\mathrm{Ru}(1)-\mathrm{N}(1)-\mathrm{C}(3)-\mathrm{C}(4)$

$-164.18(16)$

75.08(19)

$\mathrm{C}(7)-\mathrm{N}(2)-\mathrm{C}(4)-\mathrm{C}(3)$

$\mathrm{Ru}(1)-\mathrm{N}(2)-\mathrm{C}(4)-\mathrm{C}(3)$

$-46.90(17)$

$\mathrm{N}(1)-\mathrm{C}(3)-\mathrm{C}(4)-\mathrm{N}(2)$

$45.9(2)$

$\mathrm{C}(4)-\mathrm{N}(2)-\mathrm{C}(7)-\mathrm{C}(8)$

$\mathrm{C}(9)-\mathrm{N}(2)-\mathrm{C}(7)-\mathrm{C}(8)$

$-134.22(17)$

$109.75(19)$

$-16.6(2)$

$37.5(2)$

$\mathrm{N}(2)-\mathrm{C}(7)-\mathrm{C}(8)-\mathrm{S}(2)$

65.94(16)

$\mathrm{C}(6)-\mathrm{S}(2)-\mathrm{C}(8)-\mathrm{C}(7)$

$-35.65(15)$

$\mathrm{Ru}(1)-\mathrm{S}(2)-\mathrm{C}(8)-\mathrm{C}(7)$

157.61(16)

$\mathrm{C}(4)-\mathrm{N}(2)-\mathrm{C}(9)-\mathrm{C}(10)$

$-84.9(2)$

$42.98(19)$

$-131.53(17)$

106.04(19)

$-7.5(2)$

$11.38(18)$

94.19(14)

$131.77(11)$

$-145.41(11)$

$-109.83(13)$

$-27.02(14)$

Symmetry transformations used to generate equivalent atoms:

$\# 1-x+1,-y,-z+1$ 
Table A27. Crystal data and structure refinement for 11

\begin{tabular}{|c|c|c|}
\hline Identification code & \multicolumn{2}{|l|}{ cag 177lt } \\
\hline Empirical formula & \multicolumn{2}{|c|}{ C16 H28 N2 O2 Ru S3 } \\
\hline Formula weight & \multicolumn{2}{|c|}{477.65} \\
\hline Temperature & \multicolumn{2}{|l|}{$100.0 \mathrm{~K}$} \\
\hline Wavelength & \multicolumn{2}{|l|}{$0.7107 \AA$} \\
\hline Crystal system & \multicolumn{2}{|l|}{ Monoclinic } \\
\hline Space group & \multicolumn{2}{|l|}{ P $121 /$ n 1} \\
\hline \multirow[t]{3}{*}{ Unit cell dimensions } & $a=9.2404(2) \AA$ & $\alpha=90^{\circ}$ \\
\hline & $\mathrm{b}=20.7973(4) \AA$ & $\beta=94.916(2)^{\circ}$ \\
\hline & $c=10.6722(3) \AA$ & $\gamma=90^{\circ}$ \\
\hline Volume & \multicolumn{2}{|l|}{$2043.40(8) \AA^{3}$} \\
\hline Z & \multicolumn{2}{|l|}{4} \\
\hline Density (calculated) & \\
\hline Absorption coefficient & \multicolumn{2}{|l|}{$1.085 \mathrm{~mm}^{-1}$} \\
\hline$F(000)$ & \multicolumn{2}{|l|}{984} \\
\hline Theta range for data collection & \multicolumn{2}{|l|}{3.42 to $29.01^{\circ}$} \\
\hline Crystal color, habit & \multicolumn{2}{|c|}{ yellow plate } \\
\hline Crystal size & \multicolumn{2}{|c|}{$0.23 \times 0.18 \times 0.05 \mathrm{~mm}^{3}$} \\
\hline Theta range for data collection & \multicolumn{2}{|c|}{3.42 to $29.01^{\circ}$} \\
\hline Index ranges & \multicolumn{2}{|c|}{$-12<=\mathrm{h}<=11,-28<=\mathrm{k}<=23,-13<=\mathrm{l}<=7$} \\
\hline Reflections collected & \multicolumn{2}{|c|}{11721} \\
\hline Independent reflections & \multicolumn{2}{|c|}{$4763[\mathrm{R}(\mathrm{int})=0.0278]$} \\
\hline Completeness to theta $=29.0^{\circ}$ & \multicolumn{2}{|c|}{$87.5 \%$} \\
\hline Completeness to theta $=26.3^{\circ}$ & \multicolumn{2}{|c|}{$99.4 \%$} \\
\hline Absorption correction & \multicolumn{2}{|c|}{ Semi-empirical from equivalents } \\
\hline Max. and min. transmission & \multicolumn{2}{|c|}{0.958 and 0.803} \\
\hline Refinement method & \multicolumn{2}{|c|}{ Full-matrix least-squares on $\mathrm{F}^{2}$} \\
\hline Data / restraints / parameters & \multicolumn{2}{|c|}{$4763 / 3 / 223$} \\
\hline Goodness-of-fit on $\mathrm{F}^{2}$ & \multicolumn{2}{|c|}{1.049} \\
\hline Final $R$ indices $[I>2 \operatorname{sigma}(I)]$ & \multicolumn{2}{|c|}{$\mathrm{R} 1=0.0280, \mathrm{wR} 2=0.0615$} \\
\hline $\mathrm{R}$ indices (all data) & \multicolumn{2}{|c|}{$\mathrm{R} 1=0.0384, \mathrm{wR} 2=0.0667$} \\
\hline Largest diff. peak and hole & \multicolumn{2}{|c|}{0.610 and $-0.436 \mathrm{e} . \AA^{-3}$} \\
\hline
\end{tabular}


Table A28. Atomic coordinates ( $\left.\times 10^{4}\right)$ and equivalent isotropic displacement parameters $\left(\AA^{2} \times 10^{3}\right)$ for $11 \mathrm{U}(\mathrm{eq})$ is defined as one third of the trace of the orthogonalized $\mathrm{U}^{\mathrm{ij}}$ tensor.

\begin{tabular}{|c|c|c|c|c|}
\hline & $\mathrm{x}$ & $\mathrm{y}$ & $z$ & $\mathrm{U}(\mathrm{eq})$ \\
\hline $\mathrm{Ru}(1)$ & $8555(1)$ & $2214(1)$ & $5854(1)$ & $9(1)$ \\
\hline $\mathrm{S}(2)$ & $8749(1)$ & $3080(1)$ & $4411(1)$ & $13(1)$ \\
\hline $\mathrm{S}(3)$ & 7274(1) & $1336(1)$ & $4840(1)$ & $13(1)$ \\
\hline$S(1)$ & $10914(1)$ & $1845(1)$ & $5283(1)$ & $13(1)$ \\
\hline$O(1)$ & $5702(2)$ & $2767(1)$ & $6400(2)$ & $18(1)$ \\
\hline $\mathrm{N}(2)$ & 9752(2) & $2840(1)$ & $7125(2)$ & $10(1)$ \\
\hline $\mathrm{N}(1)$ & $8912(2)$ & $1515(1)$ & $7313(2)$ & $11(1)$ \\
\hline$C(15)$ & $6809(3)$ & $2539(1)$ & $6218(2)$ & $12(1)$ \\
\hline $\mathrm{C}(4)$ & 9322(3) & $2597(1)$ & $8368(2)$ & $12(1)$ \\
\hline$C(7)$ & $7490(2)$ & $1199(1)$ & $7495(2)$ & $13(1)$ \\
\hline$C(5)$ & $11383(2)$ & 2799(1) & $7110(2)$ & $13(1)$ \\
\hline $\mathrm{C}(3)$ & $9510(3)$ & 1871(1) & $8483(2)$ & $13(1)$ \\
\hline$C(11)$ & $9205(3)$ & $3514(1)$ & 6916(2) & $14(1)$ \\
\hline $\mathrm{C}(1)$ & $11351(3)$ & $1261(1)$ & $6545(2)$ & $15(1)$ \\
\hline$C(12)$ & 9094(3) & $3752(1)$ & $5547(2)$ & $14(1)$ \\
\hline$C(14)$ & $10460(3)$ & $4122(1)$ & $5256(3)$ & $17(1)$ \\
\hline$C(13)$ & $7803(3)$ & $4219(1)$ & $5378(3)$ & $19(1)$ \\
\hline$C(2)$ & $9960(3)$ & $1005(1)$ & $7011(2)$ & $14(1)$ \\
\hline $\mathrm{C}(9)$ & $5045(3)$ & $1082(1)$ & $6337(3)$ & $18(1)$ \\
\hline$C(10)$ & $6823(3)$ & $208(1)$ & $6111(3)$ & $19(1)$ \\
\hline$C(6)$ & $11896(3)$ & 2551(1) & $5886(2)$ & $15(1)$ \\
\hline $\mathrm{C}(8)$ & $6663(3)$ & $937(1)$ & $6265(2)$ & $14(1)$ \\
\hline$C(20)$ & $9639(8)$ & $744(3)$ & $2476(7)$ & $31(2)$ \\
\hline$O(20)$ & $8272(5)$ & $336(2)$ & $2748(5)$ & $36(1)$ \\
\hline$C(30)$ & $9267(8)$ & $683(3)$ & $2126(6)$ & $26(2)$ \\
\hline $\mathrm{O}(30 \mathrm{~A})$ & $8919(11)$ & $327(4)$ & $3274(9)$ & $25(2)$ \\
\hline $\mathrm{O}(30 \mathrm{~B})$ & $9472(8)$ & $421(3)$ & $3643(7)$ & $14(2)$ \\
\hline
\end{tabular}


Table A29. Bond lengths $[\AA]$ and angles $\left[{ }^{\circ}\right]$ for 11

\begin{tabular}{|c|c|}
\hline $\mathrm{Ru}(1)-\mathrm{C}(15)$ & $1.822(2)$ \\
\hline $\mathrm{Ru}(1)-\mathrm{N}(2)$ & $2.1215(19)$ \\
\hline $\mathrm{Ru}(1)-\mathrm{N}(1)$ & $2.1354(19)$ \\
\hline $\mathrm{Ru}(1)-\mathrm{S}(2)$ & $2.3854(6)$ \\
\hline $\mathrm{Ru}(1)-\mathrm{S}(3)$ & $2.3854(6)$ \\
\hline $\mathrm{Ru}(1)-\mathrm{S}(1)$ & $2.4379(6)$ \\
\hline$S(2)-C(12)$ & $1.861(2)$ \\
\hline$S(3)-C(8)$ & $1.861(3)$ \\
\hline$S(1)-C(6)$ & $1.815(2)$ \\
\hline$S(1)-C(1)$ & $1.832(3)$ \\
\hline $\mathrm{O}(1)-\mathrm{C}(15)$ & $1.158(3)$ \\
\hline$N(2)-C(11)$ & $1.500(3)$ \\
\hline$N(2)-C(4)$ & $1.504(3)$ \\
\hline $\mathrm{N}(2)-\mathrm{C}(5)$ & $1.512(3)$ \\
\hline $\mathrm{N}(1)-\mathrm{C}(2)$ & $1.490(3)$ \\
\hline$N(1)-C(7)$ & $1.496(3)$ \\
\hline$N(1)-C(3)$ & $1.515(3)$ \\
\hline$C(4)-C(3)$ & $1.522(3)$ \\
\hline $\mathrm{C}(4)-\mathrm{H}(4 \mathrm{~A})$ & 0.9700 \\
\hline $\mathrm{C}(4)-\mathrm{H}(4 \mathrm{~B})$ & 0.9700 \\
\hline$C(7)-C(8)$ & $1.559(3)$ \\
\hline $\mathrm{C}(7)-\mathrm{H}(7 \mathrm{~A})$ & 0.9700 \\
\hline $\mathrm{C}(7)-\mathrm{H}(7 \mathrm{~B})$ & 0.9700 \\
\hline$C(5)-C(6)$ & $1.517(3)$ \\
\hline $\mathrm{C}(5)-\mathrm{H}(5 \mathrm{~A})$ & 0.9700 \\
\hline $\mathrm{C}(5)-\mathrm{H}(5 \mathrm{~B})$ & 0.9700 \\
\hline$C(3)-H(3 A)$ & 0.9700 \\
\hline C(3)-H(3B) & 0.9700 \\
\hline$C(11)-C(12)$ & $1.538(3)$ \\
\hline $\mathrm{C}(11)-\mathrm{H}(11 \mathrm{~A})$ & 0.9700 \\
\hline $\mathrm{C}(11)-\mathrm{H}(11 \mathrm{~B})$ & 0.9700 \\
\hline$C(1)-C(2)$ & $1.515(3)$ \\
\hline $\mathrm{C}(1)-\mathrm{H}(1 \mathrm{~A})$ & 0.9700 \\
\hline $\mathrm{C}(1)-\mathrm{H}(1 \mathrm{~B})$ & 0.9700 \\
\hline$C(12)-C(14)$ & $1.532(3)$ \\
\hline$C(12)-C(13)$ & $1.537(3)$ \\
\hline $\mathrm{C}(14)-\mathrm{H}(14 \mathrm{~A})$ & 0.9600 \\
\hline $\mathrm{C}(14)-\mathrm{H}(14 \mathrm{~B})$ & 0.9600 \\
\hline $\mathrm{C}(14)-\mathrm{H}(14 \mathrm{C})$ & 0.9600 \\
\hline$C(13)-H(13 A)$ & 0.9600 \\
\hline $\mathrm{C}(13)-\mathrm{H}(13 \mathrm{~B})$ & 0.9600 \\
\hline $\mathrm{C}(13)-\mathrm{H}(13 \mathrm{C})$ & 0.9600 \\
\hline $\mathrm{C}(2)-\mathrm{H}(2 \mathrm{~A})$ & 0.9700 \\
\hline $\mathrm{C}(2)-\mathrm{H}(2 \mathrm{~B})$ & 0.9700 \\
\hline$C(9)-C(8)$ & $1.534(3)$ \\
\hline $\mathrm{C}(9)-\mathrm{H}(9 \mathrm{~A})$ & 0.9600 \\
\hline $\mathrm{C}(9)-\mathrm{H}(9 \mathrm{~B})$ & 0.9600 \\
\hline $\mathrm{C}(9)-\mathrm{H}(9 \mathrm{C})$ & 0.9600 \\
\hline$C(10)-C(8)$ & $1.535(3)$ \\
\hline $\mathrm{C}(10)-\mathrm{H}(10 \mathrm{~A})$ & 0.9600 \\
\hline $\mathrm{C}(10)-\mathrm{H}(10 \mathrm{~B})$ & 0.9600 \\
\hline $\mathrm{C}(10)-\mathrm{H}(10 \mathrm{C})$ & 0.9600 \\
\hline $\mathrm{C}(6)-\mathrm{H}(6 \mathrm{~A})$ & 0.9700 \\
\hline $\mathrm{C}(6)-\mathrm{H}(6 \mathrm{~B})$ & 0.9700 \\
\hline
\end{tabular}




\begin{tabular}{|c|c|}
\hline $\mathrm{C}(20)-\mathrm{O}(20)$ & $1.569(8)$ \\
\hline $\mathrm{C}(30)-\mathrm{O}(30 \mathrm{~A})$ & $1.490(10)$ \\
\hline$C(30)-O(30 B)$ & $1.703(9)$ \\
\hline $\mathrm{C}(15)-\mathrm{Ru}(1)-\mathrm{N}(2)$ & $93.36(9)$ \\
\hline$C(15)-R u(1)-N(1)$ & $100.43(9)$ \\
\hline$N(2)-R u(1)-N(1)$ & $84.94(7)$ \\
\hline$C(15)-R u(1)-S(2)$ & $88.57(8)$ \\
\hline$N(2)-R u(1)-S(2)$ & $83.46(5)$ \\
\hline$N(1)-R u(1)-S(2)$ & $165.71(5)$ \\
\hline$C(15)-R u(1)-S(3)$ & $88.10(7)$ \\
\hline $\mathrm{N}(2)-\mathrm{Ru}(1)-\mathrm{S}(3)$ & $166.21(5)$ \\
\hline$N(1)-R u(1)-S(3)$ & $81.31(5)$ \\
\hline $\mathrm{S}(2)-\mathrm{Ru}(1)-\mathrm{S}(3)$ & $110.30(2)$ \\
\hline$C(15)-R u(1)-S(1)$ & $176.13(8)$ \\
\hline$N(2)-\operatorname{Ru}(1)-S(1)$ & $85.70(5)$ \\
\hline$N(1)-R u(1)-S(1)$ & $83.23(5)$ \\
\hline$S(2)-R u(1)-S(1)$ & $87.59(2)$ \\
\hline$S(3)-R u(1)-S(1)$ & $93.71(2)$ \\
\hline$C(12)-S(2)-R u(1)$ & $99.49(8)$ \\
\hline$C(8)-S(3)-R u(1)$ & $98.26(8)$ \\
\hline$C(6)-S(1)-C(1)$ & $101.72(12)$ \\
\hline$C(6)-S(1)-R u(1)$ & $94.99(8)$ \\
\hline$C(1)-S(1)-R u(1)$ & $99.80(8)$ \\
\hline$C(11)-N(2)-C(4)$ & $109.56(18)$ \\
\hline$C(11)-N(2)-C(5)$ & $112.03(17)$ \\
\hline$C(4)-N(2)-C(5)$ & $109.23(18)$ \\
\hline$C(11)-N(2)-R u(1)$ & $109.11(14)$ \\
\hline $\mathrm{C}(4)-\mathrm{N}(2)-\mathrm{Ru}(1)$ & $101.24(13)$ \\
\hline $\mathrm{C}(5)-\mathrm{N}(2)-\mathrm{Ru}(1)$ & $115.08(14)$ \\
\hline$C(2)-N(1)-C(7)$ & $108.02(17)$ \\
\hline $\mathrm{C}(2)-\mathrm{N}(1)-\mathrm{C}(3)$ & $109.16(18)$ \\
\hline$C(7)-N(1)-C(3)$ & $111.73(18)$ \\
\hline$C(2)-N(1)-R u(1)$ & $112.94(14)$ \\
\hline$C(7)-N(1)-R u(1)$ & $108.13(14)$ \\
\hline$C(3)-N(1)-R u(1)$ & $106.91(13)$ \\
\hline $\mathrm{O}(1)-\mathrm{C}(15)-\mathrm{Ru}(1)$ & $176.6(2)$ \\
\hline$N(2)-C(4)-C(3)$ & $111.57(19)$ \\
\hline$N(2)-C(4)-H(4 A)$ & 109.3 \\
\hline $\mathrm{C}(3)-\mathrm{C}(4)-\mathrm{H}(4 \mathrm{~A})$ & 109.3 \\
\hline $\mathrm{N}(2)-\mathrm{C}(4)-\mathrm{H}(4 \mathrm{~B})$ & 109.3 \\
\hline$C(3)-C(4)-H(4 B)$ & 109.3 \\
\hline $\mathrm{H}(4 \mathrm{~A})-\mathrm{C}(4)-\mathrm{H}(4 \mathrm{~B})$ & 108.0 \\
\hline $\mathrm{N}(1)-\mathrm{C}(7)-\mathrm{C}(8)$ & $114.61(19)$ \\
\hline $\mathrm{N}(1)-\mathrm{C}(7)-\mathrm{H}(7 \mathrm{~A})$ & 108.6 \\
\hline $\mathrm{C}(8)-\mathrm{C}(7)-\mathrm{H}(7 \mathrm{~A})$ & 108.6 \\
\hline $\mathrm{N}(1)-\mathrm{C}(7)-\mathrm{H}(7 \mathrm{~B})$ & 108.6 \\
\hline $\mathrm{C}(8)-\mathrm{C}(7)-\mathrm{H}(7 \mathrm{~B})$ & 108.6 \\
\hline $\mathrm{H}(7 \mathrm{~A})-\mathrm{C}(7)-\mathrm{H}(7 \mathrm{~B})$ & 107.6 \\
\hline$N(2)-C(5)-C(6)$ & $114.48(19)$ \\
\hline $\mathrm{N}(2)-\mathrm{C}(5)-\mathrm{H}(5 \mathrm{~A})$ & 108.6 \\
\hline $\mathrm{C}(6)-\mathrm{C}(5)-\mathrm{H}(5 \mathrm{~A})$ & 108.6 \\
\hline $\mathrm{N}(2)-\mathrm{C}(5)-\mathrm{H}(5 \mathrm{~B})$ & 108.6 \\
\hline $\mathrm{C}(6)-\mathrm{C}(5)-\mathrm{H}(5 \mathrm{~B})$ & 108.6 \\
\hline$H(5 A)-C(5)-H(5 B)$ & 107.6 \\
\hline$N(1)-C(3)-C(4)$ & $112.77(19)$ \\
\hline $\mathrm{N}(1)-\mathrm{C}(3)-\mathrm{H}(3 \mathrm{~A})$ & 109.0 \\
\hline
\end{tabular}




\begin{tabular}{|c|c|}
\hline$C(4)-C(3)-H(3 A)$ & 109.0 \\
\hline $\mathrm{N}(1)-\mathrm{C}(3)-\mathrm{H}(3 \mathrm{~B})$ & 109.0 \\
\hline $\mathrm{C}(4)-\mathrm{C}(3)-\mathrm{H}(3 \mathrm{~B})$ & 109.0 \\
\hline$H(3 A)-C(3)-H(3 B)$ & 107.8 \\
\hline $\mathrm{N}(2)-\mathrm{C}(11)-\mathrm{C}(12)$ & $115.94(19)$ \\
\hline $\mathrm{N}(2)-\mathrm{C}(11)-\mathrm{H}(11 \mathrm{~A})$ & 108.3 \\
\hline $\mathrm{C}(12)-\mathrm{C}(11)-\mathrm{H}(11 \mathrm{~A})$ & 108.3 \\
\hline $\mathrm{N}(2)-\mathrm{C}(11)-\mathrm{H}(11 \mathrm{~B})$ & 108.3 \\
\hline $\mathrm{C}(12)-\mathrm{C}(11)-\mathrm{H}(11 \mathrm{~B})$ & 108.3 \\
\hline $\mathrm{H}(11 \mathrm{~A})-\mathrm{C}(11)-\mathrm{H}(11 \mathrm{~B})$ & 107.4 \\
\hline$C(2)-C(1)-S(1)$ & $109.60(17)$ \\
\hline $\mathrm{C}(2)-\mathrm{C}(1)-\mathrm{H}(1 \mathrm{~A})$ & 109.8 \\
\hline $\mathrm{S}(1)-\mathrm{C}(1)-\mathrm{H}(1 \mathrm{~A})$ & 109.8 \\
\hline $\mathrm{C}(2)-\mathrm{C}(1)-\mathrm{H}(1 \mathrm{~B})$ & 109.8 \\
\hline $\mathrm{S}(1)-\mathrm{C}(1)-\mathrm{H}(1 \mathrm{~B})$ & 109.8 \\
\hline$H(1 A)-C(1)-H(1 B)$ & 108.2 \\
\hline$C(14)-C(12)-C(13)$ & $107.70(19)$ \\
\hline$C(14)-C(12)-C(11)$ & $111.5(2)$ \\
\hline$C(13)-C(12)-C(11)$ & $107.6(2)$ \\
\hline$C(14)-C(12)-S(2)$ & $110.10(17)$ \\
\hline$C(13)-C(12)-S(2)$ & $108.06(17)$ \\
\hline$C(11)-C(12)-S(2)$ & $111.69(16)$ \\
\hline $\mathrm{C}(12)-\mathrm{C}(14)-\mathrm{H}(14 \mathrm{~A})$ & 109.5 \\
\hline $\mathrm{C}(12)-\mathrm{C}(14)-\mathrm{H}(14 \mathrm{~B})$ & 109.5 \\
\hline $\mathrm{H}(14 \mathrm{~A})-\mathrm{C}(14)-\mathrm{H}(14 \mathrm{~B})$ & 109.5 \\
\hline $\mathrm{C}(12)-\mathrm{C}(14)-\mathrm{H}(14 \mathrm{C})$ & 109.5 \\
\hline $\mathrm{H}(14 \mathrm{~A})-\mathrm{C}(14)-\mathrm{H}(14 \mathrm{C})$ & 109.5 \\
\hline $\mathrm{H}(14 \mathrm{~B})-\mathrm{C}(14)-\mathrm{H}(14 \mathrm{C})$ & 109.5 \\
\hline $\mathrm{C}(12)-\mathrm{C}(13)-\mathrm{H}(13 \mathrm{~A})$ & 109.5 \\
\hline$C(12)-C(13)-H(13 B)$ & 109.5 \\
\hline$H(13 A)-C(13)-H(13 B)$ & 109.5 \\
\hline$C(12)-C(13)-H(13 C)$ & 109.5 \\
\hline $\mathrm{H}(13 \mathrm{~A})-\mathrm{C}(13)-\mathrm{H}(13 \mathrm{C})$ & 109.5 \\
\hline$H(13 B)-C(13)-H(13 C)$ & 109.5 \\
\hline$N(1)-C(2)-C(1)$ & $113.95(19)$ \\
\hline $\mathrm{N}(1)-\mathrm{C}(2)-\mathrm{H}(2 \mathrm{~A})$ & 108.8 \\
\hline $\mathrm{C}(1)-\mathrm{C}(2)-\mathrm{H}(2 \mathrm{~A})$ & 108.8 \\
\hline $\mathrm{N}(1)-\mathrm{C}(2)-\mathrm{H}(2 \mathrm{~B})$ & 108.8 \\
\hline $\mathrm{C}(1)-\mathrm{C}(2)-\mathrm{H}(2 \mathrm{~B})$ & 108.8 \\
\hline $\mathrm{H}(2 \mathrm{~A})-\mathrm{C}(2)-\mathrm{H}(2 \mathrm{~B})$ & 107.7 \\
\hline $\mathrm{C}(8)-\mathrm{C}(9)-\mathrm{H}(9 \mathrm{~A})$ & 109.5 \\
\hline $\mathrm{C}(8)-\mathrm{C}(9)-\mathrm{H}(9 \mathrm{~B})$ & 109.5 \\
\hline $\mathrm{H}(9 \mathrm{~A})-\mathrm{C}(9)-\mathrm{H}(9 \mathrm{~B})$ & 109.5 \\
\hline $\mathrm{C}(8)-\mathrm{C}(9)-\mathrm{H}(9 \mathrm{C})$ & 109.5 \\
\hline$H(9 A)-C(9)-H(9 C)$ & 109.5 \\
\hline $\mathrm{H}(9 \mathrm{~B})-\mathrm{C}(9)-\mathrm{H}(9 \mathrm{C})$ & 109.5 \\
\hline$C(8)-C(10)-H(10 A)$ & 109.5 \\
\hline $\mathrm{C}(8)-\mathrm{C}(10)-\mathrm{H}(10 \mathrm{~B})$ & 109.5 \\
\hline $\mathrm{H}(10 \mathrm{~A})-\mathrm{C}(10)-\mathrm{H}(10 \mathrm{~B})$ & 109.5 \\
\hline $\mathrm{C}(8)-\mathrm{C}(10)-\mathrm{H}(10 \mathrm{C})$ & 109.5 \\
\hline$H(10 A)-C(10)-H(10 C)$ & 109.5 \\
\hline$H(10 B)-C(10)-H(10 C)$ & 109.5 \\
\hline$C(5)-C(6)-S(1)$ & $113.36(16)$ \\
\hline$C(5)-C(6)-H(6 A)$ & 108.9 \\
\hline$S(1)-C(6)-H(6 A)$ & 108.9 \\
\hline $\mathrm{C}(5)-\mathrm{C}(6)-\mathrm{H}(6 \mathrm{~B})$ & 108.9 \\
\hline
\end{tabular}




$\begin{array}{ll}\mathrm{S}(1)-\mathrm{C}(6)-\mathrm{H}(6 \mathrm{~B}) & 108.9 \\ \mathrm{H}(6 \mathrm{~A})-\mathrm{C}(6)-\mathrm{H}(6 \mathrm{~B}) & 107.7 \\ \mathrm{C}(9)-\mathrm{C}(8)-\mathrm{C}(10) & 107.62(19) \\ \mathrm{C}(9)-\mathrm{C}(8)-\mathrm{C}(7) & 107.4(2) \\ \mathrm{C}(10)-\mathrm{C}(8)-\mathrm{C}(7) & 113.0(2) \\ \mathrm{C}(9)-\mathrm{C}(8)-\mathrm{S}(3) & 108.57(17) \\ \mathrm{C}(10)-\mathrm{C}(8)-\mathrm{S}(3) & 108.28(17) \\ \mathrm{C}(7)-\mathrm{C}(8)-\mathrm{S}(3) & 111.80(16)\end{array}$

Symmetry transformations used to generate equivalent atoms: 
Table A30. Anisotropic displacement parameters $\left(\AA^{2} \times 10^{3}\right)$ for 11 The anisotropic displacement factor exponent takes the form: $-2 \pi^{2}\left[h^{2} a^{* 2} U^{11}+\ldots+2 h k a^{*} b^{*} U^{12}\right]$

\begin{tabular}{lcccccc}
\hline & $\mathrm{U}^{11}$ & $\mathrm{U}^{22}$ & $\mathrm{U}^{33}$ & $\mathrm{U}^{23}$ & $\mathrm{U}^{13}$ & $\mathrm{U}^{12}$ \\
\hline $\mathrm{Ru}(1)$ & $8(1)$ & $11(1)$ & $9(1)$ & $-1(1)$ & $-1(1)$ & $0(1)$ \\
$\mathrm{S}(2)$ & $16(1)$ & $14(1)$ & $10(1)$ & $0(1)$ & $-1(1)$ & $-1(1)$ \\
$\mathrm{S}(3)$ & $14(1)$ & $13(1)$ & $11(1)$ & $-2(1)$ & $-1(1)$ & $-1(1)$ \\
$\mathrm{S}(1)$ & $11(1)$ & $16(1)$ & $13(1)$ & $-2(1)$ & $2(1)$ & $0(1)$ \\
$\mathrm{O}(1)$ & $12(1)$ & $21(1)$ & $21(1)$ & $-3(1)$ & $2(1)$ & $2(1)$ \\
$\mathrm{N}(2)$ & $9(1)$ & $13(1)$ & $9(1)$ & $0(1)$ & $0(1)$ & $0(1)$ \\
$\mathrm{N}(1)$ & $9(1)$ & $13(1)$ & $11(1)$ & $2(1)$ & $-1(1)$ & $0(1)$ \\
$\mathrm{C}(15)$ & $15(1)$ & $12(1)$ & $9(1)$ & $0(1)$ & $-4(1)$ & $-4(1)$ \\
$\mathrm{C}(4)$ & $12(1)$ & $16(1)$ & $8(1)$ & $-2(1)$ & $0(1)$ & $-2(1)$ \\
$\mathrm{C}(7)$ & $12(1)$ & $14(1)$ & $13(1)$ & $4(1)$ & $1(1)$ & $0(1)$ \\
$\mathrm{C}(5)$ & $9(1)$ & $15(1)$ & $14(1)$ & $-1(1)$ & $-3(1)$ & $-2(1)$ \\
$\mathrm{C}(3)$ & $12(1)$ & $16(1)$ & $9(1)$ & $1(1)$ & $-3(1)$ & $-2(1)$ \\
$\mathrm{C}(11)$ & $15(1)$ & $12(1)$ & $14(1)$ & $-3(1)$ & $0(1)$ & $0(1)$ \\
$\mathrm{C}(1)$ & $14(1)$ & $14(1)$ & $18(1)$ & $-1(1)$ & $1(1)$ & $2(1)$ \\
$\mathrm{C}(12)$ & $16(1)$ & $12(1)$ & $13(1)$ & $-1(1)$ & $1(1)$ & $-1(1)$ \\
$\mathrm{C}(14)$ & $20(1)$ & $16(1)$ & $17(1)$ & $1(1)$ & $0(1)$ & $-2(1)$ \\
$\mathrm{C}(13)$ & $22(1)$ & $15(1)$ & $19(1)$ & $0(1)$ & $-1(1)$ & $2(1)$ \\
$\mathrm{C}(2)$ & $12(1)$ & $15(1)$ & $15(1)$ & $0(1)$ & $0(1)$ & $2(1)$ \\
$\mathrm{C}(9)$ & $14(1)$ & $20(1)$ & $20(2)$ & $0(1)$ & $0(1)$ & $-3(1)$ \\
$\mathrm{C}(10)$ & $19(1)$ & $16(1)$ & $22(2)$ & $-1(1)$ & $0(1)$ & $-4(1)$ \\
$\mathrm{C}(6)$ & $11(1)$ & $18(1)$ & $17(1)$ & $0(1)$ & $2(1)$ & $-2(1)$ \\
$\mathrm{C}(8)$ & $12(1)$ & $14(1)$ & $16(1)$ & $1(1)$ & $0(1)$ & $-1(1)$ \\
& & & & & & \\
\hline
\end{tabular}


Table A31. Hydrogen coordinates ( $\left.\times 10^{4}\right)$ and isotropic displacement parameters $\left(\AA^{2} \times 10^{3}\right)$ for 11 .

\begin{tabular}{|c|c|c|c|c|}
\hline & $\mathrm{x}$ & $y$ & $z$ & $\mathrm{U}(\mathrm{eq})$ \\
\hline $\mathrm{H}(4 \mathrm{~A})$ & 8315 & 2707 & 8453 & 15 \\
\hline $\mathrm{H}(4 \mathrm{~B})$ & 9914 & 2805 & 9044 & 15 \\
\hline $\mathrm{H}(7 \mathrm{~A})$ & 6872 & 1507 & 7875 & 15 \\
\hline$H(7 B)$ & 7663 & 845 & 8081 & 15 \\
\hline $\mathrm{H}(5 \mathrm{~A})$ & 11759 & 2518 & 7788 & 15 \\
\hline $\mathrm{H}(5 \mathrm{~B})$ & 11792 & 3223 & 7275 & 15 \\
\hline$H(3 A)$ & 10535 & 1773 & 8646 & 15 \\
\hline$H(3 B)$ & 9019 & 1720 & 9196 & 15 \\
\hline $\mathrm{H}(11 \mathrm{~A})$ & 9842 & 3802 & 7419 & 16 \\
\hline $\mathrm{H}(11 \mathrm{~B})$ & 8250 & 3544 & 7225 & 16 \\
\hline $\mathrm{H}(1 \mathrm{~A})$ & 11909 & 909 & 6233 & 18 \\
\hline $\mathrm{H}(1 \mathrm{~B})$ & 11934 & 1466 & 7231 & 18 \\
\hline $\mathrm{H}(14 \mathrm{~A})$ & 10607 & 4478 & 5825 & 26 \\
\hline $\mathrm{H}(14 \mathrm{~B})$ & 10342 & 4279 & 4407 & 26 \\
\hline $\mathrm{H}(14 \mathrm{C})$ & 11286 & 3841 & 5353 & 26 \\
\hline $\mathrm{H}(13 \mathrm{~A})$ & 7946 & 4564 & 5972 & 28 \\
\hline $\mathrm{H}(13 \mathrm{~B})$ & 6923 & 3994 & 5516 & 28 \\
\hline $\mathrm{H}(13 \mathrm{C})$ & 7732 & 4390 & 4539 & 28 \\
\hline $\mathrm{H}(2 \mathrm{~A})$ & 9492 & 724 & 6374 & 17 \\
\hline $\mathrm{H}(2 \mathrm{~B})$ & 10202 & 749 & 7759 & 17 \\
\hline$H(9 A)$ & 4509 & 943 & 5575 & 28 \\
\hline $\mathrm{H}(9 \mathrm{~B})$ & 4912 & 1536 & 6441 & 28 \\
\hline $\mathrm{H}(9 \mathrm{C})$ & 4701 & 858 & 7039 & 28 \\
\hline $\mathrm{H}(10 \mathrm{~A})$ & 6395 & -9 & 6783 & 29 \\
\hline $\mathrm{H}(10 \mathrm{~B})$ & 7835 & 99 & 6133 & 29 \\
\hline $\mathrm{H}(10 \mathrm{C})$ & 6340 & 77 & 5320 & 29 \\
\hline$H(6 A)$ & 12922 & 2447 & 6018 & 18 \\
\hline $\mathrm{H}(6 \mathrm{~B})$ & 11787 & 2890 & 5260 & 18 \\
\hline
\end{tabular}


Table A32. Torsion angles [ $\left.{ }^{\circ}\right]$ for 11.

\begin{tabular}{|c|c|}
\hline $\mathrm{C}(15)-\mathrm{Ru}(1)-\mathrm{S}(2)-\mathrm{C}(12)$ & $71.77(11)$ \\
\hline $\mathrm{N}(2)-\mathrm{Ru}(1)-\mathrm{S}(2)-\mathrm{C}(12)$ & $-21.77(9)$ \\
\hline $\mathrm{N}(1)-\mathrm{Ru}(1)-\mathrm{S}(2)-\mathrm{C}(12)$ & $-57.7(2)$ \\
\hline$S(3)-R u(1)-S(2)-C(12)$ & $159.22(8)$ \\
\hline$S(1)-R u(1)-S(2)-C(12)$ & $-107.71(8)$ \\
\hline $\mathrm{C}(15)-\mathrm{Ru}(1)-\mathrm{S}(3)-\mathrm{C}(8)$ & $-69.85(11)$ \\
\hline$N(2)-R u(1)-S(3)-C(8)$ & $26.5(2)$ \\
\hline $\mathrm{N}(1)-\mathrm{Ru}(1)-\mathrm{S}(3)-\mathrm{C}(8)$ & $31.00(9)$ \\
\hline$S(2)-R u(1)-S(3)-C(8)$ & $-157.62(8)$ \\
\hline$S(1)-R u(1)-S(3)-C(8)$ & $113.57(8)$ \\
\hline$N(2)-R u(1)-S(1)-C(6)$ & $-19.90(10)$ \\
\hline$N(1)-R u(1)-S(1)-C(6)$ & $-105.30(10)$ \\
\hline$S(2)-R u(1)-S(1)-C(6)$ & $63.72(9)$ \\
\hline$S(3)-R u(1)-S(1)-C(6)$ & $173.91(9)$ \\
\hline $\mathrm{N}(2)-\mathrm{Ru}(1)-\mathrm{S}(1)-\mathrm{C}(1)$ & $82.93(10)$ \\
\hline$N(1)-R u(1)-S(1)-C(1)$ & $-2.48(10)$ \\
\hline$S(2)-R u(1)-S(1)-C(1)$ & $166.55(8)$ \\
\hline $\mathrm{S}(3)-\mathrm{Ru}(1)-\mathrm{S}(1)-\mathrm{C}(1)$ & $-83.26(8)$ \\
\hline$C(15)-R u(1)-N(2)-C(11)$ & $-48.33(16)$ \\
\hline $\mathrm{N}(1)-\mathrm{Ru}(1)-\mathrm{N}(2)-\mathrm{C}(11)$ & $-148.52(15)$ \\
\hline $\mathrm{S}(2)-\mathrm{Ru}(1)-\mathrm{N}(2)-\mathrm{C}(11)$ & $39.84(14)$ \\
\hline$S(3)-R u(1)-N(2)-C(11)$ & $-144.07(19)$ \\
\hline$S(1)-R u(1)-N(2)-C(11)$ & $127.91(14)$ \\
\hline $\mathrm{C}(15)-\mathrm{Ru}(1)-\mathrm{N}(2)-\mathrm{C}(4)$ & $67.14(15)$ \\
\hline $\mathrm{N}(1)-\mathrm{Ru}(1)-\mathrm{N}(2)-\mathrm{C}(4)$ & $-33.05(13)$ \\
\hline$S(2)-R u(1)-N(2)-C(4)$ & $155.32(13)$ \\
\hline $\mathrm{S}(3)-\mathrm{Ru}(1)-\mathrm{N}(2)-\mathrm{C}(4)$ & $-28.6(3)$ \\
\hline $\mathrm{S}(1)-\mathrm{Ru}(1)-\mathrm{N}(2)-\mathrm{C}(4)$ & $-116.62(13)$ \\
\hline $\mathrm{C}(15)-\mathrm{Ru}(1)-\mathrm{N}(2)-\mathrm{C}(5)$ & $-175.23(16)$ \\
\hline $\mathrm{N}(1)-\mathrm{Ru}(1)-\mathrm{N}(2)-\mathrm{C}(5)$ & $84.58(15)$ \\
\hline $\mathrm{S}(2)-\mathrm{Ru}(1)-\mathrm{N}(2)-\mathrm{C}(5)$ & $-87.06(15)$ \\
\hline$S(3)-R u(1)-N(2)-C(5)$ & $89.0(3)$ \\
\hline$S(1)-R u(1)-N(2)-C(5)$ & $1.01(14)$ \\
\hline$C(15)-R u(1)-N(1)-C(2)$ & $158.65(16)$ \\
\hline $\mathrm{N}(2)-\mathrm{Ru}(1)-\mathrm{N}(1)-\mathrm{C}(2)$ & $-108.86(15)$ \\
\hline $\mathrm{S}(2)-\mathrm{Ru}(1)-\mathrm{N}(1)-\mathrm{C}(2)$ & $-73.0(3)$ \\
\hline$S(3)-R u(1)-N(1)-C(2)$ & $72.21(14)$ \\
\hline$S(1)-R u(1)-N(1)-C(2)$ & $-22.61(14)$ \\
\hline$C(15)-R u(1)-N(1)-C(7)$ & $39.17(16)$ \\
\hline $\mathrm{N}(2)-\mathrm{Ru}(1)-\mathrm{N}(1)-\mathrm{C}(7)$ & $131.66(15)$ \\
\hline $\mathrm{S}(2)-\mathrm{Ru}(1)-\mathrm{N}(1)-\mathrm{C}(7)$ & $167.50(16)$ \\
\hline$S(3)-R u(1)-N(1)-C(7)$ & $-47.26(13)$ \\
\hline $\mathrm{S}(1)-\mathrm{Ru}(1)-\mathrm{N}(1)-\mathrm{C}(7)$ & $-142.08(14)$ \\
\hline $\mathrm{C}(15)-\mathrm{Ru}(1)-\mathrm{N}(1)-\mathrm{C}(3)$ & $-81.27(15)$ \\
\hline $\mathrm{N}(2)-\mathrm{Ru}(1)-\mathrm{N}(1)-\mathrm{C}(3)$ & $11.22(14)$ \\
\hline $\mathrm{S}(2)-\mathrm{Ru}(1)-\mathrm{N}(1)-\mathrm{C}(3)$ & $47.1(3)$ \\
\hline$S(3)-R u(1)-N(1)-C(3)$ & $-167.71(14)$ \\
\hline$S(1)-R u(1)-N(1)-C(3)$ & $97.47(13)$ \\
\hline $\mathrm{N}(1)-\mathrm{Ru}(1)-\mathrm{C}(15)-\mathrm{O}(1)$ & $178(100)$ \\
\hline$C(11)-N(2)-C(4)-C(3)$ & $165.77(18)$ \\
\hline C(5)-N(2)-C(4)-C(3) & $-71.2(2)$ \\
\hline$R u(1)-N(2)-C(4)-C(3)$ & $50.63(19)$ \\
\hline $\mathrm{C}(2)-\mathrm{N}(1)-\mathrm{C}(7)-\mathrm{C}(8)$ & $-71.2(2)$ \\
\hline$C(3)-N(1)-C(7)-C(8)$ & $168.72(18)$ \\
\hline
\end{tabular}




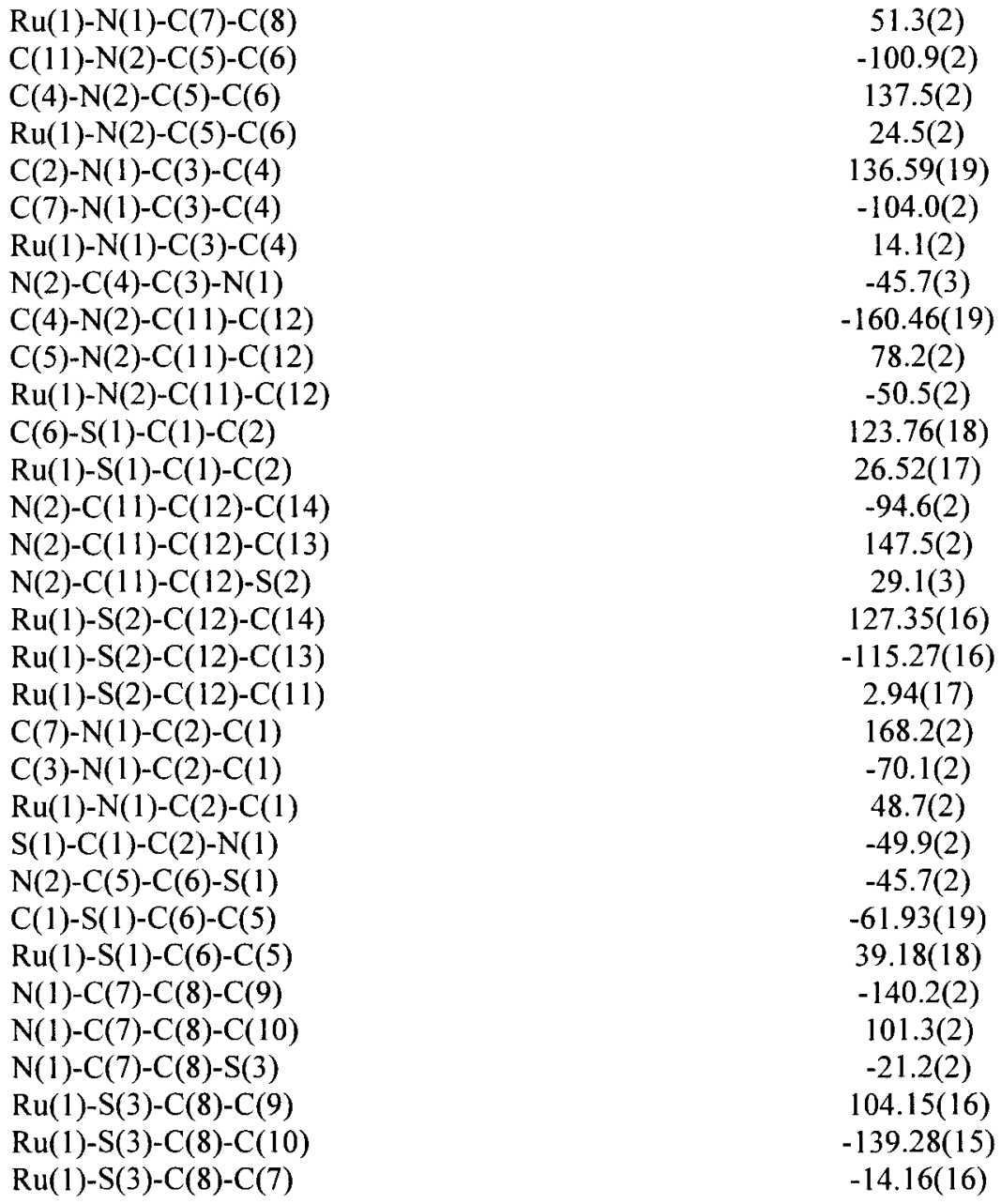

Symmetry transformations used to generate equivalent atoms: 


\section{CURRICULUM VITAE}

Mr. César Antonio, Masitas Castillo.

1800 South 2nd St. Apt 44 A, Louisville KY, Zip 40208, USA.

+1502-759-9369 camasi01@louisville.edu; cesar_masitas@yahoo.com.ar

\section{Education:}

University Degree:

Ph.D. Chemistry 2012 UNIVERSITY OF LOUISVILLE, (UofL) KY, USA.

- Dissertation title: Sulfur oxygenation of ruthenium-dithiolate nitrile hydratase mimic enhances ligand lability and hydrolysis activity.

- Mentor: Ph.D. Craig A. Grapperhaus.

- Graduate Dean's Citation. University of Louisville Spring 2012

M.S. Chemistry 2010 UNIVERSITY OF LOUISVILLE, (UofL) KY, USA.

B.S. Chemistry 2000 UNIVERSIDAD PERUANA CAYETANO HEREDIA, (UPCH) LIMA PERU.

- Licenciatura dissertation title: In vitro antimicrobial activity of 15 Peruvian plants and phytochemical study of Gentianella weberbaueri (Gilg) Fabris.

- Mentor: M.D. Ph.D. Rosario Rojas

Course:

2005 CRESTCOM INTERNATIONAL, Ltd.

Course and diploma in Bullet Proof $\mathbb{R}$ Manager.

Where I:

- Improved leadership strategies in personal and working life.

Professional experience.

In Academia: Courses taught as Teaching Assistant.

Course title

Organic Chemistry Lab.

Physical Chemistry Lab.

Organic Chemistry Lab.
Semester taught

Spring 1999- Fall 2000

Spring 1999- Fall 2000

Fall 2006 and spring 2006

Fall 2007 and summer 2011
University.

UPCH

UPCH

UofL

UofL 
In Industry:

11/2001-07/2006 CASTILLO GALERIA DE ARTE INTERNACIONAL (ART

GALLERY)

Co-Manager and logistic coordinator. (Family business)

Where:

- Organized, operated and administered the logistic of the company.

- Directed the customer service of the company.

Accomplishments: Set up and run an Art Gallery business.

06/2004-02/2005 ALS LABORATORY GROUP, LIMA PERU.

Laboratory of mineral and environmental assays for mining. Head of supply chain.

Where I:

- Coordinated and organized company supply chain.

- Reduced chemical consumable expenses with new vendors and the improvement in the logistic of the company.

Accomplishments: Design, organize and implement a warehouse for mineral sample storage. Warehouse area 6500 square feet.

12/2003-03/2004 JICA. (JAPAN INTERNATIONAL COOPERATION AGENCY). External consultant.

Where I:

- Performed a study about "Drinking water quality in Peru" and "Drinking water characteristics in Peru" in coordination with "SUNASS". The National Sanitation Service Superintendence of Peru.

- Developed, organized and implemented a quality system in the laboratory of "SUNASS".

Accomplishments: One book publication as collaborators, Sunass is owner of the manuscript rights.

\section{2/2002-09/2003 CORPORACION JOSE R. LINDLEY S.A. (CORPORATION}

INCA KOLA).

Coke, Fanta and Sprite are bottled for this company.

Co-op in quality control assurance.

Where:

- Performed and coordinated physical and chemical assays of quality control, in the pet bottle production line.

Accomplishments: Reduction of time in physical determinations report. 3h/day of saved time in Quality Assurance process. 
08/2001 - 10/2001 MOLITALIA S.A.

Food industry. Noodles and flour are produced for this company.

Co-op as Quality control supervisor.

Where:

- Inspected and performed quality controls in different stages of the production in the company in coordination with the production division.

\section{4/2001 - 07/2001 REFINERIA CAJAMARQUILLA S.A. (VOTORANTIN}

METALS).

Mining Industry. Refining zinc company.

Co-op. In assurance quality control.

Where:

- Performed analytic chemistry analysis in mineral and environmental samples.

- Analyzed and coordinated routine atomic absorption (AA), ICP and electrochemistry analysis.

Accomplishments: Acquire technical expertise in spectroscopic instrumentation and performed a professional position as analyst for two months due to regular personal went on strike.

01/1998-12/1998 QUALITY CONTROL LABORATORY. (UPCH).

Co-op in quality control of pharmaceutical products.

Where:

- Performed Chemical and Physical-Chemical Testing. (e.g. HPLC, GC, UV/VIS, IR).

- Performed the identification of active ingredients and impurities.

- Performed Physical and physical-chemical determinations (e.g. pH, viscosity, melting point, osmolality and osmolarity).

- Residual solvents (volatile organic compounds [VOC], organic volatile impurities [OVI]).

- Pharmaceutical technical procedures (e.g. disintegration, dissolution, hardness, friability).

01/1995 - 12/1997 MASITAS ING.

Design and construction of metallic structures.

I was a field supervisor, sale representative and supply chain administrator.

- Organized, operated and administered the logistic of the company.

- Trained in the construction of metallic structure and use of construction equipment. Welding experience of 3 years.

- Directed the customer service of the company.

Accomplishments: Set up and run a metallic structure company, get strong experience dealing with vendors and clients. 
Skills and training:

Workshops:

- Chemistry of Metals in Biological Systems. 15-22 May 2011, Université Catholique de Louvain, Louvain-la-Neuve, Belgium. Organized by Robert R Crichton.

- Principles of EPR Training Workshop, August 19-21, 2009, National Biomedical EPR Center, Medical College of Wisconsin, USA.

Skills

- Performed physic-chemistry determinations during my Ph.D. studies in Nitrile hydratase mimics. I am capable to analyze data, formulate hypothesis and elaborate scientific literature.

- I worked all my doctoral degree in air free synthesis and spectroscopic characterization, using physical and spectroscopic technique to analyze air sensitive compound. Daily use Schlenk line- flask and dry box. Between the techniques that I used on my research I can mention, X-ray, UV-visible, IR, ESIMS, Electrochemistry, NMR-(use of J-young tubes), GC-MS, EPR and analysis of DFT calculations.

- I can work, organize, coordinate and cooperate in interdisciplinary research in academia and industry.

- I define myself as a synthetic, analytical scientist; I am capable to synthetize complex of 12-15 steps and characterize them. In addition of perform Kinetic evaluation by UV-VIS, NMR and GC-MS as it was the case of the nitrile hydratase analogue that catalyzes the hydrolysis of nitrile to amide.

- Trained in transition metal organic synthesis with emphasis in metal, aminethiolates. Metal include ruthenium, nickel and iron.

- I have experience analytical chemistry in technical and administrative position in the food and mining industry, and in an international agency. These experiences allow me to work with independence if it is required.

- I strive for continued excellence and social work. I provided exceptional contributions to student organizations as member and Vice-president in the University of Louisville. I am Member of Golden Key and Omicron Delta Kappa Honor Societies.

- Excellent communication and networking abilities.

- I balance an effective work time with sport activities, social relations and family.

- I lead and develop research projects. I implemented improvements on the research labs (from time-cost reduction to technical protocols). I had experience in solve instrumental problems from software to hardware malfunction laboratory instruments.

- I had industrial experience with internal and external customers as head of supply chain.

- I speak English and Spanish fluently. 


\section{Papers:}

Influence of Sequential Thiolate Oxygenation on a Nitrile Hydratase Mimic Probed by Multi-edge X-ray Absorption Spectroscopy.

Shearer, J.; Callan, Paige E.; Masitas C. A.; Grapperhaus, C. A. Inorg. Chem. 2012 (submitted)

Controlled Sulfur Oxygenation of the Ruthenium Dithiolate (bmmp-TASN)RuPPh 3 under Limiting $\mathrm{O}_{2}$ Conditions Yields Thiolato/Sulfinato, Sulfenato/Sulfinato, and Bis-Sulfinato Derivatives

Masitas, C. A.; Kumar, M.; Mashuta, M. S.; Kozlowski, P. M.; Grapperhaus, C. A. Inorg. Chem. 2010, 49, 10875-10881.

Asymmetric Oxygenation of a Ruthenium Dithiolate Mimics the Mixed Sulfenato/Sulfinato Donor Sets of Nitrile Hydratase and Thiocyanate Hydrolase Masitas, C. A.; Mashuta, M. S.; Grapperhaus, C. A. Inorg. Chem. 2010, 49, 5344-5346.

\section{Conference and poster Presentation:}

- International Symposium on Molecular Coordination Chemistry at the Max Planck Institute for Bioinorganic chemistry. Nov. 2010. Mulheim and der Ruhr, Germany (Poster), the meeting was developed to honor the retirement of Prof. Karl Wieghardt.

- Gordon Research Seminars. Feb 2010 - January 2012. Ventura-California, USA (Poster-Presentation).

- Annual National Conference of the American Chemistry Society. Fall 2009. Washington DC, USA (Poster).

\section{Awards and Honors:}

- Golden Key (International Honor Society) member.

- Omicron Delta Kappa (National Leadership Society) member.

- Graduate Dean's Citation. University of Louisville Spring 2012

Languages: Spanish and English. Basic French.

Sports: Tennis, soccer, field hockey and polo.

\section{Organization (position):}

- The University of Louisville Polo Club (member); Sport organization.

- American Chemistry Society (member); Professional organization.

- $\mathrm{C}^{3}$ Cardinals celebrating cultures (Vice-president) first Hispanic recognized organization in UofL. 